\title{
BOUNDARY-LAYER RECEPTIVITY TO THREE-DIMENSIONAL ROUGHNESS ARRAYS ON A SWEPT-WING
}

\author{
A Dissertation \\ by \\ LAUREN ELIZABETH HUNT \\ Submitted to the Office of Graduate Studies of \\ Texas A\&M University \\ in partial fulfillment of the requirements for the degree of \\ DOCTOR OF PHILOSOPHY
}

December 2011

Major Subject: Aerospace Engineering 


\title{
BOUNDARY-LAYER RECEPTIVITY TO THREE-DIMENSIONAL ROUGHNESS ARRAYS ON A SWEPT-WING
}

\author{
A Dissertation \\ by \\ LAUREN ELIZABETH HUNT
}

\author{
Submitted to the Office of Graduate Studies of \\ Texas A\&M University \\ in partial fulfillment of the requirements for the degree of \\ DOCTOR OF PHILOSOPHY
}

\begin{abstract}
Approved by:
Chair of Committee, William Saric

Committee Members, Rodney Bowersox

JN Reddy

Helen Reed

Head of Department, Dimitris Lagoudas
\end{abstract}

December 2011

Major Subject: Aerospace Engineering 


\begin{abstract}
Boundary-Layer Receptivity to Three-Dimensional Roughness Arrays on a Swept-Wing. (December 2011)

Lauren Elizabeth Hunt, B.S., Arizona State University Chair of Advisory Committee: Dr. William Saric
\end{abstract}

On-going efforts to reduce aircraft drag through transition delay focus on understanding the process of boundary-layer transition from a physics-based perspective. For swept-wings subject to transition dominated by a stationary crossflow instability, one of the remaining challenges is understanding how freestream disturbances and surface features such as surface roughness create the initial amplitudes for unstable waves. These waves grow, modify the mean flow and create conditions for secondary instabilities to occur, which in turn ultimately lead to transition. Computational methods that model the primary and secondary instability growth can accurately model disturbance evolution as long as appropriate initial conditions are supplied. Additionally, transition delay using discrete roughness arrays that exploit known sensitivities to surface roughness has been demonstrated in flight and wind tunnel testing; however, inconsistencies in performance from the two test platforms indicate further testing is required. This study uses detailed hotwire boundary-layer velocity scans to quantify the relationship between roughness height and initial disturbance amplitude. Naphthalene 
flow visualization provides insight into how transition changes as a result of roughness height and spacing.

Micron-sized, circular roughness elements were applied near the leading edge of the ASU(67)-0315 model installed at an angle of attack of $-2.9^{\circ}$ in the Klebanoff-Saric Wind Tunnel. Extensive flow quality measurements show turbulence intensities less than $0.02 \%$ over the speed range of interest. A survey of multiple roughness heights for the most unstable and control wavelengths and Reynolds numbers of $2.4 \times 10^{6}, 2.8 \times 10^{6}$ and $3.2 \times 10^{6}$ was completed for chord locations of $10 \%, 15 \%$ and $20 \%$. When care was taken to measure in the region of linear stability, it was found that the disturbance amplitude varies almost linearly with roughness height. Naphthalene flow visualization indicates that moderate changes in already-low freestream turbulence levels can have a significant impact on transition behavior. 


\section{ACKNOWLEDGEMENTS}

First and foremost, I should like to thank my committee chair and advisor Dr. William Saric. Through him, I have had the opportunity to participate in several research efforts and interact with a wide-ranging spectrum of people and organizations, all of which have served to broaden my experience and understanding of the research process. I greatly appreciate his willingness to continually engage in my educational growth through extensive conversations and providing opportunities to take on grand tasks. Contributing to the reconstruction of the Klebanoff-Saric Wind Tunnel was an experience I shall not soon forget.

I would also like to thank my other committee members. In many ways, I would not be where I am today without first having worked for Dr. Helen Reed as an undergraduate. Your encouragement then and your support now have been invaluable. Special thanks are given to you and graduate student Matthew Tufts who provided computational support for this project. I also appreciate the support of Dr. Rodney Bowersox and Dr. JN Reddy throughout my research project. My thanks to Dr. Edward White, the unofficial committee member, who was another source of assistance during both tunnel construction and my research.

Rebuilding the Klebanoff-Saric Wind Tunnel was in every respect a team effort and I am indebted to all of the individuals who participated in the reconstruction effort. Over 21 students and 15 university faculty and staff members contributed to some aspect of the wind tunnel. In particular, Cecil Rhodes, Robert Downs, Matthew Kuester and Brian 
Crawford were instrumental to the project. Thank you for all your efforts. My thanks to the Oran W. Nicks Low Speed Wind Tunnel Staff who participated in tunnel construction and now continue to support facility needs.

Beyond the people directly involved with my research project, I also wish to thank fellow students Jerrod Hofferth, Alex Craig and David West, and former students Andrew Carpenter and Shane Schouten. Your technical support and friendship are appreciated. I should also like to thank Colleen Leatherman, who always makes sure operations run smoothly.

I gratefully acknowledge the following generous support: AFOSR Grant FA955005-1-0299 and Dr. Thomas Beutner for tunnel reconstruction, AFOSR Grants FA955008-1-0093 and FA9550-05-1-0044, and Dr. John Schmisseur for research support, AFRL / AFOSR Grant FA9550-09-1-0035 and Mr. Gary Dale, and NASA with Dr. Meelan Choudhari for receptivity research support. My sincere thanks for the support of the NASA Aeronautics Scholarship Program. 


\section{NOMENCLATURE}

\begin{tabular}{ll}
$A, B, n$ & Curve-fit constants \\
$A_{0}$ & Initial disturbance amplitude \\
$c$ & Streamwise chord \\
$C_{p}$ & Pressure coefficient \\
$C_{T}$ & Temperature-compensation coefficient \\
$d$ & Roughness element diameter \\
$E$ & Voltage \\
$E_{c o m p}$ & Temperature-compensated voltage \\
$f$ & Frequency \\
$L / D$ & Ratio of contraction length to diameter \\
$k$ & Roughness element height \\
$N$ & N-factor \\
$p$ & Static pressure at pressure port \\
$p_{\infty}$ & Freestream static pressure \\
$Q_{\infty}$ & Freestream velocity normal to leading edge \\
$R e_{c}$ & Chord-Reynolds number \\
$R e_{k}$ & Roughness Reynolds number based on $k$ \\
$T_{a}$ & Ambient temperature \\
$T_{w}$ & Freestream turbulence intensity \\
\hline &
\end{tabular}


Mean streamwise velocity

Mean streamwise boundary-layer velocity

Mean streamwise freestream velocity

Velocity fluctuations in global $(X, Y, Z)$ coordinates

Fraction of streamwise chord

Model-oriented coordinates: $x$ is normal to the leading edge, $y$ is normal to the chord line, $z$ is spanwise (parallel to leading edge)

Boundary-layer-oriented coordinates: $x_{t}$ is tangent to the inviscid streamline, $y_{t}$ is normal to model surface, $z_{t}$ is normal to the streamline

Global test section coordinates: $X$ is streamwise, $Y$ is normal to side-wall, $Z$ is spanwise (ceiling to floor)

Model angle of attack

Streamwise wavenumber, tangential to swept-wing streamline

Spanwise wavenumber, normal to streamline

Sweep angle

Roughness element spacing

Kinematic viscosity

Density

Non-dimensional frequency 


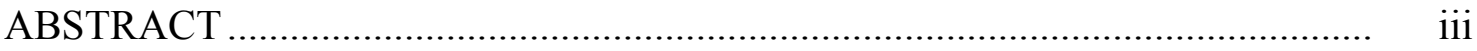

ACKNOWLEDGEMENTS ....................................................................

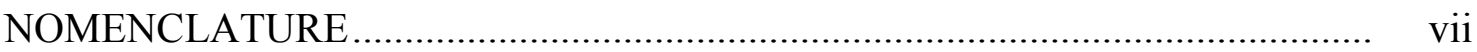

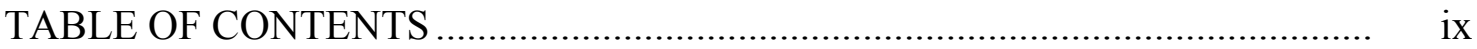

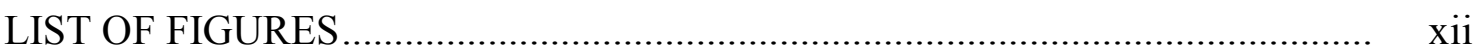

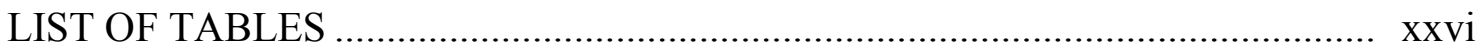

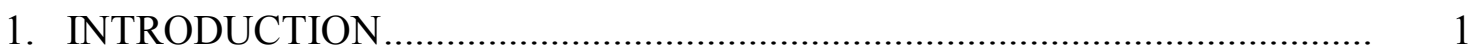

$1.1 \quad$ Background and Motivation ................................................................ 1

1.2 Swept-Wing Stability and Transition .................................................. 3

1.2.1 Crossflow Instability- Origins and Development ............................ 4

1.2.2 Transition Prediction and Disturbance Growth Models for Crossflow ................................................................................ 9

1.2.3 Laminar Flow Control for Crossflow ……………........................ 12

1.3 Receptivity Literature Review........................................................... 15

1.3.1 Receptivity Overview ............................................................ 15

1.3.2 Crossflow Receptivity Studies ................................................... 18

1.3.3 On-Going Crossflow Receptivity Work ………………………....... 25

1.4 Experimental Objectives ................................................................. 29

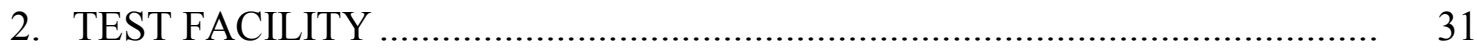

2.1 Klebanoff-Saric Wind Tunnel ............................................................ 32

2.1.1 Tunnel History …………………............................................... 32

2.1.2 KSWT Building Facility ............................................................. 36

2.1.3 Wind Tunnel .............................................................................. 37

2.1.4 Differences Between ASU and TAMU Configurations .................. 49

2.2 Computer Systems and Instrumentation ............................................. 51

2.2.1 Traverse and Sting Mount................................................................ 56 
3. EXPERIMENTAL CONFIGURATON AND DESIGN

3.1 Fundamental Requirements for Stability and Transition

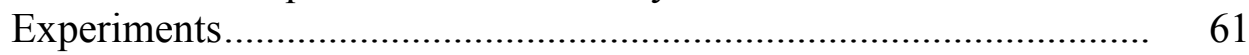

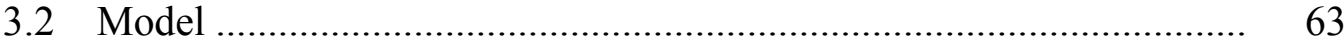

3.2.1 History and Design ................................................................... 63

3.2.2 Mounting and Tunnel Placement .................................................. 68

3.2.3 A Note on Angle-of-Attack Selection ......................................... $\quad 70$

3.2.4 Wall Liners ............................................................................ 71

3.3 Roughness Arrays …………………………………………....... 73

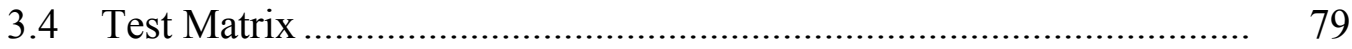

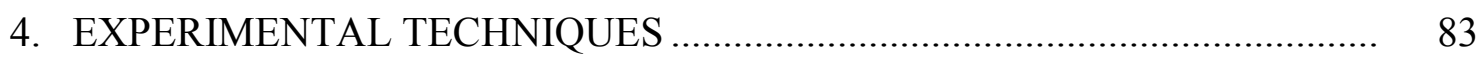

4.1 Hotwire Anemometry ...................................................................... 83

4.1.1 Hotwire Calibration ..................................................................... 84

4.1.2 Coordinate Systems..................................................................... $\quad 90$

4.1.3 Boundary-Layer Velocity Profiles................................................... 92

4.1.4 Boundary-Layer Velocity Scans .................................................... 93

4.2 Naphthalene Flow Visualization ......................................................... 94

5. RESULTS- PART I: BASELINE MEASUREMENTS....................................... 97

$5.1 \quad$ Results Overview ........................................................................... 97

5.2 KSWT Freestream Disturbance Environment..................................... $\quad 98$

5.2.1 Measurement Configuration .......................................................... 100

5.2.2 Freestream Turbulence Levels ................................................. 103

5.2.3 Comparison of ASU and TAMU Disturbance Intensities .............. 117

5.2.4 Turbulence Levels with Model and Wall Liners Installed .............. 118

5.3 Pressure Distribution ....................................................................... 123

5.4 Baseline Naphthalene Flow Visualization ........................................ 128

6. RESULTS- PART II: RECEPTIVITY OF DRE ARRAY SPACED AT THE CRITICAL WAVELENGTH................................................................. 132

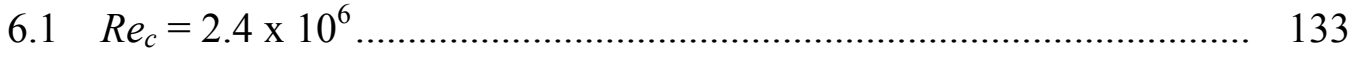

6.1.1 Naphthalene Flow Visualization.................................................. 133

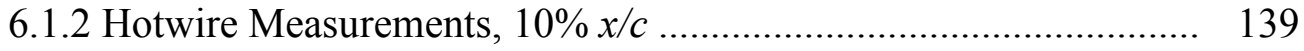

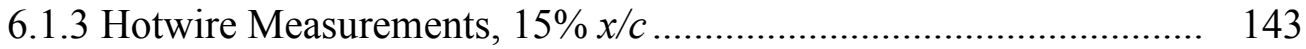

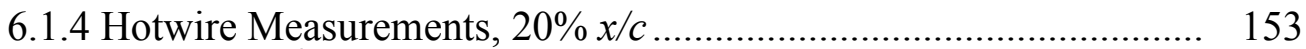

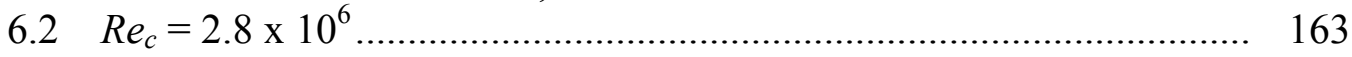

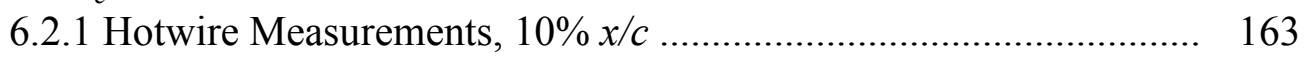


6.2.2 Hotwire Measurements, 15\% x/c ............................................. 167

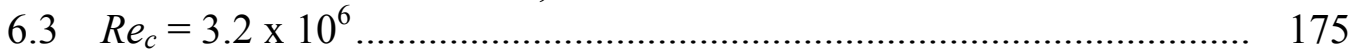

6.3.1 Naphthalene Flow Visualization............................................. 175

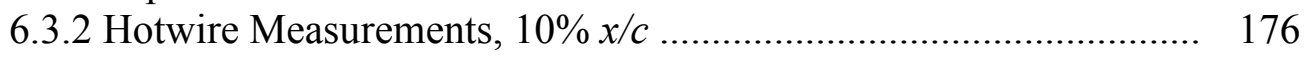

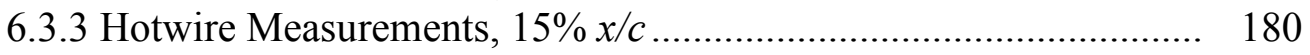

7. RESULTS- PART III: RECEPTIVITY OF DRE ARRAY SPACED AT THE CONTROL WAVELENGTH ......................................................... 189

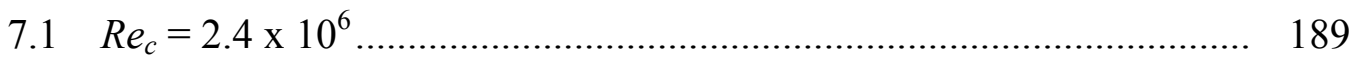

7.1.1 Naphthalene Flow Visualization................................................ 189

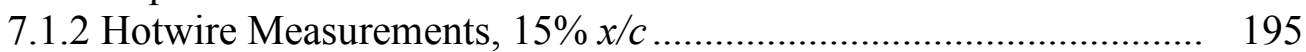

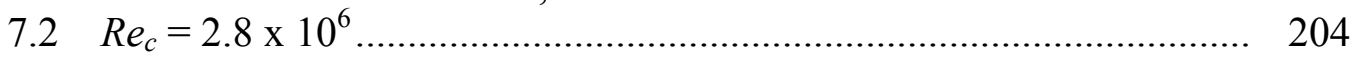

7.2.1 Naphthalene Flow Visualization ............................................... 204

7.2.2 Hotwire Measurements, 15\% x/c .............................................. 206

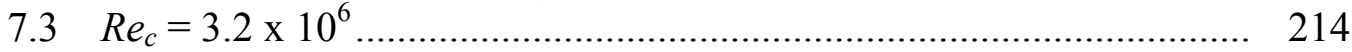

7.3.1 Naphthalene Flow Visualization................................................ 214

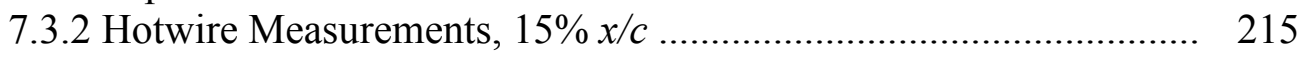

8. RESULTS- PART IV: EFFECT OF INCREASING FREESTREAM TURBULENCE ............................................................................. 221

8.1 Turbulence Generation .............................................................. 222

8.2 Naphthalene Flow Visualization ................................................ 226

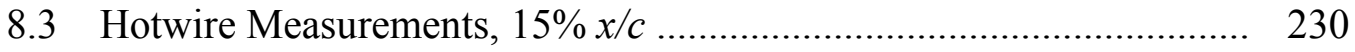

9. DISCUSSION OF RESULTS ............................................................. 237

9.1 Initial Disturbance Amplitudes ................................................ 237

9.2 Environmental Influence ....................................................... 243

10. SUMMARY AND CONCLUSIONS .................................................... 246

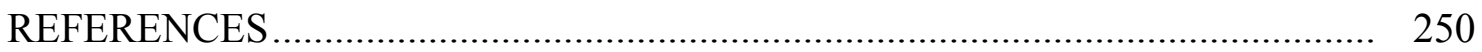

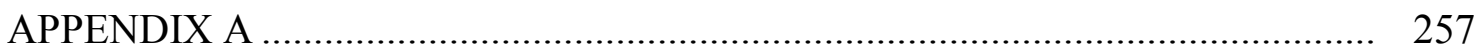

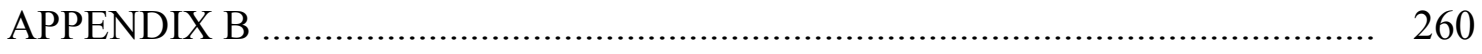

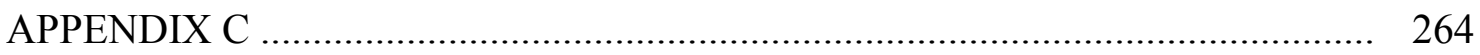

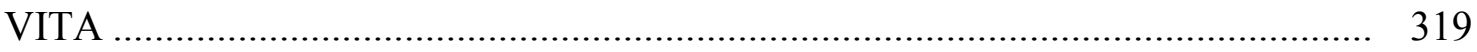




\section{LIST OF FIGURES}

FIGURE Page

1-1 Streamlines on a swept-wing ............................................................ 5

1-2 Boundary-layer velocity profiles on a swept-wing (White 2000)............. 6

1-3 Mean flow distortion from stationary crossflow vortices (left), schematic of crossflow vortices (right); figure adapted from Reibert (1996) ............ 8

1-4 Disturbance amplitudes for Orr-Sommerfeld, LPSE, NPSE (Haynes \& Reed 2000), and experiments (Reibert et al. 1996). Figure courtesy of Saric et al. (2003)

1-5 Simplified transition roadmap (Saric et al. 2002) ................................ 16

2-1 Perspective view of the UWT ........................................................ 33

2-2 Plan (top) and elevation (bottom) views of the UWT ........................... 34

2-3 Perspective view of the KSWT .................................................... 38

2-4 Plan view of the KSWT. Flow moves counter-clockwise....................... 38

2-5 Test section assembly .................................................................. 39

2-6 Upstream (left) and downstream (right) views of fan housing ................ 42

2-7 Motor and fan assembly (note: motor enclosure and nacelle not pictured) 43

2-8 Acoustic panel layout; side-walls and floor (top), ceiling (bottom) ......... 45

2-9 Example of butt-welded seam on first three screens ............................... 48

2-10 Hardware diagrams for tunnel hardware (top) and H\&S system (bottom) 54

2-11 Front view of traverse carriage. Dimensions in $\mathrm{mm}$ (White 2000).......... 57

2-12 Side view of traverse carriage. Dimensions in $\mathrm{mm}$ (White 2000) ............ 58

2-13 Plan view of sting mount. Dimensions in mm (White 2000).................. 60 
FIGURE Page

3-1 ASU(67)-0315 components........................................................... 65

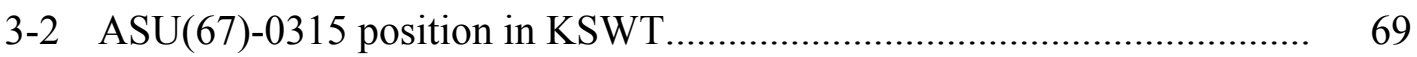

3-3 ASU(67)-0315 support brackets: top (left), bottom (right)................... 70

3-4 ASU(67)-0315 wall liner design for $\alpha=-3^{\circ}$ (contraction fairings not pictured) ............................................................... 72

3-5 ASU(67)-0315 as installed with wall liners and fairings ....................... 73

3-6 Example of appliqué DRE array applied on model............................. 75

3-7 Magnified top view of roughness elements: (a) single layer, (b) 2-layers, (c) 3 layers, (d) 4-layers. Contrast images (b) - (d) are set to show height.

3-8 Side view of roughness elements: (a) single layer, (b) 2-layers, (c) 3-layers, (d) 4-layers

4-1 Example temperature compensation

4-2 Example hotwire calibration

5-1 Freestream turbulence hotwire measurement locations 100

5-2 Crosswire mounted to side wall using aluminum stand.

5-3 Power spectral density of $u^{\prime}, v^{\prime}$ and $w^{\prime}$ at (a) $10 \mathrm{~m} / \mathrm{s}$, (b) $15 \mathrm{~m} / \mathrm{s}$, (c) $20 \mathrm{~m} / \mathrm{s}$ and (d) $25 \mathrm{~m} / \mathrm{s}$

5-4 Power spectral density of $\mathrm{u}^{\prime}$ at $20 \mathrm{~m} / \mathrm{s}$ before (15 Feb) and after (28 May) installation of turbulence manipulators

5-5 Correlation of two $\mathrm{u}^{\prime}$ signals (a) and two $\mathrm{w}^{\prime}$ signals (b) in Plane A, separated by a distance of $1.28 \mathrm{~m}$ in the $(Y, Z)$ plane at $10 \mathrm{~m} / \mathrm{s}$.

5-6 Correlation of two $\mathrm{u}^{\prime}$ signals (a) and two w' signals (b) in Plane A, separated by a distance of $1.28 \mathrm{~m}$ in the $(Y, Z)$ plane at $20 \mathrm{~m} / \mathrm{s}$.

5-7 Power spectral densities of two $u^{\prime}$ signals and their correlated and uncorrelated components at $20 \mathrm{~m} / \mathrm{s}$. 
FIGURE Page

5-8 Crosswire mounting on traverse sting........................................... 120

5-9 Spectra from crosswire measurements at $(0.257,0.826,0.051)$ for

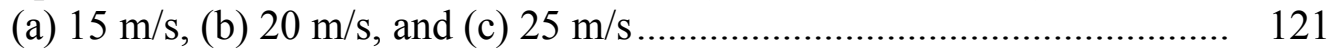

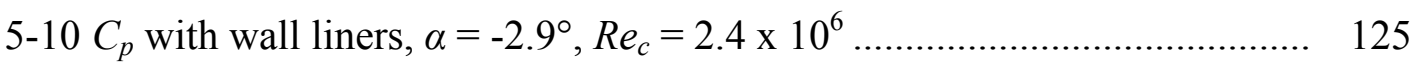

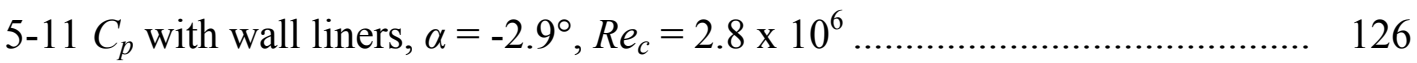

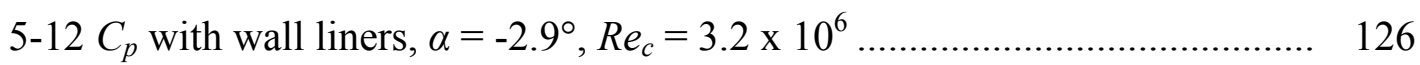

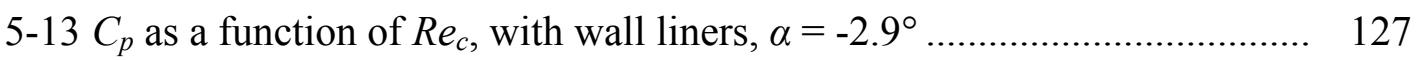

5-14 Comparison of ASU(67)-0315 at $\alpha=-2.9^{\circ}$ (TAMU) and

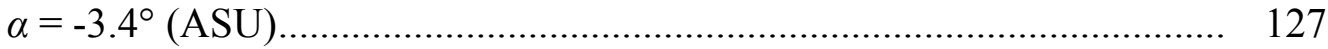

5-15 Naphthalene flow visualization of natural transition at TAMU for

$R e_{c}=2.6 \times 10^{6}, \alpha=-2.9^{\circ}$; transition location from ASU at

$R e_{c}=2.4 \times 10^{6}, \alpha=-3.4^{\circ}$, highlighted in red

5-16 Naphthalene flow visualization of natural transition at TAMU for $R e_{c}=2.6 \times 10^{6}, \alpha=-2.9^{\circ}$; wall-liners installed.....

5-17 Naphthalene flow visualization of natural transition at TAMU for $R e_{c}=2.8 \times 10^{6}, \alpha=-2.9^{\circ}$; wall-liners installed

5-18 Naphthalene flow visualization of natural transition at TAMU for $R e_{c}=3.2 \times 10^{6}, \alpha=-2.9^{\circ}$; wall-liners not installed.

6-1 NFV with appliqué roughness: $x / c=0.029, d=3 \mathrm{~mm}, \lambda=12 \mathrm{~mm}$ $R e_{c}=2.4 \times 10^{6}$; (a) $k=12 \mu \mathrm{m}$, (b) $k=24 \mu \mathrm{m}$, (c) $k=36 \mu \mathrm{m}$, (d) $k=60 \mu \mathrm{m}$

6-2 NFV of transition at ASU for $R e_{c}=2.8 \times 10^{6}$, Roughness: $x / c=0.029$, $d=3 \mathrm{~mm}, k=6 \mu \mathrm{m}, \lambda=12 \mathrm{~mm}$ (Gladden 2001)

6-3 NFV with appliqué roughness: $x / c=0.029, d=3 \mathrm{~mm}, \lambda=12 \mathrm{~mm}$ $R e_{c}=2.4 \times 10^{6}$; (a) $k=12 \mu \mathrm{m}$, (b) $k=24 \mu \mathrm{m}$, (c) $k=36 \mu \mathrm{m}$, (d) $k=47 \mu \mathrm{m}$ 
6-4 Spanwise array of 65 mean-flow boundary-layer profiles spanning $64 \mathrm{~mm}$ at $10 \% x / c . R e_{c}=2.4 \times 10^{6},[47 \mid 12]$ roughness. Mean profile shown in red.....

6-5 Spanwise array of 65 disturbance profiles spanning $64 \mathrm{~mm}$ at $10 \% x / c . R e_{c}=2.4 \times 10^{6},[47 \mid 12]$ roughness

6-6 Streamwise velocity contours at $10 \% x / c . R e_{c}=2.4 \times 10^{6}$, [47|12] roughness

6-7 Stationary crossflow mode shape at $10 \% x / c . R e_{c}=2.4 \times 10^{6}$, [47|12] roughness

6-8 Power spectral density of spanwise hotwire scan at $10 \% x / c$. $R e_{c}=2.4 \times 10^{6},[47 \mid 12]$ roughness.

6-9 Spanwise array of 65 mean-flow boundary-layer profiles spanning $64 \mathrm{~mm}$ at $15 \% x / c . R e_{c}=2.4 \times 10^{6},[12 \mid 12]$ roughness. Mean profile shown in red.....

6-10 Spanwise array of 65 mean-flow boundary-layer profiles spanning $64 \mathrm{~mm}$ at $15 \% x / c . R e_{c}=2.4 \times 10^{6},[24 \mid 12]$ roughness. Mean profile shown in red.

6-11 Spanwise array of 65 mean-flow boundary-layer profiles spanning $64 \mathrm{~mm}$ at $15 \% x / c . R e_{c}=2.4 \times 10^{6},[36 \mid 12]$ roughness. Mean profile shown in red.

6-12 Spanwise array of 65 mean-flow boundary-layer profiles spanning $64 \mathrm{~mm}$ at $15 \% x / c . R e_{c}=2.4 \times 10^{6},[47 \mid 12]$ roughness. Mean profile shown in red.

6-13 Streamwise velocity contours at $15 \% x / c . R e_{c}=2.4 \times 10^{6}$, [12|12] roughness

6-14 Streamwise velocity contours at $15 \% x / c . R e_{c}=2.4 \times 10^{6}$, [24|12] roughness.

6-15 Streamwise velocity contours at $15 \% x / c . R e_{c}=2.4 \times 10^{6}$, [36|12] roughness 


\section{FIGURE}

6-16 Streamwise velocity contours at $15 \% x / c . R e_{c}=2.4 \times 10^{6}$, [47|12] roughness

6-17 Spanwise array of 65 disturbance profiles spanning $64 \mathrm{~mm}$ at $15 \% x / c . R e_{c}=2.4 \times 10^{6},[12 \mid 12]$ roughness

6-18 Spanwise array of 65 disturbance profiles spanning $64 \mathrm{~mm}$ at $15 \% x / c . R e_{c}=2.4 \times 10^{6},[24 \mid 12]$ roughness

6-19 Spanwise array of 65 disturbance profiles spanning $64 \mathrm{~mm}$ at $15 \% x / c . R e_{c}=2.4 \times 10^{6},[36 \mid 12]$ roughness

6-20 Spanwise array of 65 disturbance profiles spanning $64 \mathrm{~mm}$ at $15 \% x / c . R e_{c}=2.4 \times 10^{6},[47 \mid 12]$ roughness.

6-21 Stationary crossflow mode shape at $15 \% x / c . R e_{c}=2.4 \times 10^{6}$, $[k \mid 12]$ roughness

6-22 Power spectral density of spanwise hotwire scan at $15 \% x / c$. $R e_{c}=2.4 \times 10^{6},[k \mid 12]$ roughness

6-23 Spanwise array of 64 mean-flow boundary-layer profiles spanning $63 \mathrm{~mm}$ at $20 \% x / c . R e_{c}=2.4 \times 10^{6},[12 \mid 12]$ roughness. Mean profile shown in red

6-24 Spanwise array of 65 mean-flow boundary-layer profiles spanning $64 \mathrm{~mm}$ at $20 \% x / c . R e_{c}=2.4 \times 10^{6},[24 \mid 12]$ roughness. Mean profile shown in red.....

6-25 Spanwise array of 65 mean-flow boundary-layer profiles spanning $64 \mathrm{~mm}$ at $20 \% x / c . R e_{c}=2.4 \times 10^{6},[36 \mid 12]$ roughness. Mean profile shown in red.

6-26 Spanwise array of 64 mean-flow boundary-layer profiles spanning $63 \mathrm{~mm}$ at $20 \% x / c . R e_{c}=2.4 \times 10^{6},[60 \mid 12]$ roughness. Mean profile shown in red.

6-27 Streamwise velocity contours at $20 \% x / c . R e_{c}=2.4 \times 10^{6}$, [12|12] roughness

6-28 Streamwise velocity contours at $20 \% x / c . R e_{c}=2.4 \times 10^{6}$, [24|12] roughness 
6-29 Streamwise velocity contours at $20 \% x / c . R e_{c}=2.4 \times 10^{6}$, [36|12] roughness

6-30 Streamwise velocity contours at $20 \% x / c . R e_{c}=2.4 \times 10^{6}$, $[60 \mid 12]$ roughness

6-31 Spanwise array of 64 disturbance profiles spanning $63 \mathrm{~mm}$ at $20 \% x / c . R e_{c}=2.4 \times 10^{6},[12 \mid 12]$ roughness

6-32 Spanwise array of 65 disturbance profiles spanning $64 \mathrm{~mm}$ at $20 \% x / c . R e_{c}=2.4 \times 10^{6},[24 \mid 12]$ roughness

6-33 Spanwise array of 65 disturbance profiles spanning $64 \mathrm{~mm}$ at $20 \% x / c . R e_{c}=2.4 \times 10^{6},[36 \mid 12]$ roughness

6-34 Spanwise array of 65 disturbance profiles spanning $64 \mathrm{~mm}$ at $20 \% x / c . R e_{c}=2.4 \times 10^{6},[60 \mid 12]$ roughness

6-35 Stationary crossflow mode shape at $20 \% x / c . R e_{c}=2.4 \times 10^{6}$, $[k \mid 12]$ roughness

6-36 Power spectral density of spanwise hotwire scan at 20\% $x / c$.

$R e_{c}=2.4 \times 10^{6},[k \mid 12]$ roughness

6-37 Spanwise array of 65 mean-flow boundary-layer profiles spanning $64 \mathrm{~mm}$ at $10 \% x / c . R e_{c}=2.8 \times 10^{6},[47 \mid 12]$ roughness. Mean profile shown in red.

6-38 Spanwise array of 65 disturbance profiles spanning $64 \mathrm{~mm}$ at $10 \% x / c . R e_{c}=2.8 \times 10^{6},[47 \mid 12]$ roughness

6-39 Streamwise velocity contours at $10 \% x / c . R e_{c}=2.8 \times 10^{6}$, [47|12] roughness

6-40 Stationary crossflow mode shape at $10 \% x / c . R e_{c}=2.8 \times 10^{6}$, [47|12] roughness

6-41 Power spectral density of spanwise hotwire scan at $10 \% x / c$. $R e_{c}=2.8 \times 10^{6},[47 \mid 12]$ roughness. 
6-42 Spanwise array of 64 mean-flow boundary-layer profiles spanning $63 \mathrm{~mm}$ at $15 \% x / c . R e_{c}=2.8 \times 10^{6},[12 \mid 12]$ roughness. Mean profile shown in red.....

6-43 Spanwise array of 65 mean-flow boundary-layer profiles spanning $64 \mathrm{~mm}$ at $15 \% x / c . R e_{c}=2.8 \times 10^{6},[24 \mid 12]$ roughness. Mean profile shown in red.

6-44 Spanwise array of 65 mean-flow boundary-layer profiles spanning $64 \mathrm{~mm}$ at $15 \% x / c . R e_{c}=2.8 \times 10^{6}$, [36|12] roughness. Mean profile shown in red.

6-45 Spanwise array of 65 mean-flow boundary-layer profiles spanning $64 \mathrm{~mm}$ at $15 \% x / c . R e_{c}=2.8 \times 10^{6},[47 \mid 12]$ roughness. Mean profile shown in red

6-46 Streamwise velocity contours at $15 \% x / c . R e_{c}=2.8 \times 10^{6}$, [12|12] roughness

6-47 Streamwise velocity contours at $15 \% x / c . R e_{c}=2.8 \times 10^{6}$, [24|12] roughness

6-48 Streamwise velocity contours at $15 \% x / c . R e_{c}=2.8 \times 10^{6}$, [36|12] roughness

6-49 Streamwise velocity contours at $15 \% x / c . R e_{c}=2.8 \times 10^{6}$, [47|12] roughness

6-50 Spanwise array of 64 disturbance profiles spanning $63 \mathrm{~mm}$ at $15 \% x / c . R e_{c}=2.8 \times 10^{6},[12 \mid 12]$ roughness

6-51 Spanwise array of 65 disturbance profiles spanning $64 \mathrm{~mm}$ at $15 \% x / c . R e_{c}=2.8 \times 10^{6},[24 \mid 12]$ roughness

6-52 Spanwise array of 65 disturbance profiles spanning $64 \mathrm{~mm}$ at $15 \% x / c . R e_{c}=2.8 \times 10^{6},[36 \mid 12]$ roughness

6-53 Spanwise array of 65 disturbance profiles spanning $64 \mathrm{~mm}$ at $15 \% x / c . R e_{c}=2.8 \times 10^{6},[47 \mid 12]$ roughness 
6-54 Stationary crossflow mode shape at $15 \% x / c . R e_{c}=2.8 \times 10^{6}$, $[k \mid 12]$ roughness

6-55 Power spectral density of spanwise hotwire scan at $15 \% x / c$.

$\operatorname{Re}_{c}=2.8 \times 10^{6},[k \mid 12]$ roughness

6-56 Close-up of naphthalene flow visualization for $R e_{c}=3.2 \times 10^{6}$, [36|12] roughness

6-57 Spanwise array of 65 mean-flow boundary-layer profiles spanning $64 \mathrm{~mm}$ at $10 \% x / c . R e_{c}=3.2 \times 10^{6},[47 \mid 12]$ roughness. Mean profile shown in red.....

6-58 Spanwise array of 65 disturbance profiles spanning $64 \mathrm{~mm}$ at $10 \% x / c . R e_{c}=3.2 \times 10^{6},[47 \mid 12]$ roughness

6-59 Streamwise velocity contours at $10 \% x / c . R e_{c}=3.2 \times 10^{6}$, [47|12] roughness

6-60 Stationary crossflow mode shape at $10 \% x / c . R e_{c}=3.2 \times 10^{6}$, [47|12] roughness

6-61 Power spectral density of spanwise hotwire scan at $10 \% x / c$. $R e_{c}=3.2 \times 10^{6},[47 \mid 12]$ roughness

6-62 Spanwise array of 65 mean-flow boundary-layer profiles spanning $64 \mathrm{~mm}$ at $15 \% x / c . R e_{c}=3.2 \times 10^{6},[12 \mid 12]$ roughness. Mean profile shown in red.....

6-63 Spanwise array of 65 mean-flow boundary-layer profiles spanning $64 \mathrm{~mm}$ at $15 \% x / c . R e_{c}=3.2 \times 10^{6},[24 \mid 12]$ roughness. Mean profile shown in red.....

6-64 Spanwise array of 65 mean-flow boundary-layer profiles spanning $64 \mathrm{~mm}$ at $15 \% x / \mathrm{c} . \operatorname{Re}_{c}=3.2 \times 10^{6},[36 \mid 12]$ roughness. Mean profile shown in red

6-65 Spanwise array of 65 mean-flow boundary-layer profiles spanning $64 \mathrm{~mm}$ at $15 \% x / \mathrm{c} . \operatorname{Re}_{c}=3.2 \times 10^{6}$, [47|12] roughness. Mean profile shown in red.... 


\section{FIGURE}

6-66 Streamwise velocity contours at $15 \% x / c . R e_{c}=3.2 \times 10^{6}$,

[12|12] roughness

6-67 Streamwise velocity contours at $15 \% x / c . R e_{c}=3.2 \times 10^{6}$, [24|12] roughness

6-68 Streamwise velocity contours at $15 \% x / c . R e_{c}=3.2 \times 10^{6}$, [36|12] roughness

6-69 Streamwise velocity contours at $15 \% x / c . R e_{c}=3.2 \times 10^{6}$, [47|12] roughness

6-70 Spanwise array of 65 disturbance profiles spanning $64 \mathrm{~mm}$ at $15 \% x / c . R e_{c}=3.2 \times 10^{6},[12 \mid 12]$ roughness

6-71 Spanwise array of 65 disturbance profiles spanning $64 \mathrm{~mm}$ at $15 \% x / c . R e_{c}=3.2 \times 10^{6},[24 \mid 12]$ roughness

6-72 Spanwise array of 65 disturbance profiles spanning $64 \mathrm{~mm}$ at $15 \% x / c . R e_{c}=3.2 \times 10^{6},[36 \mid 12]$ roughness

6-73 Spanwise array of 65 disturbance profiles spanning $64 \mathrm{~mm}$ at $15 \% x / c . R e_{c}=3.2 \times 10^{6},[47 \mid 12]$ roughness

6-74 Stationary crossflow mode shape at $15 \% x / c . R e_{c}=3.2 \times 10^{6}$, $[k \mid 12]$ roughness

6-75 Power spectral density of spanwise hotwire scan at $15 \% x / c$.

$R e_{c}=3.2 \times 10^{6},[k \mid 12]$ roughness

7-1 NFV with appliqué roughness: $x / c=0.029, d=3 \mathrm{~mm}, \lambda=6 \mathrm{~mm}$ $R e_{c}=2.4 \times 10^{6}$; (a) $k=14 \mu \mathrm{m}$, (b) $k=27 \mu \mathrm{m}$, (c) $k=42 \mu \mathrm{m}$, (d) $k=56 \mu \mathrm{m}$

7-2 Close-up of $6 \mathrm{~mm}$ streaking from NFV, $R e_{c}=2.4 \times 10^{6}$, [42|6] roughness

7-3 Spanwise array of 65 mean-flow boundary-layer profiles spanning $64 \mathrm{~mm}$ at $15 \% x / c . R e_{c}=2.4 \times 10^{6},[14 \mid 6]$ roughness. Mean profile shown in red.... 
7-4 Spanwise array of 65 mean-flow boundary-layer profiles spanning $64 \mathrm{~mm}$ at $15 \% x / c . R e_{c}=2.4 \times 10^{6},[27 \mid 6]$ roughness. Mean profile shown in red

7-5 Spanwise array of 65 mean-flow boundary-layer profiles spanning $64 \mathrm{~mm}$ at $15 \% x / c . R e_{c}=2.4 \times 10^{6},[42 \mid 6]$ roughness. Mean profile shown in red

7-6 Spanwise array of 65 mean-flow boundary-layer profiles spanning $64 \mathrm{~mm}$ at $15 \% x / c . R e_{c}=2.4 \times 10^{6},[56 \mid 6]$ roughness. Mean profile shown in red....

7-7 Streamwise velocity contours at $15 \% x / c . R e_{c}=2.4 \times 10^{6}$, [14|6] roughness

7-8 Streamwise velocity contours at $15 \% x / c . R e_{c}=2.4 \times 10^{6}$, [27|6] roughness

7-9 Streamwise velocity contours at $15 \% x / c . R e_{c}=2.4 \times 10^{6}$, $[42 \mid 6]$ roughness

7-10 Streamwise velocity contours at $15 \% x / c . R e_{c}=2.4 \times 10^{6}$, [56|6] roughness

7-11 Spanwise array of 65 disturbance profiles spanning $64 \mathrm{~mm}$ at $15 \% x / c . R e_{c}=2.4 \times 10^{6},[14 \mid 6]$ roughness

7-12 Spanwise array of 65 disturbance profiles spanning $64 \mathrm{~mm}$ at $15 \% x / c . R e_{c}=2.4 \times 10^{6},[27 \mid 6]$ roughness

7-13 Spanwise array of 65 disturbance profiles spanning $64 \mathrm{~mm}$ at $15 \% x / c . R e_{c}=2.4 \times 10^{6},[42 \mid 6]$ roughness

7-14 Spanwise array of 65 disturbance profiles spanning $64 \mathrm{~mm}$ at $15 \% x / c . R e_{c}=2.4 \times 10^{6},[56 \mid 6]$ roughness

7-15 Stationary crossflow mode shape at $15 \% x / c . R e_{c}=2.4 \times 10^{6}$, [k|6] roughness

7-16 Power spectral density of spanwise hotwire scan at $15 \% x / c$. $R e_{c}=2.4 \times 10^{6},[k \mid 6]$ roughness 
7-17 NFV with appliqué roughness: $x / c=0.029, d=3 \mathrm{~mm}, \lambda=6 \mathrm{~mm}$ $R e_{c}=2.8 \times 10^{6}$; (a) $k=14 \mu \mathrm{m}$, (b) $k=27 \mu \mathrm{m}$, (c) $k=42 \mu \mathrm{m}$, (d) $k=56 \mu \mathrm{m}$

7-18 Spanwise array of 65 mean-flow boundary-layer profiles spanning $64 \mathrm{~mm}$ at $15 \% x / c . R e_{c}=2.8 \times 10^{6},[14 \mid 6]$ roughness. Mean profile shown in red.

7-19 Spanwise array of 65 mean-flow boundary-layer profiles spanning $64 \mathrm{~mm}$ at $15 \% x / c . R e_{c}=2.8 \times 10^{6},[27 \mid 6]$ roughness. Mean profile shown in red.

7-20 Spanwise array of 65 mean-flow boundary-layer profiles spanning $64 \mathrm{~mm}$ at $15 \% x / \mathrm{c} . \operatorname{Re}_{c}=2.8 \times 10^{6},[42 \mid 6]$ roughness. Mean profile shown in red.

7-21 Spanwise array of 65 mean-flow boundary-layer profiles spanning $64 \mathrm{~mm}$ at $15 \% x / c . R e_{c}=2.8 \times 10^{6},[56 \mid 6]$ roughness. Mean profile shown in red.

7-22 Streamwise velocity contours at $15 \% x / c . R e_{c}=2.8 \times 10^{6}$, $[14 \mid 6]$ roughness

7-23 Streamwise velocity contours at $15 \% x / c . R e_{c}=2.8 \times 10^{6}$, [27|6] roughness

7-24 Streamwise velocity contours at $15 \% x / c . R e_{c}=2.8 \times 10^{6}$, [42|6] roughness

7-25 Streamwise velocity contours at $15 \% x / c . R e_{c}=2.8 \times 10^{6}$, [56|6] roughness

7-26 Spanwise array of 65 disturbance profiles spanning $64 \mathrm{~mm}$ at $15 \% x / c . R e_{c}=2.8 \times 10^{6},[14 \mid 6]$ roughness

7-27 Spanwise array of 65 disturbance profiles spanning $64 \mathrm{~mm}$ at $15 \% x / c . R e_{c}=2.8 \times 10^{6},[27 \mid 6]$ roughness

7-28 Spanwise array of 65 disturbance profiles spanning $64 \mathrm{~mm}$ at $15 \% x / c . R e_{c}=2.8 \times 10^{6},[42 \mid 6]$ roughness 
7-29 Spanwise array of 65 disturbance profiles spanning $64 \mathrm{~mm}$ at $15 \% x / c . R e_{c}=2.8 \times 10^{6},[56 \mid 6]$ roughness

7-30 Stationary crossflow mode shape at $15 \% x / c . R e_{c}=2.8 \times 10^{6}$, $[k \mid 6]$ roughness

7-31 Power spectral density of spanwise hotwire scan at $15 \% x / c$. $R e_{c}=2.8 \times 10^{6},[k \mid 6]$ roughness

7-32 NFV with appliqué roughness: $x / c=0.029, d=3 \mathrm{~mm}, \lambda=6 \mathrm{~mm}$ $R e_{c}=3.2 \times 10^{6}$; (a) $k=14 \mu \mathrm{m}$, (b) $k=27 \mu \mathrm{m}$

7-33 Spanwise array of 65 mean-flow boundary-layer profiles spanning $64 \mathrm{~mm}$ at $15 \% x / \mathrm{c} . \operatorname{Re}_{c}=3.2 \times 10^{6},[14 \mid 6]$ roughness. Mean profile shown in red.....

7-34 Spanwise array of 65 mean-flow boundary-layer profiles spanning $64 \mathrm{~mm}$ at $15 \% x / c . R e_{c}=3.2 \times 10^{6},[27 \mid 6]$ roughness. Mean profile shown in red.....

7-35 Streamwise velocity contours at $15 \% x / c . R e_{c}=3.2 \times 10^{6}$, $[14 \mid 6]$ roughness

7-36 Streamwise velocity contours at $15 \% x / c . R e_{c}=3.2 \times 10^{6}$, [27|6] roughness

7-37 Spanwise array of 65 disturbance profiles spanning $64 \mathrm{~mm}$ at $15 \% x / c . R e_{c}=3.2 \times 10^{6},[14 \mid 6]$ roughness

7-38 Spanwise array of 65 disturbance profiles spanning $64 \mathrm{~mm}$ at $15 \% x / c . R e_{c}=3.2 \times 10^{6},[27 \mid 6]$ roughness

7-39 Stationary crossflow mode shape at $15 \% x / c . R e_{c}=3.2 \times 10^{6}$, $[k \mid 6]$ roughness

7-40 Power spectral density of spanwise hotwire scan at $15 \% x / c$. $R e_{c}=3.2 \times 10^{6},[k \mid 6]$ roughness

8-1 Tape on downstream side of screen seven (view is looking upstream) 
8-2 Power spectral density of $u^{\prime}$ and $v^{\prime}$ at $15 \mathrm{~m} / \mathrm{s}$. Tape applied to screen seven.

8-3 Tape on upstream side of screen one (flow moves right to left from this angle)

8-4 Power spectral density of $u^{\prime}$ and $v^{\prime}$ at $15 \mathrm{~m} / \mathrm{s}$. Tape applied to screen one

8-5 NFV for [36|12] roughness: $x / c=0.029, R_{c}=2.4 \times 10^{6}$; (a) no tape, (b) with tape- higher turbulence

8-6 NFV for [36|12] roughness: $x / c=0.029, R_{c}=3.2 \times 10^{6}$; (a) no tape, (b) with tape- higher turbulence

8-7 Close-up of naphthalene flow visualization for $R e_{c}=3.2 \times 10^{6}$, [36|12] roughness. (a) No tape, (b) With tape- higher turbulence

8-8 Spanwise array of 65 mean-flow boundary-layer profiles spanning $64 \mathrm{~mm}$ at $15 \% \times / \mathrm{c}$ (high turbulence). $R e_{c}=2.8 \times 10^{6}$, [36|12] roughness. Mean profile shown in red.

8-9 Streamwise velocity contours at $15 \% x / c$ (high turbulence). $R e_{c}=2.8 \times 10^{6},[36 \mid 12]$ roughness

8-10 Spanwise array of 65 disturbance profiles spanning $64 \mathrm{~mm}$ at $15 \% x / c$ (high turbulence). $R e_{c}=2.8 \times 10^{6},[36 \mid 12]$ roughness.

8-11 Stationary crossflow mode shape at $15 \% x / c . R e_{c}=2.8 \times 10^{6}$, [36|12] roughness

8-12 Power spectral density of spanwise hotwire scan at $15 \% x / c$. $R e_{c}=2.8 \times 10^{6},[36 \mid 12]$ roughness

8-13 Spanwise array of 65 mean-flow boundary-layer profiles spanning $64 \mathrm{~mm}$ at $15 \% x / \mathrm{c}$ (high turbulence). $R e_{c}=3.2 \times 10^{6}$, [36|12] roughness. Mean profile shown in red.

8-14 Streamwise velocity contours at $15 \% x / c$ (high turbulence). $R e_{c}=3.2 \times 10^{6},[36 \mid 12]$ roughness 
FIGURE $\quad$ Page

8-15 Spanwise array of 65 disturbance profiles spanning $64 \mathrm{~mm}$ at $15 \% \times / c$ (high turbulence). $R e_{c}=3.2 \times 10^{6},[36 \mid 12]$ roughness.

8-16 Stationary crossflow mode shape at $15 \% x / c . R e_{c}=3.2 \times 10^{6}$, [36|12] roughness .

8-17 Power spectral density of spanwise hotwire scan at $15 \% x / c$. $R e_{c}=3.2 \times 10^{6},[36 \mid 12]$ roughness ............................................... 236 


\section{LIST OF TABLES}

TABLE Page

1-1 Comparison of parameters in receptivity experiments........................... 26

2-1 Traverse travel and step size ....................................................... 58

3-1 Surface roughness measurements on ASU(67)-0315 leading-edge insert

3-2 Measured appliqué roughness height 77

3-3 Test matrix for receptivity measurements 82

5-1 Percentage of mean streamwise velocity variations in Plane A 104

5-2 Test section disturbance intensities at $10 \mathrm{~m} / \mathrm{s}$; AC-coupled, $1 \mathrm{~Hz}-10 \mathrm{kHz}$ bandpass; Uncertainty: $\pm 0.005 \%$

5-3 Test section disturbance intensities at $15 \mathrm{~m} / \mathrm{s}$; AC-coupled, $1 \mathrm{~Hz}-10 \mathrm{kHz}$ bandpass; Uncertainty: $\pm 0.005 \%$

5-4 Test section disturbance intensities at $20 \mathrm{~m} / \mathrm{s}$; AC-coupled, $1 \mathrm{~Hz}-10 \mathrm{kHz}$ bandpass; Uncertainty: $\pm 0.005 \%$

5-5 Test section disturbance intensities at $25 \mathrm{~m} / \mathrm{s}$; AC-coupled, $1 \mathrm{~Hz}-10 \mathrm{kHz}$ bandpass; Uncertainty: $\pm 0.005 \%$

5-6 Separated turbulence and acoustic disturbance intensities 114

5-7 Comparison of UWT and KSWT transverse velocity fluctuations. 118

5-8 Turbulence intensities with model and wall liners installed 119

6-1 Maximum and normalized stationary crossflow disturbance amplitudes at $15 \% x / c . R e_{c}=2.4 \times 10^{6},[k \mid 12]$ roughness.

6-2 Stationary crossflow disturbance amplitude ratios at $15 \% x / c$. $R e_{c}=2.4 \times 10^{6},[k \mid 12]$ roughness 
6-3 Maximum and normalized stationary crossflow disturbance amplitudes at $20 \% x / c . R e_{c}=2.4 \times 10^{6},[k \mid 12]$ roughness

6-4 Stationary crossflow disturbance amplitude ratios at $20 \% x / c$.

$R e_{c}=2.4 \times 10^{6},[k \mid 12]$ roughness

6-5 Maximum and normalized stationary crossflow disturbance amplitudes at $15 \% x / c . R_{c}=2.8 \times 10^{6},[k \mid 12]$ roughness

6-6 Stationary crossflow disturbance amplitude ratios at $15 \% x / c$. $R e_{c}=2.8 \times 10^{6},[k \mid 12]$ roughness

6-7 Stationary crossflow disturbance amplitude ratios at $10 \% x / c$. [47|12] roughness

6-8 Maximum and normalized stationary crossflow disturbance amplitudes at $15 \% x / c . R e_{c}=3.2 \times 10^{6},[k \mid 12]$ roughness.

6-9 Stationary crossflow disturbance amplitude ratios at $15 \% x / c$. $\operatorname{Re}_{c}=3.2 \times 10^{6},[k \mid 12]$ roughness

7-1 Maximum and normalized stationary crossflow disturbance amplitudes at $15 \% x / c . R e_{c}=2.4 \times 10^{6},[k \mid 6]$ roughness

7-2 Maximum and normalized stationary crossflow disturbance amplitudes at $15 \% x / c . R e_{c}=2.8 \times 10^{6},[k \mid 6]$ roughness.

7-3 Maximum and normalized stationary crossflow disturbance amplitudes at $15 \% x / c, \operatorname{Re}_{c}=3.2 \times 10^{6},[k \mid 6]$ roughness.

8-1 Maximum stationary crossflow disturbance amplitudes at $15 \% x / c$, [36|12] roughness

9-1 Summary of normalized stationary crossflow disturbance amplitudes for $\lambda=12 \mathrm{~mm}$ at $15 \% x / \mathrm{c}$

9-2 Summary of normalized stationary crossflow disturbance amplitudes for $\lambda=6 \mathrm{~mm}$ at $15 \% x / c$

9-3 Summary of measurement points for Reibert (1996) and Carrillo (1996). 
9-4 Maximum stationary crossflow disturbance amplitudes for $\lambda=12 \mathrm{~mm}$ at $R e_{c}=2.4 \times 10^{6}$, estimated from Reibert (1996)

9-5 Normalized stationary crossflow disturbance amplitudes for $\lambda=12 \mathrm{~mm}$ at $R e_{c}=2.4 \times 10^{6}$, estimated from Reibert (1996)

9-6 Maximum stationary crossflow disturbance amplitudes for $\lambda=8 \mathrm{~mm}$ at $R e_{c}=2.4 \times 10^{6}$, estimated from Carrillo (1996)......

9-7 Normalized stationary crossflow disturbance amplitudes for $\lambda=8 \mathrm{~mm}$ at $R e_{c}=2.4 \times 10^{6}$, estimated from Carrillo (1996).

9-8 Summary of stationary crossflow disturbance amplitude ratios at $15 \% x / \mathrm{c}$ for $\lambda=12 \mathrm{~mm}$

9-9 Stationary crossflow disturbance amplitude ratios at $R e_{c}=2.4 \times 10^{6}$ for $\lambda=12 \mathrm{~mm}$, estimated from Reibert (1996) 


\section{INTRODUCTION}

\subsection{Background and Motivation}

In an effort to extend modern vehicle performance, emphasis in both the commercial and military sectors has been placed on design improvements that can, among other characteristics, increase aircraft efficiency. Understanding the process of laminarturbulent transition can contribute to this effort by providing a means to delay or induce transition more effectively. Both scenarios can benefit aircraft performance by either delaying transition through Laminar Flow Control (LFC) techniques, ultimately reducing skin friction drag, or inducing transition to take advantage of turbulent flow characteristics such as increased mixing rates for low-Reynolds-number combustion processes.

LFC, in particular, has seen cyclical periods of interest over the last several decades that generally correlated to fuel prices. As an example of the potential benefits, estimates indicate that maintaining the region of laminar flow on the wings of a large transport aircraft could reduce aircraft drag by $15 \%$, decreasing fuel burn in the process (Arnal \& Archambaud 2009). More recent pressures to reduce both the economic and environmental impact of fuel burn have renewed interest in applications that reduce fuel usage. Reductions in aircraft weight via improved structural materials or more efficient engines are possible techniques; however, Green (2008) reviews limitations imposed on

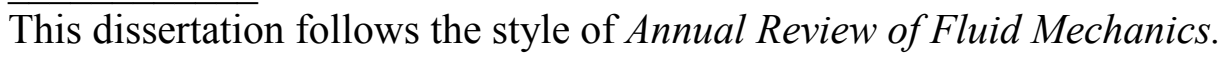


the designer and concludes that LFC is one of the most promising techniques for reducing fuel burn.

Efforts in LFC are a natural extension of decades of research dedicated to identifying the underlying phenomenon associated with boundary-layer stability and transition. Advances in understanding factors that affect the transition process have produced techniques to increase the extent of laminar flow on an airfoil (Saric \& Reed 2004, Joslin 1998). Yet, for all the stability and transition studies completed to date, a model for predicting the transition Reynolds number on a zero-pressure gradient flat plate does not currently exist (Saric et al. 2002). Difficulties that complicate current understanding include boundary-layer sensitivity to initial conditions such as freestream turbulence and noise, and nonlinear interactions in the boundary layer. Attempts to use empirical correlations have at best provided a technique for comparing stabilizing and destabilizing effects, but they have yet to yield a solution applicable to a wide variety of geometries and flight conditions. Ultimately, identifying the underlying phenomena of the transition process is a necessary component for improved LFC schemes which seek to control the instabilities whose behavior can indeed be predicted. The experimental efforts described in this study are intended to provide data that help explain a particular regime of the swept-wing transition process and be used as a validation tool for computational models. 


\subsection{Swept-Wing Stability and Transition}

There are several instability types and transition pathways (Saric et al. 2002) that may appear for a given bounded shear layer in an external flow which are dependent on Reynolds number, wall curvature, leading-edge sweep, roughness, and initial conditions. Mack (1984) provides a detailed review of the governing equations and development of instabilities. Reshotko (1976) and Kachanov (1994) also provide general overviews of boundary-layer stability and transition for external flows. Given the multitude of potential stability and transition characteristics, concentrating on the geometry and flight conditions most relevant to practical applications provides a reasonable way to narrow the research focus. Within that framework, swept-wing geometries with threedimensional boundary layers are prevalent in many commercial and military aircraft and thus warrant more detailed study.

Swept-wings have three-dimensional boundary-layers subject to streamwise (Tollmien-Schlichting), attachment line, centrifugal (Görtler) and crossflow instabilities. Of these four, the dominating instability leading to transition depends on airfoil geometry and freestream conditions. Detailed reviews of the three-dimensional boundary layer are provided in Reed \& Saric (1989) and Saric et al. (2003). Over the last several years, techniques have been developed to minimize or eliminate the effect of the first three by controlling airfoil shape to include a large extent of favorable pressure gradient followed by a small recovery region after the pressure minimum, avoid concave regions until after the pressure minimum and provide a leading-edge radius below a critical value. 
Given the ability to control the three other instabilities and the unique possibility of LFC when the crossflow instability dominates transition, the scope of this work is limited to swept-wings subject to crossflow instability. Moreover, the flight environment for these aircraft is characterized as low-disturbance, meaning small-amplitude freestream fluctuations such as turbulence or noise. The crossflow instability can manifest as either traveling or stationary waves, which develop in different ways depending on the freestream disturbance environment and surface conditions. Previous studies (Bippes 1999) have shown that the stationary crossflow wave is responsible for transition in the low-disturbance flight environment. As a result, study of the crossflow instability is further restricted to the stationary case.

\subsubsection{Crossflow Instability- Origins and Development}

Discovery of crossflow is generally attributed to Grey (1952) who noticed that transition on a swept-wing occurred much earlier than an unswept wing under the same operating conditions. Using flow visualization, streamwise streaks, attributed to stationary vortices, were observed upstream of the transition location. The theoretical basis for Gray's experiment was later provided by Stuart in Gregory et al. (1955). Sweep and a favorable pressure gradient create curved streamlines in the inviscid region around an airfoil (Figure 1-1). In the boundary layer, the pressure gradient remains, but the streamwise velocity reduces causing a secondary flow perpendicular to the external streamline to occur. This transverse flow is called crossflow. For a swept-back wing, it is directed inboard upstream of the pressure minimum and outboard downstream of the 
pressure minimum. The crossflow velocity must equal zero at the wall and approach zero at the boundary-layer edge which creates an inflection point in the crossflow velocity profile (Figure 1-2), that is subject to an inviscid instability. This instability manifests itself as a set of co-rotating vortices, which may be stationary or traveling. Although stationary and traveling modes may both be present, transition usually occurs due to one or the other, not both.

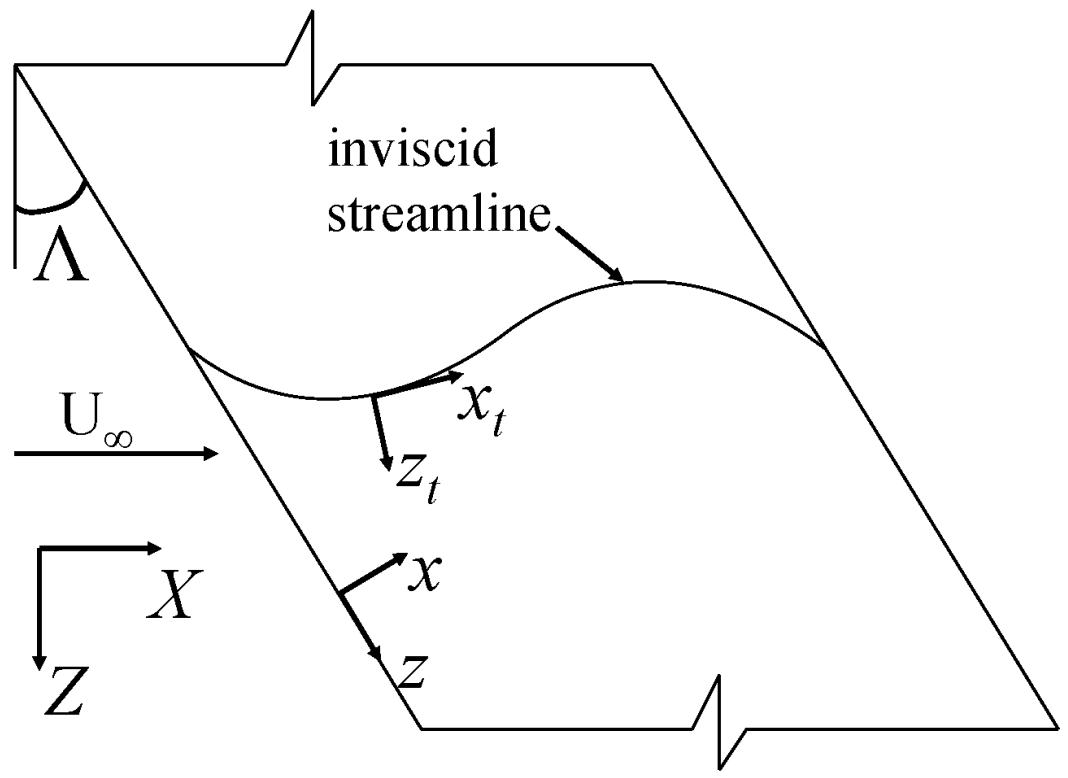

Figure 1-1. Streamlines on a swept-wing. 


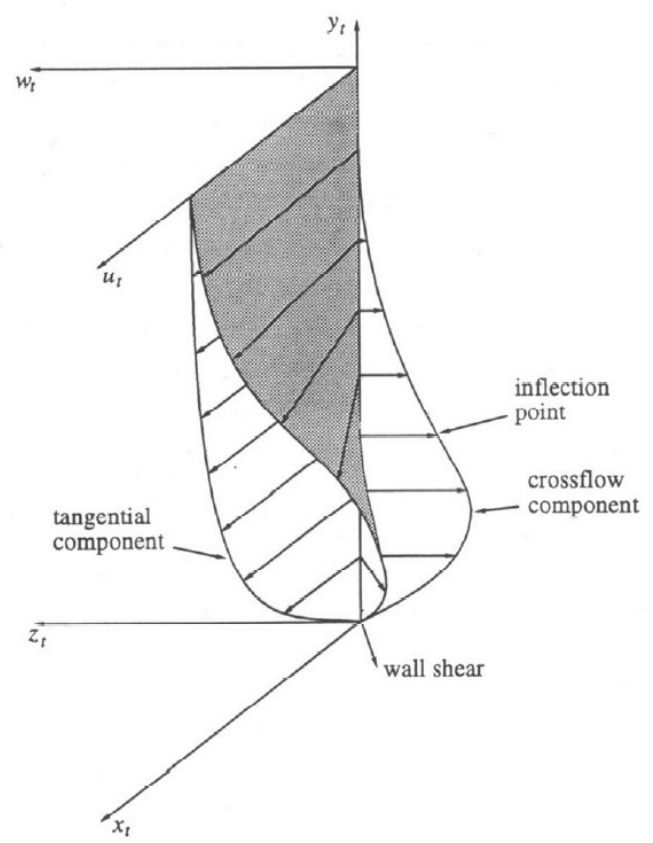

Figure 1-2. Boundary-layer velocity profiles on a swept-wing (White 2000).

Since the discovery of crossflow, much work has been invested in understanding the origins and development of the crossflow instability. Bippes (1999), Kachanov (2000) and Saric et al. (2003) provide detailed reviews of crossflow instability development based on computational and experimental evidence. For small-amplitude freestream disturbances, characteristic of the flight environment, a simplified version of the transition process can be described by several identifiable regimes: receptivity, linear disturbance growth, nonlinear growth and saturation, secondary mechanisms, followed by rapid breakdown and transition. One aspect of receptivity is the process by which freestream disturbances such as turbulence and sound enter the boundary layer and create the initial amplitude, frequency and phase for the unstable waves. It is the 
receptivity process that determines whether stationary or traveling crossflow vortices will result. Of all the crossflow development regimes, it is the least understood. A more detailed review of crossflow receptivity is provided in Section 1.3.

Once the initial conditions have been supplied, there is a small streamwise region wherein disturbance growth is linear; however the primary instability growth quickly becomes nonlinear, eventually resulting in amplitude saturation (Reibert et al. 1996). As the crossflow vortices grow, the disturbance velocities act on the basic state and begin to distort the mean flow. High-momentum fluid is convected down toward the surface where $v^{\prime}<0$ and low-momentum fluid is convected away from the wall in regions where $v^{\prime}>0$. Although the disturbance velocities $\left(v^{\prime}, w^{\prime}\right)$ are quite small, they convect $O(1)$ streamwise momentum. The stationary nature of the instability waves nearly aligned with the inviscid streamlines then allows the disturbances to act on the same fluid element over an extended distance. Figure 1-3 shows a schematic of the crossflow vortices and the resulting streamwise velocity distortions. 

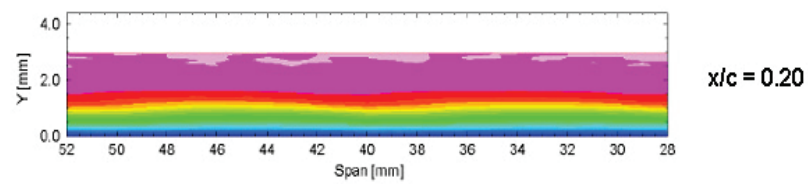

Stationary Crossflow Waves

$\operatorname{NLF}(2)-0415$ at $\alpha=-4^{\circ}, R e_{c}=2.4 \times 10^{6}$

$6 \mu \mathrm{m}$ roughness at $x / c=0.023,12 \mathrm{~mm}$ spacing
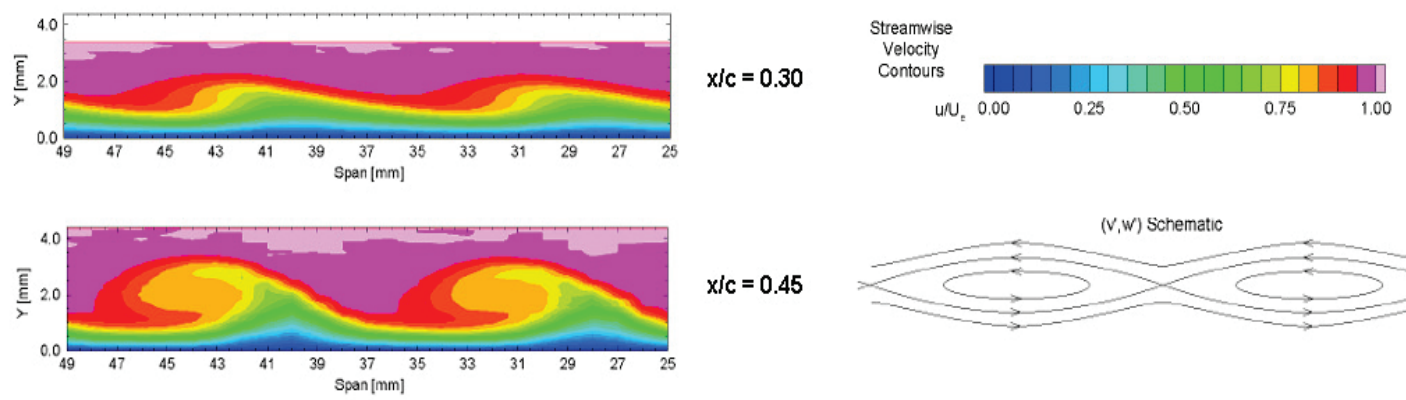

$x i c=0.45$

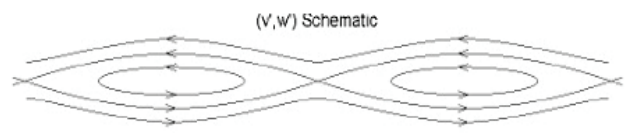

Figure 1-3. Mean flow distortion from stationary crossflow vortices (left), schematic of crossflow vortices (right); figure adapted from Reibert (1996).

As this distortion grows more extreme, alternating inflected, accelerated and decelerated streamwise velocity profiles result. The inflected profiles provide the conditions necessary for secondary inviscid instabilities to develop (Kohama et al. 1991, White \& Saric 2005). It is the secondary instability, characterized by highly amplified disturbance growth, that ultimately leads to transition, which occurs a short distance downstream once the secondary instability has appeared.

In contrast to stationary waves, growth of traveling waves is a linear process. Growth rates are usually larger compared to stationary waves. In low-turbulence environments however, the initial disturbance amplitudes for stationary waves are usually larger and the mean flow distortions provide conditions for transition before the traveling wave amplitudes are large enough to cause transition. 


\subsubsection{Transition Prediction and Disturbance Growth Models for Crossflow}

The three-dimensional Navier-Stokes equations are needed to completely model disturbance growth and evolution. These equations are initial-condition dependent and are computationally expensive to model. Traditional transition prediction schemes often use Linear Stability Theory (LST) and the $\mathrm{e}^{N}$ method as a simplified method for determining disturbance amplification associated with the primary instability and transition location.

LST describes how instability waves propagate in the boundary-layer and its basic assumption is that transition occurs because the basic state is unstable to disturbances. Use of LST requires that the disturbances be small and that parallel flow is assumed. Full details of the derivations are provided in Mack (1984). The resulting spatial Linear Stability Equations (LSE) are an eigenvalue problem that provides complex wave numbers $\alpha_{t}$ (streamwise) and $\beta_{t}$ (spanwise) and a frequency $\omega$. The imaginary component of $\alpha_{t}$ is the streamwise disturbance growth rate and the real component of $\beta_{t}$ is the spanwise spacing of the crossflow vortices. The spatial growth rates show whether disturbances are damped (stable) or amplified (unstable). Solution of the eigenvalue problem also shows that there are amplified traveling $(\omega \neq 0)$ or stationary $(\omega=0)$ waves. From the spatial growth rates $\left(\alpha_{i}\right)$, the most unstable crossflow wavelength is identified.

The $\mathrm{e}^{N}$ method, independently proposed by Smith \& Gamberoni (1956) and van Ingen (1956), uses the growth rates from LST and suggests that transition will occur after a given amplification ratio has been achieved. The correlation works well because 
the region of linear growth is large and the region of transition is small. The ratio, or $\mathrm{N}$ factor, is shown in Eqn (1-1).

$$
N=\int_{x_{0}}^{x}-\alpha_{i} d x=\ln \frac{A}{A_{0}}
$$

In Eqn (1-1), $A_{0}$ is the initial disturbance amplitude and $A$ is the amplitude at an $x$ distance downstream. Since the initial disturbance amplitude, $A_{0}$, is generally not known, this correlation can only predict relative disturbance growth and decay. The $N$-factor that ultimately results in transition is generally determined experimentally. As a result experimental and computational work cannot be compared and extrapolations from one experiment to the next cannot be used unless the governing stability conditions, such as freestream disturbances and model geometry, are matched. Reed et al. (1996) reviews use of LST and $\mathrm{e}^{N}$ and its applicability to different instabilities. When stationary crossflow vortices dominate the transition process, use of LST is limited to identification of the most unstable wavelength. Mean flow distortions imposed by the presence of the stationary waves quickly lead to nonlinear mode interactions that result in a saturation of the most unstable wavelength and growth of harmonics in wavenumber space (Reibert 1996). In these instances, LST overpredicts the amplification required to cause transition. Nonlinear parabolized stability equations (NPSE), which include curvature, nonparallel effects, and nonlinear interactions are instead used to model disturbance growth (Herbert 1997). 


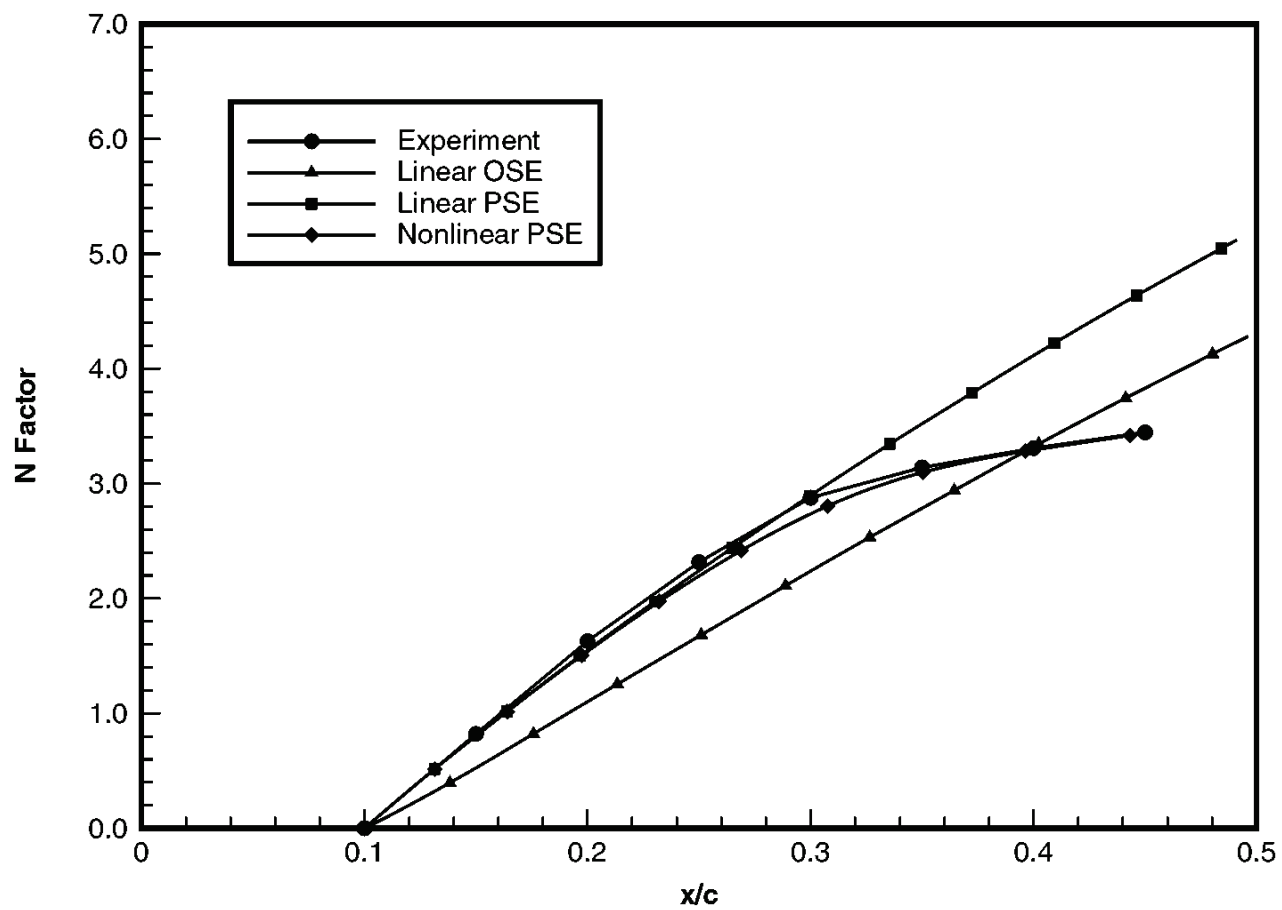

Figure 1-4. Disturbance amplitudes for Orr-Sommerfeld, LPSE, NPSE (Haynes \& Reed 2000), and experiments (Reibert et al. 1996). Figure courtesy of Saric et al. (2003).

Figure 1-4 compares an experimental set of $N$-factors with LST and NPSE predictions. It should be noted that for the LST data points in Figure 1-4,curvature is included in the computations. For the stationary crossflow instability, LST fails to predict disturbance amplitude growth even when effects of curvature are included. There is a region at early chord locations where Linear Parabolized Stability Equations (LPSE), which account for curvature and nonparallel effects but do not include nonlinear interactions, can accurately predict disturbance growth; however, as nonlinear interactions increase, disturbance amplitude begins to saturate. In the case of Figure 1-4 then, experimental values begin to depart from all but NPSE. Progress in LST and 
transition experiments targeting evolution of disturbances demonstrate that to fully explain and predict transition, initial disturbance amplitudes and nonlinear disturbance evolution models are needed (Chang \& Choudhari 2005, Saric et al. 2002). The NPSE are initial condition dependent and thus require some knowledge of the initial amplitude (i.e., the problem of receptivity) for use in a transition prediction scheme. Experimental and computational agreement has been achieved for both the primary instability growth (Haynes \& Reed 2000, Reibert et al. 1996) and secondary mechanisms (Kohama et al. 1991; Kawakami et al. 1999; Malik et al. 1999; Janke \& Balakumar 2000; Wasserman \& Kloker 2002; White \& Saric 2005) as long as appropriate initial conditions (amplitude and wavelength) are supplied (White et al. 2001). Successful agreement between experimental and computational work for crossflow instability development indicates that all of the important physics, such as curvature and nonlinear interactions are accounted for in these modeling techniques. Understanding how initial disturbance amplitudes are generated from freestream and airfoil surface conditions is one of the remaining challenges in disturbance modeling techniques.

\subsubsection{Laminar Flow Control for Crossflow}

To maximize LFC on a swept-wing, all four possible instabilities must be addressed. Attachment-line instability, Görtler instabilities and Tollmien-Schlichting instabilities can all be controlled through proper airfoil design. This involves keeping the leading edge radius below a critical value, avoiding concave geometry forward of the pressure minimum and extending the favorable pressure gradient as far aft as possible. A review 
of each transition mechanism is provided in Reed \& Saric (2008) and Saric et al. (2003). The favorable pressure gradient that stabilizes the Tollmien-Schlichting instability actually destabilizes the remaining crossflow instability. With three of the four instabilities minimized or eliminated, crossflow remains as the final challenge in sweptwing LFC.

The physics-based models used to understand disturbance growth and breakdown, coupled with the accompanying experiments provide needed insight into controlling transition location. Although, transition in a crossflow dominated flow is ultimately the result of secondary mechanisms that develop as the primary instability deforms the basic state, it is very difficult to implement any type of transition control once the secondary instability appears. This is mainly due to the highly amplified disturbance growth rates of the secondary instability, which cause breakdown and transition over a very short streamwise length scale. Delaying growth of the crossflow disturbances, which provide the conditions for the secondary mechanism to occur, is the most viable option for transition control.

Several techniques to minimize crossflow instability growth are available, depending on the design requirements and timeframe for implementation. Saric et al. (2011) list in order of technology readiness level (TRL) suction, reduction of wing sweep, reduction of leading edge surface roughness and addition of spanwise-periodic discrete roughness elements (DRE). Joslin (1998) and Saric \& Reed (2004) provide key overviews of past LFC efforts using suction. In light of design and operational complexities associated 
with the first three options, much attention has been given to LFC using spanwiseperiodic DRE.

Surface roughness is one of the key factors for determining initial disturbance amplitudes for stationary crossflow vortices (Radeztsky et al. 1999, Deyhle \& Bippes 1996). It is common practice to use artificial roughness for experiments trying to understand disturbance growth. Natural surface roughness can excite all modes, making it difficult to separate complex mode interactions and to compare with computational results which have a fixed set of modes. By using artificial surface roughness in the form of spanwise-periodic DRE, a single mode is forced resulting in a smaller set of modes to measure and identify. During these experiments, it was observed that harmonics, never subharmonics, in wavenumber space appeared for a given roughness condition (Saric \& Reed 2003). This phenomenon has been exploited for the benefit of LFC on airfoils dominated by a crossflow instability. Saric et al. (1998) showed that by using a DRE wavelength spacing less than the most unstable wavelength (critical wavelength), growth of the critical wavelength was suppressed and transition was delayed. The smaller wavelength spacing, termed the control wavelength, modifies the basic state such that the most unstable wavelength can no longer grow. The control wavelength then decays before amplitudes large enough to cause transition can occur. The result is a delay in transition location. Studies regarding the most effective placement and size for the roughness arrays have been completed (Radeztsky et al. 1999); however, the connection between initial disturbance amplitude and roughness height and shape is still not well understood. As Section 1.3 will show, this is a critical component to optimizing LFC 
techniques using these roughness arrays. Sensitivities such as roughness shape, observed in flight tests (Saric et al. 2011, Woodruff et al. 2011), indicate that a better understanding of the receptivity process is needed before LFC using DRE can be regularly implemented with success.

\subsection{Receptivity Literature Review}

\subsubsection{Receptivity Overview}

Receptivity is the first stage in all transition processes. Coined by Morkovin (1969), it describes how freestream disturbances enter the boundary-layer, interact with surface features on the airfoil and generate the initial disturbances responsible for the breakdown of laminar flow and ultimately the transition to turbulence. There is a broad spectrum of transition pathways that can occur as a result of initial disturbance amplitude. Figure 1-5 shows a simplified version of the transition paths (Saric et al. 2002, Morkovin et al. 1994). 


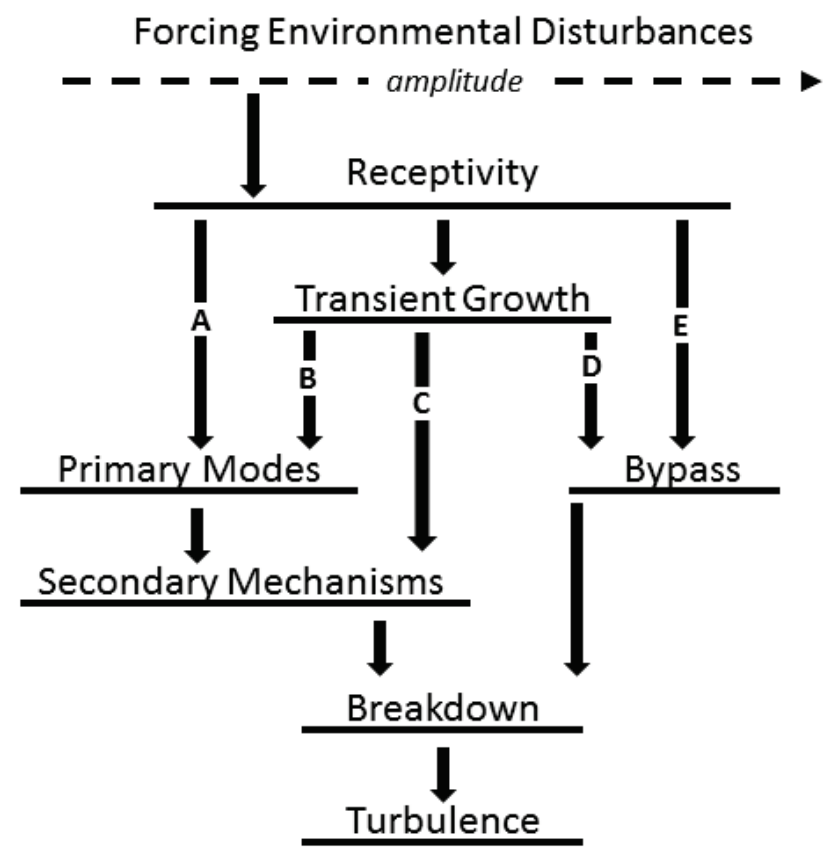

Figure 1-5. Simplified transition roadmap (Saric et al. 2002).

When the disturbances are very small, the primary instability starts as small fluctuations in the basic state and grows exponentially, leading to secondary mechanisms and finally breakdown to turbulence. The crossflow instability follows this path as long as freestream disturbances have small amplitudes. When the amplitudes grow too large, bypass can occur. In these instances, linear disturbance evolution models fail to describe disturbance growth and transition to turbulence often occurs very quickly (Morkovin 1969). Between these two extremes is the realm of transient growth, wherein stable waves interact and grow algebraically, resulting in transition to turbulence (Ellingsen \& Palm 1975, White et al. 2005). The receptivity process, then, influences every stage that follows. It becomes even more complicated when the presence of multiple instabilities is possible, as in the case of swept wings. Receptivity affects each of these instabilities 
differently and may serve as a dominating instability selection mechanism or cause competing transition paths.

As previously discussed, the receptivity stage is one of the last remaining challenges to transition control. Disturbance growth models require inputs resulting from the receptivity process. LFC optimization requires a better understanding of the factors that result in disturbance amplitude generation. Despite on-going experimental and computational efforts, it is still not well understood. Part of the difficulty comes from the scales involved. In the case of the stationary crossflow instability, the disturbance levels are too small to measure at the location where the disturbances are first generated. Measurements must occur at a downstream location after the disturbance has had time to grow. Interpretation of experimental data requires one to distinguish between effects related to receptivity and those related to disturbance growth. Another difficulty is the multiple factors that may influence the receptivity process. More than one disturbance source or receptivity site may influence the generation of the initial amplitude and in several cases, these sources can interact to form the initial amplitudes. These factors must be known to accurately model disturbance generation or to guide experimental design (Crouch \& Ng 2000). Additionally, multiple modes may be generated from the disturbance sources adding to the overall complexity of computational models and experimental data interpretation.

The first step in understanding the receptivity process is to understand disturbance sources; specifically: what the disturbance sources are, from where they come, and where and how they enter the boundary-layer. External sources may include turbulence, 
which is a rotational disturbance, and sound, an irrotational disturbance. For chemically reacting flows, concentration or temperature fluctuations can also be a factor. Bushnell (1990) notes that "the sources and thus the amplitude, spectral content, "organization/structure," and orientation of stream "turbulence" are a function of the flow medium/test apparatus." This is particularly important to recognize when choosing an experimental test facility to study an instability which may develop differently under varying environmental conditions. Carpenter et al. (2009) and Riedel \& Stizmann (1997) provide measurements of disturbance intensities in the flight environment. Section 2 addresses some of the specific issues related to wind tunnel and flight testing environments. Model features such as surface imperfections, geometry and roughness, as well as model vibration or discontinuities in surface curvature can also play a role. In flight, additional receptivity sources may come from particulates, such as bug strikes (Coleman 1959), that can introduce unintended roughness sites or additional vorticity as vortex shedding occurs around particles stuck to the surface (Bushnell 1990). These disturbance sources and their impact on the results must be carefully considered as data are compared from one test to another. As the rest of this section will show, seemingly small differences in freestream or surface conditions can have a large impact on the resulting receptivity and stability behavior.

\subsubsection{Crossflow Receptivity Studies}

To date, most of the crossflow receptivity studies have been parametric in nature, singling out the most sensitive disturbance sources and receptivity sites for instability 
generation. Of the potential disturbance sources listed previously, these studies have identified a complex relationship between freestream turbulence and surface roughness as a key factor in the receptivity process. For a given roughness height, Deyhle \& Bippes (1996) observed that transition Reynolds number decreased when freestream turbulence levels were increased. These results were observed with the same model in a single tunnel with two different turbulence levels and in two other facilities with different turbulence levels, ranging from $T u=0.08 \%$ to $0.57 \%$ with a $2 \mathrm{~Hz}-2 \mathrm{kHz}$ bandpass. In these instances $T u$ is representative of all three velocity fluctuations, as defined in Eqn. $(1-2)$.

$$
T u=\frac{1}{U_{\infty}}\left[\frac{1}{3}\left(\overline{u^{\prime 2}}+\overline{v^{\prime 2}}+\overline{w^{\prime 2}}\right)\right]^{1 / 2}
$$

Those hotwire turbulence measurements were unseparated signals that contain vortical and irrotational components representative of freestream turbulence and sound respectively. The Radeztsky et al. (1999) experiments, as well as the Deyhle \& Bippes study indicate that sound has no impact on stationary or traveling crossflow waves. The sound experiments tested SPL levels up to $113 \mathrm{~dB}$ using a combination of broadband and single frequencies in the passbands of traveling crossflow waves, destabilized Tollmien-Schlichting (T-S) waves and secondary instabilities. In all cases, the influence of forced acoustic disturbances was determined to be negligible. As a result, the effect of changing disturbance levels is attributed to the turbulent component of the signal. 
LST predicts that the traveling crossflow mode is more amplified than the stationary mode. However, experiments in low-disturbance environments almost always indicate that the stationary mode is the dominant transition mechanism (White et al. 2001). Although the growth rates are higher for traveling waves, their initial disturbance amplitudes are often smaller than the stationary mode (Saric et al. 2003). Moreover, since the stationary wave is nearly aligned with the inviscid streamlines, the same disturbance acts on a fluid element as it travels downstream, producing in an integrated effect that results in strong mean flow distortions. These mean flow distortions produce the secondary instabilities that cause transition well before the traveling wave amplitudes are large enough to cause breakdown of laminar flow. Thus, mode selection, i.e. traveling or stationary wave dominance, is the result of freestream turbulence providing the initial input. In the same Deyhle \& Bippes (1996) study mentioned above, experiments also demonstrated that freestream disturbance fluctuations determine whether stationary or traveling crossflow vortices will dominate transition. As turbulence level increased, traveling wave amplitudes also increased. For their studies, a criterion of $T u<0.2 \%$ was provided as the upper-bound for crossflow transition dominated by stationary waves for a given surface roughness. However, since the turbulent component of the overall disturbance intensity is not provided, the threshold limit is not well defined. Additionally, White et al. (2001) determined that the interaction of freestream disturbances and three-dimensional roughness may be more complex than the criteria suggested by Deyhle \& Bippes; transition induced by stationary waves with a $T u=0.3 \%$ with a highly polished leading edge was demonstrated. 
Experimental studies have established that surface roughness is a key parameter for transition. In a low-turbulence environment, Müller \& Bippes (1988) translated their swept-flat plate in the test section and observed that the wedge formation and streaking pattern remained fixed to the model, indicating that surface roughness is the primary factor in stationary crossflow wave development. Individual wedges are attributed to small differences in local surface roughness for a given test article. Dagenhart (1992) also found that the transition pattern remained the same over several tunnel entries and after cleaning the tunnel screens, although quantitative changes in freestream turbulence levels are not reported before and after the tunnel cleaning. In the case of natural roughness, transition location was delayed from $40 \% x / c$ to $61 \% x / c$ when surface finish was decreased from $9 \mu \mathrm{m}$-rms to $0.5 \mu \mathrm{m}$-rms in tests by Radeztsky et al. (1999). Lowering the surface roughness to $0.25 \mu \mathrm{m}$-rms delayed transition further to $68 \% x / c$. This effect was consistent over a wide range of chord-Reynolds numbers.

Radeztsky et al. (1999) and Deyhle \& Bippes (1996) both found that twodimensional roughness strips ranging in height from $6-50 \mu \mathrm{m}$ had no effect on transition location, except at the ends of the strip, which behave more like threedimensional roughness elements. These instances show that three-dimensional roughness is more critical for stationary crossflow development. These same studies also identified the most effective roughness location and diameter as just upstream of the branch I neutral point and at least $10 \%$ of the most amplified wavelength (with $1 / 4-1 / 3$ being ideal) respectively. Disturbance amplitudes generated by roughness upstream or downstream of the neutral point do not reach the same amplification potential either due 
to partial decay before the neutral point or delayed start of amplification after the neutral point. In the Radeztsky et al. tests, roughness placed after $10 \% x / c$ had no effect on transition location. Similarly, smaller roughness diameters fail to excite the intended crossflow modes.

Using spanwise-periodic DRE, Reibert et al. (1996) demonstrated that roughness arrays could be used to force a particular mode. Harmonics in wavenumber space were often observed, but never subharmonics. The implications of this finding lead to the LFC technique (Saric et al. 1998) described in Section 1.2.3. Transition control using the same technique has also been demonstrated in the F2 wind tunnel at ONERA using a swept wing (Arnal et al. 2011). Considerable interest in using DRE for LFC provided the opportunity to conduct flight tests at higher chord-Reynolds numbers and in an actual flight disturbance environment at Texas A\&M University (TAMU). Transition control (both advancement and delay) was demonstrated at $R e_{c}=7.5 \times 10^{6}$ (Carpenter et al. 2010); however, the experimental results indicate that there are some unresolved discrepancies in the receptivity to roughness in a flight disturbance environment. Previous work at the Arizona State University Unsteady Wind Tunnel (ASU-UWT) by Radeztsky et al. (1999) showed that isolated three-dimensional and background surface roughness amplitudes alter the transition location as long as amplitude saturation is not reached. Conversely, Reibert et al. (1996) found that changes in initial disturbance amplitude did not alter the final transition location as long as the roughness height was large enough to achieve amplitude saturation. Roughness arrays with heights from 6 $50 \mu \mathrm{m}$ were examined over a range of chord-Reynolds numbers from $1.6 \times 10^{6}-$ 
$3.2 \times 10^{6}$ and transition location did not appreciably shift. Moreover, several roughness types and shapes including appliqué, pneumatic (White \& Saric 2000) and plasma discharge (Saric \& Reed 2004) were all tested with the same results.

In contrast to the wind tunnel experiments, the flight experiments showed a sensitivity to both roughness height and shape. In the Carpenter et al. (2010) tests, appliqué roughness arrays did not move transition forward until reaching a height of $24 \mu \mathrm{m}$, at which point transition abruptly moved forward from $80 \% x / c$ to forward of $15 \% x / c$. No intermediate transition fronts were observed below a roughness height of $24 \mu \mathrm{m}$ and in each case the roughness height was well below the values provided by von Doenhoff \& Braslow (1961) for describing bypass transition (Carpenter 2009). When attempting the same change in roughness height using pneumatic DRE, transition location did not shift from the baseline case of transition at $80 \% x / c$ with no roughness applied. Roughness height was extended to $70 \mu \mathrm{m}$ for the pneumatic case, still resulting in no change from the baseline. Follow-on flight testing by Woodruff et al. (2011) found similar results. A direct numerical simulation (DNS) study by Rizzetta et al. (2010) supports experimental findings in flight that roughness height and shape do impact receptivity. Discrepancies between flight tests and previous wind tunnel tests indicate that the factors influencing the receptivity process are not fully resolved.

Detailed overviews of theoretical and computational work on receptivity of threedimensional boundary layers can be found in Saric et al. (2003) and Kachanov (2000). Several computational studies on the receptivity of roughness or the coupling of roughness and freestream acoustic disturbances have been completed (Choudhari 1993, 
Crouch 1993, Crouch 1994, Ng \& Crouch 1999). The computational results agree with earlier experimental work indicating that stationary modes should dominate in lowdisturbance environments and that the most effective placement for roughness is near the branch I neutral point. In these instances effects of curvature are not included. Later studies by Janke (2001), Collis \& Lele (1999), Bertolotti (2000) and Haynes \& Reed (2000) show that curvature and non-parallel effects are a significant factor in crossflow instability generation and development. Piot et al. (2008) examine DRE arrays of varying shape and height on a swept-cylinder and find that both traveling and stationary modes are excited downstream of the roughness element; the results also indicate that the receptivity of these roughness elements is nonlinear for the roughness heights considered. Schrader et al. (2009) examine the receptivity of freestream turbulence and roughness in the presence of crossflow. Using a swept-flat plate as a model, they consider individual cases for turbulence and roughness arrays as well as the two sources together. Their study indicates that traveling and stationary modes may be interacting. Detailed studies on the effect of freestream turbulence coupled with surface roughness on a swept-wing have not been completed to date.

The missing component from the large body of experimental receptivity studies is measurement of initial amplitudes as they relate to freestream vortical disturbances combined with roughness receptivity sites. Gaponenko et al. (2002) provide a comparison of experimental and theoretical receptivity coefficients for localized surface roughness using a swept flat plate with a pressure gradient induced by a contoured wall liner. Kurian et al. (2011) also use a swept-flat plate with contoured wall liners to 
examine the effects of freestream turbulence and surface roughness. Turbulence levels for the study range between $0.23 \%$ and $0.58 \%$. In all but one of the cases studied, traveling waves dominate the transition process. Successful measurement of the receptivity coefficient for non-localized surface roughness on a swept-wing in a lowdisturbance environment has not been accomplished to date.

\subsubsection{On-Going Crossflow Receptivity Work}

Future progress in understanding receptivity as it relates to the crossflow instability will need to proceed in a manner similar to previous transition studies, i.e. through a combination of experimental and computation efforts. Given the sensitivity to initial conditions and the small scales involved, it is unlikely that experiments alone can supply definitive receptivity coefficients for all flight regimes and airfoils of interest. However, they can provide validation data for computational models that take into account the $O(1)$ factors identified by earlier parametric receptivity studies. To that end, quantitative data for receptivity of roughness arrays on a model, where effects such as curvature are included, are still needed.

Additionally, the more recent discrepancies between wind tunnel testing at ASU and flight testing at TAMU described earlier indicate that more experiments are needed to identify the differences in the two test programs. It is difficult to obtain these answers by comparing previous experimental efforts due to variations in the wide variety of test parameters involved. Table 1-1 provides a summary of models, roughness conditions and turbulence levels from some of the experiments cited in this section. 
Table 1-1. Comparison of parameters in receptivity experiments.

\begin{tabular}{|c|c|c|c|c|c|}
\hline Model & $\begin{array}{c}\text { Surface } \\
\text { Roughness }\end{array}$ & Tunnel & $\begin{array}{c}\text { Tu } \\
\text { (separated) }\end{array}$ & $\begin{array}{c}\text { Tu } \\
\text { (unseparated) }\end{array}$ & Data source \\
\hline $\begin{array}{l}\text { Wooden plate } \\
c=0.5 \mathrm{~m}, \Lambda=45^{\circ}\end{array}$ & $\begin{array}{c}\mathrm{R}_{\mathrm{z}}=6 \mu \mathrm{m} \\
(\mathrm{pk}-\mathrm{pk})\end{array}$ & $\begin{array}{l}\text { DLR } \\
\text { NWB }\end{array}$ & $\begin{array}{c}0.07 \%{ }^{(\mathrm{a})} \\
(\mathrm{BP}: 2 \mathrm{~Hz}-2 \mathrm{kHz})\end{array}$ & $\begin{array}{c}0.08 \% \\
(\mathrm{BP}: 2 \mathrm{~Hz}-2 \mathrm{kHz})\end{array}$ & $\begin{array}{l}\text { Deyhle \& Bippes, } 1996 \\
{ }^{(a)} \text { Bippes, } 1999\end{array}$ \\
\hline $\begin{array}{l}\text { Wooden plate } \\
c=0.5 \mathrm{~m}, \Lambda=45^{\circ}\end{array}$ & $\begin{array}{c}\mathrm{R}_{\mathrm{z}}=6 \mu \mathrm{m} \\
\quad(\mathrm{pk}-\mathrm{pk})\end{array}$ & $\begin{array}{l}\text { DLR } \\
1 \mathrm{MK}\end{array}$ & $\begin{array}{c}0.12 \% \\
(\mathrm{BP}: 2 \mathrm{~Hz}-2 \mathrm{kHz})\end{array}$ & $\begin{array}{c}0.15 \% \\
(\mathrm{BP}: 2 \mathrm{~Hz}-2 \mathrm{kHz})\end{array}$ & $\begin{array}{l}\text { Deyhle \& Bippes, } 1996 \\
\text { (a) Bippes, } 1999\end{array}$ \\
\hline $\begin{array}{l}\text { Plate covered with sandpaper } \\
c=0.5 \mathrm{~m}, \Lambda=45^{\circ}\end{array}$ & $\begin{array}{l}\mathrm{R}_{\mathrm{z}}=40 \mu \mathrm{m} \\
\quad(\mathrm{pk}-\mathrm{pk})\end{array}$ & & & & \\
\hline $\begin{array}{l}\text { Aluminum plate, sanded } \\
c=0.5 \mathrm{~m}, \Lambda=45^{\circ}\end{array}$ & $\begin{array}{l}\mathrm{R}_{\mathrm{z}}=5 \mu \mathrm{m} \\
\quad(\mathrm{pk}-\mathrm{pk})\end{array}$ & & & & \\
\hline $\begin{array}{l}\text { Aluminum plate, polished } \\
c=0.5 \mathrm{~m}, \Lambda=45^{\circ}\end{array}$ & $\begin{array}{l}\mathrm{R}_{\mathrm{z}}=1.8 \mu \mathrm{m} \\
\quad(\mathrm{pk}-\mathrm{pk})\end{array}$ & & & & \\
\hline $\begin{array}{l}\text { Aluminum plate, polished } \\
c=0.5 \mathrm{~m}, \Lambda=45^{\circ}\end{array}$ & $\begin{array}{l}\mathrm{R}_{\mathrm{z}}=1.8 \mu \mathrm{m} \\
\quad(\mathrm{pk}-\mathrm{pk})\end{array}$ & $\begin{array}{c}\text { DLR } \\
1 \mathrm{MK} \\
\text { /screen }\end{array}$ & $\begin{array}{c}0.17 \%{ }^{(\mathrm{a})} \\
(\mathrm{BP}: 2 \mathrm{~Hz}-2 \mathrm{kHz})\end{array}$ & $\begin{array}{c}0.27 \% \\
(\mathrm{BP}: 2 \mathrm{~Hz}-2 \mathrm{kHz})\end{array}$ & $\begin{array}{l}\text { Deyhle \& Bippes, } \\
1996 \\
\text { (a) Bippes, } 1999\end{array}$ \\
\hline $\begin{array}{l}\text { Aluminum plate, polished } \\
c=0.5 \mathrm{~m}, \Lambda=45^{\circ}\end{array}$ & $\begin{array}{l}\mathrm{R}_{\mathrm{z}}=1.8 \mu \mathrm{m} \\
\quad(\mathrm{pk}-\mathrm{pk})\end{array}$ & $\begin{array}{c}\text { DLR } \\
\text { NWG }\end{array}$ & $\begin{array}{c}0.58 \%{ }^{(\mathrm{a})} \\
\text { (BP: } 2 \mathrm{~Hz}-2 \mathrm{kHz})\end{array}$ & $\begin{array}{c}0.57-0.7 \% \\
(\mathrm{BP}: 2 \mathrm{~Hz}-2 \mathrm{kHz})\end{array}$ & $\begin{array}{l}\text { Deyhle \& Bippes, } \\
1996 \\
\text { (a) Bippes, } 1999\end{array}$ \\
\hline $\begin{array}{l}\text { Swept-wing, NLF(2)-0415, painted } \\
c=1.83 \mathrm{~m}, \Lambda=45^{\circ} \\
\text { Swept-wing, NLF(2)-0415, sanded } \\
\text { Swept-wing, NLF(2)-0415, sanded } \\
\text { and polished }\end{array}$ & $\begin{array}{l}\mathrm{R}_{\mathrm{z}}=8-10 \mu \mathrm{m} \\
\quad(\mathrm{pk}-\mathrm{pk}) \\
\mathrm{R}_{\mathrm{q}}=0.5 \mu \mathrm{m} \\
\quad(\mathrm{rms}) \\
\mathrm{R}_{\mathrm{q}}=0.25 \mu \mathrm{m} \\
(\mathrm{rms})\end{array}$ & $\begin{array}{l}\text { ASU } \\
\text { UWT }\end{array}$ & & $\begin{array}{c}0.04 \%-0.09 \%{ }^{(\mathrm{b})} \\
\text { (BP: NR) } \\
0.038 \%^{(\mathrm{c})} \\
\text { (BP: } 1 \mathrm{~Hz}-1 \mathrm{kHz}) \\
<0.02 \%^{(\mathrm{d})} \\
(\mathrm{HP}: 2 \mathrm{~Hz})\end{array}$ & $\begin{array}{l}\text { Radeztsky et al., } 1999 \\
{ }^{(b)} \text { Dagenhart, } 1992 \\
{ }^{(c)} \text { Radeztsky, } 1994 \\
{ }^{(d)} \text { Reibert, } 1996\end{array}$ \\
\hline
\end{tabular}


Table 1-1. (continued)

\begin{tabular}{|c|c|c|c|c|c|}
\hline Model & $\begin{array}{c}\text { Surface } \\
\text { Roughness }\end{array}$ & Tunnel & $\begin{array}{c}\text { Tu } \\
\text { (separated) }\end{array}$ & $\begin{array}{c}\text { Tu } \\
\text { (unseparated) }\end{array}$ & Data source \\
\hline $\begin{array}{l}\text { Swept-wing, ASU(67)-0315, sanded } \\
\text { and polished } \\
c=1.83 \mathrm{~m}, \Lambda=45^{\circ}\end{array}$ & $\begin{array}{c}\mathrm{R}_{\mathrm{q}}=\underset{(\mathrm{rms})}{0.25 \mu \mathrm{m}} \\
\quad\end{array}$ & $\begin{array}{l}\text { ASU } \\
\text { UWT }\end{array}$ & & $\begin{array}{c}<0.02 \% \\
(\mathrm{HP}: 2 \mathrm{~Hz})\end{array}$ & White, 2000 \\
\hline $\begin{array}{l}\text { Swept-plate } \\
c=1.65 \mathrm{~m}, \Lambda=25^{\circ}\end{array}$ & NR & $\begin{array}{l}\text { ITAM } \\
\text { T-324 } \\
\end{array}$ & & $\begin{array}{c}0.06 \% \\
(\mathrm{HP}: 1 \mathrm{~Hz}) \\
\end{array}$ & Gaponenko et al., 2002 \\
\hline $\begin{array}{l}\text { Swept-plate } \\
c=2 \mathrm{~m}, \Lambda=25^{\circ}\end{array}$ & NR & $\begin{array}{l}\text { KTH } \\
\text { MTL }\end{array}$ & & $\begin{array}{c}0.2-0.58 \% \\
(\mathrm{BP}: \mathrm{NR})\end{array}$ & Kurian et al., 2011 \\
\hline $\begin{array}{l}\text { Swept-plate } \\
c=3.7 \mathrm{~m}, \Lambda=25^{\circ}\end{array}$ & $\begin{array}{l}\mathrm{R}_{\mathrm{q}}=0.3 \mu \mathrm{m} \\
(\mathrm{rms})\end{array}$ & $\begin{array}{l}\text { VPI } \\
\text { SWT }\end{array}$ & & $\begin{array}{l}0.02 \%-0.04 \% \\
(\mathrm{BP}: \mathrm{NR})\end{array}$ & Saric and Yeates, 1984 \\
\hline $\begin{array}{l}\text { Swept-plate } \\
c=0.7 \mathrm{~m} \text { (perpendicular to leading- } \\
\text { edge) } \Lambda=35-60^{\circ}\end{array}$ & & $\begin{array}{l}\text { ONERA } \\
\text { F2 }\end{array}$ & & $0.07 \%$ & Arnal et al., 2011 \\
\hline $\begin{array}{l}\text { Swept-wing, SWIFT, polished } \\
c=1.37 \mathrm{~m}, \Lambda=30^{\circ} \\
\text { Swept-wing, SWIFT, painted and } \\
\text { sanded } \\
c=1.37 \mathrm{~m}, \Lambda=30^{\circ}\end{array}$ & $\begin{array}{l}\mathrm{R}_{\mathrm{q}}=0.24 \\
(\mathrm{rms})^{(\mathrm{f})} \\
\mathrm{R}_{\mathrm{z}}=1.00 \\
(\mathrm{pk}-\mathrm{pk})^{(\mathrm{f})} \\
\mathrm{R}_{\mathrm{q}}=1.66 \\
(\mathrm{rms})^{(\mathrm{f})} \\
\mathrm{R}_{\mathrm{z}}=12.38 \\
(\mathrm{pk}-\mathrm{pk})^{(\mathrm{f})}\end{array}$ & $\begin{array}{l}\text { TAMU } \\
\text { FRL }\end{array}$ & & $\begin{array}{c}0.05 \%-0.07 \% \\
(\mathrm{BP}: 1 \mathrm{~Hz}- \\
10 \mathrm{kHz})\end{array}$ & $\begin{array}{l}\text { Martin et al., } 2008 \\
\text { Carpenter et al., } 2010\end{array}$ \\
\hline
\end{tabular}

ASU UWT: Arizona State University Unsteady Wind Tunnel; Tempe, Arizona

DLR NWB: Deutsches Zentrum für Luft- und Raumfahrt 3.25m x 2.8m low-speed wind tunnel; Braunschweig, Germany

DLR 1MK: Deutsches Zentrum für Luft- und Raumfahrt; $1 \mathrm{~m}$ x $0.7 \mathrm{~m}$ low-speed wind tunnel; Göttingen, Germany

DLR NWG: Deutsches Zentrum für Luft- und Raumfahrt 3m x 3m low-speed wind tunnel; Göttingen, Germany

ITAM T-324: Institute of Theoretical and Applied Mechanics 1m x 1m low-turbulence wind tunnel; Novosibirsk, Russia

KTH MTL: Royal Institute of Technology Minimum Turbulence Level wind tunnel; Stockholm, Sweden

ONERA F2: Office National d'Etudes et Recherches Aérospatiales subsonic continuous flow wind tunnel; Le Fauga Mauzac, France

VPI SWT: Virginia Polytechnic Institute (now Virginia Tech) Stability Wind Tunnel; Roanoke, Virginia

TAMU FRL: Texas A\&M University Flight Research Laboratory; College Station, Texas 
The entries in Table 1-1 indicate that several experimental designs and data analysis/reporting techniques are used in the literature. The abbreviation NR is used in instances where the information was not reported. It is not intended to be a complete listing, but rather a selected list that can help identify how future experiments can be improved based on the conclusions from previous receptivity experiments. Both sweptflat plates and swept-wings have been previously used in experiments, but based on the studies mentioned earlier, curvature is known to be an important factor in instability development. Swept-flat plate and swept wing experimental results may not yield the same trends. Roughness is now a known $O(1)$ factor in the receptivity process. As Table 1-1 shows, surface roughness is often reported as either a peak-to-peak value or a rootmean-square value. Reporting both values provides the best opportunity to understand and compare results. Similarly, freestream turbulence, rather than an acoustic disturbance, plays a critical role in the receptivity process. A mixture of separated and unseparated turbulence levels are described in previous literature. Unseparated signals, which include contributions of turbulence and sound, are less helpful in identifying critical thresholds for traveling and stationary mode influence. Moreover, it is not always clear from the stated values whether all velocity fluctuations (streamwise and transverse) are included in the freestream disturbance statements. These can vary based on tunnel configuration (see Section 5.2.2). Varying bandpasses also make it difficult to quantify the differences in tunnel turbulence levels. Reshotko et al. (1997) and Saric \& Reshotko (1998) discuss these differences in detail and supply recommended guidelines for disturbance intensity thresholds, identifying the individual components of the measured 
disturbances (i.e. acoustic or turbulent) and properly documenting the disturbance environment.

The key details of previous research and their impact on future studies can be summarized by the following statements:

1. The receptivity process is highly dependent on freestream and airfoil surface conditions near the leading edge

2. Freestream turbulence and surface roughness are the most important factors related to the stationary and traveling modes

3. Inconsistencies in previous experimental results suggest that extreme care in documenting the freestream environment and test conditions is necessary in future experiments

4. Validation data are still needed for swept-wings using artificial roughness arrays

\subsection{Experimental Objectives}

Previous work, both computational and experimental, has shown that meaningful transition prediction and control techniques and applications require more knowledge of the receptivity process. This is especially true given the inconsistencies observed in previous wind tunnel and flight tests. Modern prediction tools such as the NPSE require an input regarding initial disturbance amplitude that is not readily available for a wide variety of airfoil geometries and freestream conditions. LFC strategies using DRE need to incorporate the most effective shape and size to meet design objectives. Surface roughness and freestream turbulence have been identified as key factors in initial 
disturbance amplitude generation, but quantitative amplitudes are not well defined. Within the framework for established crossflow receptivity studies involving roughness on a swept-wing and freestream turbulence, this experiment seeks to obtain initial disturbance amplitudes as a function of vortical disturbances and non-localized surface roughness. The specific objective is to quantify the relationship between DRE height and initial disturbance amplitude over a range of chord-Reynolds numbers.

These data will be compiled in a database for use in theoretical and numerical simulation validation. Once these models have been validated, their use can be extended to include more realistic airfoil geometries and operating conditions. Additionally, it is expected that the role of freestream turbulence interacting with surface roughness in a low-disturbance environment will be further elucidated, enabling improved techniques for LFC and a better understanding of the discrepancies in results from previous crossflow experiments. 


\section{TEST FACILITY}

Choice of test facility is particularly important to transition and stability experiments. The freestream environment within which the experiment is conducted determines the transition pathway via the receptivity process and can influence how an instability develops along a given transition pathway. Wind tunnels introduce several disturbance sources not seen in flight, such as fan rotation, blade pitch, turning vanes, regions of separated flow, noise from the fan and motor, or low-frequency unsteady flow. The extent to which these turbulent or acoustic disturbances affect transition depends on the dominant boundary-layer instability. For example, as discussed earlier, the crossflow instability is quite sensitive to freestream turbulence. As freestream turbulence levels increase above a threshold value for a given surface roughness, traveling waves dominate the transition process. Unlike the companion stationary waves, traveling waves grow in amplitude according to linear theory. Attempts to draw conclusions regarding transition behavior for flight conditions using data obtained from higher turbulence tests where traveling waves dominate may lead to erroneous results.

Given the sensitivity to freestream disturbances for these experiments, data would ideally be collected in an environment similar to the target end-use; in this case, the lowdisturbance flight environment at chord-Reynolds and Mach numbers comparable to commercial and military aircraft specifications. However, the cost and risk associated with flight-test programs limit its use, leaving wind tunnel testing as the most viable alternative. Other advantages to testing in a wind tunnel compared to flight include the 
use of more sophisticated instrumentation and measurements, more control over test conditions and longer run times. The wind tunnel, then, can provide a platform to take detailed measurements which help us understand the physics behind a particular phenomenon. These data can then be supplied to computational models for numerical validation and to later develop flight-test programs that minimize the cost and risk by incorporating the lessons learned from tunnel testing. It must be emphasized that the advantages of wind tunnel testing are only useful provided that the disturbance environment is acceptable for the experiments of interest.

Experiments for this receptivity study are conducted in the TAMU Klebanoff-Saric Wind Tunnel (KSWT). In addition to discussing tunnel operating parameters, a brief summary of tunnel history and design changes is provided. Since the freestream disturbance environment is inherently a product of tunnel design, the overview is intended to provide perspective on flow quality as it relates to past and future transition experiments.

\subsection{Klebanoff-Saric Wind Tunnel}

\subsubsection{Tunnel History}

The KSWT is the third incarnation of the Unsteady Wind Tunnel (UWT) originally designed and built by Dr. Phillip Klebanoff at the National Bureau of Standards (NBS) in 1970. In 1984, the tunnel was relocated to Arizona State University (ASU) under the direction of Dr. William Saric. At that time, several modifications were made to the 
tunnel to improve flow quality with the intent of using the tunnel for boundary-layer stability and transition research (Saric et al. 1988, Saric 1992). Figures 2-1 and 2-2 show perspective, and plan and elevation views of the ASU-UWT respectively.

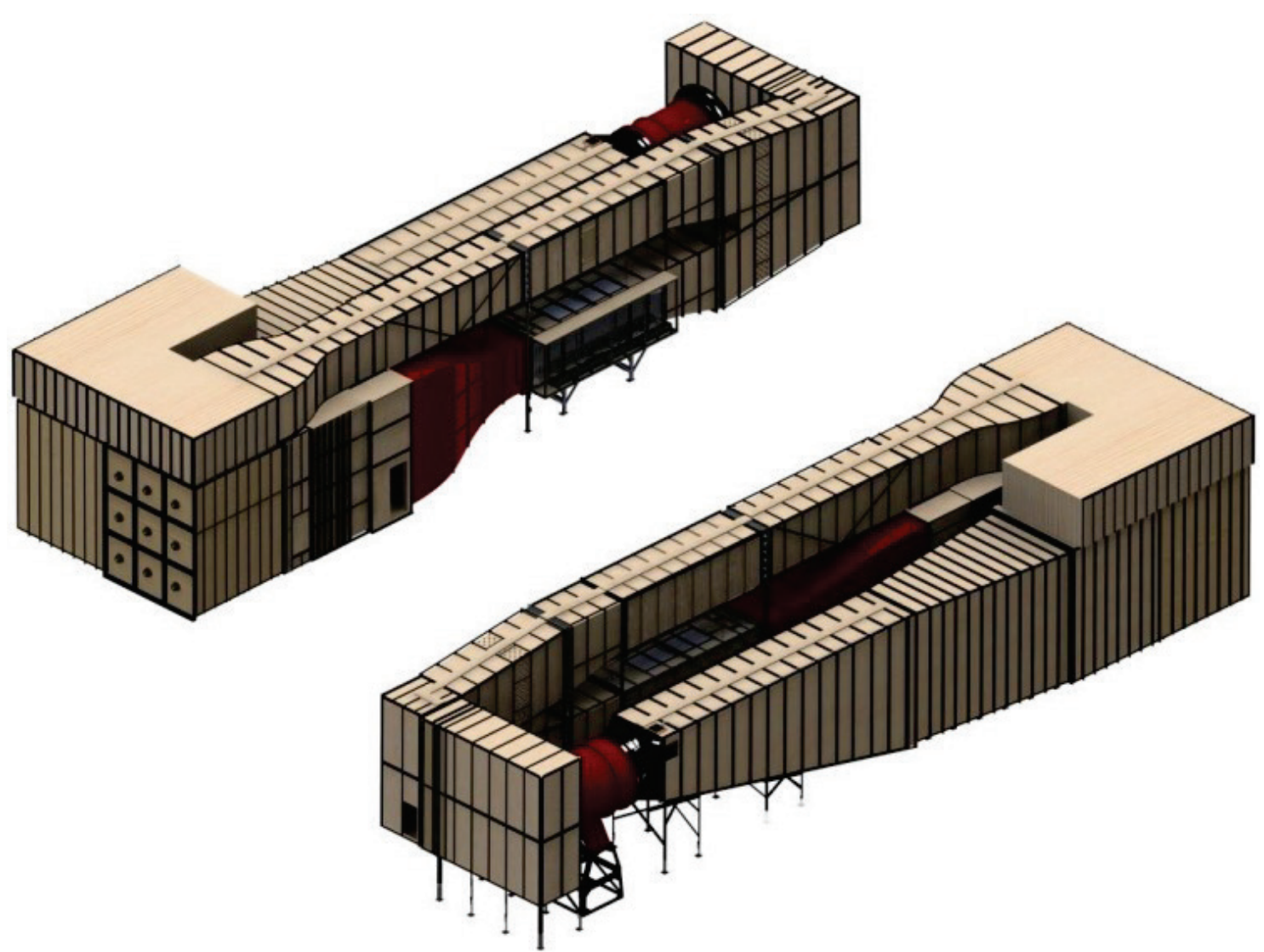

Figure 2-1. Perspective view of the UWT. 

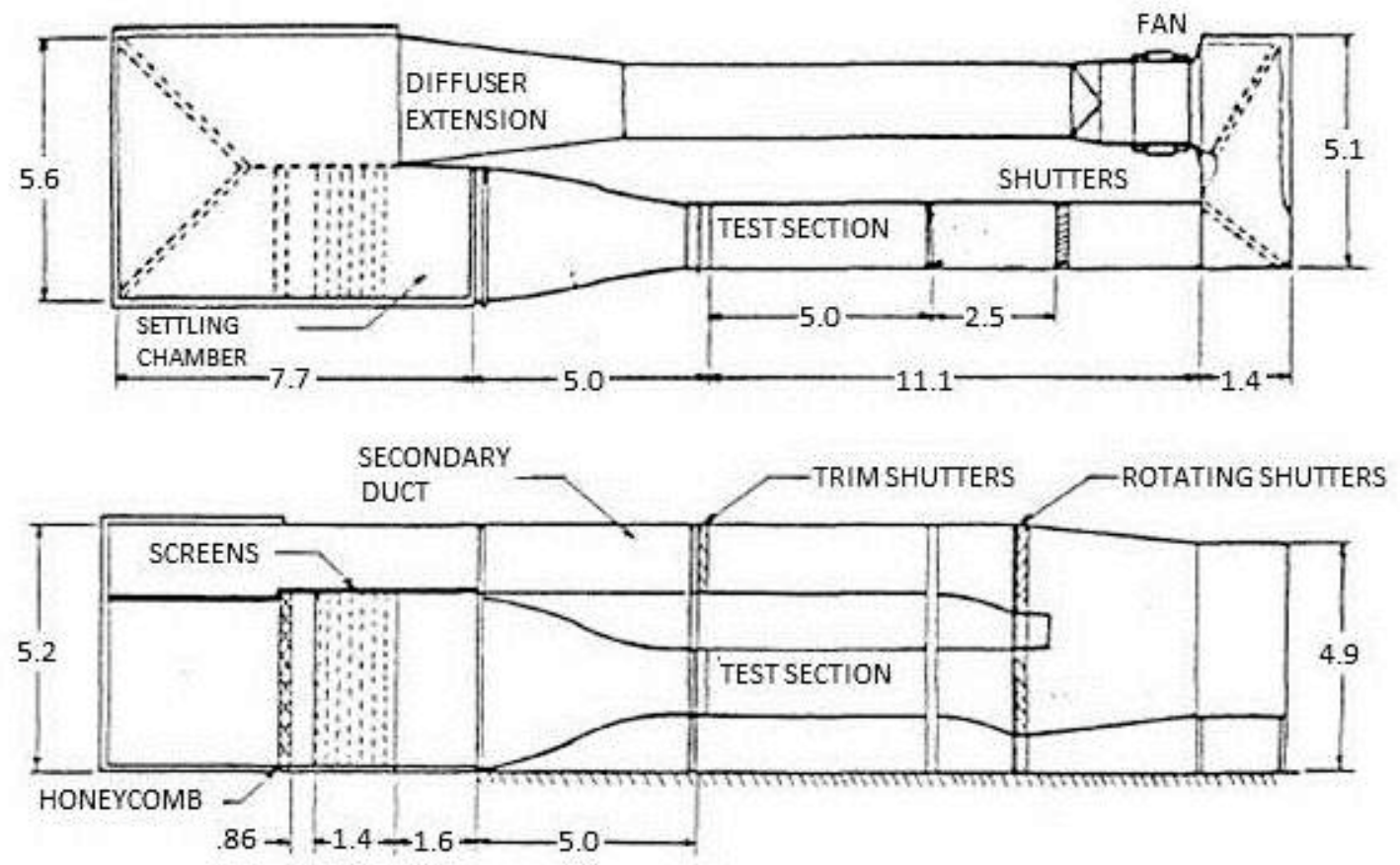

Figure 2-2. Plan (top) and elevation (bottom) views of the UWT.

At ASU, the tunnel had two operating configurations: steady and unsteady. During unsteady operations, trap doors in the plenum entrance and merging duct were opened, allowing airflow into a secondary duct that ran parallel to the primary tunnel loop. Rotating shutters located downstream of the test section generated oscillatory flows suitable for experiments such as unmanned air vehicles (UAV's) involving gusts and lulls. Although this unsteady capability was maintained during the tunnel transfer from NBS to ASU, the principal use of the UWT during its seventeen years of operation at ASU was in the steady configuration. In this operational mode, the upper duct was sealed using the trap doors and all airflow remained in the lower tunnel loop. The modifications made during the first reconstruction effort reduced disturbance levels in the tunnel from $0.1 \%$ to $0.02 \%-0.04 \%$ (Mousseux 1988) and peak-to-peak velocity 
decreased from $1 \% U_{o}$ to $0.4 \% U_{o}$ (Saric 1992), thus creating a low-turbulence environment suitable for boundary-layer stability and transition studies. The names ASU Transition Research Facility and ASU Unsteady Wind Tunnel are referenced in earlier literature and are representative of the facility in its ASU configuration. Much of the work completed in that tunnel configuration is summarized in Saric et al. (2002) and Saric et al. (2003).

In 2003, ASU decommissioned the wind tunnel in order to use the space for another building. Consequently, it was dismantled and relocated to TAMU in 2005. The second reconstruction effort provided an opportunity to use the operating experience at ASU to further improve the flow-quality environment. Although the tunnel was considered a low-disturbance facility, certain design features were identified as the most likely sources of turbulence and pressure fluctuations (Saric et al. 1988) and in several cases, those features were related to unsteady-mode components. The main objective during the TAMU reconstruction then was to reduce or eliminate these features further to minimize tunnel disturbances. In many instances at both ASU and TAMU, the design choices that reduce turbulent and acoustic disturbances come at the cost of tunnel efficiency. This is considered a necessary trade-off to achieve the desired flow quality. Reshotko et al. (1997) and Saric and Reshotko (1998) provide design guidelines for lowdisturbance wind tunnels; the UWT and KSWT use many of these design features to reduce the disturbance environment. During the second build, the decision was made to only reconstruct the primary duct with the intent of resuming stability and transition experiments as soon as possible. All components related to the unsteady configuration 
are in storage awaiting a stage-two build. Tunnel construction began mid-2008 and was complete by the end of 2009. The tunnel was operational by January 2010 after which, an extensive flow quality measurement campaign was completed. Hunt at al. (2010) provide a brief overview of the tunnel design, including differences between the ASU and TAMU configurations, and resulting flow quality measurements. An expanded set of those results related to turbulence levels are presented in Section 5. The tunnel description in Section 2.1.3 is an overview of the KSWT with an emphasis on component design as it relates to flow quality. This receptivity study is the first experiment in the tunnel since its completion.

\subsubsection{KSWT Building Facility}

A new facility to house the wind tunnel was designed and built prior to the reconstruction of the tunnel itself. All of these activities were supervised by the author. Facility design, site selection, university facilities planning and permission as well as the contractor bidding took place from June 2006 through November 2007. Building construction started in December 2007 and was completed in March of 2008. Located at the general aviation side of Easterwood Airport (KCLL) in College Station, TX, the KSWT facility joins a suite of tunnel and flight test capabilities supported by the Department of Aerospace Engineering (see http://aerospace-labs.tamu.edu). The Flight Research Laboratory (FRL), whose experiments are described in Section 1, is located in a nearby hangar. The close proximity of the flight laboratory and tunnels allows for shared resources such as staff and faculty expertise as well as on-site equipment and 
tools including forklifts and an extensive machine shop operated by the Oran W. Nicks Low-Speed Wind Tunnel.

Since the building was designed specifically to house the tunnel, lessons learned from ASU operations were also used to improve facility functionality. The $418 \mathrm{~m}^{2}$ (4500 $\mathrm{ft}^{2}$ ) building includes a two-ton overhead crane for model and test section transport, a loading dock with a $20 \mathrm{ft}$-high bay door and several areas for test article preparation. A pre-fabricated control room, which sits in-line with the test section, was installed by staff and students after facility and much of the tunnel construction was complete. It includes a second story for office space in addition to the tunnel control room and conference space on the first floor.

\subsubsection{Wind Tunnel}

The KSWT is a closed-loop, low-disturbance wind tunnel capable of generating test section speeds up to $31 \mathrm{~m} / \mathrm{s}$ that are controllable with $0.1 \mathrm{~m} / \mathrm{s}$ of the desired speed. When wall-liners and contraction fairings are installed, the maximum test section speed increases slightly. Figures $2-3$ and 2-4 show perspective and top views of the tunnel respectively. 


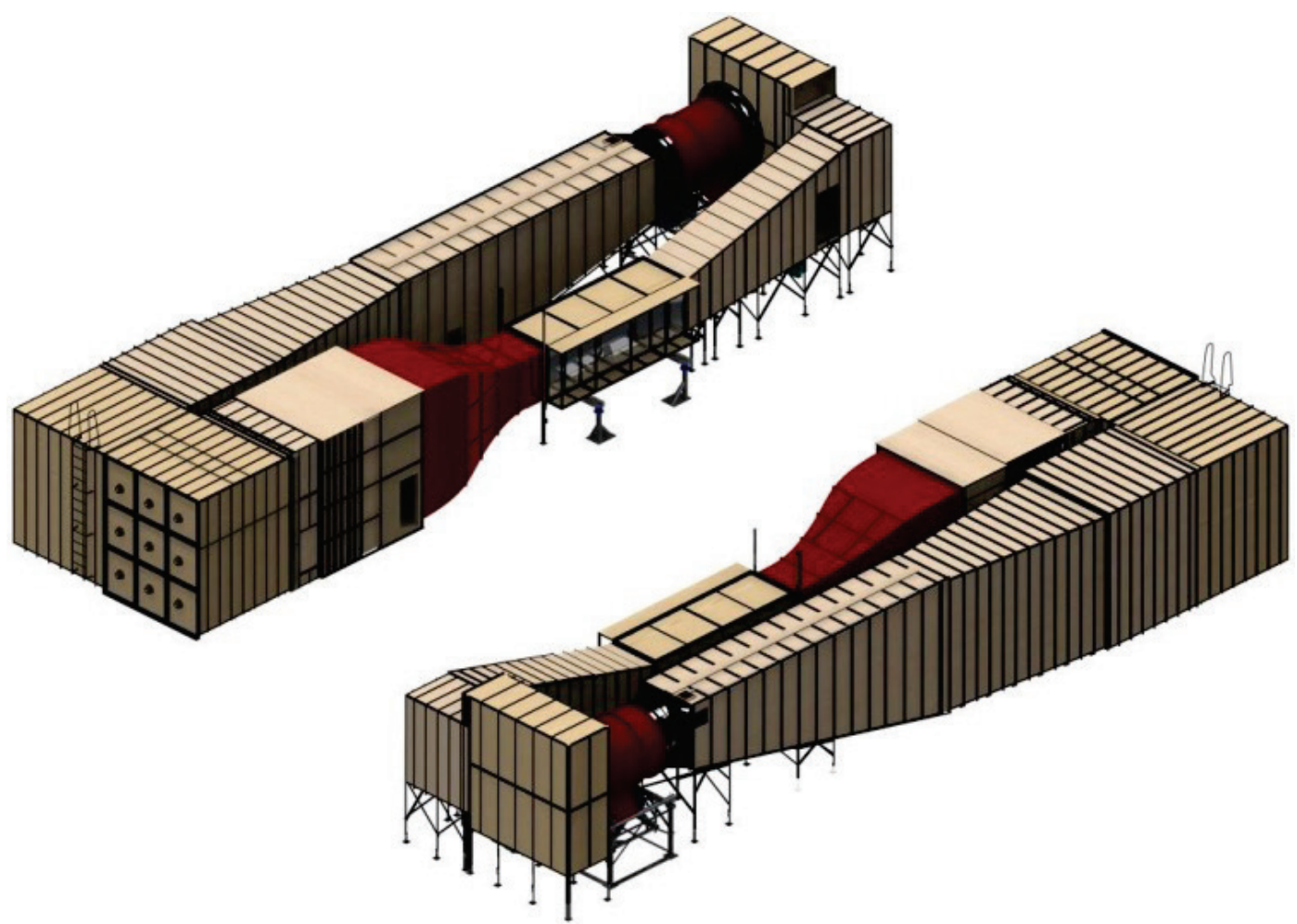

Figure 2-3. Perspective view of the KSWT.

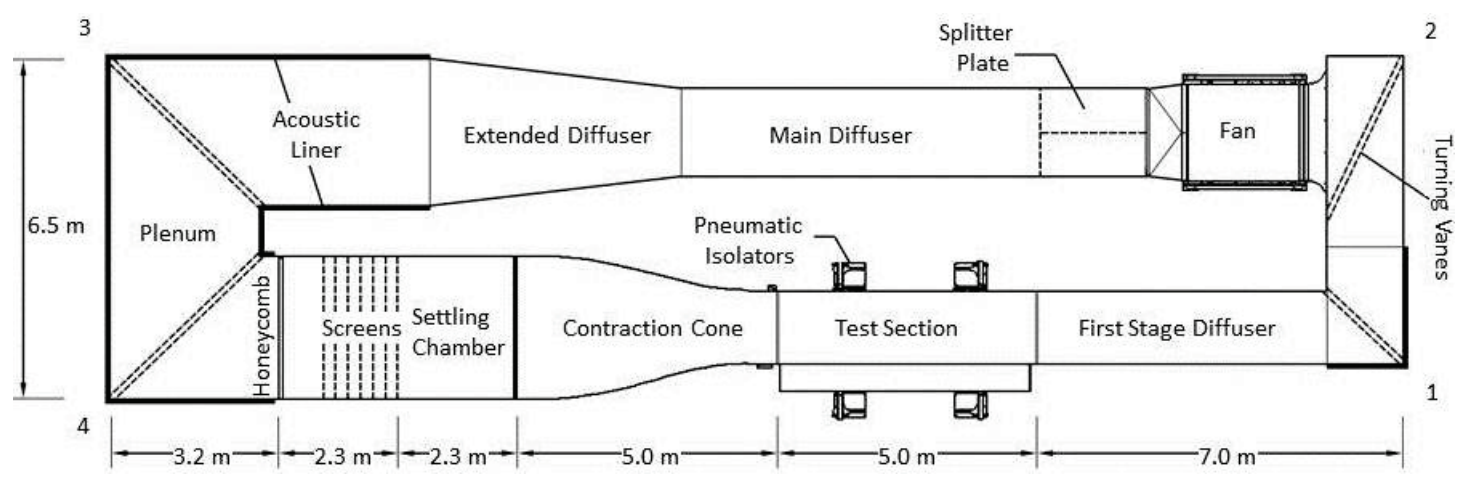

Figure 2-4. Plan view of the KSWT. Flow moves counter-clockwise. 
Constructed of a steel frame and wood paneling, the test section cross-sectional area measures $1.4 \mathrm{~m} \times 1.4 \mathrm{~m}(4.5 \mathrm{ft} \times 4.5 \mathrm{ft})$ at the entrance and has a length of $4.9 \mathrm{~m}(16 \mathrm{ft})$. The floor slopes slightly to account for displacement thicknesses associated with boundary-layer growth on the tunnel walls. The exit plane is $1.41 \mathrm{~m} \times 1.4 \mathrm{~m}$ (4.64 ft x $4.5 \mathrm{ft})$. Figure 2-5 shows the full test section assembly. Four pedestals form the

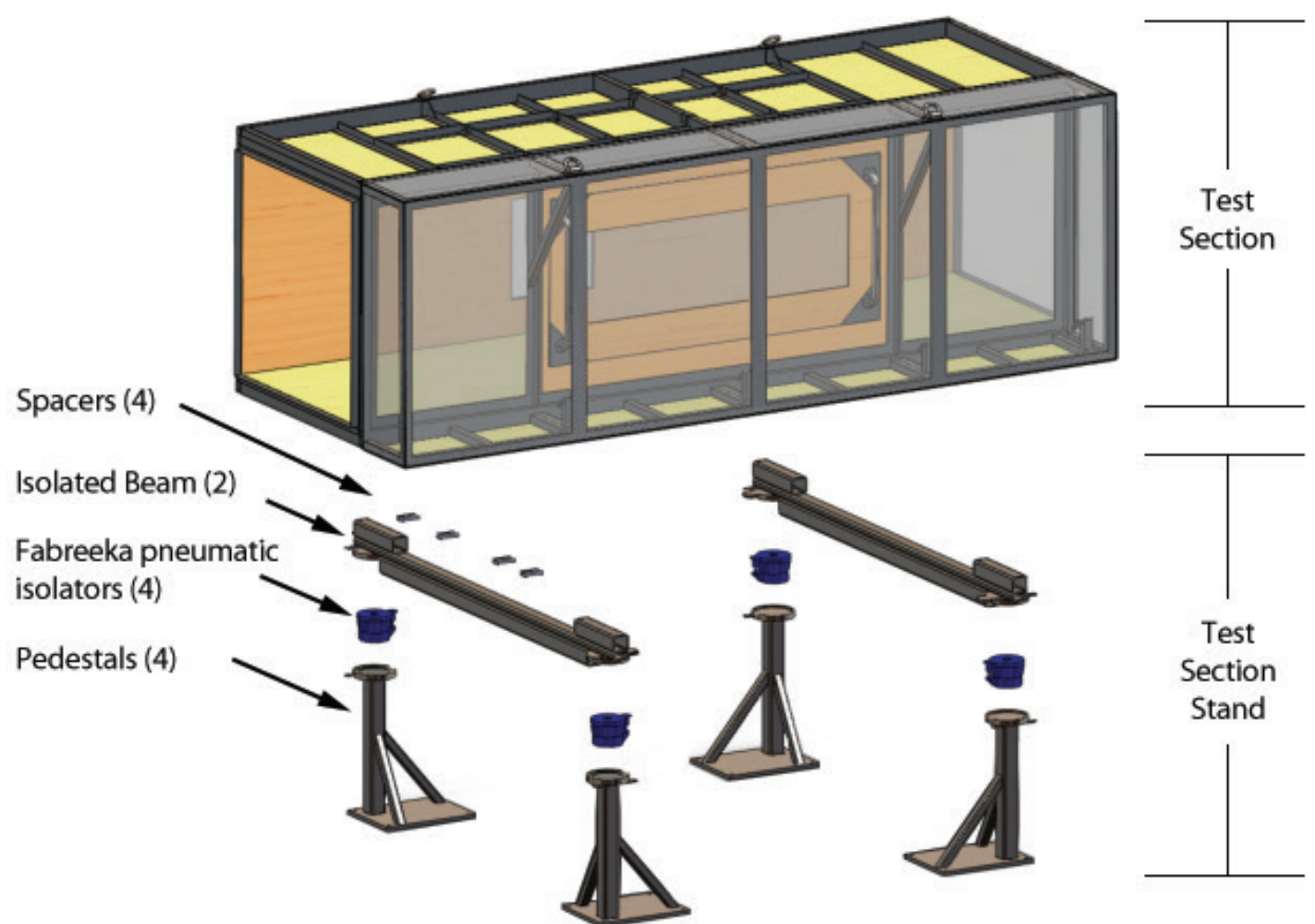

Figure 2-5. Test section assembly.

support base, upon which four Fabreeka ${ }^{\circledR}$ Precision-Aire ${ }^{\mathrm{TM}}$ PAL 21 pneumatic isolators, two isolator beams, four spacers and the test section rest. The pneumatic isolators are intended to prevent transmission of structural vibration from the ground or other tunnel 
components to the test section. Minimizing vibration is critical for stability experiments, such as those involving the Tollmien-Schlichting instability, where leading-edge vibrations can act as a receptivity site (Saric 2007). Similarly, flanges on both streamwise ends of the test section do not rigidly connect to the contraction cone and diffuser to avoid another transmission source. The four spacers in Figure 2-5 are located only on the upstream isolator beam and are used to properly level the sloping floor. Beyond the pedestals, which are anchored to the floor, none of the other test section components are attached to each other. The reasons for this are two-fold. First, there are actually two interchangeable test sections that allow for continuous tunnel operation. While the first is in use, the second can undergo model installation and preparation or removal. Model installation and alignment is often an extended process; having two test sections minimizes tunnel downtime. Historically, one test section has been used for swept-wing studies whereas the second was used for flat-plate experiments. The interior dimensions and entrance and exit flanges for the two test sections are identical. There are, however, small differences in access panels and steel support structures used for model mounting and access. Secondly, mating the isolator beams to the test section increases the total lifting weight for the overhead crane used to move the components. With models installed, the test section alone can be very close to the maximum two-ton limit on the crane. A series of three interchangeable windows are available for use depending on the given test: a glass window for optical access, a solid wood panel with slots for infrared cameras and a traversing mechanism used for detailed boundary-layer scans. Details on the traverse, which is used extensively in these measurements, are 
provided in Section 2.2.1. Venting occurs approximately $2.54 \mathrm{~m}$ (100 in) downstream of test section, resulting in a test section that is slightly below atmospheric pressure during tunnel runs.

The Buffalo Forge (now Howden Buffalo) nine-bladed, adjustable-pitch-vaneaxial fan is located downstream of corner two on the return leg of the tunnel. It is powered by a 150 HP, 1750 RPM Emerson Industrial Controls direct-current, variable-RPM motor through a belt-drive system. Maximum RPM with the fan installed is 1300. Current motor control is approximately \pm 1 RPM. An unpublished study at ASU examined the relationship between blade pitch and turbulence intensity in the tunnel. The optimal setting for tunnel efficiency does not necessarily correspond to the lowest turbulence levels. Current pitch settings are the same as those selected at ASU and are a compromise between reducing disturbances and maintaining preferred test section speeds. The fan housing also includes eleven stators just downstream of the blades that help remove swirl produced by the fan and a nacelle that promotes flow recovery downstream of the stator hub. Figure 2-6 shows the upstream and downstream sides of the fan. The nacelle also covers the power transmission cartridge (PTC) that includes the belt pulleys and shaft thrust bearings in the belt drive system. The fan housing is sealed around the belts via fairings made of sheet metal and shaped like NACA airfoils. They span from the fan housing up to the PTC and are shown in Figure 2-6. In addition to sealing the tunnel, the fairings eliminate additional swirl that would have been introduced into the freestream by the rotating belts. Similar to the test section assembly, streamwise ends of the fan housing are not rigidly connected to the upstream and 
downstream components to minimize transmission of vibration from the motor to the tunnel. The motor sits below the fan housing in a steel frame (Figure 2-7). A wood enclosure internally coated with acoustic foam surrounds the frame to minimize acoustic disturbances that might otherwise impact the freestream environment. The motor support design also allows for inclusion of the same pneumatic isolators used on the test section, should the need arise. To-date, that option has not been utilized.
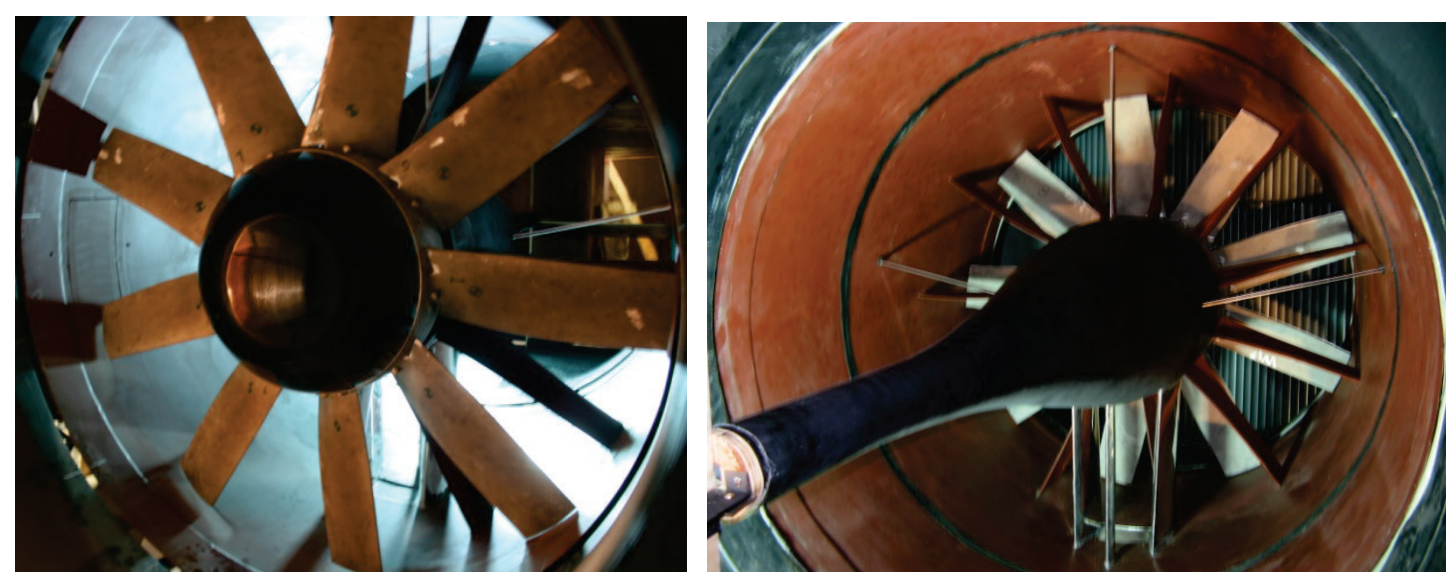

Figure 2-6. Upstream (left) and downstream (right) views of fan housing. 


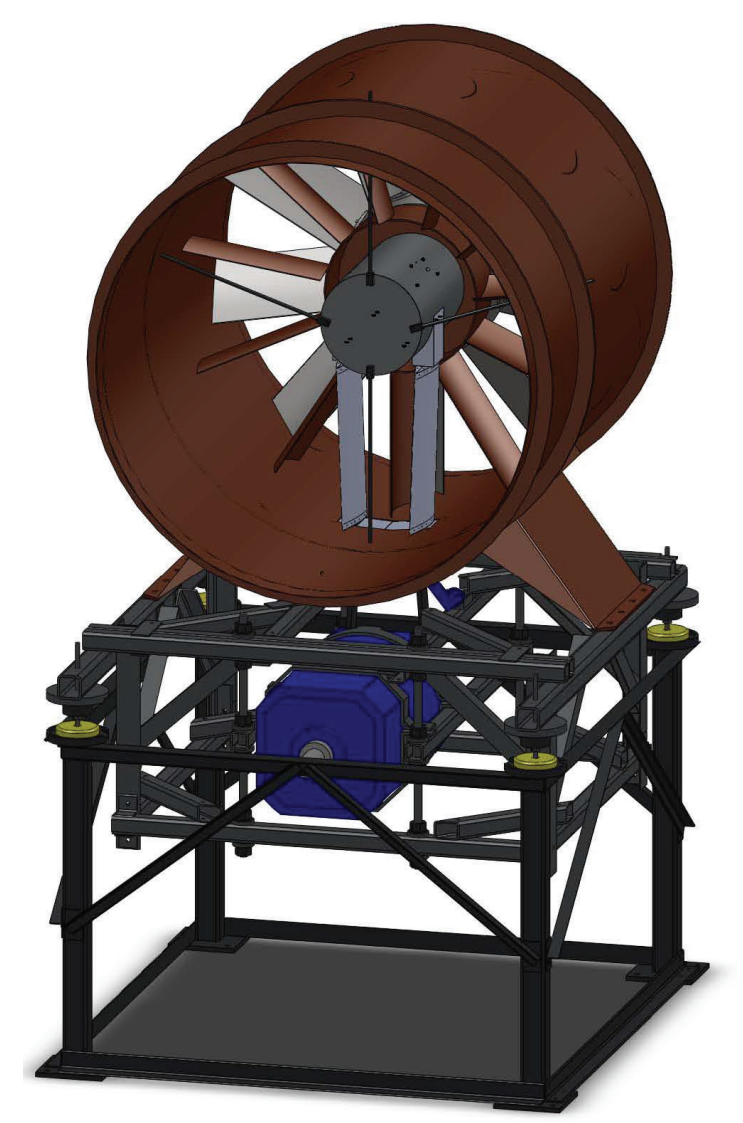

Figure 2-7. Motor and fan assembly (note: motor enclosure and nacelle not pictured).

The main and extended diffusers have $12^{\circ}$ diffuser angles, whereas the first stage diffuser has a $10^{\circ}$ angle. In all three instances, the larger angles are susceptible to separation. Barlow et al. (1999) recommend that the effective cone angle not exceed $5^{\circ}$ to avoid separation. Reshotko et al. (1997) recommend a half angle less than $3^{\circ}$. A partial splitter plate and two screens were installed in the main diffuser at ASU after discovering a separated zone at the downstream end of the diffuser during flow-quality testing. During the rebuild at TAMU, the diffuser was checked again, with the same outcome. As a result, the screens and splitter plate were once again included to provide 
better pressure recovery. The splitter plate also helps break-up large scale turbulence from the fan. Using screens in high-velocity regions of the tunnel is another example of the trade-off in design between flow quality and tunnel efficiency. The pressure drop across the screens helps keep the boundary-layer attached to the tunnel walls, but also results in power losses. Diffuser dimensions and shapes at TAMU were constricted by legacy components from ASU and NBS as well as the available building space. As a partial solution to pressure fluctuations measured during the flow quality measurements (see Section 5.2), full-length splitter plates were installed in all three diffusers, reducing the effective diffuser angle by half in each case.

The plenum section is covered in two different acoustic treatments. Thirty-seven Modex $^{\mathrm{TM}}$ Broadband plates from RPG Diffusor Systems are mounted in the plenum. Many of the instabilities studied in the KSWT have passbands between $50-400 \mathrm{~Hz}$. Using a combination of pistonic vibration, damped bending modes and porous absorption, these broadband absorbers are effective in the $50-5000 \mathrm{~Hz}$ range. Moreover, maximum panel depth is $0.1 \mathrm{~m}$ (4.25 in), making it an ideal solution for the space constrained tunnel. Figure 2-8 illustrates the panel layout in the plenum. Panel layout is designed to cover as much surface area as possible and to locate panels as close as possible to corners where low-frequency content is often present to obtain maximum benefit from the absorptive capabilities. 

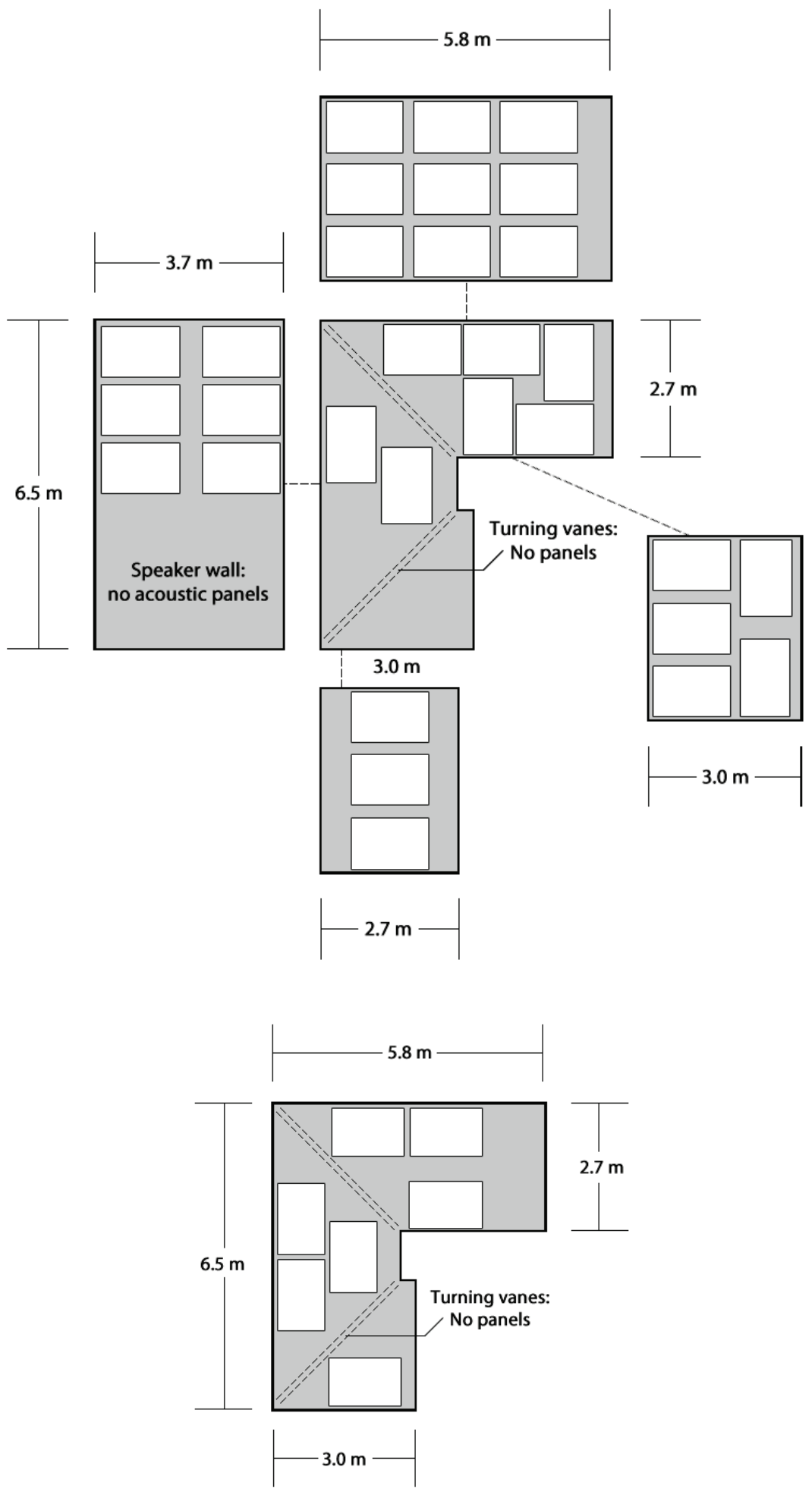

Figure 2-8. Acoustic panel layout; side-walls and floor (top), ceiling (bottom). 
Dense foam (PL1625LNA) from dB Engineering with a depth equal to the Modex plates and an absorptive range primarily above $500 \mathrm{~Hz}$ surrounds the panels, acting as a secondary acoustic treatment and providing a means to eliminate steps along the tunnel walls. Duct Mate turning vanes with a perforated front panel and absorbent lining are located in corners 1, 3 and 4.These turning vanes are not expected to remove lowfrequency content, but rather lower the overall background noise levels. Turning vanes in corner two were not replaced. The upgrade would have required a complete reconstruction of the contoured foam that provides the contraction from the rectangular duct to the circular fan housing. Three other Modex plates and accompanying foam are located in corner one to absorb noise propagating in the upstream direction, which can also affect the transition process (Saric et al. 2002).

The remaining components of the test leg are critical to flow uniformity and reduction of turbulence levels. A Hexcel aluminum honeycomb, which reduces flow angularity and turbulent scales, is located $0.3 \mathrm{~m}$ (12 in) downstream of corner four and has an $L / D$ of 12 . Each hexagonal cell is $6.35 \mathrm{~mm}(0.25 \mathrm{in})$ in maximum width. A series of seven, tensioned screens is located $0.91 \mathrm{~m}$ (36 in) downstream of the honeycomb to further reduce turbulent scales and increase flow uniformity. Each screen measures $2.74 \mathrm{~m} \times 3.66 \mathrm{~m}(9 \mathrm{ft} \times 12 \mathrm{ft})$ in cross-section and is spaced $0.23 \mathrm{~m}(9 \mathrm{in})$ from the previous screen. All are woven from $0.165 \mathrm{~mm}(0.0065 \mathrm{in})$ diameter stainless steel wire on a 30 wire/inch mesh giving an open-area ratio of $65 \%$. The ASU configuration included five butt-welded and two seamless screens from Albany International in Wisconsin. During the move from ASU to TAMU, two of the original seven (one 
seamless and one welded) were damaged during transport. It can be expensive and difficult to obtain seamless screens for large ducts. However, Watmuff $(1998,2006)$ notes that seemingly small freestream nonuniformities produced by tunnel screens can impact the resulting boundary-layer stability. After an extensive search, seamless replacements were ordered from Dorstener Wire Tech and manufactured in Germany. The new screens are woven to ISO 9044:1999 and ASTM E 2016-06 standards. These standards do allow for a certain number of defects in each screen including pin holes and creepers. An additional specification for the screen order required that no pin holes be present. The current TAMU configuration includes three of the original butt-welded screens from ASU followed by four seamless screens (three new ones and one ASU original). Figure 2-9 shows an example of the welded screens. The spacing of the honeycomb and screens from the ASU configuration was retained at TAMU and is based on distances necessary for adequate turbulence-wake decay. For tensioning purposes, each screen has a steel frame with nuts welded to the edge of the top and south sides. Connecting studs are torqued between $15-25$ in-lbs. False walls extend from screen to screen, eliminating nonuniformities introduced by the frame. 


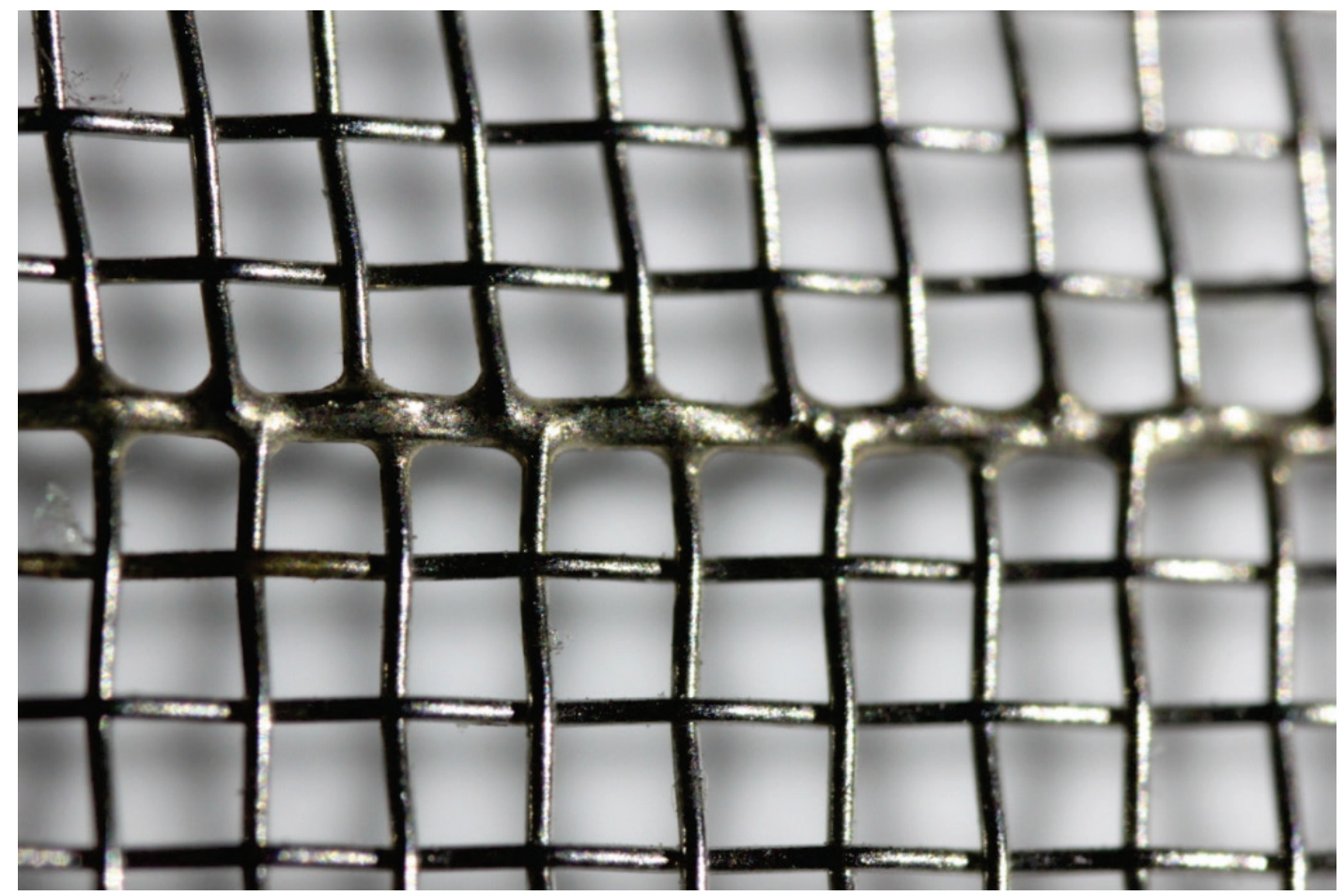

Figure 2-9. Example of butt-welded seam on first three screens.

A settling chamber $2.2 \mathrm{~m}(7.2 \mathrm{ft})$ in length is located after the last screen, followed by the contraction cone. In the settling chamber, viscous dissipation acts to further reduce disturbance energy. To minimize mismatched turbulence intensities, the contraction ratio is 5.33. A fifth-degree polynomial shape that exhibits zero-slope and zero-curvature at the entrance and exit and an $L / D$ of 1.25 was chosen to minimize the possibility of a separation bubble that could introduce a pressure fluctuation in the tunnel. Tan-Atichat et al. (1980) provide extensive guidance on contraction cone design as it relates to freestream turbulence. 


\subsubsection{Differences Between ASU and TAMU Configurations}

Approximately half of the ASU tunnel components were moved to TAMU. It was more cost effective to rebuild sections such as the plenum and extended diffuser than to carefully disassembly and move them. In these instances, the same interior dimensions were preserved. However, ASU operating experience and flow quality measurements (Saric et al. 1988, Saric 1992) indicated that there were four distinct components that would benefit from a redesign during the TAMU reconstruction effort.

A significant portion of the ASU streamwise velocity fluctuations were comprised of a low-frequency pressure fluctuation. Two locations were suspected sources of separation regions or bubbles resulting in the low-frequency content: the large diffuser angle in the merging duct just downstream of the test section and in the steep contraction from corner one to corner two. The original design of both regions was based on an effort to accommodate both the steady and unsteady operating modes. With the secondary duct sealed (steady-configuration), the diffuser angle in the merging duct was $23^{\circ}$, well above the recommended angles for keeping flow attached. In the TAMU design, the shutters and merging duct are no longer present. The first stage diffuser has been reshaped to reduce the diffuser angle to $10^{\circ}$. A splitter plate in the component further reduces it to $5^{\circ}$. Reducing the diffuser size also condensed the corner 1 crosssectional area. The contraction ratio decreased from 2.2 to 1.4 going into corner two. Additionally, contraction shape was changed to a fifth-degree polynomial on all surfaces, similar to the contraction cone design. The third modification resulted from a small pocket of increased disturbance levels measured on the non-test side of the test 
section at ASU. The suspected source was poor flow recovery around the sharp interior turn between corners three and four. To remedy the situation, the tunnel width was

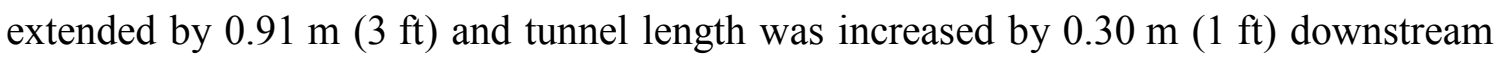
of corner four. Finally, the motor was relocated outside the fan housing altering the motor-fan configuration from a direct-drive to a belt-drive. The primary motivation for this modification was to reduce turbulence and improve experimental control. Without a tunnel cooling system, the hot turbulent jets introduced from the air-cooled motor resulted in large temperature swings during extended tunnel runs. Multi-hour hotwire scans at chord-Reynolds numbers upwards of $3.2 \times 10^{6}$ were not often completed at ASU due to the extensive tunnel heating, but are now regularly achievable. Removing the motor and enclosing it in an acoustically lined box also reduced a source of noise in the tunnel.

Beyond the major tunnel modifications, significant upgrades were also implemented for almost every remaining component. The pneumatic isolators described in the previous section are replacements for the isolated concrete foundation at ASU. In the current set-up, transmission of ground vibrations to the test section should be further reduced. Similarly, the acoustic panels in the plenum and in corner one replaced $0.08 \mathrm{~m}$ (3 in) foam common to sound studios that would have been most effective in the kilohertz range. The turning vanes, new screens and splitter plates described in Section 2.1.3 are also upgrades to the ASU arrangement. Flow quality measurements, including a comparison of ASU and TAMU turbulence levels, are provided in Section 5.2. 


\subsection{Computer Systems and Instrumentation}

Freestream static pressure, dynamic pressure, temperature and all test data are collected using three National Instruments (NI) USB-6211 data acquisition (DAQ) boards. Simultaneous use of each board provides a collective channel set of: 24 analog differential inputs, 6 analog outputs, 12 digital inputs and 12 digital outputs. Each 16-bit board is multiplexed with an aggregate sampling rate of $250 \mathrm{kHz}$. All of the KSWT tunnel control and acquisition systems are completely automated. The previous ASU arrangement used GPIB communication protocols for all DAQ hardware, traverse and motor controls. In that configuration, for example, tunnel and acquisition commands could only be performed in series. The current system uses serial lines for the traverse and motor controls and USB connections for the DAQ hardware. Changes in tunnel operation or traverse movement and data acquisition can now be performed in parallel. Test parameters are entered into a console window the tunnel operator monitors throughout the run. Using the collected data, the motor RPM can be set to maintain constant velocity, Reynolds number or fan speed. Tunnel control and signal acquisition programs are all in-house routines written in $\mathrm{C}++$ by students Brian Crawford and Robert Downs.

The Pitot-static tube is unit PAE-12-M-W from United Sensor and is located 9" downstream of the test section entrance. It connects to a MKS Baratron 1000-torr absolute pressure transducer (390HA-01000SP05) and MKS 270B signal conditioner for tunnel static pressure and a MKS 10-torr differential pressure transducer (698A11TRA) and MKS 670 signal conditioner for dynamic pressure. The accuracy of each pressure 
transducer is $0.05 \%$ of the reading. An Omega RTD (PR-11-2-100-1/8-9-E) is mounted three inches below the Pitot-static tube. It acquires freestream temperature with an accuracy of $0.15^{\circ} \mathrm{C}$. Using these components, uncertainty associated with a given freestream velocity measurement is $0.1 \mathrm{~m} / \mathrm{s}$. For the velocity ratio measurements (see Section 4.1.1), an additional MKS Baratron 10-torr differential pressure transducer (398HD-00010SP05) and 670 signal conditioner are used.

Since the reconfiguration from direct to belt-drive resulted in a significant design change from the manufacturer's motor design, an additional monitoring system is included during tunnel operations. Referred to as the Health and Status (H\&S) system, it acquires temperatures from thermocouples located on the fan shaft bearings, motor support frame and motor casing, while a RTD measures ambient temperature in the motor enclosure. A separate pressure transducer monitors the regulated pressure feeding the pneumatic isolators ensuring that test section vibration control is still active. Two permanently installed accelerometers attached to the PTC frame and stator hub can also be connected to the program to observe real-time vibrations in the fan housing. 
This diagnostic tool may help indicate changes in flow quality related to fan and motor performance. All of the measuring devices connect to a NI SC-2345 signal conditioning unit and a NI PCI-6221 DAQ board. All voltage signals are sent to a custom-built program in LabVIEW. During a given tunnel run, these parameters are monitored and acquired as needed. Warning lights appear when a given temperature or pressure is out-of-range. Figure 2-10 shows block diagrams for the main tunnel control and $\mathrm{H} \& S$ hardware.

Only the instrumentation and hardware needed for this experiment are included in this section; however, both the KSWT and FRL share a large inventory of instrumentation needed for boundary-layer stability and transition measurements. A comprehensive listing of the full inventory is provided on the joint laboratory website http://www.flight.tamu.edu. There are three main types of measurements used in this study: pressure coefficient, naphthalene flow visualization and streamwise boundarylayer velocity profiles. The instrumentation associated with each is described below. 

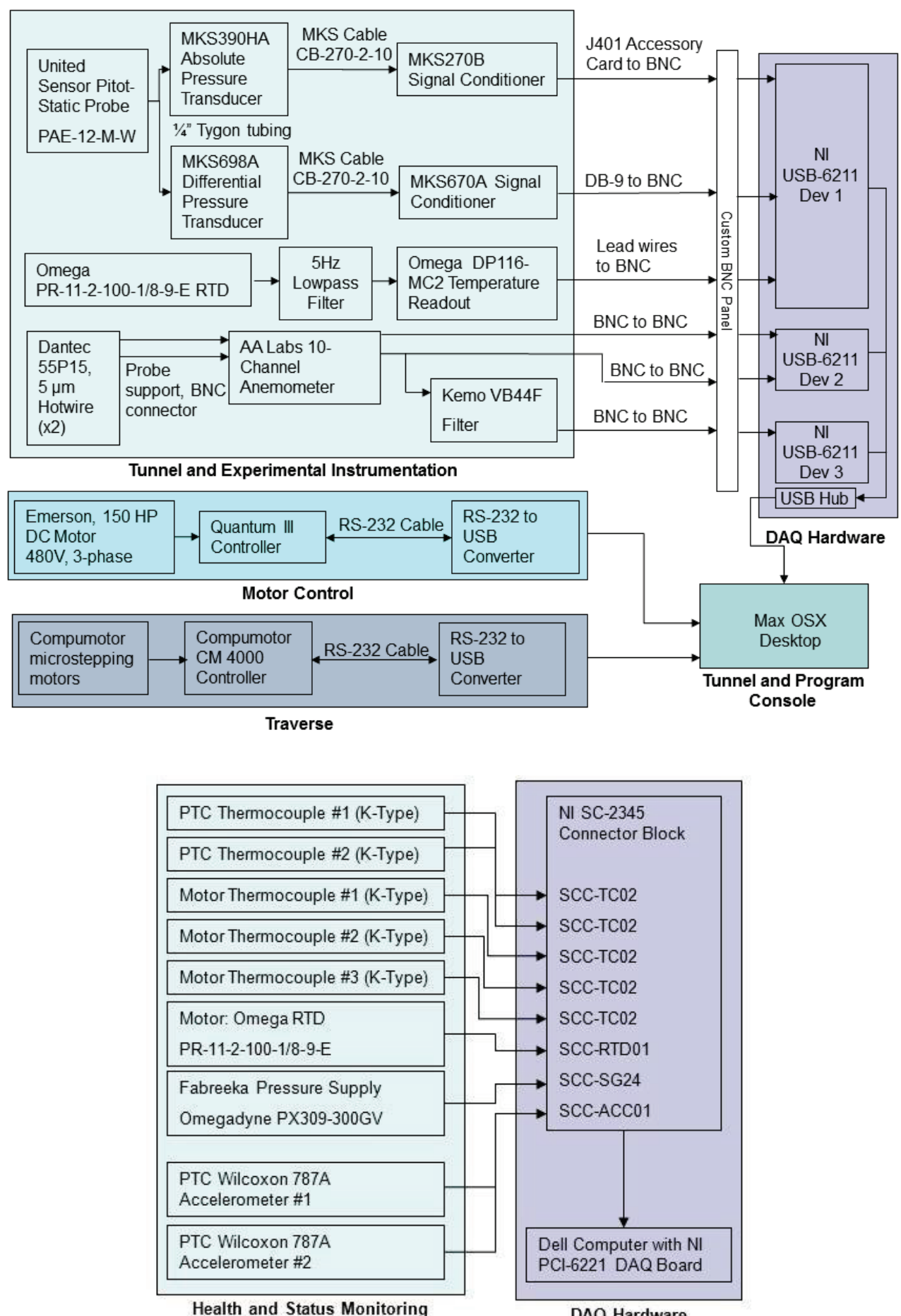

Health and Status Monitoring

DAQ Hardware

Figure 2-10. Hardware diagrams for tunnel hardware (top) and H\&S system (bottom). 
Pressure coefficient distributions on the test side of the model are obtained using a Pressure Systems Electronic Pressure Scanner (32HD-0102001000). The ESP-32HD unit has 32-channels that provide simultaneous measurement of one row of pressure ports. Since the ASU(67)-0315 model has two rows of pressure ports, the tubing is switched from one row to another between measurements. The reference pressure for this unit is directly tied to the static pressure line from the Pitot-static tube listed above. The range and accuracy of this unit is +/- 10 in-WC (water column) and 0.02 in-WC respectively. This is the only piece of experimental instrumentation that is not directly acquired through the main computer console. Use of the EPS scanner comes with proprietary software needed to convert the pressure transducer voltage signal to a meaningful pressure difference. In this instance, a separate computer was used to acquire the pressure data and the acquisition timestamps were matched to the tunnel control logs for final calculation of the pressure coefficient.

Beyond the source materials of naphthalene and acetone, the only instrumentation used for the flow visualization technique is a Canon EOS Rebel T1i camera and two external flashes, which provide an oblique source of lighting. The purpose and application of this measurement technique is provided in Section 4.2.

Boundary-layer velocity profiles are obtained using two channels from an AA Labs 1003 Anemometer. These units have a standard frequency response of $\mathrm{DC}-300 \mathrm{kHz}$. Dantec Dynamic 55P15 boundary-layer probes are connected to $4 \mathrm{~mm}$ Dantec probe supports $(55 \mathrm{H} 21)$. To obtain stationary crossflow initial disturbance amplitudes, the primary measurement of interest is the mean velocity profile (DC-component of the 
hotwire signal). However, the boundary-layer hotwire voltage is also tied to a Stewart VBF44 filter and amplifier for more resolved AC signal measurement. The signal conditioner has two lowpass and two highpass channels or a total of two bandpass channels with a cutoff frequency range of $1 \mathrm{~Hz}-255 \mathrm{kHz}$ in four decade ranges and a gain range of -20 to $70 \mathrm{~dB}$. Freestream turbulence measurements use both the Dantec Dynamics 55P15 straightwires and 55P61 crosswires. In both cases, wire diameter is $5 \mu \mathrm{m}$.

Surface roughness and roughness array shape are both tracked. A Mitutoyo SJ-400 Surface Roughness tester measures surface roughness, waviness and steps with a 1.25 $\mathrm{nm}$ resolution. This instrument is used for all surface roughness measurements on polished aluminum surfaces. A Keyence VK-9700 Violet Laser Color 3D laser scanning microscope provides roughness height and surface topology for the appliqué DRE. The system has a $1 \mathrm{~nm}$ resolution with a $0.014 \mu \mathrm{m}$ repeatability.

\subsubsection{Traverse and Sting Mount}

Detailed boundary-layer scans on arbitrary shapes using hotwire anemometry are made possible through use of a high-precision traverse that is capable of simultaneous movement along three axes. The bulk of the traverse system is externally mounted to the test section frame to minimize blockage effects, which may influence the local and global pressure fields near the airfoil. A total of four lead screws drive the traverse in the streamwise, wall-normal and spanwise directions. Two stainless steel rails support a carriage driven by a single lead screw in the streamwise direction. The carriage, in turn, 
supports two lead screws for spanwise movement and one for wall-normal movement. Figures 2-11 and 2-12 show front and side views of the carriage. Compumotor microstepping motors with a resolution of 50,000 steps per turn are connected to the lead screws. 1000-line Renco digital encoders track position, while a Compumotor CM4000 4-axis microprocessor controls the motors. Total travel and minimum step-sizes are provided in Table 2-1. With hotwires $5-\mu \mathrm{m}$ in diameter and $1-\mathrm{mm}$ in length, the minimum steps in the wall-normal and spanwise directions are a measure of the traverse resolution.

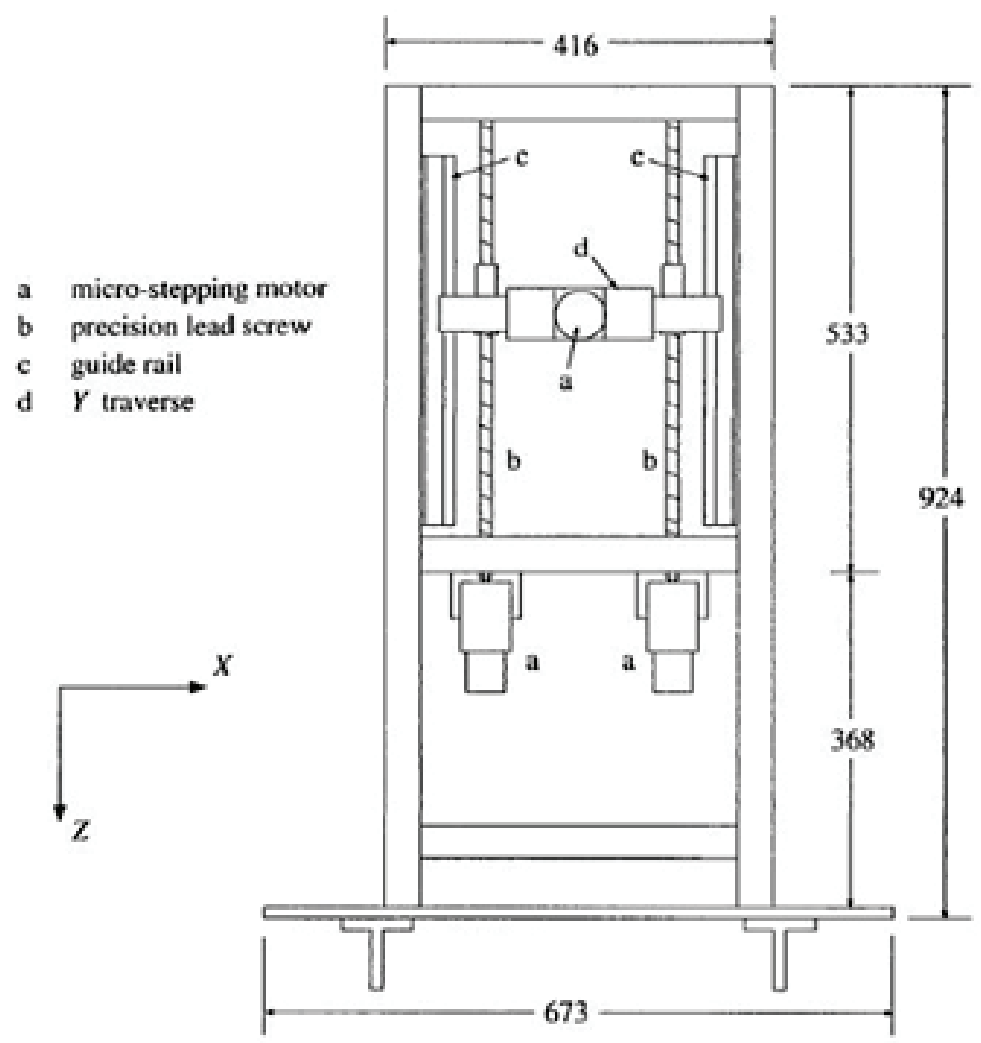

Figure 2-11. Front view of traverse carriage. Dimensions in mm (White 2000). 


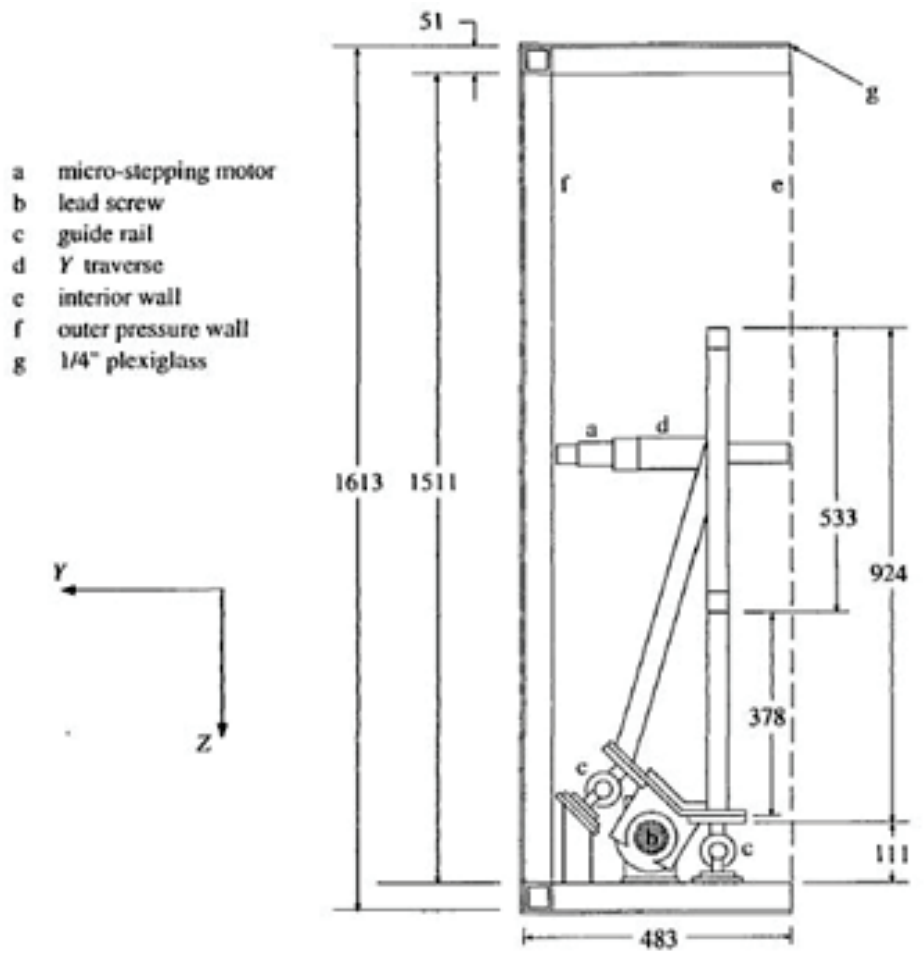

Figure 2-12. Side view of traverse carriage. Dimensions in $\mathbf{m m}$ (White 2000).

Table 2-1. Traverse travel and step size.

\begin{tabular}{|l|c|c|c|}
\hline & $X$ (streamwise) & $Y$ (wall-normal) & $Z$ (spanwise) \\
\hline Total travel $[\mathrm{mm}]$ & 1250 & 100 & 175 \\
\hline Minimum step $[\mu \mathrm{m}]$ & 11.9 & 0.64 & 1.27 \\
\hline
\end{tabular}


Bolted to a carriage platform (component $d$ in Figure 2-12), the sting mount is the only traverse component exposed to the test section interior. It enters through a zippered acrylic slot, which closes the opening on either side of the sting as the traverse moves in the streamwise direction. The entire acrylic window moves in unison with the traverse spanwise motors to further minimize the required slot opening. The entire traverse mechanism is enclosed in a pressure box made from Plexiglas bolted to the test section frame. This prevents a secondary source of tunnel venting by eliminating mass flow through the zipper opening. Figure 2-13 shows a plan view of the sting. The streamlined body is shaped like a long thin tapered airfoil and made from aluminum and carbon composite to help minimize structural or aerodynamic induced vibration, which can introduce erroneous readings in the $\mathrm{AC}$ component of the hotwire measurements. Two $4 \mathrm{~mm}$ diameter hotwire probe supports are mounted to the sting. When both probe supports are aligned with the streamwise direction, the hotwire tips are separated by a distance of $170 \mathrm{~mm}$. The boundary-layer probe mount can rotate in the $X$ - $Y$ plane to account for local surface curvature on the model. With hotwires installed, the probe tips extend upwards of $114 \mathrm{~mm}$ in front of the sting. Originally designed at ASU, this is the same traverse used in experiments at the UWT from 1994 onward. More detailed information on its design can be found in Radeztsky (1994) and Reibert (1996). 


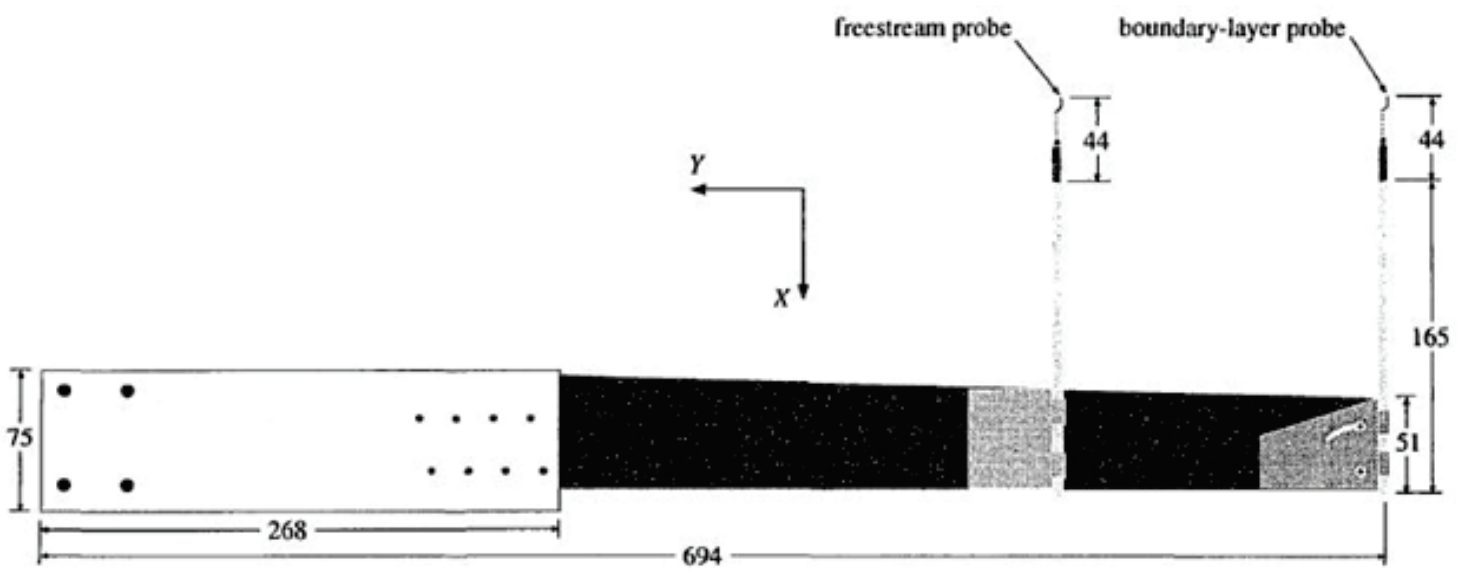

Figure 2-13. Plan view of sting mount. Dimensions in $\mathrm{mm}$ (White 2000).

The Compumotor microprocessor connects to the tunnel control computer through a serial cable. The tunnel operator need only supply a desired position, after which, the Compumotor microprocessor handles all communication with the individual motors. Although the traverse can be manually positioned for a given measurement, most of the traverse movements are automated through boundary-layer scanning programs that integrate the hotwire signal acquisition and traverse movement commands. In these instances, the tunnel operator provides a set of input parameters for a given set of boundary-layer scans and the scanning programs dictate all traverse movements until the routine is complete. Details of the hotwire measurement techniques are provided in Section 4.1. 


\section{EXPERIMENTAL CONFIGURATION AND DESIGN}

\subsection{Fundamental Requirements for Stability and Transition Experiments}

Stability and transition experiments require, to the extent possible, a configuration conducive to conducting well controlled and well documented measurements. Indeed, the planning and set-up involved in such a process is as often as intricate as the data collection itself. It is for this reason that the Boundary Layer Transition Study Group (Reshotko 1975) identified four essential elements to a well posed experiment (Reshotko 1976):

1. Identification and avoidance (if possible) of facility dependent characteristics

2. Detailed reporting of model design including surface conditions, materials and internal structure that may affect the transition process

3. Experimental and computational identification and understanding of disturbance coupling

4. Measurement redundancy (e.g. same experiment conducted in several tunnels to eliminate facility dependent results)

Implementation and documentation of these guidelines is intended to help identify what aspect of the results is significant to the transition process itself rather than an artifact of the experimental design. Section 2 addressed some of the issues related to facility selection. Details on the flow quality of the KSWT used in this experiment are provided in Section 5.2. The next section describes model design. One of the unique aspects of this experiment is the ability to compare experimental trends between the tunnel 
configuration at ASU and TAMU. There are many similarities in experimental design such as the same model and measurement techniques. At the same time, the turbulence levels in the tunnel are not equivalent. Interestingly, previous experiments have examined the effect of changing freestream turbulence, but in these studies, the turbulence levels were high enough to cause transition dominated by traveling waves. This is one of the first experiments where trends based on different, but extremely low turbulence levels can be compared. Section 5.2.3 provides a comparison of freestream turbulence levels from ASU and TAMU.

Beyond the attention to factors that influence the stability characteristics, there are several desirable configuration features from both an experimental and computational standpoint that make interpretation of the results easier. Model geometry should isolate the instability of interest, but at the same time be as simple as possible unless there is evidence that it affects the stability. For crossflow studies, using a swept-wing rather than a flat plate with a wall bump ensures that the effect of curvature is taken into account. Conversely, features such as taper are not included to help enforce a spanwise invariant flowfield. This simplifies the computations required. If taper is later found to be a significant factor, another experimental design would be required. Contoured wall liners matching the inviscid streamlines are also needed to create spanwise uniform flow. For the experimentalist, choosing conditions that promote strong crossflow instability growth, such as sweep or angle of attack, to allow for easier disturbance detection and large boundary-layers for well-resolved velocity profiles are also needed. Saric (2007) recommends at least $50-100$ measurement points in each profile to enable detailed 
comparisons with simulations. Computer modeling of the experiment may also require specification of the freestream environment for program inputs and a description of the measurement coordinate systems to properly match reference frames. The extent to which these factors can be experimentally documented helps increase the fidelity of the validation cases.

\subsection{Model}

\subsubsection{History and Design}

This experiment uses the ASU(67)-0315 airfoil for all tests. Originally designed at ASU by Mark Reibert, it is the same model used for the crossflow breakdown studies by White \& Saric (2005). The outer-mold-line (OML) is a slightly-modified version of the NLF(2)-0415 test article used in previous crossflow studies by Dagenhart \& Saric (1999), Reibert et al. (1996) and Radeztsky et al. (1999) at ASU. The design philosophy behind the choice of the NLF(2)-0415 is shared with the ASU(67)-0315; namely, provide a model that minimizes presence of attachment-line, T-S or Görtler instabilities and encourages growth of crossflow disturbances (Dagenhart \& Saric 1999, White 2000). After several years of testing on the NLF(2)-0415, the ASU(67)-0135 was fabricated to provide the same strong crossflow growth at a negative angle of attack under zero-lift conditions to simplify experimental set-up and to incorporate a modular leading edge that could accept multiple roughness inserts. Since the tests by Radeztsky et al. (1999) indicated that surface roughness was less important aft of $10 \%$-chord, the 
ASU(67)-0315 fabrication technique was also modified to incorporate lighter materials with more relaxed tolerances downstream of $20 \%$ chord, reducing model mass from approximately $725 \mathrm{~kg}$ to $350 \mathrm{~kg}$.

The ASU(67)-0315 has a $1.83 \mathrm{~m}$ streamwise constant-chord length, $45^{\circ}$ leading-edge sweep and a pressure minimum at $71 \%$ chord. Swept-airfoil coordinates are provided in Appendix A. From the leading edge to $10 \% x / c$, the model is machined out of solid aluminum. The test article is outfitted with an interchangeable leading edge that is centered at mid-span, extends to $20 \% x / c$ and is $0.762 \mathrm{~m} \mathrm{(30} \mathrm{in)} \mathrm{in} \mathrm{swept-span.} \mathrm{Also}$ machined out of aluminum, it is designed to integrate almost seamlessly with the main body. The leading edge insert also includes a smaller slot that accepts different roughness inserts, which can vary in element diameter, spacing and distance from the leading edge. These smaller inserts are generally referred to as the roughness insert. Centered at $2.9 \% \mathrm{x} / \mathrm{c}$, this smaller insert slot is $46 \mathrm{~mm}$ in chord and $716 \mathrm{~mm}$ in span. Figure 3-1 shows the test article components. 


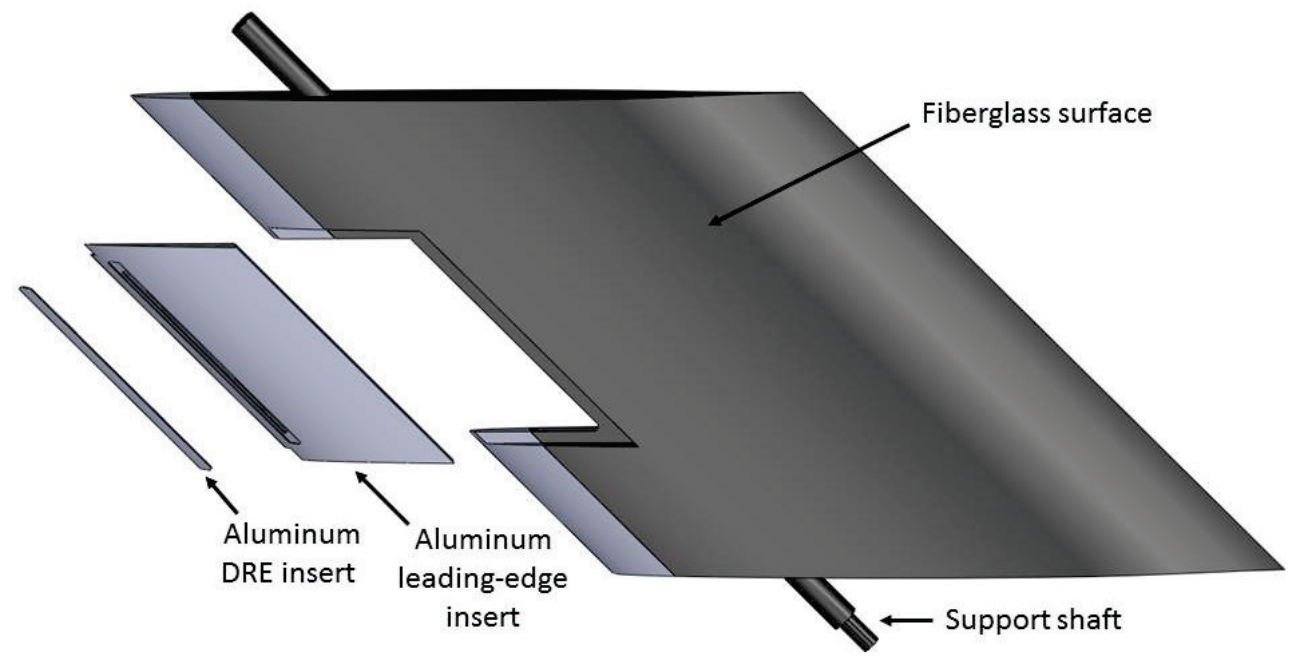

Figure 3-1. ASU(67)-0315 components.

Other tests have used pneumatic and plasma actuator inserts as the roughness source. This experiment only uses a solid aluminum insert, for both the baseline (natural surface roughness) and appliqué roughness tests. The rest of the model is comprised of a foam core and aluminum frame, covered in fiberglass. On the suction side (test side), the fiberglass surface is painted black to increase the contrast during flow visualization tests. Two rows of 29 pressure ports each, one above and one below the leading edge insert, are located on the suction side between the leading edge and $90 \% x / c$. Pressure port locations are shown in Appendix B with the pressure distribution data.

Having control over surface finish is important for all stability and transition experiments, but particularly for receptivity studies. Previous research has already established that surface non-uniformities and other sources of irregularity can introduce unintended receptivity sites, thus generating instabilities. All aluminum surfaces on the 
leading edge are first wet-sanded starting at a grit of 800 and incrementally increased to 3600. From 800 - 2000 grit, waterproof P-grade aluminum oxide sandpaper from Norton Abrasives is used. The finer sandpaper comes from the Micro-Mesh ${ }^{\circledR}$ product line from Micro-Surface Finishing Products. Use of mechanical sanding devices must be used with extreme care. Since material is removed much faster compared to hand-sanding, it is easier to unevenly remove aluminum resulting in a locally deformed airfoil geometry. The ASU(67)-0315 is manually sanded using flexible-foam-sanding blocks with each stroke extending the full span of the leading edge insert to avoid local nonuniformities. The surface is then polished first using Granitize XMP metal polish (grades medium, fine and superfine). A coat of Mother's Mag and Aluminum Polish is the final surface treatment. The extensive set of sanding and polishing steps is intended to minimize the scales involved in the background surface roughness. When these are small enough, the DRE applied to the model become the dominant roughness source. Table 3-1 shows the roughness measurements resulting from an average of fifteen measurements taken in various locations on the leading edge insert using the Mitutoyo SJ-400 Surface Roughness tester with a $2-\mu \mathrm{m}$-radius stylus tip and a range of $80 \mu \mathrm{m}$. The tester was set to P-mode, which does not filter any of the results. Prior to taking measurements on the aluminum surface, a calibration specimen was used to verify the accuracy of the roughness tester. Carpenter (2009) provides an extensive comparison of surface roughness measurements for the FRL flight tests and commercially available aircraft. 
Table 3-1. Surface roughness measurements on ASU(67)-0315 leading edge insert.

\begin{tabular}{|l|l|}
\hline RMS roughness, $\mathrm{P}_{\mathrm{q}}[\mu \mathrm{m}-\mathrm{rms}]$ & 0.3 \\
\hline Mean of absolute deviations, $\mathrm{P}_{\mathrm{a}}[\mu \mathrm{m}]$ & 0.2 \\
\hline Maximum peak-to-peak value, $\mathrm{P}_{\mathrm{y}}[\mu \mathrm{m}]$ & 2.3 \\
\hline Five-point average of the maximum peak-to-peak values, $\mathrm{P}_{\mathrm{z}}[\mu \mathrm{m}]$ & 1.8 \\
\hline
\end{tabular}

Once installed, the leading-edge insert and main body interface is covered with Bondo and sanded to eliminate junctions. Since the automotive body-filler does not begin until aft of $20 \% x / c$ in the measurement region of interest, the increased surface roughness is not expected to affect the receptivity measurements. As a side note on model preparation, the sanding and polishing supplies and techniques are the same as those used on the SWIFT flight test program (Carpenter 2009, Carpenter et al. 2010) and the profilometer is the same unit used in the current experiment. There are only two differences in the supplies and tools used. First, after sanding with the Micro-Mesh ${ }^{\circledR}$ sheets, jeweler's rouge was used to cut and polish the surface followed by the Mother's polishing treatment on the SWIFT model. Second, a $5 \mu$ m-radius tip would have been used to complete the roughness measurements.

During long-term storage and transport from ASU to TAMU, a small grouping of what appears to be oxidized divots and a small, but deep scratch appeared near the leading edge. All of the features are small scale surface defects (less than $0.5 \mathrm{~mm}$ in length or width), but they were deep enough to remain even after the extensive sanding and polishing procedure described above. Further sanding was not implemented after examining the scale of the roughness to avoid removing a large amount of material and potentially altering the shape of the leading edge. The small defects are clustered in a 
patch $270 \mathrm{~mm}$ below the top of the leading edge insert between $4 \%$ and $5.4 \% x / c$. The patch area is approximately $45 \mathrm{~mm}$ x $35 \mathrm{~mm}$. Subsequent naphthalene flow visualization did not indicate any wedges originating from this area, confirming that the small scales involved did not impact future measurements. Hotwire scans were always conducted well below this region.

\subsubsection{Mounting and Tunnel Placement}

A $76.2 \mathrm{~mm}\left(3^{\prime \prime}\right)$ diameter shaft centered at $1 / 4$-chord in the swept-direction runs through the airfoil parallel to the leading edge. The shaft center is located $0.765 \mathrm{~m}$ from the back wall in the test section (non-test side) and $0.605 \mathrm{~m}$ from the front. The model is purposely placed off-center to avoid nodes associated with large scale vortical motion in the test section (Saric 2007). Shaft distance was calculated based on measured distances from the leading and trailing edges to the non-test side of the test section. Figure 3-2 shows the model placement and measurements used to calculate angle of attack. 


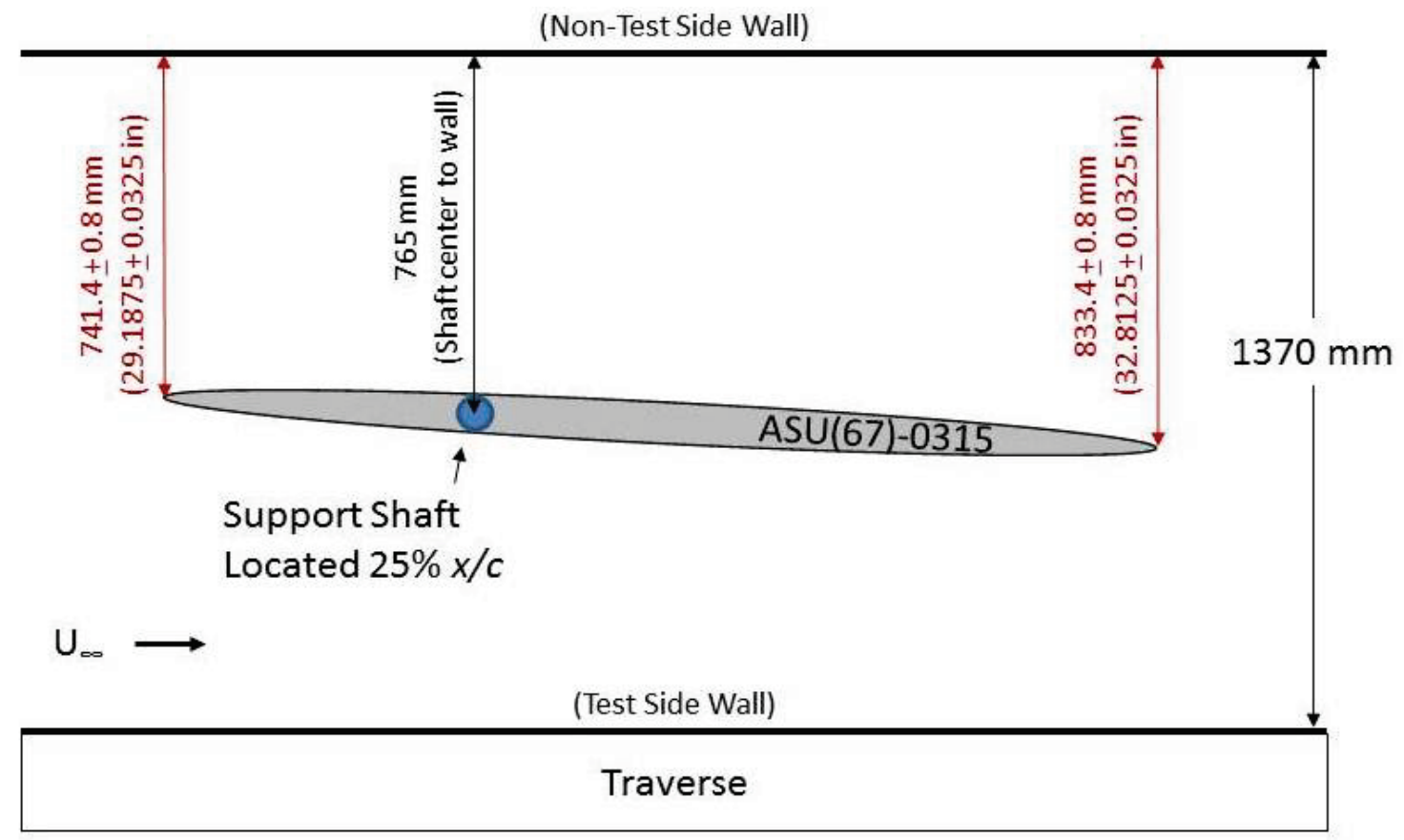

Figure 3-2. ASU(67)-0315 position in KSWT.

The steel test section frame and wood paneling can have small degree of waviness

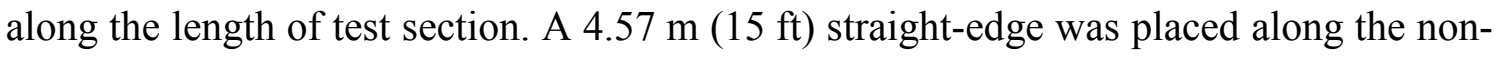
test side wall to average-out local variations in the wall. Measurements from the leading and trailing edge were all taken relative to the straight edge to obtain a more accurate angle of attack. The model is set to a constant, streamwise angle of attack of $-2.9^{\circ}$. At mid-span, the model is approximately $0.72 x / c$ downstream of the test section entrance. A thrust-bearing mounted below the test section supports the model shaft and locks the angle of attack setting. A second brace located on the top of the test section prevents model tilt and vibration. Figure 3-3 shows both support pieces. 

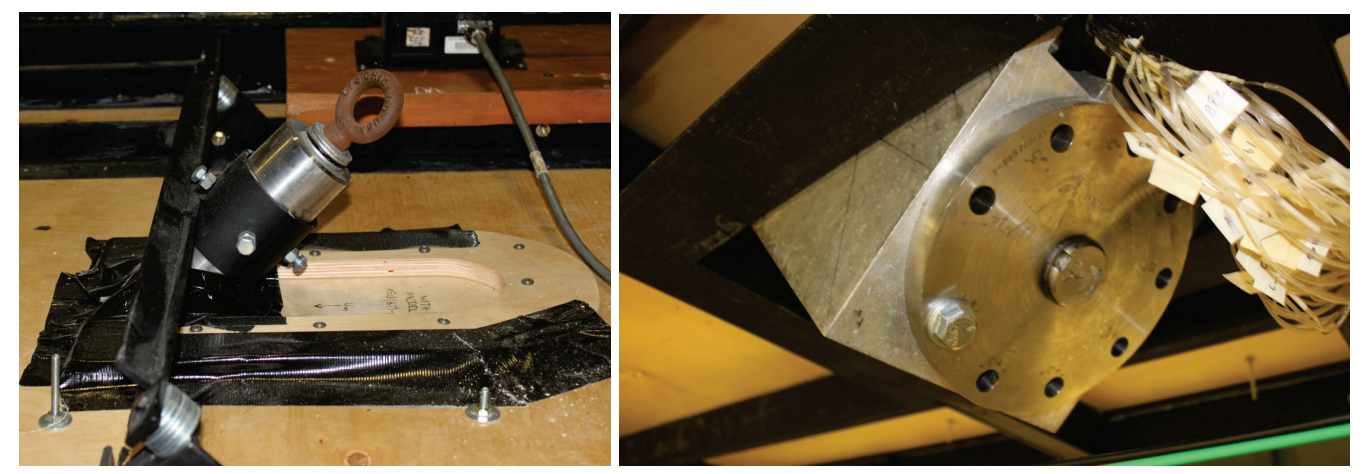

Figure 3-3. ASU(67)-0315 support brackets: top (left), bottom (right).

\subsubsection{A Note on Angle-of-Attack Selection}

White \& Saric (2005) note that the ASU(67)-0315 was designed to have a zero-lift condition at $\alpha=-3^{\circ}$ and that after a machining error, the final angle of attack was set in the tunnel to $\alpha=-3.4^{\circ}$. Zero lift is a desirable design feature for experimental set-up because it greatly simplifies wall liner construction. The streamline discontinuity that occurs between the pressure and suction sides when lift is present can be difficult to incorporate into the wall liner design. The original wall liners from ASU were not moved to TAMU. During the wall liner redesign process, it was discovered that a miscommunication between experimental and computational definitions of angle of attack resulted in a non-zero lift alignment in the tunnel. Most computational simulations of streamlines use a model-oriented reference frame (see Section 4.1.2), where $x$ is normal to the leading edge, to simplify the calculations. When aligning the model in the test section, it is more convenient to use global test section coordinates where $X$ is in the streamwise direction. Model angle of attack for these two reference frames is not the same. The zero-lift design at $-3^{\circ}$ used the reference frame where $x$ is normal to the 
leading edge. The model should have been placed at $-2.1^{\circ}$ in the test section (global coordinate system) to meet the zero-lift condition. Understanding this difference helps explain the pressure distribution offset between the computational and experimental results shown in White \& Saric (2005). The difference was originally attributed to use of an inviscid simulation which does not take into account displacement thickness associated with boundary-layer growth on the model and tunnel walls. Resolution of this discrepancy is attributed to Dr. Helen Reed and her graduate student Matthew Tufts who completed all the computational aspects for this experiment. To be clear, all chord and angle of attack references in this document are in the streamwise (swept) direction unless specifically designated as normal-to-the-leading edge.

A complete understanding of the earlier discrepancies was not known until after wall liner construction had begun. As a result, the TAMU experimental angle of attack was left at $\alpha=-3^{\circ}$ (streamwise AoA). Careful measurements indicated that the final placement of the ASU(67)-0315 mounted in the KSWT resulted in $\alpha=-2.9^{\circ}$ (streamwise AoA).

\subsubsection{Wall Liners}

Contoured wall liners attached to the tunnel floor and ceiling simulate an infinite swept-wing, avoiding spanwise variation of the basic state. Wall liner design was based on the model placement in the tunnel and a streamwise angle of attack of $-3^{\circ}$. The airfoil mesh was created using ANSYS Gambit, Version 2.3.16. Fluent, Version 12.0.16 generated the inviscid streamlines. Using first TechPlot360 and then SolidWorks 2009, 
the results were converted into solid parts for machining. The final liners were built from layers of Trymer 2000 foam, machine cut to match the streamlines, glued together and then coated in fiberglass to allow tunnel users to walk on the liners during configuration changes. Figure 3-4 shows the SolidWorks version of the liners inside the test section. Inclusion of the liners reduces the total area of the test section entrance from $1.88 \mathrm{~m}^{2}$ to $1.50 \mathrm{~m}^{2}$. The exit area is reduced from $1.94 \mathrm{~m}^{2}$ to $1.46 \mathrm{~m}^{2}$. A contraction fairing extends from the contraction inflection point to its exit to avoid any steps at the entrance of the test section. With the fairing and liners installed, the contraction ratio is increased to 6.9. Figure 3-5 shows an "as-installed" picture of the contraction fairing, wall liners and model.

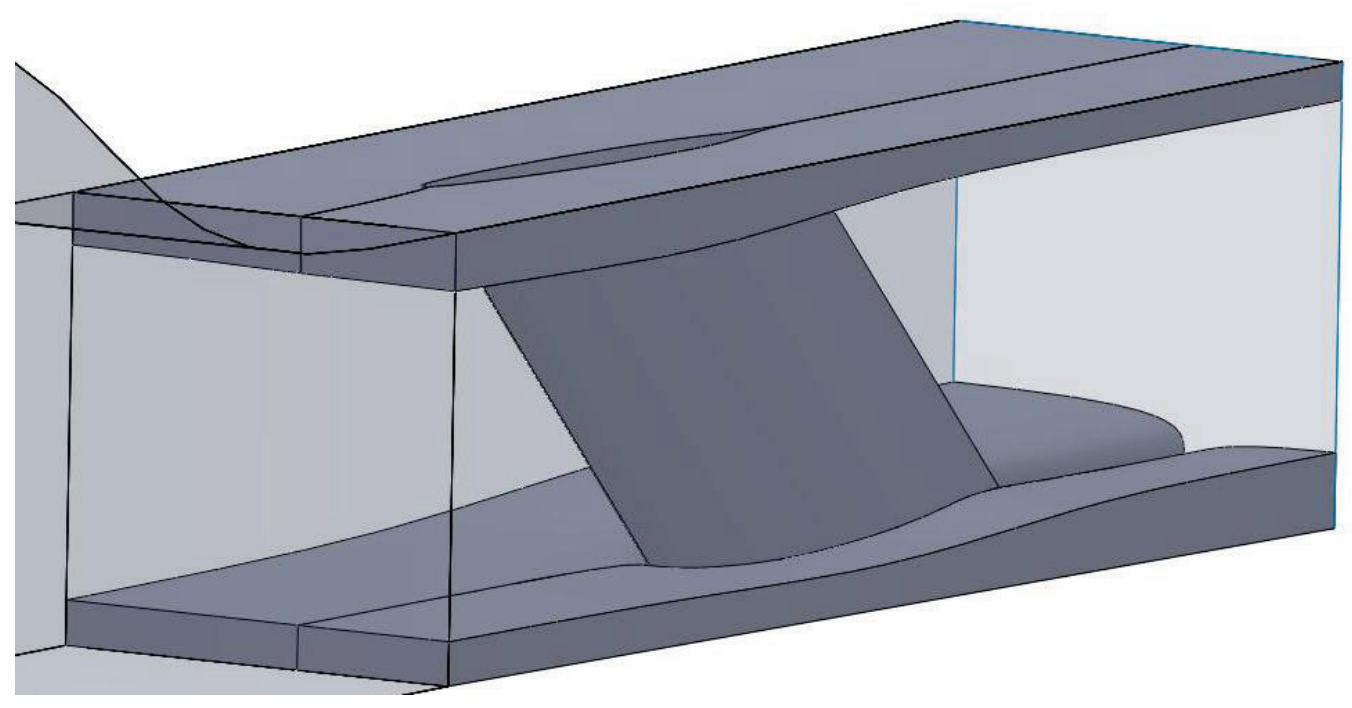

Figure 3-4. ASU(67)-0315 wall liner design for $\alpha=-3^{\circ}$ (contraction fairings not pictured). 


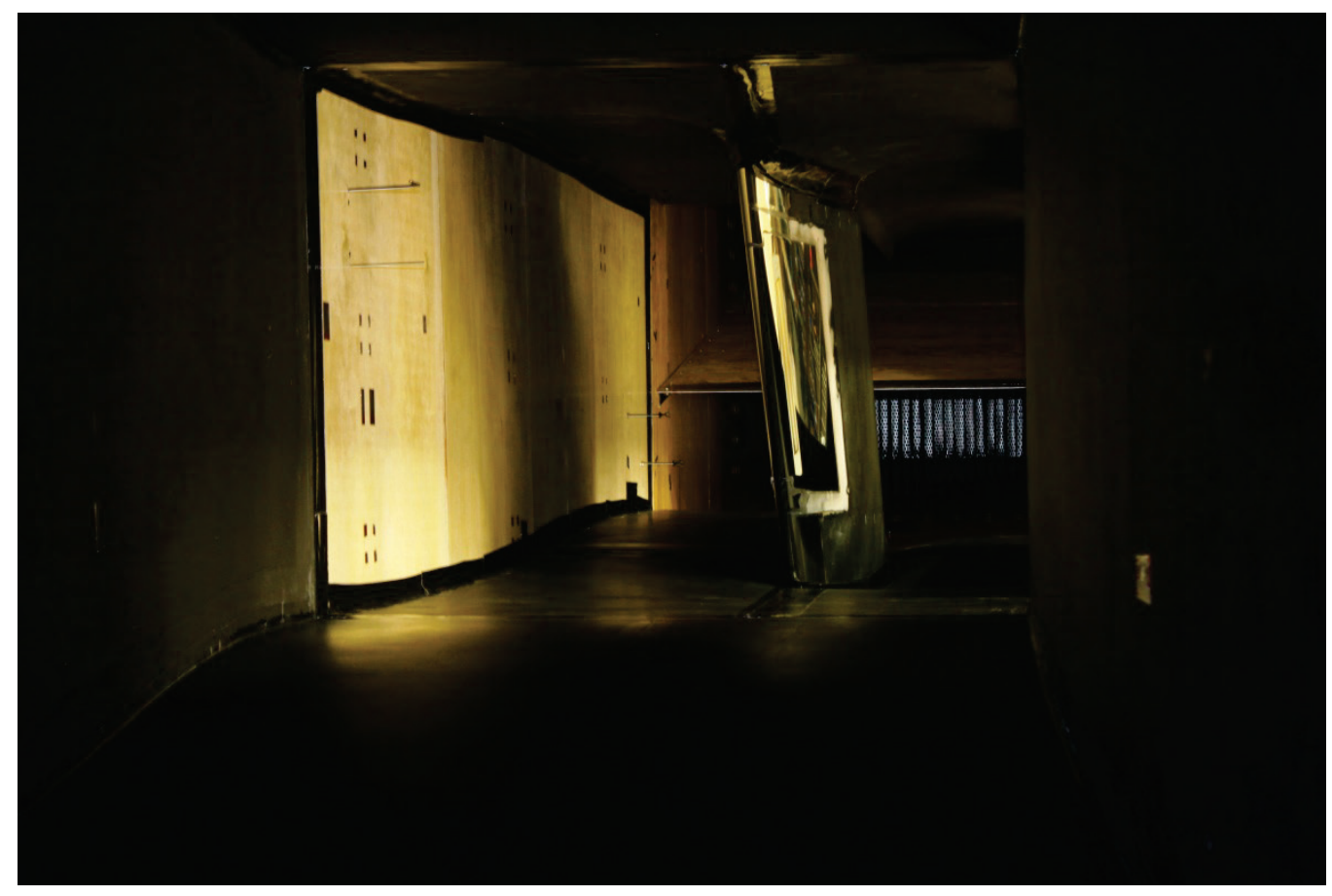

Figure 3-5. ASU(67)-0315 as installed with wall liners and fairings.

Wall liners on the side walls are not ideal for an experimental setting where optical access and measurement tools such as the traverse are needed. Instead, accompanying computational efforts account for the pressure influence on the model by including the side walls in both basic state and stability calculations.

\subsection{Roughness Arrays}

Natural surface roughness can excite all modes for the crossflow instability. Use of artificial roughness in the form of spanwise-periodic DRE simplifies the problem by limiting the experimental focus to a single or minimal set of modes, which can be more easily compared with computational results. Applying the DRE along the full span of the 
roughness insert produces an invariant disturbance field along a span of constant chord. Additionally, since the use of spanwise-periodic DRE has been used to demonstrate transition control (Saric et al. 1998), there is additional motivation for understanding how this particular roughness configuration, rather than natural surface roughness, performs. The neutral stability point for the ASU(67)-0315 is located at $0.025 x / c$ (White 2000). All roughness elements for this experiment are placed at $2.9 \% x / c$. This location was based on DRE placement for the pneumatic insert, which was machined at ASU. Measurements at TAMU show that these holes were centered at 2.9\%. Appliqué roughness was placed to match this location, providing one-to-one comparisons of roughness shape should multiple roughness types be used. Although this position is slightly off-center from the ideal roughness location at the neutral stability point, it is still within the band of effective DRE placement (Radeztsky et al. 1999).

The shape and layout of the appliqué roughness elements are designed in-house and sent to Redd Europe Inc. where the pattern is applied to a dry-transfer sheet. Figure 3-6 shows an example of what the appliqué DRE look like when applied to the model. 


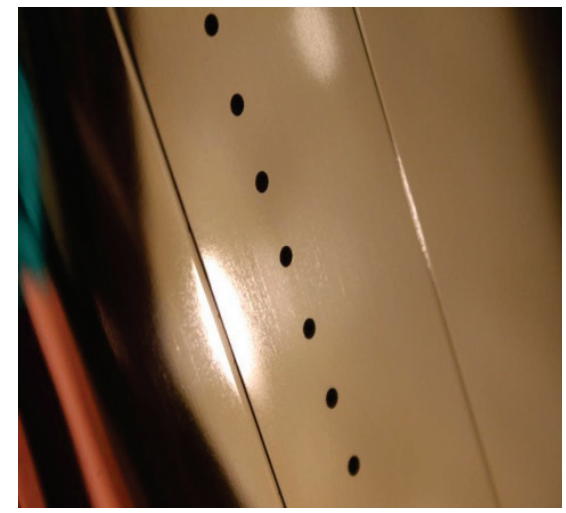

Figure 3-6. Example of appliqué DRE array applied on model.

DRE diameter is fixed at $3 \mathrm{~mm}$ for this experiment and center-to-center spacings of $12 \mathrm{~mm}$ and $6 \mathrm{~mm}$ (critical and control wavelengths respectively) are used. Variations less than $0.05 \mathrm{~mm}$ are measured in the diameter and spacing of the dry-transfer sheet. DRE height is usually consistent for a given batch, but can vary from one order to the next. This inconsistency is related to the manufacturing process and is not specifiable when ordering. Orders from the same company during ASU testing were on the order of $6 \mu \mathrm{m}$ for a single layer. More recent orders from TAMU have ranged from $12-14 \mu \mathrm{m}$. DRE height is measured for each new order. To increase roughness height, the appliqué DRE are layered. 

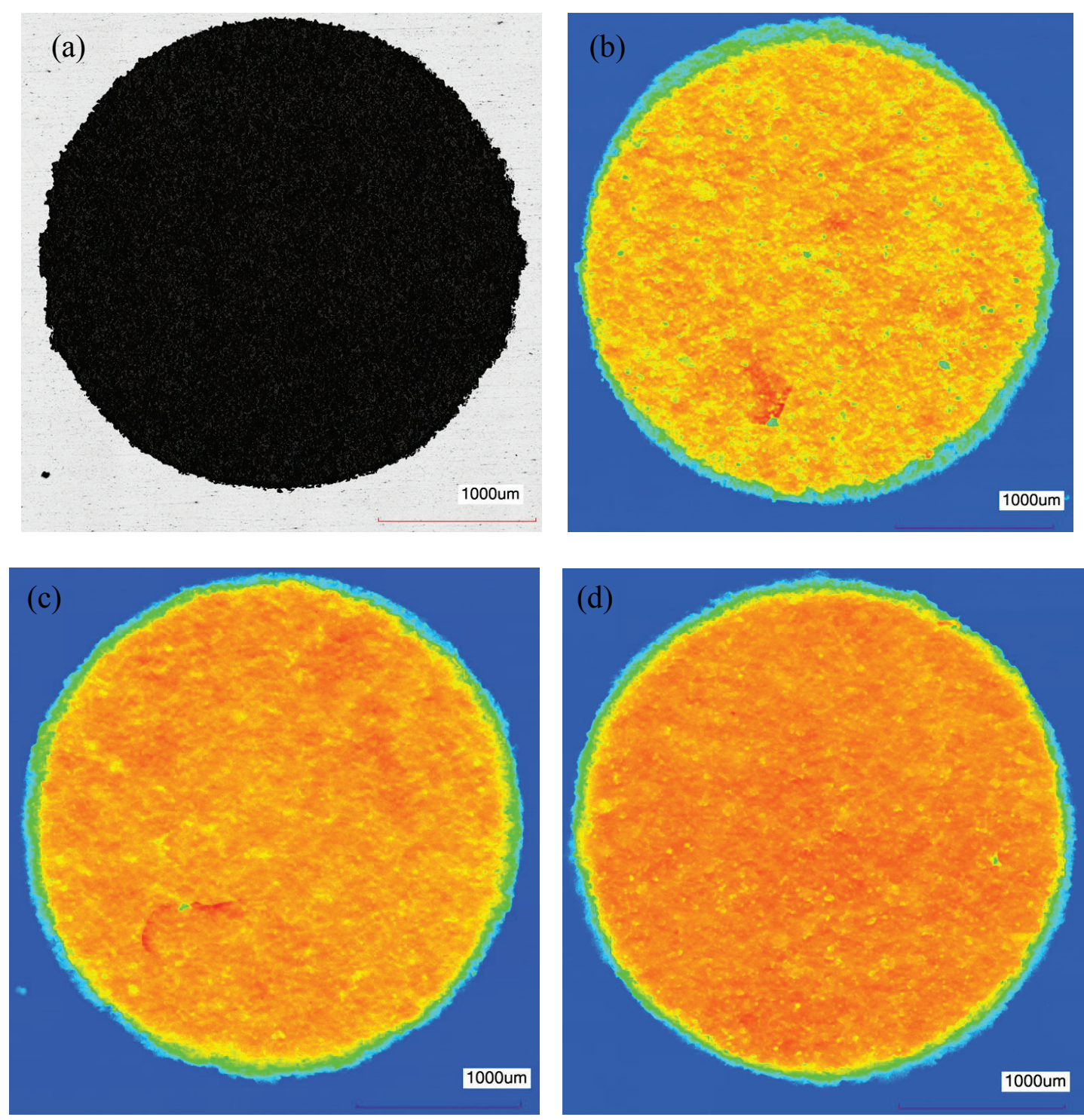

Figure 3-7. Magnified top view of roughness elements: (a) single layer, (b) 2-layers, (c) 3-layers, (d) 4-layers. Contrast images (b) - (d) are set to show height.

Magnified versions of single, double, triple and quadruple layer DRE taken from a Keyence VK-9700 3D Laser Scanning Microscope are shown in Figure 3-7.The singlelayer DRE clearly shows irregular edges that are not visible without the $20 \mathrm{x}$ magnification. The contrast images (b) - (d) in Figure 3-7 are set to highlight differences 
in height. For each layer beyond the single application, a thin rim appears around the edge of the roughness element. As additional layers are added, the dry-transfer material below compresses and spreads. This is more clearly shown when viewed with a crosssectional profile (Figure 3-8). Multiple tiers are visible, representing each new layer. For the set of samples prepared for the laser scanning microscope, maximum DRE diameter varied no more than $65 \mu \mathrm{m}$. The maximum difference in DRE diameter from the top to the bottom of a given roughness element was $240 \mu \mathrm{m}$, which occurred for the four-layer case. Table 3-2 lists the measured heights for the two roughness configurations measured here: $6 \mathrm{~mm}$ and $12 \mathrm{~mm}$ wavelengths.

Table 3-2. Measured appliqué roughness height.

\begin{tabular}{|c|c|c|}
\hline \multirow{2}{*}{$\begin{array}{c}\text { Number } \\
\text { of Layers }\end{array}$} & \multicolumn{2}{|c|}{ Roughness height, $k[\mu \mathrm{m}]$} \\
\cline { 2 - 3 } & $\lambda=6 \mathrm{~mm}$ & $\lambda=12 \mathrm{~mm}$ \\
\hline 1 & 14 & 12 \\
\hline 2 & 27 & 24 \\
\hline 3 & 42 & 36 \\
\hline 4 & 56 & 47 \\
\hline
\end{tabular}

An additional source of variation in roughness height can come from the pressure applied during application. Differences on the order of 1-2 $\mu \mathrm{m}$ are possible when comparing roughness heights applied by two different people. To help mitigate these variations, all roughness for these tests is applied by the same person. The profilometer used to measure background surface roughness is not used for the DRE as it tends to scratch the appliqué, limiting its use in establishing DRE height. 


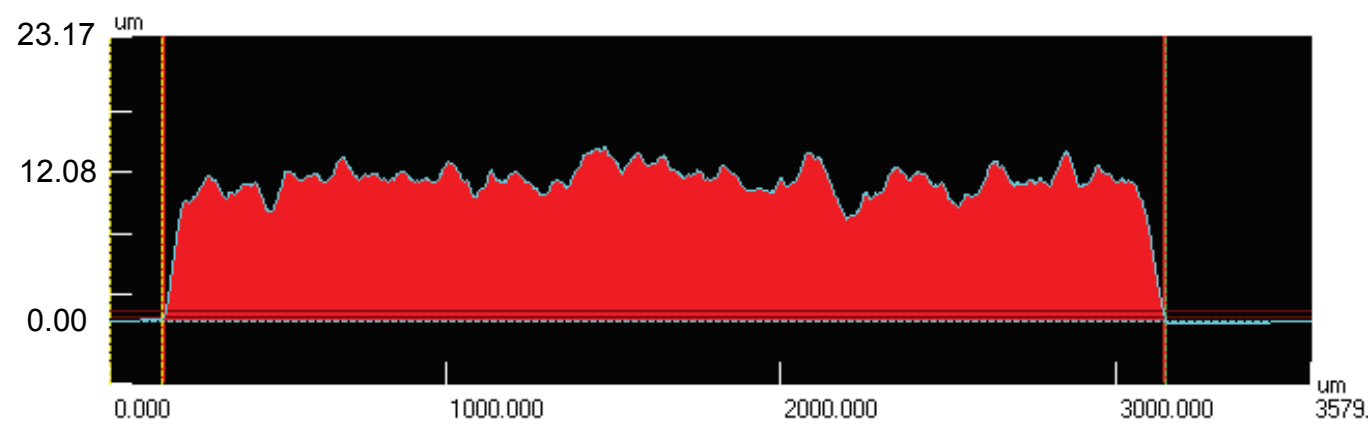

(a)

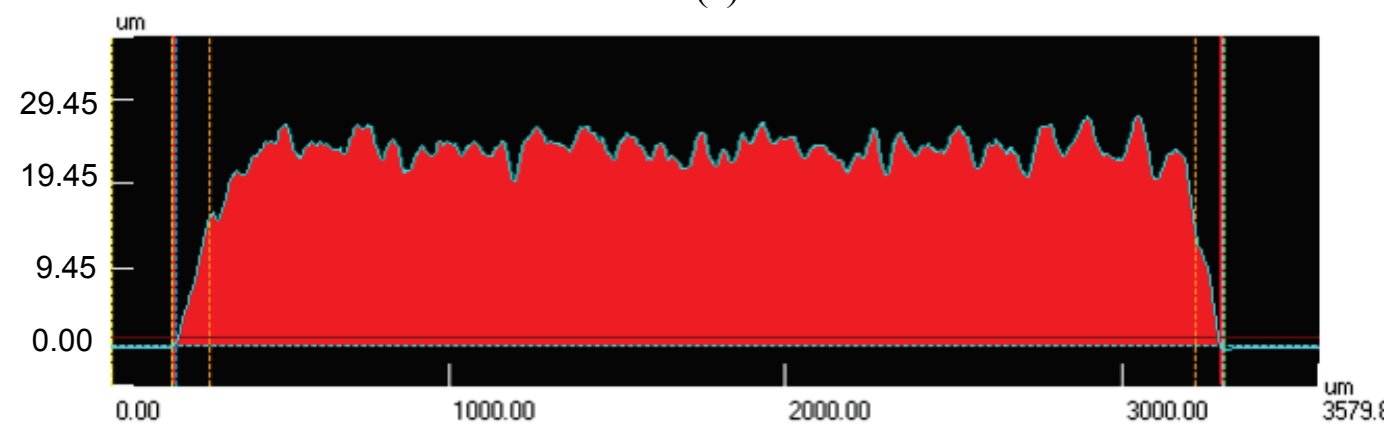

(b)

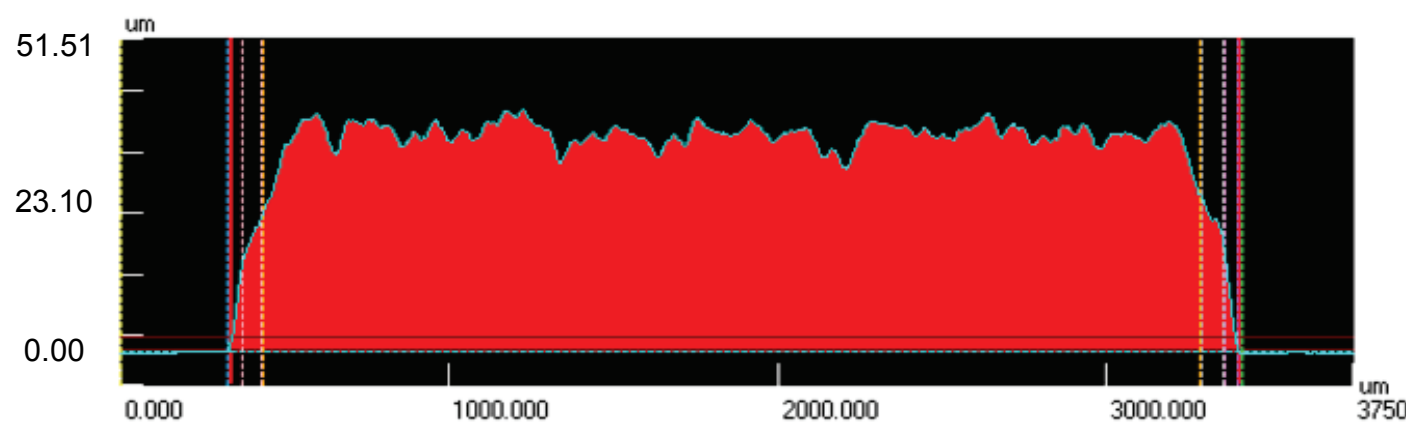

(c)

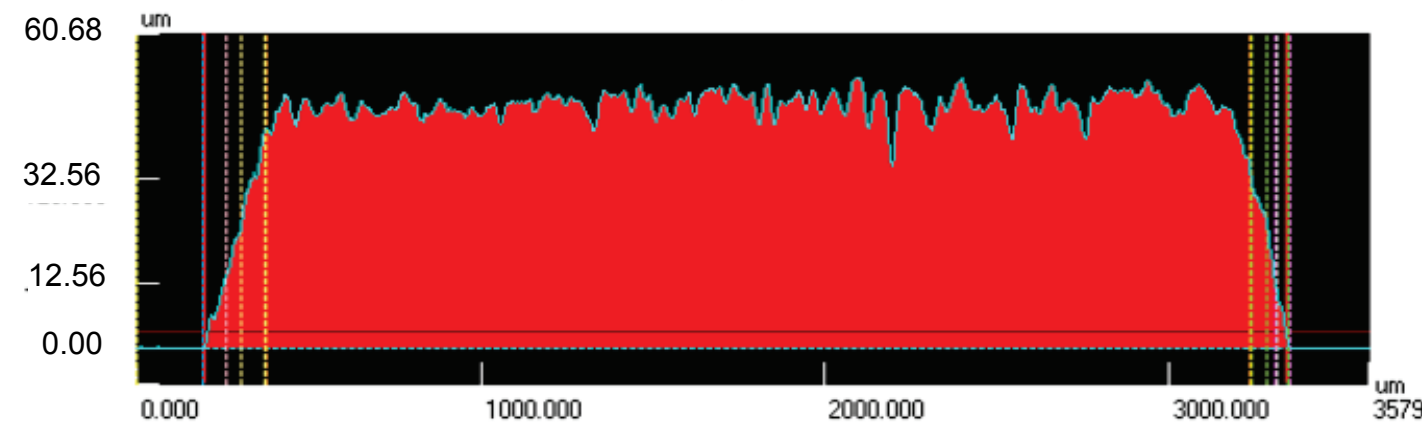

(d)

Figure 3-8. Side view of roughness elements: (a) single layer, (b) 2-layers, (c) 3-layers, (d) 4-layers. 


\subsection{Test Matrix}

Test points are selected based on the experimental objectives, tunnel limitations and measurement feasibility. One of the primary objectives of this study is to provide experimental data for use as a validation tool in DNS and NPSE codes. Ideally, from a computational standpoint, the measurements would be taken as close to the roughness array as possible and in a Reynolds number range and freestream environment relevant to flight conditions and operations. Experimentally, it is not always possible to provide those conditions. Appropriate choice of test facility is perhaps the most important decision that need be made. However, there are several other factors that directly impact the final test matrix.

The KSWT, like many of the tunnels with low-turbulence levels, does not have the speed capability to reach typical flight Reynolds numbers. The philosophy in these instances is to provide detailed data for a specific test condition, which can be used as a validation case for a given code. Once the code has been verified under those conditions, it can be extended to model higher Reynolds numbers and more complex geometries. With wall liners installed, a chord-Reynolds number of $3.8 \times 10^{6}$ is possible at 1100 RPM. This Reynolds number is not sustainable for extended tunnel runs needed during hotwire scans which can last up to five hours. The tunnel is not temperature controlled, resulting in a temperature drift over time. Total drift depends on tunnel rpm and ambient laboratory temperature. Temperature restrictions on tunnel components and instrumentation are the limiting factor in choice of maximum sustainable Reynolds number. Pressure transducers connected to the Pitot-static tube in the test section require 
that tunnel temperature not exceed $50^{\circ} \mathrm{C}$. In the ASU configuration where the motor was installed inside the tunnel, this meant that tests were rarely conducted above $R e_{c}=2.8 \times 10^{6}$ due to excessive heating in the tunnel. More commonly, tests were run from $1.6 \times 10^{6}-2.4 \times 10^{6}$. The current KSWT configuration supports an extended Reynolds number range that allows for regular testing at $R e_{c}=3.2 \times 10^{6}$.

Beyond tunnel heating, there are additional trade-offs in choice of Reynolds number. A higher Reynolds number will result in larger disturbance amplitudes, which are easier to measure. Conversely, the smaller boundary-layer thicknesses associated with increased Reynolds number will limit how many points can realistically be measured, reducing the fidelity of the profile. Boundary-layer thicknesses are on the order of $1-3 \mathrm{~mm}$ between $10 \%$ and $20 \%$-chord for this test configuration. With the traverse capability described in Section 2.2.1 and the small spatial resolution of the hotwire, it is possible to obtain enough measurement points in the boundary-layer even at Reynolds numbers of $3.2 \times 10^{6}$. The main factors in final selection of Reynolds number for this experiment were measureable disturbance amplitudes and ability to compare with previous ASU experiments.

For receptivity experiments, selection of measurement location is also critical. Another experimental objective was to determine the relationship between initial disturbance amplitude and roughness height. The ideal location would be as close to the roughness array as possible. However, disturbance amplitudes are well below the measurable range at this position. Care must be taken at downstream chord locations to ensure that measurements are within the linear stability range. Figure 1-4 shows a plot of 
$N$-factor as a function of chord-location from ASU experiments using the NLF(2)-0415 model. In this example, there is a range below $25 \% x / c$ where disturbance amplitude increases linearly from one chord location to the next. Beyond $25 \% x / c$, nonlinear disturbance evolution occurs. To eliminate nonlinear stability activity as a potential source affecting interpretation of measured disturbance amplitude, measurements must be taken in a region where growth of instability waves is known to be a linear process. The extent of the linear region will change based on factors such as Reynolds number, angle of attack and roughness height.

Table 3-3 shows the final test matrix for this set of measurements. Ideally, the roughness height for both the $6 \mathrm{~mm}$ and $12 \mathrm{~mm}$ would be the same. Differences in batch processing from the manufacturer do not always make this possible. Roughness height and disturbance amplitudes are normalized in the results section to eliminate this difference.

The roughness elements for all cases are appliqué circles with a $3 \mathrm{~mm}$ diameter located at $2.9 \% x / c$. Spanwise spacing varies between the critical wavelength of $12 \mathrm{~mm}$ and the control wavelength at $6 \mathrm{~mm}$. Each bullet in Table 3-3 indicates that a $64 \mathrm{~mm}$ spanwise hotwire scan was completed, providing 65 boundary-layer profiles with $1 \mathrm{~mm}$ spacing. Naphthalene flow visualization (NFV) images are available for every $R e_{c}=2.4 \times 10^{6}$ case. Standard practice included completing an NFV run after each new roughness layer was added to ensure no wedges were present in the measurement region and to track transition location since no hotwire measurements are taken downstream of 
$20 \% x / c$. NFV was not completed for the higher Reynolds number cases in most instances. Exceptions are discussed in the results section.

Table 3-3. Test matrix for receptivity measurements.

\begin{tabular}{|c|c|c|c|c|c|}
\hline$x / c$ & $k$ & $\lambda$ & \multicolumn{3}{|c|}{$\operatorname{Re}_{c}$} \\
\cline { 4 - 6 } & {$[\mu \mathrm{m}]$} & {$[\mathrm{mm}]$} & $2.4 \times 10^{6}$ & $2.8 \times 10^{6}$ & $3.2 \times 10^{6}$ \\
\hline $10 \%$ & 47 & 12 & $\bullet$ & $\bullet$ & $\bullet$ \\
\hline \multirow{5}{*}{$15 \%$} & 12 & 12 & $\bullet$ & $\bullet$ & $\bullet$ \\
\cline { 2 - 6 } & 24 & 12 & $\bullet$ & $\bullet$ & $\bullet$ \\
\cline { 2 - 6 } & 36 & 12 & $\bullet$ & $\bullet$ & $\bullet$ \\
\cline { 2 - 6 } & 47 & 12 & $\bullet$ & $\bullet$ & $\bullet$ \\
\cline { 2 - 6 } & $36^{* *}$ & 12 & & $\bullet$ & $\bullet$ \\
\cline { 2 - 6 } & 14 & 6 & $\bullet$ & $\bullet$ & $\bullet$ \\
\cline { 2 - 6 } & 27 & 6 & $\bullet$ & $\bullet$ & $\bullet$ \\
\cline { 2 - 6 } & 42 & 6 & $\bullet$ & $\bullet$ & \\
\cline { 2 - 6 } & 56 & 6 & $\bullet$ & $\bullet$ & \\
\hline $20 \%$ & 12 & 12 & $\bullet$ & & \\
\cline { 2 - 6 } & 24 & 12 & $\bullet$ & & \\
\cline { 2 - 6 } & 36 & 12 & $\bullet$ & & \\
\cline { 2 - 6 } & 60 & 12 & $\bullet$ & & \\
\hline
\end{tabular}

Same $36 \mu \mathrm{m}$ case at $15 \% x / c$, with increased turbulence levels

For one roughness configuration, a preliminary exploration on the effects of increased turbulence was also completed. Measurements at $10 \% x / c$ for a single DRE layer $(10 \mu \mathrm{m})$ were attempted; however, disturbance levels were below the measurable range and as result, are not reported. Measurements for the higher Reynolds-numbercases at $20 \%$ chord were not taken. At $R e_{c}=3.2 \times 10^{6}$, nonlinear stability effects were visible by a roughness height of $36 \mu \mathrm{m}$ and at $20 \% x / c$, nonlinear stability behavior was present for the $60 \mu \mathrm{m}$ case at $R e_{c}=2.4 \times 10^{6}$. It is likely then, that the majority of the points at $2.8 \times 10^{6}$ and $3.2 \times 10^{6}$ would not be useful for roughness height comparisons. 


\section{EXPERIMENTAL TECHNIQUES}

Two primary experimental techniques are used for this experiment: naphthalene flow visualization and hotwire anemometry. Use of each technique is detailed in the following sections.

\subsection{Hotwire Anemometry}

The stationary disturbance $\left(v^{\prime}, w^{\prime}\right)$ caused by the crossflow instability can often be too small to measure directly. Additionally, the boundary-layer thickness is too small to resolve $v^{\prime}$. However, the streamwise mean flow distortions induced by the transverse velocity disturbances are measureable. Hotwire anemometry is one of the most suitable techniques for measuring these velocity variations. The small wire size of $5 \mu \mathrm{m}$ provides the needed spatial resolution to obtain multiple points in these thin boundary-layers. The $1 \mathrm{~mm}$ length of the sensor is also much smaller than the wavelengths of interest for this experiment. Additionally, hotwires do not require any type of particle seeding to obtain measurements. This is particularly important for low-turbulence wind tunnels where cleanliness is a key factor in maintaining the desired flow quality. Hotwires also provide the needed frequency response should features such as traveling waves, secondary instability or freestream turbulence be studied.

The basic premise behind hotwire operation is convective cooling from the air to a heated wire. For this study, a Constant Temperature Anemometer (CTA) is used to maintain a fixed wire temperature. The resistance of the wire material (often tungsten or 
platinum) is proportional to wire temperature. As the flow mass flow rate changes, wire temperature and thus resistance also change. In CTA systems, bridge voltage is adjusted through a feedback loop to ensure that wire resistance is held constant. Perry (1982) and Bruun (1995) provide detailed instruction on the principles behind and use of hotwire anemometry.

Care must be taken to determine to what degree intrusive measurement devices influence the local flow field. Saric (2007) suggests a procedure to investigate the influence of the traversing hotwire on the local pressure coefficient. In the ASU tests, a flat-plate with a fixed hotwire located a $U / U_{e}=0.3$ was used to measure the disturbance amplitude from a Tollmien- Schlichting wave. The traversing hotwire was then stepped toward the fixed probe and the local pressure coefficient was measured. In those tests, the local pressure coefficient varied less than 0.005 (Radeztsky 1994), indicating that the use of hotwires and traverse do not pose a significant risk to the reliability of the results. Furthermore, the excellent agreement between the experimental measurements by Reibert et al. (1996) and Haynes \& Reed (2000) in mapping the primary instability growth suggest that the hotwire is a suitable tool for these measurements.

\subsubsection{Hotwire Calibration}

Hotwire calibration is a multi-stage process involving hotwire/anemometer set-up, a one-time velocity ratio measurement, a velocity and voltage correlation and temperature compensation. All hotwires are first configured according to the directions in the AN1003 manual using overheat ratios of 1.8. After tuning, time responses on the order of 
$5 \mu$ s were achieved. In addition to the external signal conditioning units available, the AN-1003 also provides options to apply signal filters, gain and offsets. No frequency cutoff options are used, but gain and offset are applied to each channel prior to hotwire calibration to best match the voltage range of the DAQ hardware $(+/-10 \mathrm{~V})$, increasing the measurement resolution.

Hotwires are mounted to the traverse sting for boundary-layer scans. The traverse and sting do not have the range to position the hotwires in the same plane as the test section Pitot-static tube, which along with the test section RTD, serves as the reference velocity during calibration. Instead, temporary Pitot-static tubes were placed in the calibration positions for the hotwires, providing a ratio between velocities measured at the test section entrance and at the hotwire calibration position. This is a necessary correction since presence of the model and tunnel walls near the hotwires influences the local velocity field. The hotwire calibration position is defined at $60 \% x / c$, midspan with full retraction of the sting. In this location, the boundary-layer hotwire is $49 \mathrm{~mm}$ above the model surface and the freestream hotwire is $222 \mathrm{~mm}$ above the surface. To obtain velocity ratios between these two points and the test section Pitot-static tube, tunnel speed was varied from $2.0 \mathrm{~m} / \mathrm{s}$ to $32.0 \mathrm{~m} / \mathrm{s}$ in $2 \mathrm{~m} / \mathrm{s}$ increments. Velocity ratios below a freestream speed of $10 \mathrm{~m} / \mathrm{s}$ started to vary slightly from a linear fit and are attributed to unsteadiness and head loss in the tunnel at low speeds. The final curve fit only included data from $10.0 \mathrm{~m} / \mathrm{s}$ to $32.0 \mathrm{~m} / \mathrm{s}$. The velocity ratio between the Pitot-static tubes in the upstream and boundary-layer hotwire position is $1.114 \pm 0.031$ and for the upstream and freestream hotwire position is $1.069 \pm 0.035$, where the error is the standard error of the 
regression. Including the full data set from $2.0 \mathrm{~m} / \mathrm{s}$ to $32.0 \mathrm{~m} / \mathrm{s}$ does not substantially change the velocity ratio (1.114 to 1.115 in the case of the boundary-layer position, no change for the freestream position), but does increase the error to \pm 0.058 and \pm 0.070 respectively. These ratios are very similar to those recorded by White (2000). The measured velocity ratio for each hotwire position is applied as a velocity correction during calibration to ensure that the speed corresponding to hotwire voltage is correct.

The relationship between voltage and velocity is nonlinear and requires an individual calibration for each wire and CTA channel combination. In many instances, an empirical correlation based on a polynomial curve fit provides sufficient accuracy (Perry 1982). This methodology was originally used at ASU for hotwire measurements by Radeztsky (1994) and Reibert (1996). Without a source of active cooling, the temperature variations in the KSWT also impact the hotwire measurements. Radeztsky et al. (1993) applied a linear correction to voltage drift based on changes in freestream temperature during a hotwire scan. The current KSWT hotwire calibration and temperature compensation algorithms come directly from White (2000) who reports in detail the procedure for combining the two independent procedures into one process. A summary of that procedure is provided below.

For hotwire calibration schemes, Bearman (1971) takes King's Law, which describes the relationship between heat transfer and fluid properties and writes it in terms of voltage, velocity and temperature as shown in Eqn (4-1). 


$$
E^{2}=A\left(T_{w}-T_{a}\right)+B\left(T_{w}-T_{a}\right) U^{1 / n}
$$

In this equation, $E$ is voltage, $U$ is velocity, and $T_{w}$ and $T_{a}$ are wire and ambient temperatures respectively. $A, B$ and $n$ are constants determined experimentally. By measuring the voltage at the same velocity for a high and low temperature, a temperature-compensation coefficient $C_{T}(U)$, shown in Eqn (4-2), can be derived from Eqn (4-1).

$$
C_{T}(U)=\frac{E_{h}^{2}-E_{l}^{2}}{T_{h}-T_{l}}=-A-B U^{1 / n}
$$

The temperature-compensation coefficient is then used to provide a compensated voltage, as given by:

$$
E_{\text {comp }}^{2}=E^{2}+C_{T}(U)\left(T_{\text {comp }}-T\right)
$$

where the subscript "comp" is the temperature compensated voltage and an arbitrary temperature compensation temperature. Setting $\left(T_{w}-T_{a}\right)$ to $\left(T_{w}-T_{\text {comp }}\right)$ in Eqn (4-1) results in the following simplification:

$$
U=\left(A^{\prime}+B^{\prime} E_{c o m p}^{2}\right)^{n^{\prime}}
$$

where $A^{\prime}, B^{\prime}$ and $n^{\prime}$ are also experimentally determined coefficients. Hotwire calibration and velocity measurements proceed as follows. The tunnel is run at 100-200 rpm for a few minutes prior to initiating the calibration program. This ensures that air is fully circulating in the tunnel and that any minor changes in tunnel temperature are stabilized. 
Tunnel rpm is then varied from 100 to $1100 \mathrm{rpm}$ in $100 \mathrm{rpm}$ increments. After stabilizing at each new rpm, hotwire voltage, and freestream velocity and temperature are acquired for $10 \mathrm{~s}$ at $1000 \mathrm{~Hz}$. The velocity ratios obtained for each hotwire position are applied to each acquired velocity at the given rpm. Once the ramp-up sequence is complete, the tunnel rpm is fixed at 1100 while the tunnel heats. This stage will last for a maximum of 20 minutes or until a $6^{\circ} \mathrm{C}$ temperature change is measured, whichever comes first. The tunnel is then ramped down using the test section velocities measured during ramp-up for a given rpm. Freestream speed, temperature and hotwire voltages are acquired. Velocity ratios are applied to the measured velocity for each hotwire. The actual velocity on the ramp-down many vary a little from the ramp-up, so after all acquisition is complete, voltages and velocities are adjusted using linear interpolation to match the exact speed during ramp-up. $C_{T}$ is calculated using Eqn (4-2) and a curve fit is then used to solve for coefficients $A, B$ and $n . C_{T}$ and the voltages from the ramp down $\left(E_{h}\right)$ are then used to calculate $E_{\text {comp }}$ in Eqn (4-3). Finally, a nonlinear curve fit using $E_{\text {comp }}$ and the measured velocity values $(U)$ is applied to calculate the parameters $A^{\prime}, B^{\prime}$ and $n^{\prime}$ in Eqn. 4.4. Plots of Eqns (4-2) and (4-4) with the calculated coefficients are provided at the end of the calibration procedure. Examples are shown in Figures 4-1 and 4-2. 


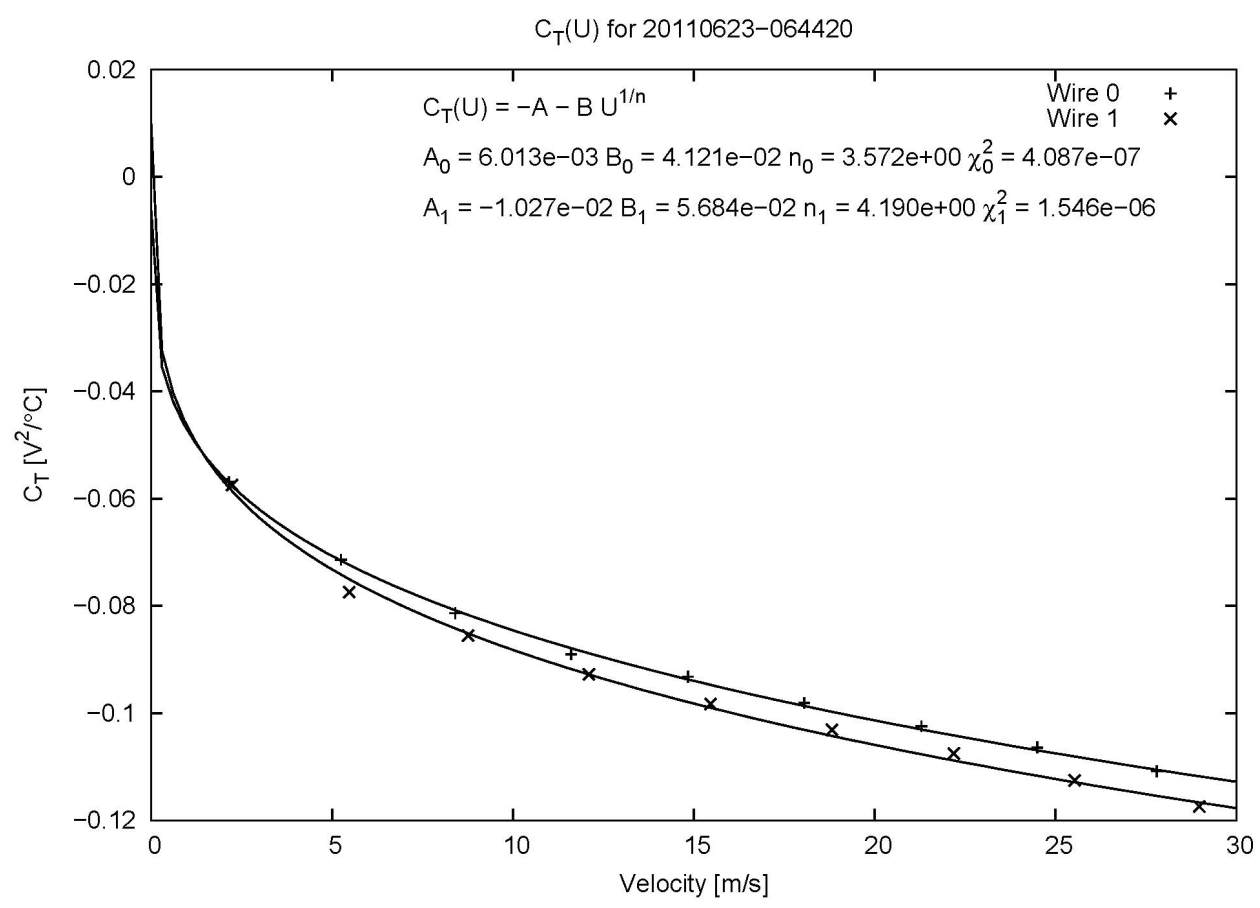

Figure 4-1. Example temperature compensation.

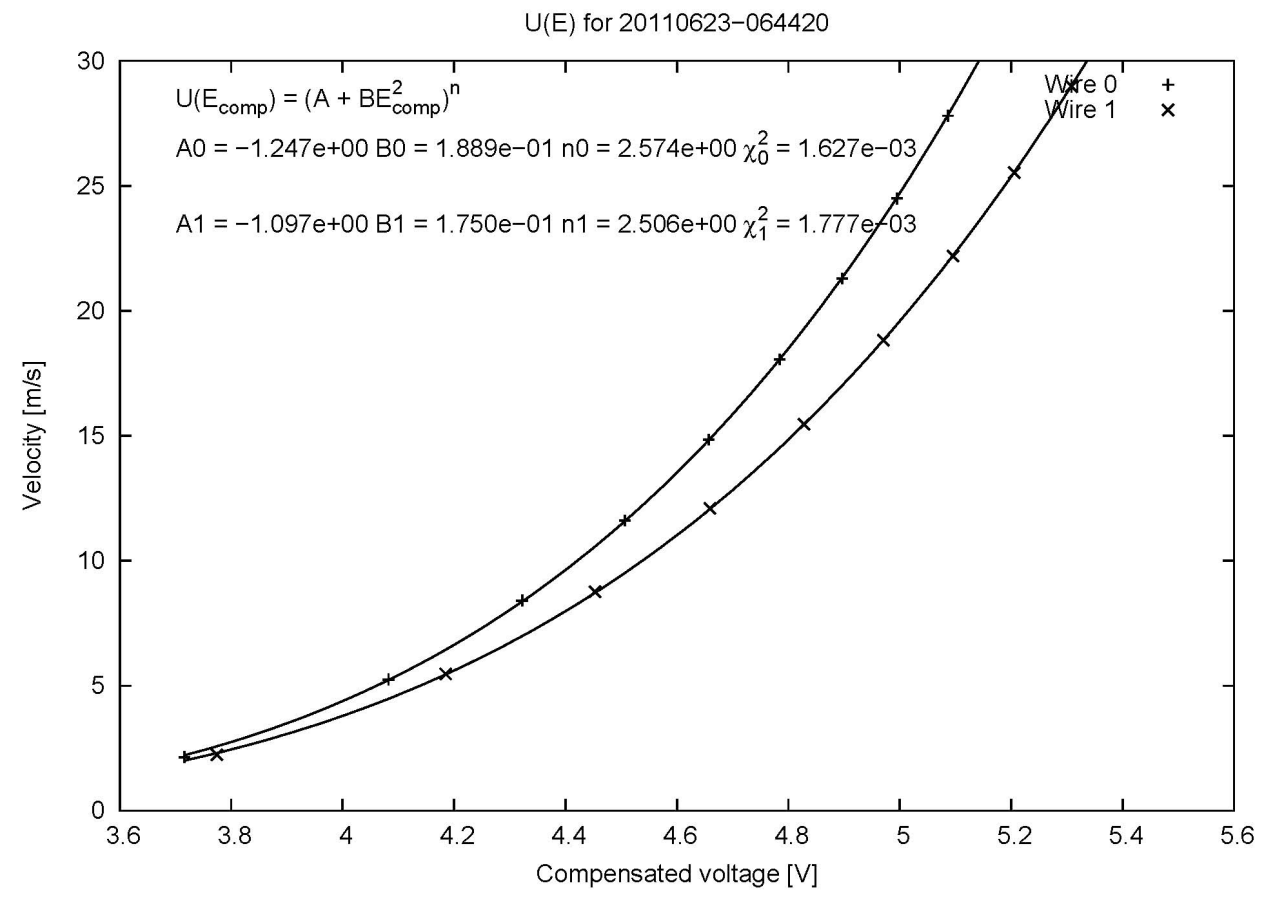

Figure 4-2. Example hotwire calibration. 
For each voltage measured after the calibration is complete, an iterative process using Eqns (4-2), (4-3) and (4-4) is required since velocity is needed to generate a temperature-compensation coefficient. This iterative procedure usually converges within four iterations and has no substantial impact on the processing time to convert raw voltages to velocity. Voltages are always converted to velocity before any averages are taken.

\subsubsection{Coordinate Systems}

Prior to discussing the hotwire boundary-layer profile and scanning techniques, an understanding of coordinate systems used in the tunnel and experimental configuration is needed. Several reference frames are present for a given experimental set-up. The global test section coordinates, denoted $(X, Y, Z)$ are fixed relative to the test section and are shown in Figure 1-1. Flow is moving from left to right with $X$ describing the streamwise direction, $Y$ normal to the side wall and $Z$ pointing to the ground. Also shown in Figure 1-1 is the model-oriented system $(x, y, z)$ in which $x$ is normal to the leading edge, $z$ runs parallel to the leading edge and $y$ is normal to the unswept chordline. Boundary-layer or streamline-oriented coordinates provide yet another reference frame $\left(x_{t}, y_{t}, z_{t}\right)$, where $x_{t}$ is tangent to the inviscid streamline, $z_{t}$ is normal to the streamline and $y_{t}$ is normal to the model surface. These definitions are all consistent with previous ASU experiments in the UWT.

Most computational efforts use either model-oriented or stream-oriented coordinates. The traverse is aligned with the global coordinates $(X, Y, Z)$. While it would 
be possible to program movements for any reference frame, moving in the $y_{t}$ direction would require adjustments in $X$ and $Z$ that are below the minimum obtainable step size provided in Table 2.1. Rather than introduce additional uncertainty in the measurements by trying to move the traverse in streamline-oriented coordinates, profiles are obtained in $Y$ to maximize the available resolution. Moving the traverse in the streamline direction $z_{t}$ is within available tolerances, but because this movement would not occur over lines of constant-chord, features such as model curvature present additional difficulties to the experimentalist. Instead, all measurements are made along lines of constant-streamwise chord equivalent to $z$ in the model-oriented system. A coordinate transformation is required for any computational model that uses alternate coordinate systems. However, since computational results can be acquired in almost any reference frame, this should not pose an unreasonable burden.

After hotwire calibration, the traverse moves the hotwires to the desired measurement location. Two manual rotations in the $(X, Y, Z)$ frame are required. The hotwire is first rotated about the $Z$-axis in the $X-Y$ plane towards the model until the hotwire tines are parallel with the surface. This rotation ensures that the hotwire itself, rather than the tines, probe support or sting impact the surface first. Rotation angle varies based on chord location. This is why scans are completed in $z$ rather than $z_{t}$; movement along $z_{t}$ would require additional adjustments to avoid unintended contact with the model. A second rotation about the probe axis occurs until the tines on the hotwire are equidistant from the surface. Misalignment of the tines can result in spatial averaging across the boundary-layer height. This rotation is also chord-dependent. 


\subsubsection{Boundary-Layer Velocity Profiles}

A single boundary-layer velocity profile is obtained using two hotwires attached to the traverse sting mount. Following hotwire calibration and hotwire positioning at the desired chord and span a velocity-profile may be acquired. For a given profile, the boundary-layer (BL) and freestream (FS) hotwires both start in the freestream. Just outside the boundary layer, the edge velocity, and the ratio of the mean velocities from both hotwires are measured. The traverse then moves toward the model in the negative $Y$ direction, acquiring $U_{B L}$ and $U_{F S}$ at each point. The boundary-layer velocity is normalized first by $U_{F S}$ and then the ratio of $U_{B L} / U_{F S}$ measured at the start of the profile. This two-part normalization is needed since tunnel velocity is adjusted to preserve Reynolds number when freestream temperature changes. Small step sizes in $\mathrm{Y}$ are desired in the boundary-layer to better resolve the profile, especially as the wire moves closer to the wall. To reduce the total number of points required for a profile, larger step sizes are used near the boundary-layer edge. The step size then decreases as measured velocity decreases by the following relationship:

$$
\Delta_{\text {step }}=\Delta_{\text {initalstep }}\left(\frac{U_{B L}}{U_{e}}\right)^{1.75}
$$

The initial step size is set by the user. As the normalized boundary-layer velocity decreases, the step size also decreases, increasing the number of points taken over a given distance. A minimum step size is also supplied by the user to ensure that steps smaller than the hotwire diameter are not taken. As an example, profiles at $15 \% x / c$ for 
$R e_{c}=2.4 \times 10^{6}$ usually started with a $0.070 \mathrm{~mm}$ step and decreased until reaching the minimum step size of $0.010 \mathrm{~mm}$. Small variations in hotwire position and the wall surface make it difficult to know where the wall is located before the start of a profile. After each new measurement point, the normalized boundary-layer velocity is checked. When $U_{B L} / U_{e}$ is less than or equal to 0.20 , the profile measurements stop and the hotwire returns to the freestream. Data collection is terminated below this point to prevent radiation from the hotwire to the model from influencing the measurements. This can occur when measurements are taken within $150 \mu \mathrm{m}$ from the wall (Saric 2007). Using the normalized velocity data between 0.2 and 0.5 , a second-order curve fit is applied to the data to extrapolate distance to the wall.

\subsubsection{Boundary-Layer Velocity Scans}

The primary purpose of using hotwire anemometry is to obtain measurements of the mean-flow distortions in the streamwise direction. To accomplish this, a spanwise series of velocity profiles is needed. The $64 \mathrm{~mm}$ spanwise measurement range was selected to cover multiple wavelengths, as a means to provide an average disturbance amplitude. This minimizes errors that may occur from interpreting distortion strength from a single wavelength span. With $1 \mathrm{~mm}$ incremental steps, five and ten full wavelengths are measured for the critical and control wavelengths respectively. Of course, the more profiles obtained, the better the average; however, a compromise between number of profiles and total test runtime must be made. An average of 50 measurements in the boundary-layer, with an additional 25 - 35 in the freestream, is acquired for each profile. 
Each point is measured for 2 seconds with a sampling rate of $10 \mathrm{kHz}$. Total runtime for the scans is as long as five hours. Including hotwire calibration and hotwire positioning, an average of $6-7$ hours is needed to provide a single disturbance profile and amplitude. Moreover, the freestream temperature in the tunnel must not exceed the operating limit of $50^{\circ} \mathrm{C}$ for the pressure transducers. In the summer, when ambient laboratory temperature is warm, it is not uncommon to come close to reaching this limit for long scans at $R e_{c}=3.2 \times 10^{6}$.

Since step size is dependent on the normalized boundary-layer velocity and distance from the wall where $U_{B L} / U_{e}=0.2$ will vary based on the mean-flow distortions, the step size and total number of steps is different for each profile in the scan. Calculation of a mean profile, disturbance profile and mode shape requires data spaced at equal intervals. Once the wall distance has been determined for each profile, the data are interpolated to provide normalized velocities and wall distances on an evenly spaced grid so that manipulation of the 65 profiles to provide, for example, the mean velocity profile is straight-forward.

\subsection{Naphthalene Flow Visualization}

Hotwire anemometry could also be used to determine transition location on the model. However, this technique is best suited to test plans that require measurements at multiple streamwise locations. The hotwire must be manually adjusted at each chord location to account for surface curvature. Determining the transition location via 
hotwires would be a time-intensive process taking measurements in regions that are beyond the scope of receptivity measurements.

Naphthalene flow visualization is another technique to determine where transition has occurred on the model. Near room temperature, naphthalene sublimes at a rate proportional to shear stress. Regions of higher shear stress, such as a turbulent wedge, will cause the crystals to sublime faster, providing a well-defined image of laminar and turbulent regions. Since full-chord scans are beyond the scope of this study, naphthalene flow visualization provides a method to determine where transition occurs for a given roughness configuration and identify premature turbulent wedges in measurement regions of interest. Wedges that occur forward of the mean transition location may indicate problems with the roughness insert installation or roughness application. This technique provides immediate feedback on how the overall transition pattern is affected by particular roughness elements, whereas the detailed hotwire scans provide quantitative data on the initial disturbance amplitudes resulting from the DRE.

Naphthalene crystals are dissolved in acetone and sprayed aft of $20 \%$-chord to avoid introducing an unintended source of surface roughness. Radeztsky et al. (1999) demonstrated that small roughness placed aft of $10 \%$-chord has no effect on the transition process; in that study, flow visualization results were also cross-referenced with hotwire measurements to confirm that presence of the additional roughness did not alter the transition location. For the crossflow instability, the secondary instability responsible for breakdown and transition occurs over a very short distance downstream, ensuring that this technique is an appropriate transition detection tool. 
For each run, naphthalene is sprayed over the full span of the model between pressure port rows and from $20 \%$-chord to model trailing edge. The spray pattern always moves in the spanwise direction, parallel to the leading edge to avoid confusing streaks from the mean flow distortion with thickness variations that may occur during application. Once on-condition, chord markers along the full-span of the model are used to identify where transition occurs. White regions where naphthalene is still present indicate laminar regions and black regions where the model surface is exposed indicate turbulent regions. When stationary crossflow vortices dominate the transition process, the transition front is generally characterized by a jagged, sawtooth pattern, which occurs due to variations in local surface roughness near the leading edge. In addition to transition location, streaking in the laminar regions is also identifiable. When artificial roughness is used, the streaks occur at a regular wavelength. The alternating light and dark regions are the result of low-momentum and high-momentum regions that occur as stationary crossflow vortices distort the basic state. Turbulent wedges always appear to start in a low-momentum region (White \& Saric 2000). The secondary instability, which ultimately causes transition, spatially coincides with the low-shear regions. Each time the roughness configuration is altered (i.e. a new insert is installed or a new applique layer is applied), naphthalene flow visualization is completed. 


\section{RESULTS- PART I: BASELINE MEASUREMENTS}

\subsection{Results Overview}

Experimental results are separated into four main sections. Section 5 provides all measurements related to baseline data. This includes measurement of freestream turbulence, pressure distributions and transition location without artificial roughness. The first two are needed to verify that the test platform reflects the planned experimental design. Freestream turbulence levels must be low enough to ensure that stationary, rather than traveling, waves dominate the transition process. The pressure distribution measurements indicate whether an assumption of spanwise uniform flow is valid. This design feature is not an absolute requirement for a stability experiment, but confirming this aspect simplifies the computational effort considerably. NFV to detect transition location in the absence of artificial roughness provides a means to determine how transition location changes when DRE arrays are applied. Section 6 includes the NFV and hotwire scan results for DRE spaced at the critical wavelength $\left(\lambda_{\text {crit }}=12 \mathrm{~mm}\right)$, followed by control wavelength spacing $\left(\lambda_{\text {cont }}=6 \mathrm{~mm}\right)$ in Section 7 . Preliminary results showing the effect of increasing turbulence levels in the tunnel are presented in Section 8. Finally, a detailed comparison of these results and those obtained in previous ASUUWT testing is provided in Section 9. 


\subsection{KSWT Freestream Disturbance Environment}

Until the discrepancies between previous wind tunnel and flight experiments are resolved, documenting the freestream disturbance environment is critical for identifying the factors that influence the receptivity process. As discussed in Section 1.3.3, a review of previous literature demonstrates that there are many ways to document turbulence levels. Detailed reporting of those values and the process by which they were attained is necessary for meaningful comparisons of experimental data. This includes understanding what factors may influence a given measurement. For example, a straightwire oriented in the streamwise direction measures a disturbance intensity that includes both acoustic and turbulent contributions. To examine the impact of freestream turbulence on initial disturbance amplitudes, the role of turbulence alone must be assessed. Similarly, a certain portion of the signal is dominated by electronic noise. This is perhaps less important in wind tunnels where the signal-to-noise ratio will be higher due to higher turbulence levels, but in flight, were total disturbance intensities (sound, turbulence and electronic noise) are the same order of magnitude or lower than many wind tunnel turbulence levels, it may contribute to a larger portion of the total signal. Additionally, measurements should involve both streamwise and transverse directions. Measuring only one velocity fluctuation component can result in misleading interpretations of turbulence levels. Deyhle \& Bippes (1996) report measuring streamwise velocity fluctuations that were smaller than the transverse components because of the higher contraction ratio in their wind tunnel. Measurements in the UWT and KSWT indicate the opposite trend. Including only the streamwise component would have signaled turbulence levels that 
were too low and too high for the DLR and ASU experiments respectively. It is also for this reason that it is helpful to see all three velocity components rather than group them into a single value as done in Eqn. 1.2. Observations on the degree to which the turbulence is isotropic can be made when all three directions are included. Regardless of which measurements are made for a given test, perhaps the most important aspect is documenting the final approach so that results can be viewed within the context of what is and is not known regarding the disturbance environment.

After tunnel reconstruction was completed, extensive flow-quality measurements were taken in the empty KSWT test section. A combination of hotwire anemometry and microphones were used to determine mean flow uniformity, disturbance intensities and the acoustic signature of the tunnel over the speed range of $5-25 \mathrm{~m} / \mathrm{s}$. A set of turbulence and acoustic measurements is provided in Hunt et al. (2010). The scope herein is limited to measurements that indicate the turbulence levels in the tunnel, since that is the most relevant parameter for the crossflow instability. An expanded set of turbulence measurements is provided here. Section 5.2.3 provides a comparison of turbulence levels in the ASU-UWT and TAMU-KSWT and discusses the impact of the more recent tunnel modifications. A limited number of measurements were made with the ASU(67)-0315, wall liners and contraction fairings installed to confirm that the flow quality had not been substantially altered from the empty test section configuration; these are presented in Section 5.2.4. Correlation studies to measure turbulent scales and electronic noise were not part of this flow quality assessment. Should future work indicate that these are significant factors, additional measurements can be made. 


\subsubsection{Measurement Configuration}

The computer controlled traverse does not have the range to span the full length, width and height of the test section. Instead, mounts were manually fixed to several locations in the test section to measure streamwise, spanwise and wall-normal velocity fluctuations. Three streamwise measurement planes were selected, with nine different grid points in each plane. Figure 5-1 displays each location along the test section. Measurement locations are normalized by the test section length scales $(X / L, Y / W, Z / H)$.

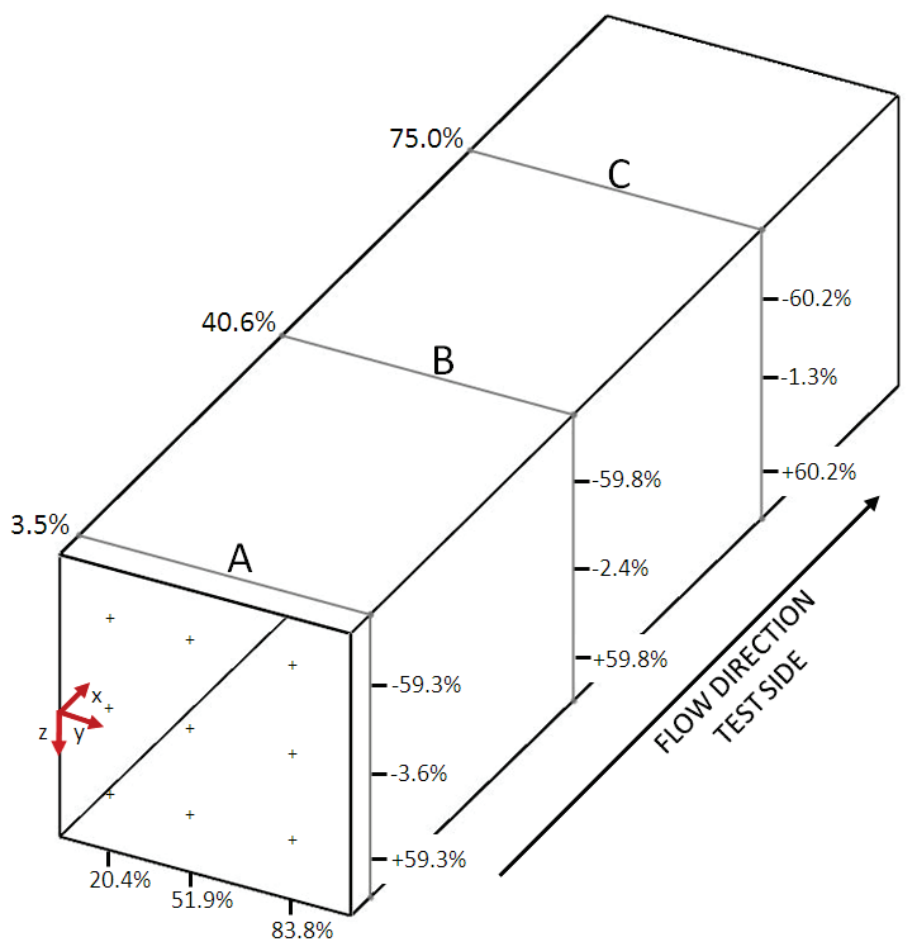

Figure 5-1. Freestream turbulence hotwire measurement locations. 
Each point is purposely located away from planes of symmetry to avoid nodes resulting from large scale in-plane vortical motion in the test section (Saric 2007). Two mounts, one $0.28 \mathrm{~m}$ (11 in) tall and the other $0.71 \mathrm{~m}$ (28 in), comprised of a symmetric aluminum strut welded to a rectangular base were made to hold the standard Dantec Dynamic hotwire supports. Threaded inserts were installed on the floor, ceiling and non-test-side wall to ensure that mounts could be repeatedly placed in the same location. The stands were used for both straightwire and crosswire measurements. Figure 5-2 shows an example of a crosswire mounted to one of the stands just below the Pitot-static tube and RTD in position $(0.035,0.204,-0.036)$.

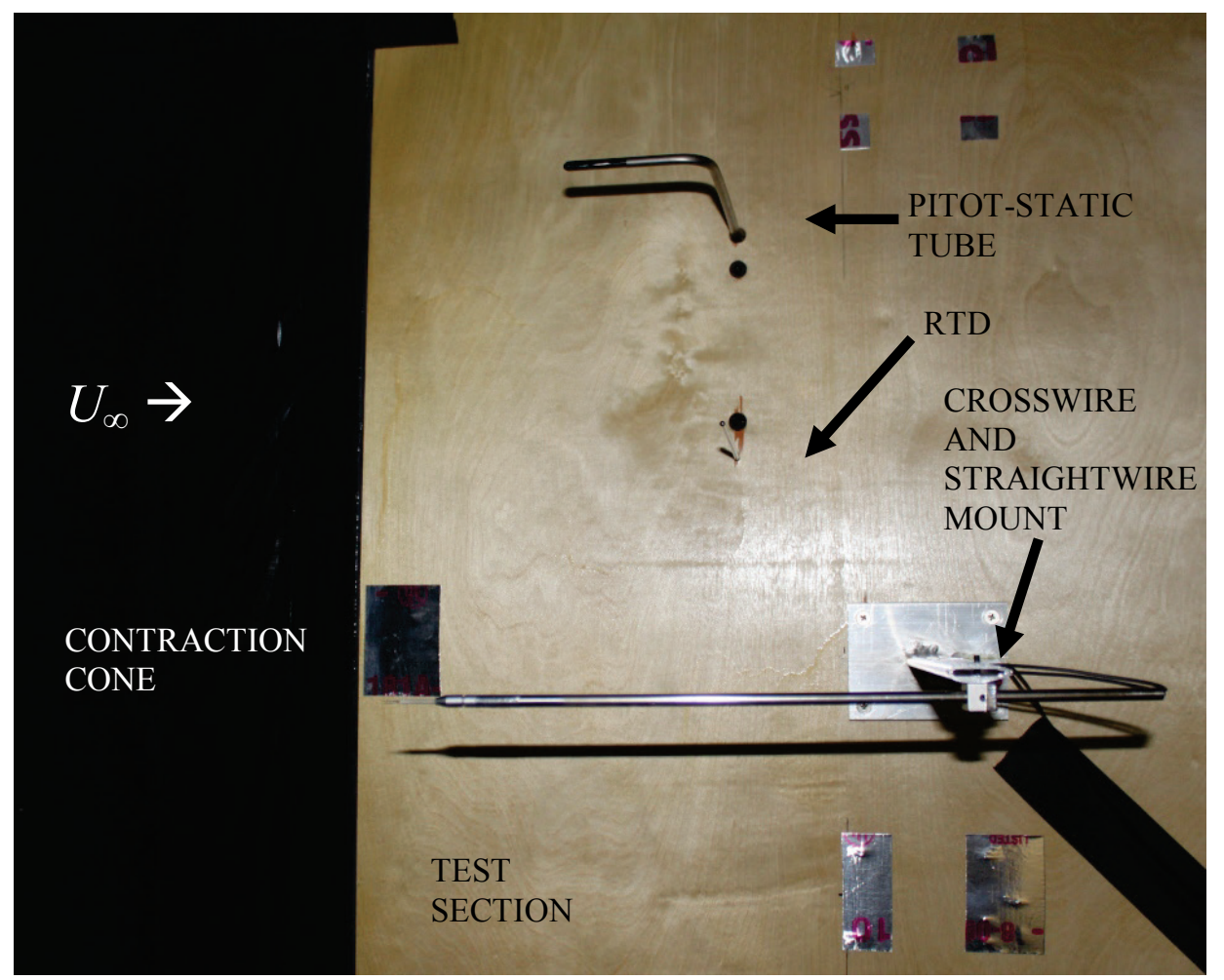

Figure 5-2. Crosswire mounted to side wall using aluminum stand. 
When mated to the ceiling or floor, the mounts are rigid in the $(X, Z)$ plane. A small degree of vibration in the $Y$ direction results in additional peaks in the hotwire spectra. To avoid introducing this additional source of error when crosswires measuring $u^{\prime}$ and $v^{\prime}$ were used, the mount was rotated to the side wall as shown in Figure 5-2. In this location, stand vibration would occur in the Z-direction. To summarize then, streamwise velocity fluctuations, $u^{\prime}$, were measured using a combination of floor, ceiling and sidewall mounting locations. All wall-normal velocity fluctuations, $v^{\prime}$, were taken from sidewall mounted stands. Ceiling and floor mounts were used to obtain spanwise velocity fluctuations, $w^{\prime}$. Holes were not drilled into the test-side wall, which would have been necessary to measure $v^{\prime}$ at $Y=0.838$. The test-side wall includes large acrylic windows and a centered panel with either a glass surface or frame for the traverse. Drilling holes into these surfaces would have compromised the functionality and durability of each window. As a result, $v^{\prime}$ data were not collected at this location for all $X$ and $Z$. However, comparison of transverse velocity fluctuations in other parts of the test section indicates that $w^{\prime}$ may be a good indication of expected turbulence levels in the wall-normal direction.

Streamwise mean and fluctuating velocity measurements were obtained using straightwires and crosswires. Redundant measurements using different probes provide a means of independent verification. Transverse measurements came from crosswires only. The vortical and irrotational components are unseparated in the reported results unless otherwise stated. Disturbance intensities are shown as a percentage of the rmsvelocity fluctuation divided by the mean streamwise velocity. Each acquisition is 
sampled at $25 \mathrm{kHz}$ for $60 \mathrm{~s}$. To obtain velocity fluctuations, the anemometer voltage is filtered with a bandpass of $1 \mathrm{~Hz}-10 \mathrm{kHz}, \mathrm{AC}$ coupled and amplified with a gain of 30 $\mathrm{dB}$. At the time of these measurements, temperature compensation had not yet been incorporated into the tunnel acquisition programs. Before the measurements were taken, all hotwires were mounted at $(0.035,0.204,-0.036)$ in the same plane as the test section Pitot-static tube. A simplified calibration routine using rpm settings from 100 to 1200 $\mathrm{rpm}$ in $100 \mathrm{rpm}$ increments was implemented to correlate velocity and anemometer voltage. A fifth-degree polynomial curve-fit was applied to the data. After calibration, the mount was moved to the desired test location. Since temperature compensation was not included for these tests, test section temperature was monitored and hotwires were recalibrated if significant temperature drifts occurred.

\subsubsection{Freestream Turbulence Levels}

To obtain mean streamwise velocity variations in plane A from Figure 5-1, a straightwire located at $(0.035,0.204,-0.593)$ was used as the reference point. Mean velocities from the other eight locations were then normalized by the reference velocity. Table 5-1 shows the mean streamwise velocity variation. 
Table 5-1. Percentage of mean streamwise velocity variations in Plane A.

\begin{tabular}{|c|c|c|c|c|c|}
\hline \multicolumn{2}{|c|}{$(0.035,0.204,-0.593)$} & \multicolumn{2}{|c|}{$(0.035,0.519,-0.593)$} & \multicolumn{2}{|c|}{$(0.035,0.838,-0.593)$} \\
\hline $10 \mathrm{~m} / \mathrm{s}$ & Reference & $10 \mathrm{~m} / \mathrm{s}$ & $0.00 \%$ & $10 \mathrm{~m} / \mathrm{s}$ & $-0.80 \%$ \\
\hline $15 \mathrm{~m} / \mathrm{s}$ & Reference & $15 \mathrm{~m} / \mathrm{s}$ & $-0.40 \%$ & $15 \mathrm{~m} / \mathrm{s}$ & $0.40 \%$ \\
\hline $20 \mathrm{~m} / \mathrm{s}$ & Reference & $20 \mathrm{~m} / \mathrm{s}$ & $-0.60 \%$ & $20 \mathrm{~m} / \mathrm{s}$ & $0.30 \%$ \\
\hline $25 \mathrm{~m} / \mathrm{s}$ & Reference & $25 \mathrm{~m} / \mathrm{s}$ & $-0.70 \%$ & $25 \mathrm{~m} / \mathrm{s}$ & $0.10 \%$ \\
\hline \multicolumn{2}{|c|}{$(0.035,0.204,-0.036)$} & \multicolumn{2}{|c|}{$(0.035,0.519,-0.036)$} & \multicolumn{2}{|c|}{$(0.035,0.838,-0.036)$} \\
\hline $10 \mathrm{~m} / \mathrm{s}$ & $0.30 \%$ & $10 \mathrm{~m} / \mathrm{s}$ & $0.50 \%$ & $10 \mathrm{~m} / \mathrm{s}$ & $0.40 \%$ \\
\hline $15 \mathrm{~m} / \mathrm{s}$ & $0.00 \%$ & $15 \mathrm{~m} / \mathrm{s}$ & $-0.20 \%$ & $15 \mathrm{~m} / \mathrm{s}$ & $-0.30 \%$ \\
\hline $20 \mathrm{~m} / \mathrm{s}$ & $-0.10 \%$ & $20 \mathrm{~m} / \mathrm{s}$ & $-0.50 \%$ & $20 \mathrm{~m} / \mathrm{s}$ & $-0.50 \%$ \\
\hline $25 \mathrm{~m} / \mathrm{s}$ & $0.00 \%$ & $25 \mathrm{~m} / \mathrm{s}$ & $-0.70 \%$ & $25 \mathrm{~m} / \mathrm{s}$ & $0.60 \%$ \\
\hline \multicolumn{2}{|c|}{$(0.035,0.204,0.593)$} & \multicolumn{2}{|c|}{$(0.035,0.519,0.593)$} & \multicolumn{2}{|c|}{$(0.035,0.838,0.593)$} \\
\hline $10 \mathrm{~m} / \mathrm{s}$ & $0.90 \%$ & $10 \mathrm{~m} / \mathrm{s}$ & $0.70 \%$ & $10 \mathrm{~m} / \mathrm{s}$ & $0.30 \%$ \\
\hline $15 \mathrm{~m} / \mathrm{s}$ & $0.30 \%$ & $15 \mathrm{~m} / \mathrm{s}$ & $0.00 \%$ & $15 \mathrm{~m} / \mathrm{s}$ & $-0.40 \%$ \\
\hline $20 \mathrm{~m} / \mathrm{s}$ & $0.10 \%$ & $20 \mathrm{~m} / \mathrm{s}$ & $-0.30 \%$ & $20 \mathrm{~m} / \mathrm{s}$ & $-0.70 \%$ \\
\hline $25 \mathrm{~m} / \mathrm{s}$ & $-0.10 \%$ & $25 \mathrm{~m} / \mathrm{s}$ & $-0.40 \%$ & $25 \mathrm{~m} / \mathrm{s}$ & $-1.00 \%$ \\
\hline
\end{tabular}

All points have an absolute mean variation less than $1 \%$, with an average of $0.4 \%$ over the tested speed range. Based on Table 5-1, there do not appear to be any trends regarding velocity variations as a function of location or tunnel speed. Disturbance intensities for each measurement point shown in Figure 5-1 are listed in Tables 5-2 - 5-5 for $10-25 \mathrm{~m} / \mathrm{s}$. 
Table 5-2. Test section disturbance intensities at $10 \mathrm{~m} / \mathrm{s}$; AC-coupled, $1 \mathrm{~Hz}-10 \mathrm{kHz}$ bandpass; Uncertainty: $\pm 0.005 \%$.

\begin{tabular}{|c|c|c|c|c|c|c|c|c|c|c|}
\hline & \multicolumn{3}{|c|}{ Plane A, $X=0.035$} & \multicolumn{3}{|c|}{ Plane B, $X=0.406$} & \multicolumn{3}{|c|}{ Plane C, $X=0.750$} \\
\hline & & $\begin{array}{c}Y= \\
0.204\end{array}$ & $\begin{array}{c}Y= \\
0.519\end{array}$ & $\begin{array}{c}Y= \\
0.838\end{array}$ & $\begin{array}{c}Y= \\
0.204\end{array}$ & $\begin{array}{c}Y= \\
0.519\end{array}$ & $\begin{array}{c}Y= \\
0.838\end{array}$ & $\begin{array}{c}Y= \\
0.204\end{array}$ & $\begin{array}{c}Y= \\
0.519\end{array}$ & $\begin{array}{c}Y= \\
0.838\end{array}$ \\
\hline \multirow{3}{*}{$\begin{array}{c}Z= \\
-0.593\end{array}$} & $u_{r m s}^{\prime} / U_{o}$ & $0.053 \%$ & $0.045 \%$ & $0.044 \%$ & $0.048 \%$ & $0.048 \%$ & $0.055 \%$ & $0.061 \%$ & $0.052 \%$ & $0.080 \%$ \\
\hline & $v_{r m s}^{\prime} / U_{o}$ & $0.016 \%$ & $0.030 \%$ & - & $0.021 \%$ & $0.013 \%$ & - & $0.034 \%$ & $0.018 \%$ & - \\
\hline & $w_{r m s}^{\prime} / U_{o}$ & $0.025 \%$ & $0.019 \%$ & $0.027 \%$ & $0.024 \%$ & $0.017 \%$ & $0.047 \%$ & $0.036 \%$ & $0.028 \%$ & $0.058 \%$ \\
\hline \multirow{3}{*}{$\begin{array}{c}Z= \\
-0.036\end{array}$} & $u_{r m s}^{\prime} / U_{o}$ & $0.048 \%$ & $0.047 \%$ & $0.044 \%$ & $0.046 \%$ & $0.047 \%$ & $0.050 \%$ & $0.046 \%$ & $0.041 \%$ & $0.058 \%$ \\
\hline & $v_{r m s}^{\prime} / U_{o}$ & $0.013 \%$ & $0.012 \%$ & - & $0.015 \%$ & $0.010 \%$ & - & $0.028 \%$ & $0.012 \%$ & - \\
\hline & $w_{r m s}^{\prime} / U_{o}$ & $0.014 \%$ & $0.012 \%$ & $0.016 \%$ & $0.014 \%$ & $0.012 \%$ & $0.019 \%$ & $0.018 \%$ & $0.014 \%$ & $0.032 \%$ \\
\hline \multirow{3}{*}{$\begin{array}{c}Z= \\
0.593\end{array}$} & $u_{r m s}^{\prime} / U_{o}$ & $0.054 \%$ & $0.053 \%$ & $0.062 \%$ & $0.057 \%$ & $0.047 \%$ & $0.058 \%$ & $0.060 \%$ & $0.050 \%$ & $0.091 \%$ \\
\hline & $v_{r m s}^{\prime} / U_{o}$ & $0.019 \%$ & $0.016 \%$ & - & $0.037 \%$ & $0.014 \%$ & - & $0.038 \%$ & $0.022 \%$ & - \\
\hline & $w_{r m s}^{\prime} / U_{o}$ & $0.023 \%$ & $0.024 \%$ & $0.036 \%$ & $0.018 \%$ & $0.025 \%$ & $0.037 \%$ & $0.021 \%$ & $0.026 \%$ & $0.067 \%$ \\
\hline
\end{tabular}


Table 5-3. Test section disturbance intensities at $15 \mathrm{~m} / \mathrm{s} ;$ AC-coupled, $1 \mathrm{~Hz}-10 \mathrm{kHz}$ bandpass; Uncertainty: $\pm 0.005 \%$.

\begin{tabular}{|c|c|c|c|c|c|c|c|c|c|c|}
\hline & \multicolumn{3}{|c|}{ Plane A, $X=0.035$} & \multicolumn{3}{|c|}{ Plane B, $X=0.406$} & \multicolumn{3}{|c|}{ Plane C, $X=0.750$} \\
\hline & & $\begin{array}{c}Y= \\
0.204\end{array}$ & $\begin{array}{c}Y= \\
0.519\end{array}$ & $\begin{array}{c}Y= \\
0.838\end{array}$ & $\begin{array}{c}Y= \\
0.204\end{array}$ & $\begin{array}{c}Y= \\
0.519\end{array}$ & $\begin{array}{c}Y= \\
0.838\end{array}$ & $\begin{array}{c}Y= \\
0.204\end{array}$ & $\begin{array}{c}Y= \\
0.519\end{array}$ & $\begin{array}{c}Y= \\
0.838\end{array}$ \\
\hline \multirow{3}{*}{$\begin{array}{c}Z= \\
-0.593\end{array}$} & $u_{r m s}^{\prime} / U_{o}$ & $0.075 \%$ & $0.072 \%$ & $0.063 \%$ & $0.067 \%$ & $0.072 \%$ & $0.081 \%$ & $0.080 \%$ & $0.075 \%$ & $0.093 \%$ \\
\hline & $v_{r m s}^{\prime} / U_{o}$ & $0.020 \%$ & $0.017 \%$ & - & $0.022 \%$ & $0.014 \%$ & - & $0.038 \%$ & $0.023 \%$ & - \\
\hline & $w_{r m s}^{\prime} / U_{o}$ & $0.016 \%$ & $0.020 \%$ & $0.032 \%$ & $0.023 \%$ & $0.020 \%$ & $0.055 \%$ & $0.038 \%$ & $0.032 \%$ & $0.057 \%$ \\
\hline \multirow{3}{*}{$\begin{array}{c}Z= \\
-0.036\end{array}$} & $u_{r m s}^{\prime} / U_{o}$ & $0.082 \%$ & $0.073 \%$ & $0.074 \%$ & $0.069 \%$ & $0.069 \%$ & $0.075 \%$ & $0.069 \%$ & $0.064 \%$ & $0.079 \%$ \\
\hline & $v_{r m s}^{\prime} / U_{o}$ & $0.066 \%$ & $0.012 \%$ & - & $0.032 \%$ & $0.011 \%$ & - & $0.028 \%$ & $0.015 \%$ & - \\
\hline & $w_{r m s}^{\prime} / U_{o}$ & $0.013 \%$ & $0.015 \%$ & $0.014 \%$ & $0.016 \%$ & $0.016 \%$ & $0.018 \%$ & $0.020 \%$ & $0.017 \%$ & $0.034 \%$ \\
\hline \multirow{3}{*}{$\begin{array}{c}Z= \\
0.593\end{array}$} & $u_{r m s}^{\prime} / U_{o}$ & $0.077 \%$ & $0.071 \%$ & $0.079 \%$ & $0.077 \%$ & $0.069 \%$ & $0.075 \%$ & $0.077 \%$ & $0.067 \%$ & $0.101 \%$ \\
\hline & $v_{r m s}^{\prime} / U_{o}$ & $0.018 \%$ & $0.015 \%$ & - & $0.036 \%$ & $0.013 \%$ & - & $0.036 \%$ & $0.023 \%$ & - \\
\hline & $w_{r m s}^{\prime} / U_{o}$ & $0.013 \%$ & $0.022 \%$ & $0.020 \%$ & $0.014 \%$ & $0.018 \%$ & $0.031 \%$ & $0.023 \%$ & $0.027 \%$ & $0.065 \%$ \\
\hline
\end{tabular}


Table 5-4. Test section disturbance intensities at $20 \mathrm{~m} / \mathrm{s} ;$ AC-coupled, $1 \mathrm{~Hz}-10 \mathrm{kHz}$ bandpass; Uncertainty: $\pm 0.005 \%$.

\begin{tabular}{|c|c|c|c|c|c|c|c|c|c|c|}
\hline & \multicolumn{3}{|c|}{ Plane A, $X=0.035$} & \multicolumn{3}{|c|}{ Plane B, $X=0.406$} & \multicolumn{3}{|c|}{ Plane C, $X=0.750$} \\
\hline & & $\begin{array}{c}Y= \\
0.204\end{array}$ & $\begin{array}{c}Y= \\
0.519\end{array}$ & $\begin{array}{c}Y= \\
0.838\end{array}$ & $\begin{array}{c}Y= \\
0.204\end{array}$ & $\begin{array}{c}Y= \\
0.519\end{array}$ & $\begin{array}{c}Y= \\
0.838\end{array}$ & $\begin{array}{c}Y= \\
0.204\end{array}$ & $\begin{array}{c}Y= \\
0.519\end{array}$ & $\begin{array}{c}Y= \\
0.838\end{array}$ \\
\hline \multirow{3}{*}{$\begin{array}{c}Z= \\
-0.593\end{array}$} & $u_{r m s}^{\prime} / U_{o}$ & $0.101 \%$ & $0.106 \%$ & $0.087 \%$ & $0.095 \%$ & $0.103 \%$ & $0.113 \%$ & $0.100 \%$ & $0.102 \%$ & $0.116 \%$ \\
\hline & $v_{r m s}^{\prime} / U_{o}$ & $0.017 \%$ & $0.016 \%$ & - & $0.025 \%$ & $0.017 \%$ & - & $0.039 \%$ & $0.020 \%$ & - \\
\hline & $w_{r m s}^{\prime} / U_{o}$ & $0.018 \%$ & $0.015 \%$ & $0.037 \%$ & $0.024 \%$ & $0.019 \%$ & $0.063 \%$ & $0.039 \%$ & $0.031 \%$ & $0.058 \%$ \\
\hline \multirow{3}{*}{$\begin{array}{c}Z= \\
-0.036\end{array}$} & $u_{r m s}^{\prime} / U_{o}$ & $0.108 \%$ & $0.106 \%$ & $0.107 \%$ & $0.098 \%$ & $0.091 \%$ & $0.101 \%$ & $0.100 \%$ & $0.094 \%$ & $0.102 \%$ \\
\hline & $v_{r m s}^{\prime} / U_{o}$ & $0.028 \%$ & $0.016 \%$ & - & $0.044 \%$ & $0.015 \%$ & - & $0.034 \%$ & $0.017 \%$ & - \\
\hline & $w_{r m s}^{\prime} / U_{o}$ & $0.020 \%$ & $0.015 \%$ & $0.018 \%$ & $0.019 \%$ & $0.019 \%$ & $0.021 \%$ & $0.025 \%$ & $0.020 \%$ & $0.034 \%$ \\
\hline \multirow{3}{*}{$\begin{array}{c}Z= \\
0.593\end{array}$} & $u_{r m s}^{\prime} / U_{o}$ & $0.105 \%$ & $0.106 \%$ & $0.102 \%$ & $0.102 \%$ & $0.097 \%$ & $0.100 \%$ & $0.104 \%$ & $0.089 \%$ & $0.118 \%$ \\
\hline & $v_{r m s}^{\prime} / U_{o}$ & $0.027 \%$ & $0.019 \%$ & - & $0.042 \%$ & $0.016 \%$ & - & $0.039 \%$ & $0.025 \%$ & - \\
\hline & $w_{r m s}^{\prime} / U_{o}$ & $0.020 \%$ & $0.022 \%$ & $0.023 \%$ & $0.017 \%$ & $0.021 \%$ & $0.032 \%$ & $0.023 \%$ & $0.029 \%$ & $0.062 \%$ \\
\hline
\end{tabular}


Table 5-5. Test section disturbance intensities at $25 \mathrm{~m} / \mathrm{s}$; AC-coupled, $1 \mathrm{~Hz}-10 \mathrm{kHz}$ bandpass; Uncertainty: $\pm 0.005 \%$.

\begin{tabular}{|c|c|c|c|c|c|c|c|c|c|c|}
\hline & \multicolumn{3}{|c|}{ Plane A, $X=0.035$} & \multicolumn{3}{|c|}{ Plane B, $X=0.406$} & \multicolumn{3}{|c|}{ Plane C, $X=0.750$} \\
\hline & & $\begin{array}{c}Y= \\
0.204\end{array}$ & $\begin{array}{c}Y= \\
0.519\end{array}$ & $\begin{array}{c}Y= \\
0.838\end{array}$ & $\begin{array}{c}Y= \\
0.204\end{array}$ & $\begin{array}{c}Y= \\
0.519\end{array}$ & $\begin{array}{c}Y= \\
0.838\end{array}$ & $\begin{array}{c}Y= \\
0.204\end{array}$ & $\begin{array}{c}Y= \\
0.519\end{array}$ & $\begin{array}{c}Y= \\
0.838\end{array}$ \\
\hline \multirow{3}{*}{$\begin{array}{c}Z= \\
-0.593\end{array}$} & $u_{r m s}^{\prime} / U_{o}$ & $0.138 \%$ & $0.134 \%$ & $0.125 \%$ & $0.121 \%$ & $0.133 \%$ & $0.131 \%$ & $0.123 \%$ & $0.130 \%$ & $0.144 \%$ \\
\hline & $v_{r m s}^{\prime} / U_{o}$ & $0.021 \%$ & $0.018 \%$ & - & $0.029 \%$ & $0.024 \%$ & - & $0.047 \%$ & $0.025 \%$ & 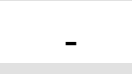 \\
\hline & $w_{r m s}^{\prime} / U_{o}$ & $0.021 \%$ & $0.018 \%$ & $0.046 \%$ & $0.026 \%$ & $0.021 \%$ & $0.068 \%$ & $0.042 \%$ & $0.031 \%$ & $0.061 \%$ \\
\hline \multirow{3}{*}{$\begin{array}{c}Z= \\
-0.036\end{array}$} & $u_{r m s}^{\prime} / U_{o}$ & $0.138 \%$ & $0.127 \%$ & $0.136 \%$ & $0.131 \%$ & $0.125 \%$ & $0.137 \%$ & $0.130 \%$ & $0.119 \%$ & $0.125 \%$ \\
\hline & $v_{r m s}^{\prime} / U_{o}$ & $0.040 \%$ & $0.021 \%$ & - & $0.034 \%$ & $0.019 \%$ & - & $0.041 \%$ & $0.027 \%$ & - \\
\hline & $w_{r m s}^{\prime} / U_{o}$ & $0.021 \%$ & $0.020 \%$ & $0.025 \%$ & $0.022 \%$ & $0.023 \%$ & $0.025 \%$ & $0.026 \%$ & $0.023 \%$ & $0.036 \%$ \\
\hline \multirow{3}{*}{$\begin{array}{c}Z= \\
0.593\end{array}$} & $u_{r m s}^{\prime} / U_{o}$ & $0.141 \%$ & $0.135 \%$ & $0.136 \%$ & $0.129 \%$ & $0.124 \%$ & $0.129 \%$ & $0.133 \%$ & $0.113 \%$ & $0.149 \%$ \\
\hline & $v_{r m s}^{\prime} / U_{o}$ & $0.023 \%$ & $0.021 \%$ & - & $0.038 \%$ & $0.019 \%$ & - & $0.044 \%$ & $0.028 \%$ & - \\
\hline & $w_{r m s}^{\prime} / U_{o}$ & $0.018 \%$ & $0.021 \%$ & $0.025 \%$ & $0.018 \%$ & $0.028 \%$ & $0.033 \%$ & $0.023 \%$ & $0.030 \%$ & $0.063 \%$ \\
\hline
\end{tabular}


Over the full speed range measured, streamwise disturbance intensities are elevated compared to the spanwise and wall-normal directions. Transverse velocity fluctuations are the same order of magnitude and are generally below the recommended $0.05 \%$ cutoff recommended by Saric \& Reshotko (1998) for stability experiments where freestream turbulence is a factor. The lower corner, where $(Y, Z)=(0.838,0.593)$ is the one exception. Slightly higher transverse fluctuations appear in this region. The source of the higher disturbance intensity has not been resolved; however, the interior door of the settling chamber, which is located in the same relative section before the contraction, may be a contributing factor. There are no signification differences in the junctions or paneling leading up to this corner. Higher turbulence levels in this location may not pose a significant problem since most models are mounted near $Y=0.563$, which is closer to the center measurement points in the above tables. Additionally, transverse fluctuations do not appreciably increase as tunnel speed increases.

To determine whether the streamwise turbulence levels are low enough for these crossflow experiments, the individual contribution of the rotational and irrotational components must be understood. Based on the extensive tests by Tan-Atichat et al. (1980), the KSWT contraction upstream of the test-section should result in streamwise turbulence intensities that are smaller than the transverse directions. The expectation, then, is that a majority of the streamwise disturbance intensity is related to the irrotational (acoustic) portion of the signal. A similar situation was detected during flow quality measurements at ASU (Saric et al. 1988, Saric 1992). Supporting evidence for this conclusion is provided below. 
Power spectral densities (PSD's) for $u^{\prime}, v^{\prime}$ and $w^{\prime}$ at point $(0.406,0.519,-0.024)$ are shown in Figure 5-3. At low frequencies $(<20 \mathrm{~Hz})$, the streamwise fluctuations have much larger amplitudes compared to the spanwise and wall-normal directions. As speed increases, the low-frequency content in $u^{\prime}$ continues to increase, while the $v^{\prime}$ and $w^{\prime}$ amplitudes remain relatively unchanged. Peaks greater than $20 \mathrm{~Hz}$ correlate to either power, motor, blade passing or stator passing frequencies and their harmonics. The one exception to this is at $10 \mathrm{~m} / \mathrm{s}$ where the motor rotation is approximately $8 \mathrm{~Hz}$ in the present configuration. These trends are representative of spectra from all data points taken. Appendix C provides the full set of spectral plots for $10 \mathrm{~m} / \mathrm{s}$ to $25 \mathrm{~m} / \mathrm{s}$. Changes in streamwise velocity fluctuations were tracked as turbulence reducing features such as the honeycomb and extra screens in the screen cage were installed. These updates yielded no significant change to the streamwise disturbance levels. Figure 5-4 compares the frequency content before and after these turbulence manipulators were installed. The PSD's show a decrease in high frequency content $(f>20 \mathrm{~Hz})$, while low-frequency content remains unchanged providing corroborating evidence that the high streamwise velocity fluctuations are the result of a pressure fluctuation. 


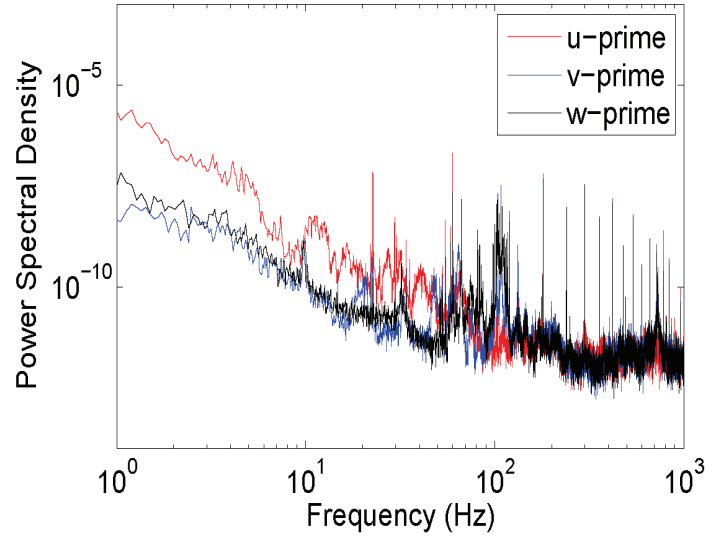

(a)

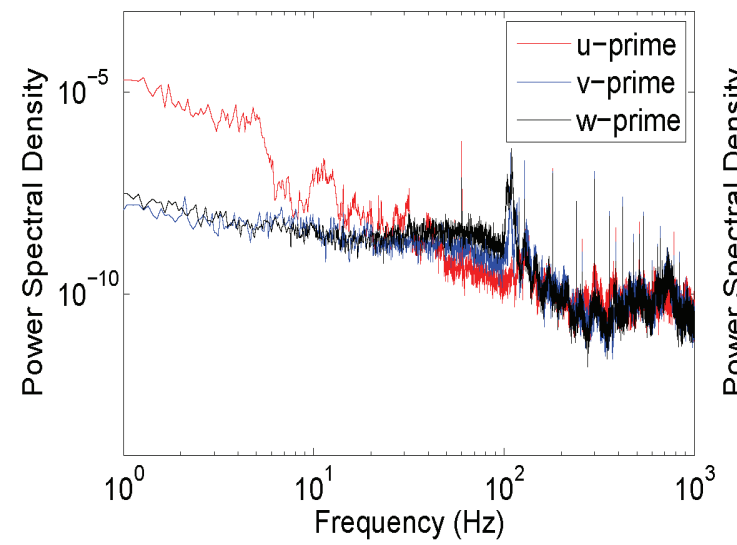

(c)

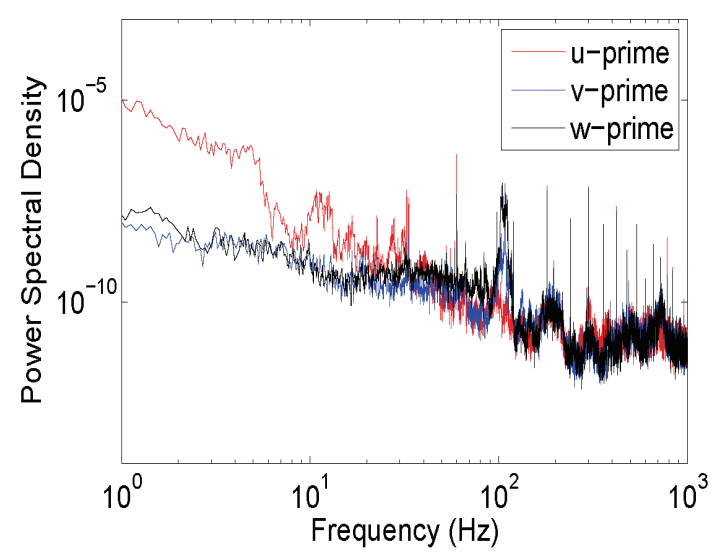

(b)

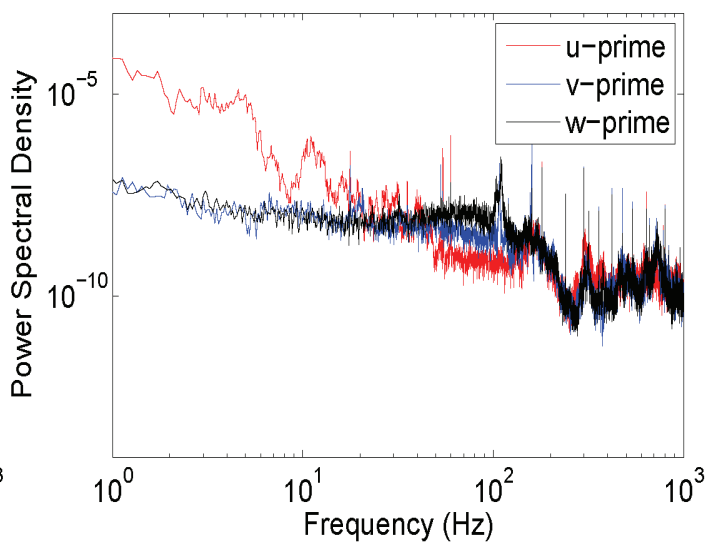

(d)

Figure 5-3. Power spectral density of $u^{\prime}, v^{\prime}$ and $w^{\prime}$ at (a) $10 \mathrm{~m} / \mathrm{s}$, (b) $15 \mathrm{~m} / \mathrm{s}$, (c) $20 \mathrm{~m} / \mathrm{s}$ and (d) $25 \mathrm{~m} / \mathrm{s}$. 


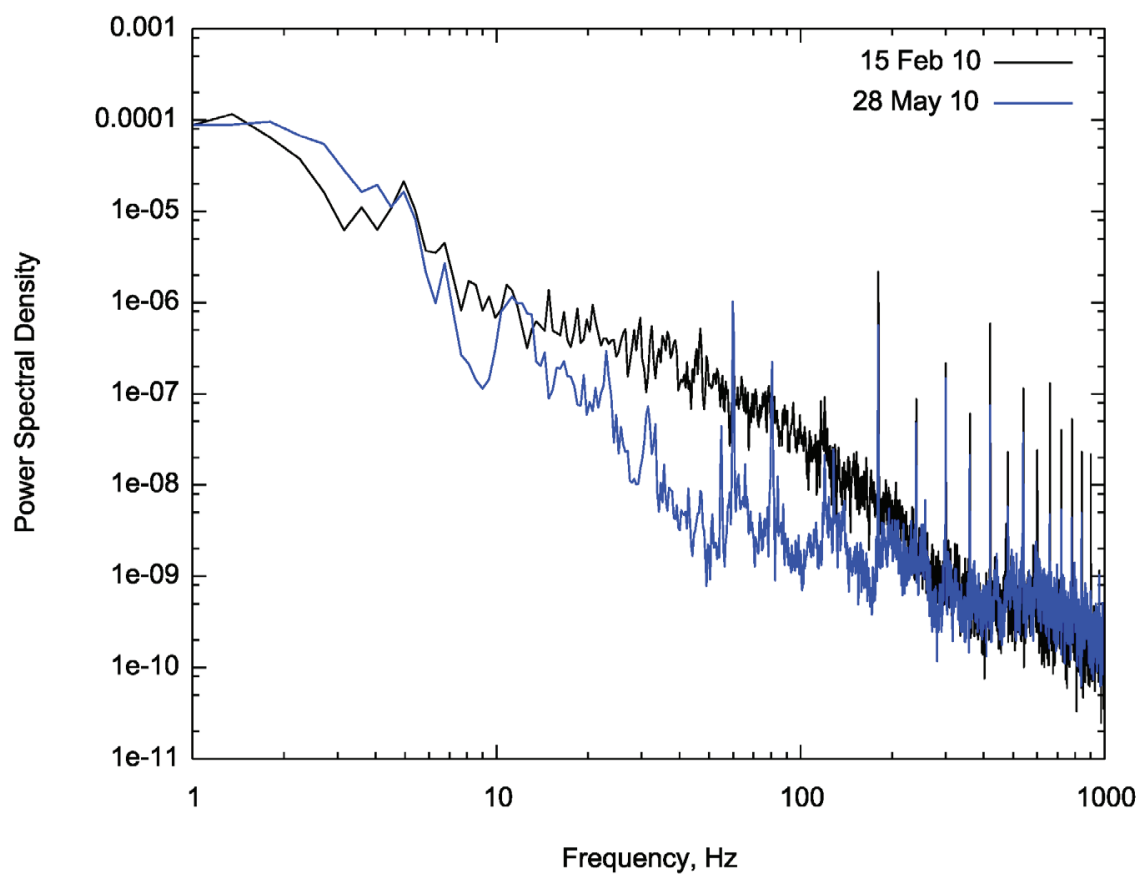

Figure 5-4. Power spectral density of $u^{\prime}$ at $20 \mathrm{~m} / \mathrm{s}$ before (15 Feb) and after (28 May) installation of turbulence manipulators.

Correlations of two signals in the same plane can also help determine whether the disturbance intensity is the result of acoustic or turbulent fluctuations. When the separation distance of the hotwires is large, a strong correlation indicates a signal dominated by pressure fluctuations. Figures 5-5 and 5-6 show correlations of $u^{\prime}$ and $w^{\prime}$ at $10 \mathrm{~m} / \mathrm{s}$ and $20 \mathrm{~m} / \mathrm{s}$ respectively for two crosswires mounted in Plane A at points $(0.035,0.204,0.593)$ and $(0.035,0.838,-0.593)$. At both speeds, a strong correlation is observed between the streamwise signals, whereas little correlation exists for the transverse fluctuations. Similar results are obtained at other speeds. In addition to again confirming that most of the streamwise signal is acoustic in nature, it also shows that the transverse disturbance intensities are more representative of turbulence levels in the wall-normal and spanwise directions rather that a total disturbance source. 

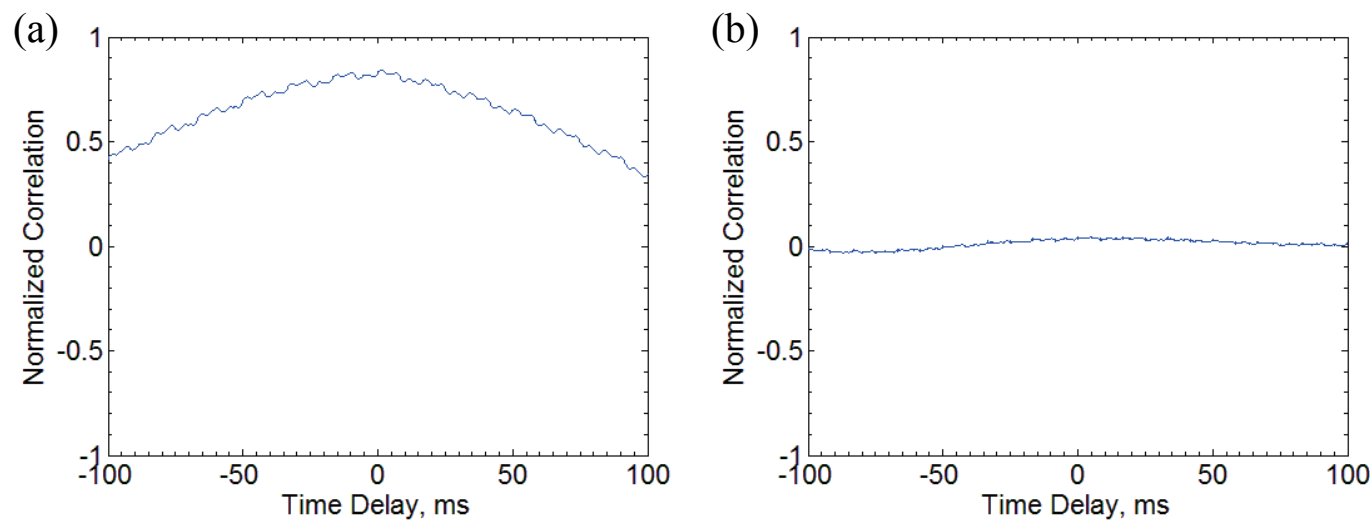

Figure 5-5. Correlation of two $u^{\prime}$ signals (a) and two $w^{\prime}$ signals (b) in Plane A, separated by a distance of $1.28 \mathrm{~m}$ in the $(Y, Z)$ plane at $10 \mathrm{~m} / \mathrm{s}$.
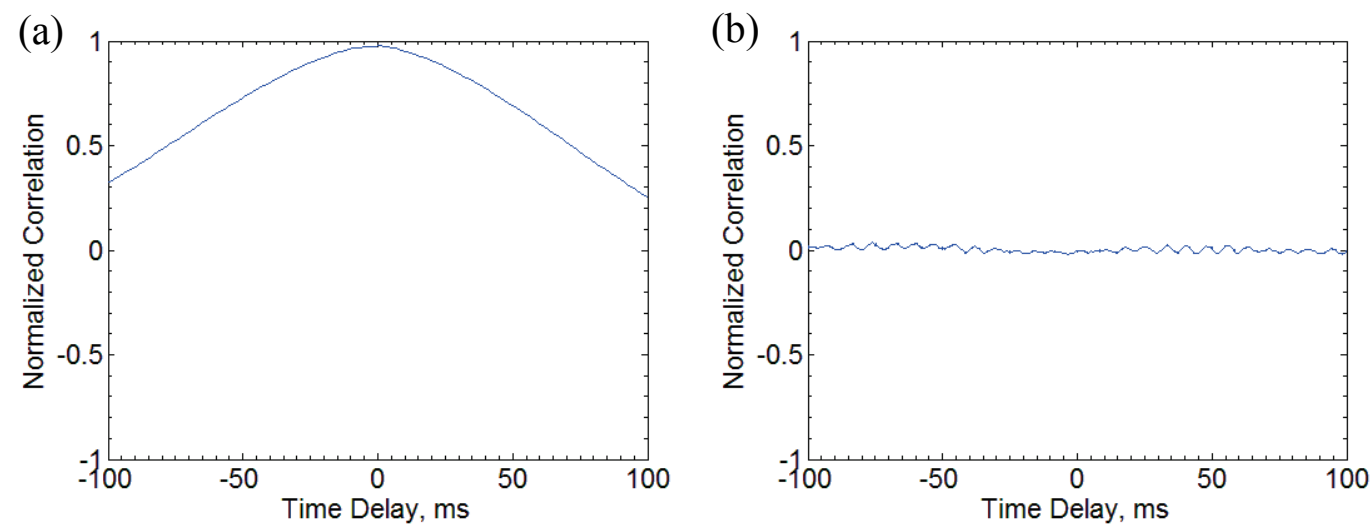

Figure 5-6. Correlation of two u' signals (a) and two w' signals (b) in Plane A, separated by a distance of $1.28 \mathrm{~m}$ in the $(Y, Z)$ plane at $20 \mathrm{~m} / \mathrm{s}$. 
Naguib et al. (1996) suggest an optimal filtering technique that can separate the total disturbance intensity into acoustic (correlated) and turbulent (uncorrelated) components. Using two straightwires in Plane $A$ at points $(0.035,0.204,0.593)$ and $(0.035,0.838,-0.593)$, turbulence intensities in the streamwise direction were obtained. Table 5-6 provides the results for $10 \mathrm{~m} / \mathrm{s}$ to $25 \mathrm{~m} / \mathrm{s}$.

Table 5-6. Separated turbulence and acoustic disturbance intensities.

\begin{tabular}{|c|c|c|c|c|}
\hline & & $U[\mathrm{~m} / \mathrm{s}]$ & $u^{\prime}[\mathrm{m} / \mathrm{s}]$ & $\% u^{\prime} / U$ \\
\hline \multirow{4}{*}{$10 \mathrm{~m} / \mathrm{s}$} & HW0 & 9.98 & 0.0051 & 0.051 \\
\hline & HW1 & 9.51 & 0.0055 & 0.058 \\
\hline & Correlated & \multirow{2}{*}{9.74} & 0.0039 & 0.040 \\
\hline & Uncorrelated & & 0.0039 & 0.040 \\
\hline \multirow{4}{*}{$15 \mathrm{~m} / \mathrm{s}$} & HW0 & 14.88 & 0.0124 & 0.083 \\
\hline & HW1 & 14.83 & 0.0123 & 0.083 \\
\hline & Correlated & \multirow{2}{*}{14.85} & 0.0121 & 0.081 \\
\hline & Uncorrelated & & 0.0025 & 0.017 \\
\hline \multirow{4}{*}{$20 \mathrm{~m} / \mathrm{s}$} & HW0 & 19.81 & 0.0208 & 0.105 \\
\hline & HW1 & 18.53 & 0.0178 & 0.096 \\
\hline & Correlated & \multirow{2}{*}{19.17} & 0.0173 & 0.090 \\
\hline & Uncorrelated & & 0.004 & 0.021 \\
\hline \multirow{4}{*}{$25 \mathrm{~m} / \mathrm{s}$} & HW0 & 24.58 & 0.0364 & 0.148 \\
\hline & HW1 & 22.90 & 0.0291 & 0.127 \\
\hline & Correlated & \multirow{2}{*}{23.74} & 0.0283 & 0.119 \\
\hline & Uncorrelated & & 0.0068 & 0.029 \\
\hline
\end{tabular}

The uncorrelated component, which is representative of the turbulence intensity in the streamwise direction is the same order of magnitude as the transverse velocity fluctuations shown in Tables 5-2 -5-5. The majority of the disturbance intensity for the streamwise levels comes from acoustic fluctuations in the tunnel. Figure 5-7 shows 
spectra for the original hotwire signals and the correlated and uncorrelated components at $20 \mathrm{~m} / \mathrm{s}$. At low frequencies, the hotwire signal is dominated by the correlated components. At higher frequencies, the hotwires are dominated by the uncorrelated parts. This is consistent with changes in higher frequency content, but none at low frequencies, shown in Figure 5-4, as turbulence manipulators were added to the tunnel. Hunt et al. (2010) also show supporting microphone measurements that detect the lowfrequency content, again indicating that it is the result of a pressure fluctuation in the tunnel.

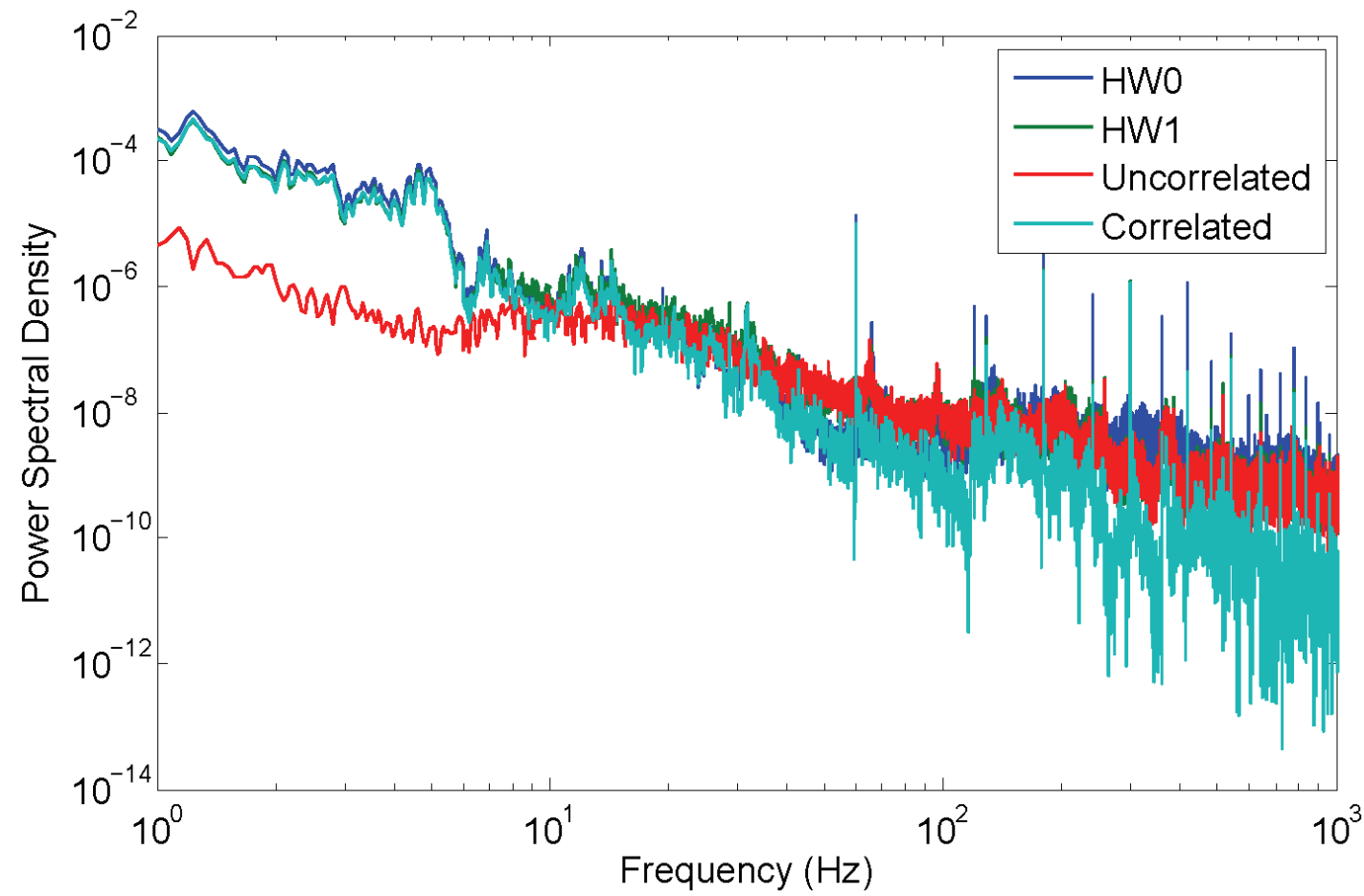

Figure 5-7. Power spectral densities of two $u^{\prime}$ signals and their correlated and uncorrelated components at $20 \mathrm{~m} / \mathrm{s}$.

Several attempts to identify the source of the low-frequency fluctuations were made during the flow quality assessment. Separated regions in the tunnel, structural vibration 
and irregular fan oscillations were all considered. Single-filament silk strands attached to a rod and portable hotwires were used in all diffusers and in the plenum to detect separated regions. Hotwires and naphthalene flow visualization were employed in the settling chamber and contraction cone to also try and detect separated regions. No significant deviations were identified. Vortex generators and screens were placed in the stage one diffuser downstream of the test section with no substantial change in the streamwise disturbance intensities. Spectra from accelerometers on the test section and fan housing did not show similar frequency content. A proximity sensor for the fan shaft installed in the PTC also provided negative results. Although the source of the pressure fluctuation has not yet been identified, the scale and wavelength of these acoustic disturbances are much larger than the turbulent scales that affect the crossflow instability. Presence of the low-frequency acoustic disturbance is not expected to impact this experiment.

The power spectral densities and correlation studies support the earlier conclusion indicating that the higher disturbance intensities measured in the streamwise direction are the result of a low-frequency pressure fluctuation and that transverse disturbance intensities are the best indicators of turbulence levels in the test section. Saric \& Reshotko (1998) recommend that turbulence levels be no greater than $0.05 \%$ for stability experiments where low-turbulence levels are required. Using this criterion, the KSWT is considered a suitable platform for crossflow instability measurements.

It is of interest to note that despite efforts to eliminate as much structural vibration as possible, the mounts used to support the hotwire probes are subject to varying degrees 
of vibration from nearby tunnel and flight operations. Subsequent measurements intended to evaluate repeatability have shown that abnormally high disturbance intensities can occur, for example, when the Oran W. Nicks Low-Speed Wind tunnel is operating at speeds greater than $70 \mathrm{~m} / \mathrm{s}$. These higher values are not due to increased turbulence in the tunnel, but rather probe vibration skewing the results. The present experimental measurements rely on the DC component of the signal and as a result, are less impacted by these external influences. However, as future turbulence intensities or traveling crossflow disturbances are measured, external environmental factors should be considered when evaluating the results.

\subsubsection{Comparison of ASU and TAMU Disturbance Intensities}

Table 5-7 compares the ASU UWT turbulence levels cited in Saric et al. (1988) and Saric (1992) with the TAMU KSWT turbulence levels. To make the comparison easier, the middle point in Plane B $(0.406,0.519,-0.024)$ is provided as a point characteristic of average levels in the test section. Only transverse fluctuations, which are most representative of turbulence levels are assessed. The comparison is not exactly one-toone since different bandpasses are used. However, the TAMU bandpass is more inclusive of frequency content, ensuring that instances of lower KSWT turbulence intensities are indeed lower. 
Table 5-7. Comparison of UWT and KSWT transverse velocity fluctuations.

\begin{tabular}{|r|c|c|c|c|}
\hline \multirow{2}{*}{$\begin{array}{c}\text { Freestream } \\
\text { Speed } \\
U_{o}(\mathrm{~m} / \mathrm{s})\end{array}$} & \multicolumn{2}{|c|}{$\begin{array}{c}\text { ASU Disturbance intensities } \\
\text { AC coupled, } 2 \mathrm{~Hz}-1 \mathrm{kHz} \text { bandpass }\end{array}$} & \multicolumn{2}{c|}{$\begin{array}{c}\text { TAMU Disturbance intensities } \\
\text { AC coupled, } 1 \mathrm{~Hz}-10 \mathrm{kHz} \text { bandpass }\end{array}$} \\
\cline { 2 - 5 } & $\begin{array}{c}\text { Wall-normal } \\
\text { Fluctuation } \\
v_{r m s}^{\prime} / U_{o}\end{array}$ & $\begin{array}{c}\text { Spanwise } \\
\text { Fluctuation } \\
w_{r m s}^{\prime} / U_{o}\end{array}$ & $\begin{array}{c}\text { Wall-normal } \\
\text { Fluctuation } \\
v_{r m s}^{\prime} / U_{o}\end{array}$ & $\begin{array}{c}\text { Spanwise } \\
\text { Fluctuation } \\
w_{r m s}^{\prime} / U_{o}\end{array}$ \\
\hline 5.0 & $0.007 \%$ & $0.007 \%$ & $0.015 \%$ & $0.013 \%$ \\
\hline 10.0 & $0.014 \%$ & $0.014 \%$ & $0.010 \%$ & $0.012 \%$ \\
\hline 15.0 & $0.014 \%$ & $0.014 \%$ & $0.011 \%$ & $0.016 \%$ \\
\hline 20.0 & Not provided & Not provided & $0.015 \%$ & $0.019 \%$ \\
\hline 25.0 & $0.032 \%$ & $0.032 \%$ & $0.019 \%$ & $0.023 \%$ \\
\hline
\end{tabular}

Most of the crossflow experiments operate in the range of $20-29 \mathrm{~m} / \mathrm{s}$. In these instances, the TAMU turbulence intensities are lower compared to ASU test conditions. For flight experiments at the FRL, Carpenter (2009) measured disturbance intensities (unseparated signal) in the streamwise direction between $0.05 \%$ and $0.07 \%$ for speeds between 81 and $86 \mathrm{~m} / \mathrm{s}$, with a bandpass of $1 \mathrm{~Hz}-10 \mathrm{kHz}$. Since the overall disturbance intensities were small enough for crossflow experiments, no attempt to separate the vortical and irrotational components or understand the influence of electronic noise was made. It is likely then, that the turbulence levels in flight are several orders of magnitude smaller than either tunnel configuration.

\subsubsection{Turbulence Levels with Model and Wall Liners Installed}

Model and wall liner installation covered all but three of the original mounting locations for the hotwire stand. Point $(0.035,0.204,-0.593)$ near the test section entrance on the non-test-side wall was used to recheck $u^{\prime}$ and $v^{\prime}$ fluctuations. With the floor mount covered, $w^{\prime}$ could not be measured without introducing interference from stand vibration. 
Additional $u^{\prime}$ and $v^{\prime}$ measurements were taken on the sting mount to examine freestream turbulence near the receptivity measurement region. On the sting mount, the crosswire was attached to the freestream hotwire mounting location (Figure 5-8). The traverse was positioned at midspan on the model and approximately halfway between the model and test section wall. Streamwise and wall-normal velocity fluctuations were acquired in this configuration at three locations that approximately correspond to $-14 \% x / c$ (forward of the leading edge), $0 \% x / c$ (at the leading edge) and $20 \% x / c$. Table $5-8$ provides the transverse turbulence intensities for these four points in the current experimental configuration and Figure 5-9 shows the spectra for point $(0.257,0.826,0.051)$. Only speeds in the experimental range of interest were checked.

Table 5-8. Turbulence intensities with model and wall liners installed.

\begin{tabular}{|c|c|c|c|c|}
\hline \multirow{2}{*}{$\begin{array}{c}\text { Test } \\
\text { Section } \\
\text { Speed } \\
{[\mathrm{m} / \mathrm{s}]}\end{array}$} & \multicolumn{4}{|c|}{$\begin{array}{c}\% v_{r m s}^{\prime} / U_{o} \\
\text { AC-coupled, } 1-10000 \mathrm{~Hz} \text { Bandpass }\end{array}$} \\
\cline { 2 - 5 } & Non-test-side mount & Traverse & Traverse & Traverse \\
& $(0.035,0.204,-0.036)$ & $(0.257,0.826,0.051)$ & $(0.308,0.826,0.051)$ & $(0.382,0.826,0.051)$ \\
\hline 15 & $0.013 \%$ & $0.018 \%$ & $0.019 \%$ & $0.020 \%$ \\
\hline 20 & $0.016 \%$ & $0.019 \%$ & $0.022 \%$ & $0.022 \%$ \\
\hline 25 & $0.019 \%$ & $0.022 \%$ & $0.025 \%$ & $0.026 \%$ \\
\hline
\end{tabular}



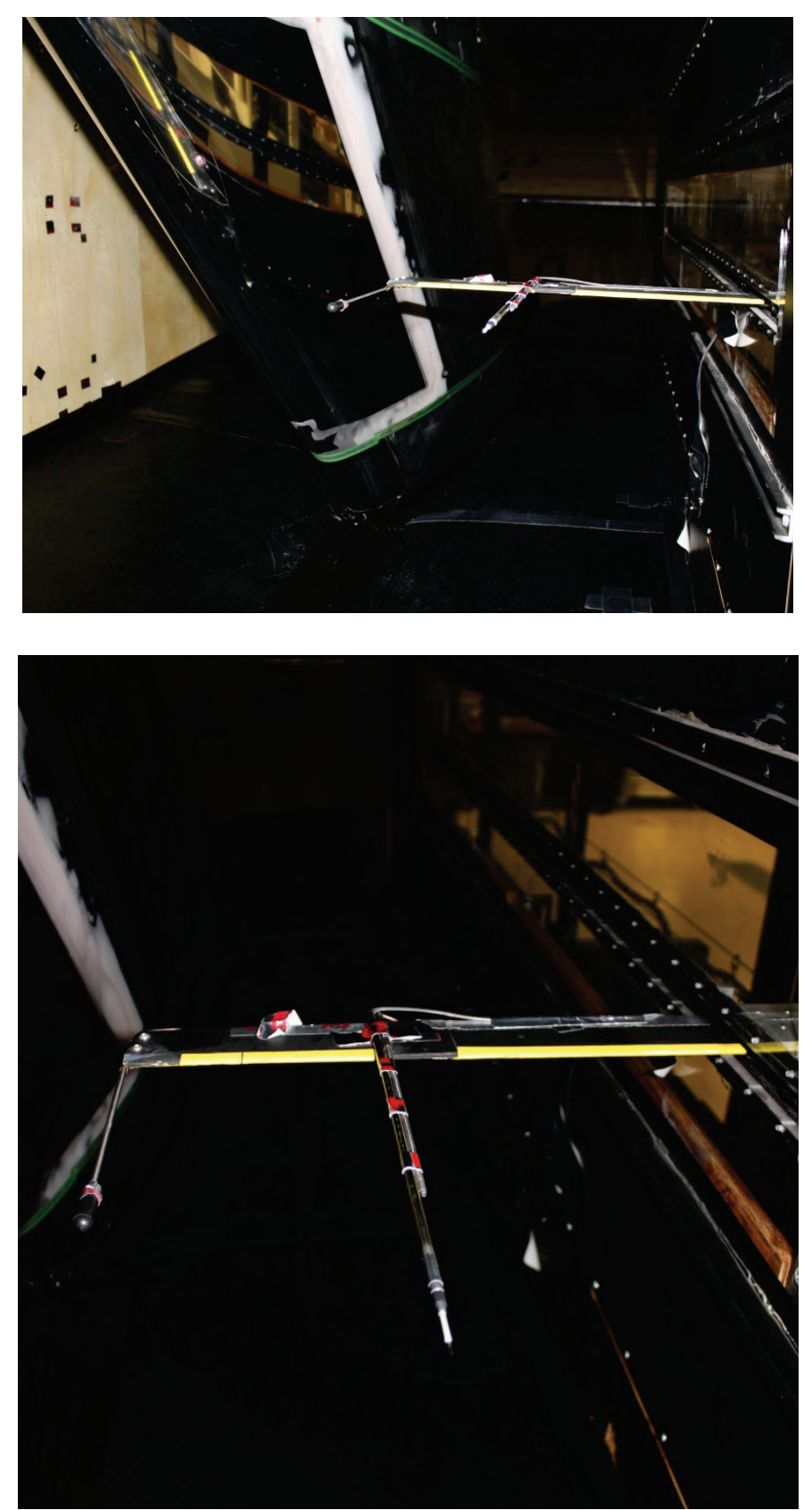

Figure 5-8. Crosswire mounting on traverse sting. 


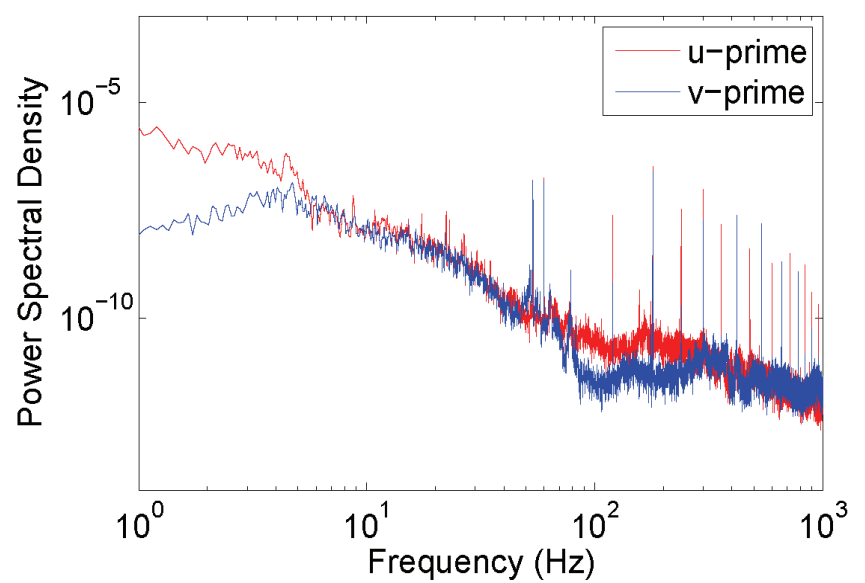

(a)

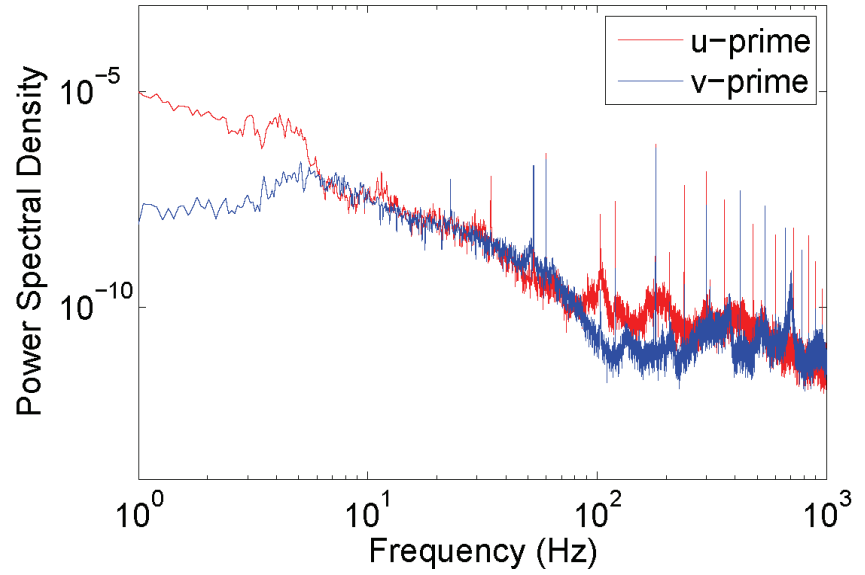

(b)

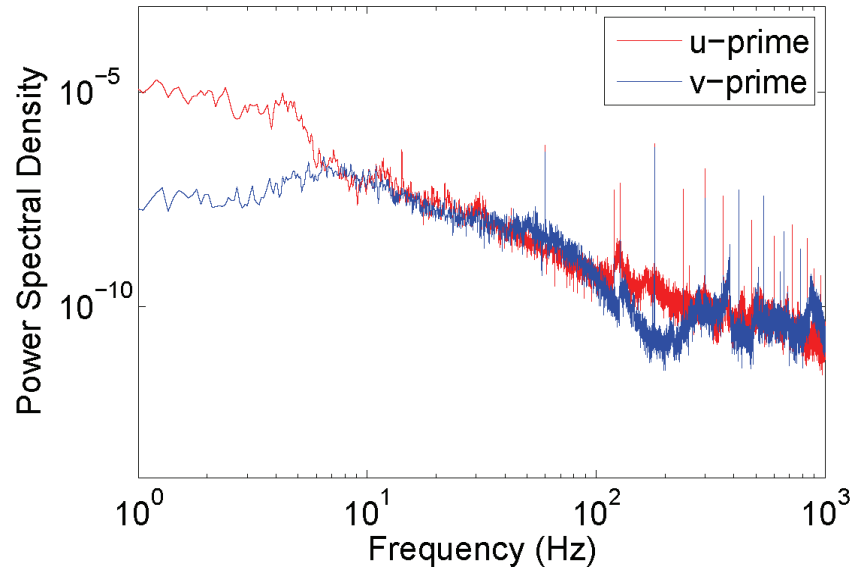

(c)

Figure 5-9. Spectra from crosswire measurements at $(0.257,0.826,0.051)$ for (a) $15 \mathrm{~m} / \mathrm{s}$, (b) $20 \mathrm{~m} / \mathrm{s}$, and (c) $25 \mathrm{~m} / \mathrm{s}$. 
Transverse velocity fluctuations are the same order of magnitude as the original empty test section flow quality measurements, indicating that addition of the model, wall liners and contraction fairings did not appreciably alter the freestream disturbance environment. Similar to the empty test section spectra, low-frequency content $(1-10 \mathrm{~Hz})$ is present in the streamwise direction, but not in the transverse. Addition of the wall liners seems to have eliminated the $10-20 \mathrm{~Hz}$ content originally present in the empty test section. Most of the peaks are associated with motor RPS, BPF, SPF or power. Results for the other two traverse locations are similar. Interestingly, Dagenhart (1992) notes that using a bandpass of $0.1 \mathrm{~Hz}-1 \mathrm{kHz}$, freestream turbulence, as indicated by transverse fluctuation measurements, is less than $0.04 \%$ over the full speed range of the tunnel. Addition of the NLF(2)-0415 and associated wall liners increased turbulence intensity to approximately $0.09 \%$. Data are not provided using a highpass of $2 \mathrm{~Hz}$, as done for the empty test section measurements at ASU, so there is no indication as to how much of the increase is due to content between 0.1 and $2 \mathrm{~Hz}$. However, this suggests that the turbulence levels in the KSWT configuration with the ASU(67)-0315 may be as much as half the original ASU swept-wing freestream turbulence intensities. Radeztsky (1994), Reibert (1996) and White (2000) appear to quote freestream turbulence measurements based on the original flow quality measurements without the swept-wing and wall liners installed. 


\subsection{Pressure Distribution}

Pressure measurements on the upper and lower pressure port rows were taken prior to starting any boundary-layer hotwire scans to determine whether the assumption of an infinite span swept-wing was valid for the experimental configuration. A pressure difference between the individual ports and test section freestream static pressure was measured and then normalized by the streamwise freestream speed as given by

$$
C_{p, 3 D}=\frac{p-p_{\infty}}{0.5 \rho U_{\infty}^{2}}
$$

where $C_{p}$ is the pressure coefficient, $p$ is static pressure at the port, $p_{\infty}$ is freestream static pressure, $\rho$ is density and $U_{\infty}$ is freestream speed. The $C_{p}$ as defined in Eqn. $5-1$ is referred to as the $3 \mathrm{D}$ case because the normalizing dynamic pressure is calculated using a freestream velocity parallel to the streamwise direction ( $X$ in the global coordinate system). All pressure distributions shown here are presented using this definition. Computationally, it is often convenient to calculate $C_{p}$ using a freestream velocity normal to the leading edge. The relationship between these two values is

$$
C_{p, 3 D}=\frac{p-p_{\infty}}{0.5 \rho\left(\frac{Q_{\infty}}{\cos \Lambda}\right)^{2}}=(\cos \Lambda)^{2} \frac{p-p_{\infty}}{0.5 \rho Q_{\infty}^{2}}=(\cos \Lambda)^{2} C_{p, 2 D}
$$

where $\Lambda$ is sweep angle and $Q_{\infty}$ is freestream velocity normal to the leading edge. For a sweep-angle of $45^{\circ}, C_{p, 2 D}$ is one-half $C_{p, 3 D}$. 
Pressure distributions were acquired for $\operatorname{Re}_{c}=2.4 \times 10^{6}, 2.8 \times 10^{6}$ and $3.2 \times 10^{6}$ and are shown in Figures 5-10-5-12. The datum for $0.03 x / c$ on the lower row was removed after abnormally high pressures were observed. The outlier is most likely the result of a clogged pressure port. Attempts to clean the port with acetone and pressurized air did not change the results. As designed, the pressure minimum occurs just aft of $70 \% x / c$. Both the upper and lower pressure distributions nominally agree for all Reynolds numbers indicating that the assumption of spanwise uniform flow is valid. Variations between the upper and lower rows are within the range of differences previously observed for the ASU experimental configurations. Uncertainty in the measurement decreases as Reynolds number increases since the accuracy of the pressure transducer is given as a percentage of the full scale. Figure 5-12 also includes a computational pressure distribution for same experimental angle of attack and Reynolds number. The viscous simulation assumes an infinite span wing and incorporates the tunnel side walls based on the dimensions provided in Figure 3-2. Experimental and computational results also agree, indicating that the experimental flowfield can be computationally replicated. Data tables for the three experimental cases are provided in Appendix B. Figure 5-13 compares the pressure distributions from the upper pressure port row for the three Reynolds numbers. The $C_{p}$ are almost exactly the same. Small variations are the result of differences in displacement thickness from boundary-layer growth on the tunnel walls and model. As a point of interest, the data at $R e_{c}=2.4 \times 10^{6}$ for the current experiment and from the previous ASU configuration at a slightly different angle of attack (White \& 
Saric 2005) are plotted in Figure 5-14. The two data sets are quite similar. Appendix B also includes the raw data from White \& Saric (2005).

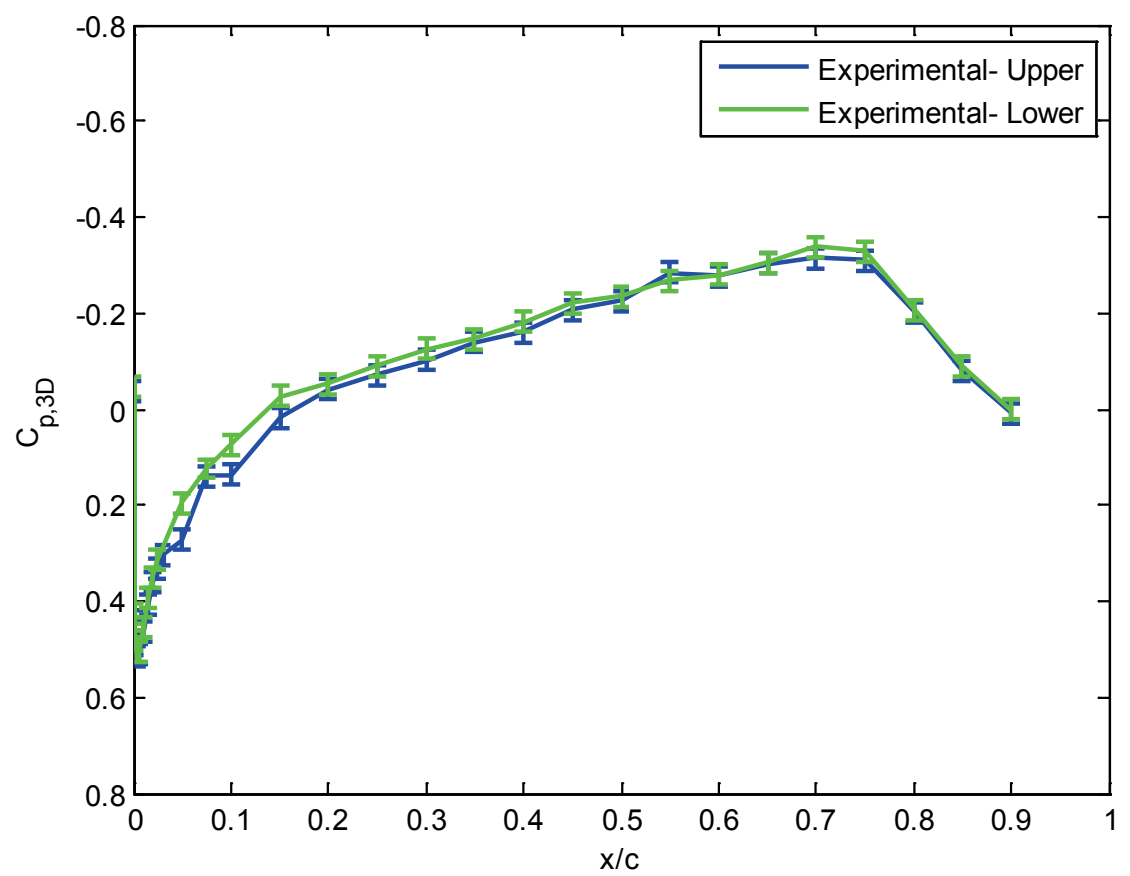

Figure 5-10. $C_{p}$ with wall liners, $\alpha=-2.9^{\circ}, R_{c}=2.4 \times 10^{6}$. 


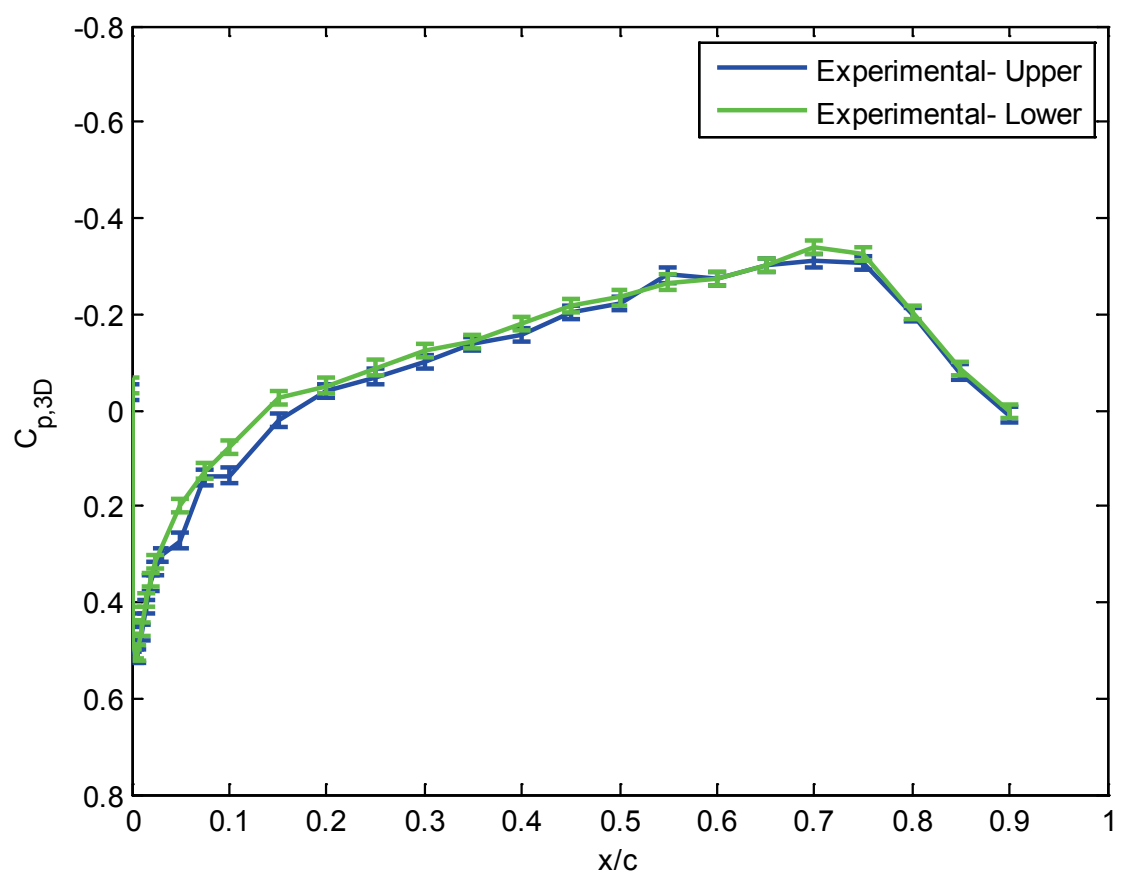

Figure 5-11. $C_{p}$ with wall liners, $\alpha=-2.9^{\circ}, R e_{c}=2.8 \times 10^{6}$.

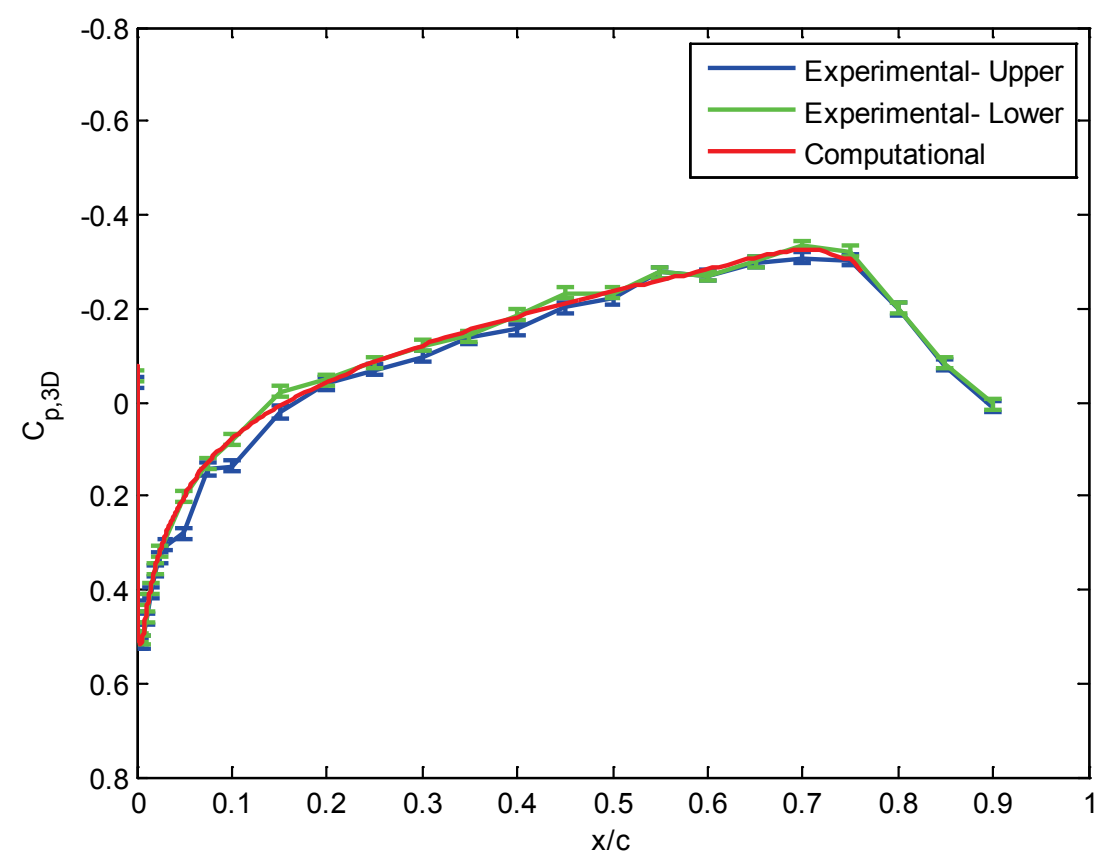

Figure 5-12. $C_{p}$ with wall liners, $\alpha=-2.9^{\circ}, R_{c}=3.2 \times 10^{6}$. 


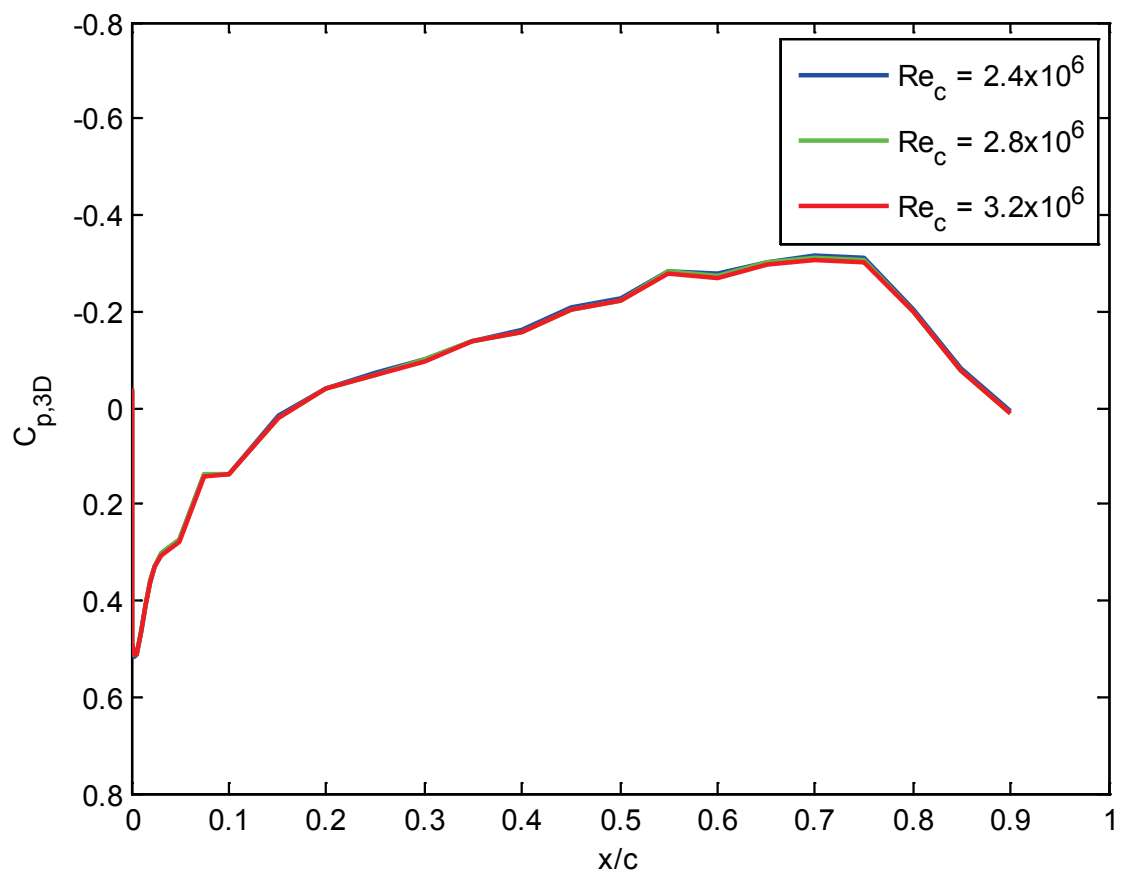

Figure 5-13. $C_{p}$ as a function of $R e_{c}$, with wall liners, $\alpha=-2.9^{\circ}$.

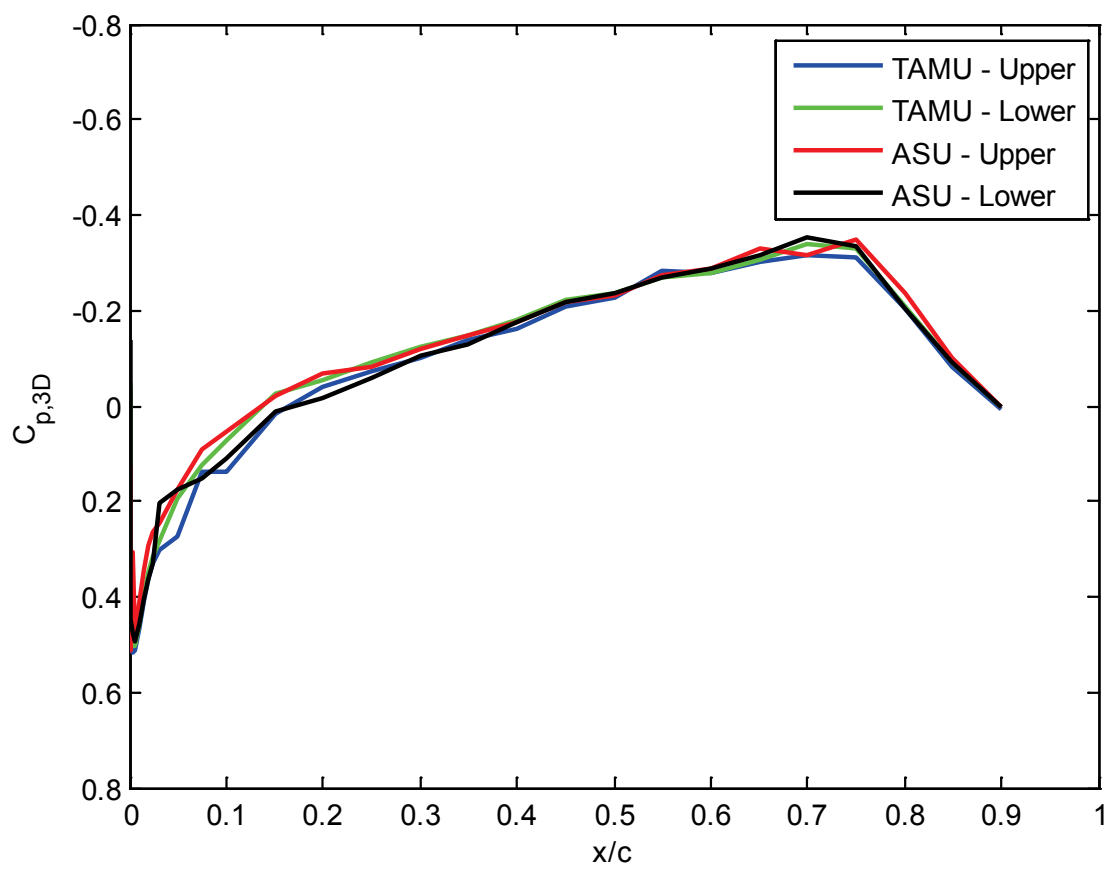

Figure 5-14. Comparison of ASU(67)-0315 at $\alpha=-2.9^{\circ}$ (TAMU) and $\alpha=-3.4^{\circ}$ (ASU). 


\subsection{Baseline Naphthalene Flow Visualization}

Although hotwire measurements with no artificial roughness are not part of the test matrix, a limited degree of NFV was completed with and without wall liners to determine the natural transition location in the absence of artificial roughness. Figure 5-15 shows the natural transition location on the model at $R e_{c}=2.6 \times 10^{6}$ without wall liners installed. Flow direction is left to right. Chord location is marked with white lines at the top of the image. Black lines indicating constant chord are visible in areas where the naphthalene remains. Shadows from the test section frame and reflections from the control room are visible in the image. Under these flow conditions, the transition front occurs just forward of $80 \% x / c$. Irregular wedges, characteristic of transition dominated by the stationary crossflow instability are not observed. Past the pressure-minimum at $71 \% x / c$, the relatively uniform front is indicative of transition due to the TollmienSchlichting instability which results from the decelerating boundary layer. Irregular streaking, due to stationary crossflow waves, is visible. When artificial roughness is not present the spacing of naturally occurring stationary crossflow waves will vary based on minor differences in local surface roughness near the leading edge (Radeztsky et al. 1999). As DRE are applied, streaking at regular intervals is expected. A single wedge in the upper span region starts at approximately $68 \% x / c$ and is most likely due to a small deviation in the surface roughness upstream of that location. NFV was not completed for $R e_{c}=2.4 \times 10^{6}$, the first test point. However, decreasing Reynolds number is a stabilizing effect. Transition would occur no earlier than the location shown in Figure $5-15$. 


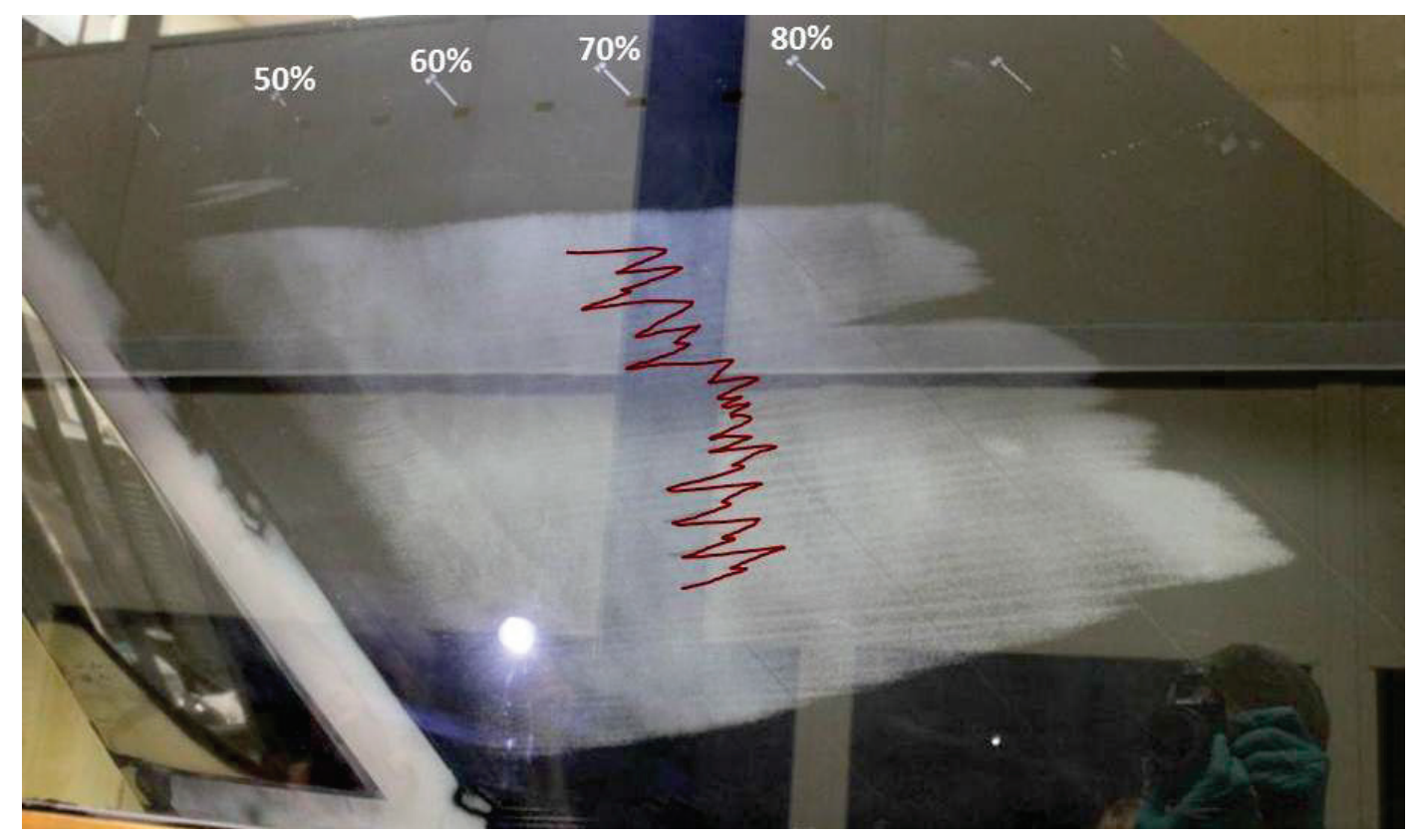

Figure 5-15. Naphthalene flow visualization of natural transition at TAMU for $R e_{c}=2.6 \times 10^{6}$, $\alpha=-2.9^{\circ}$; transition location from ASU at $R e_{c}=2.4 \times 10^{6}, \alpha=-3.4^{\circ}$, highlighted in red.

The red lines in Figure 5-15 highlight the natural transition location of the model at ASU at an angle of attack of $-3.4^{\circ}$ and $R e_{c}=2.4 \times 10^{6}$. There is a $20 \%-25 \% x / c$ difference in transition location between the two configurations. Whereas the lower angle of attack in the TAMU configuration is stabilizing, the higher Reynolds number at TAMU is destabilizing. Surface finish in each case is the same. Although differences in angle of attack must be considered, another possible explanation is the difference in flow quality between the ASU and TAMU configurations. Additional tests, not completed for this study, at the same angle of attack or supporting stability calculations should help resolve the primary factor involved.

Figure 5-16 shows the same test after wall liners and contraction fairings are installed. As before, transition occurs just forward of $80 \% x / c$ for the lower half-span of 
the model. Two more distinct wedges in the upper span region have appeared after wall liner installation. Their position is still quite far aft, providing a large usable measurement region. Hotwire scans are all taken well below the spanwise location of these wedges, ensuring that the most uniform region is selected for measurements.

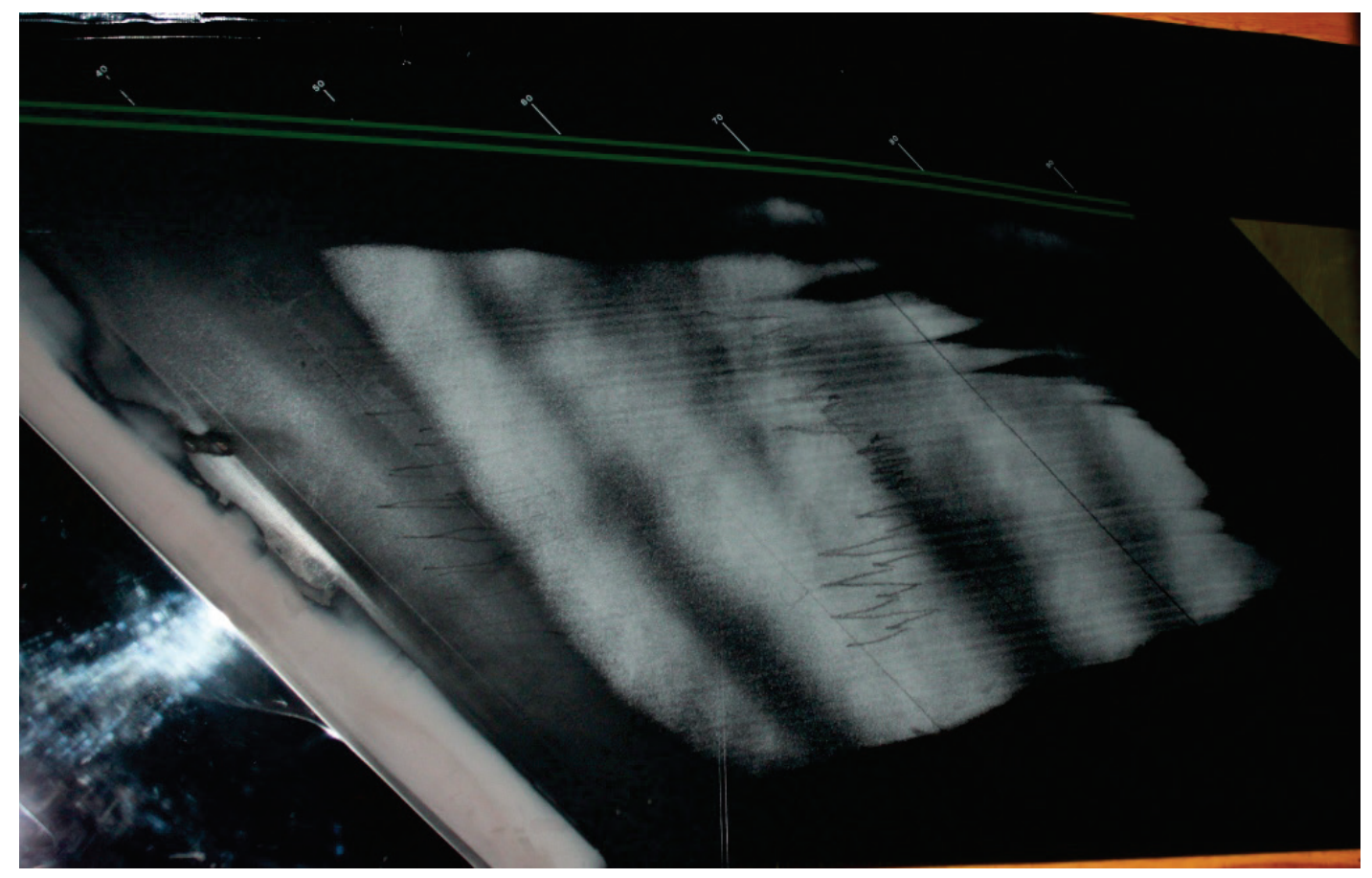

Figure 5-16. Naphthalene flow visualization of natural transition at TAMU for $\operatorname{Re}_{c}=2.6 \times 10^{6}$, $\alpha=-2.9^{\circ}$; wall-liners installed.

Flow visualization for $R e_{c}=2.8 \times 10^{6}$ and $R e_{c}=3.2 \times 10^{6}$ are shown in Figure 5-17 and Figure 5-18 respectively. The three wedges from Figure 5-16 have moved forward at $R e_{c}=2.8 \times 10^{6}$. Transition in the lower half-span, the hotwire measurement region, is between $65 \%$ and $75 \% x / c$. At $R e_{c}=3.2 \times 10^{6}$, transition is now between $55 \%$ and $60 \%$ $x / c$. It is not until reaching this higher Reynolds number that the characteristic irregular 
saw-tooth pattern documented at ASU is observed here at TAMU. In this case, transition occurs as a result of the stationary crossflow wave.

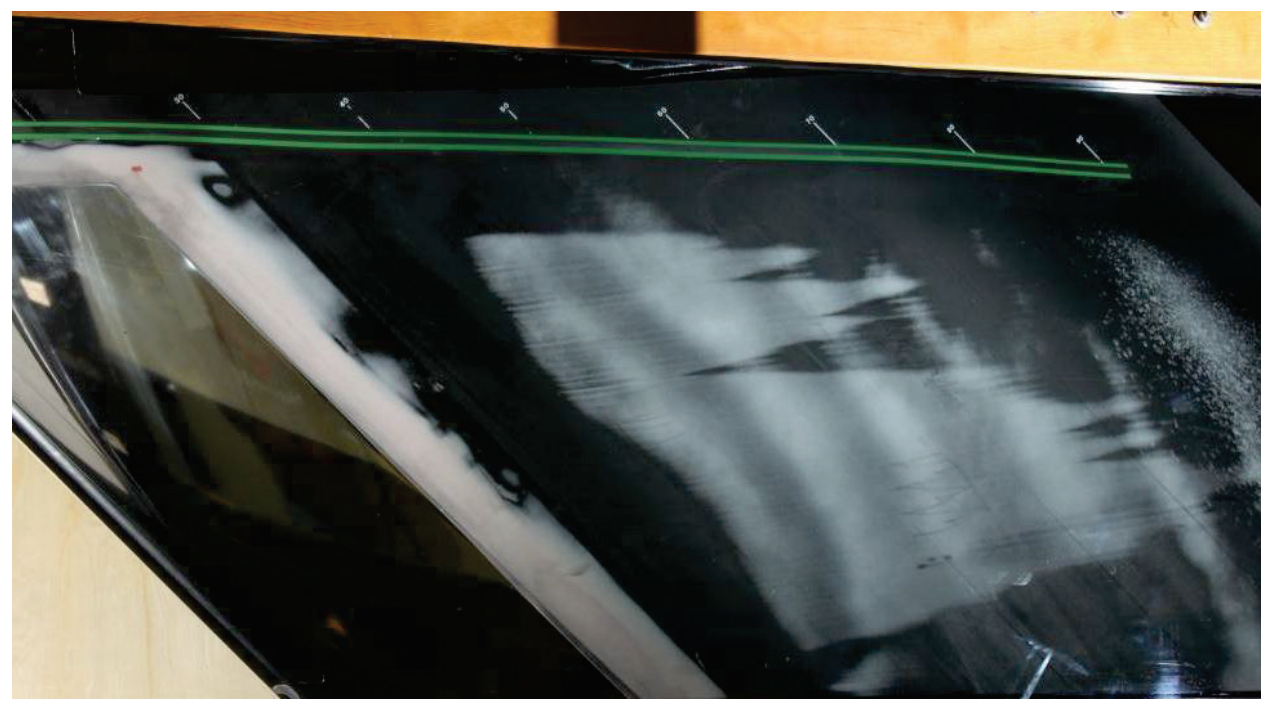

Figure 5-17. Naphthalene flow visualization of natural transition at TAMU for $R_{c}=2.8 \times 10^{6}$, $\alpha=-\mathbf{2 . 9 ^ { \circ }}$; wall-liners installed.

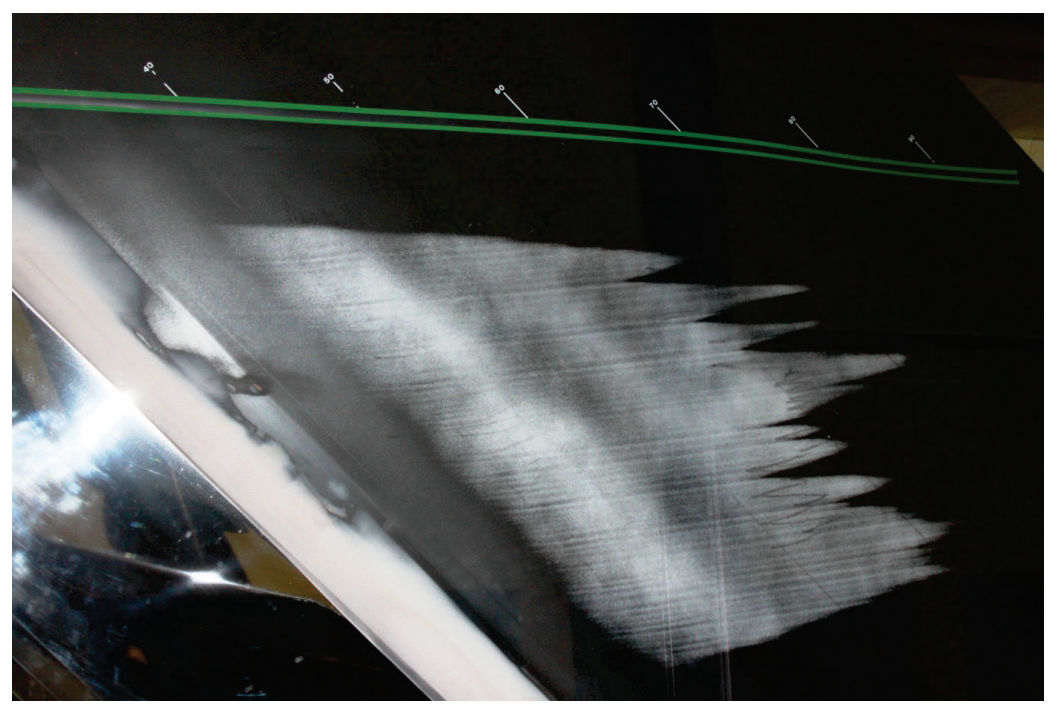

Figure 5-18. Naphthalene flow visualization of natural transition at TAMU for $R_{c}=3.2 \times 10^{6}$, $a=-2.9^{\circ}$ : wall-liners not installed. 


\section{RESULTS- PART II: RECEPTIVITY OF DRE ARRAY SPACED AT THE CRITICAL WAVELENGTH}

Stability characteristics for a given model at a constant angle of attack, sweep and roughness configuration are not necessarily the same as Reynolds number increases. NFV and hotwire results from the test matrix provided in Table 3-3 are grouped and discussed as a function of Reynolds number. Roughness notation follows the same format used in previous TAMU flight testing and ASU UWT tests. For example, [48|12] implies a roughness height, $k$, of $48 \mu \mathrm{m}$ spaced at $12 \mathrm{~mm}$. When multiple roughness heights are included on a plot, each $k$ is listed individually.

It should be noted that all of the NFV runs with artificial roughness applied were taken in conjunction with on-going hotwire measurements. After each new layer was applied, an NFV run was completed at a single Reynolds number to ensure that no wedges were present in the measurement region and to track transition location. In these instances, the traverse door was installed, providing limited optical access compared to the all-glass window used for the natural transition NFV runs discussed in Section 5. The smaller Plexiglas window does not have a large enough area to allow full span pictures. Camera angles were varied to capture as much information as possible during a particular run. NFV images discussed next are not necessarily taken from the same perspective for each case. Instead, the best image from each set is selected. Other features obscuring the full model shot include a black zipper that runs through the center of the window and regions where the Plexiglas is scratched due to traverse movement. 
As described in Section 2.2.1, the entire window translates in the $Z$ direction while the traverse moves to minimize the opening needed for the sting mount. Over time, movement of the window in its slot has resulted in isolated scratched areas that appear as a blurred spot in the photograph. Additionally, hotwire probe supports, located at $60 \%$ $x / c$ and their shadows are visible in some of the images. The Plexiglas pressure box provides a second reflective surface beyond the traverse window. External flashes set at oblique angles and low overhead lighting were used to limit and control lighting sources, but reflections of the test section support bars, camera operator and control room are visible in some images. Chord-locations starting at $30 \% x / c$ and set at $10 \%$ intervals are indicated by black lines drawn on the model surface. In many of the images, a red-line was added during post-processing to make identification easier. Finally, irregular black marks near 30\%-chord and 60\%-chord may be visible in certain images. These are traces of previous transition locations at ASU, not features of the current NFV.

\section{$6.1 R e_{c}=2.4 \times 10^{6}$}

\subsubsection{Naphthalene Flow Visualization}

Figure 6-1 shows the NFV results at $R e_{c}=2.4 \times 10^{6}$ with a wavelength spacing of $12 \mathrm{~mm}$, as roughness is height is increased from a starting height of $12 \mu \mathrm{m}$. Compared to Figure 5-16 with no artificial roughness applied, transition has moved forward from approximately $80 \% x / c$ to $60 \% x / c$ with the application of the first roughness layer that is $12 \mu \mathrm{m}$ high. As roughness height is increased, transition moves forward approximately 
$5 \% x / c$ for each new layer added up to $36 \mu \mathrm{m}$. Were this trend to continue, at $60 \mu \mathrm{m}$ transition should occur near $40 \% x / c$. Instead, transition occurs at approximately $45 \% x / c$ indicating that some nonlinear process has occurred. In each image, streamwise streaking spaced at $12 \mathrm{~mm}$ is visible. For the first three layers, individual wedges characteristic of stationary-crossflow-dominated transition are evident; however, the transition front is quite regular. At ASU, the transition front for stationary crossflow vortices was described as an irregular saw-tooth pattern for roughness heights as low as $6 \mu \mathrm{m}$. Figure 6-2 shows an example image from NFV at ASU.

This non-uniform saw-tooth pattern was observed at ASU for Radeztsky (1994) and Reibert (1996) on the NLF(2)-0415 and for White (2000) and Gladden (2001) on tests with the ASU(67)-0315 model. These irregularities were attributed to small differences in local surface roughness, which would produce small spanwise differences in the initial amplitudes. Since the irregular transition front was observed for two different models at several different angles-of-attack and over a range of roughness heights, the increased uniformity in the current tests is attributed to decreased turbulence levels in the wind tunnel. Lower turbulence levels reduce the sensitivity to small deviations in each roughness element. It is not until roughness height reaches $60 \mu \mathrm{m}$ in Figure 6-1 that the irregular wedges observed at ASU are also seen in the present case. To obtain a $60 \mu \mathrm{m}$ roughness element, five layers of appliqué DRE are applied. It is difficult to maintain diameter and spacing tolerances with five layers of appliqué roughness elements. In this instance, the higher disturbance amplitude generated by the taller $60 \mu \mathrm{m}$ roughness element may have increased the sensitivity to small changes in each roughness element. 
(a)

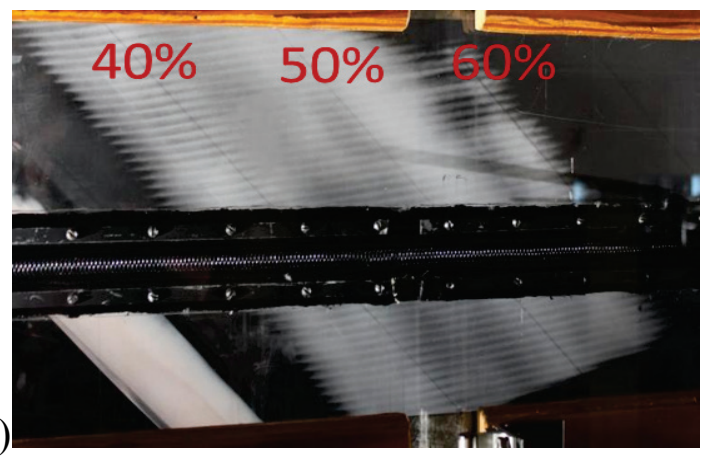

(b)

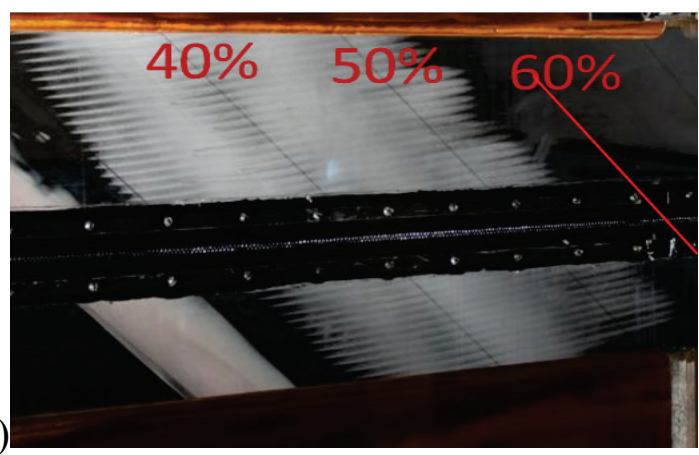

(c)

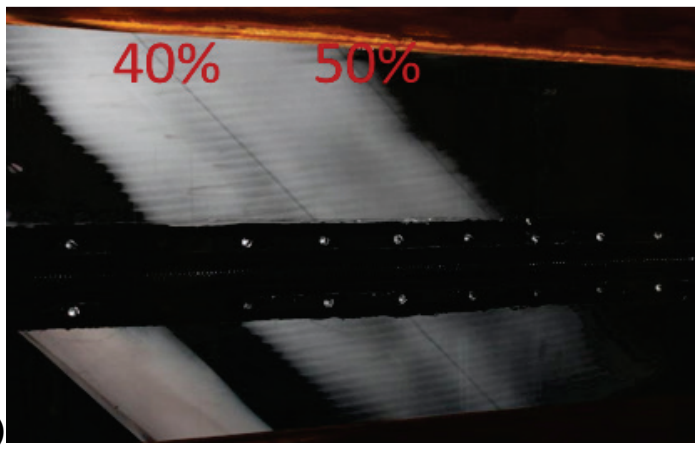

(d)

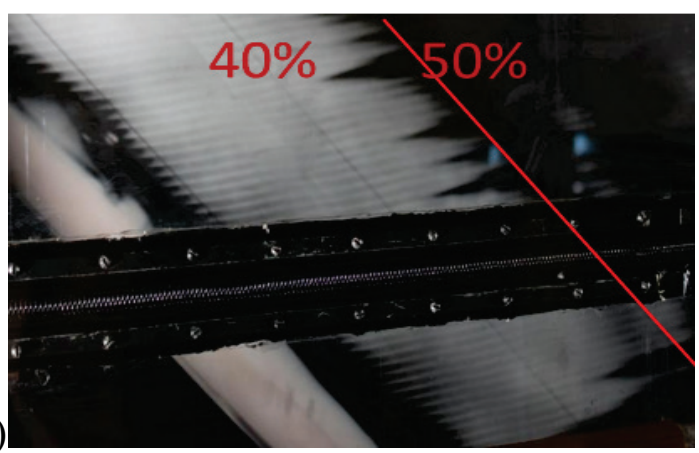

Figure 6-1. NFV with appliqué roughness: $x / c=0.029, d=3 \mathrm{~mm}, \lambda=12 \mathrm{~mm}$, $R e_{c}=2.4 \times 10^{6}$; (a) $k=12 \mu \mathrm{m}$, (b) $k=24 \mu \mathrm{m}$, (c) $k=36 \mu \mathrm{m}$, (d) $k=60 \mu \mathrm{m}$. 


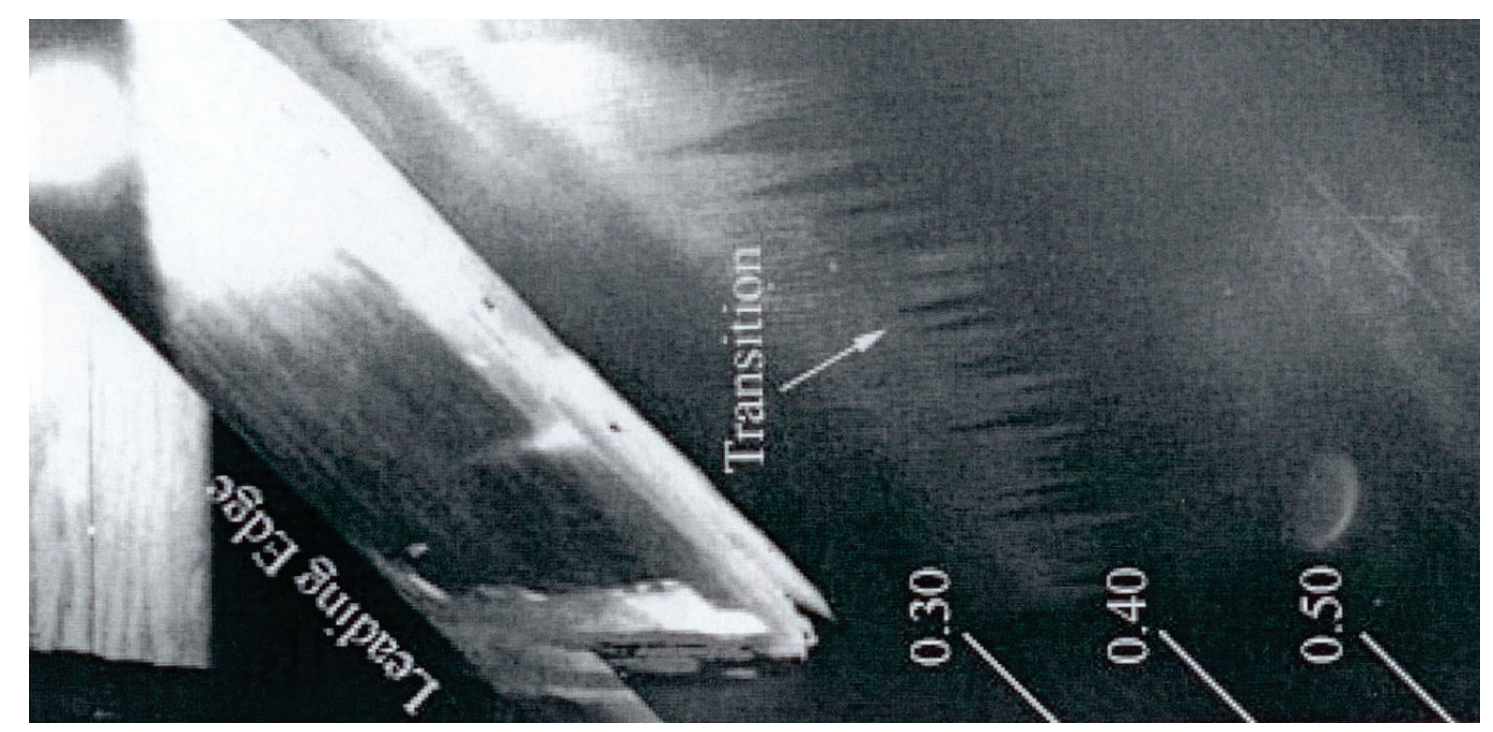

Figure 6-2. NFV of transition at ASU for $R_{c}=2.8 \times 10^{6}$, Roughness: $x / c=0.029, d=3 \mathrm{~mm}$, $k=6 \mu \mathrm{m}, \lambda=12 \mathrm{~mm}$ (Gladden 2001).

Figure 6-3 is a reapplication of the roughness elements starting from $12 \mu \mathrm{m}$ and ending this time at $47 \mu \mathrm{m}$. Roughness shape, spacing and location is the same as in Figure 6-2. In this case, transition appears to move back as a second layer is applied, then forward for the remaining two layers. It is not well understood why this occurs. Aside from a few isolated wedges, the images in Figure 6-3 also display the rather uniform transition front with small sawtooth wedges.

It is interesting to note that for supercritical wavelength spacing tested at ASU, the transition front was quite spanwise uniform. The reason for the difference between the critical and supercritical cases is also not well understood. Both Carrillo (1996) and Reibert (1996) mentioned that the transition front also became more uniform as Reynolds number increased. Initial disturbance amplitudes increase as Reynolds number increases. Thus at higher Reynolds numbers, the receptivity process would be less 
sensitive to small differences in local surface roughness or in each roughness element. Using the same model as White and Gladden in the KSWT, the transition front appears much more uniform in span for all Reynolds numbers, although the characteristic sawtooth pattern is still present. Irregular wedges are observed only in instances where roughness height is very large (Figure 6-1d) or turbulence levels in the tunnel are increased (see Section 8).

Both Carrillo and Reibert observed that for spacing equal to or larger than the critical wavelength, transition did not change as roughness height increased. Reibert's reported values change less than $3 \% x / c$ at $R e_{c}=2.4 \times 10^{6}$ and less than $4 \% x / c$ at $R e_{c}=3.2 \times 10^{6}$ when roughness height was increased from $6 \mu \mathrm{m}$ to $48 \mu \mathrm{m}$. Larger differences are observed in the KSWT tests at $2.4 \times 10^{6}$. Increasing roughness height from $12 \mu \mathrm{m}$ to $60 \mu \mathrm{m}$ produced up to a $15 \% x / \mathrm{c}$ change in the transition location for the roughness in Figure 6-1. In Figure 6-3, the transition location decreases by approximately $10 \% x / c$ when roughness is increased from $12 \mu \mathrm{m}$ up to $47 \mu \mathrm{m}$. The absolute difference in transition location for the current tests may not seem large compared to the initial decrease from natural roughness to the first layer; however, it is up to a factor of five times larger than the original ASU tests. 
(a)

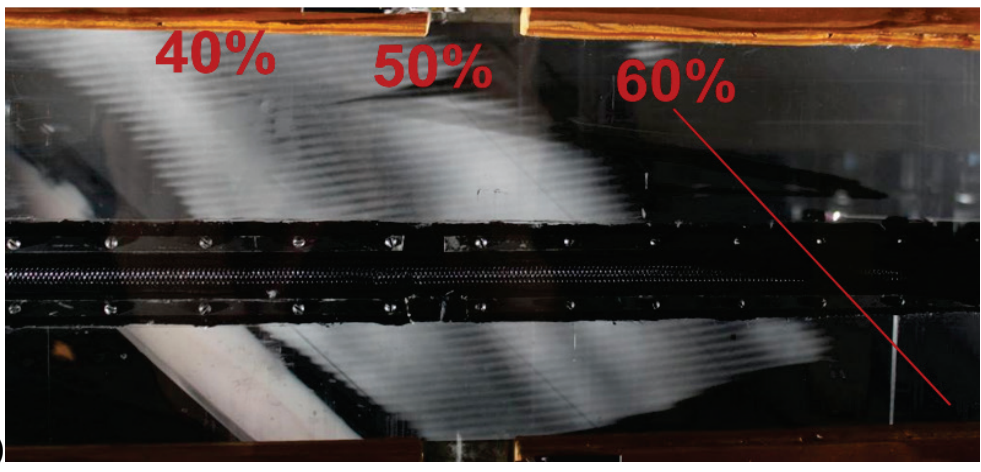

(b)

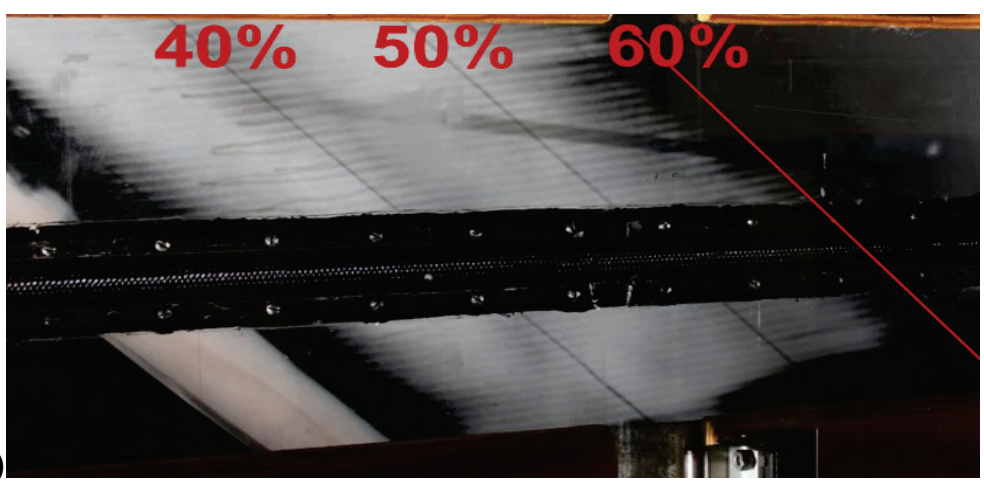

(c)

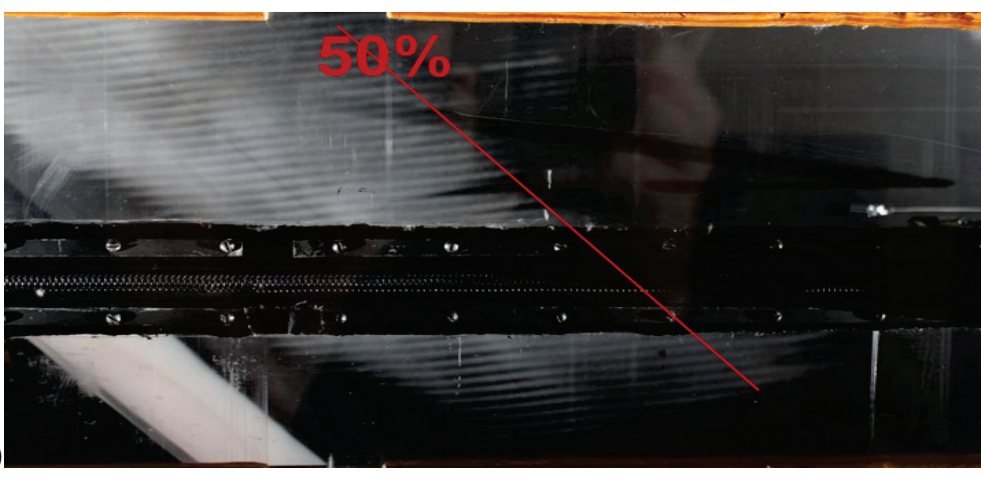

(d)

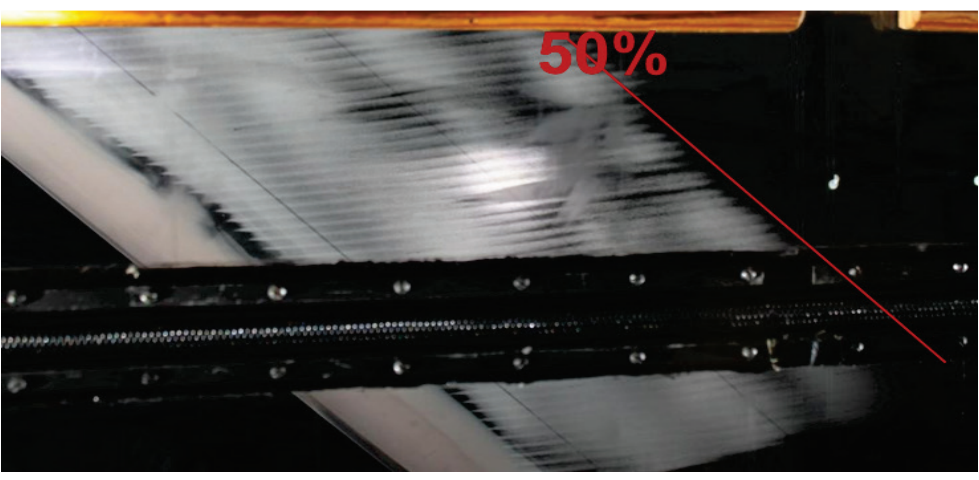

Figure 6-3. NFV with appliqué roughness: $x / c=0.029, d=3 \mathrm{~mm}, \lambda=12 \mathrm{~mm}$, $R e_{c}=2.4 \times 10^{6}$; (a) $k=12 \mu \mathrm{m}$, (b) $k=24 \mu \mathrm{m}$, (c) $k=36 \mu \mathrm{m}$, (d) $k=47 \mu \mathrm{m}$. 
The significance of many of these differences in transition location cannot be determined until the detailed boundary-layer measurements are compared. However, it is clear that the current test configuration at the KSWT shows evidence that unlike previous UWT results, transition location does change with increasing roughness height and that the transition front is much more uniform compared to instances at ASU with both the ASU(67)-0315 and NLF(2)-0415 models.

\subsubsection{Hotwire Measurements, $10 \% x / c$}

Only one roughness height, $k=47 \mu \mathrm{m}$, is presented in full detail at this chord location. Earlier attempts at $10 \% x / c$ were made with a $12 \mu \mathrm{m}$ single DRE layer, but the disturbances were too small to reliably measure. Figure 6-4 shows the 65 mean-flow boundary-layer profiles taken at constant chord. Each profile is acquired $1 \mathrm{~mm}$ in span away from the previous profile, providing a total measurement span of $64 \mathrm{~mm}$. The mean of the 65 profiles is also included in the plot. Even at this early chord location, the initial disturbance amplitude associated with the large roughness has started to broaden the profiles from the mean, indicating the start of distortions to the basic state. Figure 6-5 displays the disturbance profiles created by subtracting the mean boundary-layer velocity profile from each original profile. Note that the mean velocity profile does not represent the basic state in the absence of artificial roughness or the crossflow instability but rather, the basic state and a mean distortion mode. Streamwise velocity contours, created from the profiles in Figure 6-4 are shown in Figure 6-6. They indicate the beginnings of a $12 \mathrm{~mm}$ periodicity in the basic state over the $64 \mathrm{~mm}$ span. At $10 \% x / \mathrm{c}$ 
though, the peaks are not well formed and there are a few irregularities in the contours suggesting that the disturbance amplitude is still weak. The mode shape, calculated by taking the root-mean-square of the disturbance profiles, is shown in Figure 6-7. For this test condition, the maximum disturbance amplitude is 0.0178 . Recall that the mode shape is representative of the total disturbance signal, which may contain multiple modes. Using the boundary-layer height at which the maximum disturbance amplitude is measured, the mode shape can be spatially decomposed using the technique described by Reibert (1996). The relative amplitude of each individual mode is found by taking the square-root of the area under each peak. It should be stressed that all the spectra presented here come from 60 profiles taken at the boundary-layer height equal to the maximum disturbance amplitude. As a result, there are a limited number of points used to determine amplitudes for individual modes. Figure 6-8 shows the PSD for the 10\% $x / c$ case. As expected, a peak at $12 \mathrm{~mm}$, the forced wavelength, is dominant. A smaller peak at $6 \mathrm{~mm}$, the first harmonic, is also present. Similar to previous studies at ASU, no subharmonics are observed in the spectra. Without additional roughness heights, little can be said regarding the receptivity process itself; however this location serves as an additional measurement point in the database needed for computational validation. Multiple roughness heights are available at $15 \% x / c$ and $20 \% x / c$. 


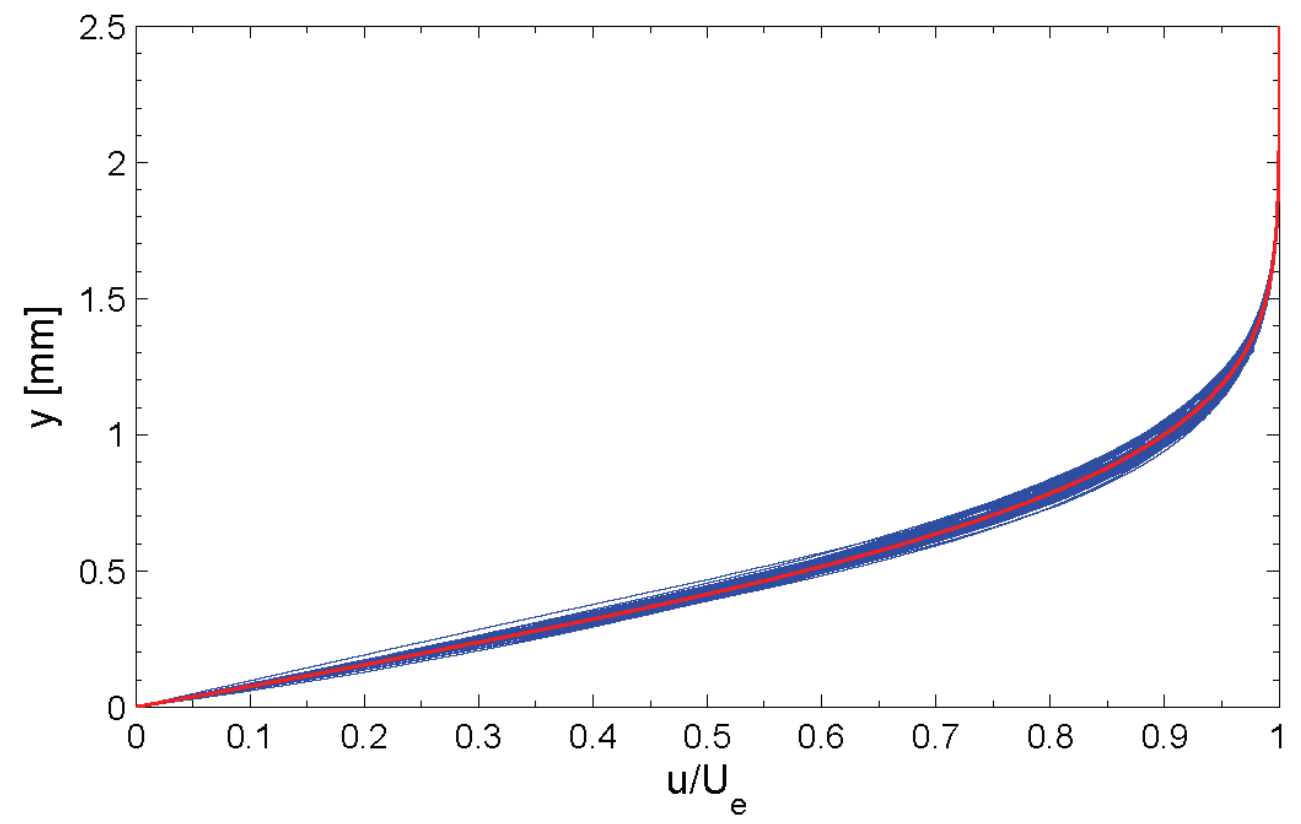

Figure 6-4. Spanwise array of 65 mean-flow boundary-layer profiles spanning $64 \mathrm{~mm}$ at $10 \% x / c . R e_{c}=2.4 \times 10^{6},[47 \mid 12]$ roughness. Mean profile shown in red.

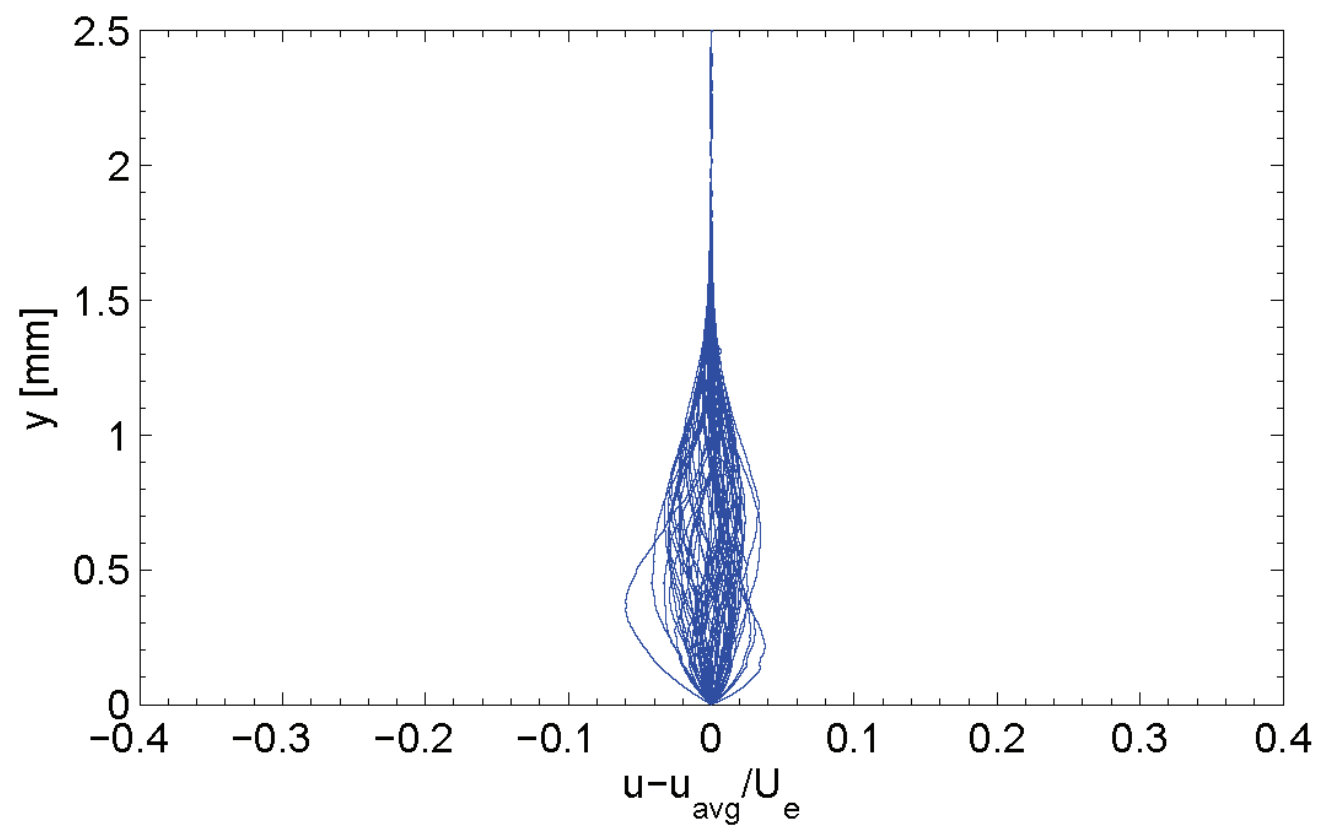

Figure 6-5. Spanwise array of 65 disturbance profiles spanning $64 \mathrm{~mm}$ at $10 \% x / c$.

$$
R e_{c}=2.4 \times 10^{6},[47 \mid 12] \text { roughness. }
$$




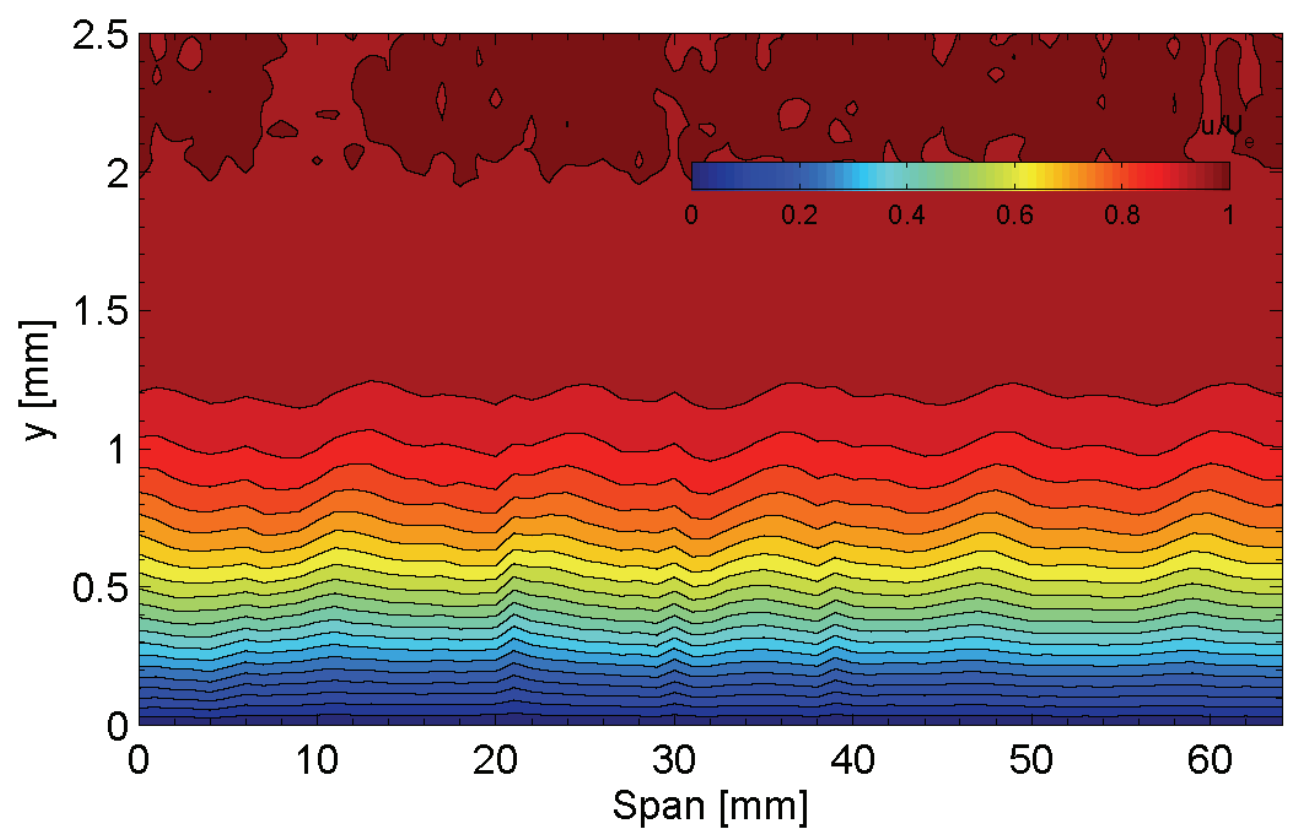

Figure 6-6. Streamwise velocity contours at $10 \% x / c . R e_{c}=2.4 \times 10^{6},[47 \mid 12]$ roughness.

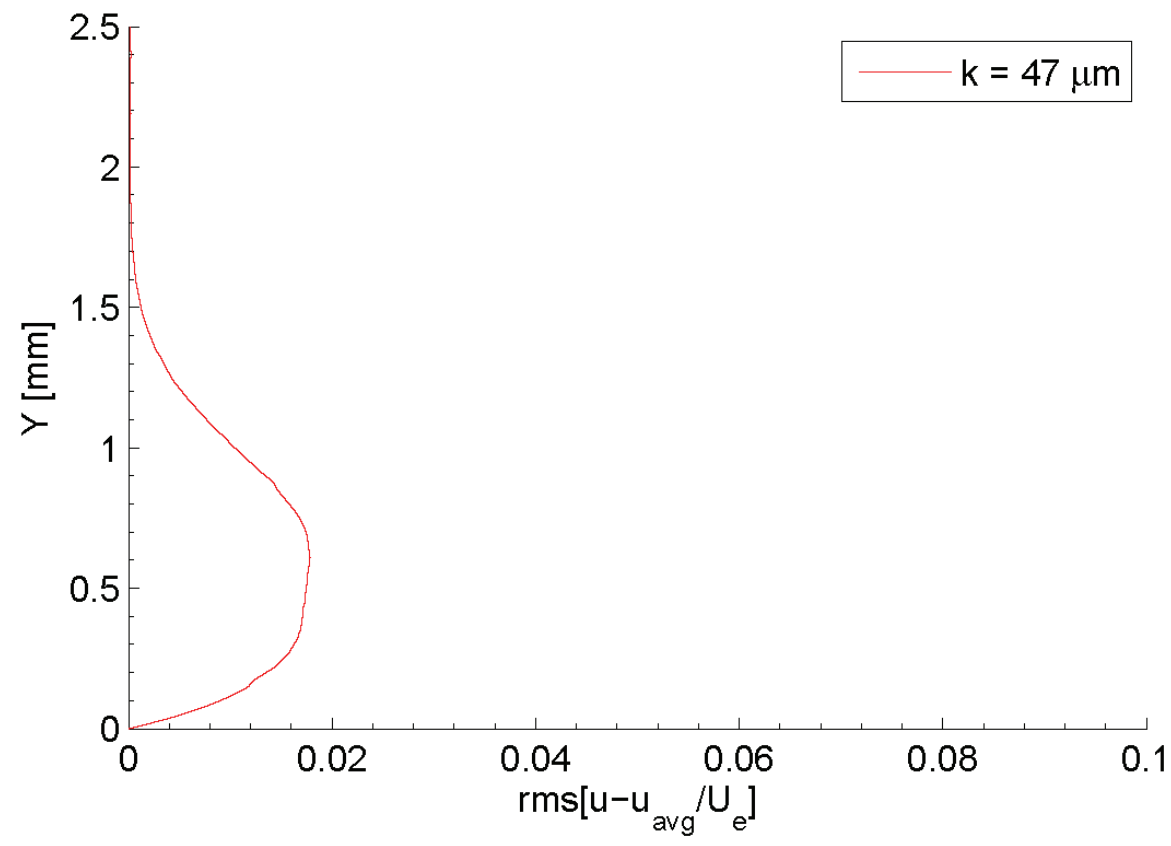

Figure 6-7. Stationary crossflow mode shape at $10 \% x / c . R e_{c}=2.4 \times 10^{6},[47 \mid 12]$ roughness. 


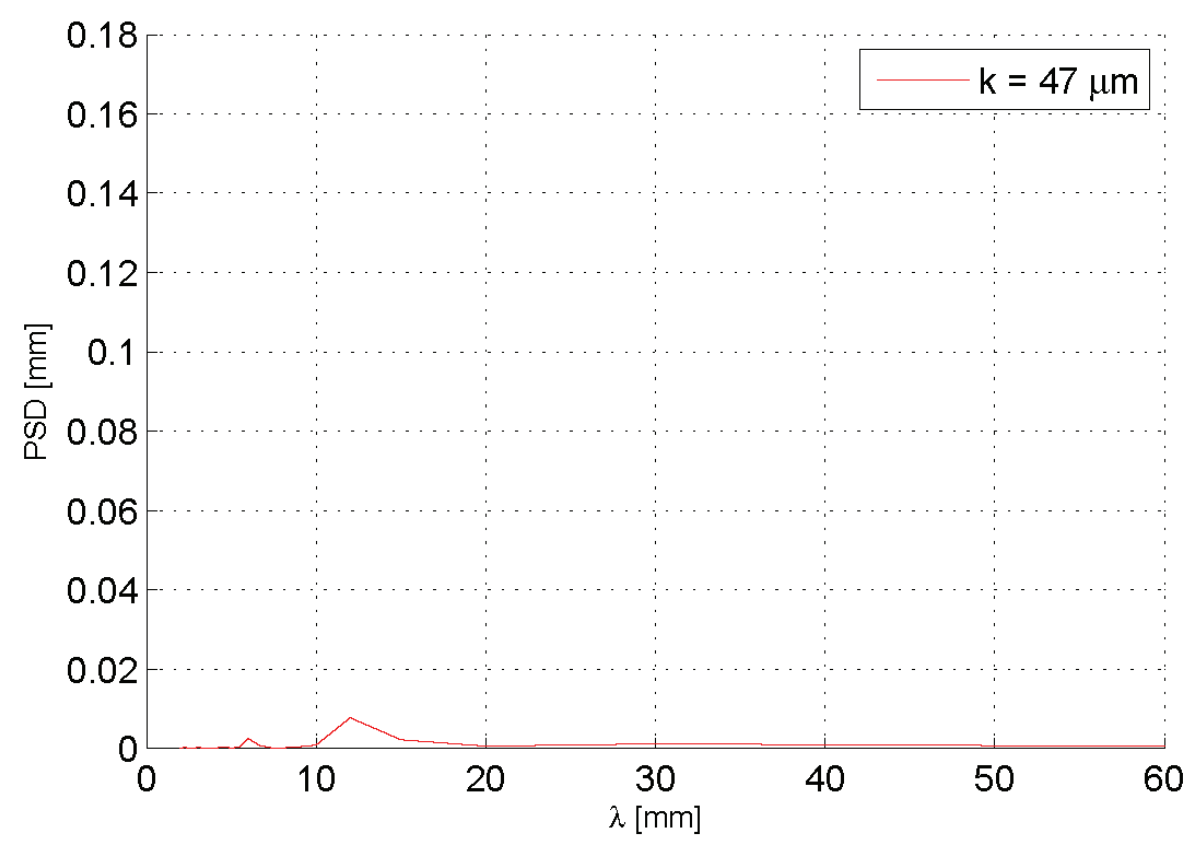

Figure 6-8. Power spectral density of spanwise hotwire scan at $10 \% x / c$. $R e_{c}=2.4 \times 10^{6},[47 \mid 12]$ roughness.

\subsubsection{Hotwire Measurements, $15 \% x / c$}

At $15 \% x / c$, DRE roughness heights included cases for $12 \mu \mathrm{m}, 24 \mu \mathrm{m}, 36 \mu \mathrm{m}$ and $47 \mu \mathrm{m}$. Figures $6-9-6-12$ show the mean-flow boundary layer profiles at $15 \% x / c$. As roughness height increases, the range of accelerated and decelerated profiles broadens and strongly inflected profiles begin to surface. At this early chord location, none of the mean profiles appear inflected- an indication that nonlinear stability effects have not yet begun to appear.

The streamwise velocity contours for each roughness height are shown in Figures 6-13 - 6-16. A $12 \mathrm{~mm}$ periodicity is visible for each roughness height. As roughness height increases, the $12 \mathrm{~mm}$ peaks and valleys also increase as a result of the more 
strongly accelerated and decelerated velocity profiles. No evidence of roll-over, often seen at later chord locations, is observed here, even at the $47 \mu \mathrm{m}$ roughness height.

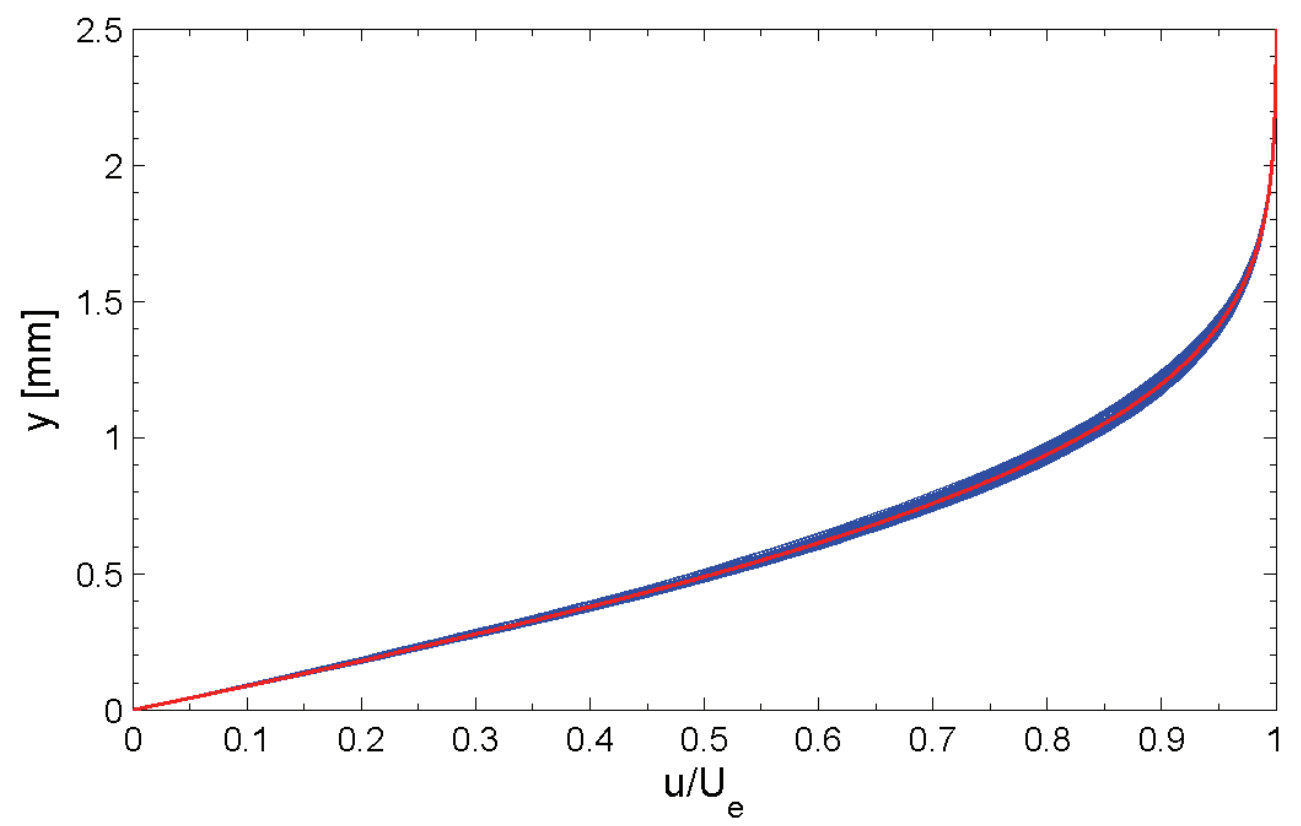

Figure 6-9. Spanwise array of 65 mean-flow boundary-layer profiles spanning $64 \mathrm{~mm}$ at $15 \% x / c . R e_{c}=2.4 \times 10^{6},[12 \mid 12]$ roughness. Mean profile shown in red. 


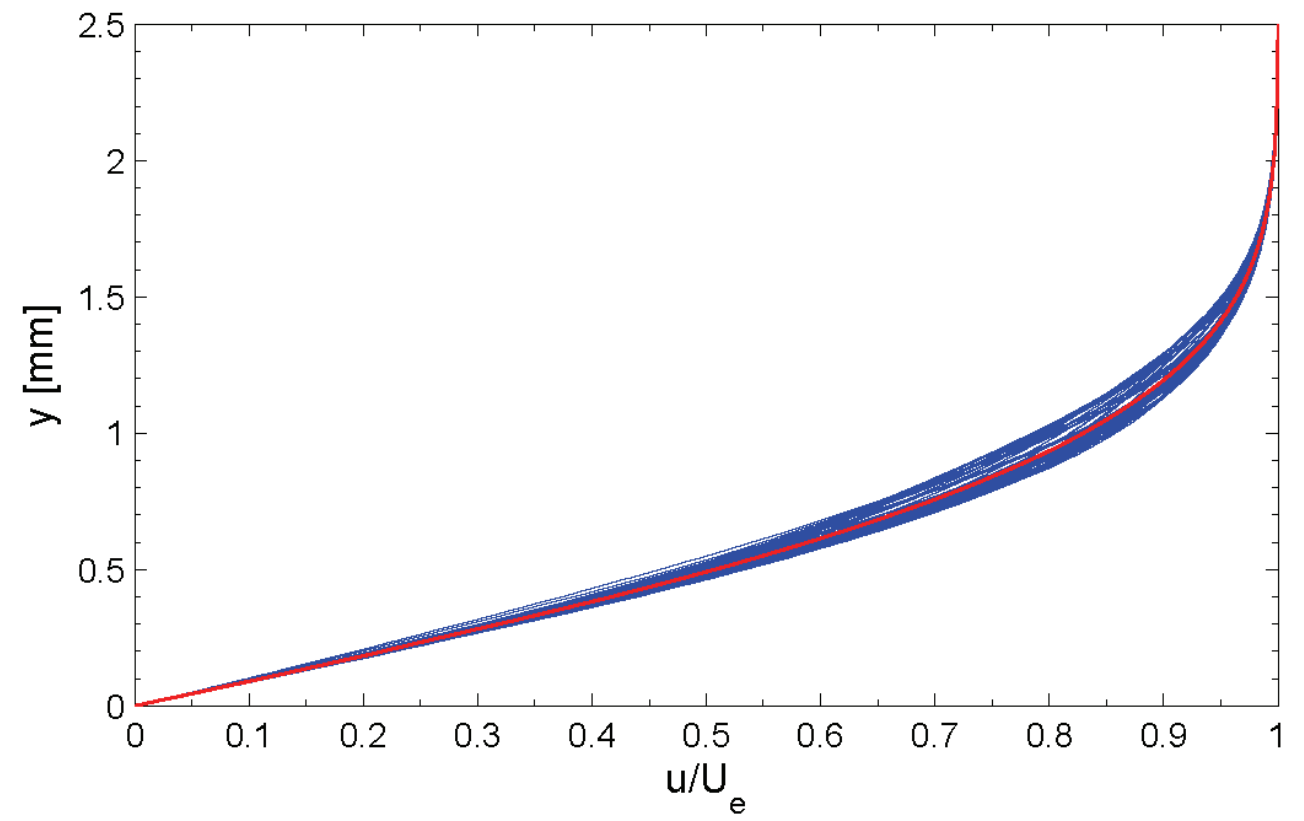

Figure 6-10. Spanwise array of 65 mean-flow boundary-layer profiles spanning $64 \mathrm{~mm}$ at $15 \% x / c . R e_{c}=2.4 \times 10^{6},[24 \mid 12]$ roughness. Mean profile shown in red.

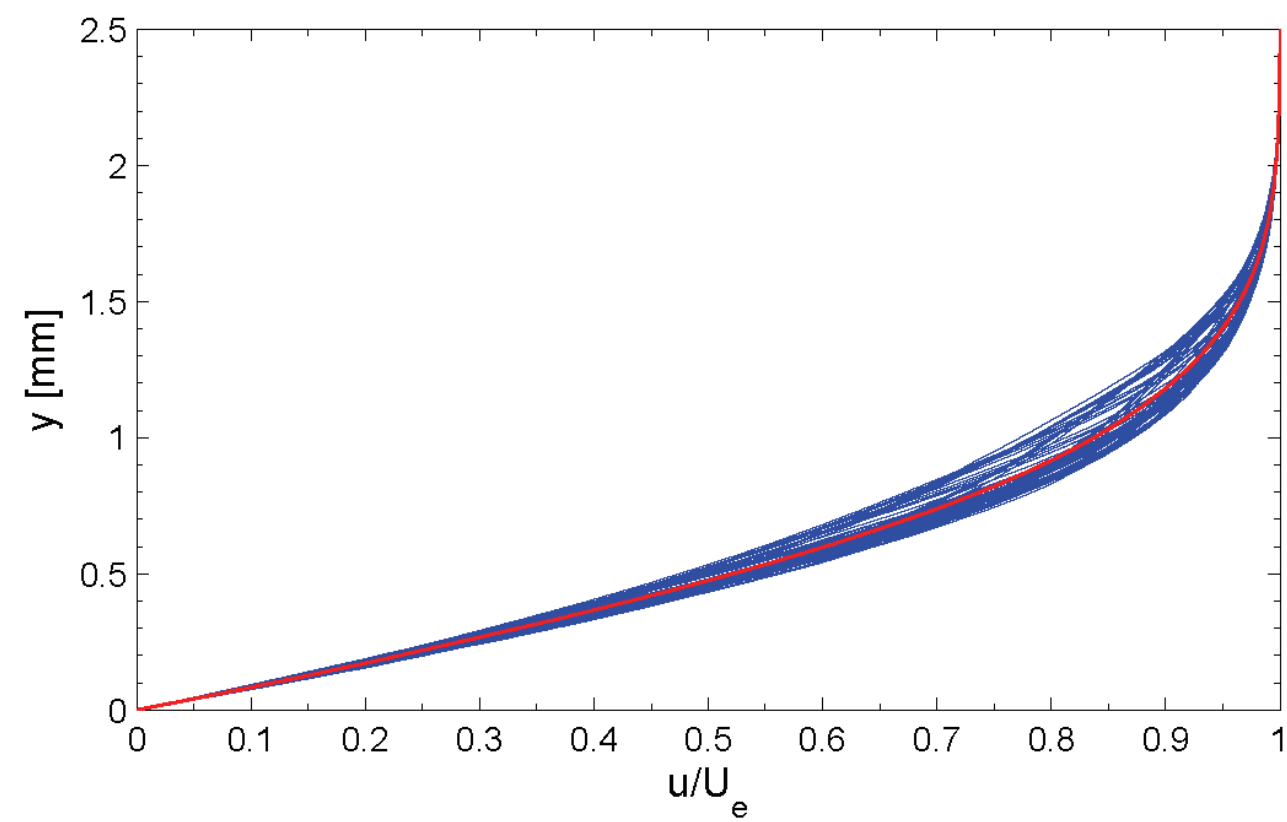

Figure 6-11. Spanwise array of 65 mean-flow boundary-layer profiles spanning $64 \mathrm{~mm}$ at $15 \% x / c . R e_{c}=2.4 \times 10^{6},[36 \mid 12]$ roughness. Mean profile shown in red. 


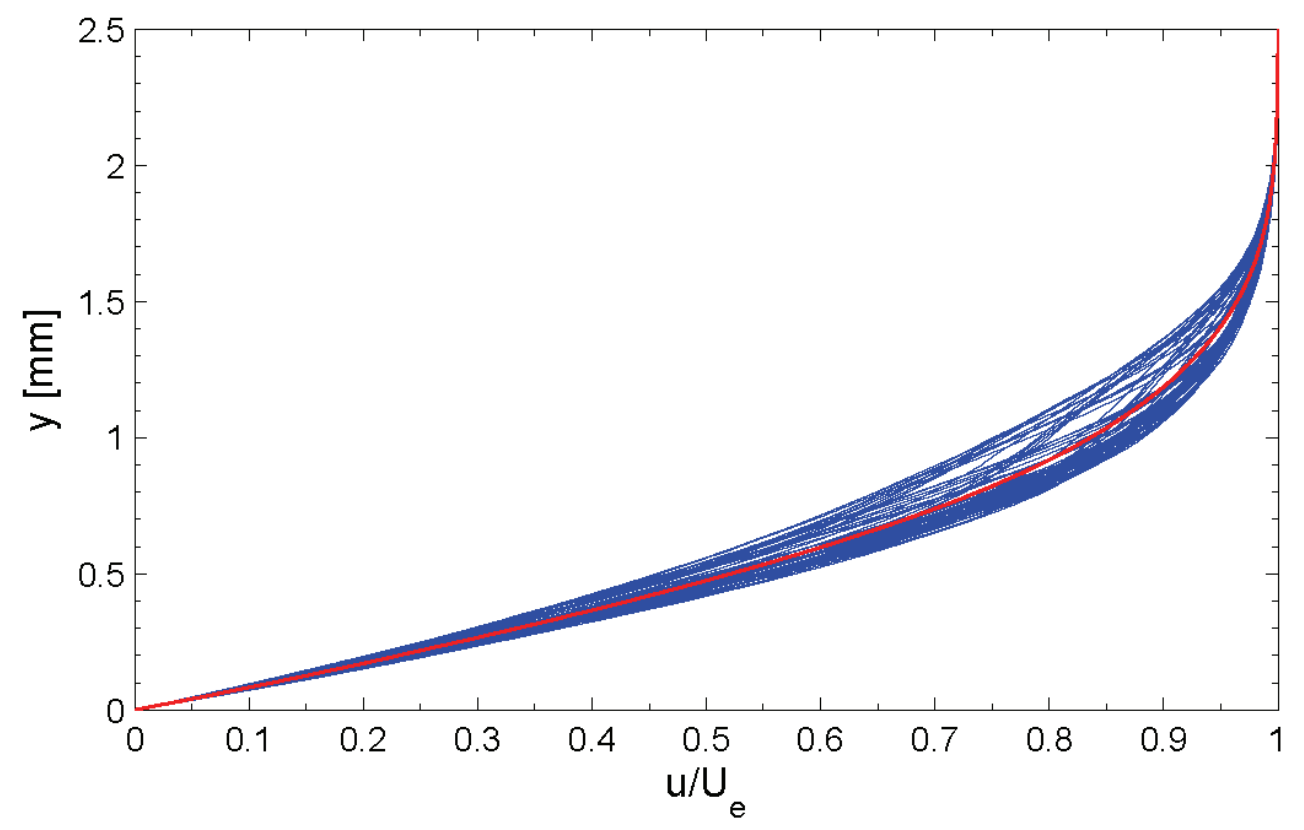

Figure 6-12. Spanwise array of 65 mean-flow boundary-layer profiles spanning $64 \mathrm{~mm}$ at $15 \% x / c . R e_{c}=2.4 \times 10^{6},[47 \mid 12]$ roughness. Mean profile shown in red.

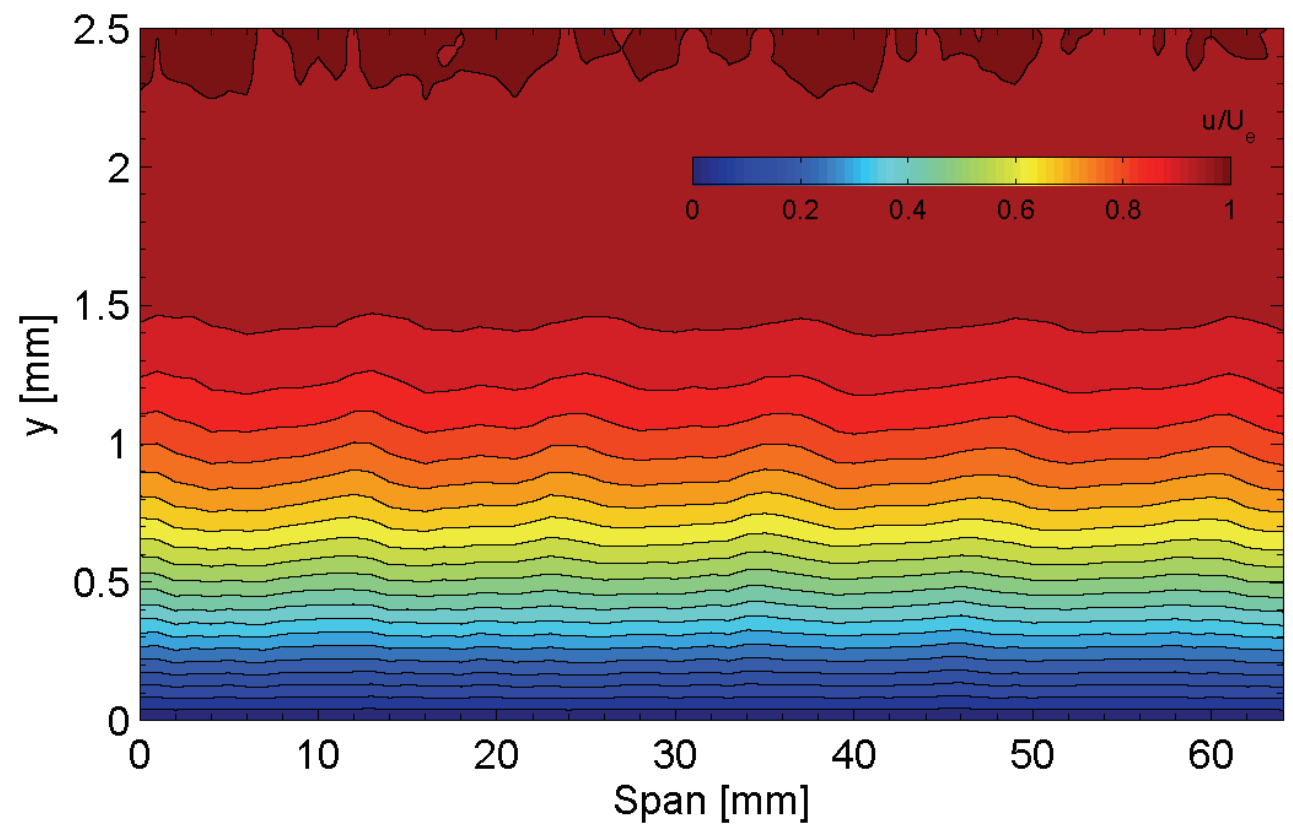

Figure 6-13. Streamwise velocity contours at $15 \% x / c . R e_{c}=2.4 \times 10^{6},[12 \mid 12]$ roughness. 


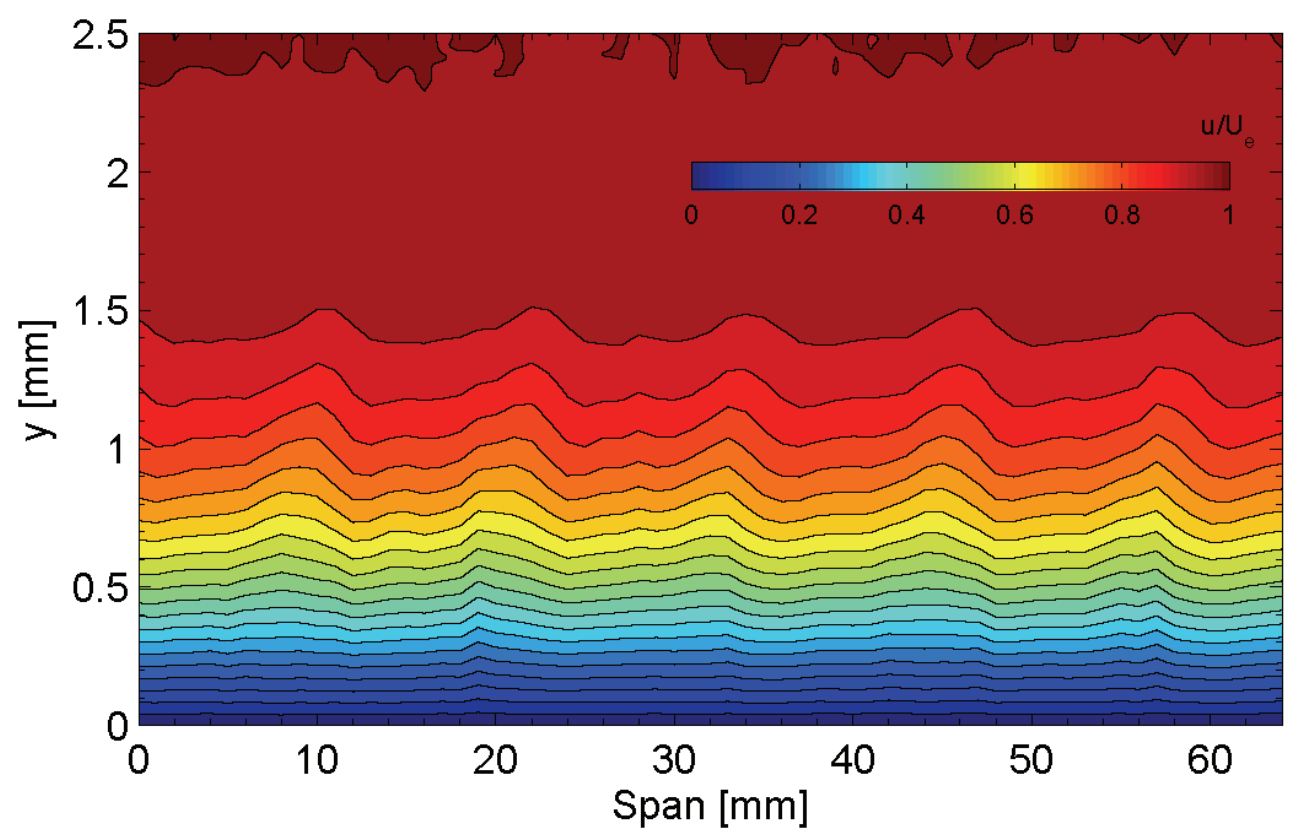

Figure 6-14. Streamwise velocity contours at $15 \% x / c . R e_{c}=2.4 \times 10^{6},[24 \mid 12]$ roughness.

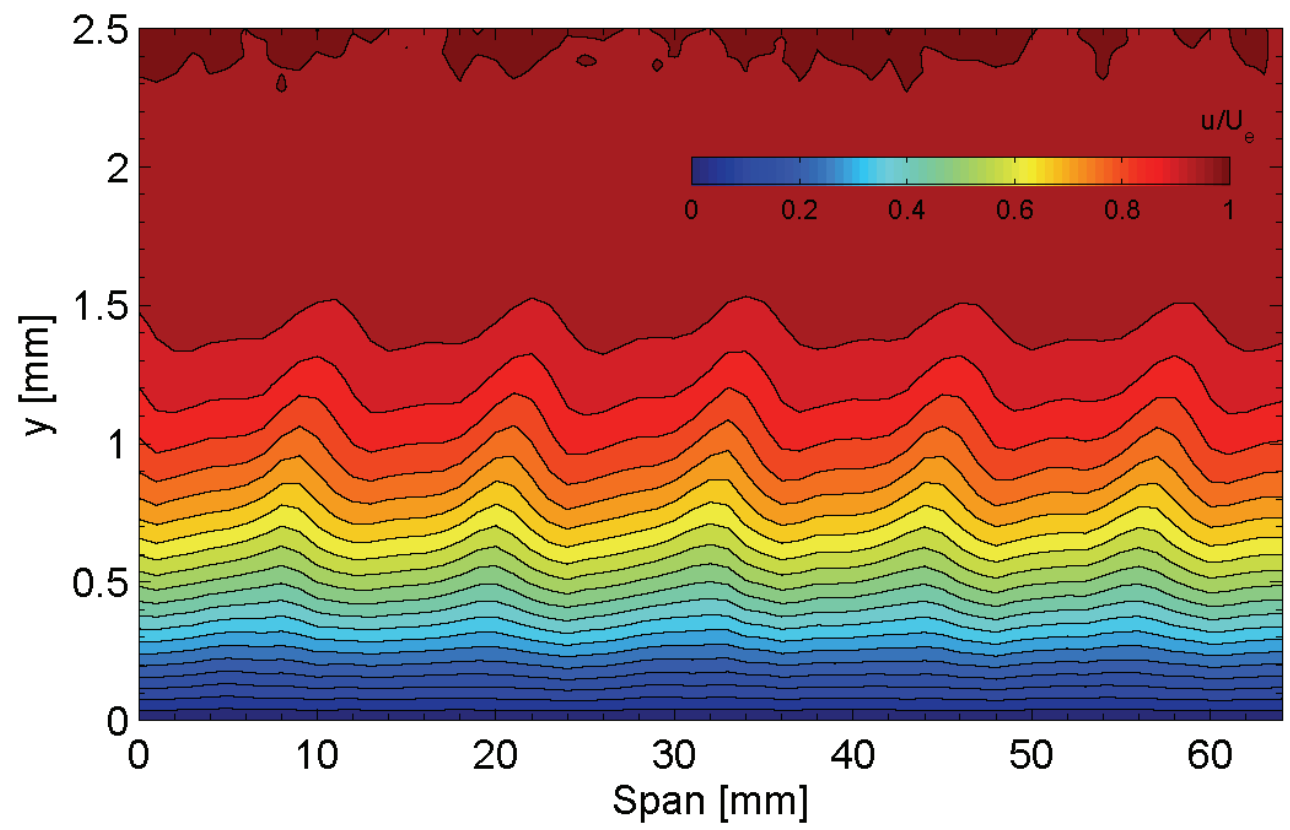

Figure 6-15. Streamwise velocity contours at $15 \% x / c . R e_{c}=2.4 \times 10^{6},[36 \mid 12]$ roughness. 


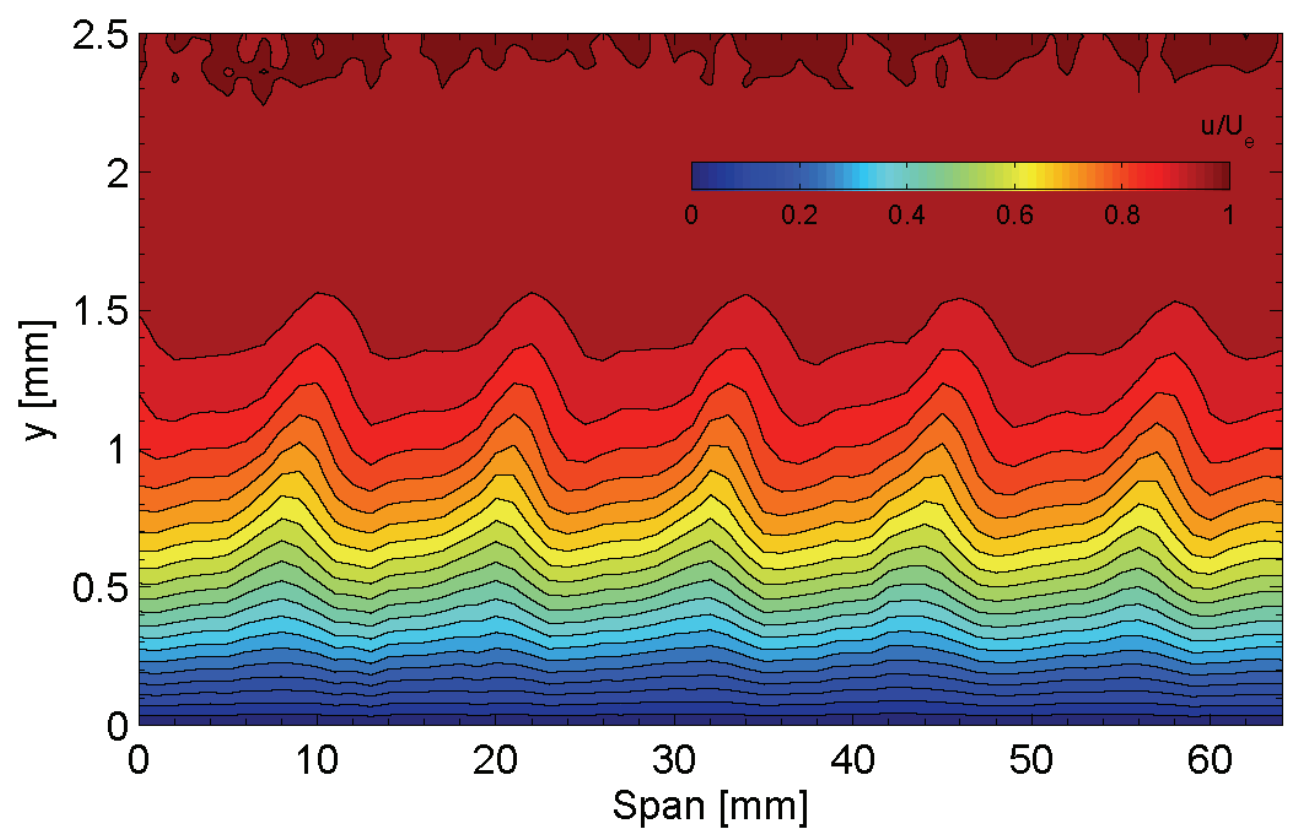

Figure 6-16. Streamwise velocity contours at $15 \% x / c . R e_{c}=2.4 \times 10^{6},[47 \mid 12]$ roughness.

Figures 6-17 - 6-20 show the disturbance profiles for each roughness height. With the mean boundary-layer profile removed, it is much easier to see how increased roughness height has resulted in larger deviations from the mean velocity profile. Figure 6-21 displays the resulting mode shape for each roughness height configuration. At this Reynolds number and chord location, none of the mode shapes display convex curvature or secondary lobes, both indicators of nonlinear stability activity. Differences in disturbance amplitudes should be related to the receptivity process rather than instability growth and basic state distortions. The maximum disturbance amplitude and its normalized value are shown in Table 6-1. The third column in Table 6-1 is derived by normalizing the maximum disturbance amplitude twice: first by the roughness height 
and then by the normalized $12 \mu \mathrm{m}$ roughness case. The resulting value in column three is represented by Eqn 6-11.

Table 6-1. Maximum and normalized stationary crossflow disturbance amplitudes at $15 \% x / c, R_{c}=2.4 \times 10^{6},[k \mid 12]$ roughness.

\begin{tabular}{|c|c|c|}
\hline$k[\mu \mathrm{m}]$ & {$\left[\left(u-u_{\text {avg }}\right) / U_{e}\right]_{\mathrm{rms}, \max }$} & {$\left[\left(u-u_{\text {avg }}\right) / U_{e}\right]_{\mathrm{rms}, k / k o}$} \\
\hline 12 & 0.0113 & 1.00 \\
\hline 24 & 0.0228 & 1.01 \\
\hline 36 & 0.0313 & 0.92 \\
\hline 47 & 0.0422 & 0.95 \\
\hline
\end{tabular}

$$
u_{\text {max }, \text { norm }}=\left[\frac{\left(u-u_{\text {avg }}\right)}{U_{e}}\right]_{r m s, k} /\left[\frac{\left(u-u_{\text {avg }}\right)}{U_{e}}\right]_{r m s, k=12 \mu m}
$$

Roughness height appears to increase almost linearly with each additional layer of DRE. Although maximum disturbance amplitude is presented for all test conditions, disturbance amplitudes can also be quantified by calculating the average and root-meansquare of the mode. The normalized disturbance amplitudes in these instances were similar to those presented here. Figure 6-22 shows the PSD for each roughness height at $15 \% x / c$ and $R e_{c}=2.4 \times 10^{6}$. Similar to the $10 \%$ scan, a predominant peak occurs at $12 \mathrm{~mm}$ followed by a smaller peak at $6 \mathrm{~mm}$. Finding the area under each peak and taking the square-root of that value provides the maximum disturbance amplitude for each mode present. Table 6-2 shows the ratio of $12 \mathrm{~mm}$ to $6 \mathrm{~mm}$ disturbance amplitudes. For each roughness height, the disturbance amplitude ratio is approximately 2, with a 
maximum change of $10 \%$ from the $12 \mu \mathrm{m}$ case. A more detailed discussion on the significance of these results is postponed until data from all chord locations and Reynolds numbers are presented.

Table 6-2. Stationary crossflow disturbance amplitude ratios at $15 \% x / c, R_{c}=2.4 \times 10^{6},[k \mid 12]$ roughness.

\begin{tabular}{|c|c|}
\hline$k[\mu \mathrm{m}]$ & {$\left[\left(u-u_{\text {avg }}\right) / U_{e}\right]_{\mathrm{rms}, \lambda=12} /\left[\left(u-u_{\text {avg }}\right) / U_{e}\right]_{\mathrm{rms}, \lambda=6}$} \\
\hline 12 & 2.06 \\
\hline 24 & 1.94 \\
\hline 36 & 2.27 \\
\hline 47 & 2.15 \\
\hline
\end{tabular}

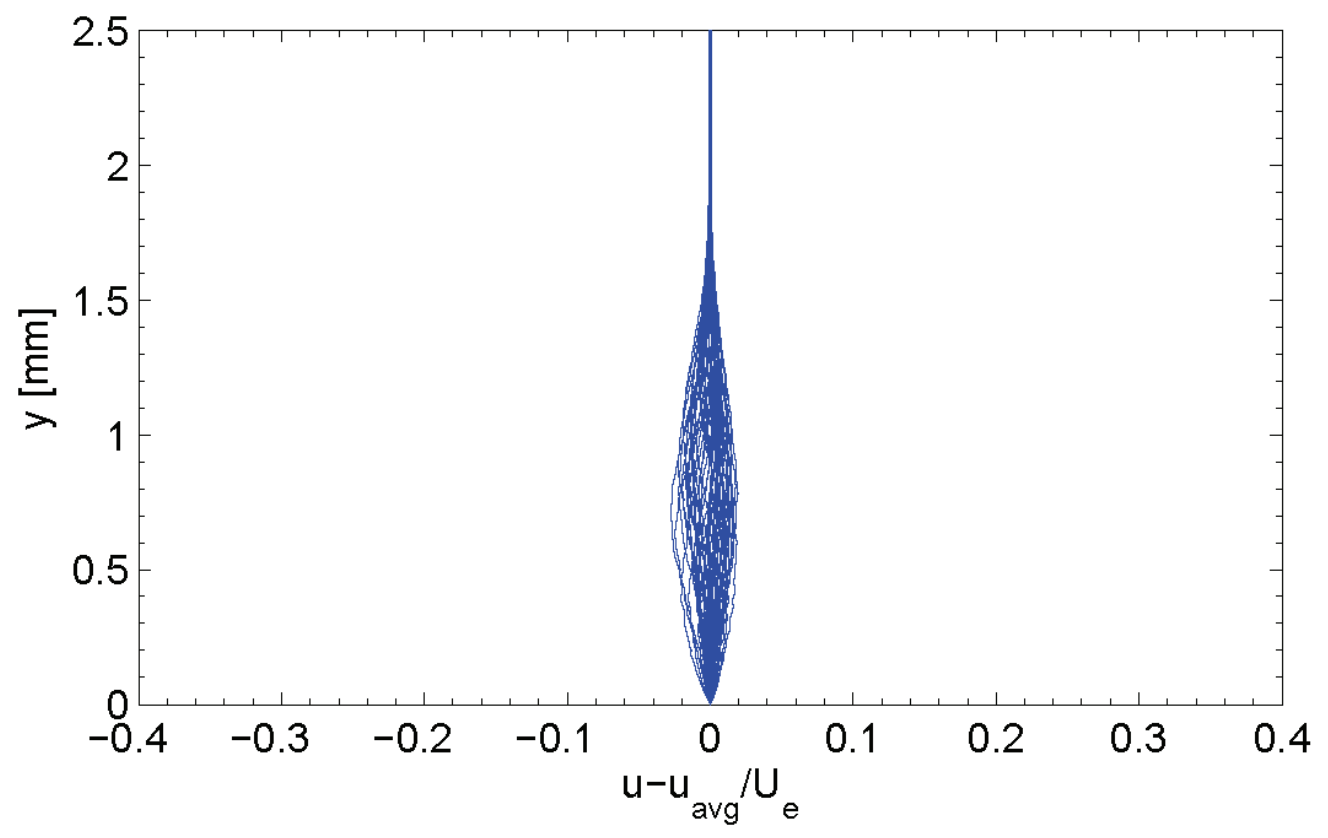

Figure 6-17. Spanwise array of 65 disturbance profiles spanning $64 \mathrm{~mm}$ at $15 \% x / c$. $R e_{c}=2.4 \times 10^{6},[12 \mid 12]$ roughness. 


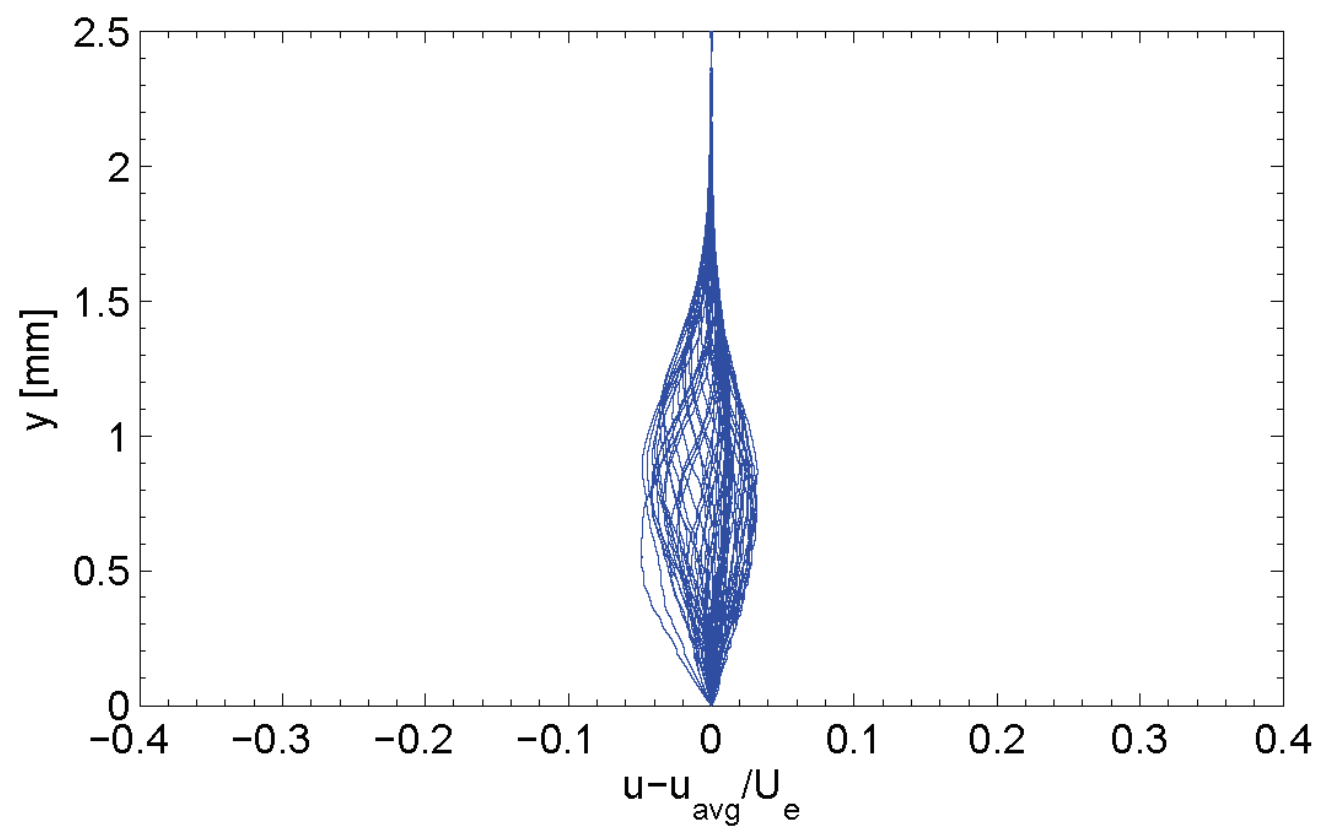

Figure 6-18. Spanwise array of 65 disturbance profiles spanning $64 \mathrm{~mm}$ at $15 \% x / c$. $R e_{c}=2.4 \times 10^{6},[24 \mid 12]$ roughness.

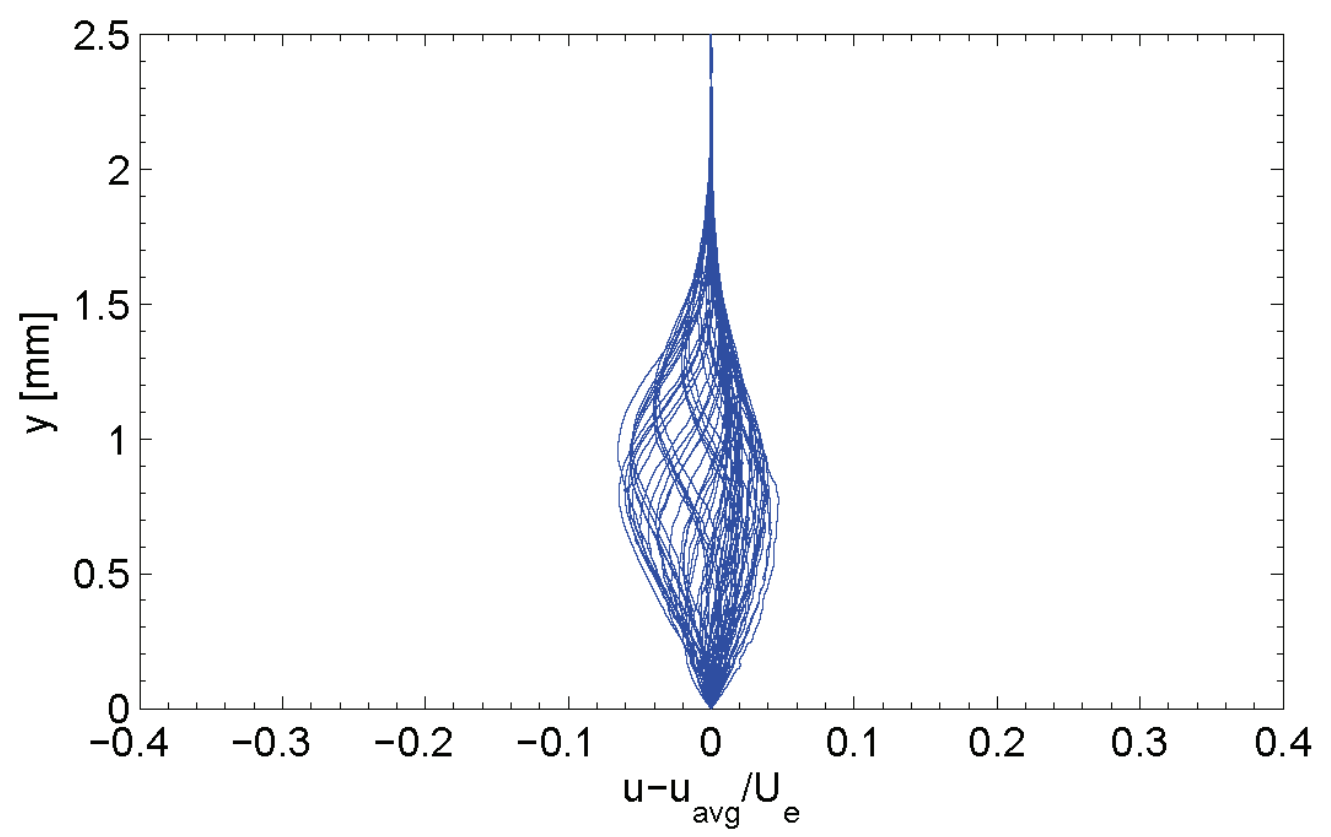

Figure 6-19. Spanwise array of 65 disturbance profiles spanning $64 \mathrm{~mm}$ at $15 \% x / c$. $R e_{c}=2.4 \times 10^{6},[36 \mid 12]$ roughness. 


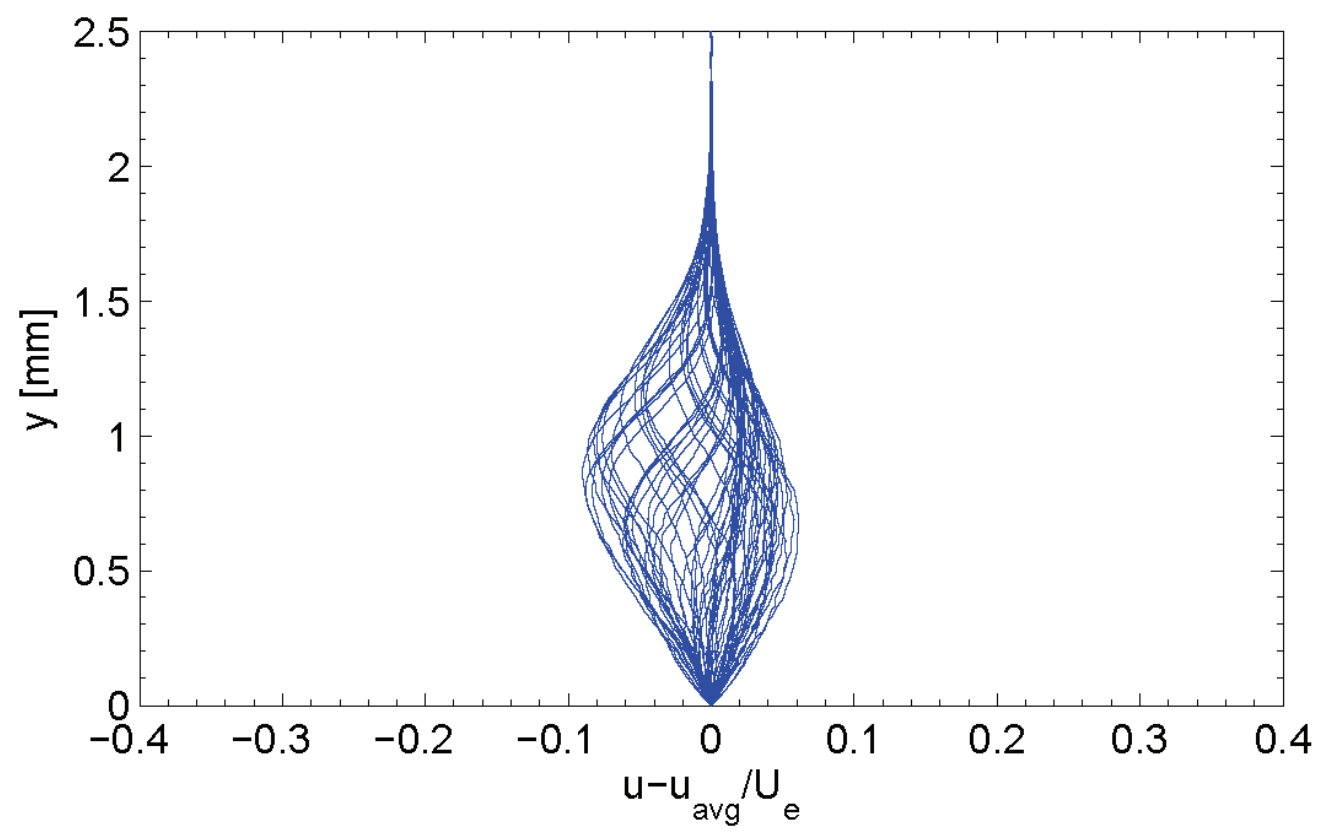

Figure 6-20. Spanwise array of 65 disturbance profiles spanning $64 \mathrm{~mm}$ at $15 \% x / c$. $R e_{c}=2.4 \times 10^{6},[47 \mid 12]$ roughness.

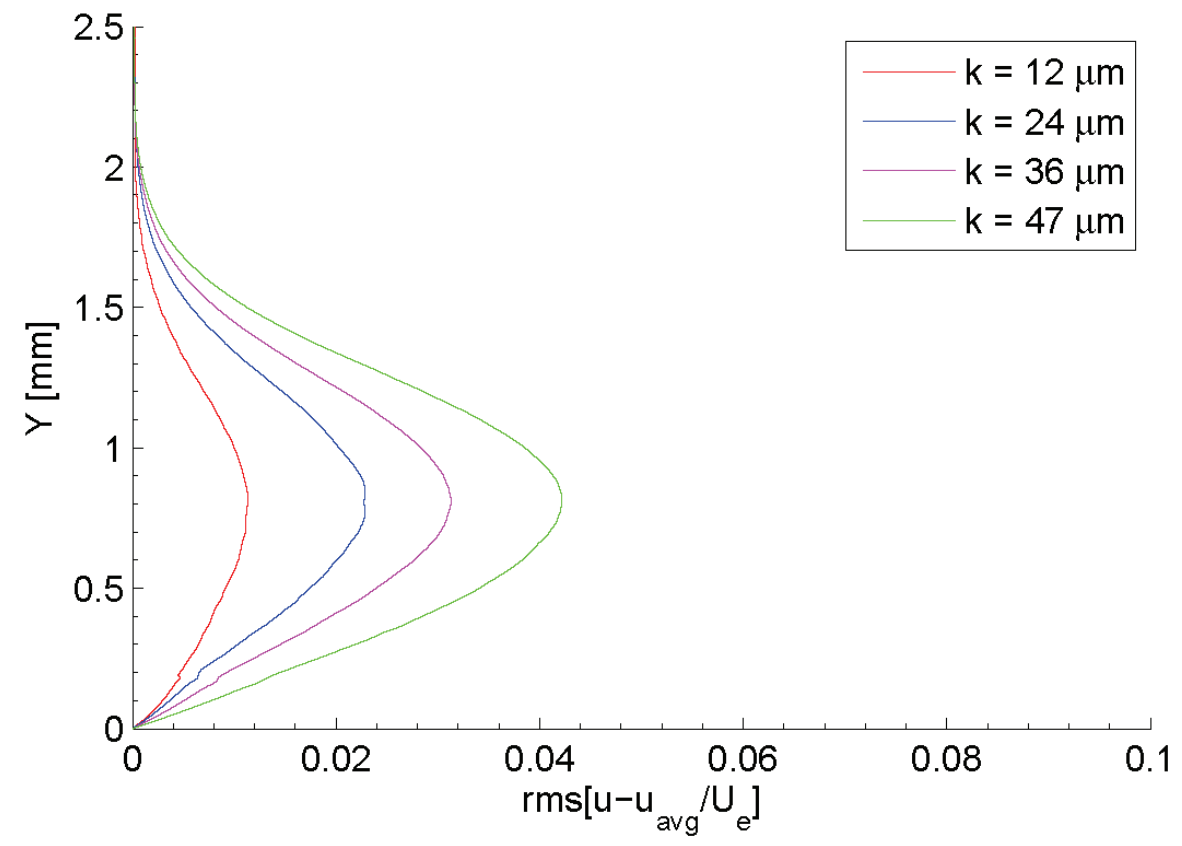

Figure 6-21. Stationary crossflow mode shape at $15 \% x / c . R e_{c}=2.4 \times 10^{6},[k \mid 12]$ roughness. 


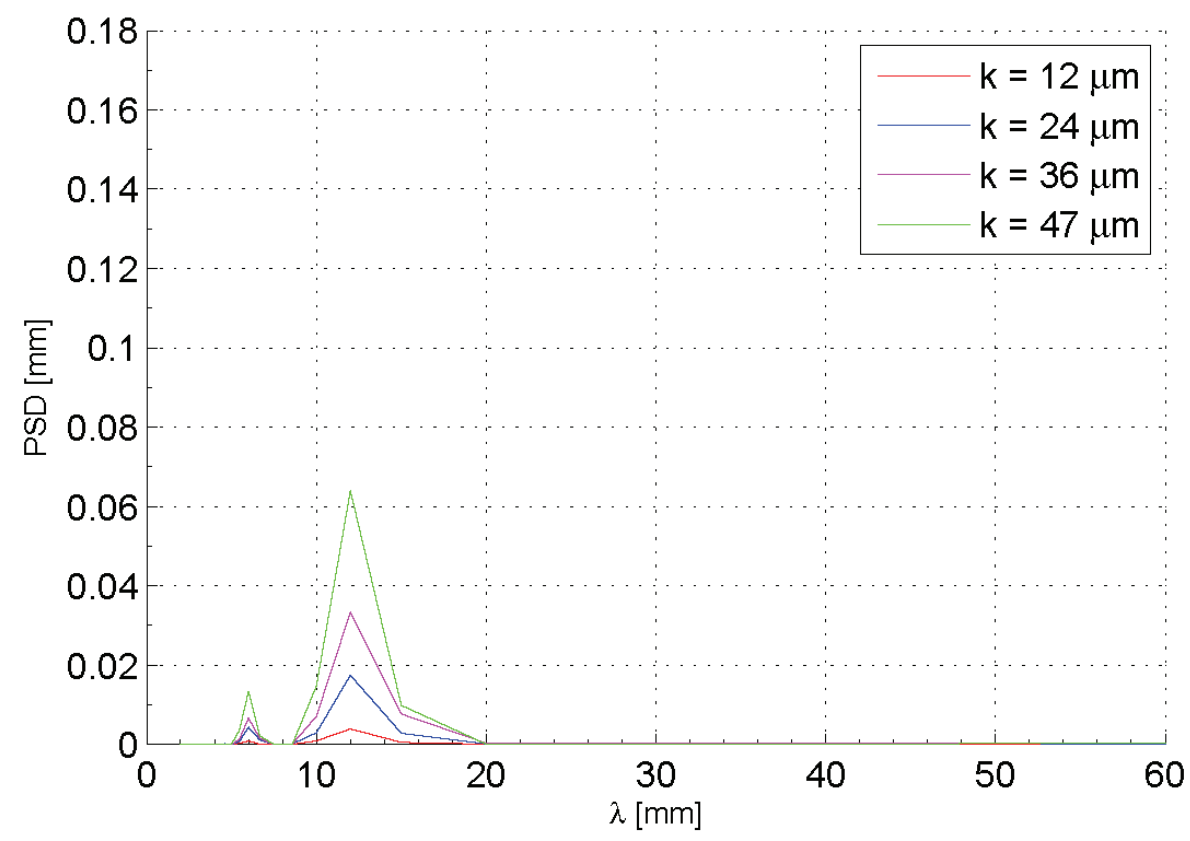

Figure 6-22. Power spectral density of spanwise hotwire scan at $15 \% x / c$. $R e_{c}=2.4 \times 10^{6},[k \mid 12]$ roughness.

\subsubsection{Hotwire Measurements, $20 \% x / c$}

At $20 \% x / c$, four different roughness heights were tested: $12 \mu \mathrm{m}, 24 \mu \mathrm{m}, 36 \mu \mathrm{m}$ and $60 \mu \mathrm{m}$. It was the first chord location measured in the test matrix. For this first set of measurements, roughness height was increased from three layers to five layers, rather than continuing to a fourth, in order to match as closely as possible the maximum height often used in the ASU swept-wing studies. It was not until more detailed roughness height measurements were made that the discrepancy in roughness height from batch to batch was discovered. In this case, the roughness height exceeds the maximum measured values at ASU by $12 \mu \mathrm{m}$. Figures 6-23-6-26 show the boundary-layer profiles from the spanwise hotwire scan for each roughness configuration. Mean-flow distortion continues 
to increase downstream based on two different actions. First, the crossflow disturbance grows until reaching the saturation amplitude or the branch II neutral point, allowing a stronger mean-flow distortion as low-momentum fluid is convected away from the surface and high-momentum fluid is forced down toward the surface. Second, since the disturbance wave is stationary and nearly aligned with the potential flow direction, the disturbance essentially acts on the same fluid element as it progresses downstream, providing another opportunity to distort the basic state. Figure $6-23$, then, shows a broader set of boundary-layer profiles compared to the same single layer at $15 \% x / c$. Inflected profiles are more pronounced at all of the roughness heights at $20 \% x / c$.

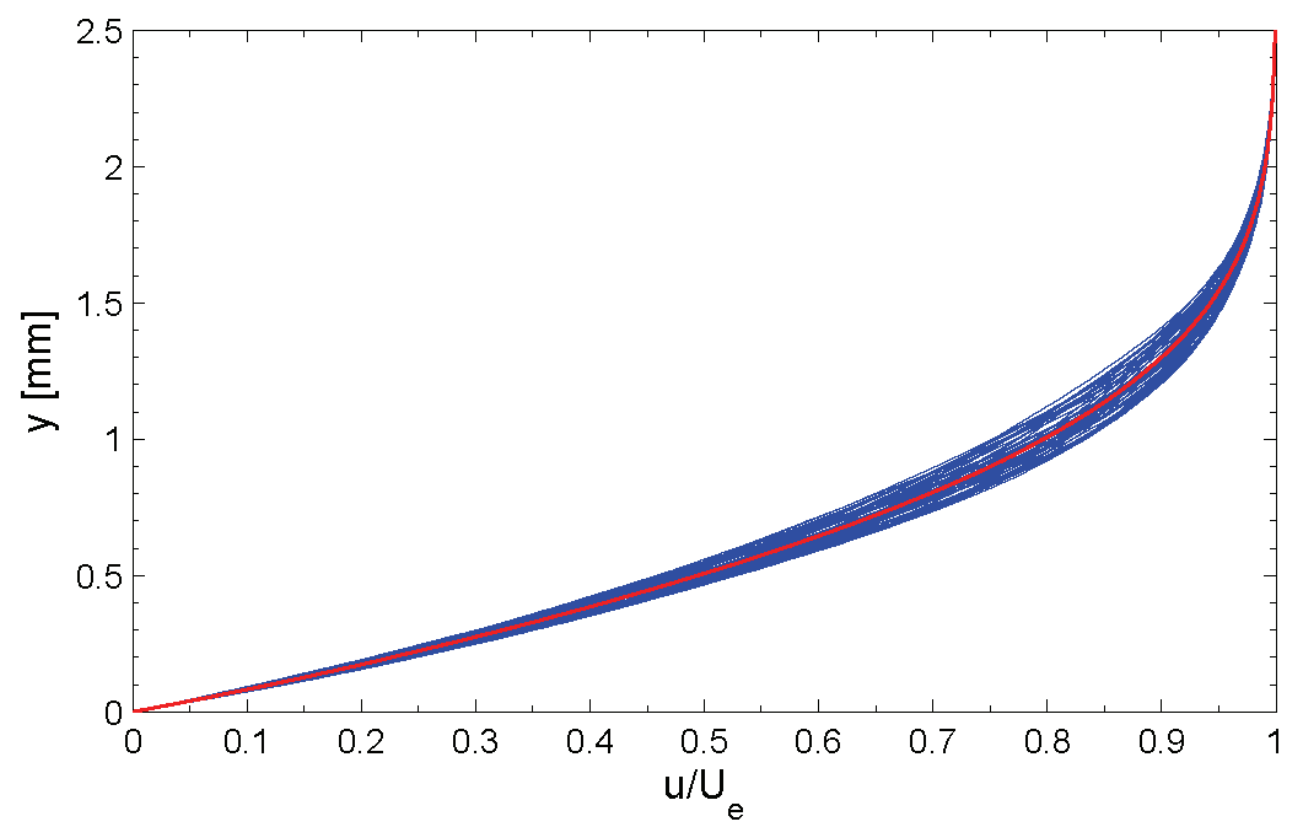

Figure 6-23. Spanwise array of 64 mean-flow boundary-layer profiles spanning $63 \mathrm{~mm}$ at $20 \% x / c . R e_{c}=2.4 \times 10^{6},[12 \mid 12]$ roughness. Mean profile shown in red. 


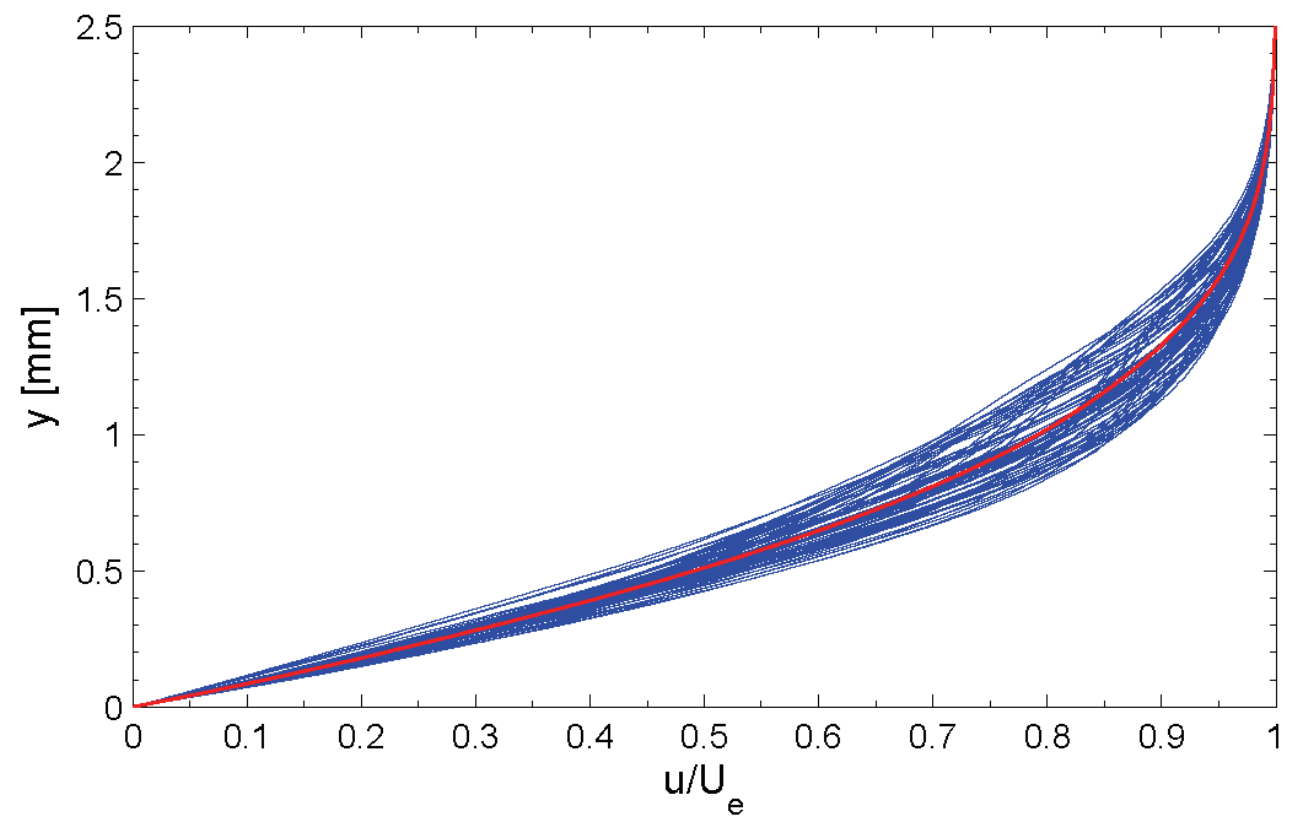

Figure 6-24. Spanwise array of 65 mean-flow boundary-layer profiles spanning $64 \mathrm{~mm}$ at $20 \% x / c . R e_{c}=2.4 \times 10^{6},[24 \mid 12]$ roughness. Mean profile shown in red.

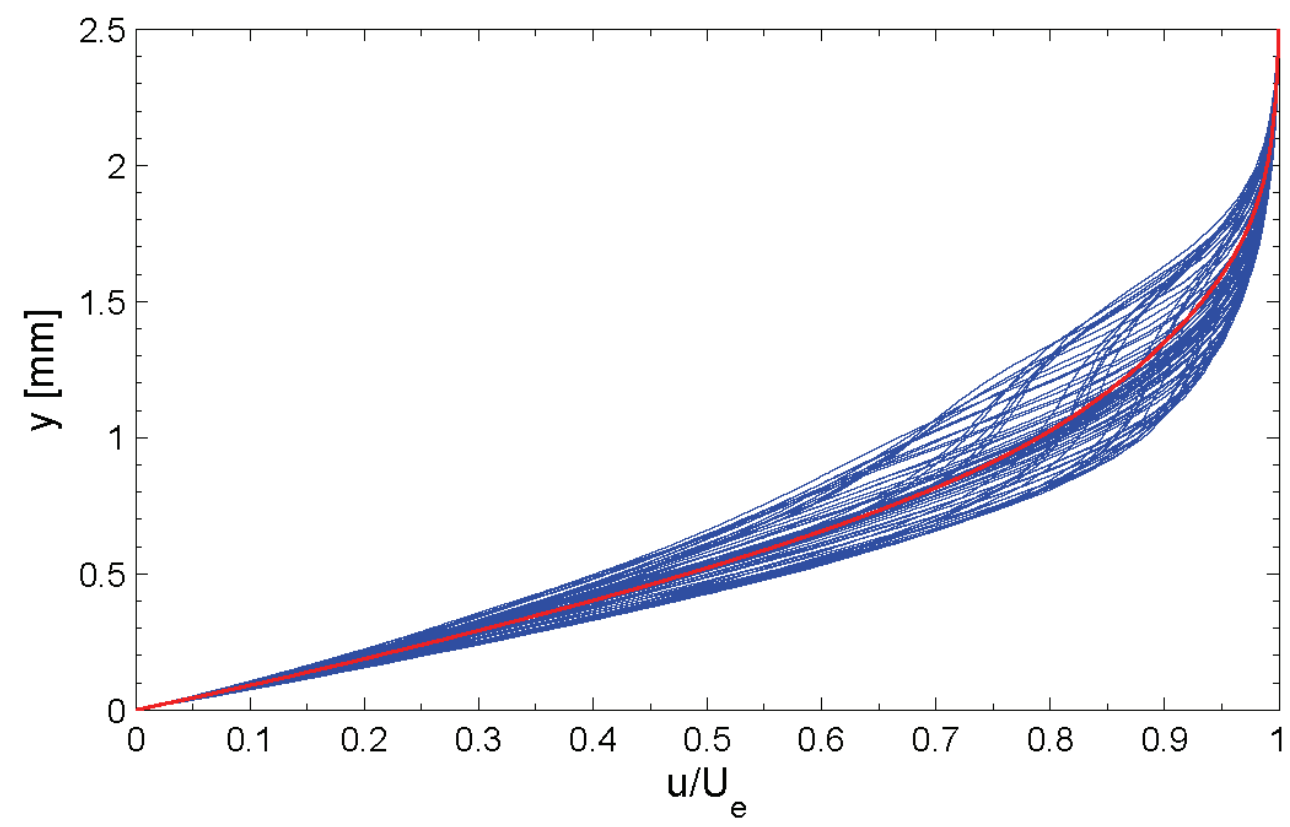

Figure 6-25. Spanwise array of 65 mean-flow boundary-layer profiles spanning $64 \mathrm{~mm}$ at $20 \% x / c . R e_{c}=2.4 \times 10^{6},[36 \mid 12]$ roughness. Mean profile shown in red. 


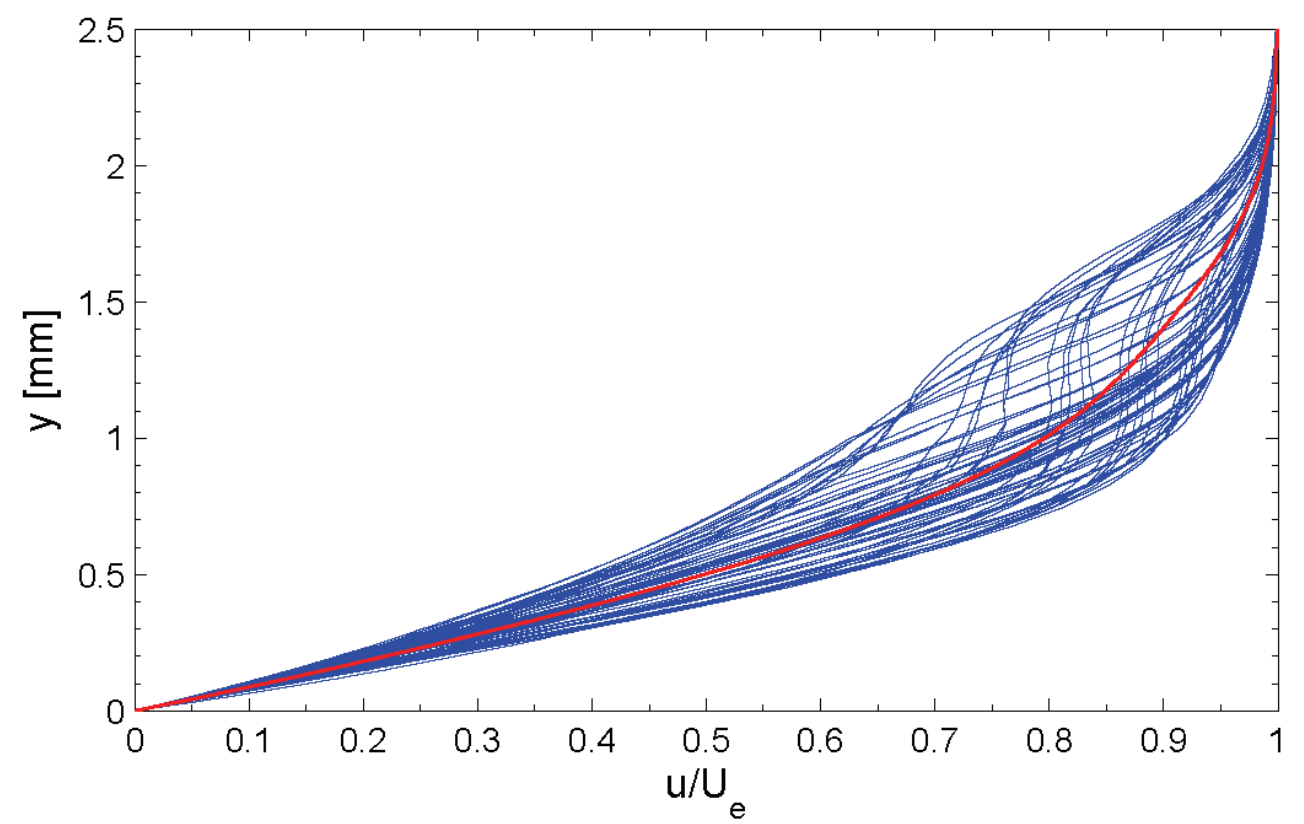

Figure 6-26. Spanwise array of 64 mean-flow boundary-layer profiles spanning $63 \mathrm{~mm}$ at $20 \% x / c . R e_{c}=2.4 \times 10^{6},[60 \mid 12]$ roughness. Mean profile shown in red.

Figures 6-27 - 6-30 show the streamwise velocity contours for this chord location. A $12 \mathrm{~mm}$ spanwise periodicity is present for each roughness height. No evidence of rollover is observed at this downstream location. The disturbance profiles are provided in Figures 6-31 - 6-34 and the final mode shape for each roughness height is shown in Figure 6-35. The first three layers are still within the linear stability range; however, at $60 \mu \mathrm{m}$, a second lobe is starting to develop indicating that modal interactions are starting to influence the growth of a particular mode. Once this occurs, differences in maximum disturbance amplitudes cannot be solely attributed to changes in the receptivity process. Thus, values for the $60 \mu \mathrm{m}$ case are included here, but do not contribute to the discussion on the receptivity process as function of roughness height. This is also the reason that the bulk of measurements occurred at $15 \% x / c$ for other Reynolds numbers and wavelengths. 
At this location, the boundary-layer is thick enough and the mean-flow distortion is large enough to reliably measure maximum disturbance amplitudes for all roughness heights (unlike certain roughness heights at $10 \% x / c$ ), but not so large that nonlinear mode interactions are starting to occur as in the case of the $20 \% x / c$ measurements. These effects become more pronounced at higher Reynolds numbers since increasing Reynolds number is a destabilizing effect. One might expect then that nonlinear stability effects would occur for smaller roughness heights at higher Reynolds numbers, making $20 \% x / c$ a non-ideal location to observe the effects of the receptivity process.

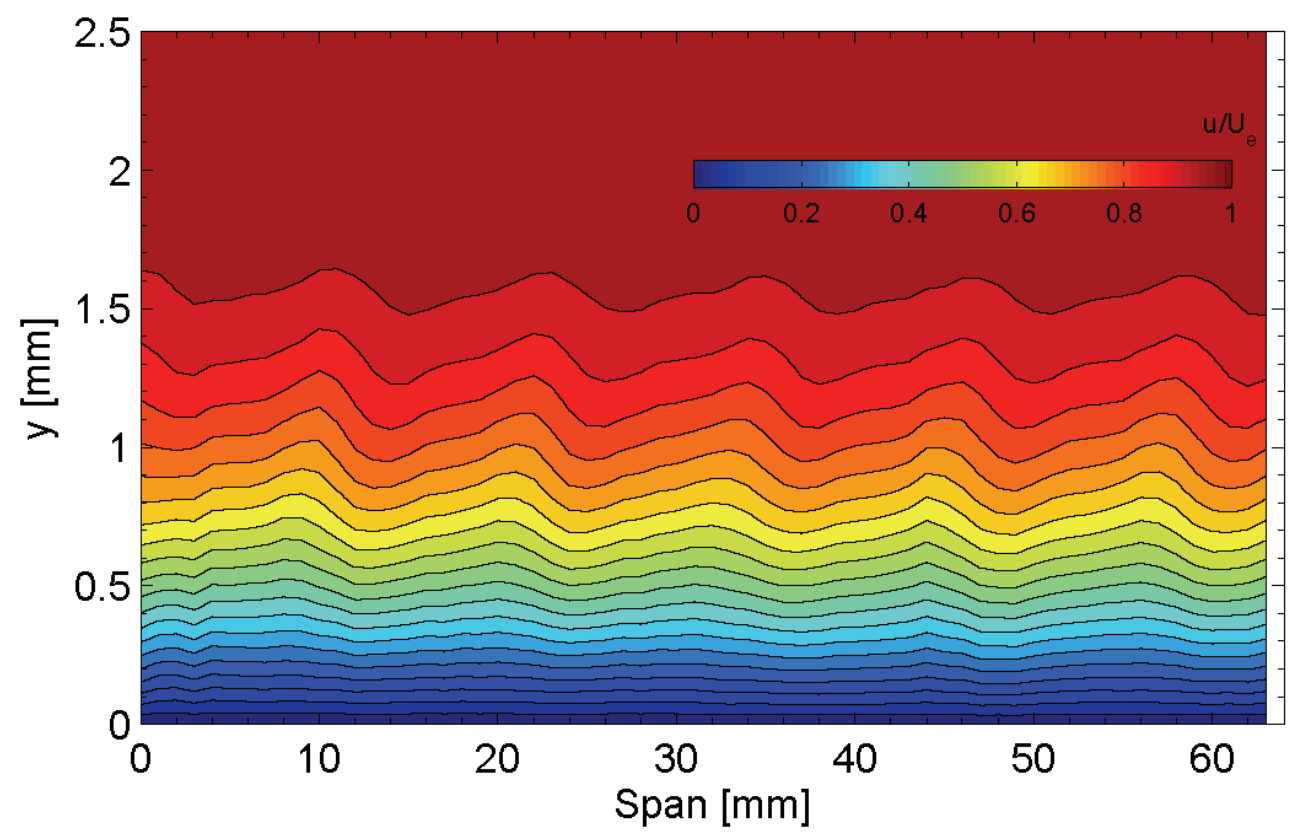

Figure 6-27. Streamwise velocity contours at $20 \% x / c . R e_{c}=2.4 \times 10^{6},[12 \mid 12]$ roughness. 


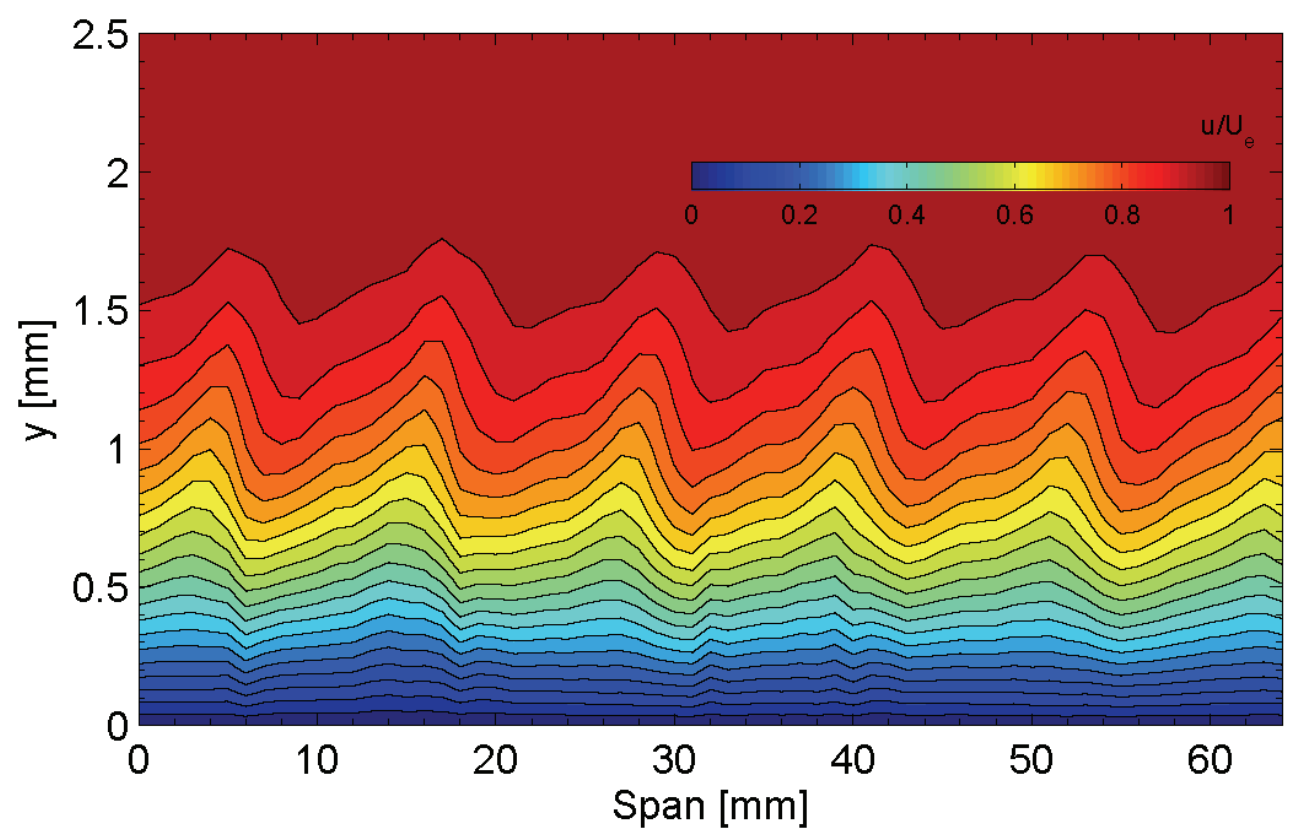

Figure 6-28. Streamwise velocity contours at $20 \% x / c . R e_{c}=2.4 \times 10^{6},[24 \mid 12]$ roughness.

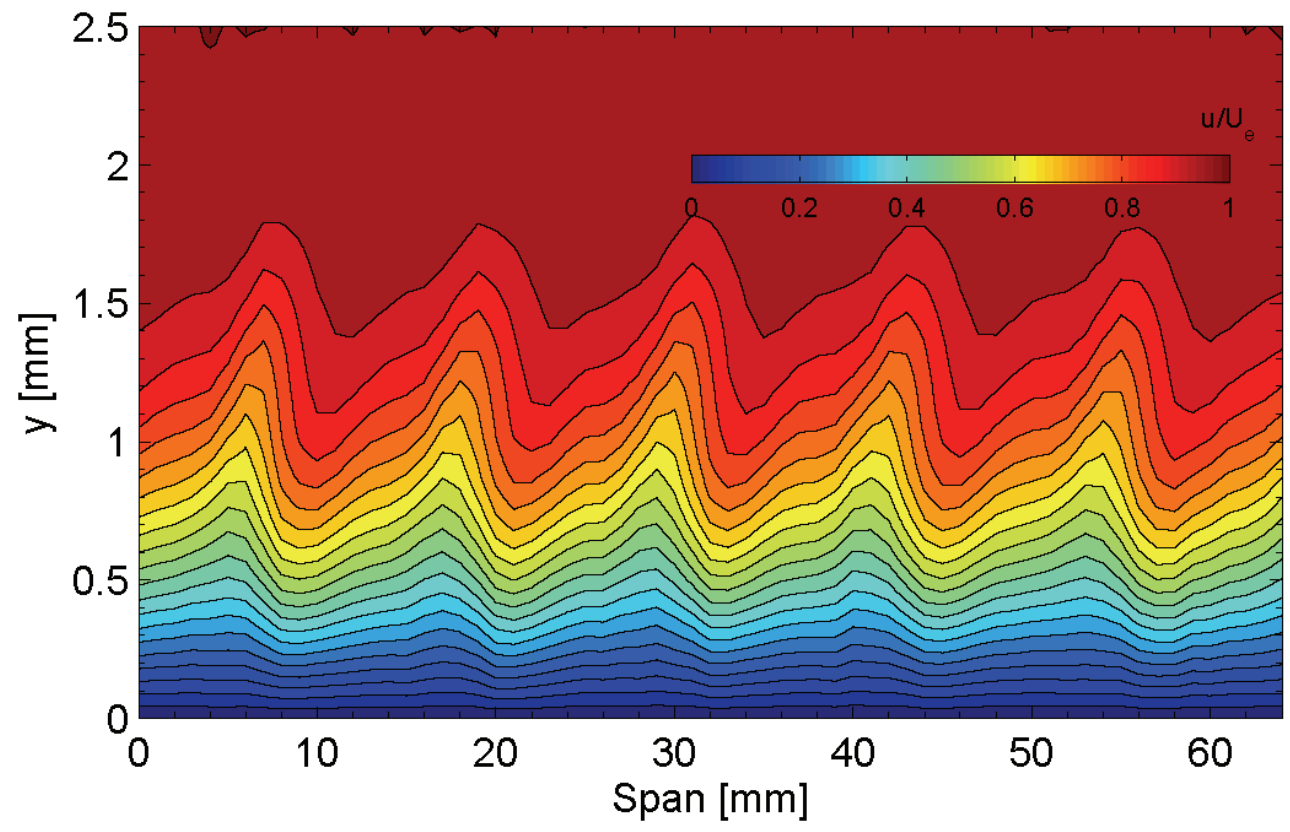

Figure 6-29. Streamwise velocity contours at $20 \% x / c . R e_{c}=2.4 \times 10^{6},[36 \mid 12]$ roughness. 


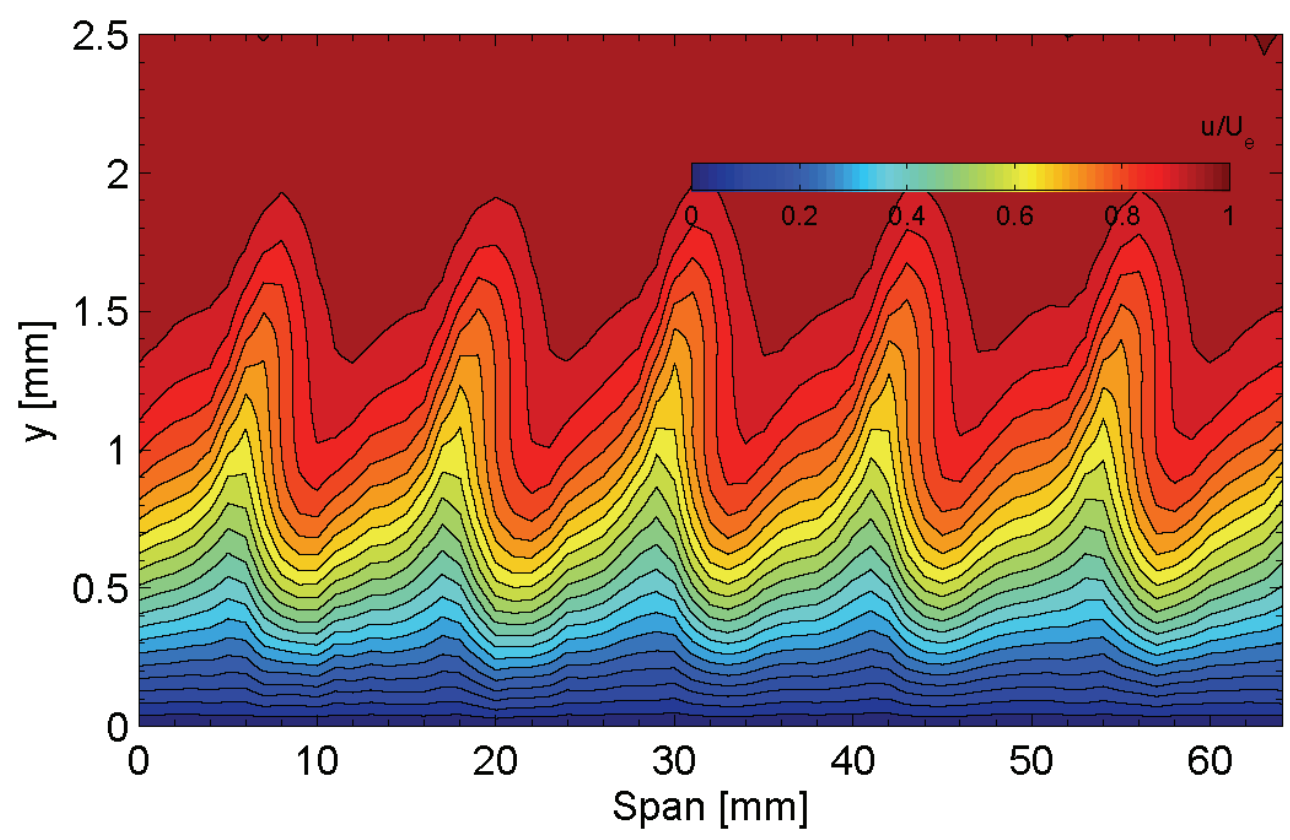

Figure 6-30. Streamwise velocity contours at $20 \% x / c . R e_{c}=2.4 \times 10^{6},[60 \mid 12]$ roughness.

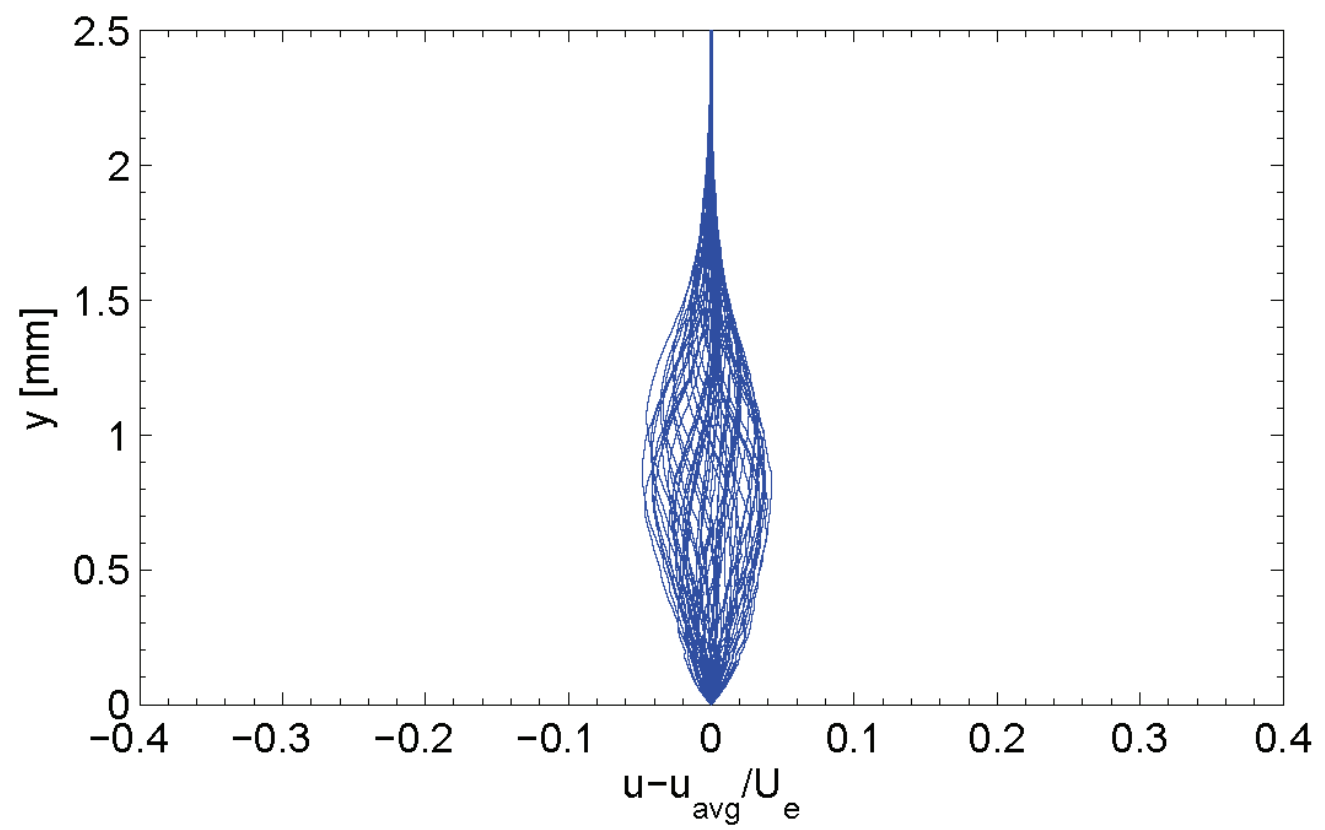

Figure 6-31. Spanwise array of 64 disturbance profiles spanning $63 \mathrm{~mm}$ at $20 \% x / c$. $R e_{c}=2.4 \times 10^{6},[12 \mid 12]$ roughness. 


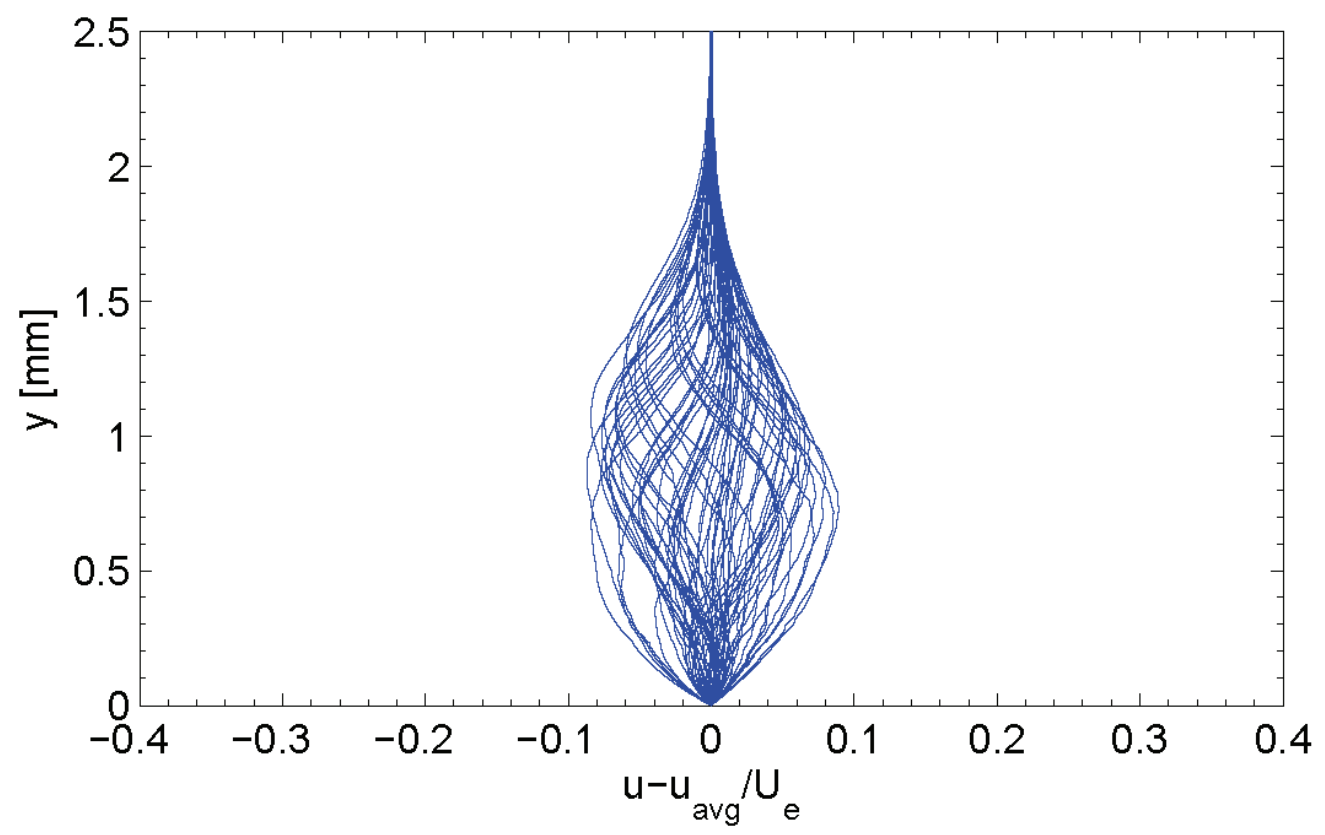

Figure 6-32. Spanwise array of 65 disturbance profiles spanning $64 \mathrm{~mm}$ at $20 \% x / c$. $R e_{c}=2.4 \times 10^{6},[24 \mid 12]$ roughness.

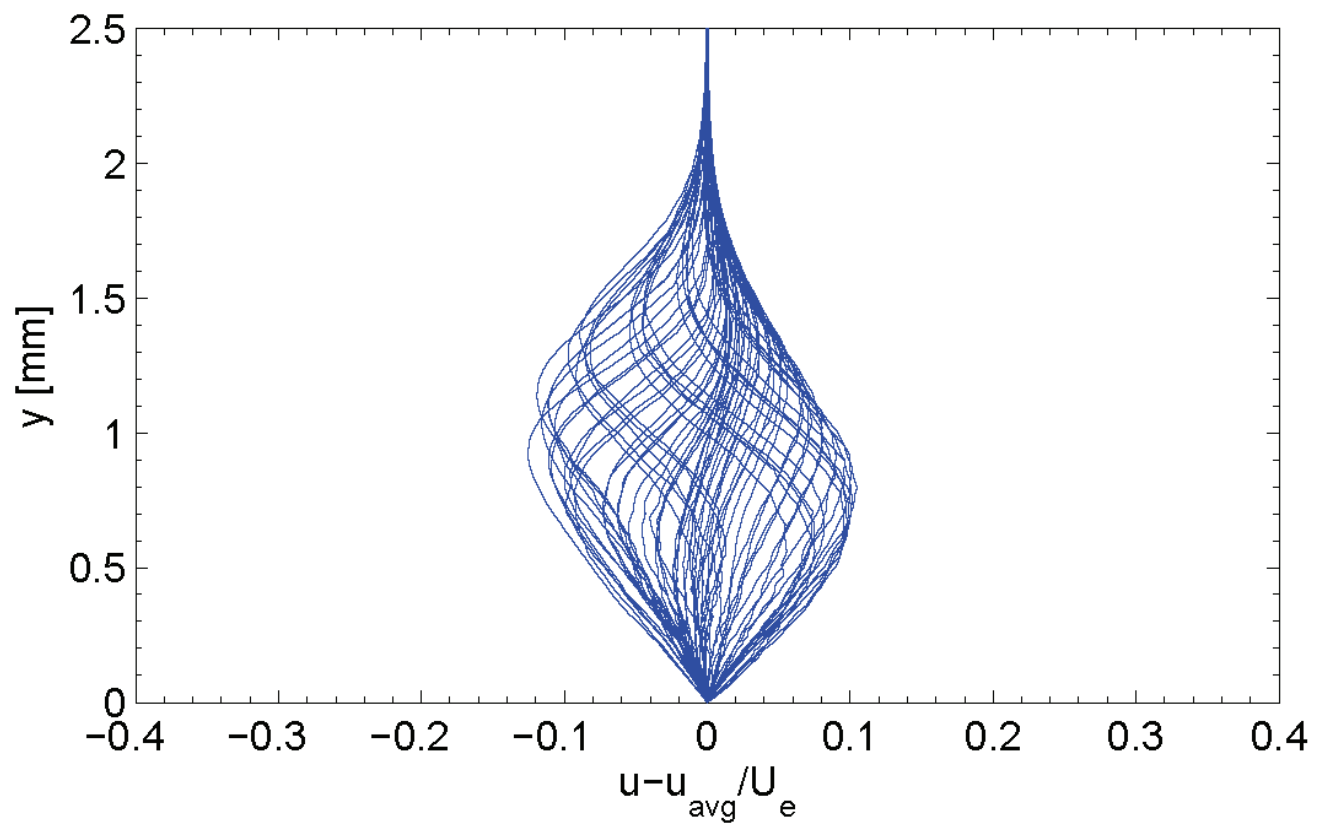

Figure 6-33. Spanwise array of 65 disturbance profiles spanning $64 \mathrm{~mm}$ at $20 \% x / c$. $R e_{c}=2.4 \times 10^{6},[36 \mid 12]$ roughness. 


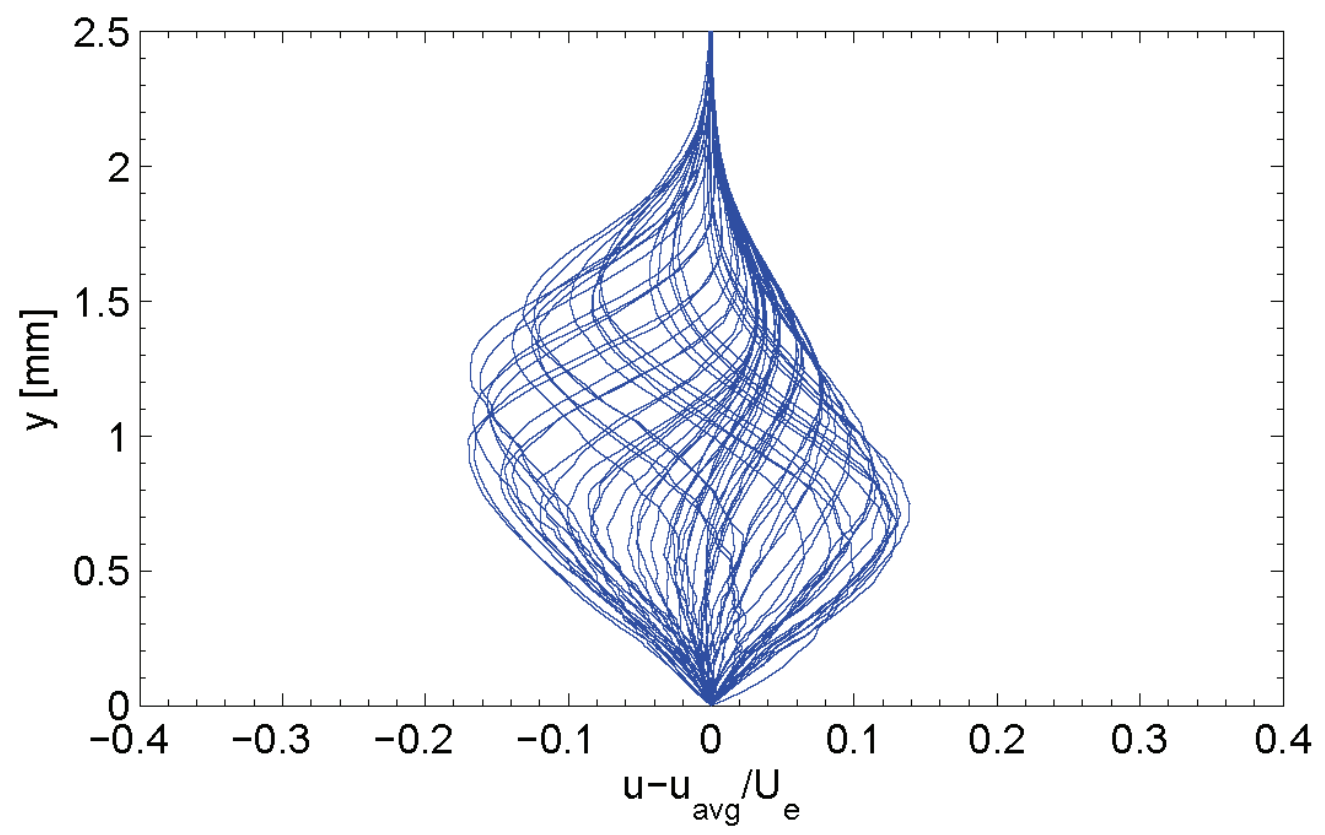

Figure 6-34. Spanwise array of 65 disturbance profiles spanning $64 \mathrm{~mm}$ at $20 \% x / c$. $R e_{c}=2.4 \times 10^{6},[60 \mid 12]$ roughness.

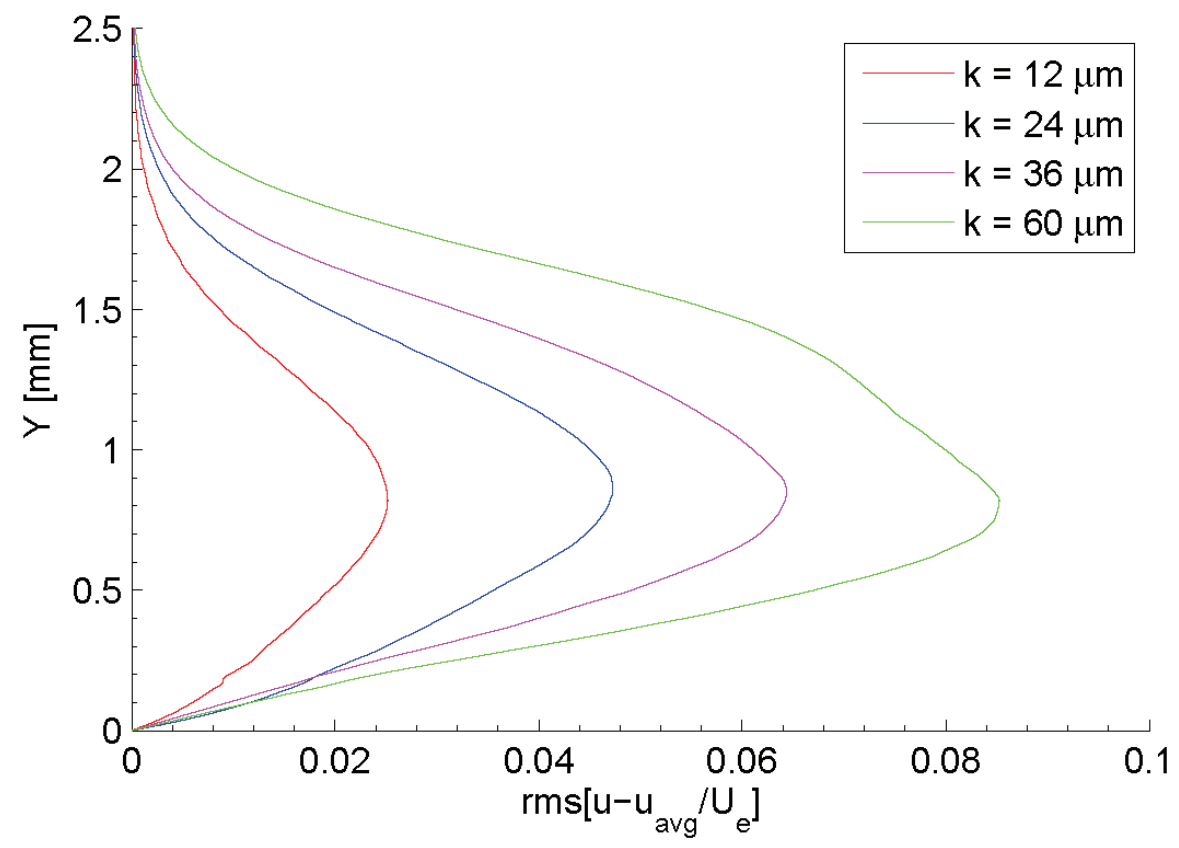

Figure 6-35. Stationary crossflow mode shape at $20 \% x / c . R e_{c}=2.4 \times 10^{6},[k \mid 12]$ roughness. 
Table 6-3. Maximum and normalized stationary crossflow disturbance amplitudes at $20 \% x / c, R e_{c}=2.4 \times 10^{6},[k \mid 12]$ roughness.

\begin{tabular}{|c|c|c|}
\hline$k[\mu \mathrm{m}]$ & {$\left[\left(u-u_{a v g}\right) / U_{e}\right]_{\mathrm{rms}, \max }$} & {$\left[\left(u-u_{\text {avg }}\right) / U_{e}\right]_{\mathrm{rms}, k / k o}$} \\
\hline 12 & 0.0252 & 1.00 \\
\hline 24 & 0.0473 & 0.94 \\
\hline 36 & 0.0644 & 0.85 \\
\hline 60 & 0.0853 & $0.68^{*}$ \\
\hline
\end{tabular}

*Indicates nonlinear stability present

Table 6-3 lists the maximum and normalized disturbance amplitudes measured at $20 \% x / c$. The first three roughness heights appear, at most, to be weakly nonlinear. Although the normalized disturbance amplitude for $k=60 \mu \mathrm{m}$ is substantially lower than the other roughness heights, the role of receptivity in creating this value is unknown since nonlinear stability is present. The spatial spectrum for each roughness height is displayed in Figure 6-36. Once again, a dominant peak at $12 \mathrm{~mm}$ followed by a smaller peak at $6 \mathrm{~mm}$ is present. For the largest roughness case, it also appears that a smaller $4 \mathrm{~mm}$ mode appears. However, the amplitude is small enough that is it within the noise limit of the spectra. Table 6-4 shows the ratio of $12 \mathrm{~mm}$ to $6 \mathrm{~mm}$ disturbance amplitudes calculated from the PSD. The ratios are slightly higher than the measurements made at 15\%-chord, but not substantially different.

Table 6-4. Stationary crossflow disturbance amplitude ratios at $20 \% x / c, R e_{c}=2.4 \times 10^{6},[k \mid 12]$ roughness.

\begin{tabular}{|c|c|}
\hline$k[\mu \mathrm{m}]$ & {$\left[\left(u-u_{\text {avg }}\right) / U_{e}\right]_{\mathrm{rms}, \lambda=12} /\left[\left(u-u_{\text {avg }}\right) / U_{e}\right]_{\mathrm{rms}, \lambda=6}$} \\
\hline 12 & 2.24 \\
\hline 24 & 2.45 \\
\hline 36 & 2.48 \\
\hline 60 & 2.72 \\
\hline
\end{tabular}




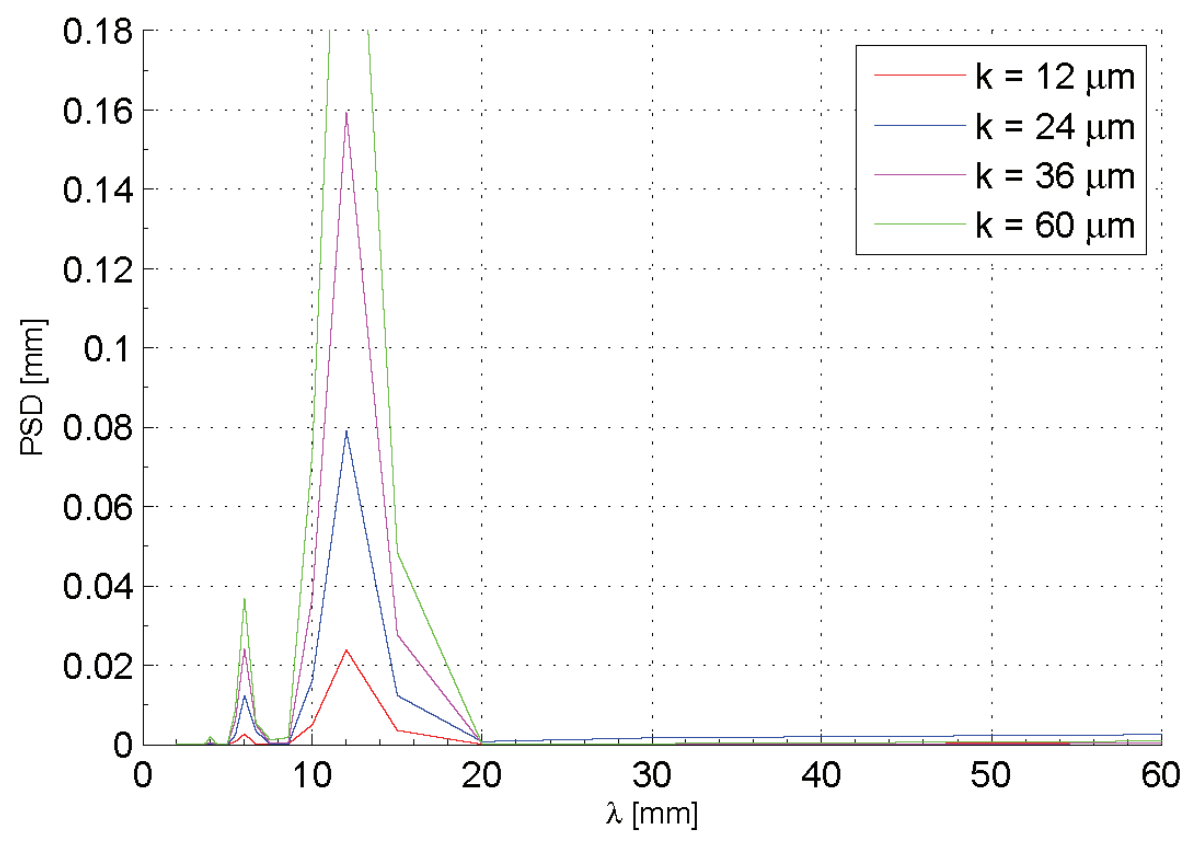

Figure 6-36. Power spectral density of spanwise hotwire scan at $20 \% x / c$. $R e_{c}=2.4 \times 10^{6},[k \mid 12]$ roughness.

\section{$6.2 R e_{c}=2.8 \times 10^{6}$}

Naphthalene flow visualization was not completed for cases where $R e_{c}=2.8 \times 10^{6}$. A single roughness height at $10 \% x / c$, followed by four roughness heights at $15 \% x / c$ were surveyed using hotwire anemometry.

\subsubsection{Hotwire Measurements, $10 \% x / c$}

Figures 6-37 - 6-40 show the boundary-layer profiles, streamwise velocity contours, disturbance shape and mode shape respectively for a $47 \mu \mathrm{m}$ roughness height at $R e_{c}=2.8 \times 10^{6}$. Measurements are taken at $10 \% x / c$. The $12 \mathrm{~mm}$ distortion observed in the streamwise velocity contours is, as expected, stronger for the higher Reynoldsnumber case. Maximum disturbance amplitude for this test condition is 0.0263 . The 
spatial mode decomposition is shown in Figure 6-41. Similar to $R_{c}=2.4 \times 10^{6}$, little can be said regarding the receptivity process at this location since other roughness heights are not available. This point does serve as an additional verification point for numerical simulations.

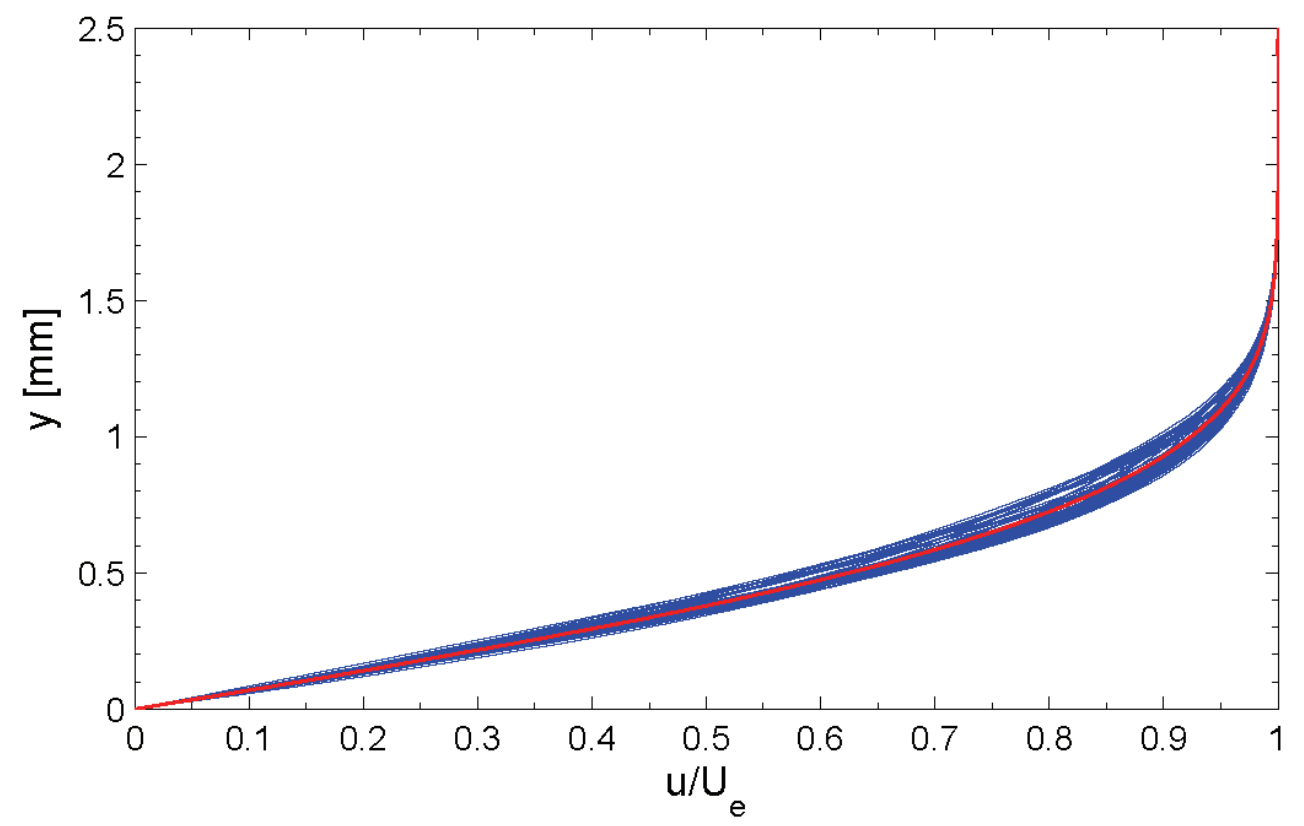

Figure 6-37. Spanwise array of 65 mean-flow boundary-layer profiles spanning $64 \mathrm{~mm}$ at $10 \% x / c . R e_{c}=2.8 \times 10^{6},[47 \mid 12]$ roughness. Mean profile shown in red. 


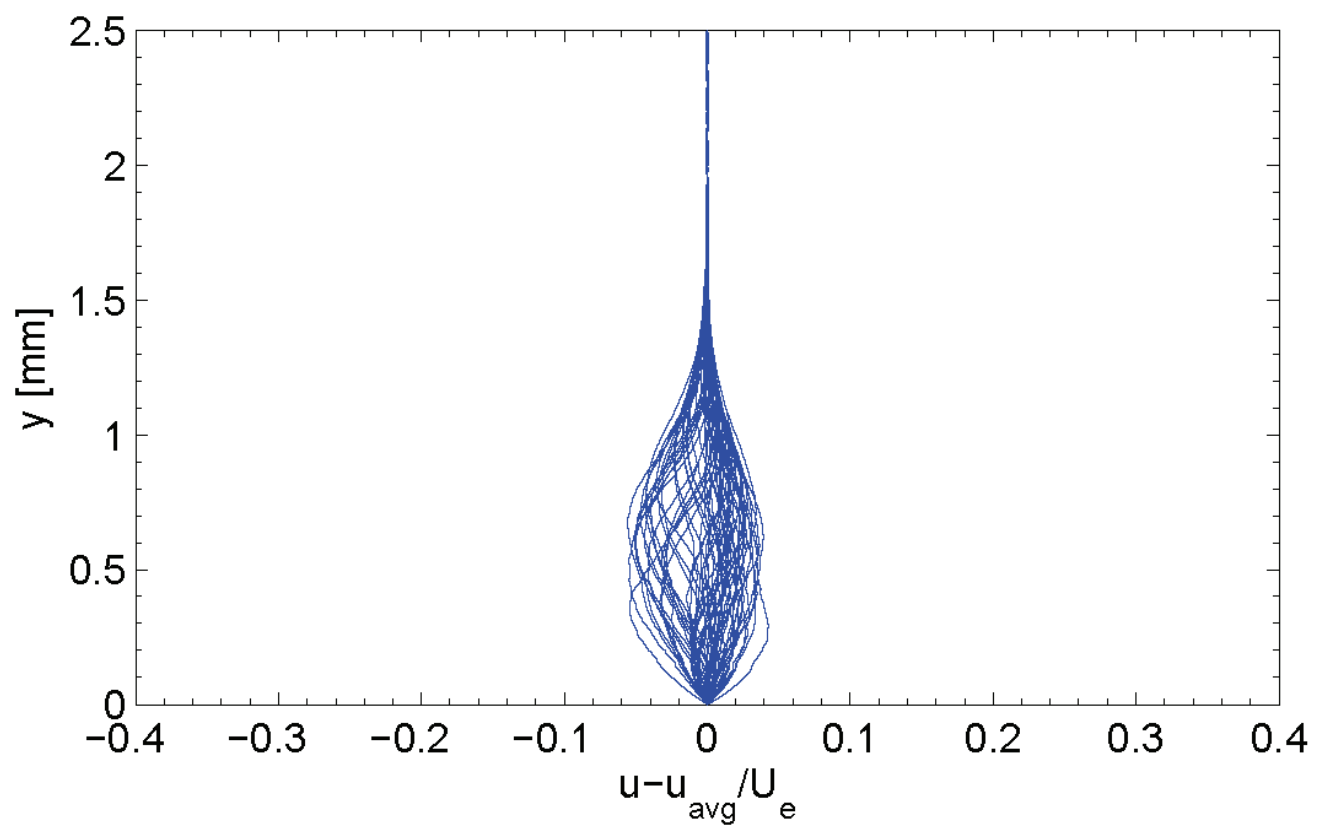

Figure 6-38. Spanwise array of 65 disturbance profiles spanning $64 \mathrm{~mm}$ at $10 \% x / c$. $R e_{c}=2.8 \times 10^{6},[47 \mid 12]$ roughness.

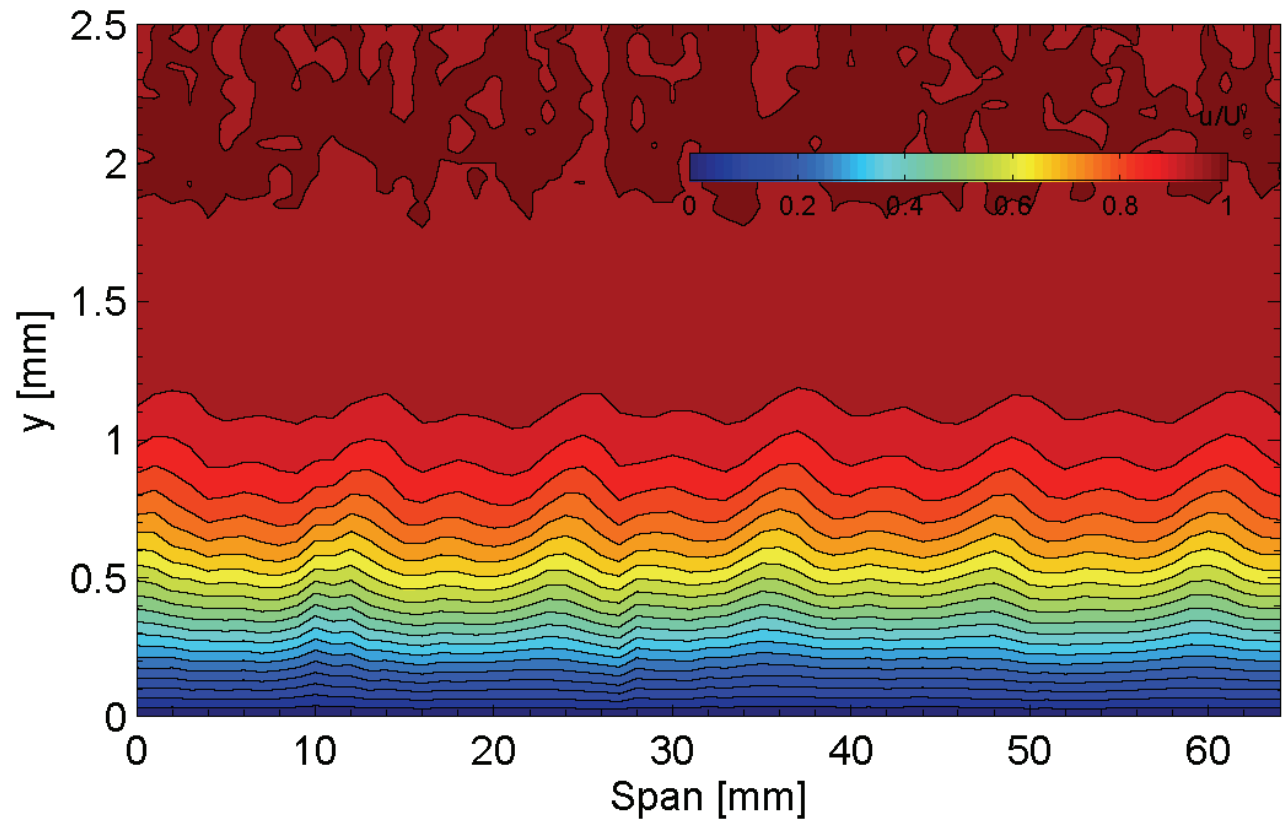

Figure 6-39. Streamwise velocity contours at $10 \% x / c . R e_{c}=2.8 \times 10^{6},[47 \mid 12]$ roughness. 


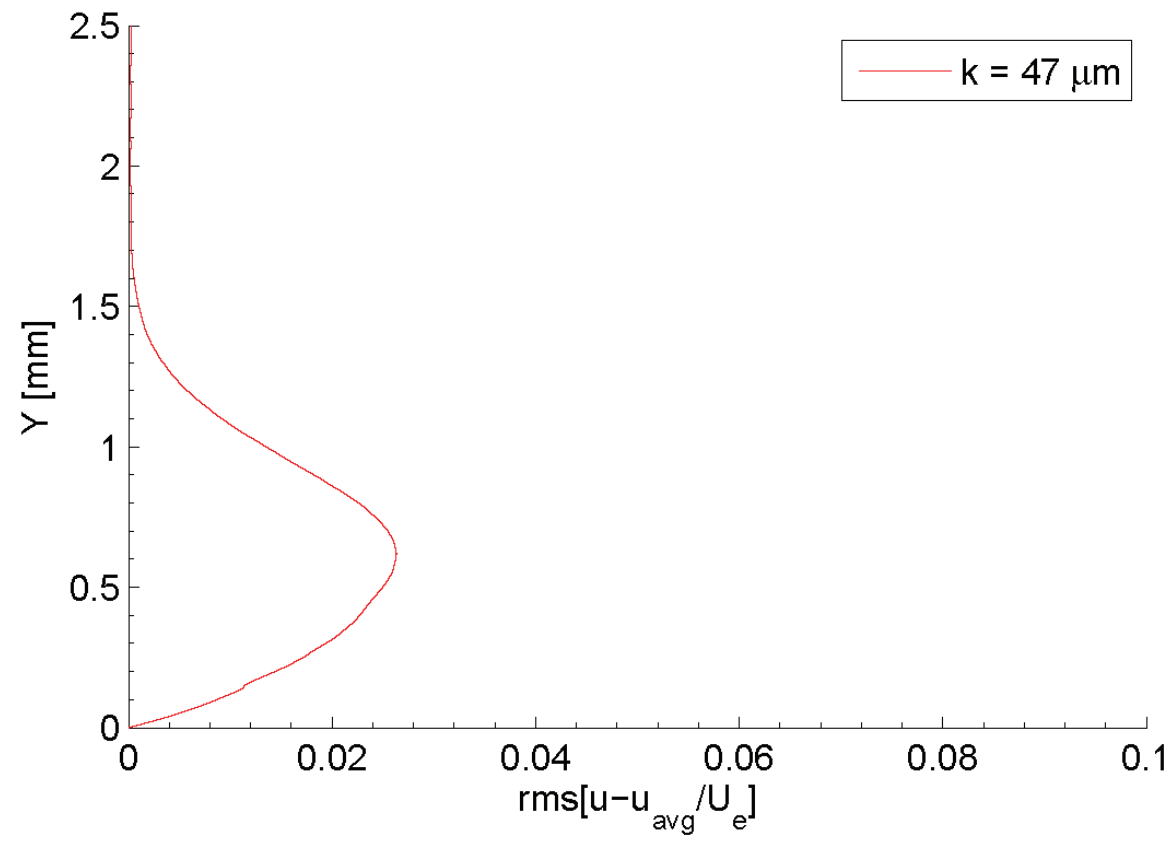

Figure 6-40. Stationary crossflow mode shape at $10 \% x / c . R e_{c}=2.8 \times 10^{6},[47 \mid 12]$ roughness.

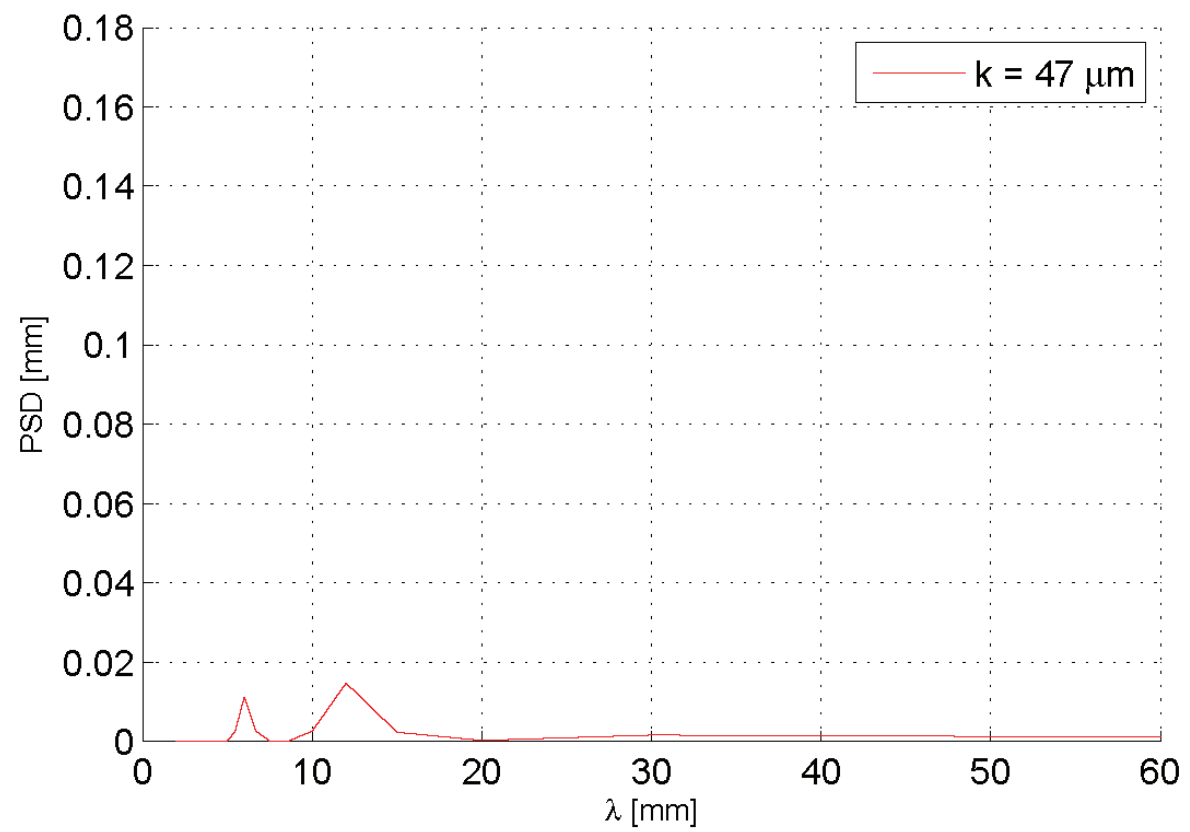

Figure 6-41. Power spectral density of spanwise hotwire scan at $10 \% x / c$. $R e_{c}=2.8 \times 10^{6},[47 \mid 12]$ roughness. 


\subsubsection{Hotwire Measurements, $15 \% x / c$}

At this Reynolds number, roughness heights ranged from $12 \mu \mathrm{m}$ to $47 \mu \mathrm{m}$. Figures 6-42 - 6-45 show the boundary layer profiles as roughness height is increased. By the time $47 \mu \mathrm{m}$ is reached, the mean-velocity profiles have been highly distorted and inflectional profiles are growing stronger. The streamwise velocity contours are shown in Figures 6-46-6-49. In addition to the $12 \mathrm{~mm}$ periodicity, a strong $6 \mathrm{~mm}$ periodicity is also visible. The amplitude of both the $6 \mathrm{~mm}$ and $12 \mathrm{~mm}$ peaks increases as roughness height increases. Disturbance shapes are shown in Figures 6-50 - 6-53 and Figure 6-54 provides the mode shapes. No evidence of a second lobe is visible for all four roughness heights, indicating that trends observed as roughness height is increased are the result of the receptivity process.

Table 6-5. Maximum and normalized stationary crossflow disturbance amplitudes at $15 \% x / c, R e_{c}=2.8 \times 10^{6},[k \mid 12]$ roughness.

\begin{tabular}{|c|c|c|}
\hline$k[\mu \mathrm{m}]$ & {$\left[\left(u-u_{\text {avg }}\right) / U_{e}\right]_{\mathrm{rms}, \max }$} & {$\left[\left(u-u_{\text {avg }}\right) / U_{e}\right]_{\mathrm{rms}, k / k o}$} \\
\hline 12 & 0.0174 & 1.00 \\
\hline 24 & 0.0347 & 1.00 \\
\hline 36 & 0.0490 & 0.94 \\
\hline 47 & 0.0618 & 0.89 \\
\hline
\end{tabular}

Table 6-5 lists the maximum and normalized disturbance amplitudes measured over the full height range. Similar to the lower Reynolds number case, the normalized disturbance amplitudes show, at most, a weakly nonlinear trend as roughness height increases. As an aside, the $36 \mu \mathrm{m}$ roughness height scan was conducted a second time to examine repeatability in the results. The same maximum disturbance amplitude was obtained for this configuration. Figure 6-55 shows the PSD for the spanwise hotwire 
scan. Both the $6 \mathrm{~mm}$ and $12 \mathrm{~mm}$ peaks are present. Unlike the results at $R e_{c}=2.4 \times 10^{6}$, the $6 \mathrm{~mm}$ mode is much more predominant. This is demonstrated more quantitatively by comparing the $12 \mathrm{~mm}$ to $6 \mathrm{~mm}$ amplitude ratio in Table 6-6. At $R e_{c}=2.8 \times 10^{6}$, the maximum disturbance amplitude from the $6 \mathrm{~mm}$ wave is almost as large as the forced wavelength of $12 \mathrm{~mm}$.

Table 6-6: Stationary crossflow disturbance amplitude ratios at $15 \% x / c . R e_{c}=2.8 \times 10^{6},[k \mid 12]$ roughness.

\begin{tabular}{|c|c|}
\hline$k[\mu \mathrm{m}]$ & {$\left[\left(u-u_{\text {avg }}\right) / U_{e}\right]_{\mathrm{rms}, \lambda=12} /\left[\left(u-u_{\text {avg }}\right) / U_{e}\right]_{\mathrm{rms}, \lambda=6}$} \\
\hline 12 & 1.17 \\
\hline 24 & 1.18 \\
\hline 36 & 1.09 \\
\hline 47 & 0.97 \\
\hline
\end{tabular}

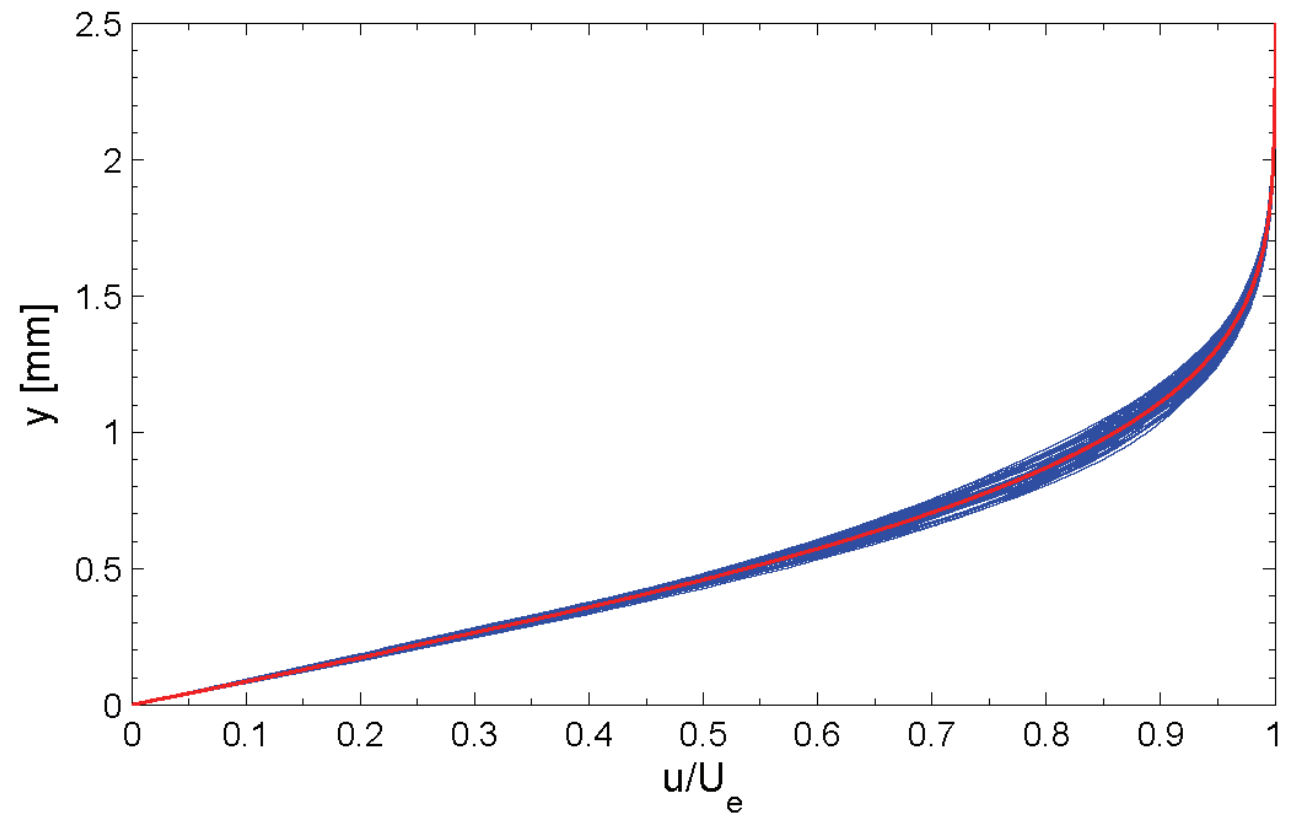

Figure 6-42. Spanwise array of 64 mean-flow boundary-layer profiles spanning $63 \mathrm{~mm}$ at $15 \% x / c . R e_{c}=2.8 \times 10^{6},[12 \mid 12]$ roughness. Mean profile shown in red. 


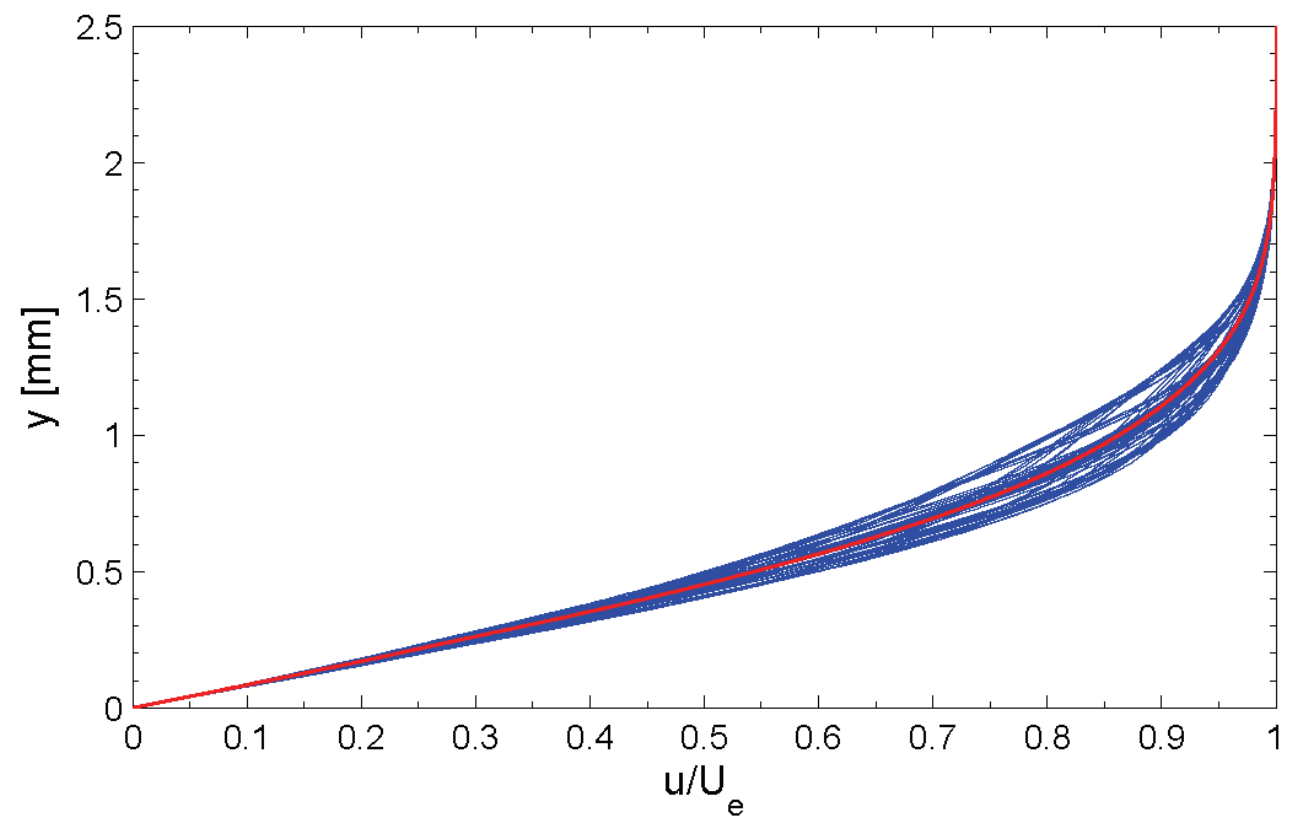

Figure 6-43. Spanwise array of 65 mean-flow boundary-layer profiles spanning $64 \mathrm{~mm}$ at $15 \% x / c . R e_{c}=2.8 \times 10^{6},[24 \mid 12]$ roughness. Mean profile shown in red.

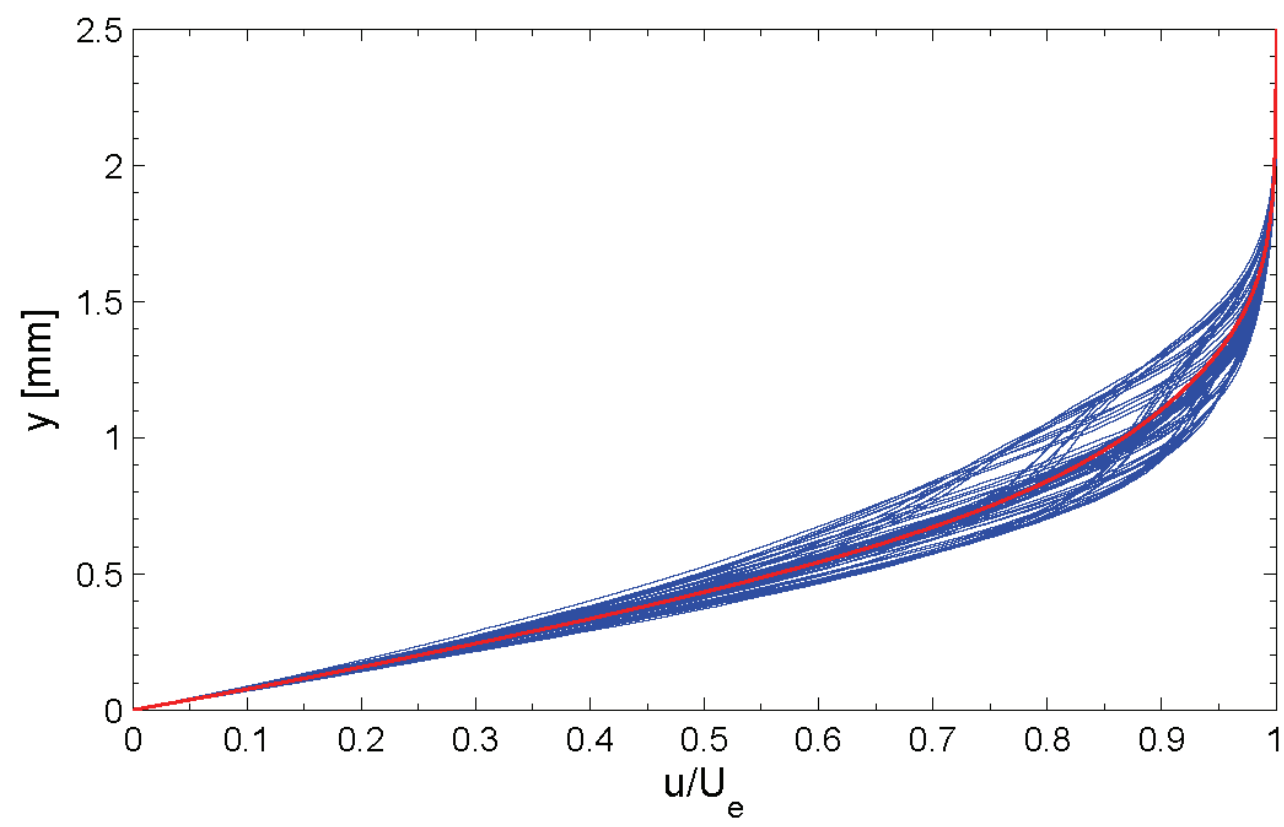

Figure 6-44. Spanwise array of 65 mean-flow boundary-layer profiles spanning $64 \mathrm{~mm}$ at $15 \% x / c . R e_{c}=2.8 \times 10^{6},[36 \mid 12]$ roughness. Mean profile shown in red. 


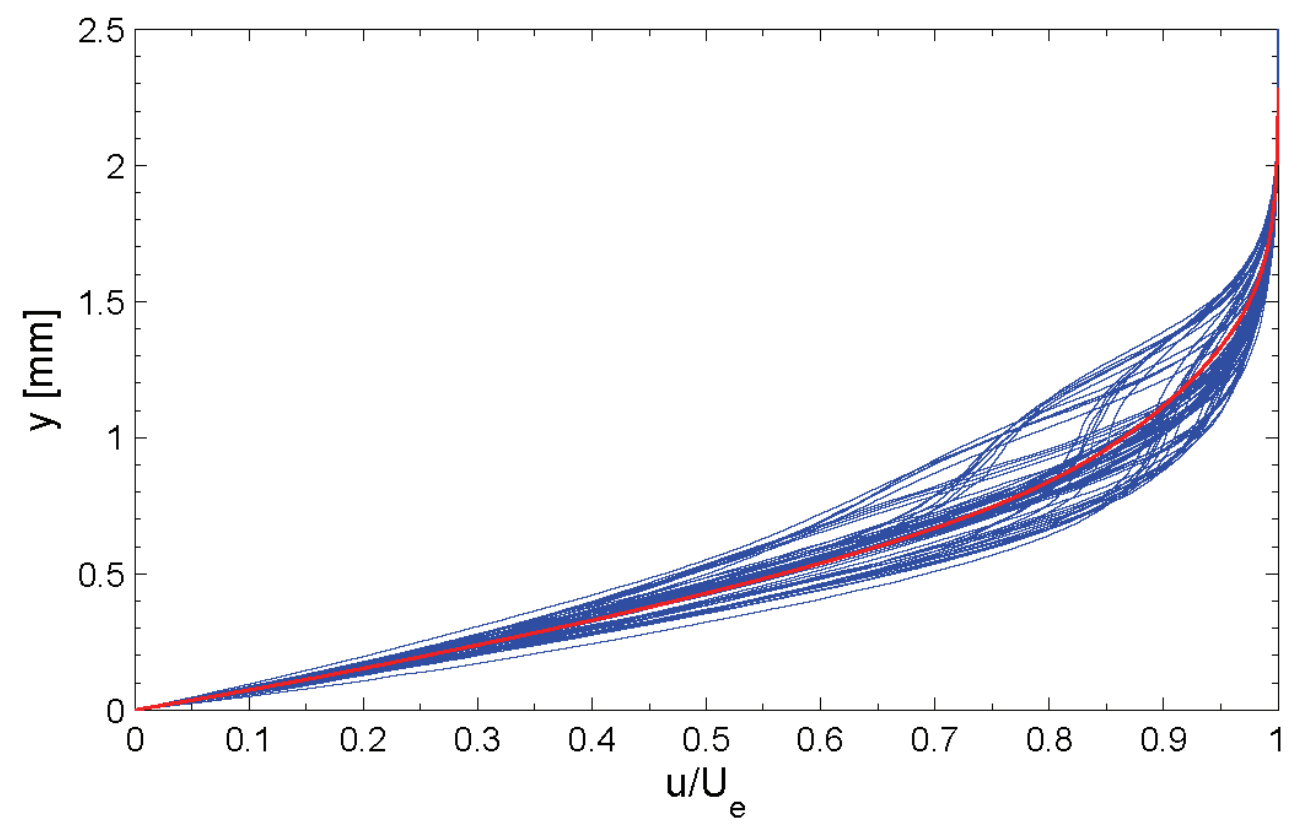

Figure 6-45. Spanwise array of 65 mean-flow boundary-layer profiles spanning $64 \mathrm{~mm}$ at $15 \% x / c . R e_{c}=2.8 \times 10^{6},[47 \mid 12]$ roughness. Mean profile shown in red.

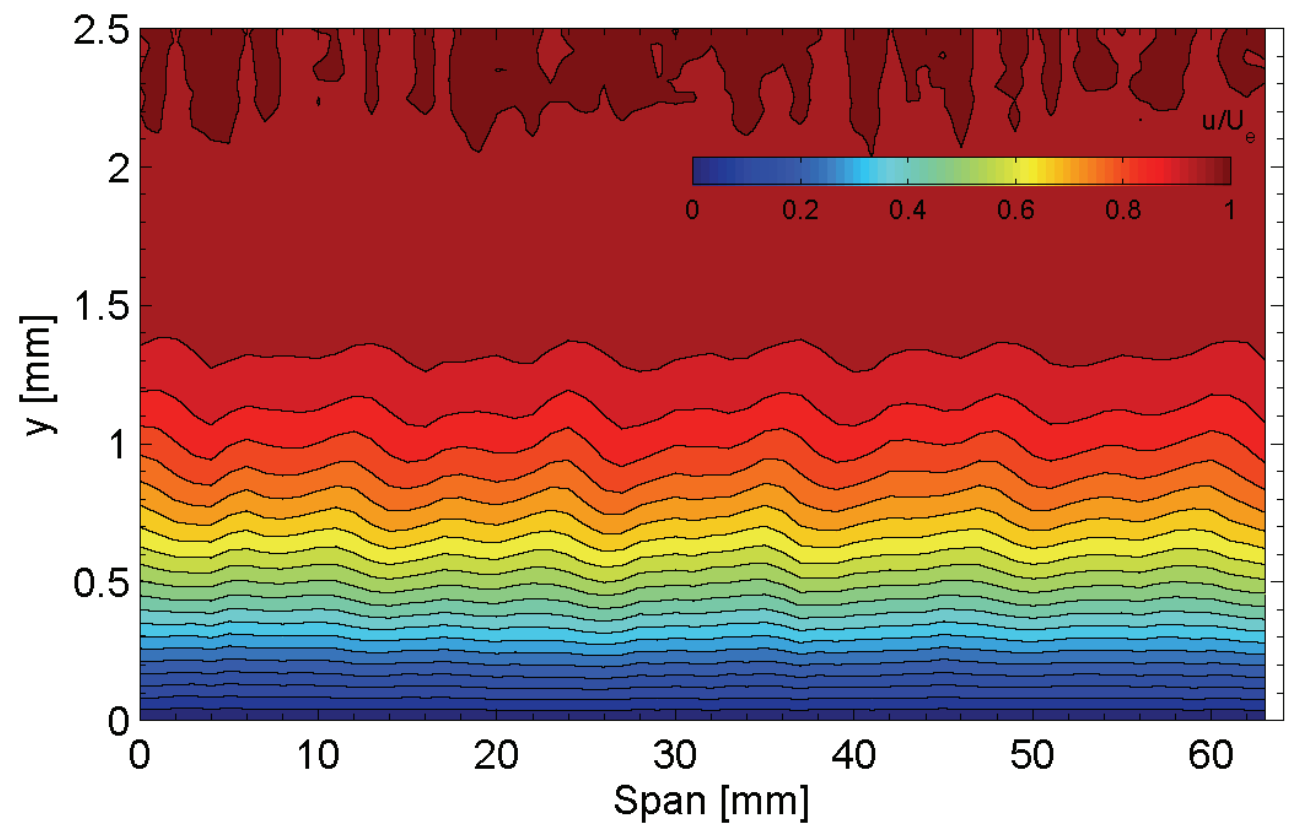

Figure 6-46. Streamwise velocity contours at $15 \% x / c . R e_{c}=2.8 \times 10^{6},[12 \mid 12]$ roughness. 


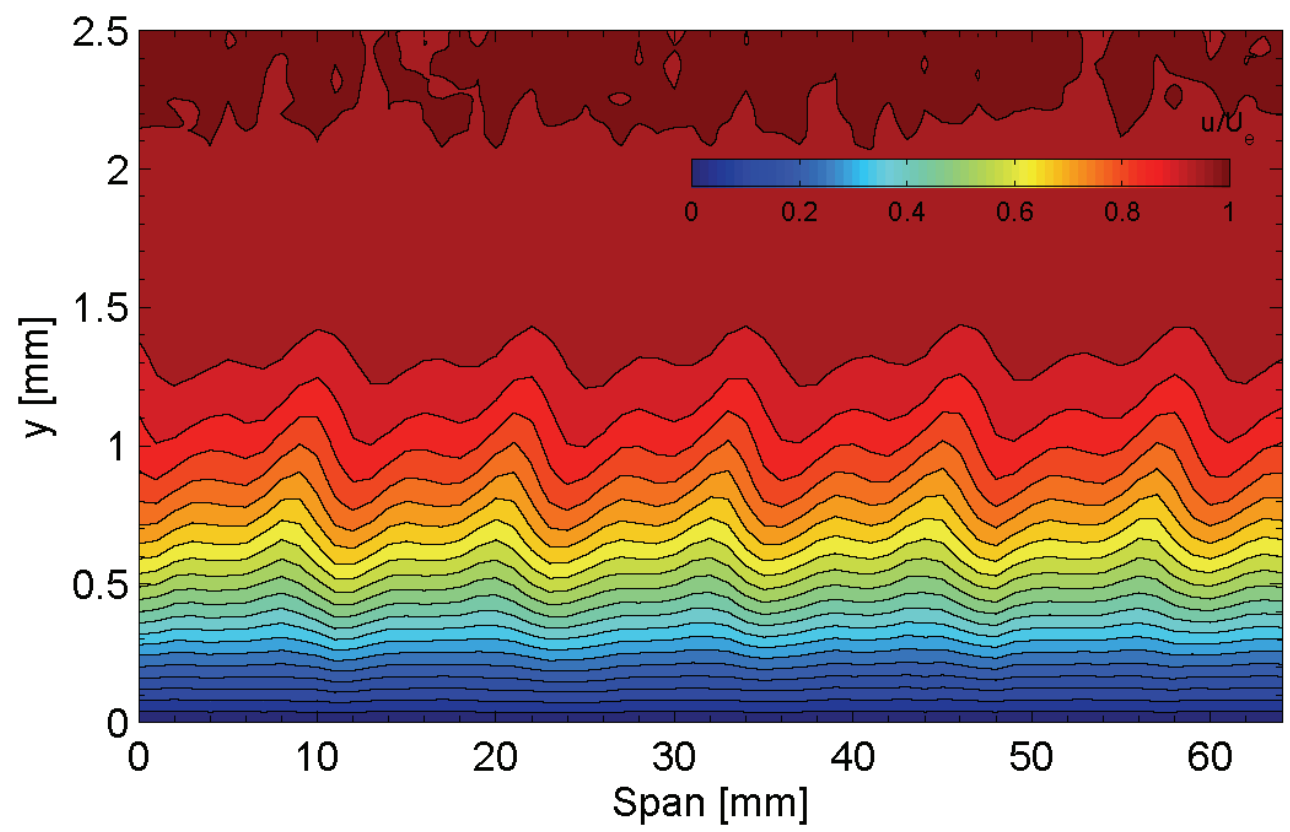

Figure 6-47. Streamwise velocity contours at $15 \% x / c . R e_{c}=2.8 \times 10^{6},[24 \mid 12]$ roughness.

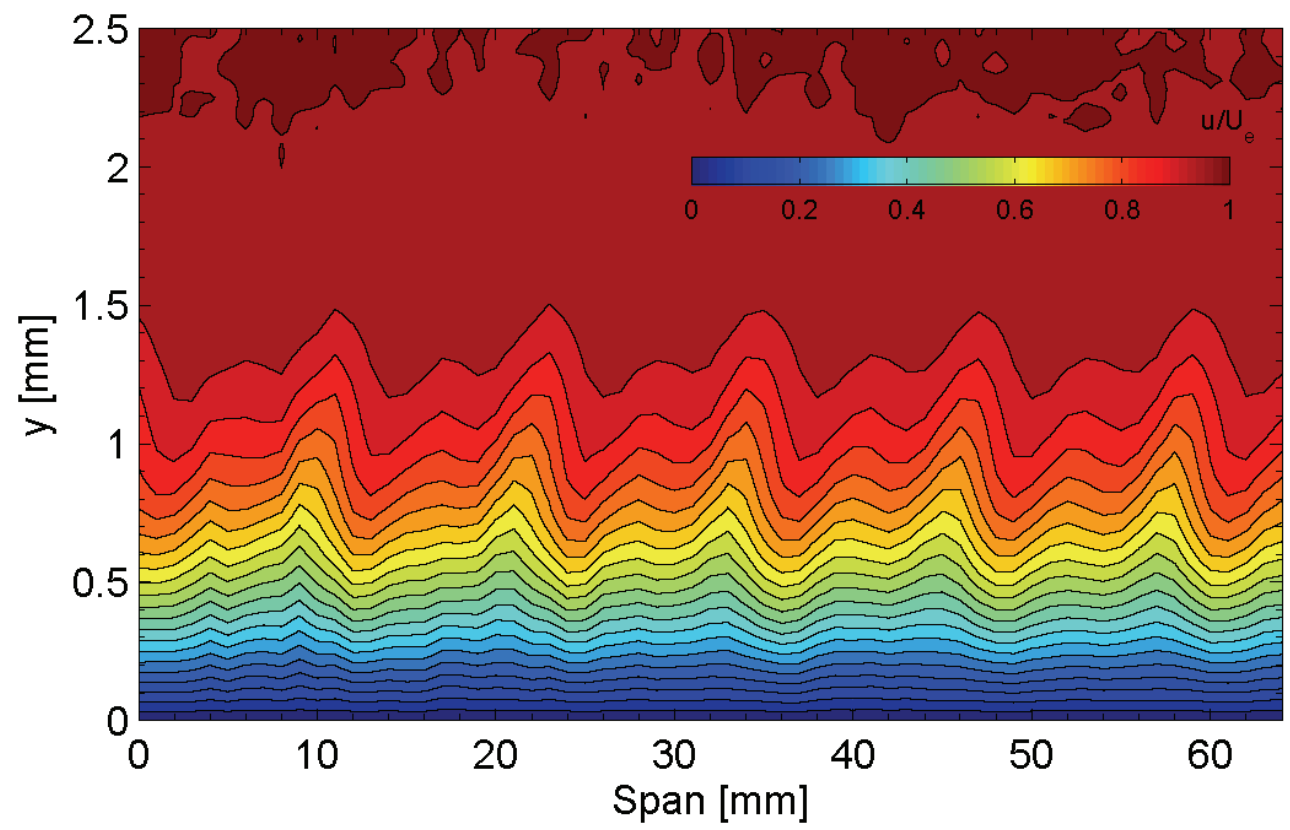

Figure 6-48 Streamwise velocity contours at $15 \% x / c . R e_{c}=2.8 \times 10^{6},[36 \mid 12]$ roughness. 


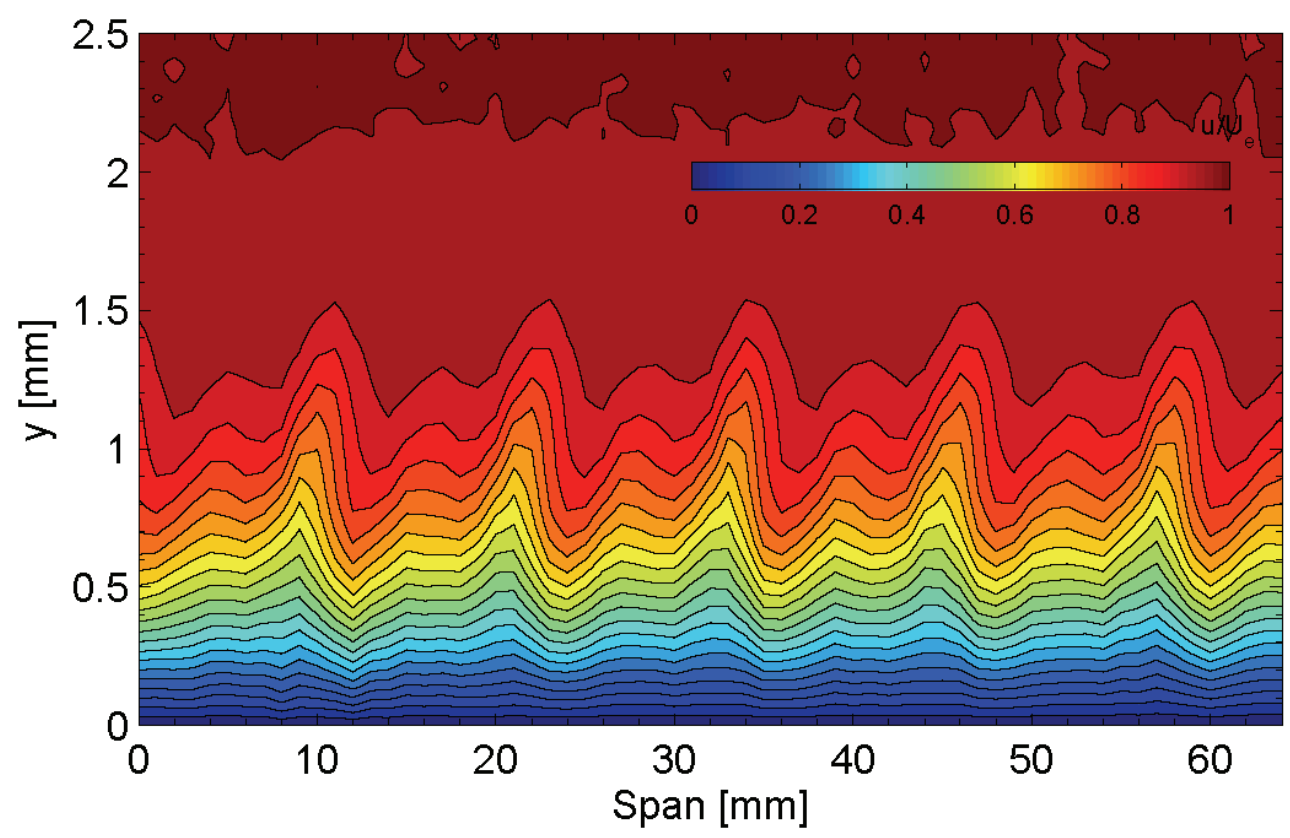

Figure 6-49. Streamwise velocity contours at $15 \% x / c . R e_{c}=2.8 \times 10^{6},[47 \mid 12]$ roughness.

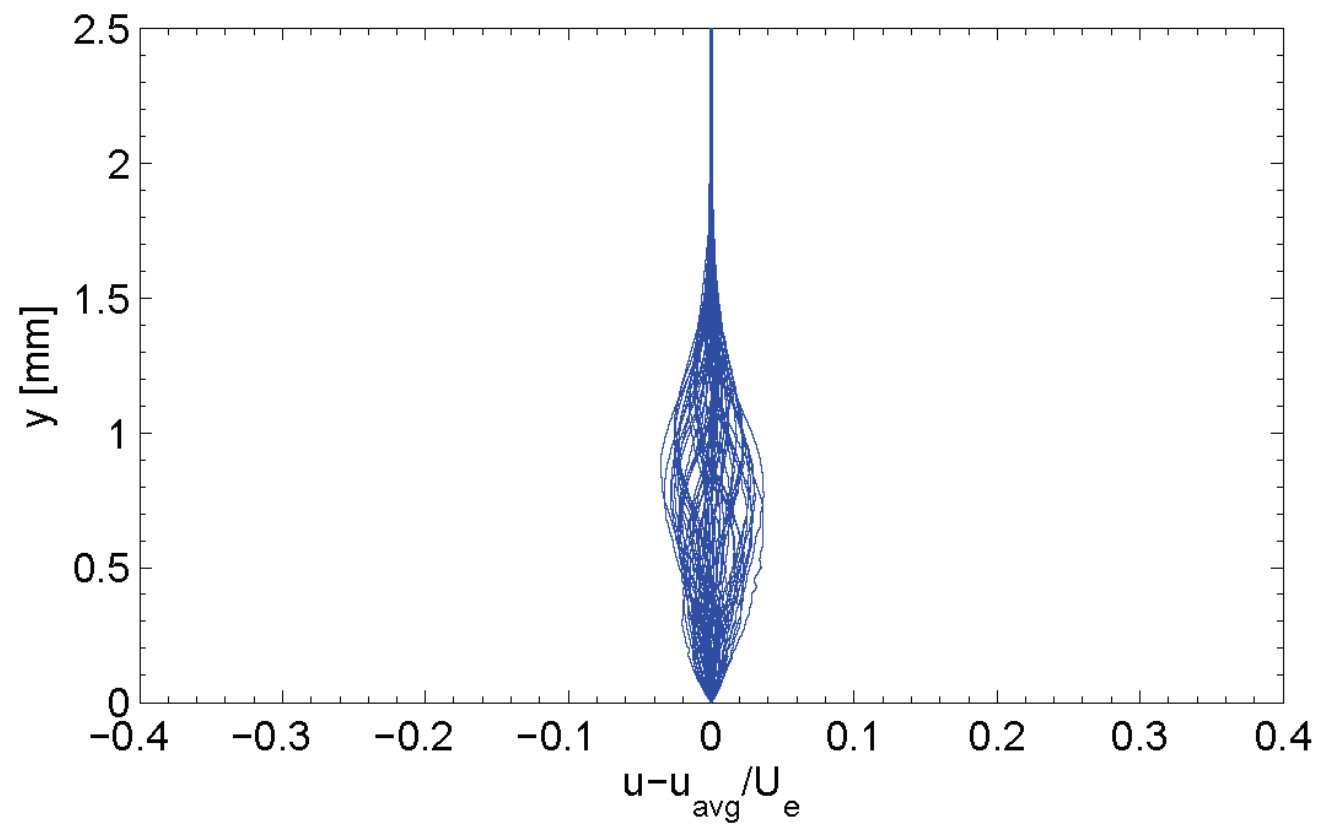

Figure 6-50. Spanwise array of 64 disturbance profiles spanning $63 \mathrm{~mm}$ at $15 \% x / c$. $R e_{c}=2.8 \times 10^{6},[12 \mid 12]$ roughness. 


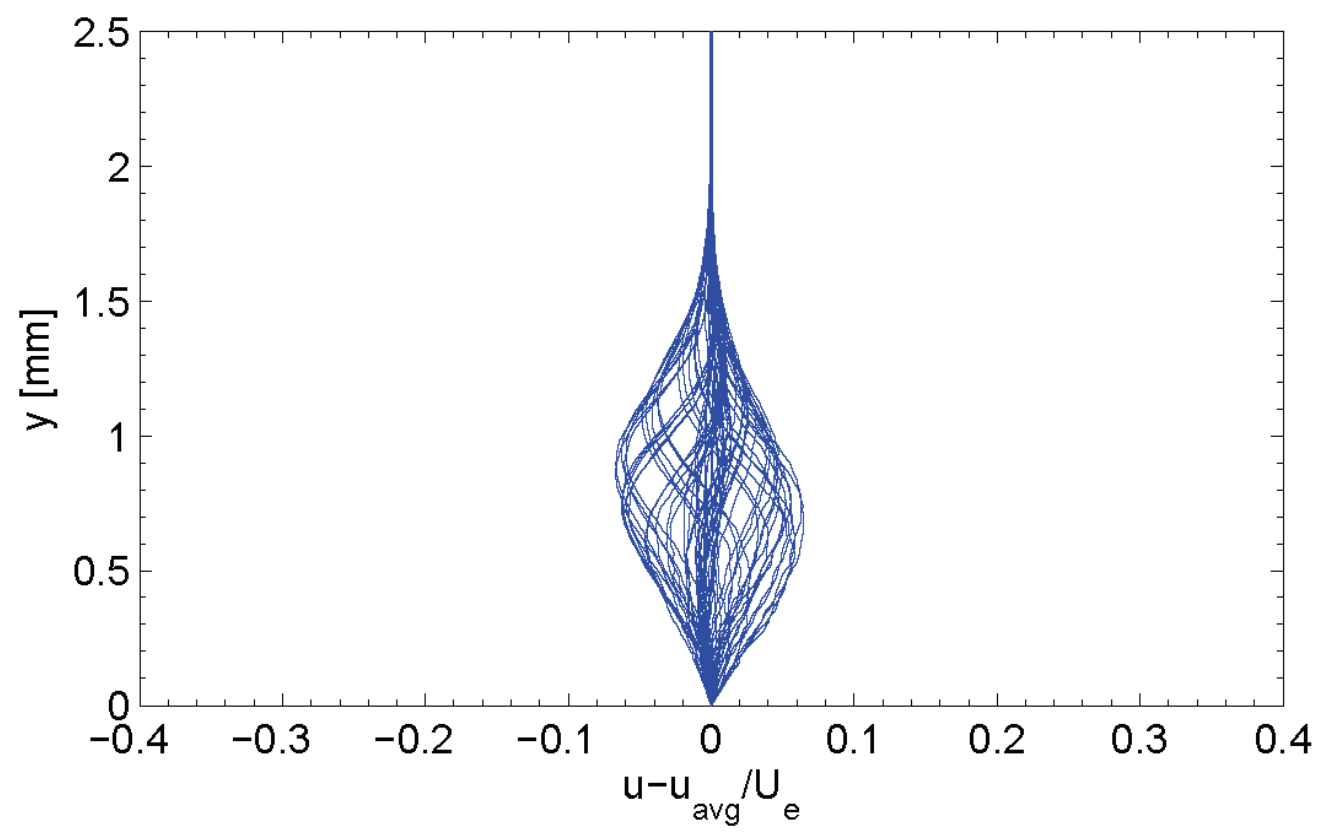

Figure 6-51. Spanwise array of 65 disturbance profiles spanning $64 \mathrm{~mm}$ at $15 \% x / c$. $R e_{c}=2.8 \times 10^{6},[24 \mid 12]$ roughness.

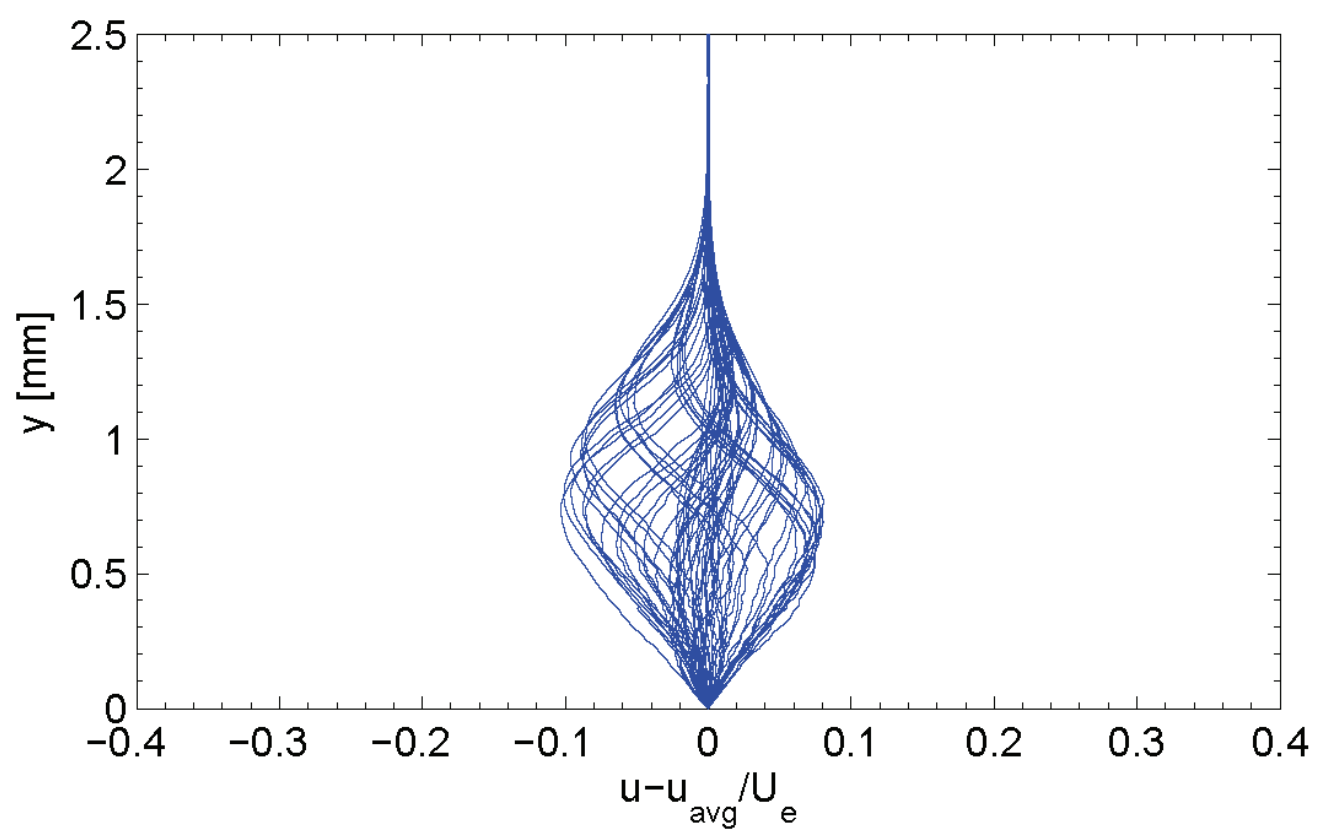

Figure 6-52. Spanwise array of 65 disturbance profiles spanning $64 \mathrm{~mm}$ at $15 \% x / c$. $R e_{c}=2.8 \times 10^{6},[36 \mid 12]$ roughness. 


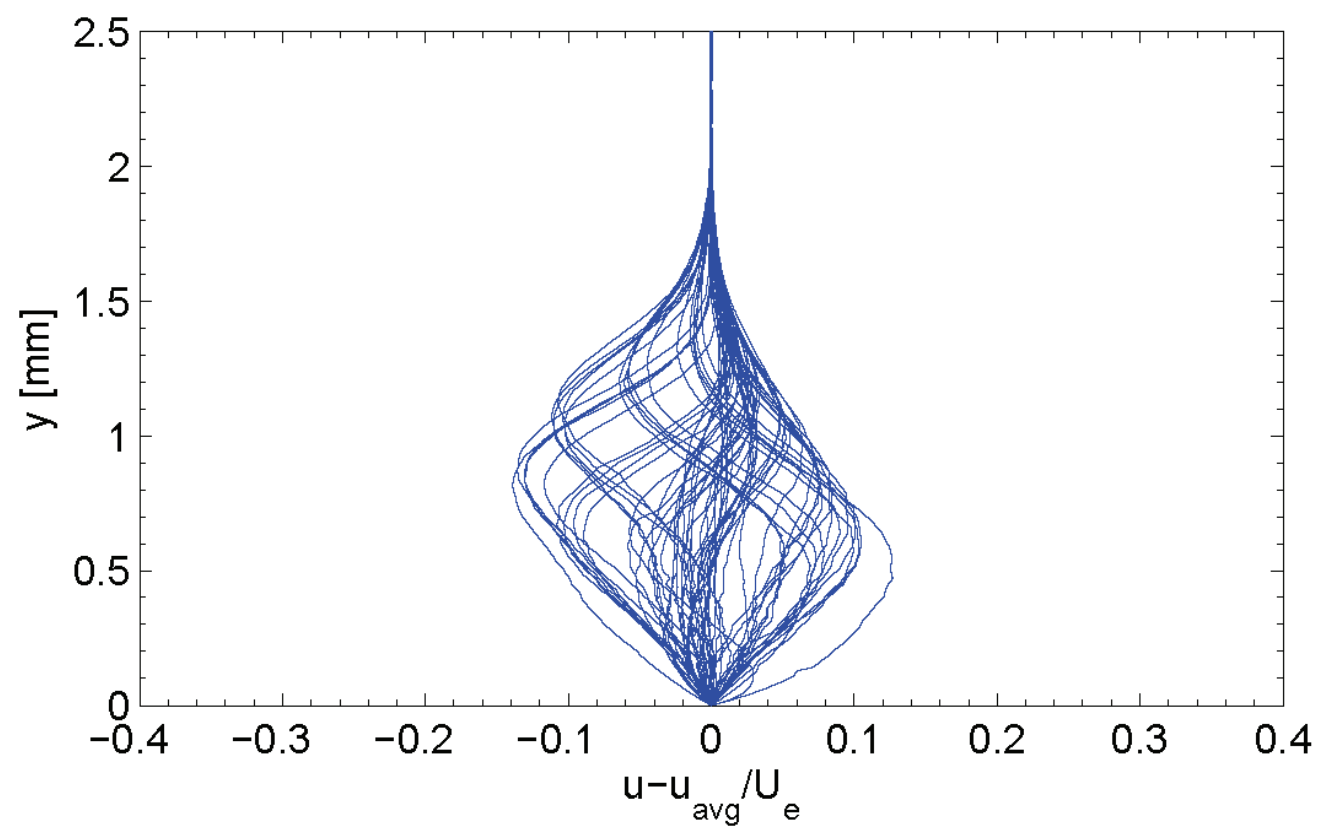

Figure 6-53. Spanwise array of 65 disturbance profiles spanning $64 \mathrm{~mm}$ at $15 \% x / c$. $R e_{c}=2.8 \times 10^{6},[47 \mid 12]$ roughness.

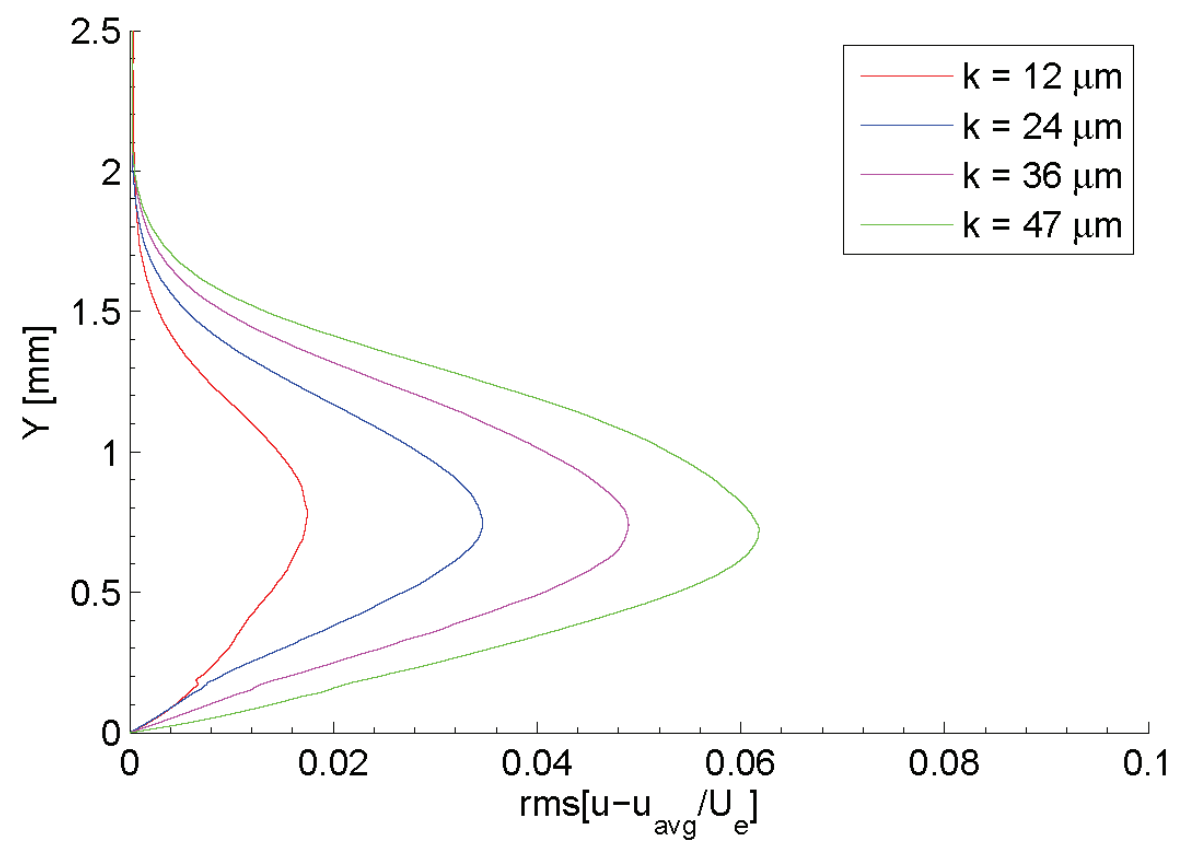

Figure 6-54. Stationary crossflow mode shape at $15 \% x / c . R e_{c}=2.8 \times 10^{6},[k \mid 12]$ roughness. 


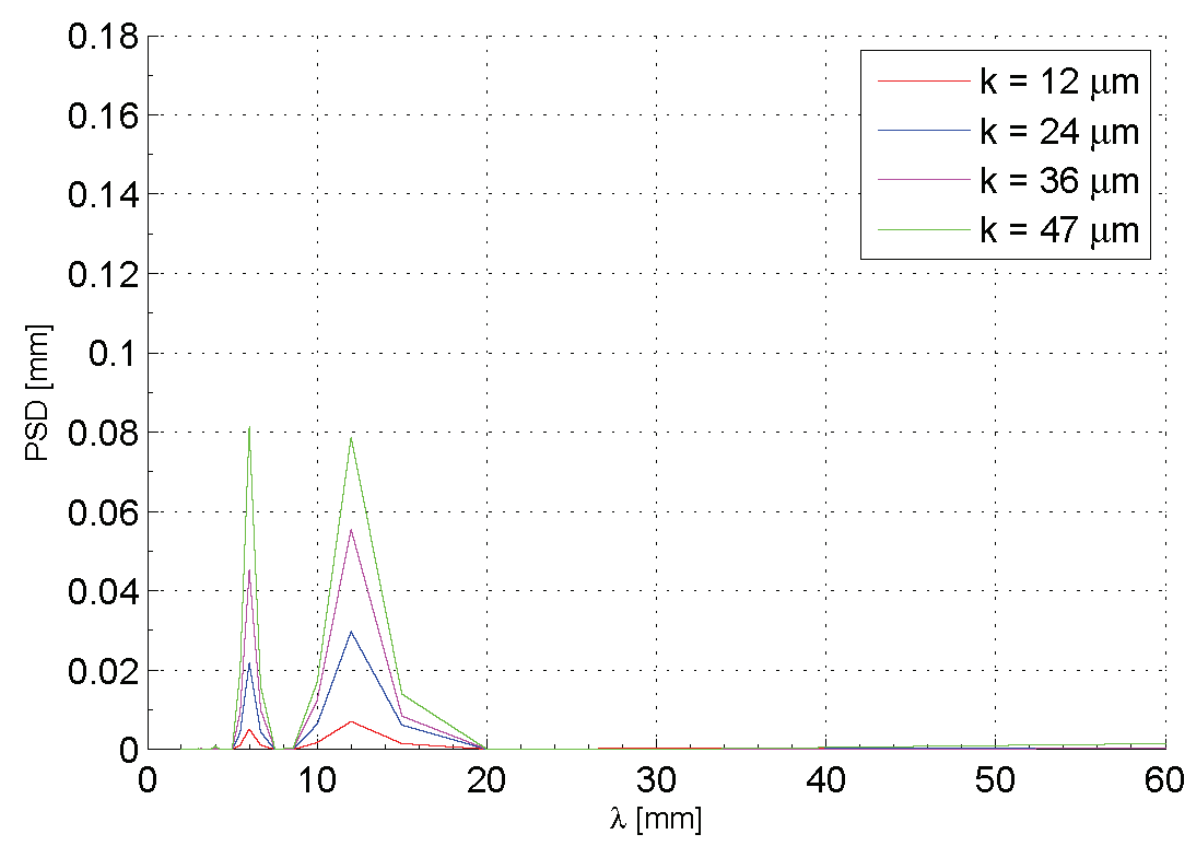

Figure 6-55. Power spectral density of spanwise hotwire scan at $15 \% x / c$. $\operatorname{Re}_{c}=2.8 \times 10^{6},[k \mid 12]$ roughness.

$6.3 R e_{c}=3.2 \times 10^{6}$

\subsubsection{Naphthalene Flow Visualization}

NFV was not completed at $R e_{c}=3.2 \times 10^{6}$ for each new appliqué roughness layer, except for $k=36 \mu \mathrm{m}$. The DRE are still spaced at $12 \mathrm{~mm}$, similar to the other Reynolds numbers. However, in this instance, flow visualization images show the presence of a $6 \mathrm{~mm}$ wave between $20 \%$ and $30 \% x / c$. The $6 \mathrm{~mm}$ spacing fades away by $30 \% x / c$, leaving only the critical $12 \mathrm{~mm}$ wave. Figure 6-56 shows a close-up of this phenomenon. A spacing smaller than the forcing wavelength was never observed in any instance of naphthalene flow visualization at ASU. These wavenumber harmonics have however, been detected in hotwire measurements by Saric \& Yeates (1984). More discussion on 
these results is provided in Sections 8 and 9. The presence of the $6 \mathrm{~mm}$ wave in Figure 6-56 is the result of a strong growth rate compared to the $12 \mathrm{~mm}$ under these flow conditions at early chord locations. By $25 \% x / c$, the $6 \mathrm{~mm}$ wave decays, while the $12 \mathrm{~mm}$ continues to grow. Extensive $6 \mathrm{~mm}$ streaking was not observed at $R e_{c}=2.4 \times 10^{6}$

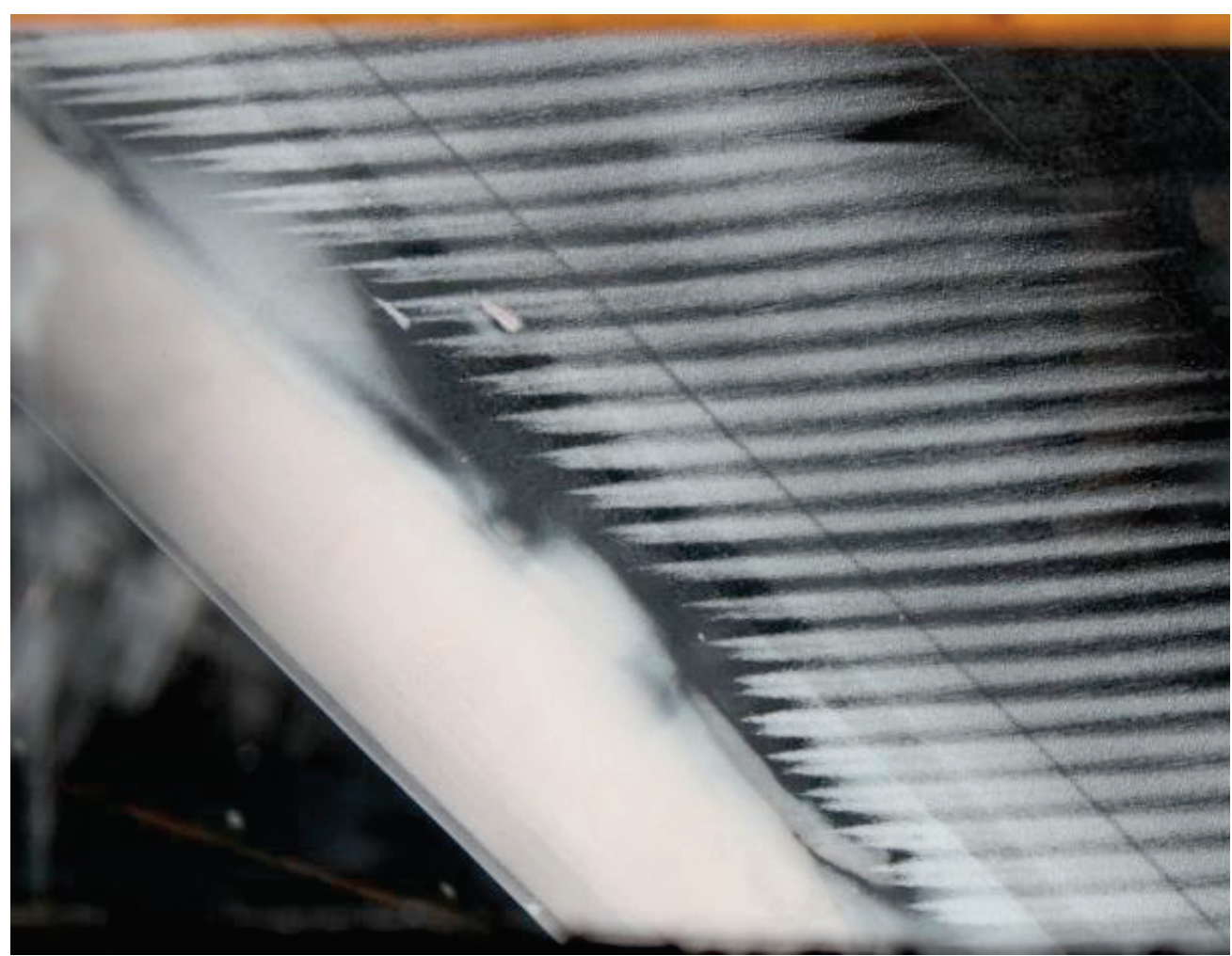

Figure 6-56. Close-up of naphthalene flow visualization for $\operatorname{Re}_{c}=3.2 \times 10^{6},[36 \mid 12]$ roughness.

\subsubsection{Hotwire Measurements, $10 \% x / c$}

As in the previous two cases, data for only a single roughness configuration at $10 \% x / c$ is presented. Figures 6-57 - 6-60 show the boundary-layer profiles, disturbance shape, streamwise velocity contours and mode shape respectively. Both a $6 \mathrm{~mm}$ and 
$12 \mathrm{~mm}$ periodicity are present in the streamwise velocity contours. Given both the $6 \mathrm{~mm}$ wave growth rate at $10 \% x / \mathrm{c}$ and observation of a $6 \mathrm{~mm}$ streak in the naphthalene image, the presence of the $6 \mathrm{~mm}$ periodicity in the contour plots is not unexpected. The maximum disturbance amplitude for this configuration is 0.0383 . Figure 6-61 shows the spatial decomposition of the total disturbance signal and Table 6-7 shows the disturbance amplitude ratio for $10 \% x / c$. For comparison purposes, the results from $R e_{c}=2.4 \times 10^{6}$ and $R e_{c}=2.8 \times 10^{6}$ at $10 \% x / c$ are also included in the table. As Reynolds number increases, the amplitude of the $6 \mathrm{~mm}$ wave relative to the $12 \mathrm{~mm}$ wave also increases. At $R e_{c}=3.2 \times 10^{6}$, the disturbance amplitude of the $6 \mathrm{~mm}$ mode is larger than the forced $12 \mathrm{~mm}$ wave. This is consistent with both $N$-factor calculations and the naphthalene flow visualization discussed earlier.

Table 6-7. Stationary crossflow disturbance amplitude ratios at $10 \% x / c,[47 \mid 12]$ roughness.

\begin{tabular}{|c|c|}
\hline$R e_{c}$ & {$\left[\left(u-u_{\text {avg }}\right) / U_{e}\right]_{\mathrm{rms}, \lambda=12} /\left[\left(u-u_{\text {avg }}\right) / U_{e}\right]_{\mathrm{rms}, \lambda=6}$} \\
\hline $2.4 \times 10^{6}$ & 1.73 \\
\hline $2.8 \times 10^{6}$ & 1.09 \\
\hline $3.2 \times 10^{6}$ & 0.84 \\
\hline
\end{tabular}




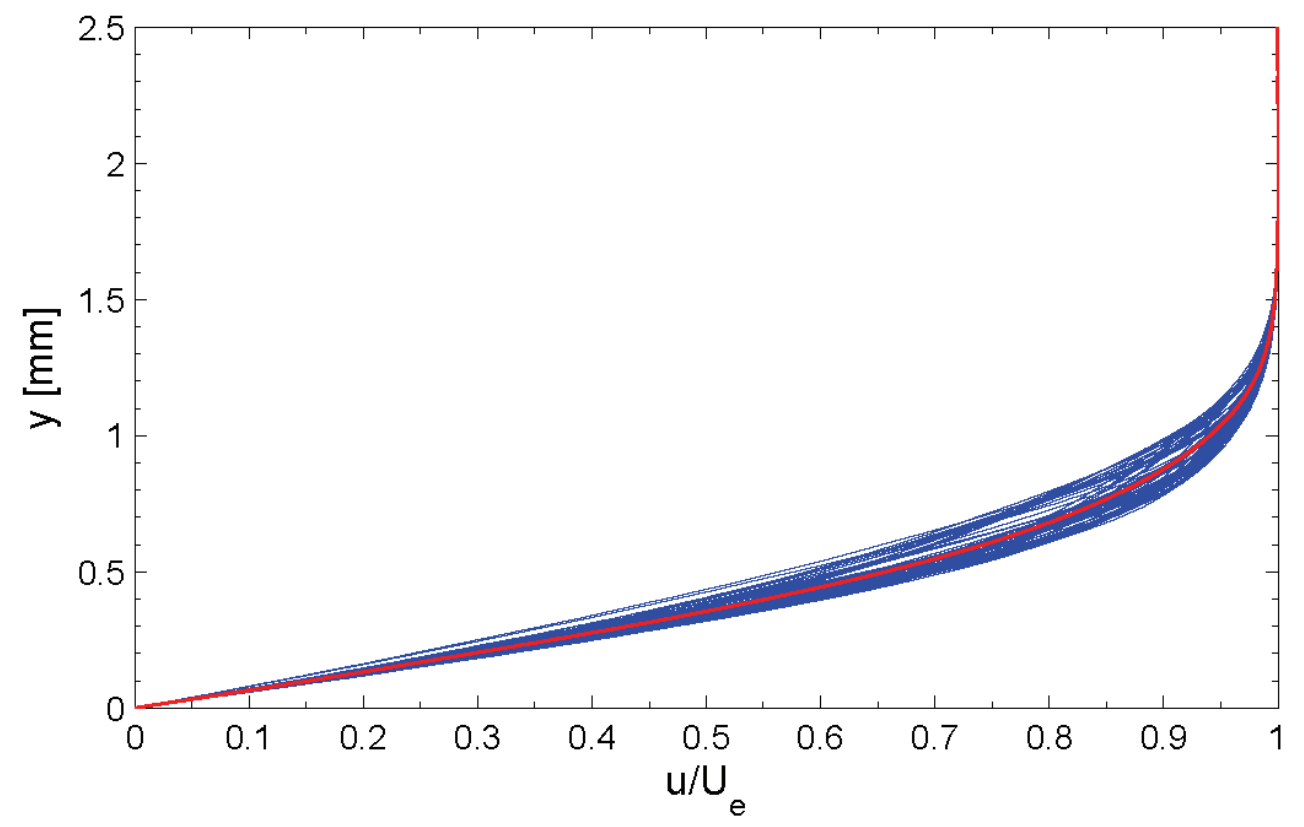

Figure 6-57. Spanwise array of 65 mean-flow boundary-layer profiles spanning $64 \mathrm{~mm}$ at $10 \% x / c . R e_{c}=3.2 \times 10^{6},[47 \mid 12]$ roughness. Mean profile shown in red.

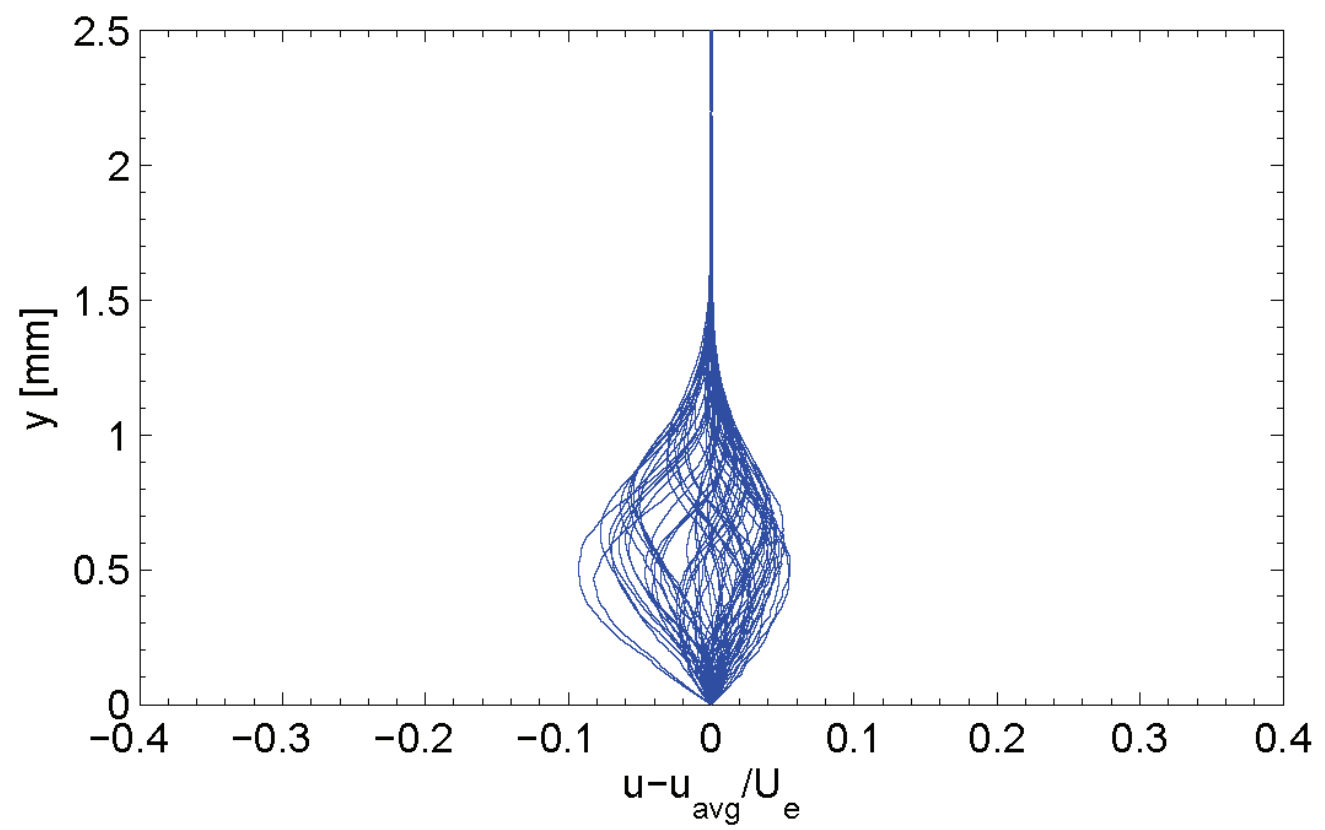

Figure 6-58. Spanwise array of 65 disturbance profiles spanning $64 \mathrm{~mm}$ at $10 \% x / c$. $R e_{c}=3.2 \times 10^{6},[47 \mid 12]$ roughness. 


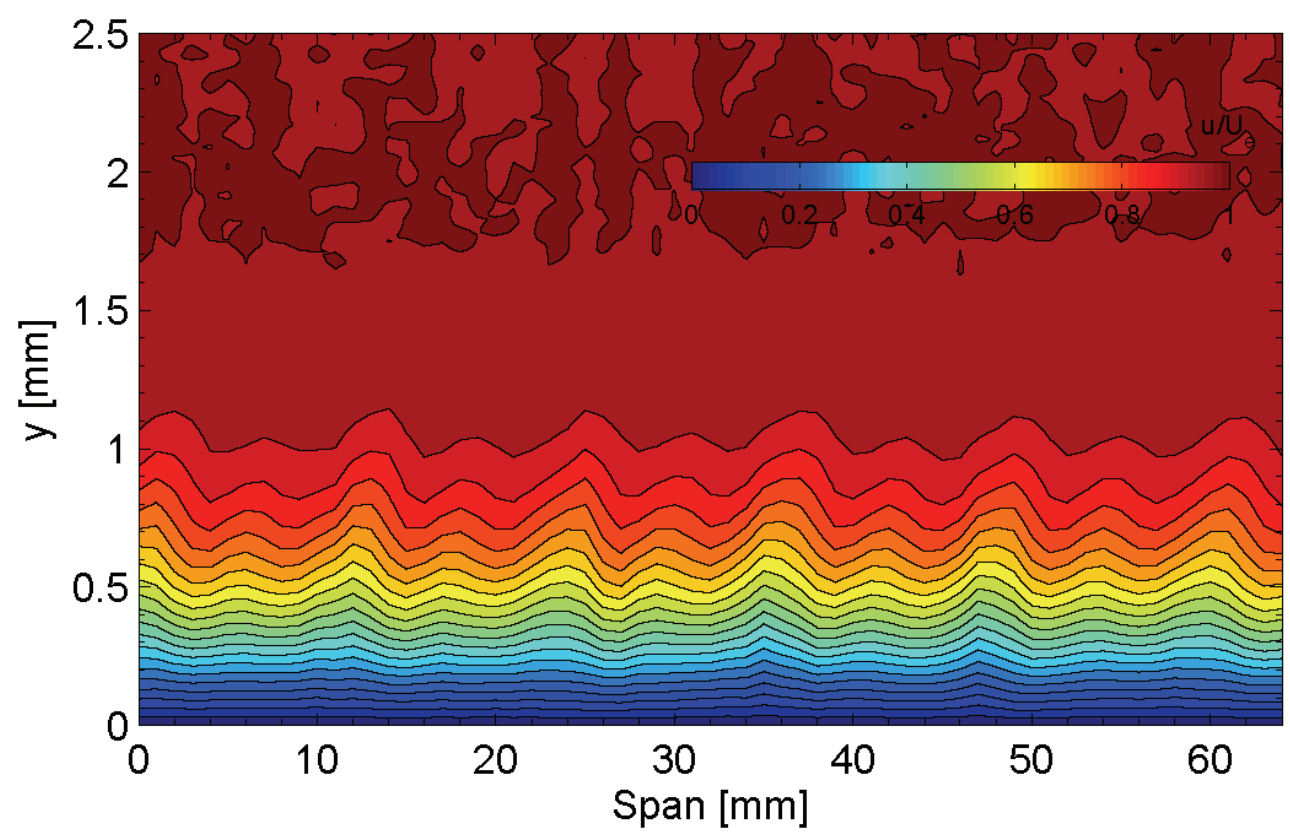

Figure 6-59. Streamwise velocity contours at $10 \% x / c . R e_{c}=3.2 \times 10^{6},[47 \mid 12]$ roughness.

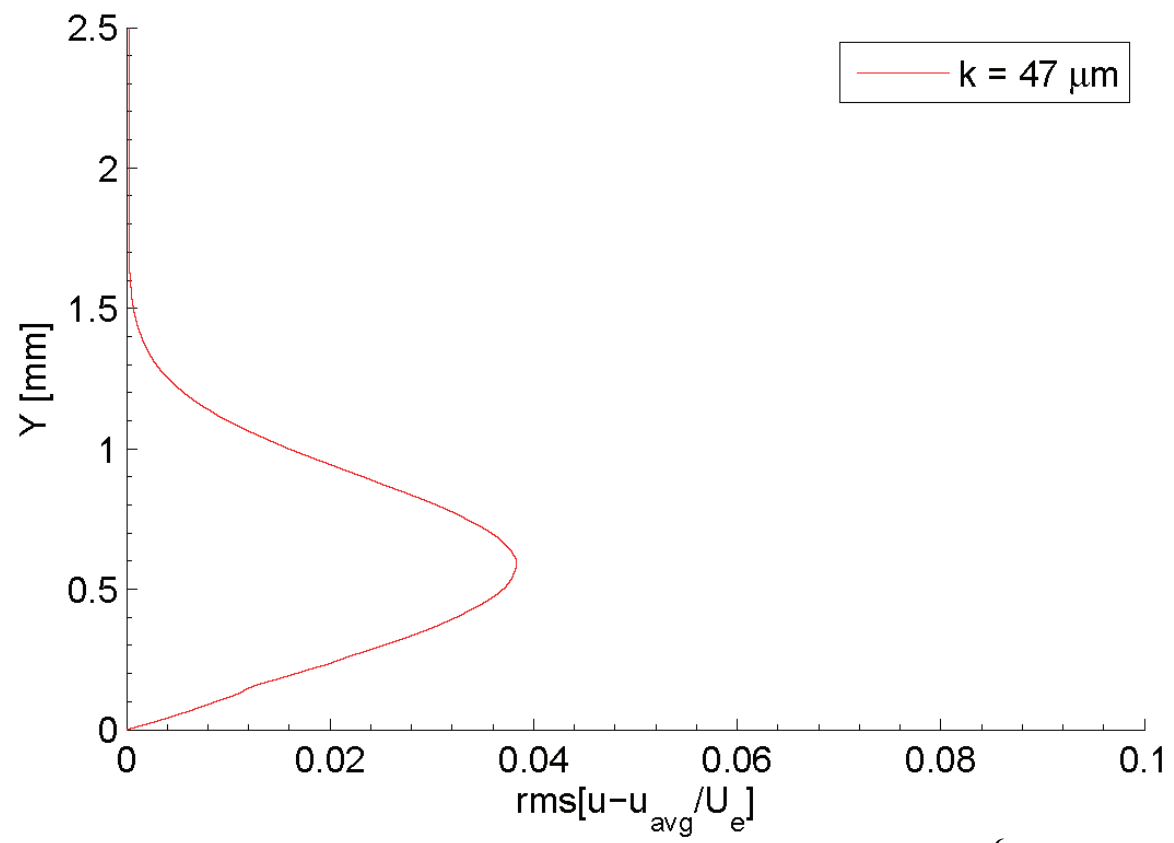

Figure 6-60. Stationary crossflow mode shape at $10 \% x / c . R e_{c}=3.2 \times 10^{6},[47 \mid 12]$ roughness. 


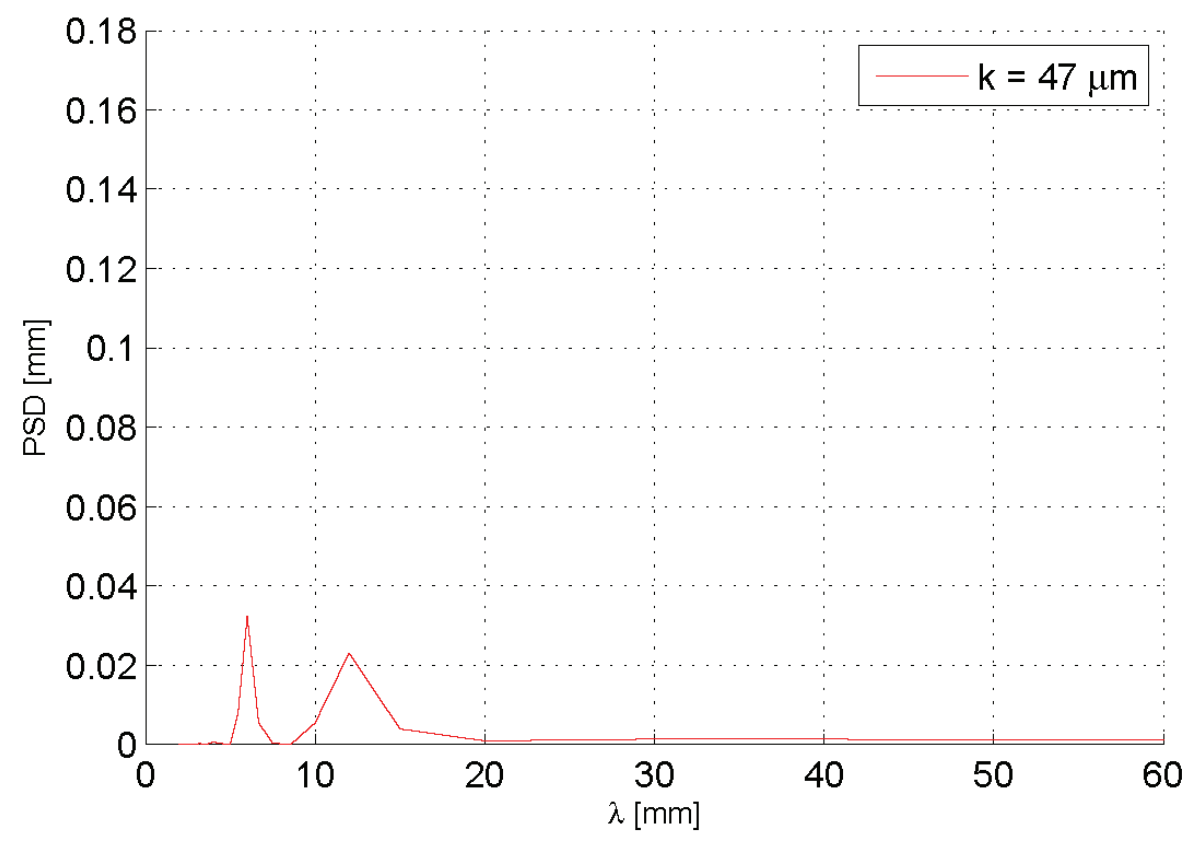

Figure 6-61. Power spectral density of spanwise hotwire scan at $10 \% x / c$. $R e_{c}=3.2 \times 10^{6},[47 \mid 12]$ roughness.

\subsubsection{Hotwire Measurements, 15\% x/c}

Measurements at 15\%-chord included roughness height configurations of $12 \mu \mathrm{m}$, $24 \mu \mathrm{m}, 36 \mu \mathrm{m}$ and $47 \mu \mathrm{m}$. Figures 6-62 - 6-65, 6-66 - 6-69, 6-70 - 6-73 and 6-74 show the boundary-layer velocity profiles, streamwise velocity contours, disturbance shape and mode shape respectively for each roughness height. Similar to $R e_{c}=2.8 \times 10^{6}$, the $6 \mathrm{~mm}$ periodicity is observed in addition to the $12 \mathrm{~mm}$ distortion. Even at the higher Reynolds number, the rollover, characteristic of nonlinear stability is not present in the streamwise velocity contour plots. However, the mode shapes for each roughness height indicate that nonlinear stability occurs once the roughness height has reached $36 \mu \mathrm{m}$, leaving the last two scans outside the scope of the receptivity analysis. Table 6-8 shows 
the maximum and normalized disturbance amplitude as roughness height increases. It is interesting to note that as strong nonlinear mode interactions start to occur, the initial disturbance amplitude rapidly departs from the almost linear relationship observed in the other cases.

Table 6-8. Maximum and normalized stationary crossflow disturbance amplitudes at $15 \% x / c, R e_{c}=3.2 \times 10^{6},[k \mid 12]$ roughness.

\begin{tabular}{|c|c|c|}
\hline$k[\mu \mathrm{m}]$ & {$\left[\left(u-u_{\text {avg }}\right) / U_{e}\right]_{\mathrm{rms}, \max }$} & {$\left[\left(u-u_{\text {avg }}\right) / \mathrm{U}_{e}\right]_{\mathrm{rms}, k / k o}$} \\
\hline 12 & 0.0307 & 1.00 \\
\hline 24 & 0.0577 & 0.94 \\
\hline 36 & 0.0756 & 0.82 \\
\hline 47 & 0.0873 & 0.71 \\
\hline
\end{tabular}

Figure 6-75 shows the PSD for the four roughness heights. At $15 \% x / c$, the $6 \mathrm{~mm}$ wave is still stronger than the $12 \mathrm{~mm}$. At downstream locations, the $12 \mathrm{~mm}$ wave would be expected to overtake the $6 \mathrm{~mm}$ mode. Table 6-9 shows the disturbance amplitude ratio for the $12 \mathrm{~mm}$ and $6 \mathrm{~mm}$ waves.

Table 6-9. Stationary crossflow disturbance amplitude ratios at $15 \% x / c, R_{c}=3.2 \times 10^{6},[k \mid 12]$ roughness.

\begin{tabular}{|c|c|}
\hline$k[\mu \mathrm{m}]$ & {$\left[\left(u-u_{\text {avg }}\right) / U_{e}\right]_{\mathrm{rms}, \lambda=12} /\left[\left(u-u_{\text {avg }}\right) / U_{e}\right]_{\mathrm{rms}, \lambda=6}$} \\
\hline 12 & 0.74 \\
\hline 24 & 0.55 \\
\hline 36 & 0.59 \\
\hline 47 & 0.49 \\
\hline
\end{tabular}




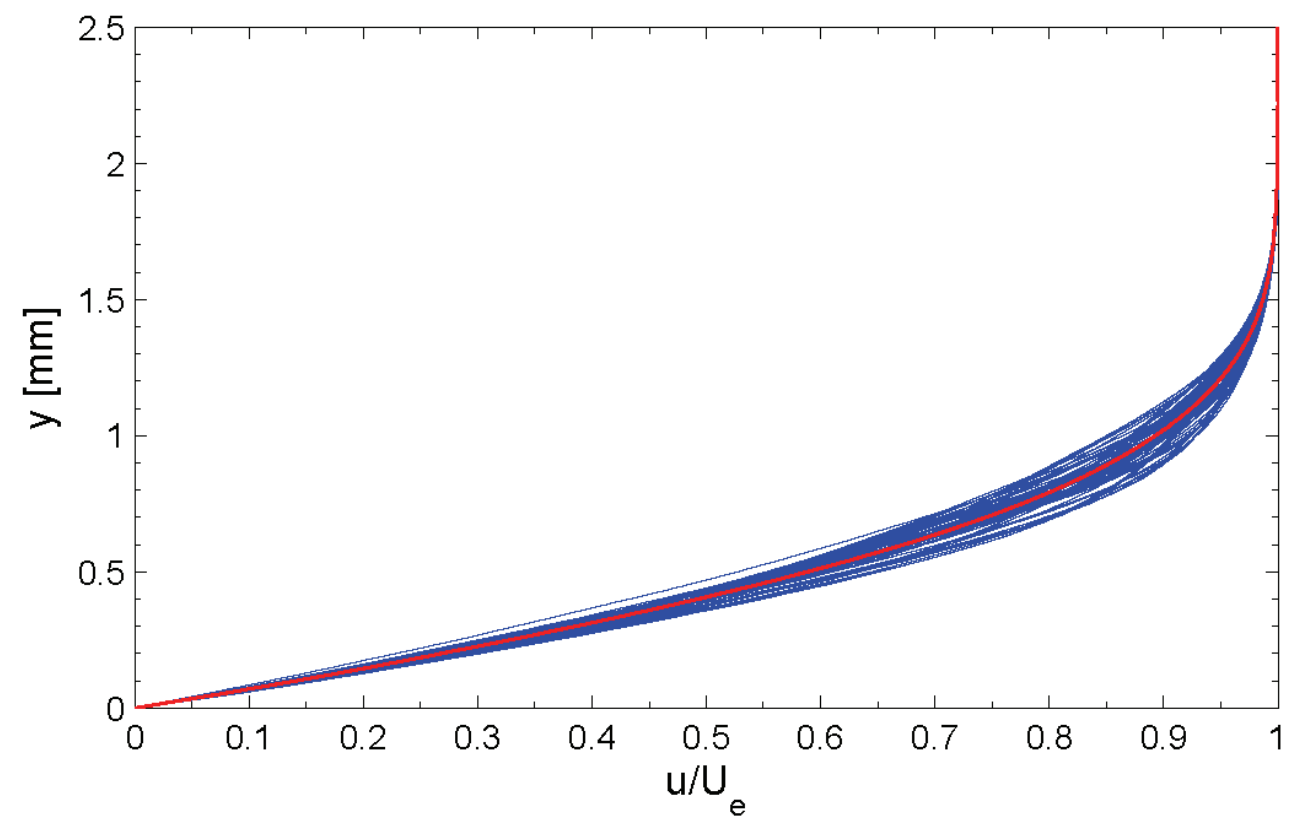

Figure 6-62. Spanwise array of 65 mean-flow boundary-layer profiles spanning $64 \mathrm{~mm}$ at $15 \% x / c . R e_{c}=3.2 \times 10^{6},[12 \mid 12]$ roughness. Mean profile shown in red.

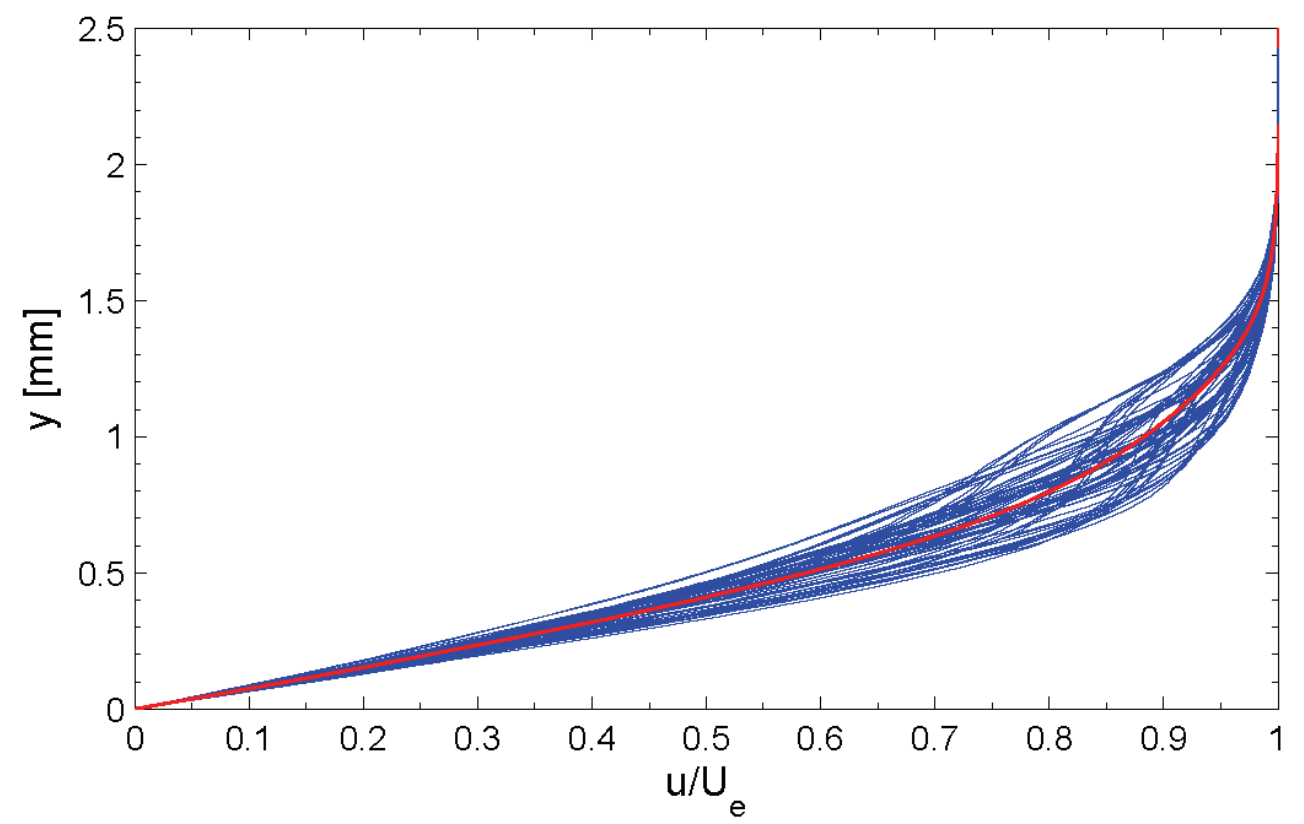

Figure 6-63. Spanwise array of 65 mean-flow boundary-layer profiles spanning $64 \mathrm{~mm}$ at $15 \% x / c . R e_{c}=3.2 \times 10^{6},[24 \mid 12]$ roughness. Mean profile shown in red. 


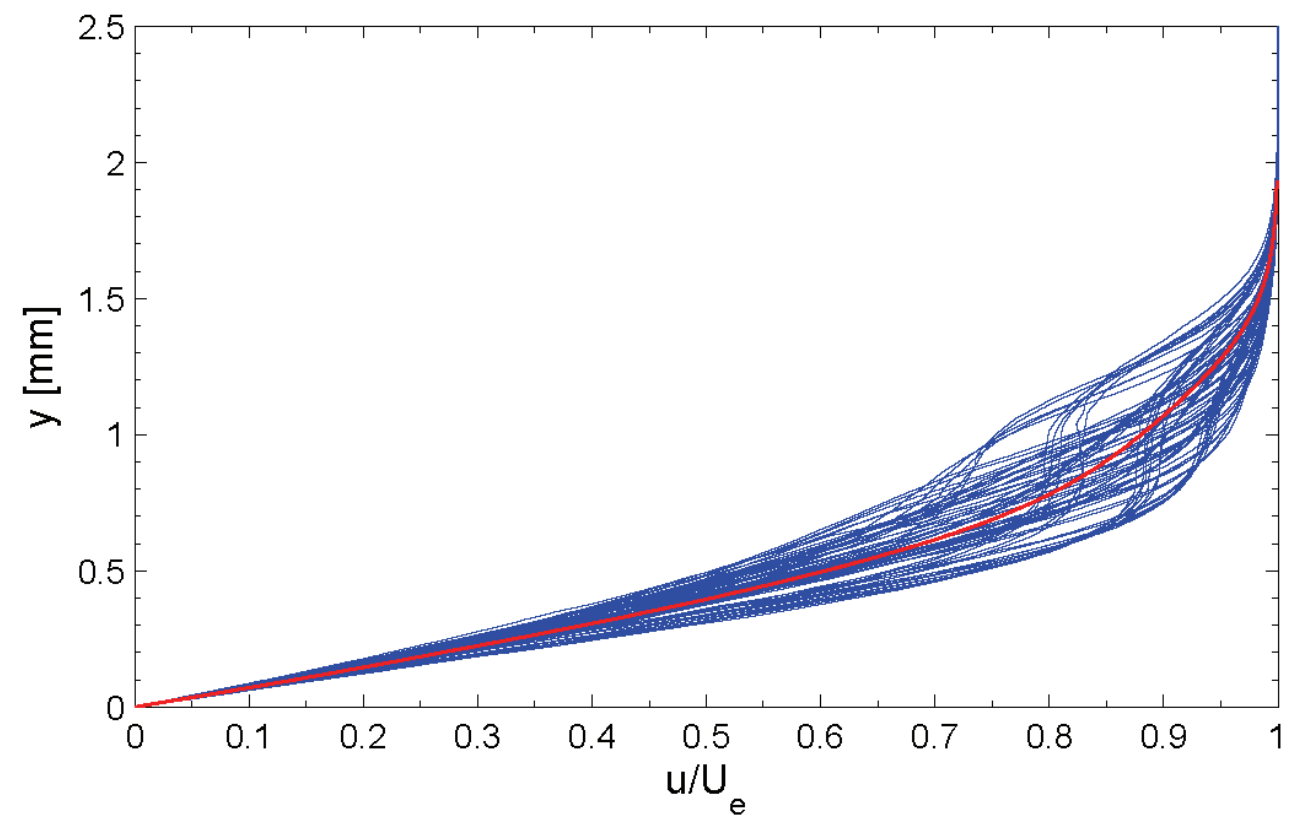

Figure 6-64. Spanwise array of 65 mean-flow boundary-layer profiles spanning $64 \mathrm{~mm}$ at $15 \% x / c . R e_{c}=3.2 \times 10^{6},[36 \mid 12]$ roughness. Mean profile shown in red.

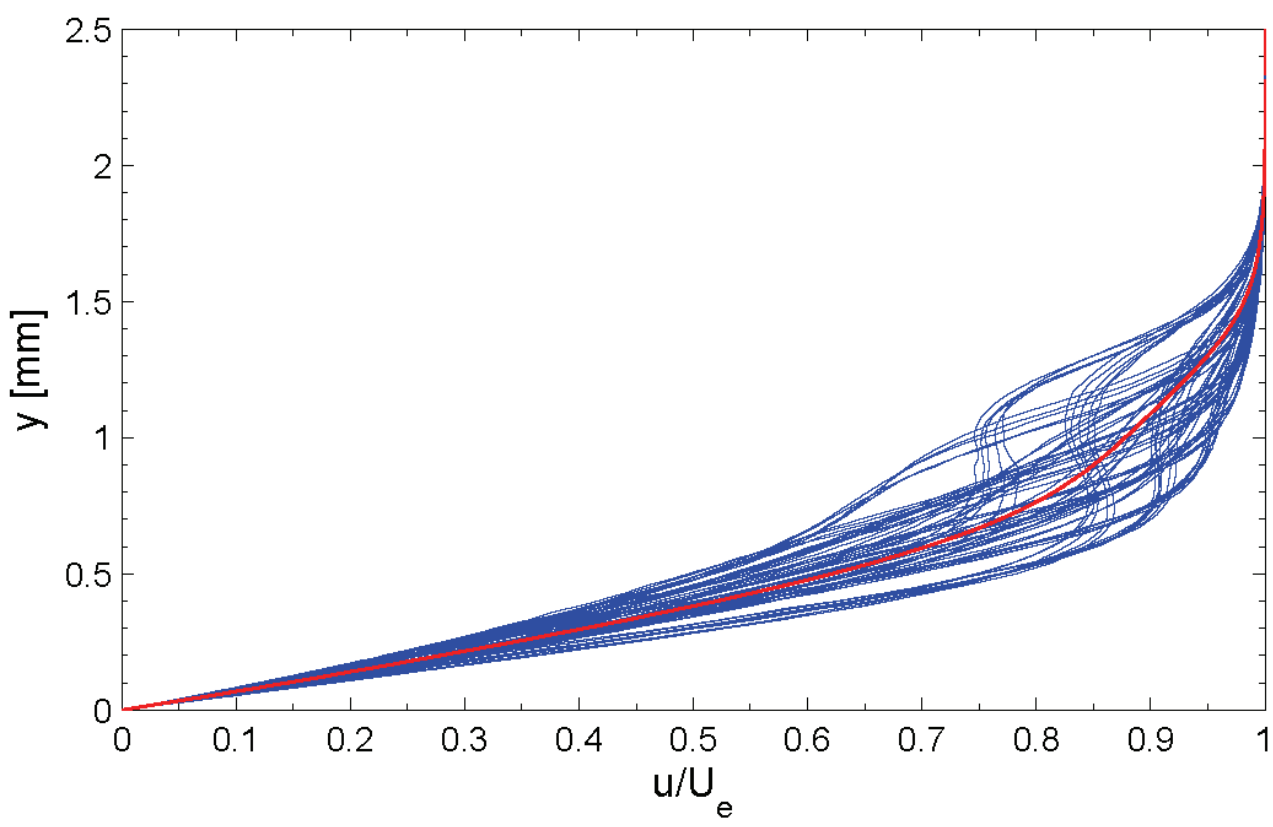

Figure 6-65. Spanwise array of 65 mean-flow boundary-layer profiles spanning $64 \mathrm{~mm}$ at $15 \% x / c . R e_{c}=3.2 \times 10^{6},[47 \mid 12]$ roughness. Mean profile shown in red. 


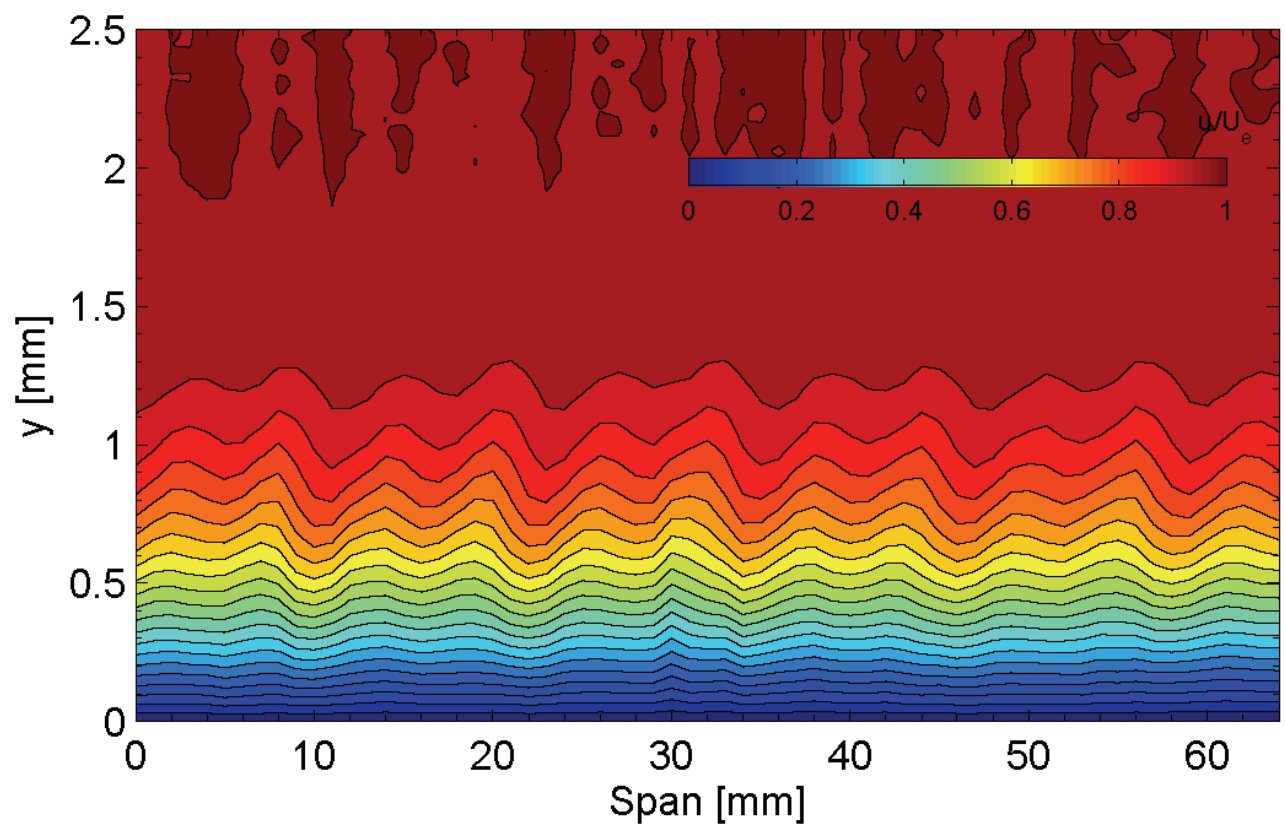

Figure 6-66. Streamwise velocity contours at $15 \% x / c . R_{c}=3.2 \times 10^{6},[12 \mid 12]$ roughness.

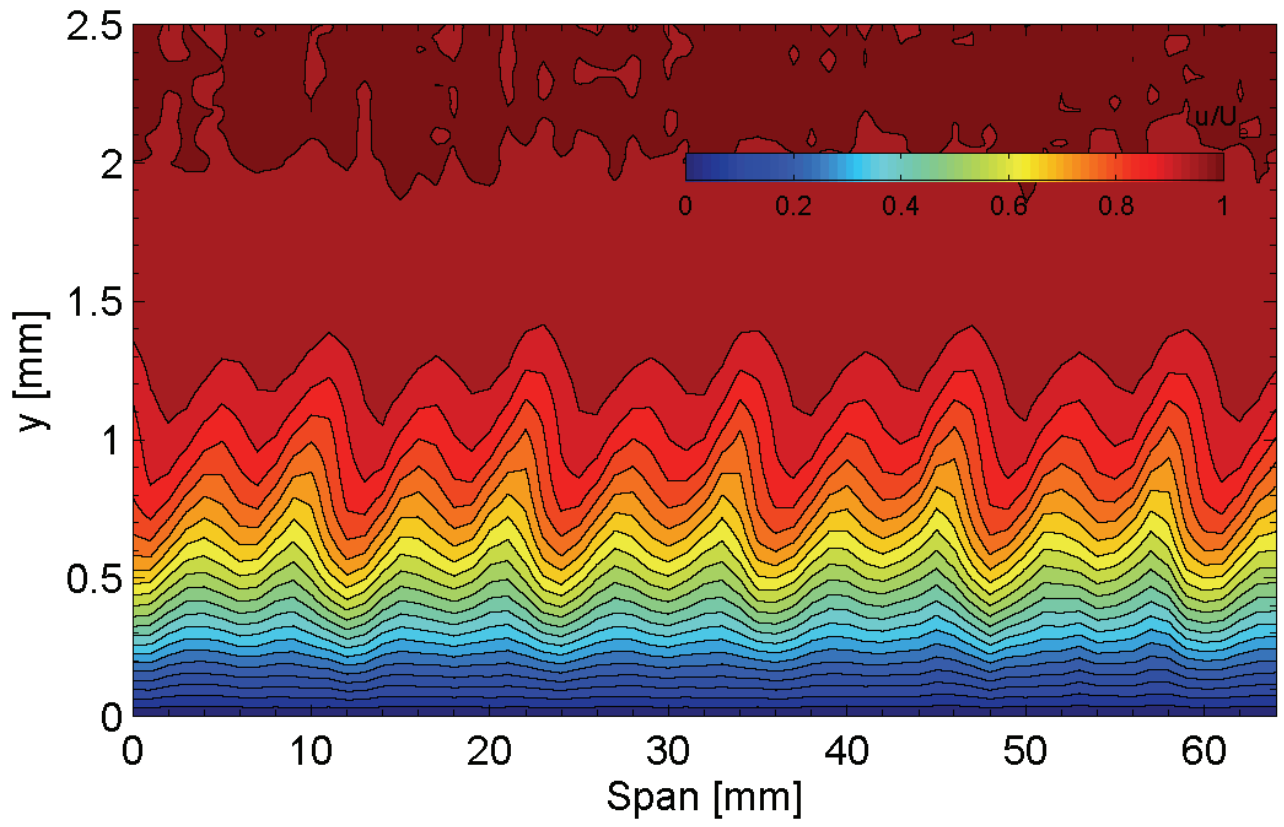

Figure 6-67. Streamwise velocity contours at $15 \% x / c . R e_{c}=3.2 \times 10^{6},[24 \mid 12]$ roughness. 


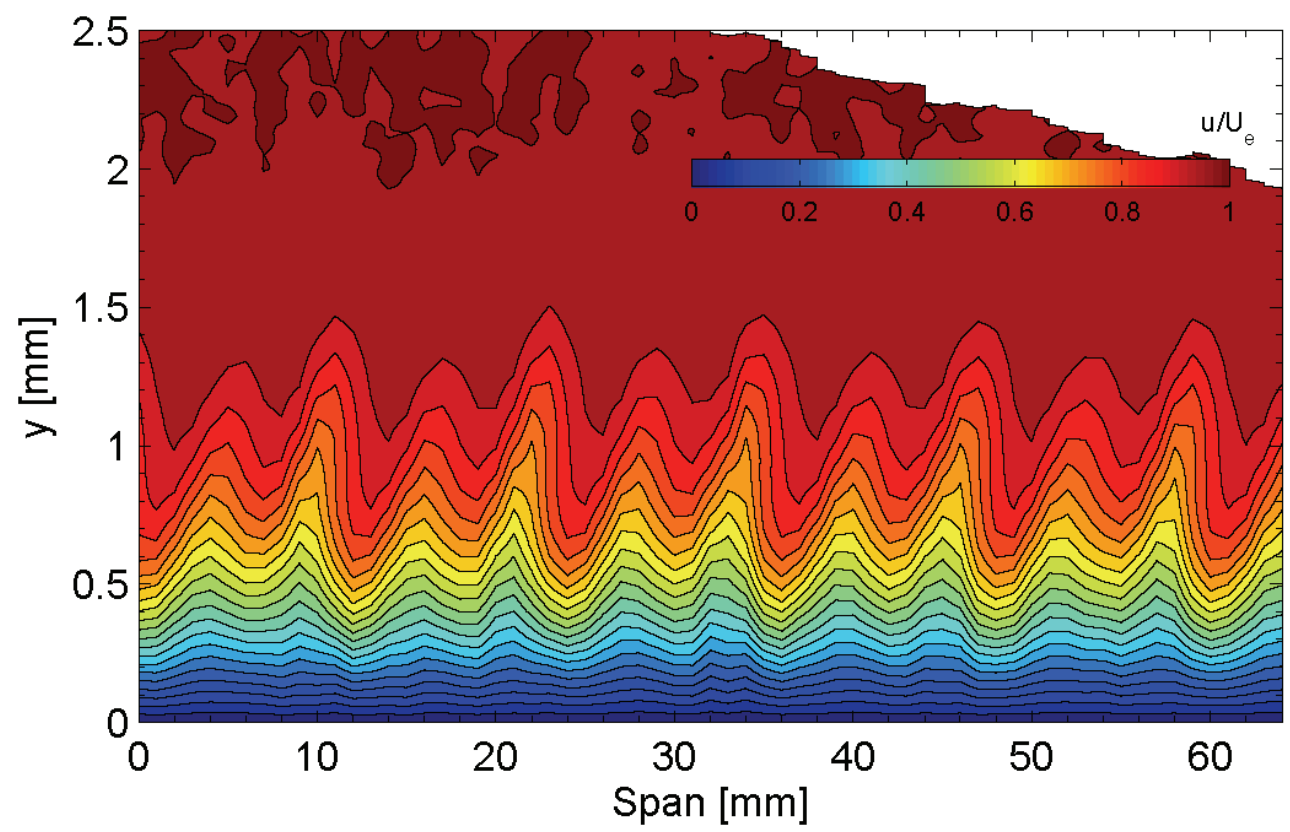

Figure 6-68. Streamwise velocity contours at $15 \% x / c . R e_{c}=3.2 \times 10^{6},[36 \mid 12]$ roughness.

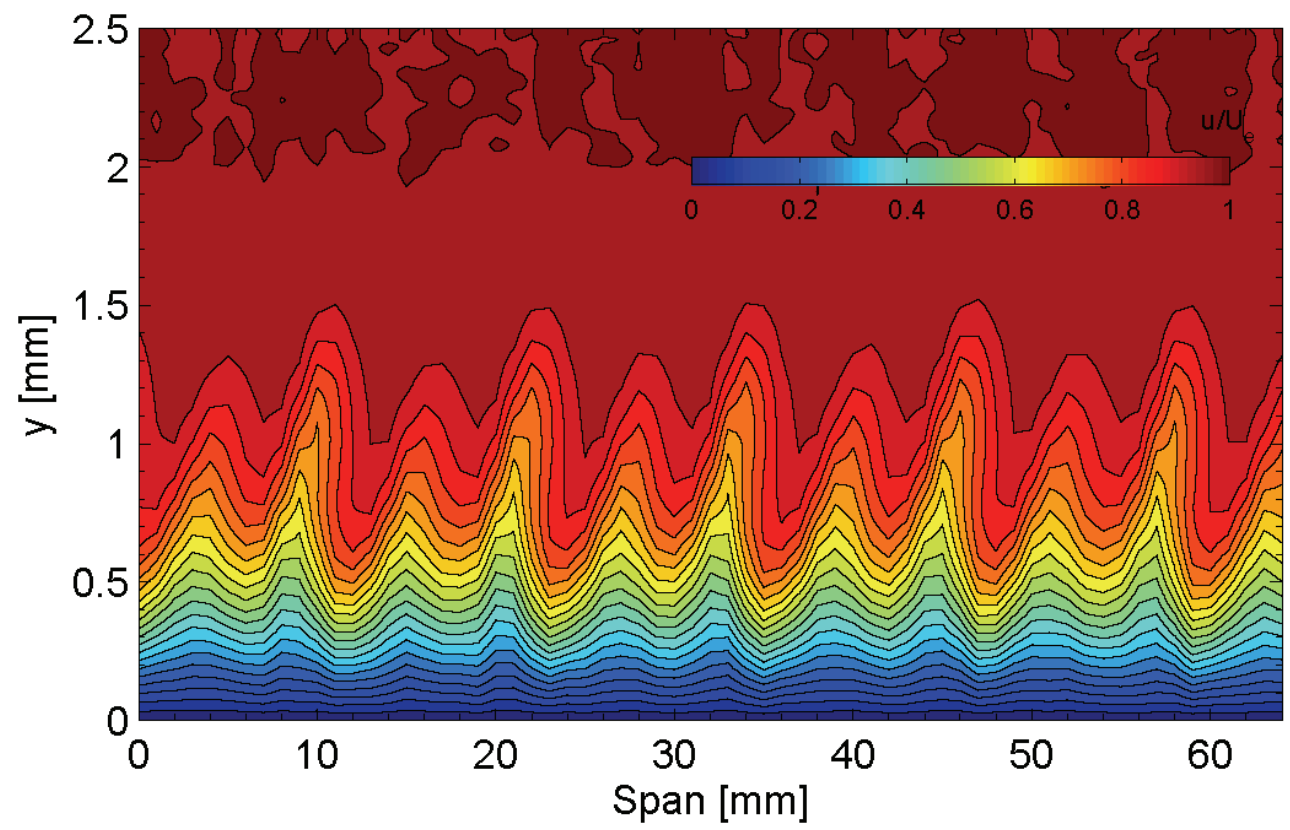

Figure 6-69. Streamwise velocity contours at $15 \% x / c . R e_{c}=3.2 \times 10^{6},[47 \mid 12]$ roughness. 


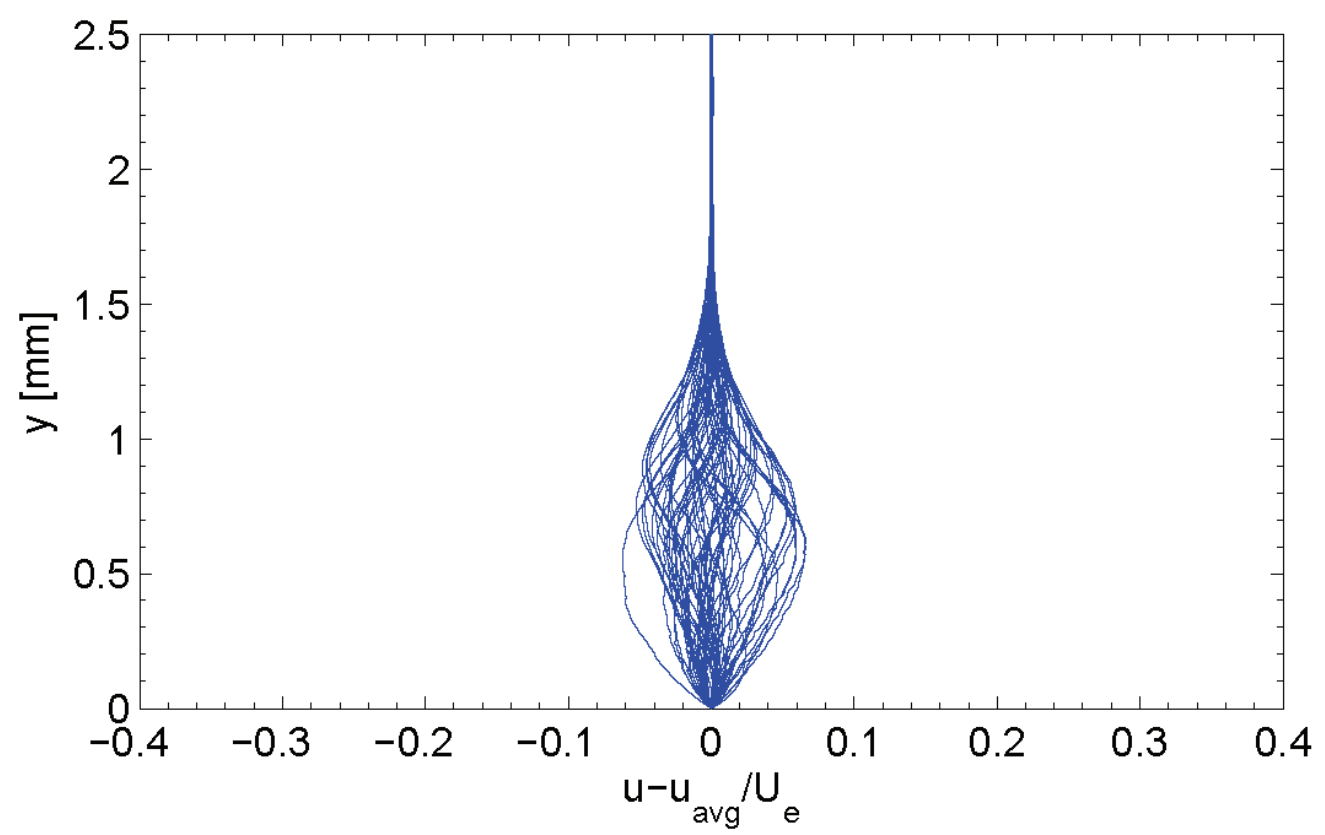

Figure 6-70. Spanwise array of 65 disturbance profiles spanning $64 \mathrm{~mm}$ at $15 \% x / c$. $R e_{c}=3.2 \times 10^{6},[12 \mid 12]$ roughness.

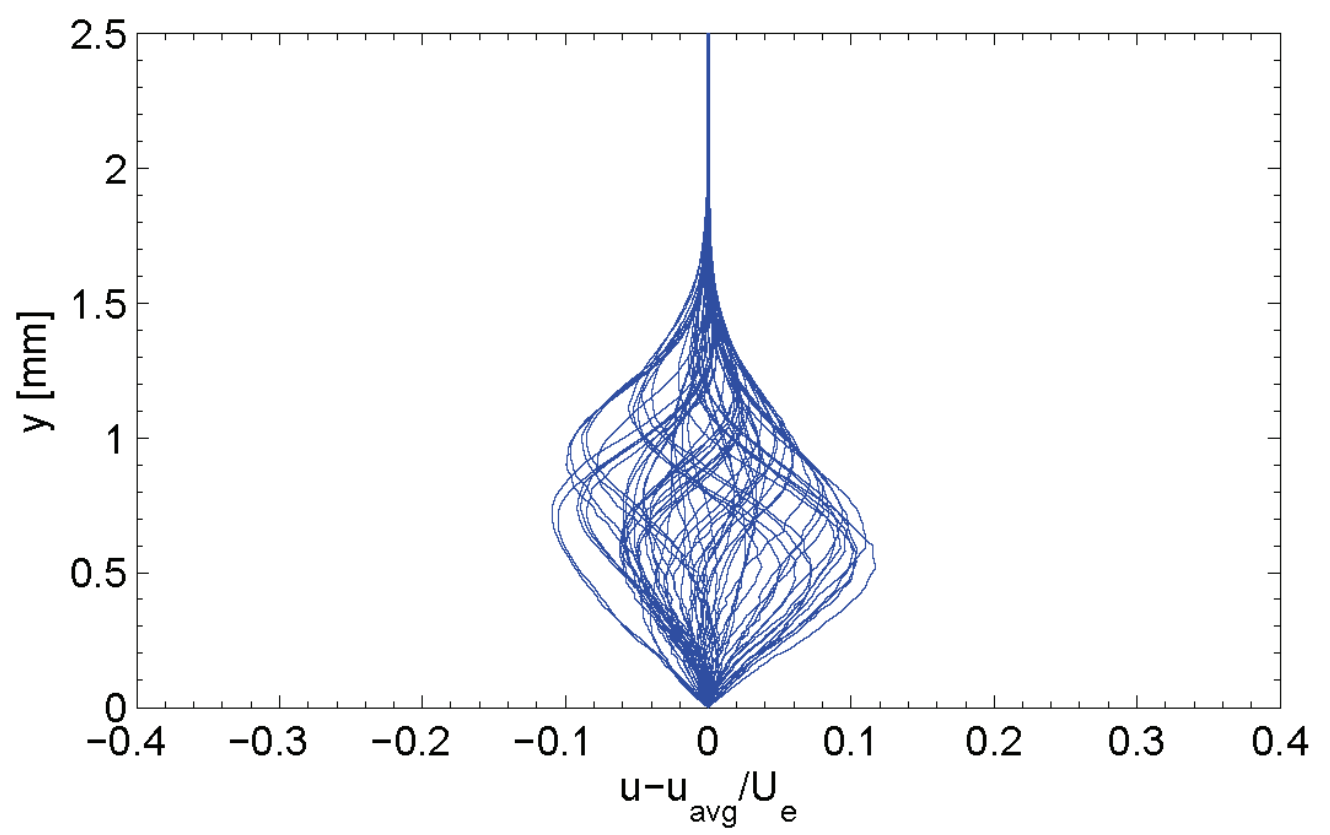

Figure 6-71. Spanwise array of 65 disturbance profiles spanning $64 \mathrm{~mm}$ at $15 \% x / c$. $R e_{c}=3.2 \times 10^{6},[24 \mid 12]$ roughness. 


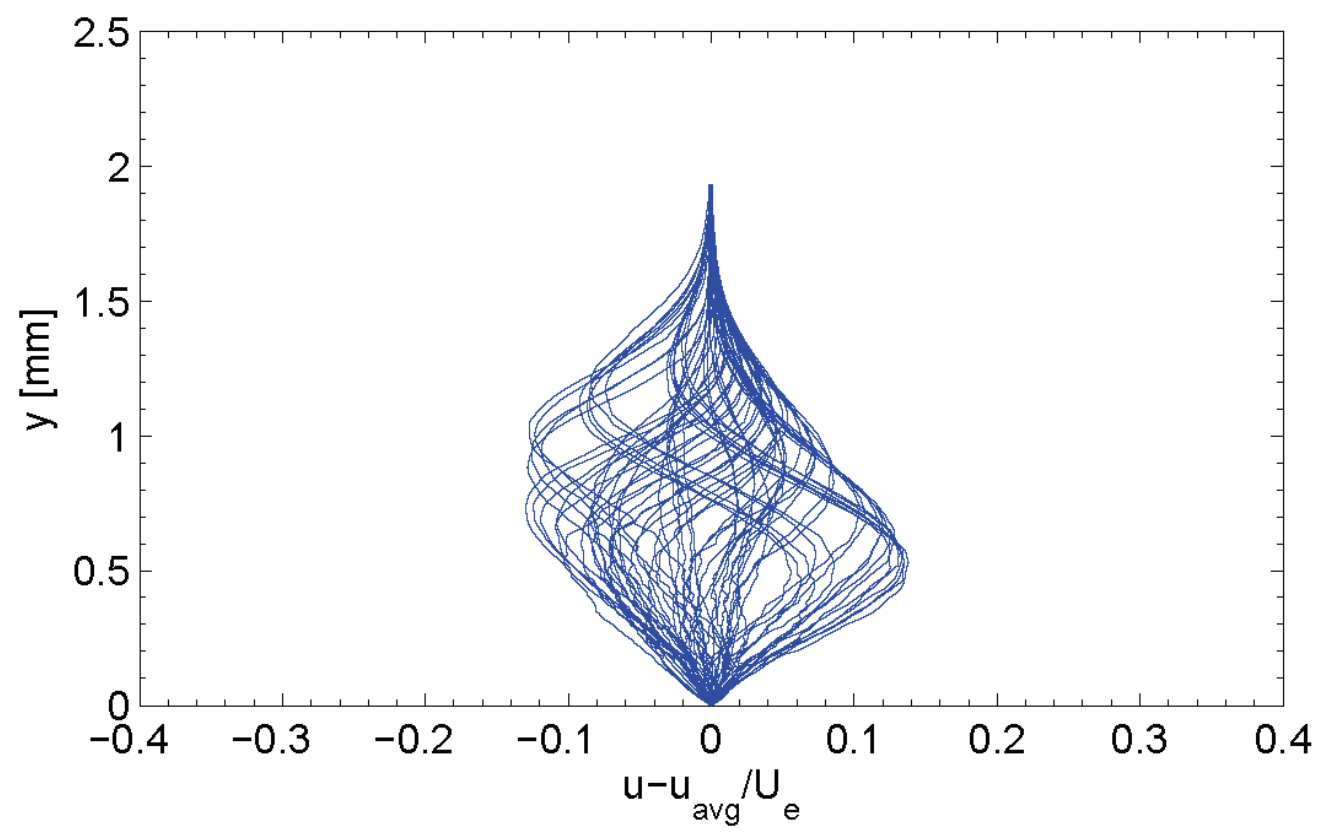

Figure 6-72. Spanwise array of 65 disturbance profiles spanning $64 \mathrm{~mm}$ at $15 \% x / c$. $R e_{c}=3.2 \times 10^{6},[36 \mid 12]$ roughness.

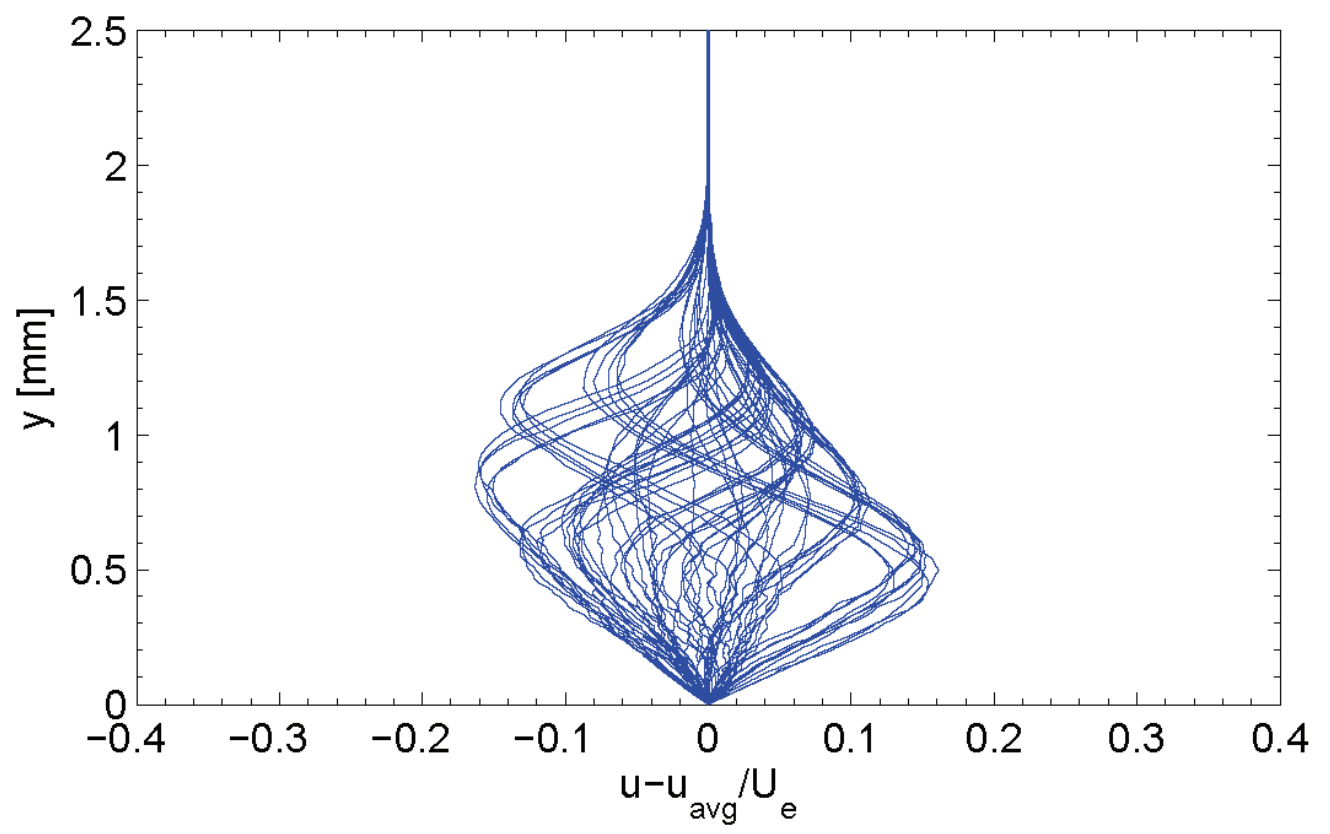

Figure 6-73. Spanwise array of 65 disturbance profiles spanning $64 \mathrm{~mm}$ at $15 \% x / c$. $R e_{c}=3.2 \times 10^{6},[47 \mid 12]$ roughness. 


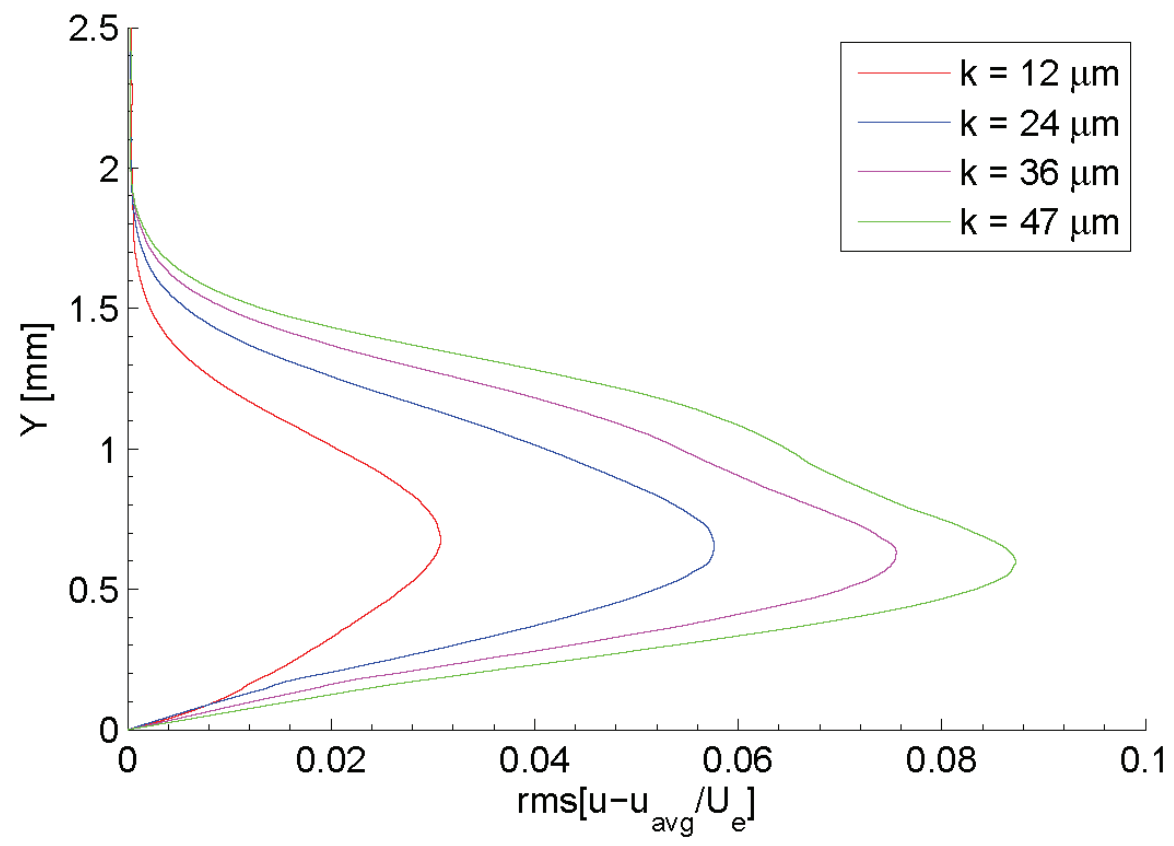

Figure 6-74. Stationary crossflow mode shape at $15 \% x / c . R e_{c}=3.2 \times 10^{6},[k \mid 12]$ roughness.

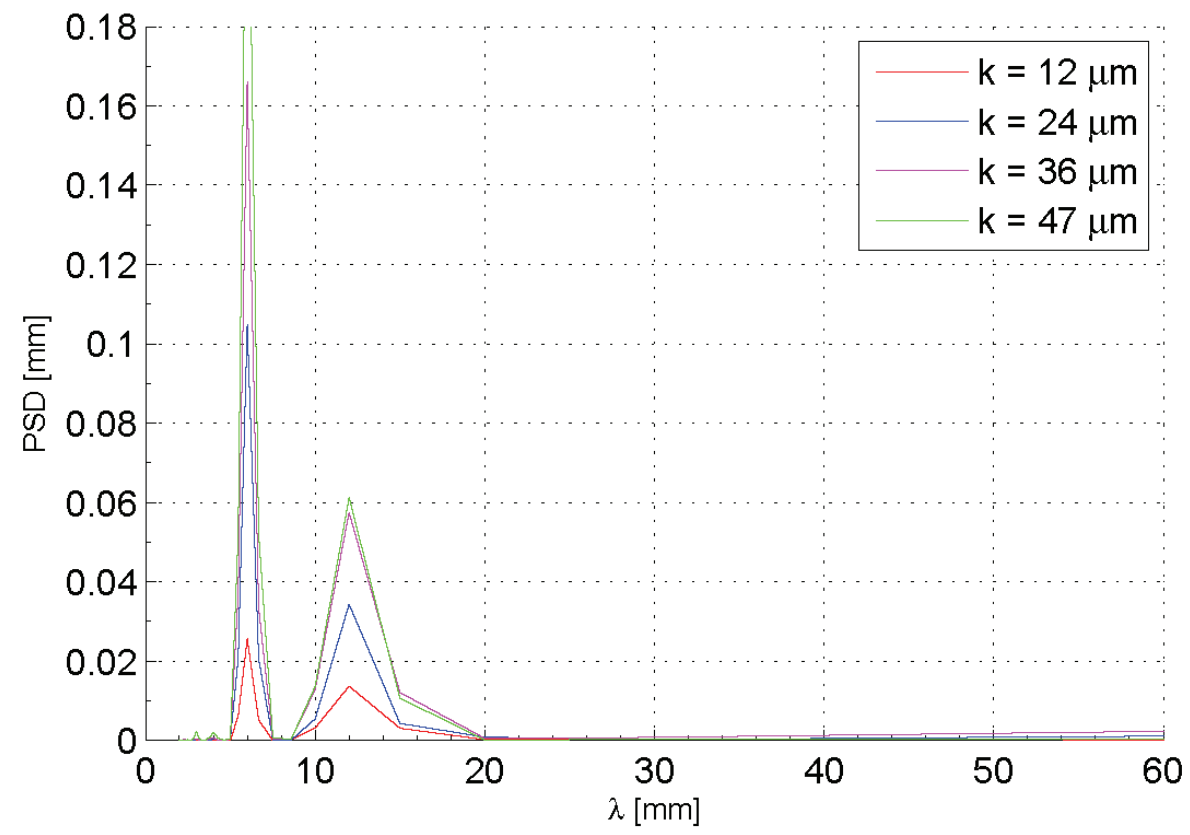

Figure 6-75. Power spectral density of spanwise hotwire scan at $15 \% x / c$. $R e_{c}=3.2 \times 10^{6},[k \mid 12]$ roughness. 


\section{RESULTS- PART III: RECEPTIVITY OF DRE ARRAY SPACED AT THE CONTROL WAVELENGTH}

Control DREs were applied after all measurements with the critically spaced wavelength were complete. Spacing for all data in this section is fixed at $6 \mathrm{~mm}$. The DRE batch for a single-layer application in this configuration is slightly higher than the critical wavelength, with a starting height of $14 \mu \mathrm{m}$ instead of $12 \mu \mathrm{m}$. Again, this is a design feature that is not controlled by the manufacturer. Based on experience with the critical DRE, NFV was completed for all Reynolds numbers. Hotwire scans and NFV were stopped for a given roughness height if the previous data set indicated nonlinear stability activity taking place. For all Reynolds numbers, hotwire scans were only completed at $15 \% x / c$, as this location provided the best results for the critical wavelength.

\section{$7.1 R e_{c}=2.4 \times 10^{6}$}

\subsubsection{Naphthalene Flow Visualization}

NFV for $k=14 \mu \mathrm{m}, 27 \mu \mathrm{m}, 42 \mu \mathrm{m}$ and $56 \mu \mathrm{m}$ at $R e_{c}=2.4 \times 10^{6}$ is shown in Figure 7-1. Recall from Figure 5-16 that natural transition for this condition is past the pressure minimum near $80 \% x / c$. Subcritical forcing is designed to grow faster than the naturally most unstable wavelength, modify the mean flow such that the critical wavelength can no longer grow and then decay before the control mode grows large enough to cause 
transition itself. For the first and second layers of DRE $(k=14 \mu \mathrm{m}$ and $27 \mu \mathrm{m}$ respectively), transition occurs between $70 \%$ and $75 \% x / c$, slightly forward of the natural transition location. A few large wedges occur before this location and are most likely due to small variations in the DRE application at the relevant upstream location. These wedges do not persist for higher roughness heights, indicating that the disturbance source was eliminated. When new layers are added, a DRE does not always fully adhere to the DRE below resulting in a partial application. In these instances, the DRE stack at that single location is replaced. It is possible then, as new layers are applied to replace partial applications or add height, that defective DRE are eliminated in the process. At $k=42 \mu \mathrm{m}$, transition occurs between $60 \%$ and $75 \% x / c$. For this roughness height, several large wedges occur forward of the natural transition location, again most likely due to small variations in a limited number of DRE. The full array is always inspected after wedges such as these are observed; however, no detectable defects were present. By the fourth layer, $k=56 \mu \mathrm{m}$, transition occurs between $55 \%$ and $60 \% x / c$. 

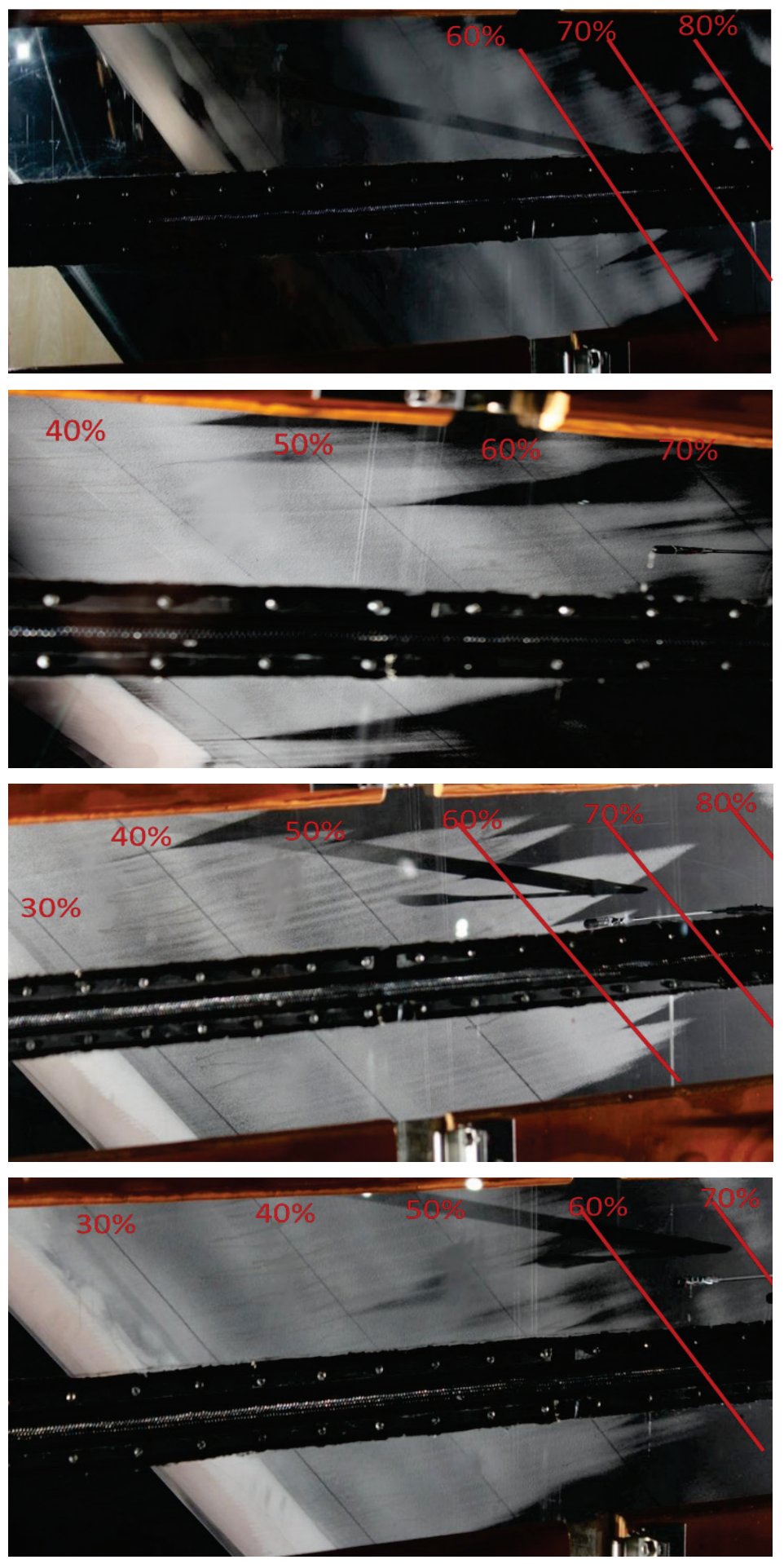

Figure 7-1. NFV with appliqué roughness: $x / c=0.029, d=3 \mathrm{~mm}, \lambda=6 \mathrm{~mm}$, $R e_{c}=2.4 \times 10^{6}$; (a) $k=14 \mu \mathrm{m}$, (b) $k=27 \mu \mathrm{m}$, (c) $k=42 \mu \mathrm{m}$, (d) $k=56 \mu \mathrm{m}$. 
Based on the four roughness heights tested, it appears that the control DRE intended to delay transition, instead bring it forward, particularly for the last two roughness heights. There are a number of factors that could influence these results. Wavelengths excited by the DRE are very important. If array spacing or DRE height are non-uniform, the most unstable $12 \mathrm{~mm}$ wave may not be fully suppressed. This is also why an emphasis on number of layers in addition to actual roughness height is given above. As previously discussed, the difficulty in maintaining roughness diameter and spacing increases as the number of layers increases. However, flow visualization results near $20 \% x / c$ show very uniform $6 \mathrm{~mm}$ streaks at each roughness height with no indication of a $12 \mathrm{~mm}$ bias. Figure 7-2 shows one such example. Hotwire measurements described in the next section also confirm that wavelengths larger than $6 \mathrm{~mm}$ are not present. The $6 \mathrm{~mm}$ streaks eventually give way to larger wavelengths, consistent with previous testing at ASU where control DRE were effective (Carrillo 1996). Irregularities in the array are most likely not the cause of early transition. 


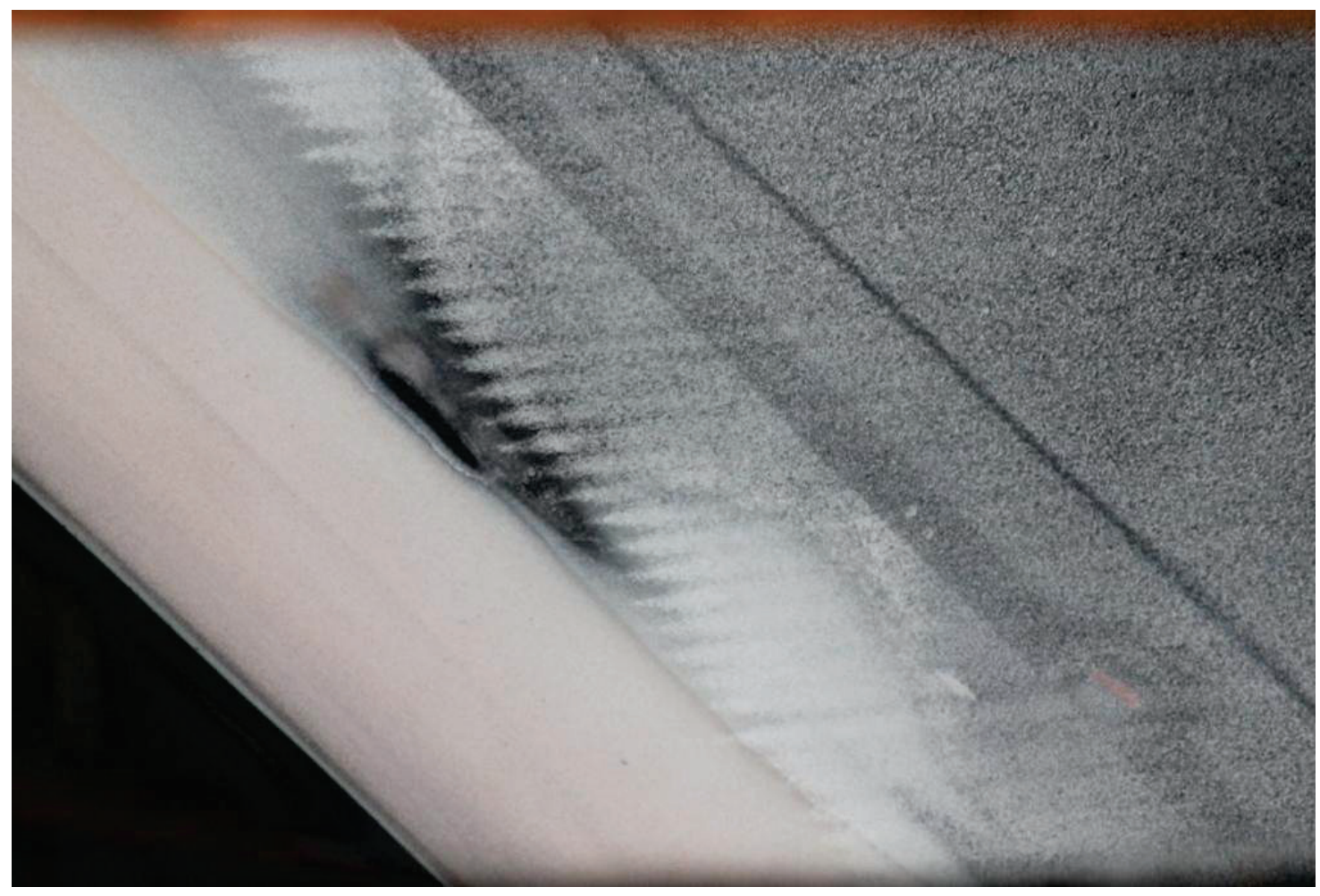

Figure 7-2. Close-up of $6 \mathrm{~mm}$ streaking from $\mathrm{NFV}, \boldsymbol{R e}_{c}=2.4 \times 10^{6},[42 \mid 6]$ roughness.

Another factor may be that the initial disturbance amplitudes generated by the artificial roughness are too large. Carrillo (1996) used $8 \mathrm{~mm}$ wavelength roughness to suppress the most unstable $12 \mathrm{~mm}$ wave and another highly unstable $9 \mathrm{~mm}$ wave on the NLF(2)-0415. For small roughness heights $(k=6 \mu \mathrm{m})$, transition was delayed past the pressure minimum. When the roughness height was increased to $48 \mu \mathrm{m}$, transition moved forward of the location where natural transition without artificial roughness occurred. At the time, this result was attributed to early amplitude saturation of the $8 \mathrm{~mm}$ wave compared to the $6 \mu \mathrm{m}$ case, which allowed long wavelength background disturbances to grow and cause early transition. No intermediate roughness heights between $6 \mu \mathrm{m}$ and $48 \mu \mathrm{m}$ were documented for the tests in Carrillo, so the threshold for 
viable control DREs has not been established. The minimum height available for the control DRE in the current tests is $14 \mu \mathrm{m}$. It is possible that the minimum available roughness height is already too large for the control scenario. Carpenter (2009) also describes at least one instance where control DRE at larger roughness heights appeared to move transition forward rather than delay it. Moreover, in all of the previously successful DRE control demonstrations (Saric et al. 1998, Saric et al. 2011), natural transition occurred closer to $30 \%-50 \% x / c$. In flight, Saric et al. (2011) observed laminar flow back to $80 \% x / c$ for the highly polished leading edge on SWIFT. Background surface roughness near the leading edge was increased to bring natural transition forward to $30 \% x / c$ before any attempts at demonstrating control were made. The purpose of a highly polished leading edge is to minimize the background disturbances such that the applied artificial roughness is the dominant forcing mechanism. One would expect the initial disturbance amplitudes created by the highly polished leading edge for the natural transition case to be much smaller than amplitudes generated by any artificial roughness. It is perhaps not entirely unexpected then, that control DRE used in a configuration (i.e. surface finish, angle of attack, Reynolds number and freestream disturbance levels) where natural transition occurs aft of the pressure minimum were at best ineffective in changing transition location and at worst moved it forward. However, more testing is needed before this issue is fully resolved.

As a final observation, the extent to which control DRE were demonstrated on this particular airfoil at ASU is not well documented. Most of the control work was completed on the NLF(2)-0415. Theses by Gladden (2001) and White (White 2000, 
White \& Saric 2005) are the two primary experimental efforts that use the ASU(67)-0315 and of those two, Gladden is the only one to use the $8 \mathrm{~mm}$ control spacing. In that study, Gladden assesses the effectiveness of the control wavelength relative to the critical wavelength transition location rather than natural transition. To this author's knowledge, Gladden is the only published case where $8 \mathrm{~mm}$ spacing is used on the ASU(67)-0315. Private communications from William Saric indicate that plasma DRE using $6 \mathrm{~mm}$ spacing were also successful. These comments are provided only to suggest that there may be some unknown differences between the experimental configurations that are limiting the success of the control DRE at the KSWT.

\subsubsection{Hotwire Measurements, $15 \% x / c$}

Figures 7-3 - 7-6, 7-7 - 7-10 and 7-11 - 7-14 show the boundary-layer profiles, streamwise velocity contours and disturbance shapes respectively. Similar to the $12 \mathrm{~mm}$ forcing, the accelerated and decelerated profiles continue to broaden as roughness height increases. Inflectional profiles begin to appear as early as $k=27 \mu \mathrm{m}$. A $6 \mathrm{~mm}$ periodicity is evident in the streamwise velocity contours and no smaller wavelengths are observed. Mode shapes for the four roughness heights are shown in Figure 7-15. Up to a roughness height of $42 \mu \mathrm{m}$, the characteristic shape of the total disturbance mode is different compared to the critical wavelength spacing. It is not until reaching a roughness height of $56 \mu \mathrm{m}$ that the mode shape more closely resembles those of the critical wavelength. Except for one instance, the shape does not persist for the higher Reynolds number cases. The reason for the different mode shape is not well understood. Table 7-1 shows 
the maximum and normalized disturbance amplitudes for these roughness heights at $R e_{c}=2.4 \times 10^{6}$.

Table 7-1. Maximum and normalized stationary crossflow disturbance amplitudes at $15 \% x / c, R_{c}=2.4 \times 10^{6},[k \mid 6]$ roughness.

\begin{tabular}{|c|c|c|}
\hline$k[\mu \mathrm{m}]$ & {$\left[\left(u-u_{\text {avg }}\right) / U_{e}\right]_{\mathrm{rms}, \max }$} & {$\left[\left(u-u_{\text {avg }}\right) / U_{e}\right]_{\mathrm{rms}, k / k o}$} \\
\hline 14 & 0.0153 & 1.00 \\
\hline 27 & 0.0273 & 0.93 \\
\hline 42 & 0.0385 & 0.84 \\
\hline 56 & 0.0452 & 0.74 \\
\hline
\end{tabular}

Disturbance amplitude appears to drop-off slightly as roughness height is increased. The third DRE layer, $k=42 \mu \mathrm{m}$, is closest to the final height tested for the critical wavelength data set. Comparison of the first three layers for $\lambda=6 \mathrm{~mm}$ with the $\lambda=12 \mathrm{~mm}$ data shows a similar trend with a slightly larger decrease in disturbance amplitude as roughness height is increased. A comparison to ASU measurements is provided in Section 9. Figure 7-16 shows the PSD for this test point. A peak at $6 \mathrm{~mm}$ is the only dominant feature in the spectra. Unlike the $12 \mathrm{~mm}$ case, no wavenumber harmonics (wavelengths less than $6 \mathrm{~mm}$ ) are observed. Also of importance, no wavenumber subharmonics are shown. Presence of a peak at a wavelength greater than $6 \mathrm{~mm}$ would have indicated that the control DREs were not effectively suppressing the larger wavelengths. 


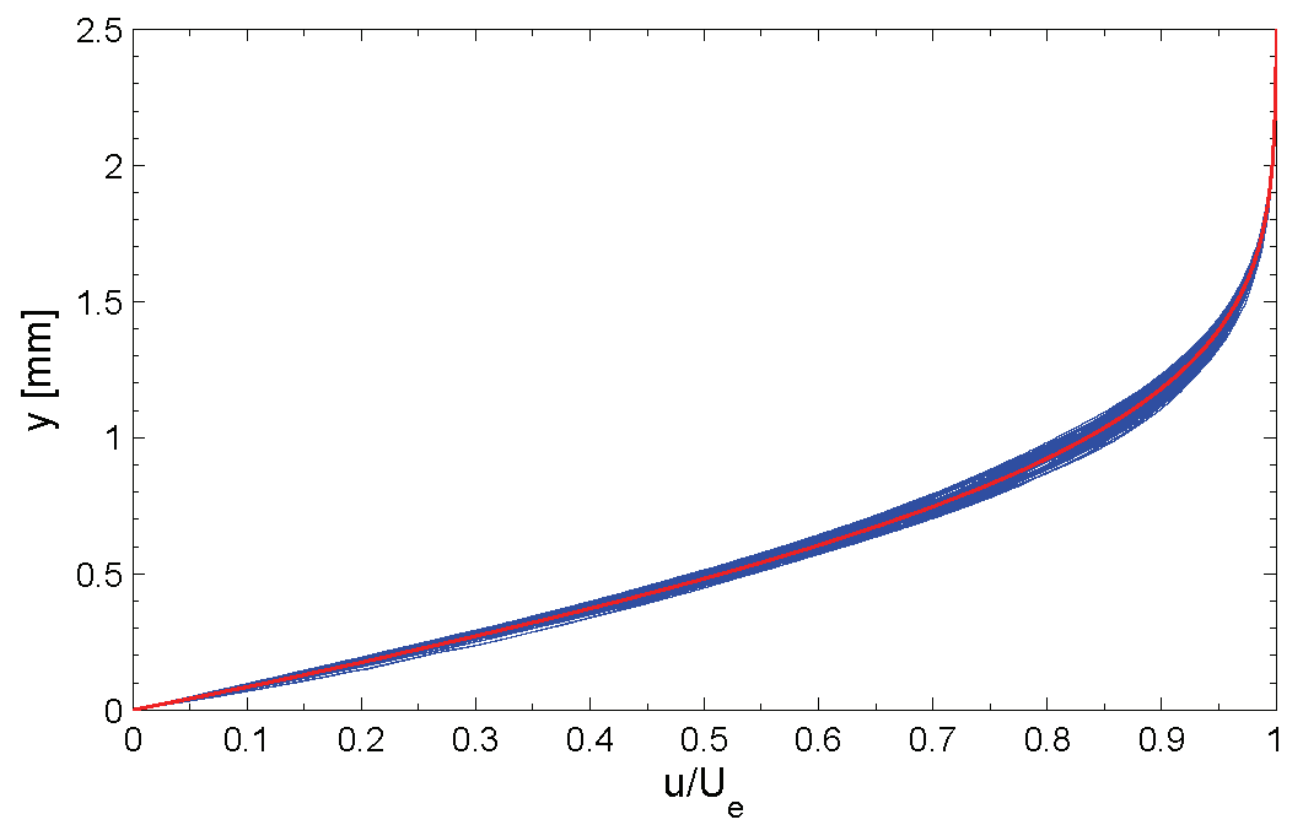

Figure 7-3. Spanwise array of 65 mean-flow boundary-layer profiles spanning $64 \mathrm{~mm}$ at $15 \% x / c . R e_{c}=2.4 \times 10^{6},[14 \mid 6]$ roughness. Mean profile shown in red.

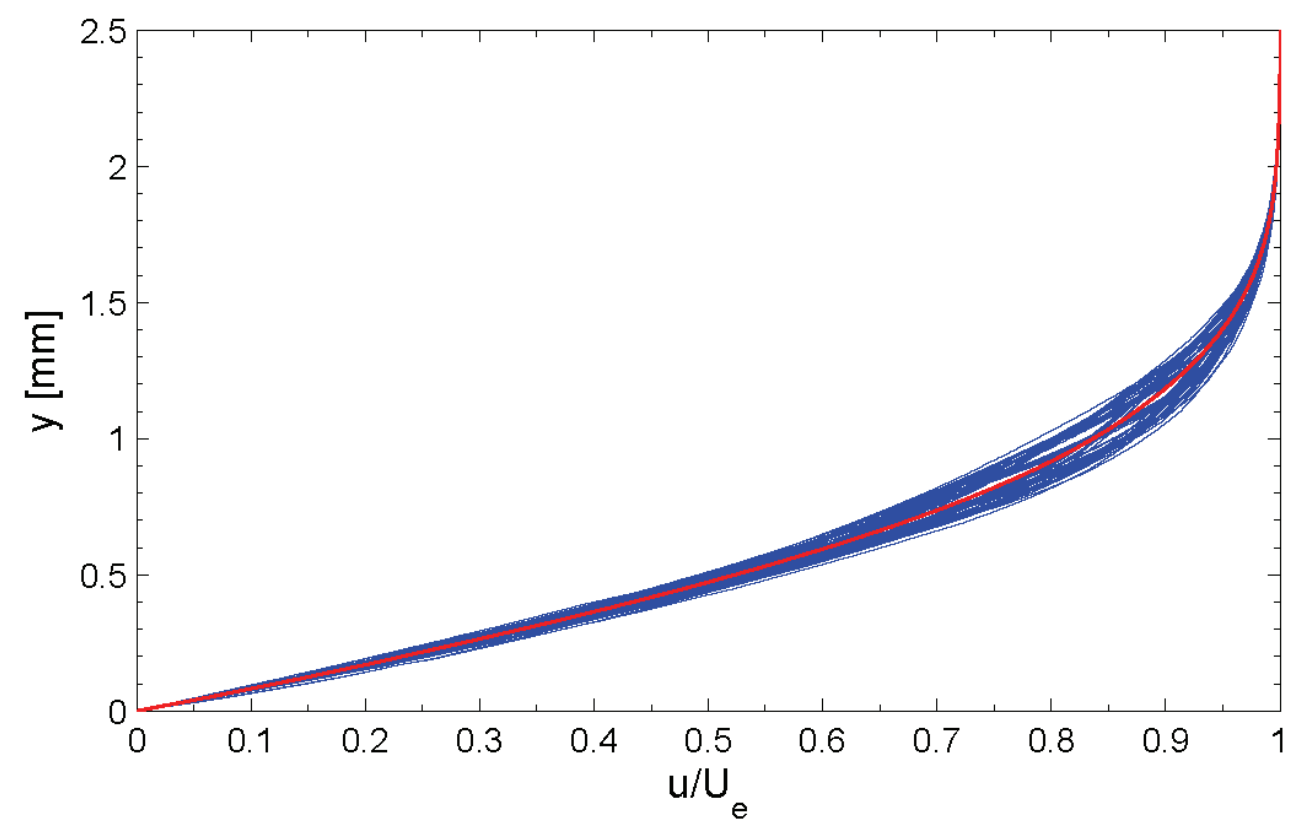

Figure 7-4. Spanwise array of 65 mean-flow boundary-layer profiles spanning $64 \mathrm{~mm}$ at $15 \% x / c . R e_{c}=2.4 \times 10^{6},[27 \mid 6]$ roughness. Mean profile shown in red. 


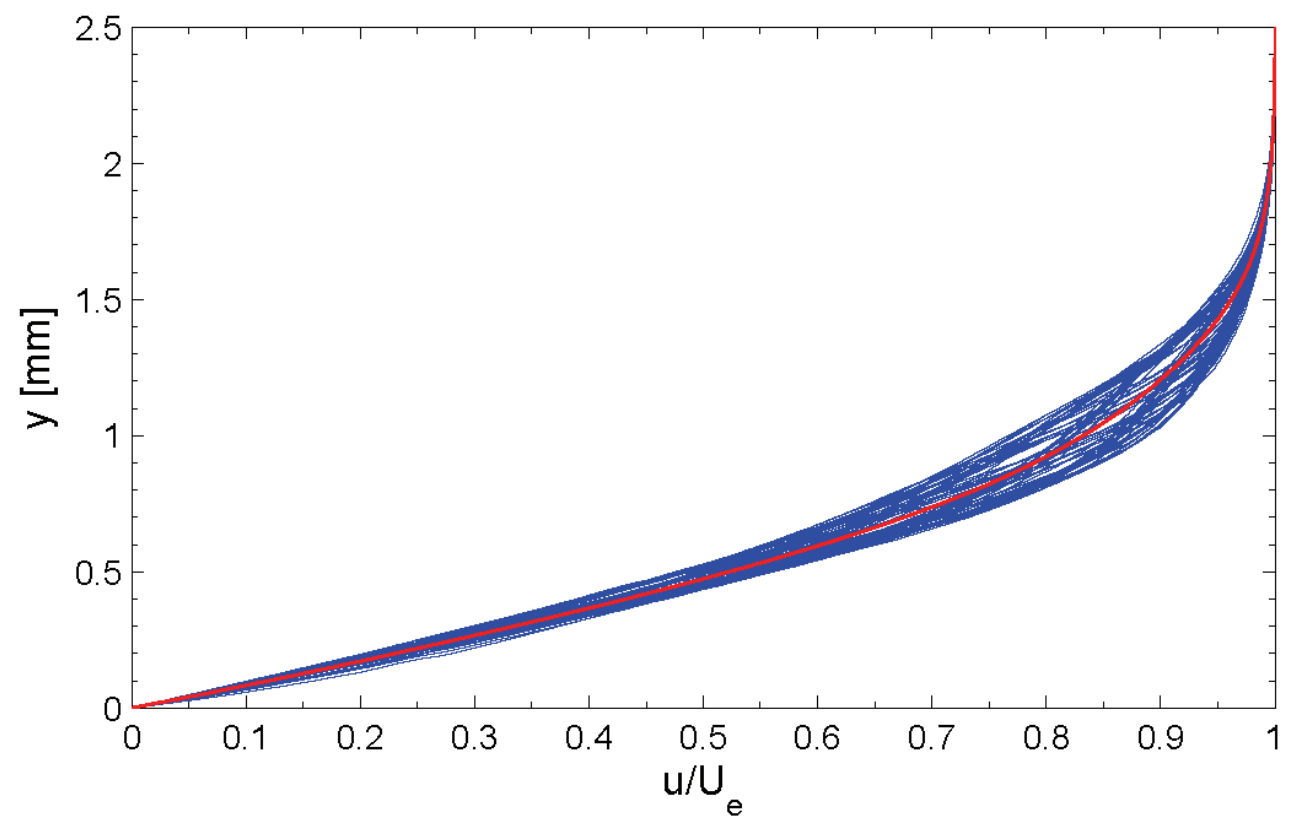

Figure 7-5. Spanwise array of 65 mean-flow boundary-layer profiles spanning $64 \mathrm{~mm}$ at $15 \% x / c . R e_{c}=2.4 \times 10^{6},[42 \mid 6]$ roughness. Mean profile shown in red.

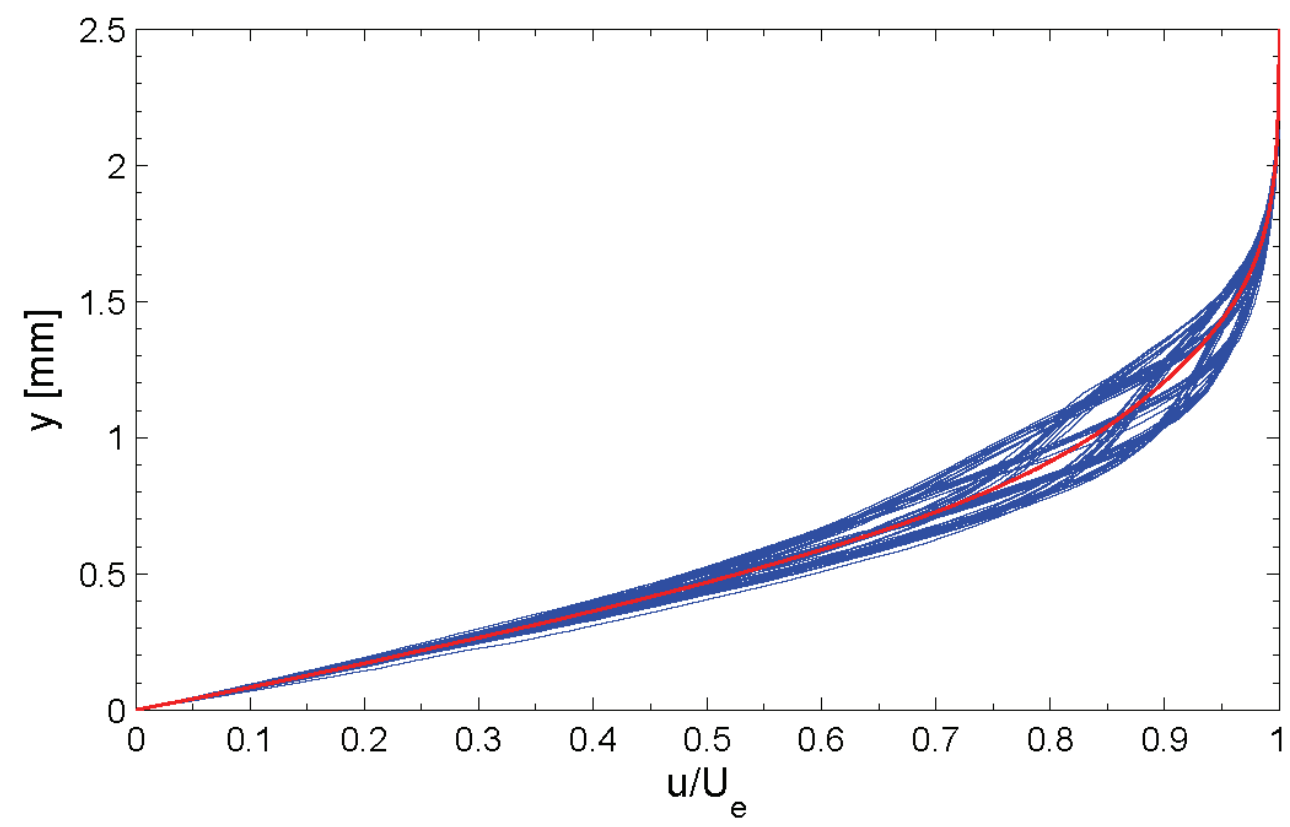

Figure 7-6. Spanwise array of 65 mean-flow boundary-layer profiles spanning $64 \mathrm{~mm}$ at $15 \% x / c . R e_{c}=2.4 \times 10^{6},[56 \mid 6]$ roughness. Mean profile shown in red. 


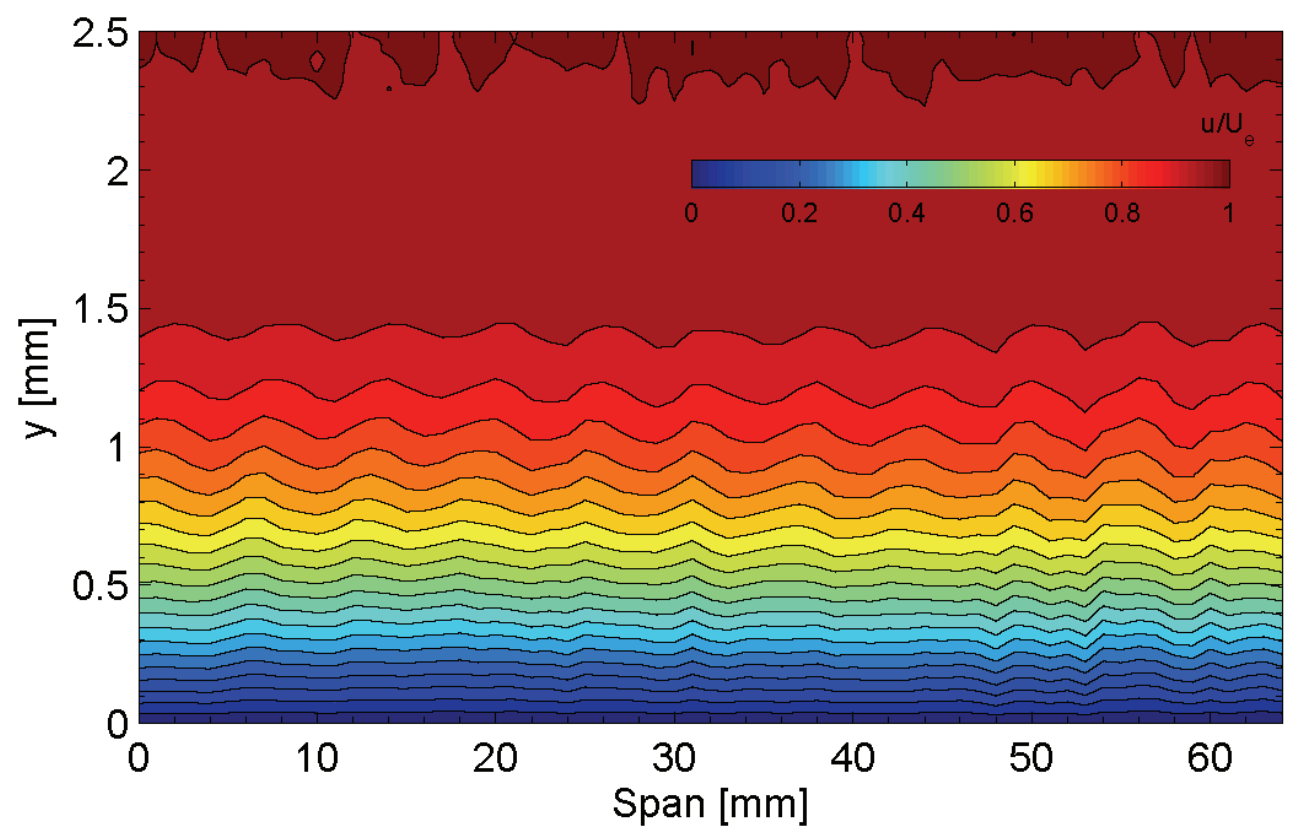

Figure 7-7. Streamwise velocity contours at $15 \% x / c . R e_{c}=2.4 \times 10^{6},[14 \mid 6]$ roughness.

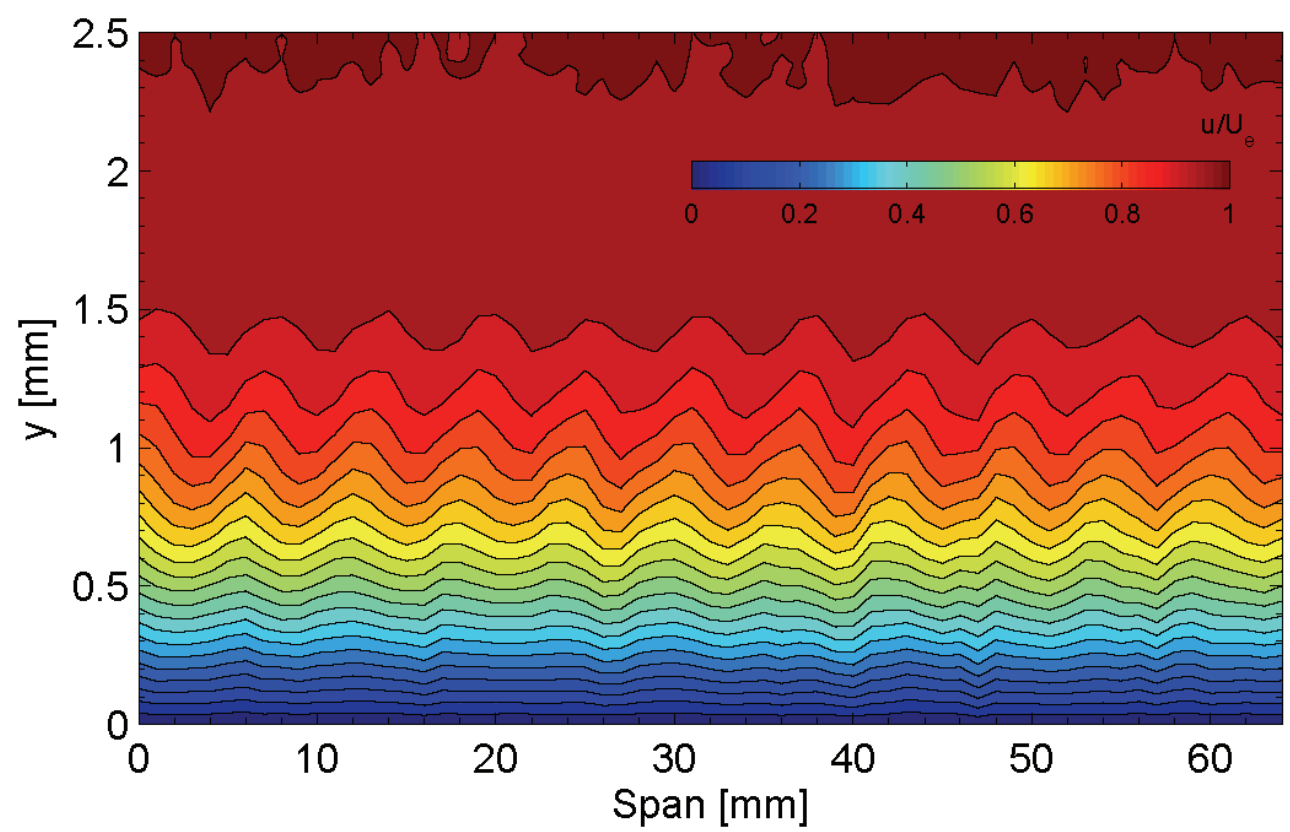

Figure 7-8. Streamwise velocity contours at $15 \% x / c . R_{c}=2.4 \times 10^{6},[27 \mid 6]$ roughness. 


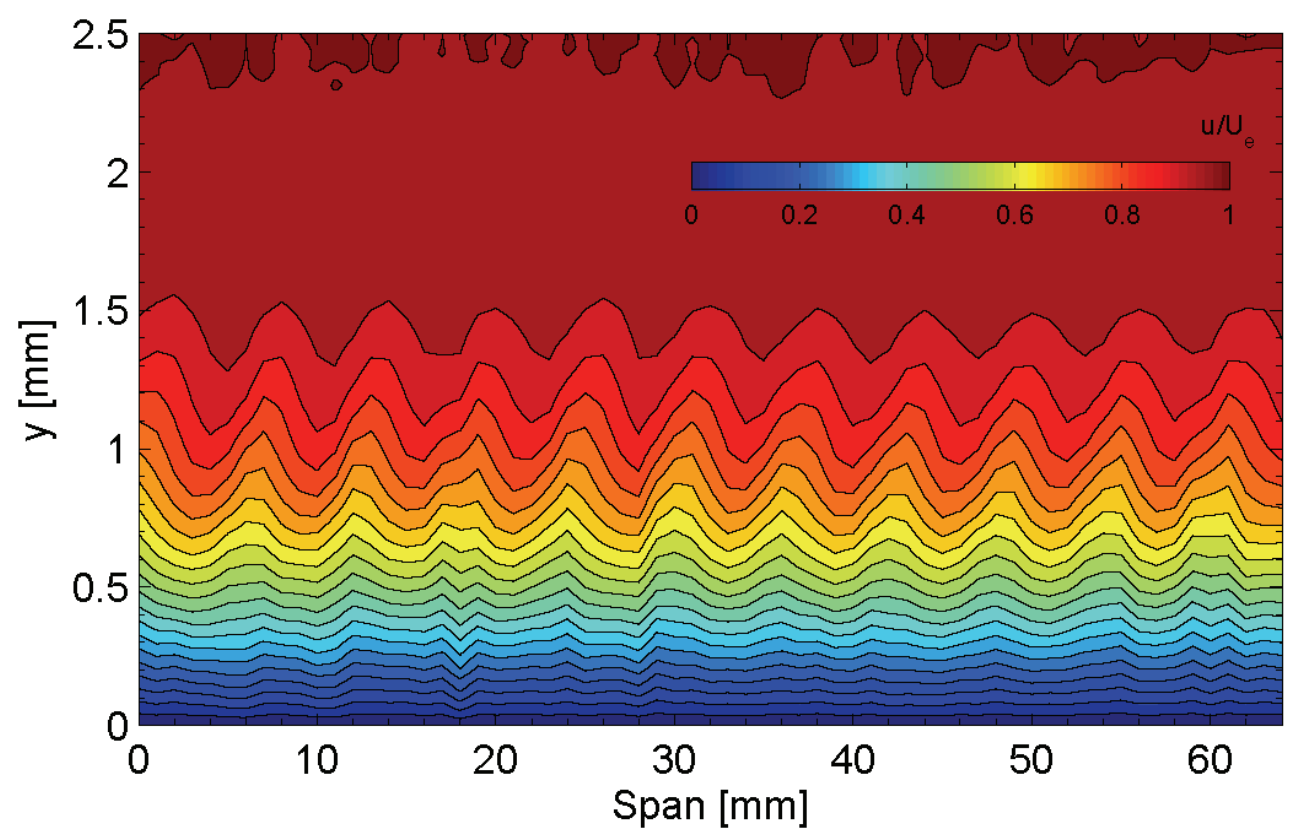

Figure 7-9. Streamwise velocity contours at $15 \% x / c . R e_{c}=2.4 \times 10^{6},[42 \mid 6]$ roughness.

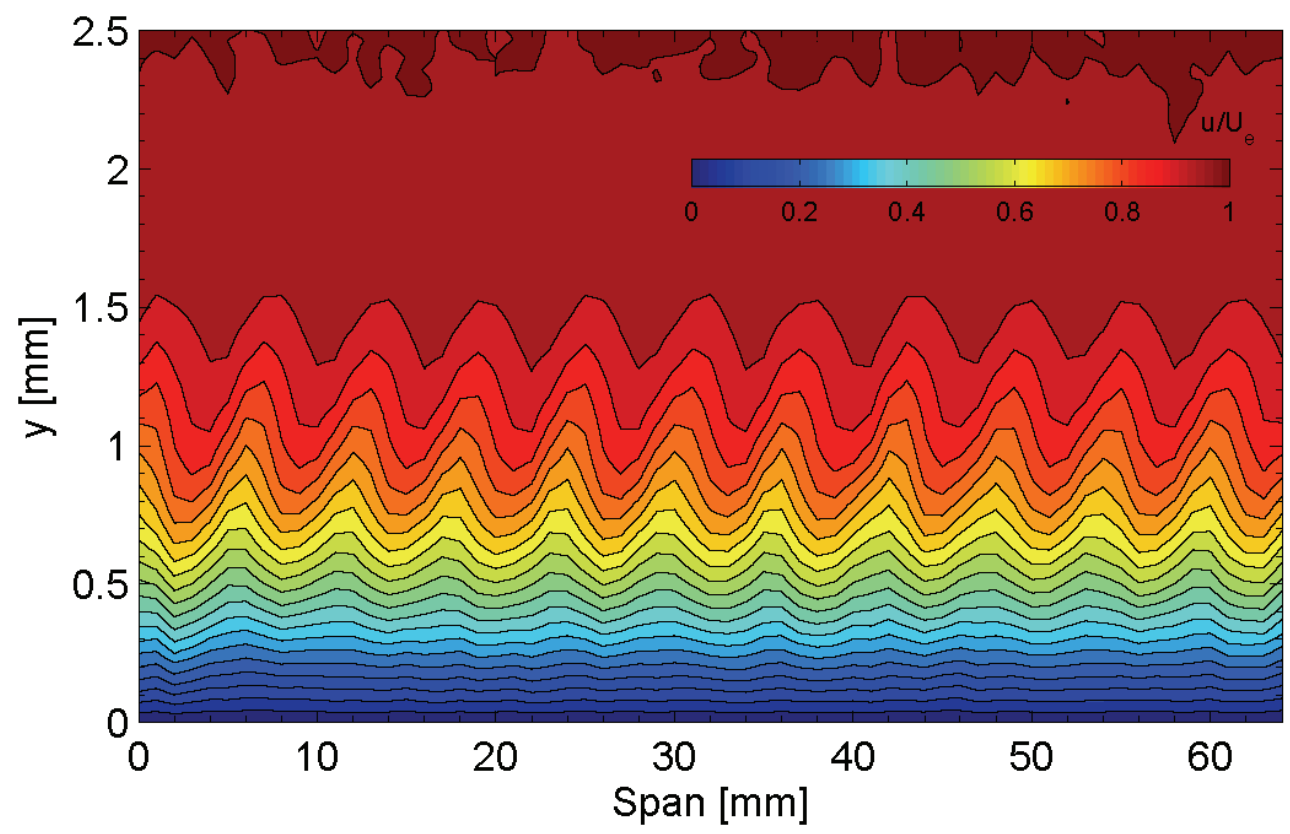

Figure 7-10. Streamwise velocity contours at $15 \% x / c . R e_{c}=2.4 \times 10^{6},[56 \mid 6]$ roughness. 


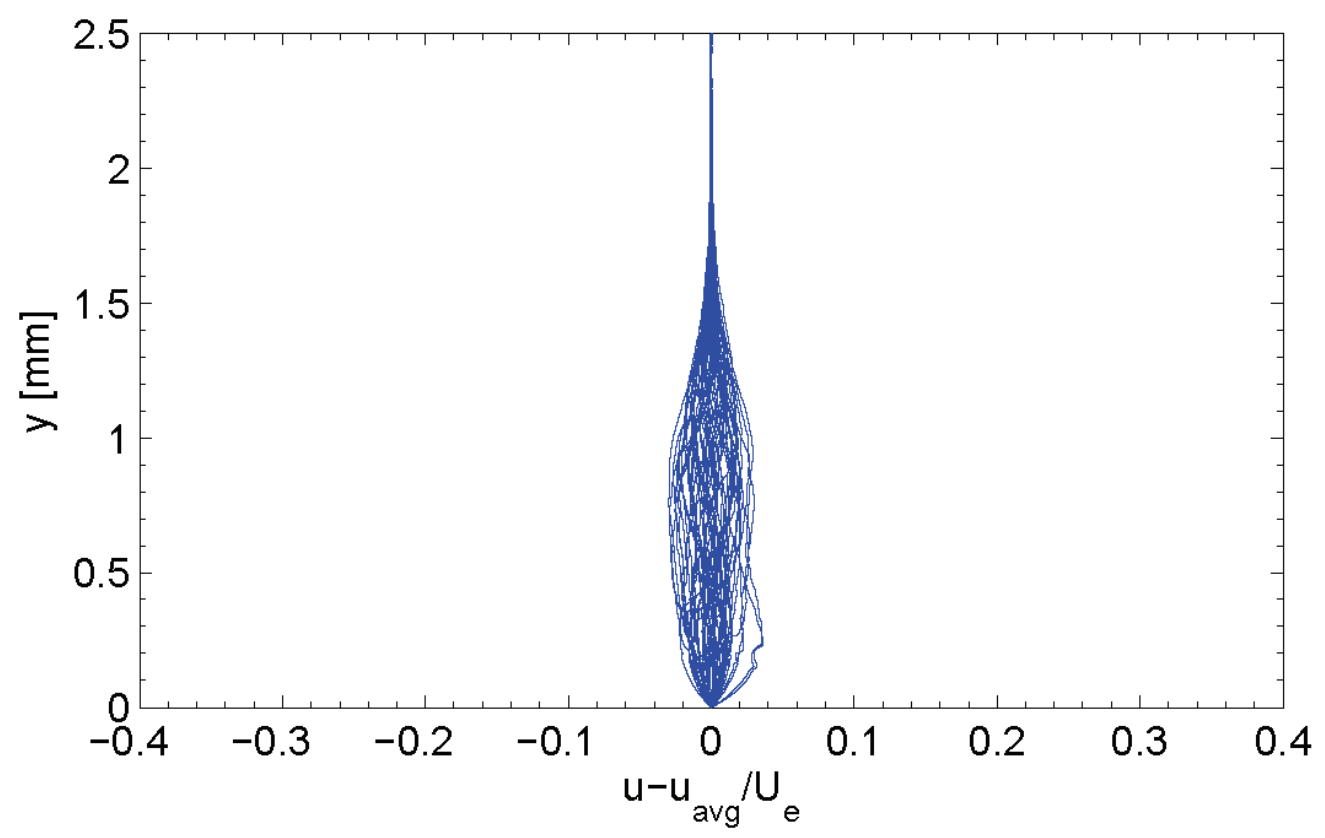

Figure 7-11. Spanwise array of 65 disturbance profiles spanning $64 \mathrm{~mm}$ at $15 \% x / c$. $R e_{c}=2.4 \times 10^{6},[14 \mid 6]$ roughness.

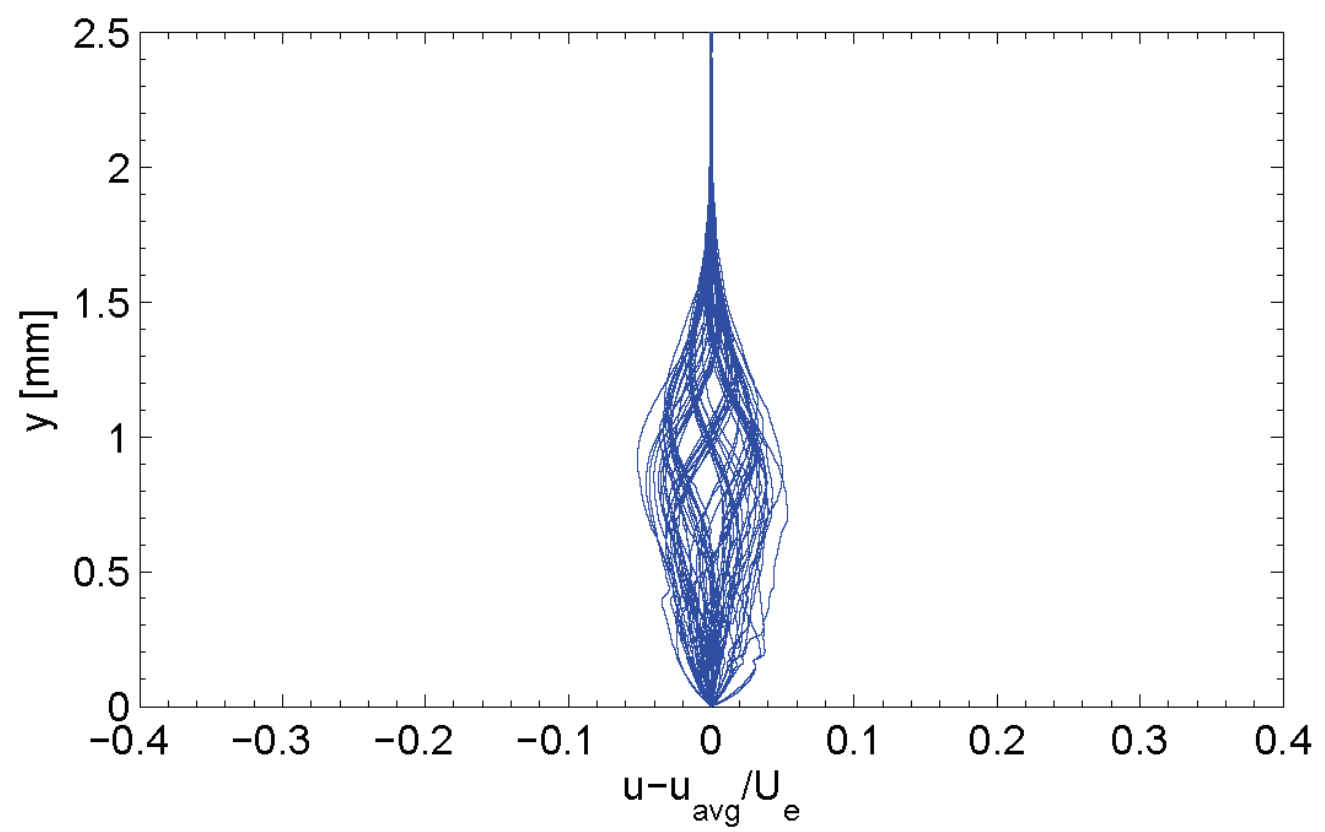

Figure 7-12. Spanwise array of 65 disturbance profiles spanning $64 \mathrm{~mm}$ at $15 \% x / c$. $R e_{c}=2.4 \times 10^{6},[27 \mid 6]$ roughness. 


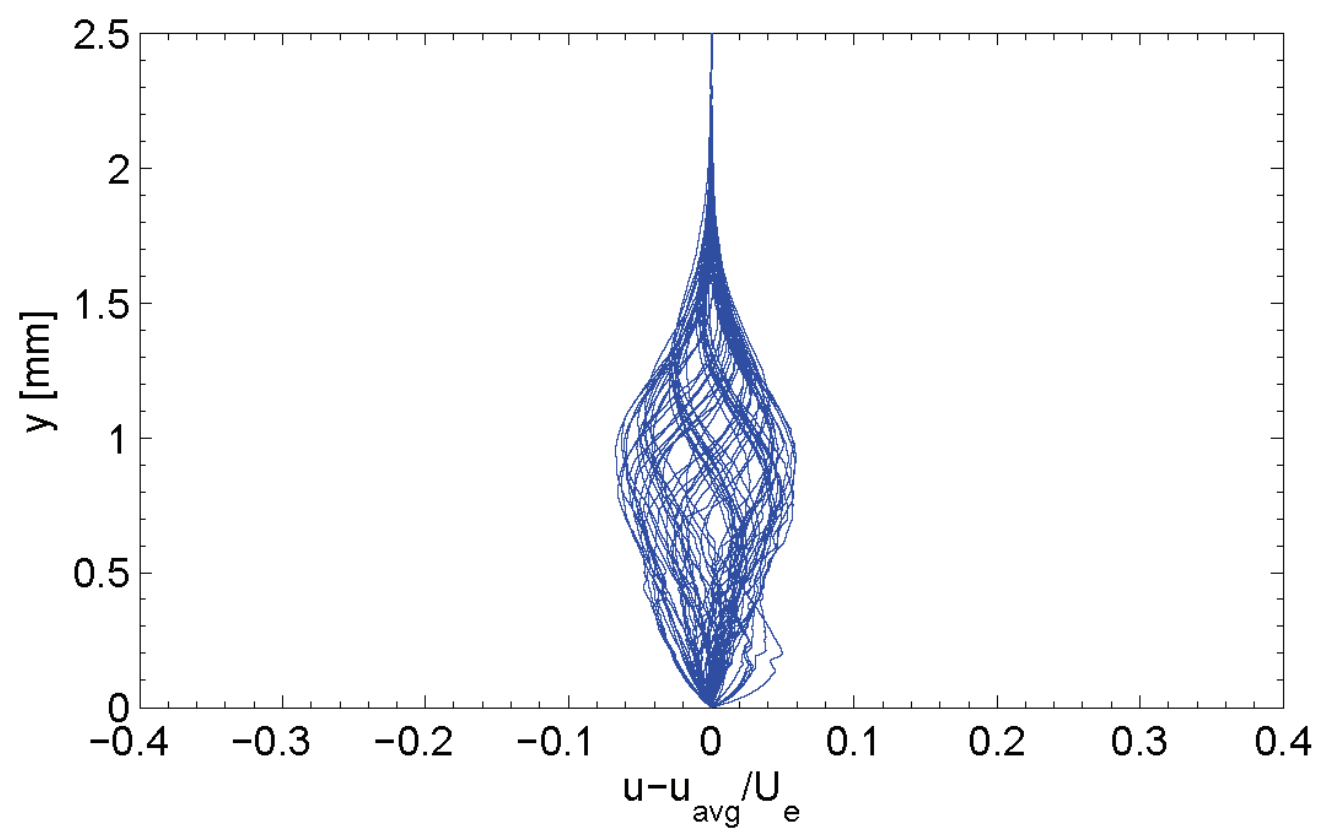

Figure 7-13. Spanwise array of 65 disturbance profiles spanning $64 \mathrm{~mm}$ at $15 \% x / c$. $R e_{c}=2.4 \times 10^{6},[42 \mid 6]$ roughness.

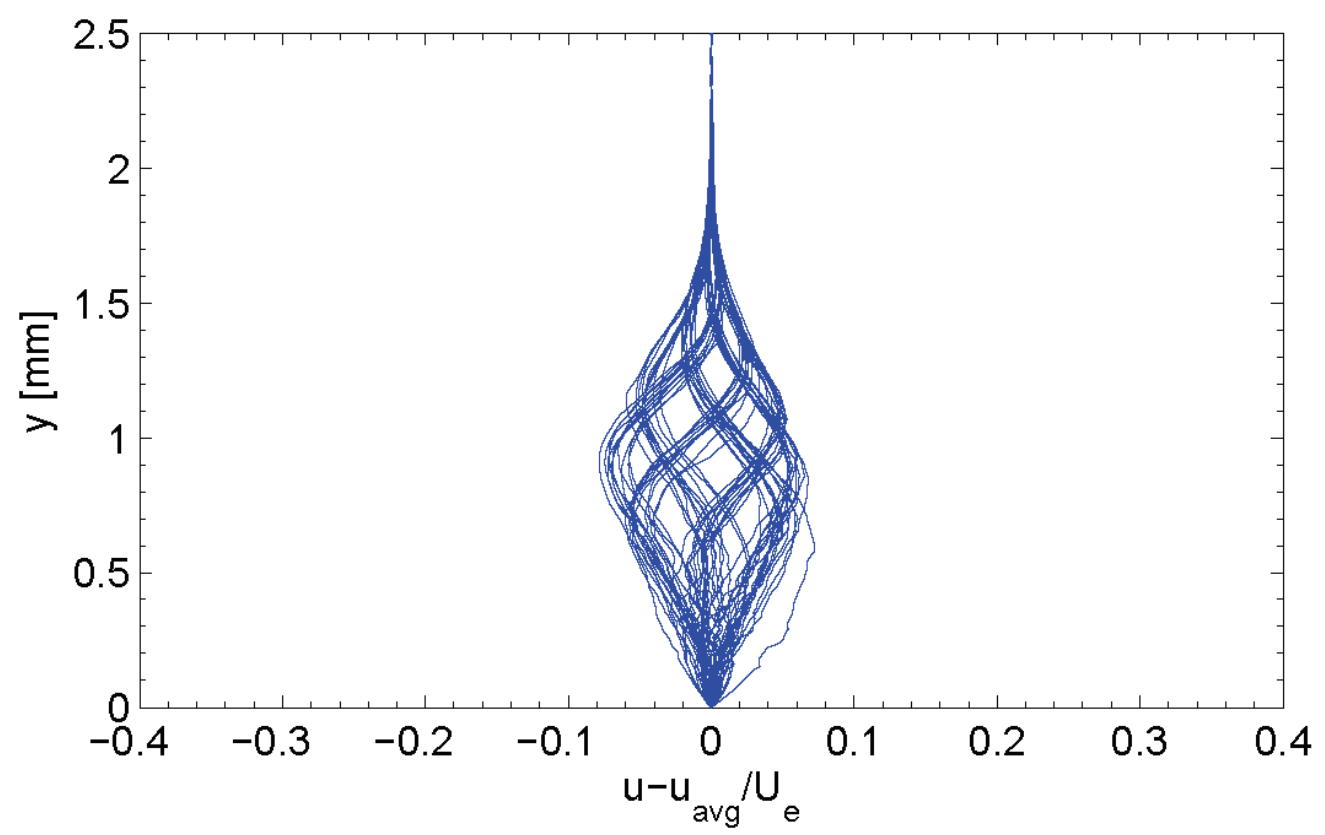

Figure 7-14. Spanwise array of 65 disturbance profiles spanning $64 \mathrm{~mm}$ at $15 \% \mathrm{x} / \mathrm{c}$. $R e_{c}=2.4 \times 10^{6},[56 \mid 6]$ roughness. 


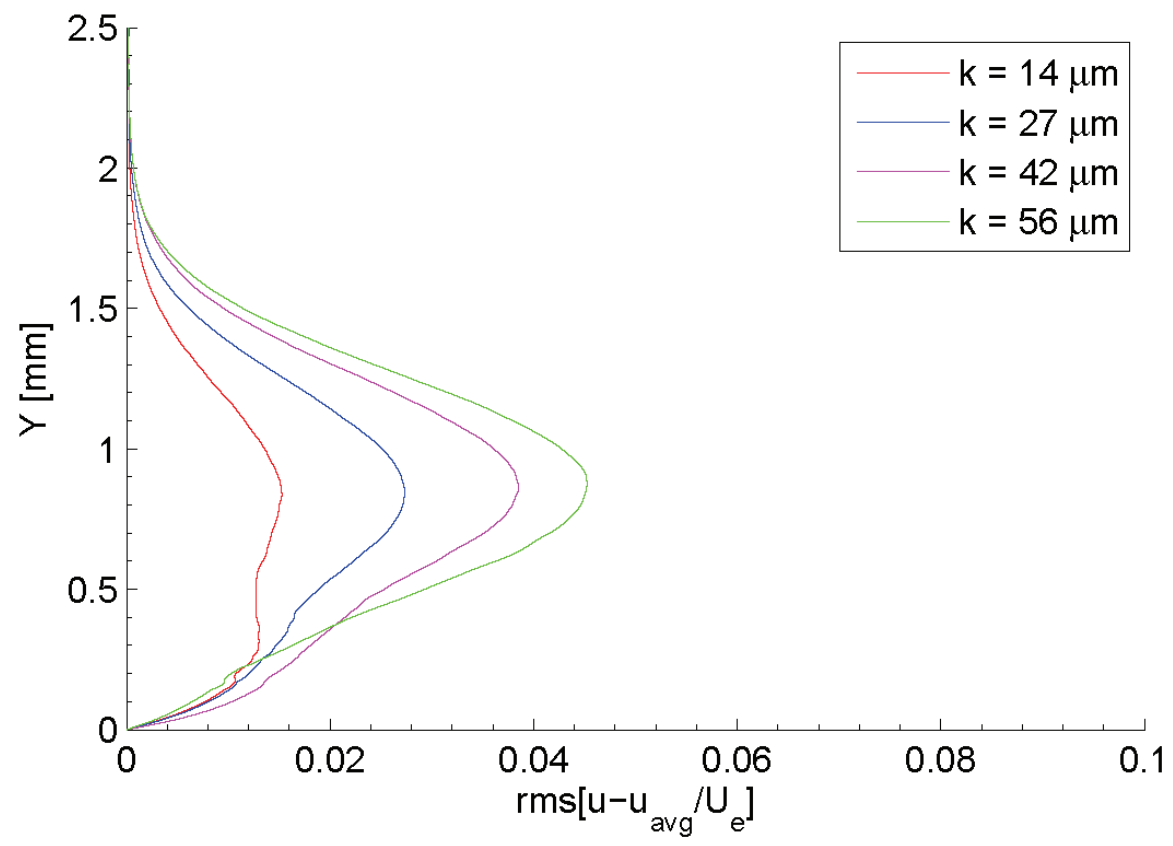

Figure 7-15. Stationary crossflow mode shape at $15 \% x / c . R e_{c}=2.4 \times 10^{6},[k \mid 6]$ roughness.

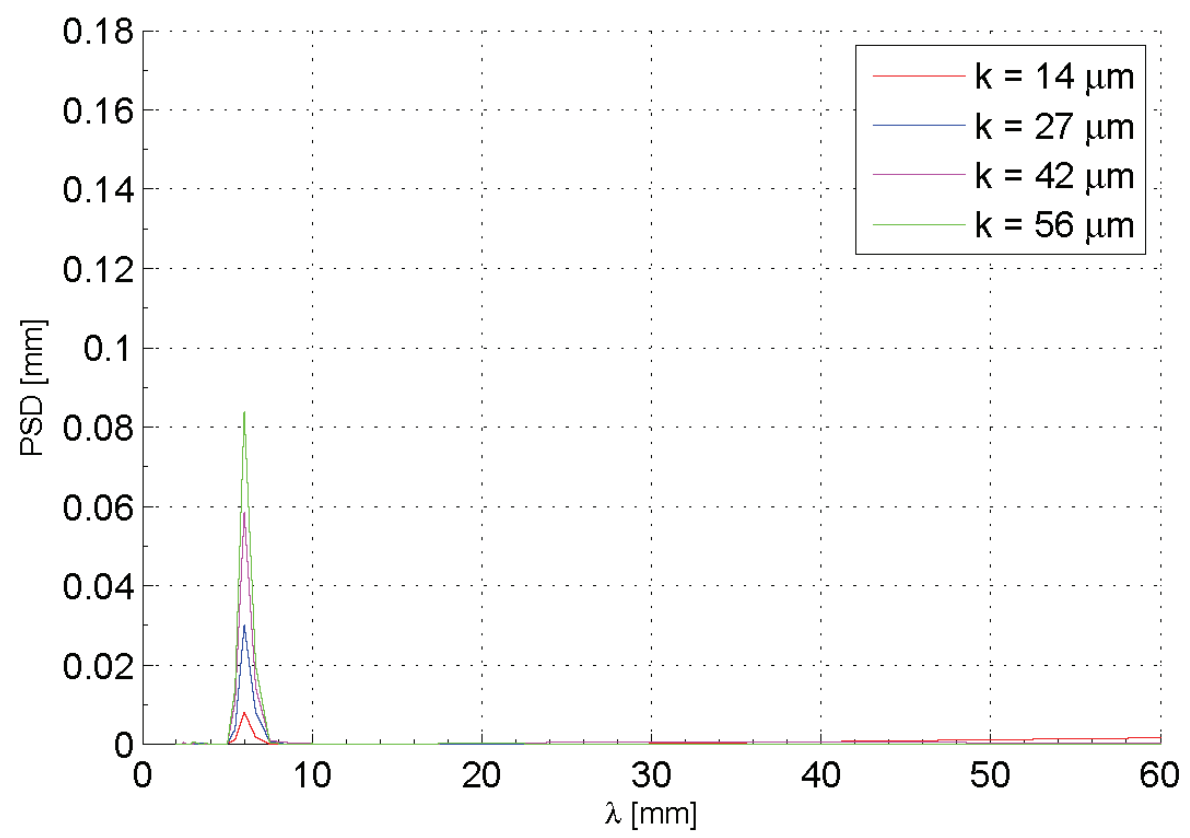

Figure 7-16. Power spectral density of spanwise hotwire scan at $15 \% x / c$. $R e_{c}=2.4 \times 10^{6},[k \mid 6]$ roughness. 


\section{$7.2 R e_{c}=2.8 \times 10^{6}$}

\subsubsection{Naphthalene Flow Visualization}

Figure 7-17 shows the NFV for each roughness height at $R e_{c}=2.8 \times 10^{6}$. Except for a few isolated wedges, transition occurs between $45 \%$ and $50 \% x / c$ for all roughness heights, indicating that transition location does not significantly change as roughness height increases. This is near the same range of difference in transition location that Reibert (1996) reported as roughness height was increased from $6 \mu \mathrm{m}$ to $48 \mu \mathrm{m}$ using critically spaced DRE. However, transition location is forward of the natural transition baseline for all cases. Figure 5-17 shows that transition occurs near $70 \% x / c$ in the lower span for this Reynolds number. In this instance transition has clearly moved forward from the baseline case, despite use of a control wavelength. Similar to $R e_{c}=2.4 \times 10^{6}$, regular $6 \mathrm{~mm}$ streaking is observed along the full span between $20 \%$ and $30 \% x / c$. More unstable wavelengths are not detected in the flow visualization. Carrillo (1996) also observed that when Reynolds number increased to $2.8 \times 10^{6}$ transition location did not change at low roughness heights and at larger roughness heights it moved forward. As before, it is possible that the initial disturbance amplitude generated by these roughness heights is too large for control. 

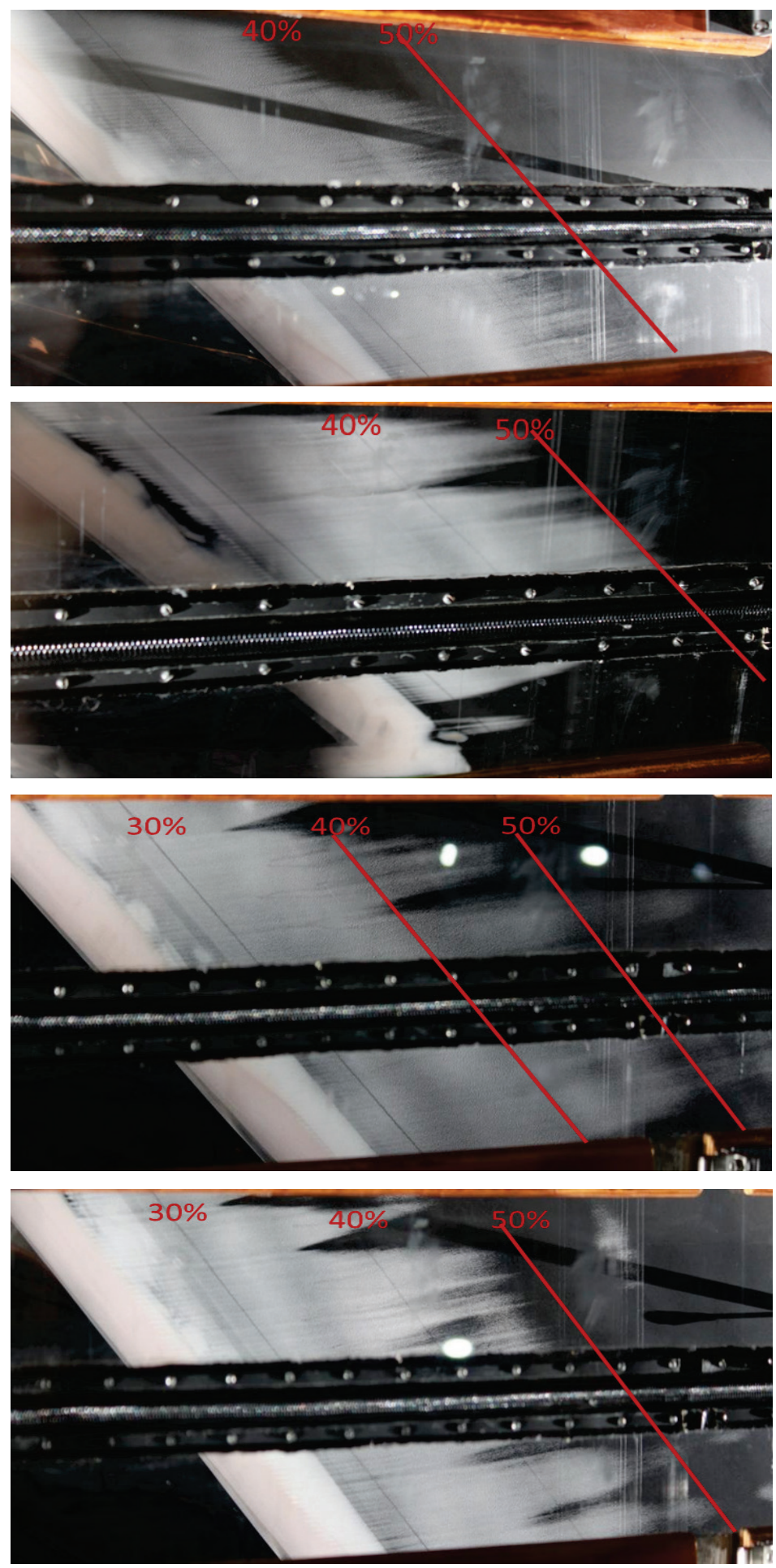

Figure 7-17. NFV with appliqué roughness: $x / c=0.029, d=3 \mathrm{~mm}, \lambda=6 \mathrm{~mm}$, $R e_{c}=2.8 \times 10^{6}$; (a) $k=14 \mu \mathrm{m}$, (b) $k=27 \mu \mathrm{m}$, (c) $k=42 \mu \mathrm{m}$, (d) $k=56 \mu \mathrm{m}$. 


\subsubsection{Hotwire Measurements, $15 \% x / c$}

Boundary-layer velocity profiles, streamwise velocity contours and disturbance shapes are shown in Figures 7-18 - 7-21, 7-22 - 7-25 and 7-26 - 7-29 respectively. Strongly inflected profiles are evident for the larger roughness heights. Inflectional mean profiles provide the first indications that nonlinear mode interactions may be occurring. A $6 \mathrm{~mm}$ periodicity is present in all of the streamwise velocity contours. The amplitude of each peak increases with roughness height, but roll-over or smaller wavelengths do not appear. Figure 7-30 includes the mode shapes for this test point. At $k=14 \mu \mathrm{m}$, the same slightly irregular mode shape observed for $R e_{c}=2.4 \times 10^{6}$ occurs again. The profiles for $k=27 \mu \mathrm{m}$ and $k=42$ return to the shape detected during critical wavelength measurements. At the final height, a secondary lobe is visible. Consistent with the inflectional mean profile discussed earlier, nonlinear stability interactions render this data point outside the scope of the receptivity analysis. Table 7-2 shows the maximum and normalized disturbance amplitudes for all roughness heights.

Table 7-2. Maximum and normalized stationary crossflow disturbance amplitudes at $15 \% x / c, R_{c}=2.8 \times 10^{6},[k \mid 6]$ roughness.

\begin{tabular}{|c|c|c|}
\hline$k[\mu \mathrm{m}]$ & {$\left[\left(u-u_{a v g}\right) / U_{e}\right]_{\mathrm{rms}, \max }$} & {$\left[\left(u-u_{\text {avg }}\right) / U_{e}\right]_{\mathrm{rms}, k / k o}$} \\
\hline 14 & 0.0313 & 1.00 \\
\hline 27 & 0.0591 & 0.98 \\
\hline 42 & 0.0797 & 0.85 \\
\hline 56 & 0.0890 & $0.71^{*}$ \\
\hline
\end{tabular}

*Indicates nonlinear stability present 
Comparing only the first three normalized amplitudes, the relationship between roughness height and disturbance amplitude is weakly nonlinear. The PSD for this case is shown in Figure 7-31. Once again, the $6 \mathrm{~mm}$ wave is the only significant peak.

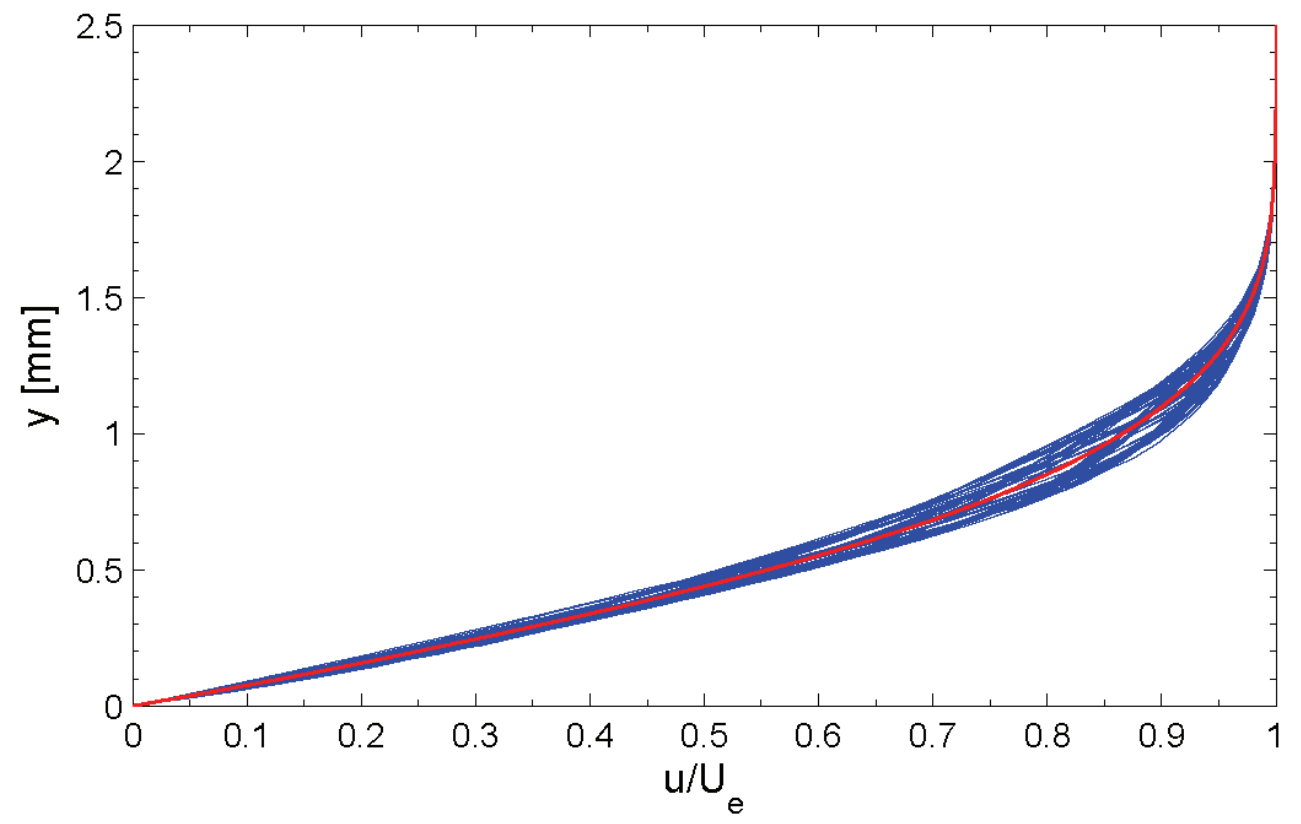

Figure 7-18. Spanwise array of 65 mean-flow boundary-layer profiles spanning $64 \mathrm{~mm}$ at $15 \% x / c . R e_{c}=2.8 \times 10^{6},[14 \mid 6]$ roughness. Mean profile shown in red. 


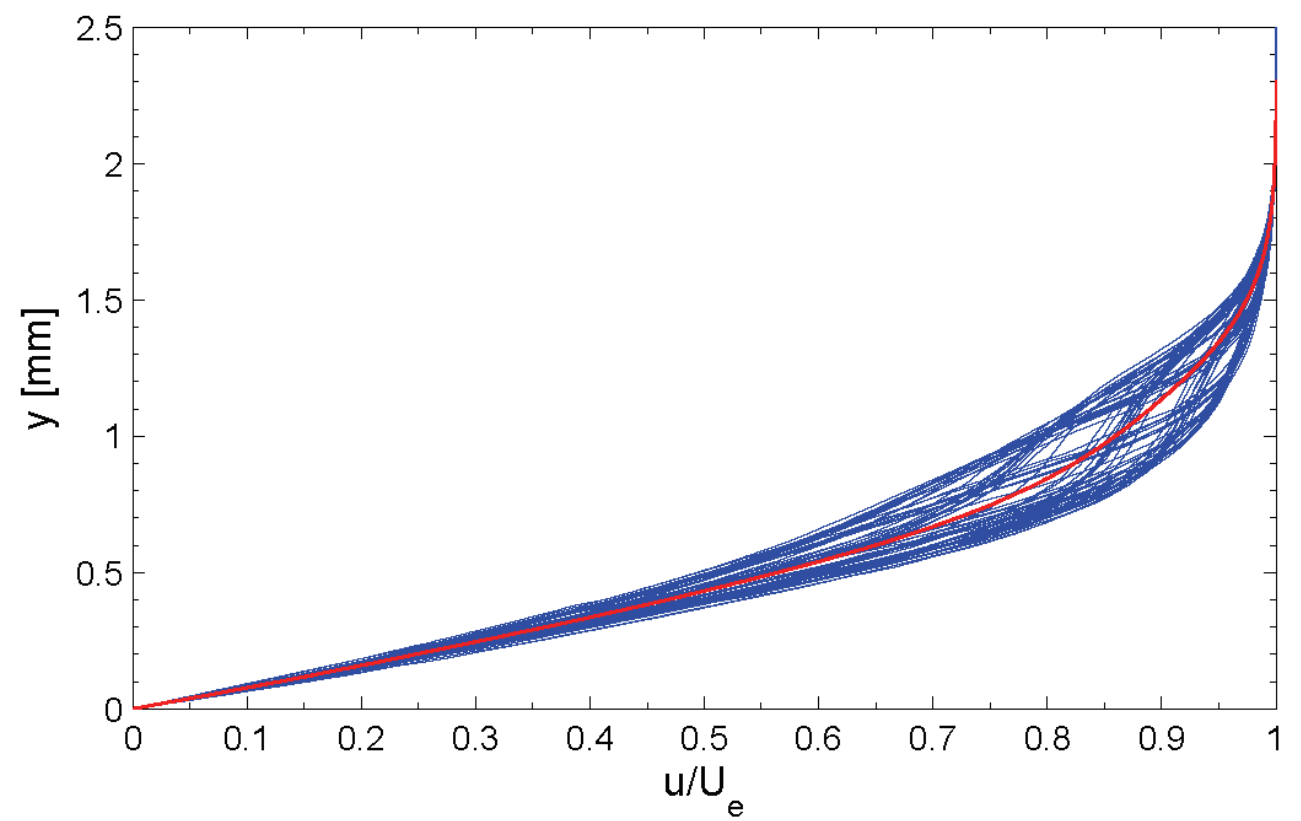

Figure 7-19. Spanwise array of 65 mean-flow boundary-layer profiles spanning $64 \mathrm{~mm}$ at $15 \% x / c . R e_{c}=2.8 \times 10^{6},[27 \mid 6]$ roughness. Mean profile shown in red.

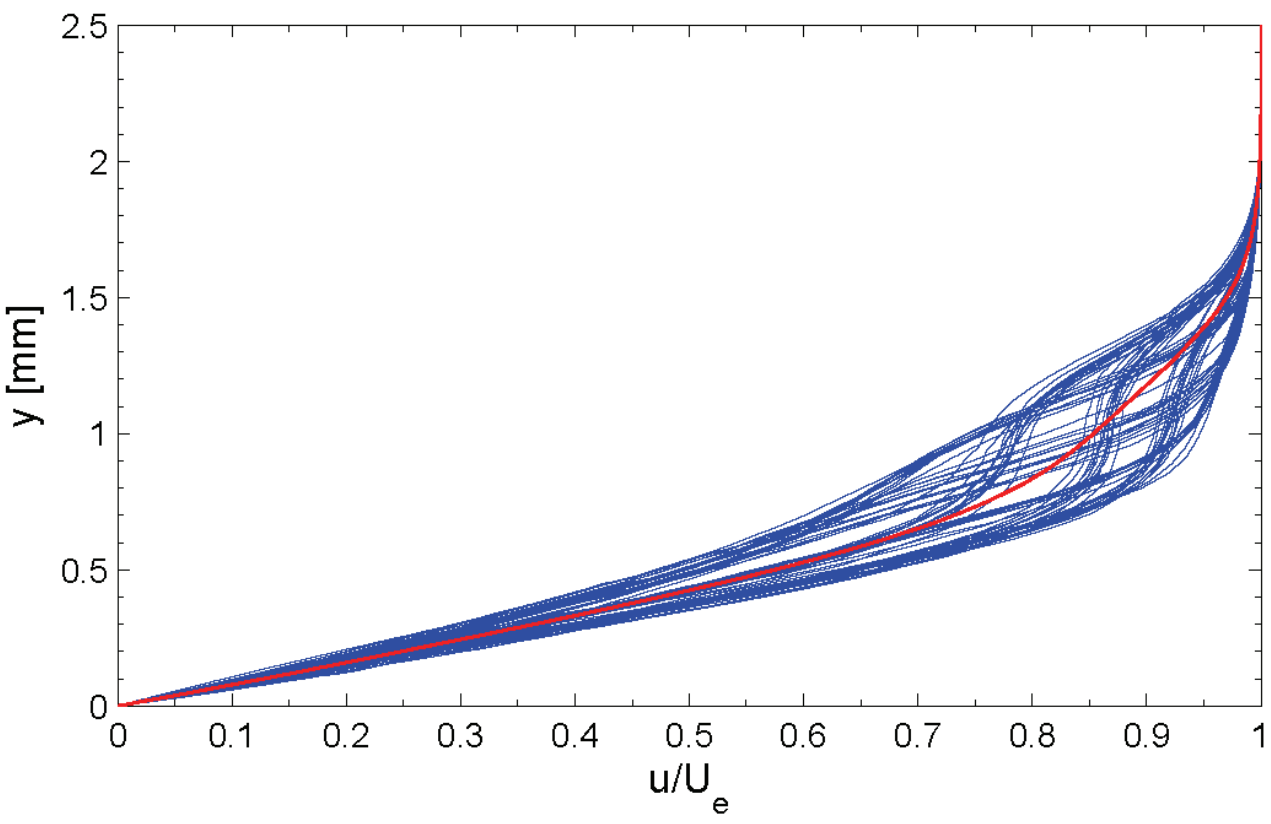

Figure 7-20. Spanwise array of 65 mean-flow boundary-layer profiles spanning $64 \mathrm{~mm}$ at $15 \% x / c . R e_{c}=2.8 \times 10^{6},[42 \mid 6]$ roughness. Mean profile shown in red. 


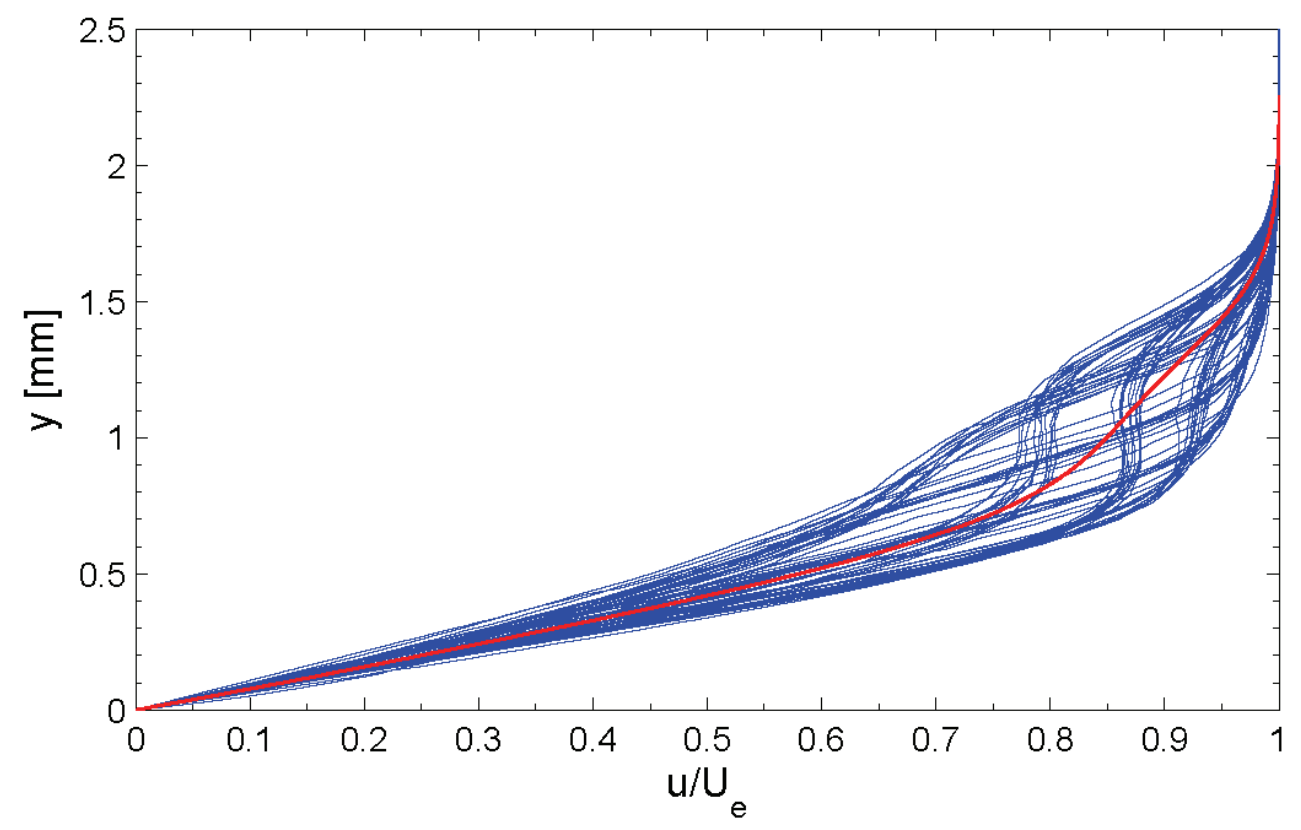

Figure 7-21. Spanwise array of 65 mean-flow boundary-layer profiles spanning $64 \mathrm{~mm}$ at $15 \% x / c . R e_{c}=2.8 \times 10^{6},[56 \mid 6]$ roughness. Mean profile shown in red.

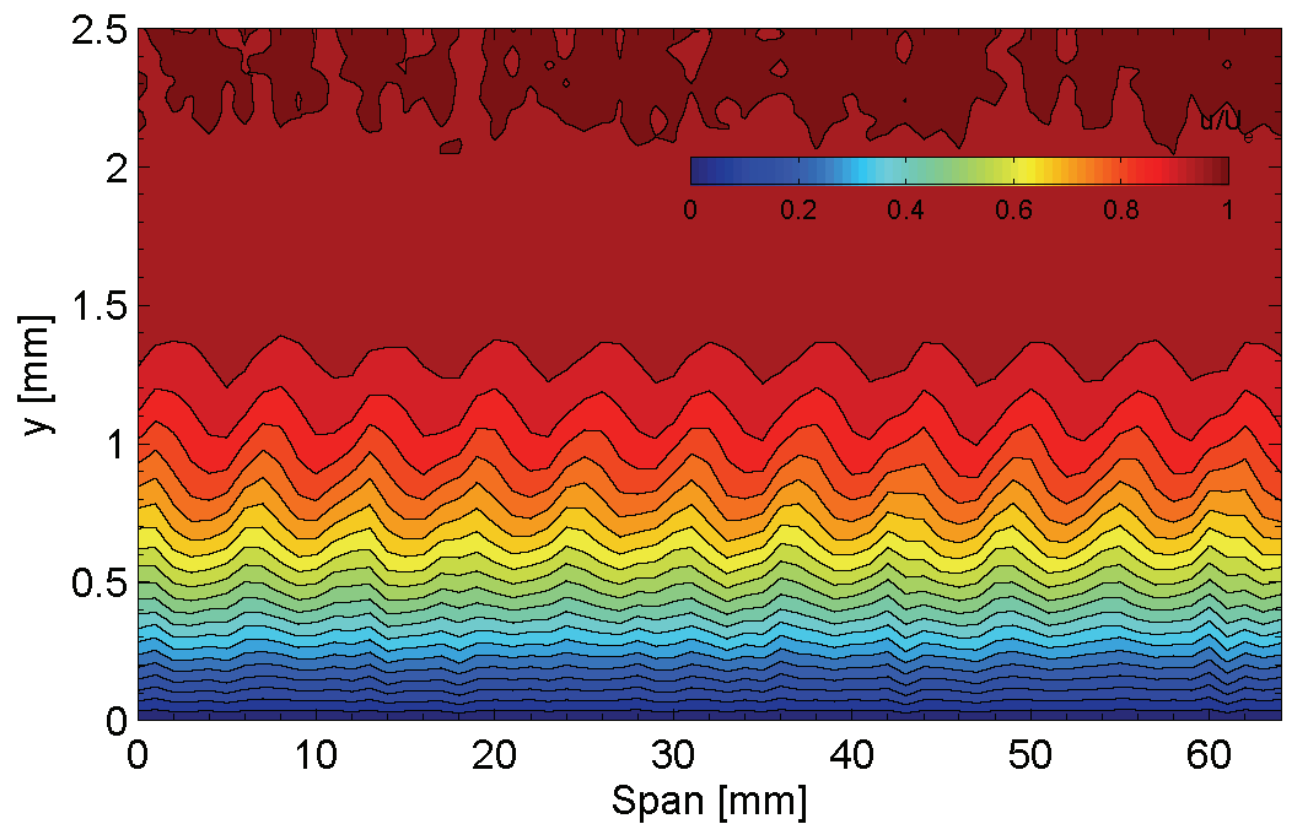

Figure 7-22. Streamwise velocity contours at $15 \% x / c . R e_{c}=2.8 \times 10^{6},[14 \mid 6]$ roughness. 


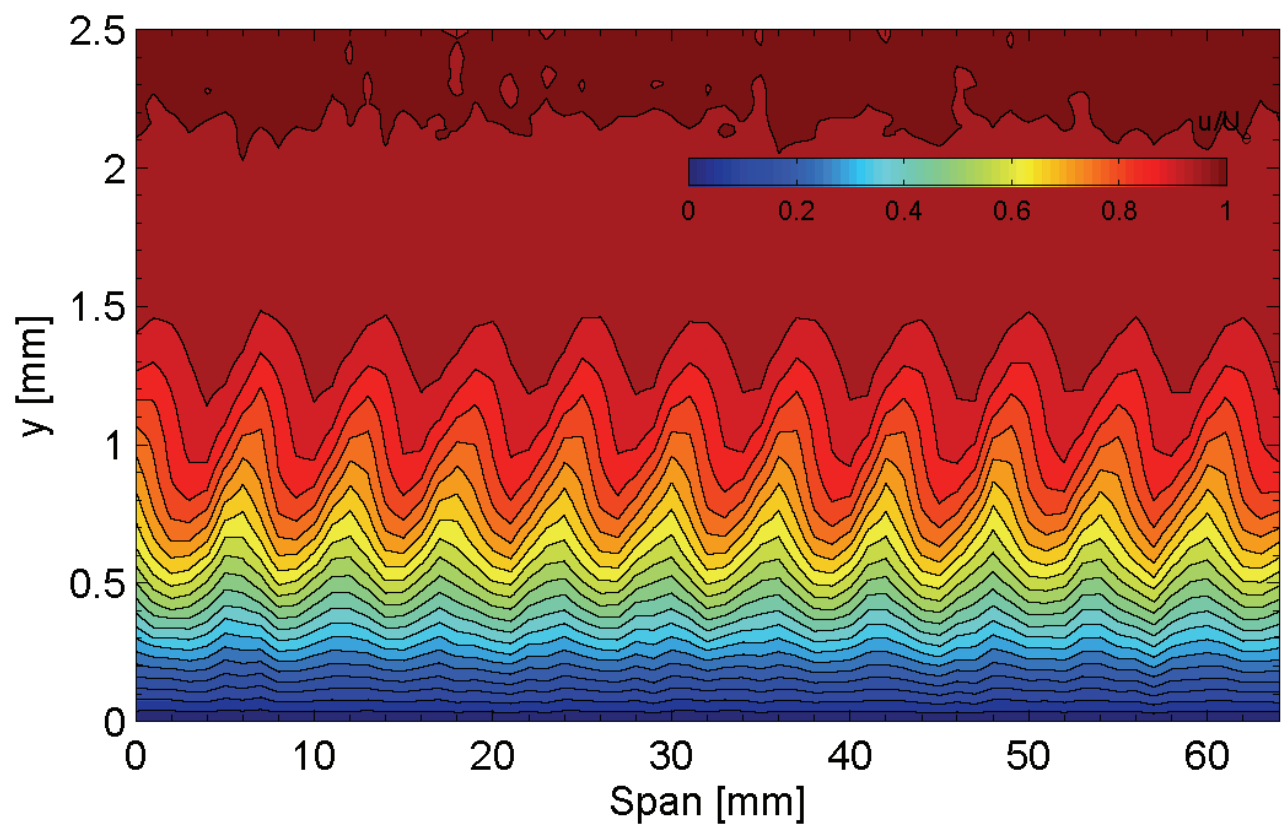

Figure 7-23. Streamwise velocity contours at $15 \% x / c . R e_{c}=2.8 \times 10^{6},[27 \mid 6]$ roughness.

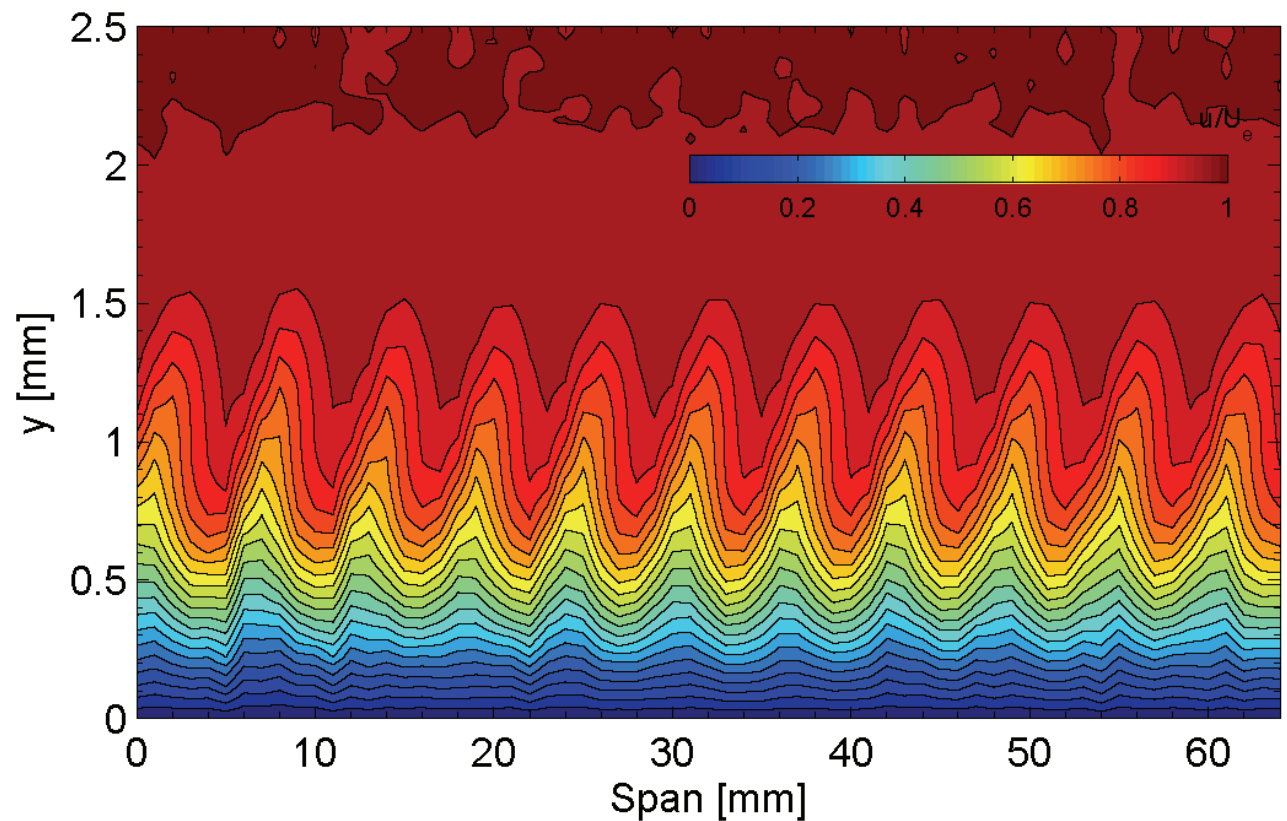

Figure 7-24. Streamwise velocity contours at $15 \% x / c . R e_{c}=2.8 \times 10^{6},[42 \mid 6]$ roughness. 


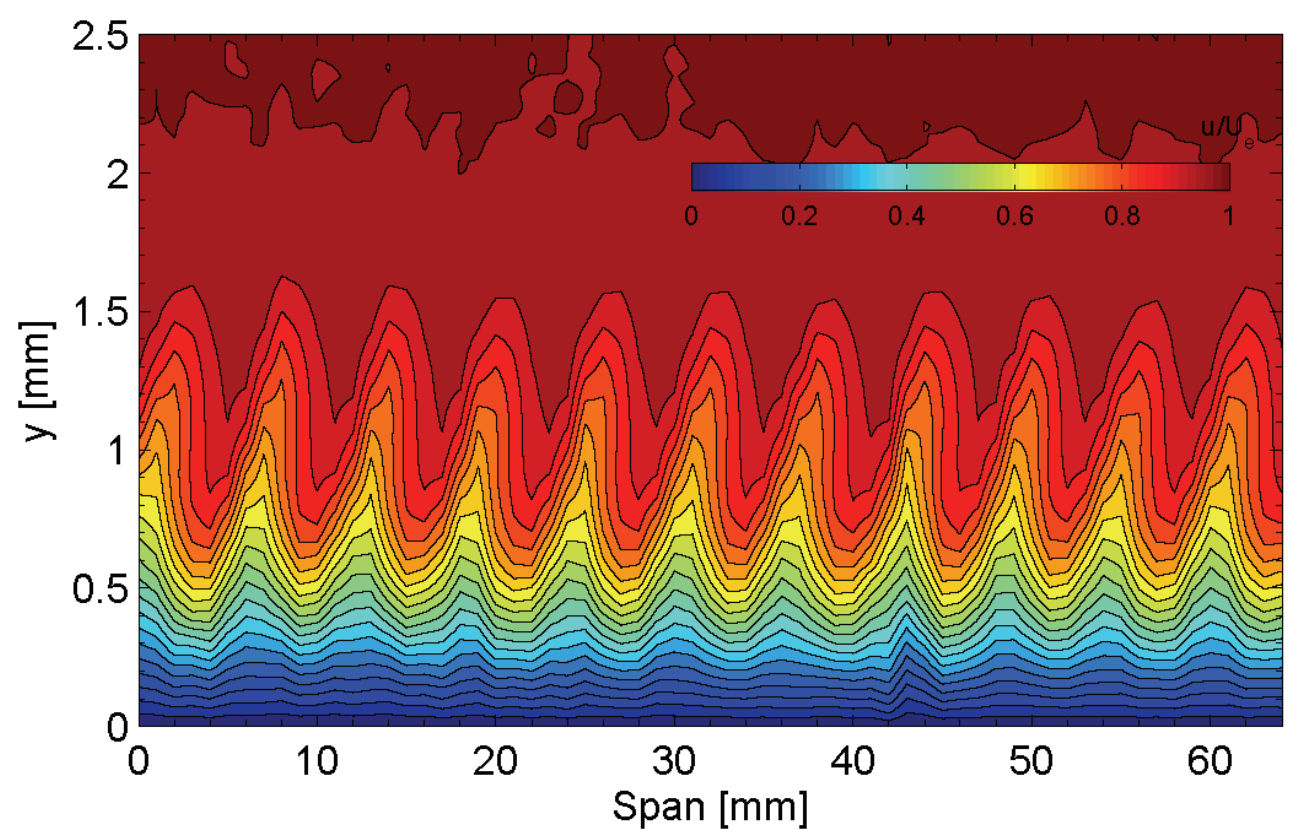

Figure 7-25. Streamwise velocity contours at $15 \% x / c . R e_{c}=2.8 \times 10^{6},[56 \mid 6]$ roughness.

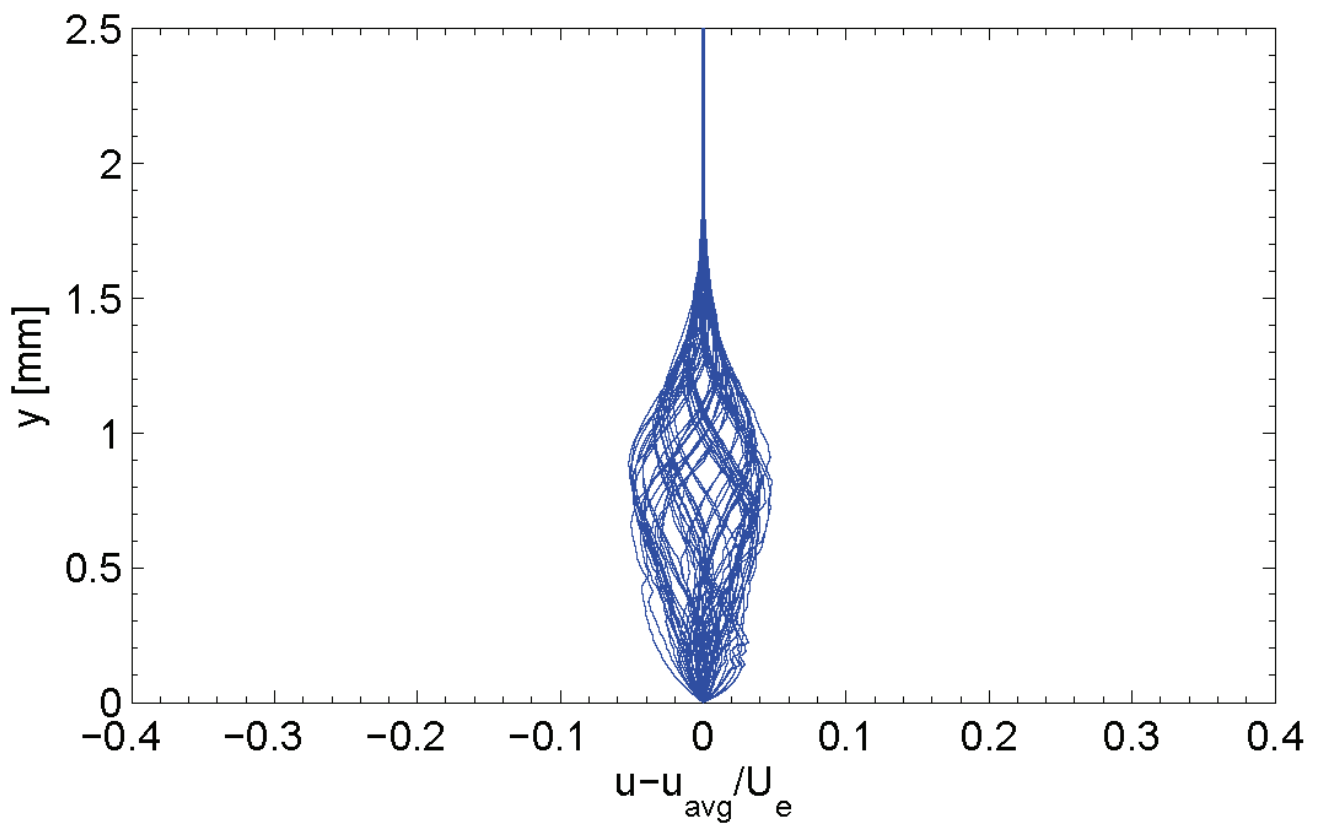

Figure 7-26. Spanwise array of 65 disturbance profiles spanning $64 \mathrm{~mm}$ at $15 \% x / c$. $R e_{c}=2.8 \times 10^{6},[14 \mid 6]$ roughness. 


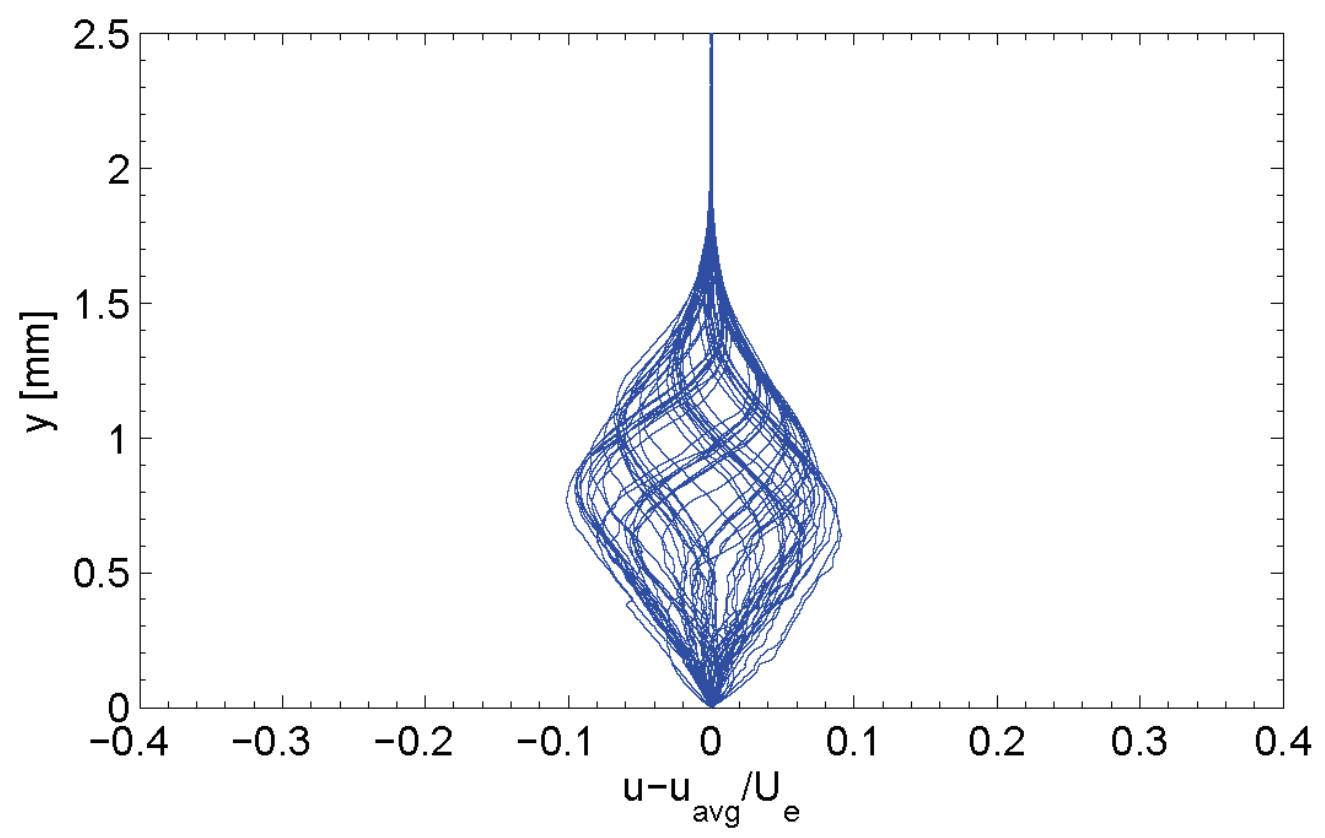

Figure 7-27. Spanwise array of 65 disturbance profiles spanning $64 \mathrm{~mm}$ at $15 \% x / c$. $R e_{c}=2.8 \times 10^{6},[27 \mid 6]$ roughness.

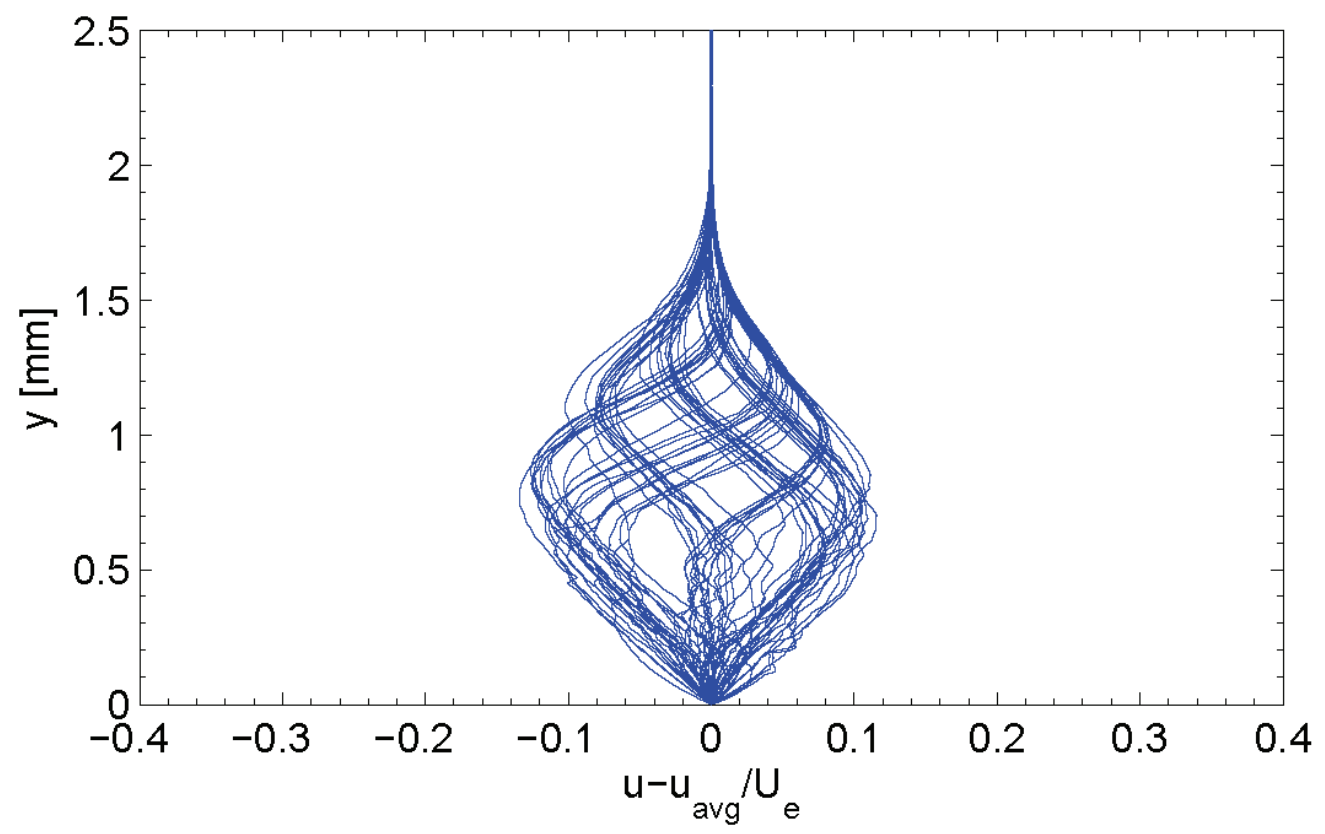

Figure 7-28. Spanwise array of 65 disturbance profiles spanning $64 \mathrm{~mm}$ at $15 \% x / c$. $R e_{c}=2.8 \times 10^{6},[42 \mid 6]$ roughness. 


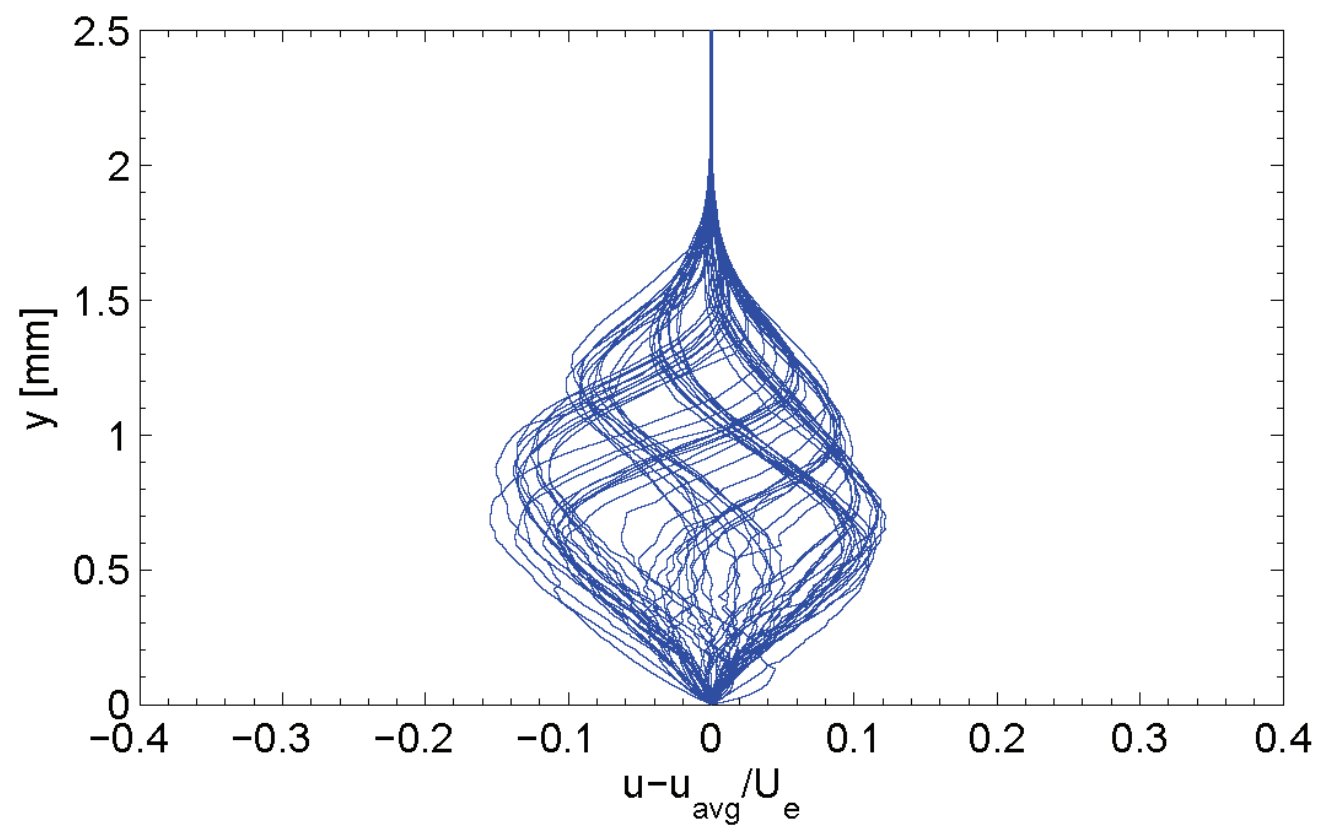

Figure 7-29. Spanwise array of 65 disturbance profiles spanning $64 \mathrm{~mm}$ at $15 \% x / c$. $R e_{c}=2.8 \times 10^{6},[56 \mid 6]$ roughness.

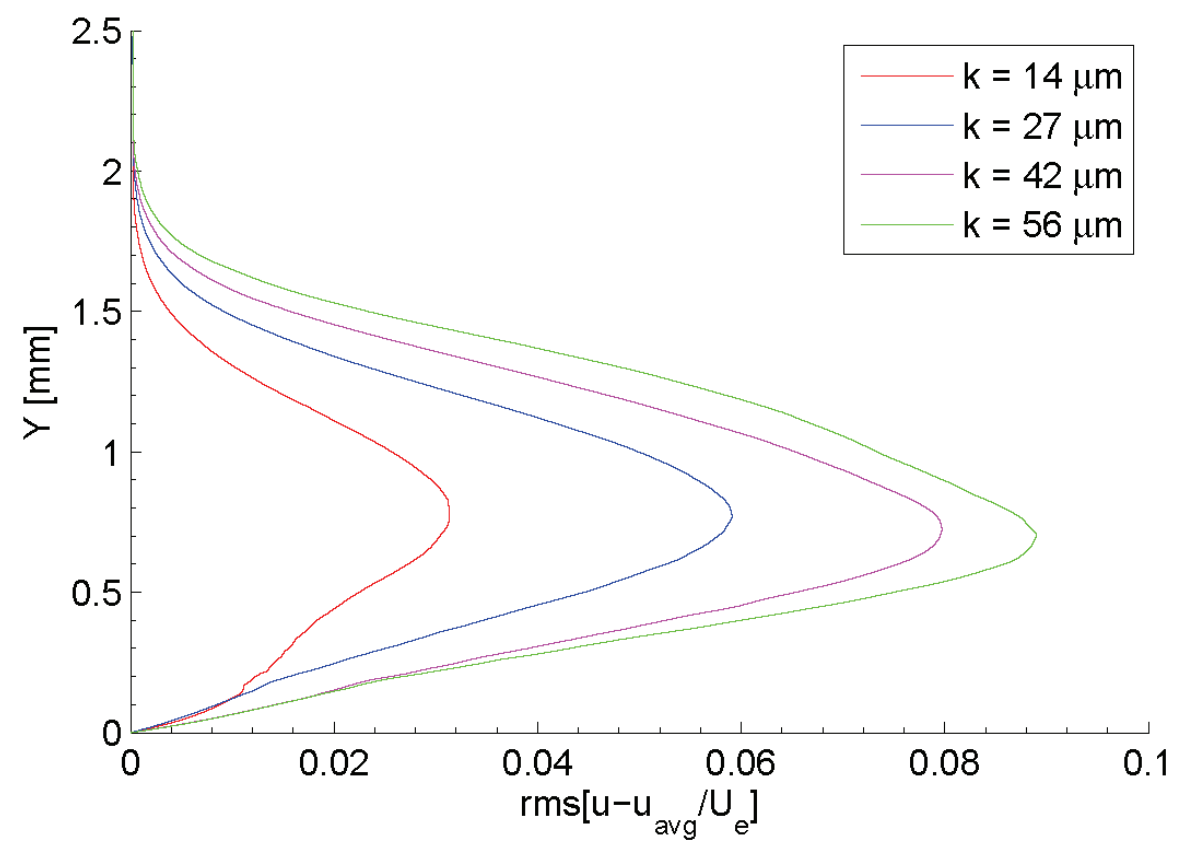

Figure 7-30. Stationary crossflow mode shape at $15 \% x / c . R e_{c}=2.8 \times 10^{6},[k \mid 6]$ roughness. 


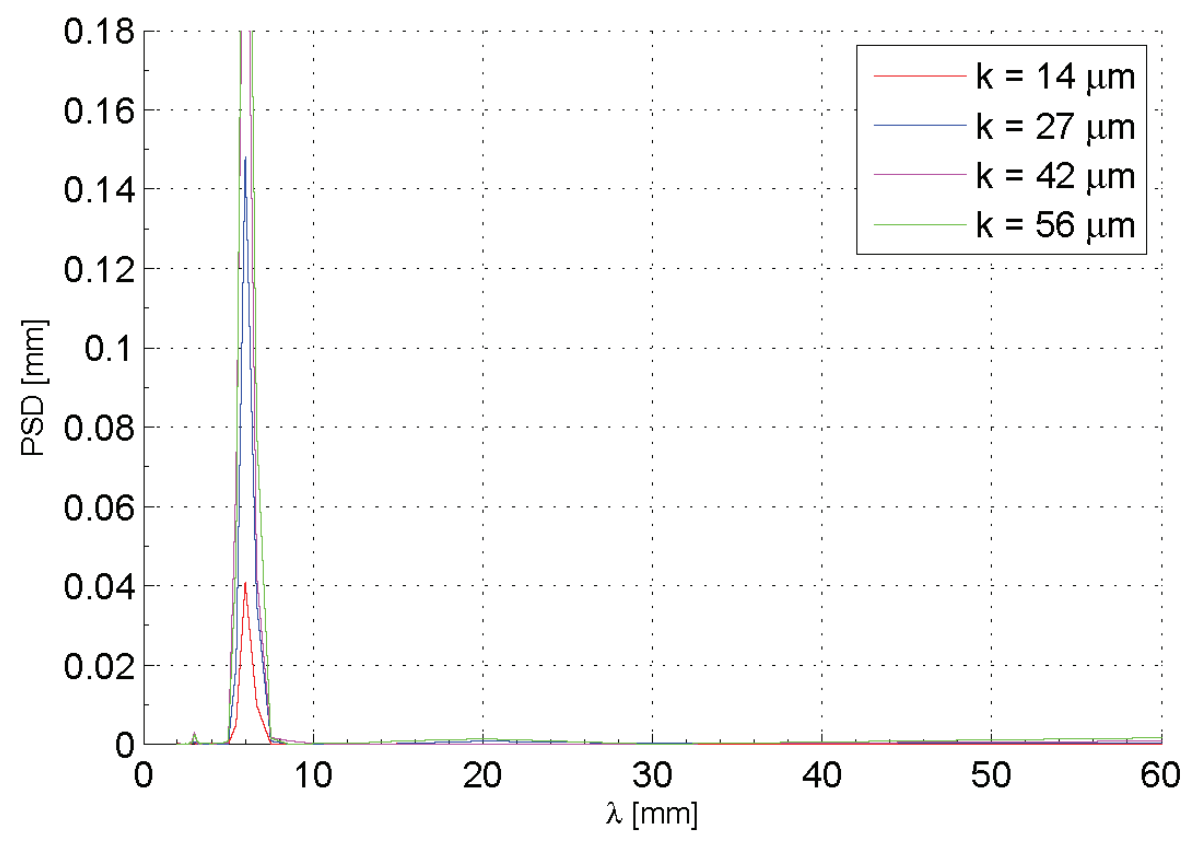

Figure 7-31. Power spectral density of spanwise hotwire scan at $15 \% x / c$. $R e_{c}=2.8 \times 10^{6},[k \mid 6]$ roughness.

$7.3 R e_{c}=3.2 \times 10^{6}$

\subsubsection{Naphthalene Flow Visualization}

For $R e_{c}=3.2 \times 10^{6}$, only two roughness heights were tested after hotwire measurements indicated nonlinear stability activity from the second DRE layer. Figure 7-32 displays the NFV for the two roughness heights. Transition occurs between $43 \%$ and $45 \% \mathrm{x} / \mathrm{c}$ for $k=14 \mu \mathrm{m}$ and moves slightly forward to approximately $40 \% \mathrm{x} / \mathrm{c}$ for $k=27 \mu \mathrm{m}$. A small patch in the lower span region puts transition at $50 \%$ and $45 \% \mathrm{x} / \mathrm{c}$ for $k=14 \mu \mathrm{m}$ and $k=27 \mu \mathrm{m}$ respectively. Natural transition for this Reynolds number is located between $55 \%$ and $60 \% x / c$. Regular streaking spaced at $6 \mathrm{~mm}$ uniformly occurs along the span. These test points follow the same trends as the $R e_{c}=2.8 \times 10^{6}$ case. 
(a)

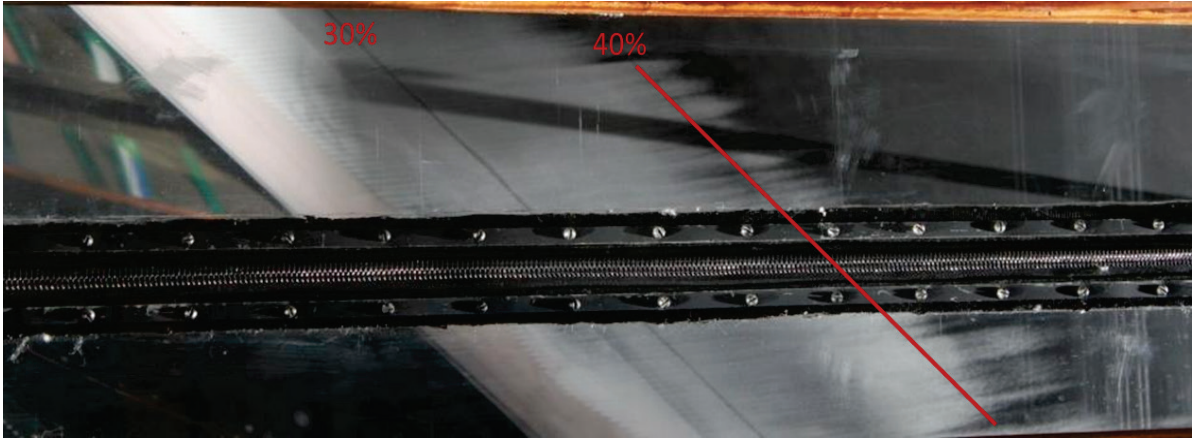

(b)

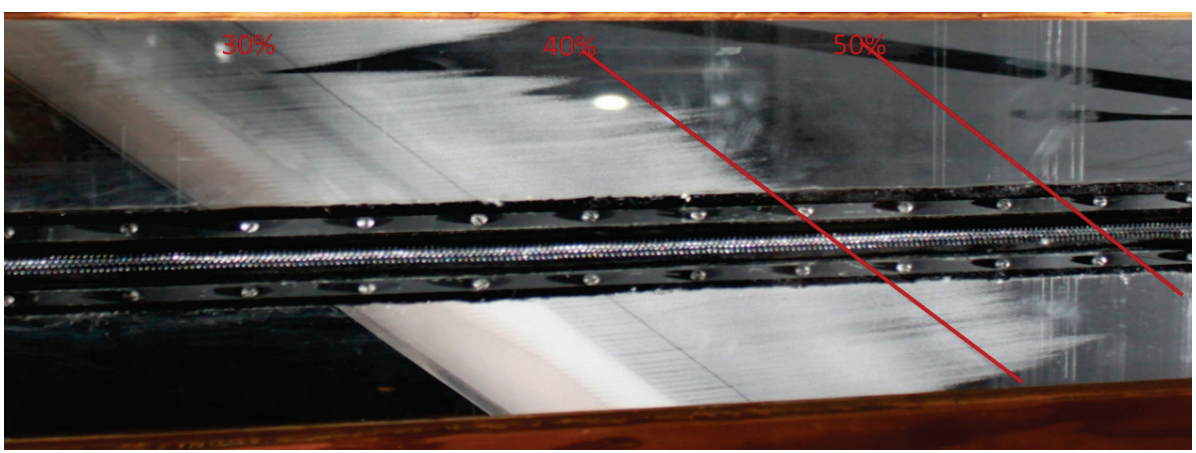

Figure 7-32. NFV with appliqué roughness: $x / c=0.029, d=3 \mathrm{~mm}, \lambda=6 \mathrm{~mm}$, $\operatorname{Re}_{c}=3.2 \times 10^{6}$; (a) $k=14 \mu \mathrm{m}$, (b) $k=27 \mu \mathrm{m}$.

\subsubsection{Hotwire Measurements, $15 \% x / c$}

Figures 7-33 - 7-34, 7-35 - 7-36, 7-37 - 7-38 display the boundary-layer velocity profiles, streamwise velocity contours and disturbance shapes, followed by the mode shapes in Figure 7-39. By the second roughness layer, the velocity profiles are as strongly inflectional as $k=56 \mu \mathrm{m}$ for $R e_{c}=2.8 \times 10^{6}$. Appearance of a secondary lobe occurs at $k=27 \mu \mathrm{m}$. The resulting disturbance amplitudes are shown in Table 7-3. Hotwire scans for the third and fourth layers were not taken at this test condition since they would have no bearing on the receptivity measurements. 
Table 7-3. Maximum and normalized stationary crossflow disturbance amplitudes at $15 \% x / c, \operatorname{Re}_{c}=3.2 \times 10^{6},[k \mid 6]$ roughness.

\begin{tabular}{|c|c|c|}
\hline$k[\mu \mathrm{m}]$ & {$\left[\left(u-u_{\text {avg }}\right) / U_{e}\right]_{\mathrm{rms}, \max }$} & {$\left[\left(u-u_{\text {avg }}\right) / U_{e}\right]_{\mathrm{rms}, k / k o}$} \\
\hline 14 & 0.0313 & 1.00 \\
\hline 27 & 0.0591 & $0.98^{*}$ \\
\hline 42 & - & - \\
\hline 56 & - & - \\
\hline
\end{tabular}

*Indicates nonlinear stability present

Figure 7-40 displays the PSD for these two roughness cases. Again, the $6 \mathrm{~mm}$ mode is dominant. A small peak at $3 \mathrm{~mm}$ also appears for $k=27 \mu \mathrm{m}$, but its amplitude is negligible compared to the $6 \mathrm{~mm}$ mode.

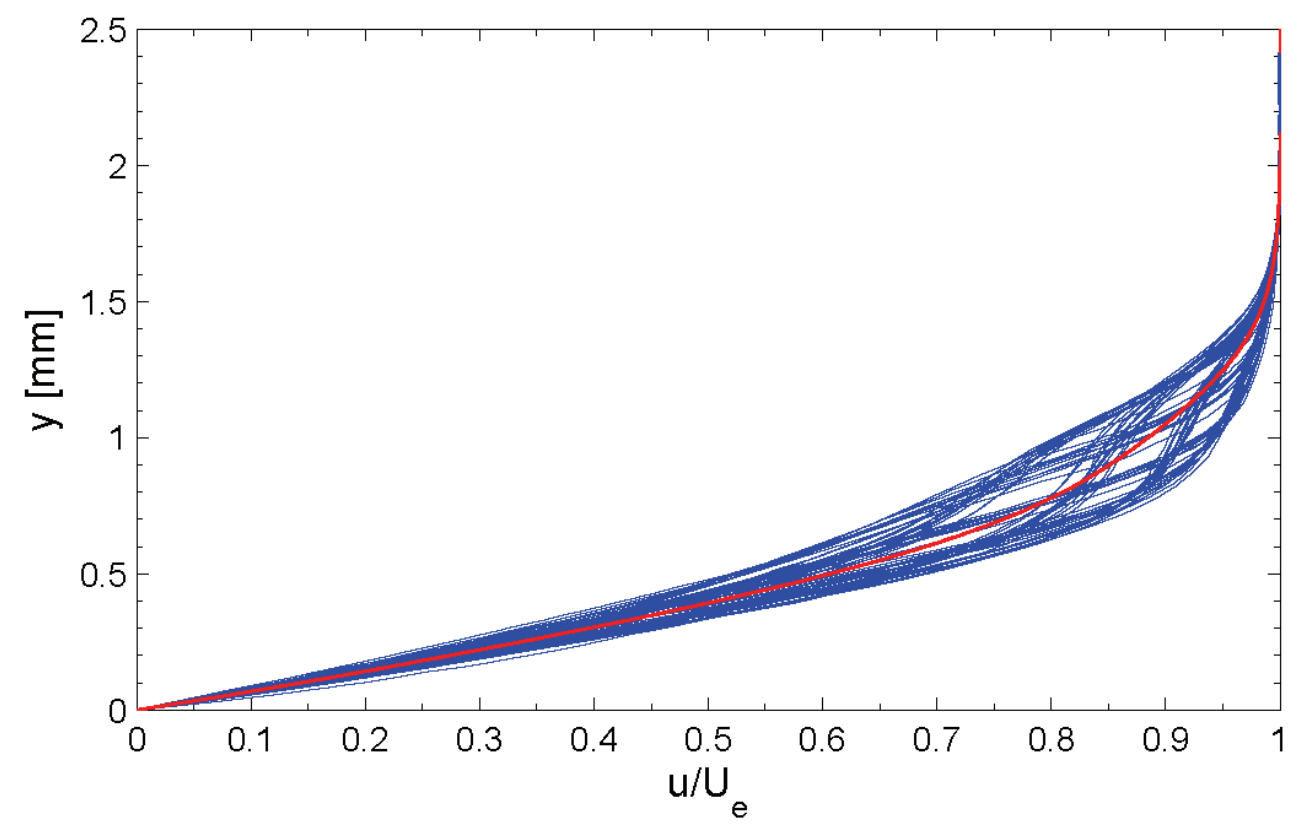

Figure 7-33. Spanwise array of 65 mean-flow boundary-layer profiles spanning $64 \mathrm{~mm}$ at $15 \% x / c . R e_{c}=3.2 \times 10^{6},[14 \mid 6]$ roughness. Mean profile shown in red. 


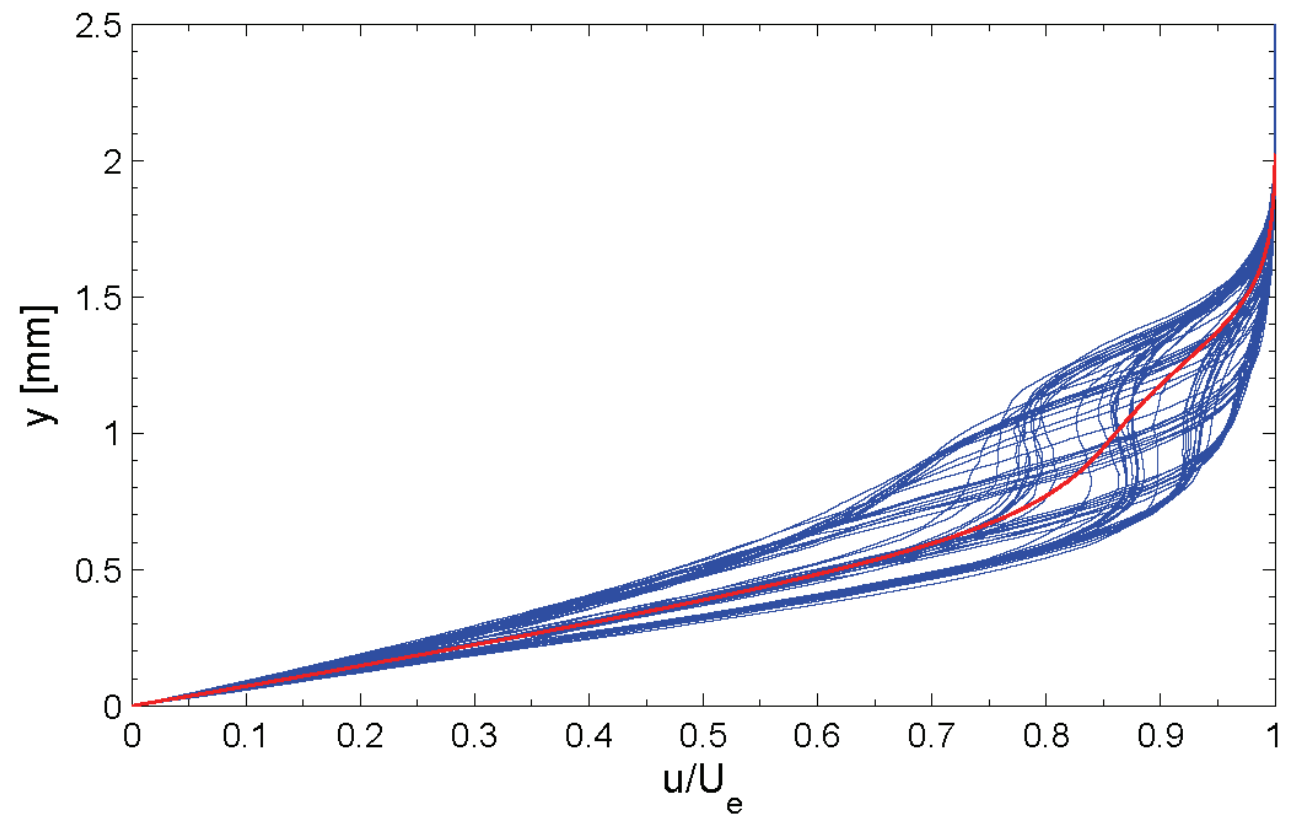

Figure 7-34. Spanwise array of 65 mean-flow boundary-layer profiles spanning $64 \mathrm{~mm}$ at $15 \% x / c . R_{c}=3.2 \times 10^{6},[27 \mid 6]$ roughness. Mean profile shown in red.

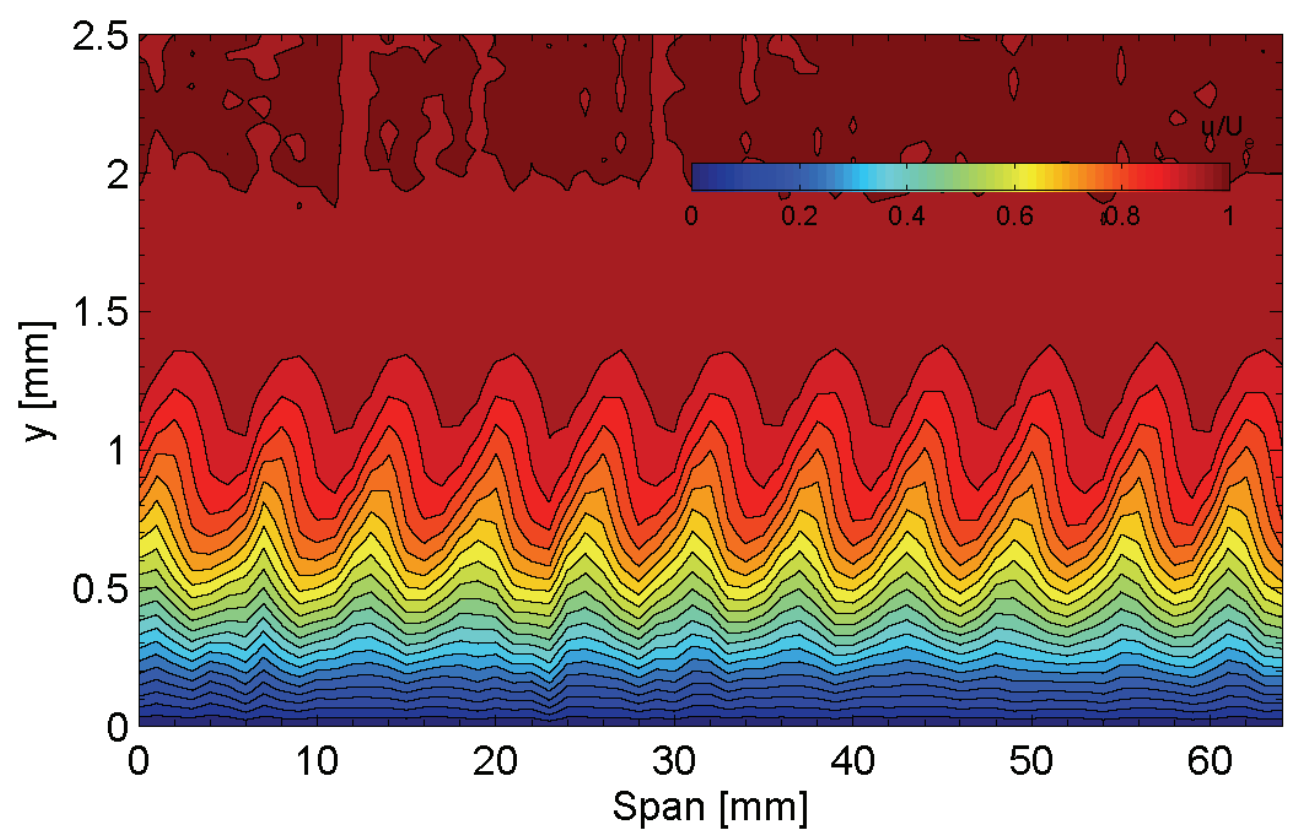

Figure 7-35. Streamwise velocity contours at $15 \% x / c . R e_{c}=3.2 \times 10^{6},[14 \mid 6]$ roughness. 


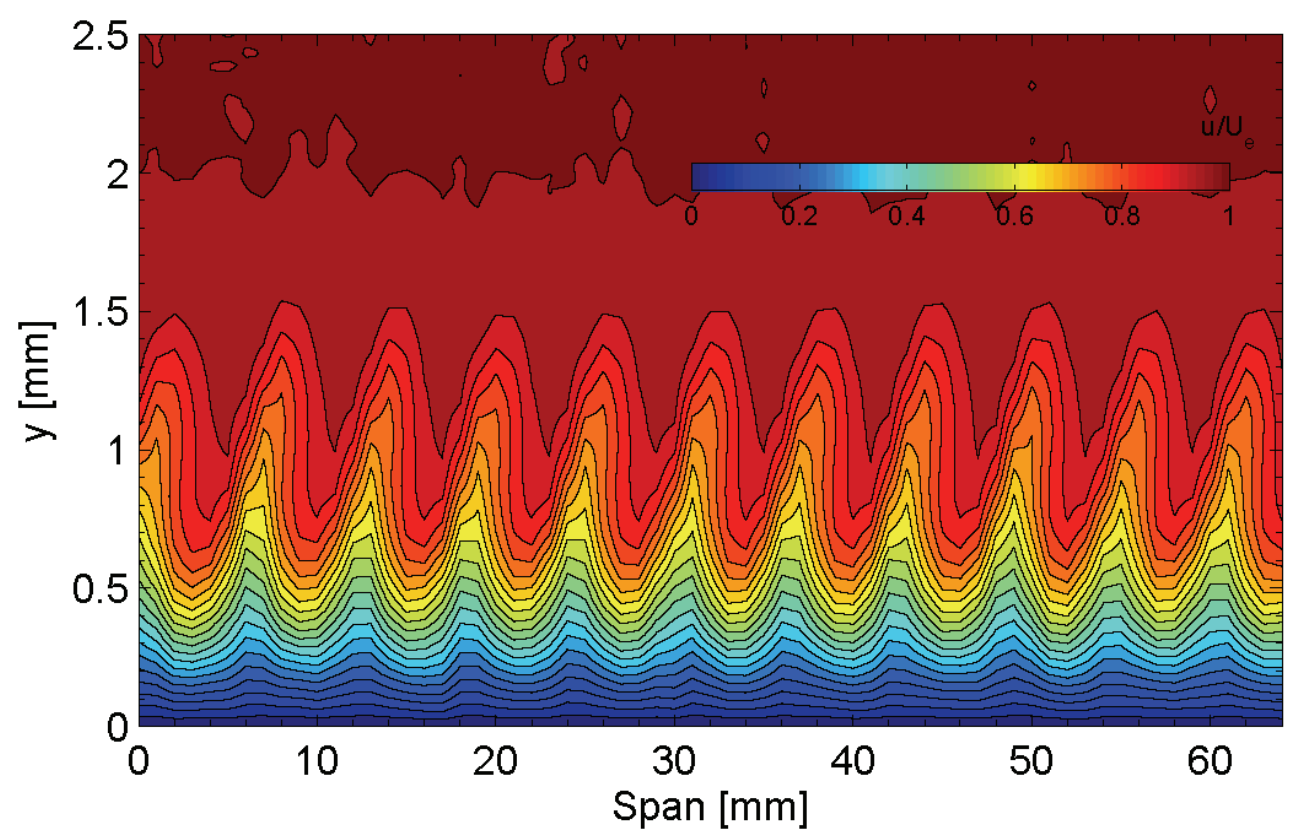

Figure 7-36. Streamwise velocity contours at $15 \% x / c . R e_{c}=3.2 \times 10^{6},[27 \mid 6]$ roughness.

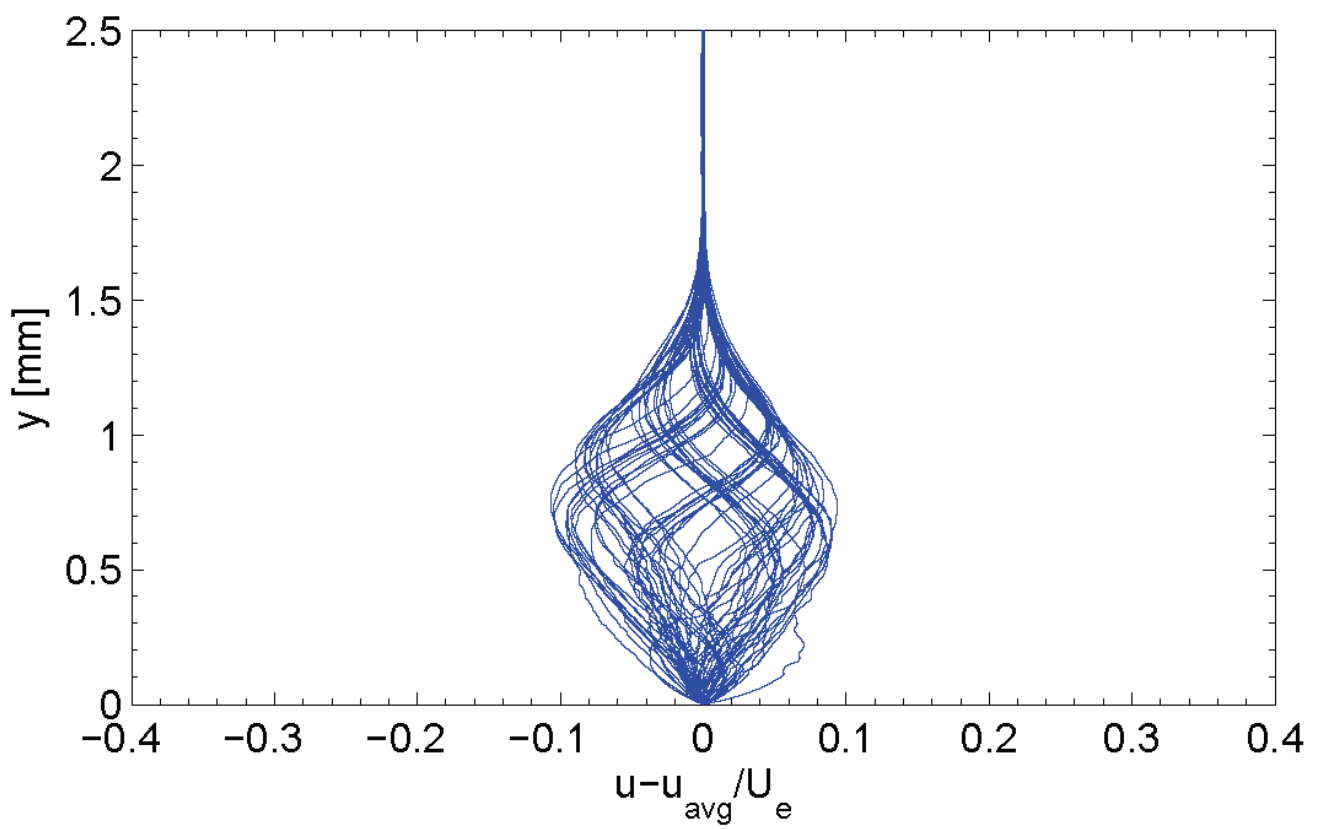

Figure 7-37. Spanwise array of 65 disturbance profiles spanning $64 \mathrm{~mm}$ at $15 \% x / c$. $R e_{c}=3.2 \times 10^{6},[14 \mid 6]$ roughness. 


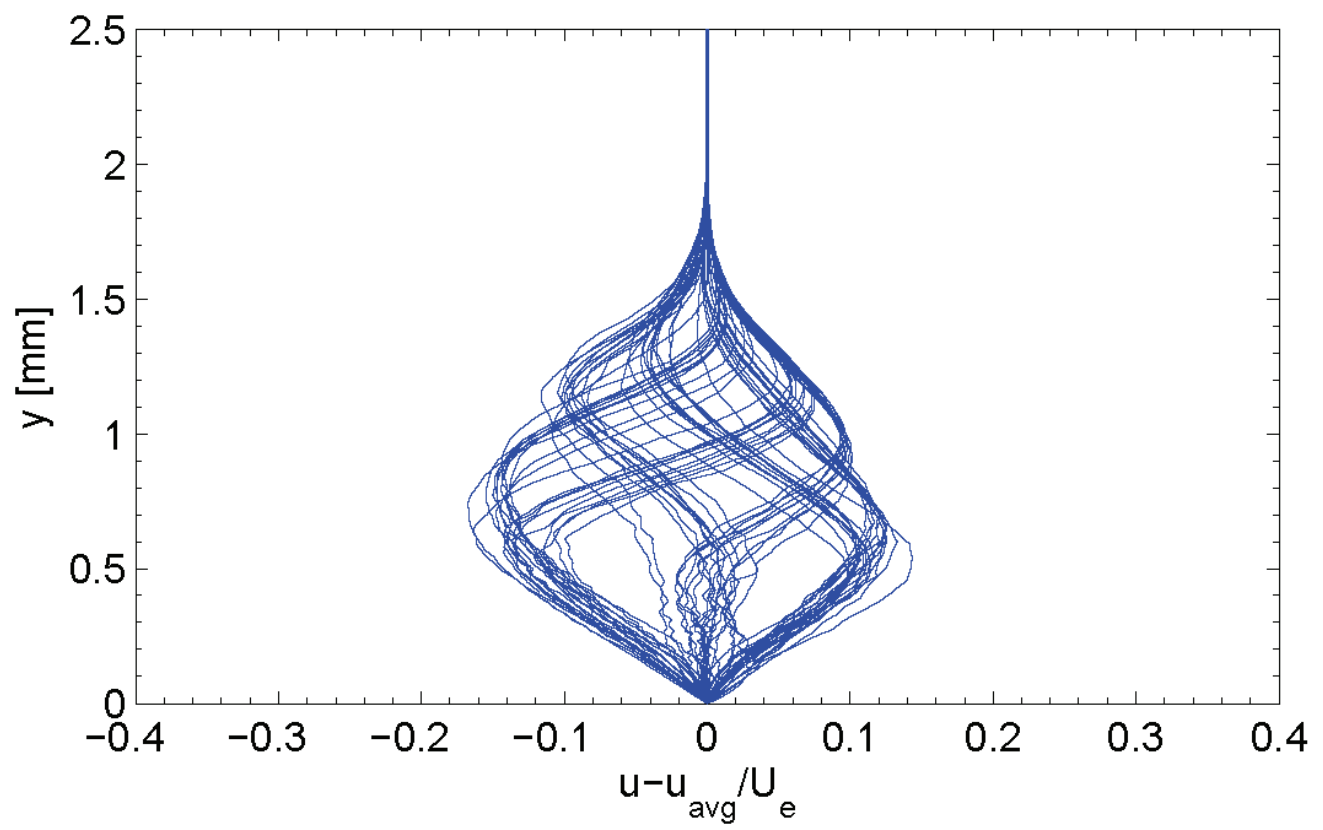

Figure 7-38. Spanwise array of 65 disturbance profiles spanning $64 \mathrm{~mm}$ at $15 \% x / c$. $R e_{c}=3.2 \times 10^{6},[27 \mid 6]$ roughness.

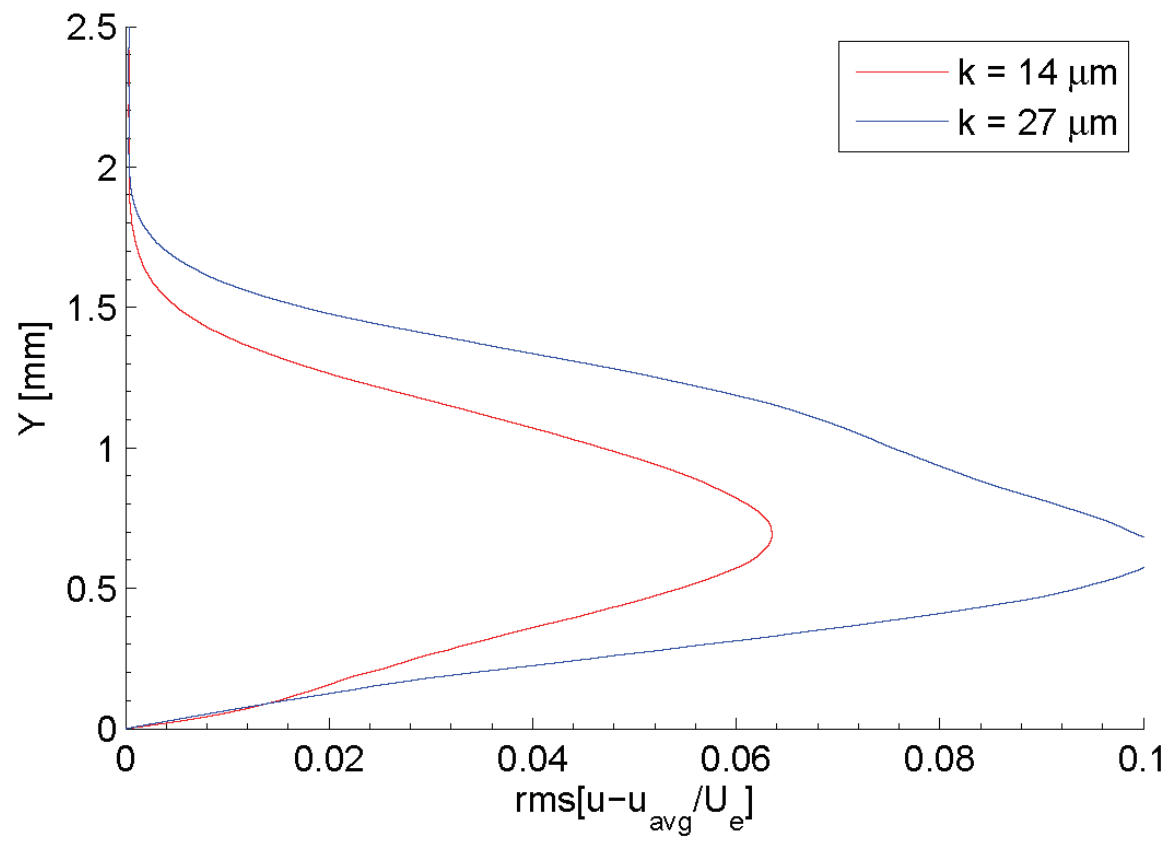

Figure 7-39. Stationary crossflow mode shape at $15 \% x / c . R_{c}=3.2 \times 10^{6},[k \mid 6]$ roughness. 


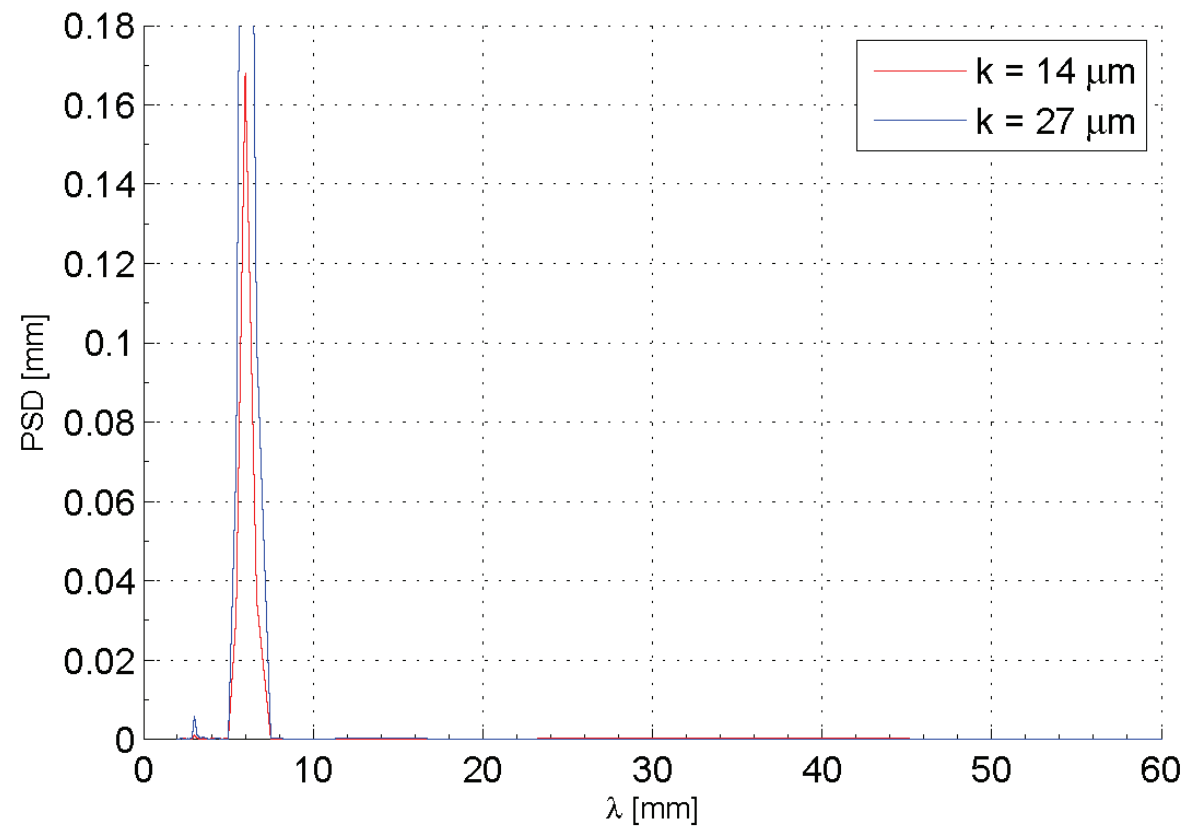

Figure 7-40. Power spectral density of spanwise hotwire scan at $15 \% x / c$. $R e_{c}=3.2 \times 10^{6},[k \mid 6]$ roughness. 


\section{RESULTS- PART IV: EFFECT OF INCREASING FREESTREAM TURBULENCE}

A review of the NFV results in the UWT and KSWT indicate a number of differences in observed results and trends. In Section 5, a comparison of transition location for the ASU(67)-0315 installed in the UWT and KSWT indicated that transition was $20 \%-25 \%$ aft in the KSWT configuration. One of the possible explanations supplied was the effect of decreased turbulence levels in the tunnel. At TAMU, transition moves upstream as critically-spaced DRE height is increased. This result directly contrasts the observation at ASU that transition location did not substantially change as roughness height increased. Additionally, in all the cases at ASU for both the NLF(2)-0415 and ASU(67)-0315, the transition front for stationary crossflow dominated transition was described as an irregular sawtooth pattern. In the KSWT, the sawtooth pattern remains, but it is much more uniform. Irregular wedges are observed only in instances where roughness height is very large (Figure 6-1d). Minor alterations to the freestream turbulence were attempted to determine what, if any, of these differing observations were the result of different freestream turbulence levels. The following sections describe how turbulence levels were increased and the resulting impact on NFV and hotwire measurements. 


\subsection{Turbulence Generation}

Recall that flow quality results discussed in Section 5 indicate that freestream turbulence levels in the speed range of interest for this experiment are up to $25 \%$ lower than measured in the UWT. Based on the comments of Dagenhart (1992) also discussed in Section 5.2.4, the difference in turbulence levels with swept-wings and wall liners installed may actually be upwards of 50\% - 75\%. Although these are significant changes, it should be stressed that in both wind tunnels, the freestream environment is lowdisturbance and that in all cases, stationary crossflow waves dominated transition.

Kapton tape was used to generate additional turbulence in the freestream. The $6.4 \mathrm{~mm}(0.25 \mathrm{in})$ wide tape was first placed on the downstream side of screen seven. Spaced $40.5 \mathrm{~mm}$ from center-to-center, tape was only applied in the horizontal direction (along $Y$ in global test section coordinates). The spacing was chosen such that once passing through the contraction, the equivalent spacing would be $12 \mathrm{~mm}$, the most unstable wavelength. To avoid excessive blockage on the screen, tape extended to only $2 / 3$ of the screen width with one end located next to the south wall (test-side in the test section). Additionally, only the minimum number of needed strips covering the span of the hotwire scan was applied. Figure 8-1 shows an example of the Kapton tape applied to the last screen. 


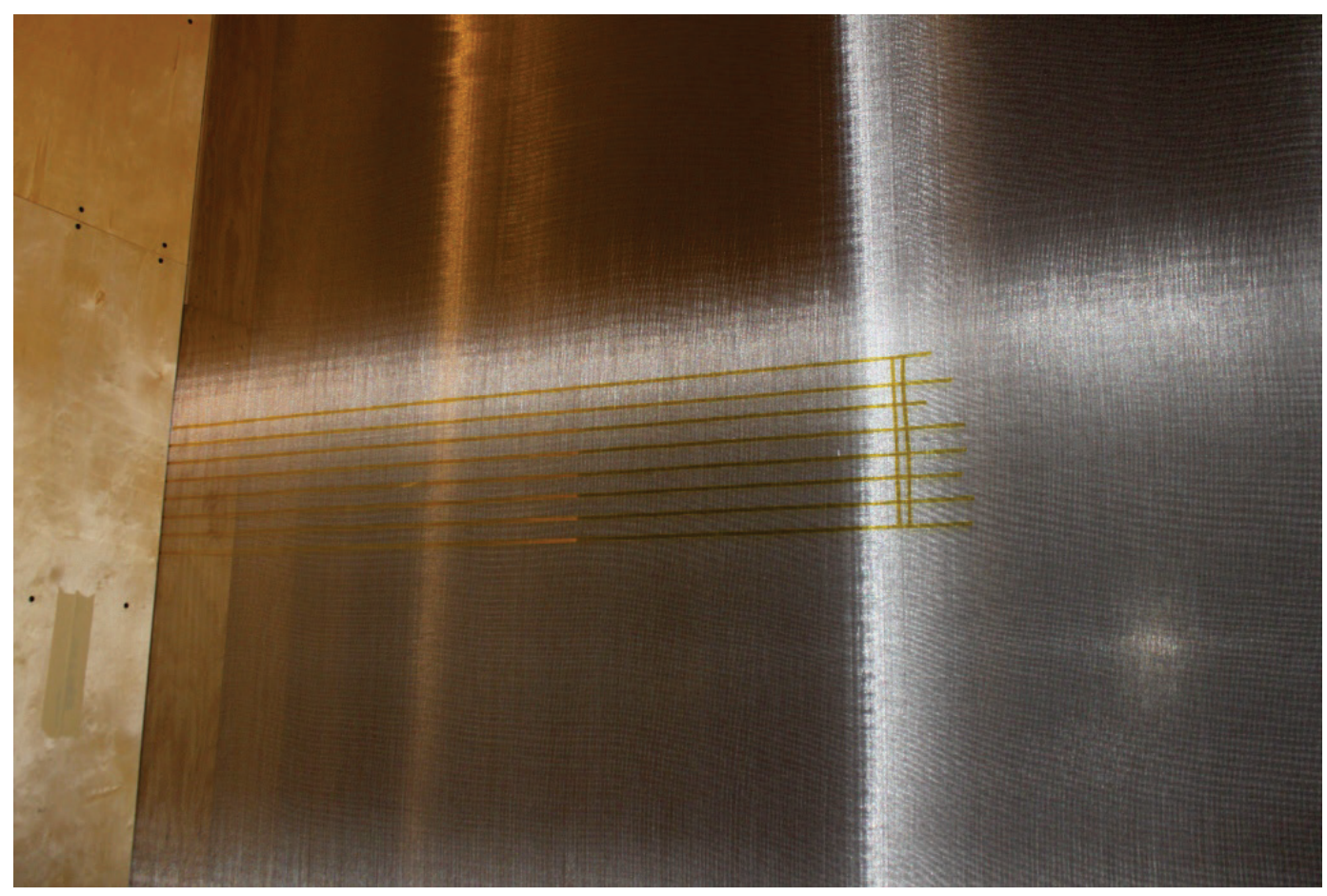

Figure 8-1. Tape on downstream side of screen seven (view is looking upstream).

A crosswire, set-up to acquire streamwise and wall-normal velocities was located at $(X, Y, Z)=(0.261,0.822,0.000)$. Disturbance intensities were measured in several different span locations (along $Z$ ) to determine the influence of the taped turbulence grid. Sampling time and rate, filtering and calibration procedures from the initial flow quality measurements discussed in Section 5 were also used here. Running at $15 \mathrm{~m} / \mathrm{s}$, the transverse velocity fluctuation, $v^{\prime}{ }_{r m s} / U_{o}$ ranged from $0.271 \%-0.335 \%$ and averaged $0.305 \%$, more than a factor of ten larger than without the tape. Figure $8-2$ displays the spectra resulting from this configuration. A large amount of broadband content is present for frequencies less than $100 \mathrm{~Hz}$. Removing the tape and retaking the measurements immediately reduced the turbulence intensity to previous flow quality measurement levels of $0.02 \%$ and a power spectrum similar to those shown in Figure 5-9, confirming 
that the large fluctuations were indeed a result of the tape, rather than, for example, a calibration error. This increase in turbulence was too large to meet the planned objectives. At these values, issues such as traveling waves may become a significant factor.

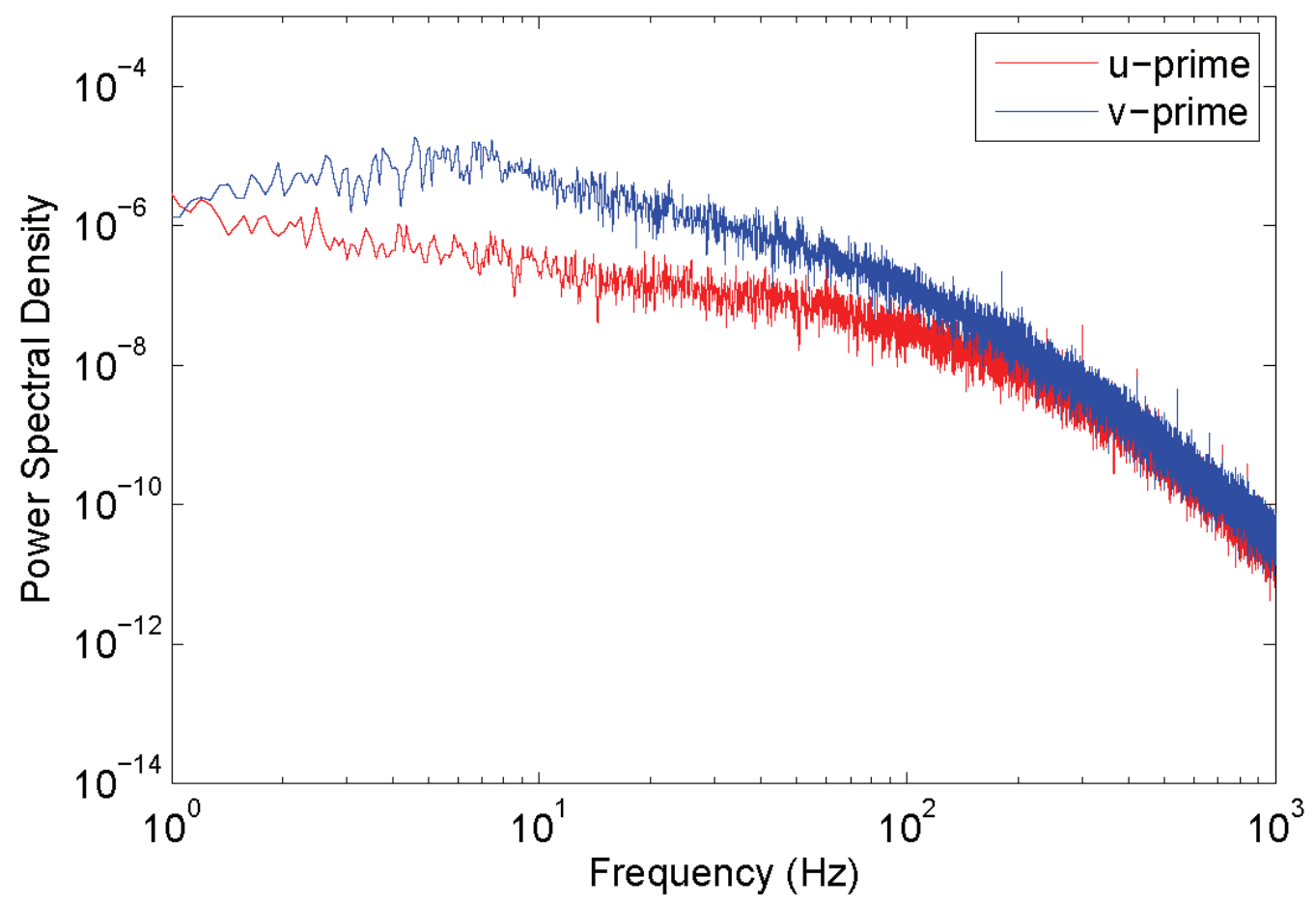

Figure 8-2. Power spectral density of $u^{\prime}$ and $v^{\prime}$ at $15 \mathrm{~m} / \mathrm{s}$. Tape applied to screen seven.

A second attempt at modestly increasing turbulence levels was made with the same materials and spacing, but with the tape located on the upstream side of screen one instead (Figure 8-3). Turbulence measurements taken at a freestream speed of $15 \mathrm{~m} / \mathrm{s}$ were now a constant $0.034 \%$ at all measurement points. It is understandable that turbulence intensities generated by tape upstream of the screen cage produce more 
uniform velocity fluctuations. The six screens following the first tend to average-out large differences in the wake behind the tape. An unintended benefit of this exercise was a demonstration of the impact a well-designed screen cage can have on the freestream disturbance environment. Turbulence introduced before the screens reduces the levels from $0.305 \%$ down to $0.034 \%$. The final test configuration increased turbulence intensity from an average of $0.02 \%$ to $0.034 \%$. Figure $8-4$ shows the PSD from one test point. A limited number of NFV and hotwire scans were obtained for this freestream condition. For all cases, the DRE configuration is fixed with a wavelength spacing of $12 \mathrm{~mm}$ and a roughness height of $36 \mathrm{~mm}$. Since only a few measurement points were taken, results are discussed together rather than by Reynolds number.

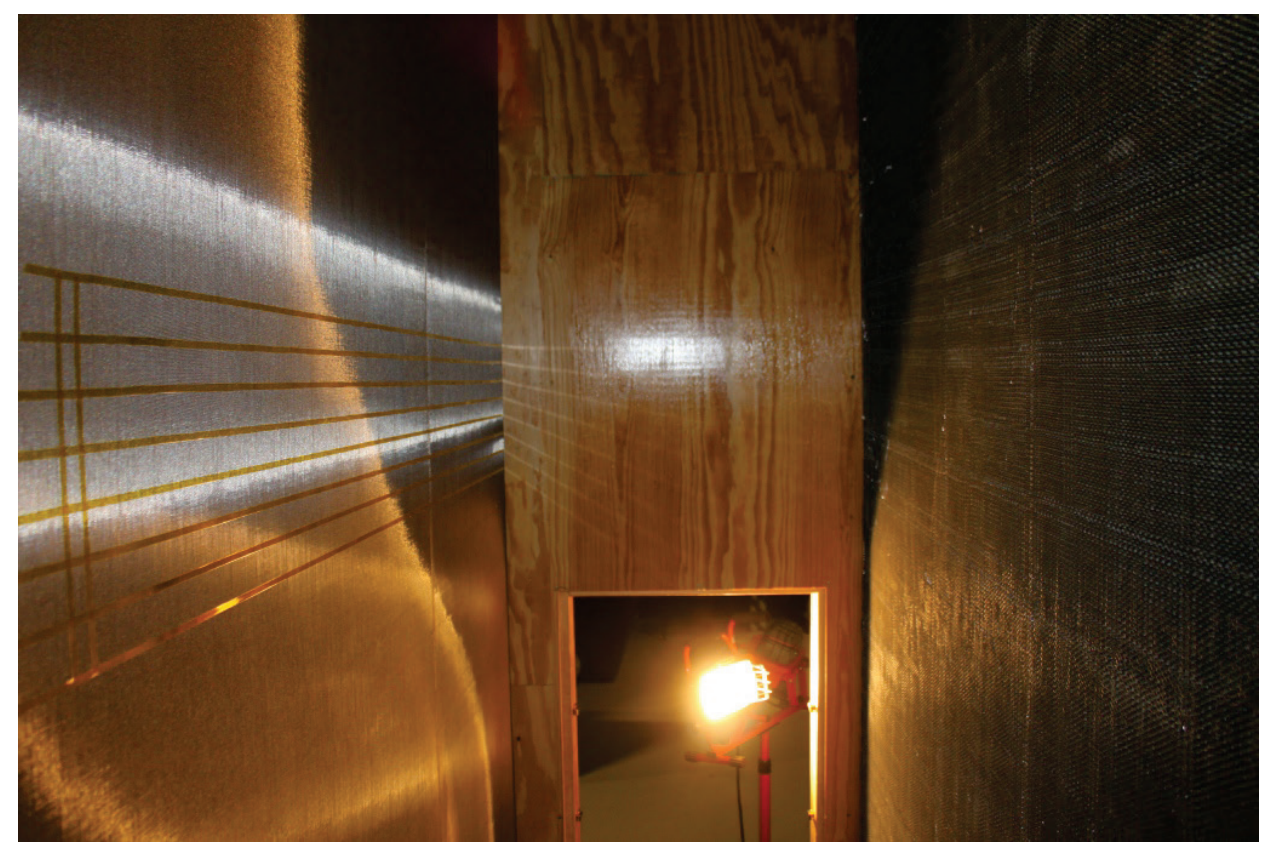

Figure 8-3. Tape on upstream side of screen one (flow moves right to left from this angle). 


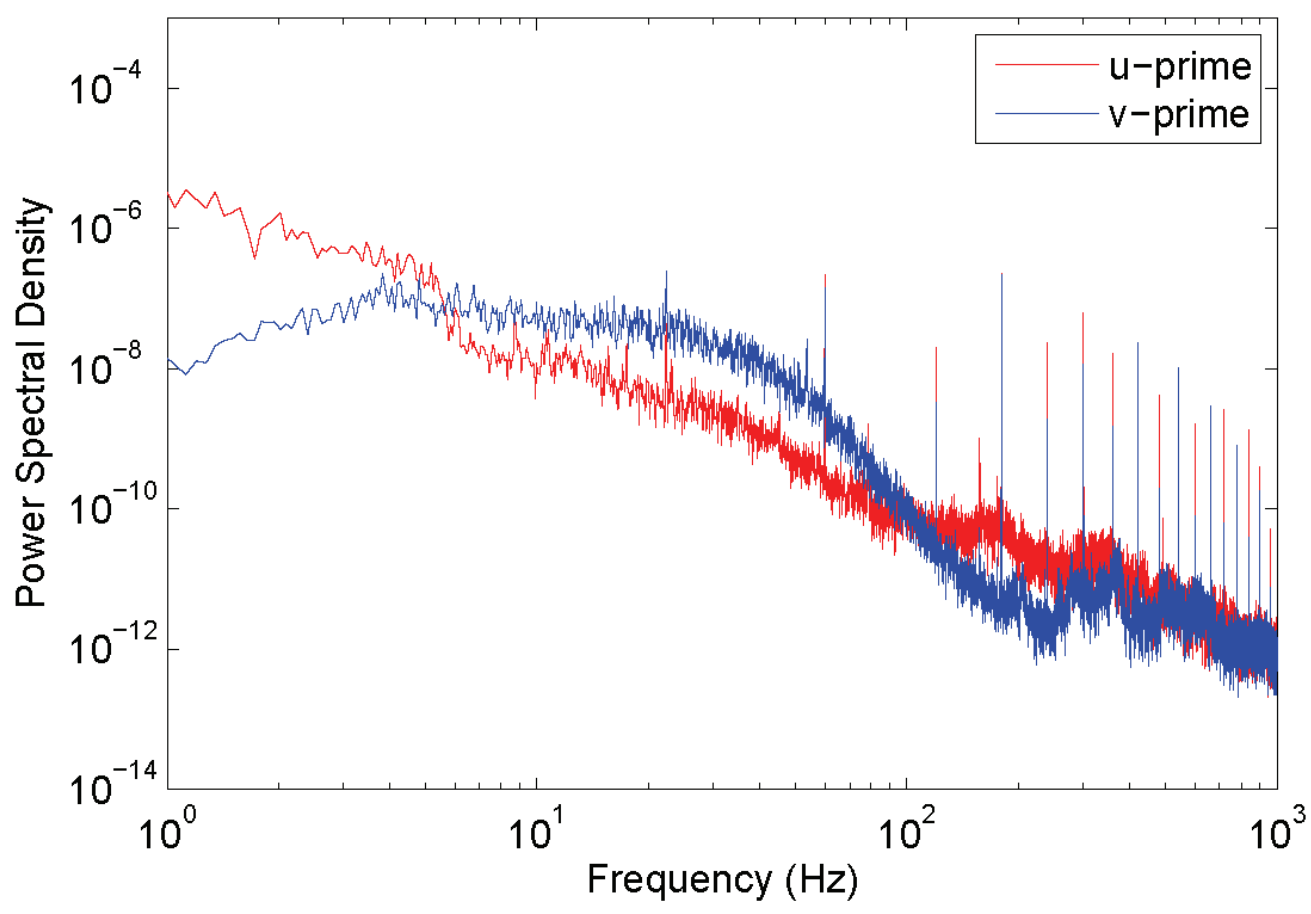

Figure 8-4. Power spectral density of $u^{\prime}$ and $v^{\prime}$ at $15 \mathrm{~m} / \mathrm{s}$. Tape applied to screen one.

\subsection{Naphthalene Flow Visualization}

Figures 8-5 and 8-6 show NFV results for $R e_{c}=2.4 \times 10^{6}$ and $3.2 \times 10^{6}$ with and without the increased turbulence levels. Flow visualization without the tape was completed immediately after the higher turbulence case to ensure that operating conditions were as similar as possible. For both Reynolds numbers, it appears as though transition is delayed when freestream turbulence is increased. It was expected that increasing turbulence would increase initial disturbance amplitudes, causing transition to occur earlier. Increasing the turbulence seems to have two other effects. First, at $R e_{c}=3.2 \times 10^{6}$ the once uniform transition front becomes much more ragged with increased turbulence, more closely resembling the patterns observed at the UWT. 
Second, the $6 \mathrm{~mm}$ streaking originally observed in Figure 6-56 is now more pronounced.

Figure 8-7 provides a comparison of the $6 \mathrm{~mm}$ streaking with and without the increased turbulence.

(a)

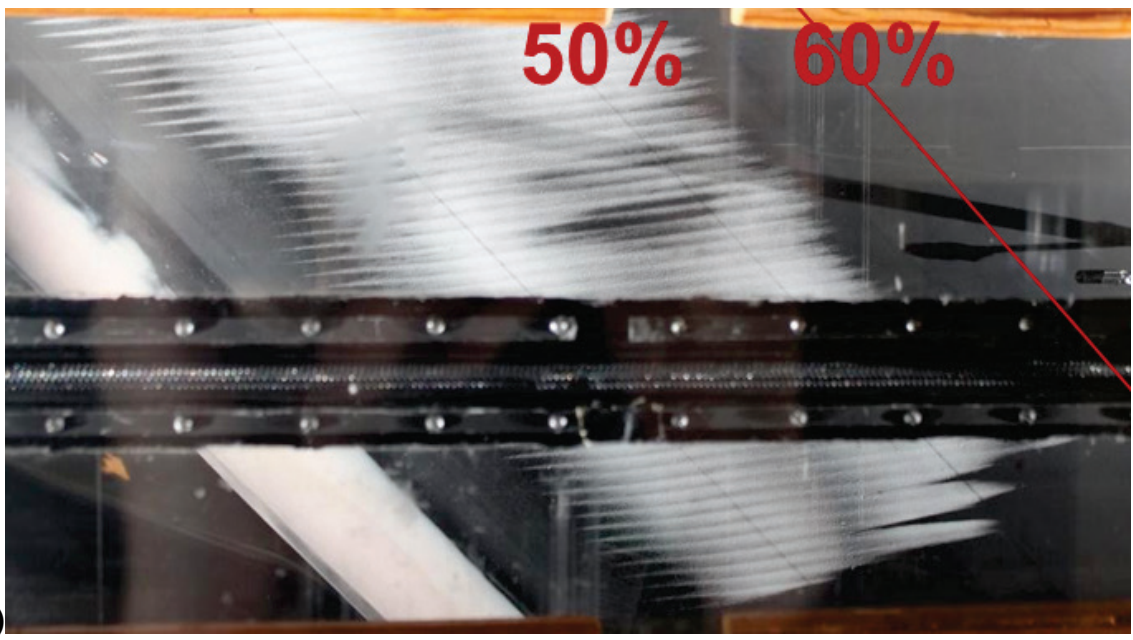

(b)

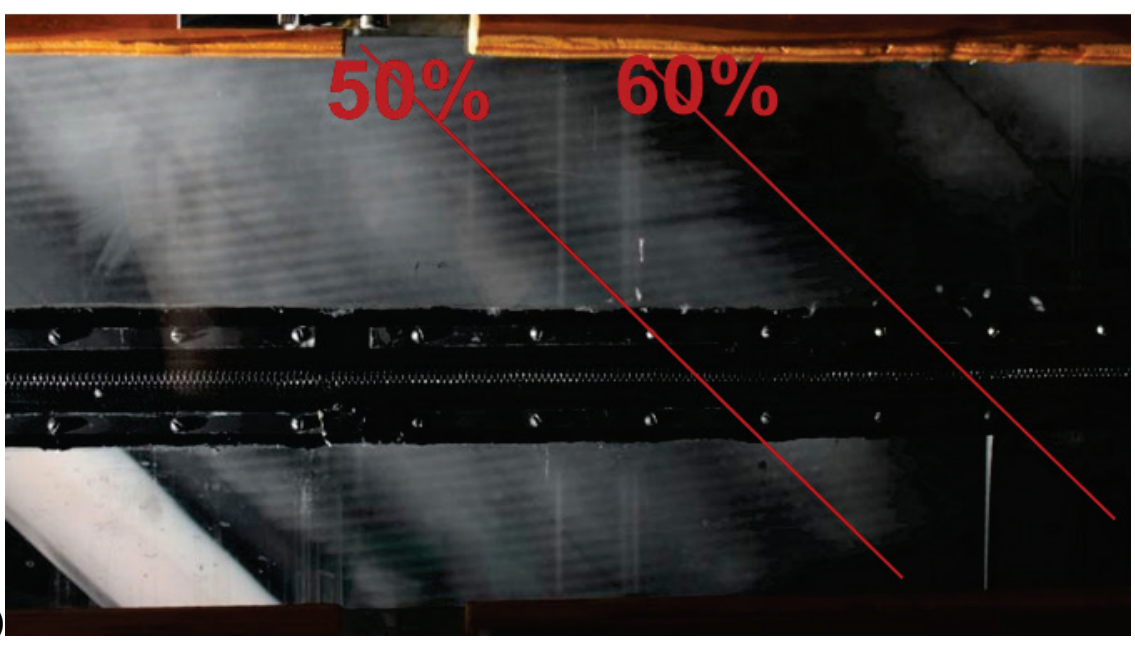

Figure 8-5. NFV for $[36 \mid 12]$ roughness: $x / c=0.029, R e_{c}=2.4 \times 10^{6}$; (a) no tape, (b) with tape- higher turbulence. 
(a)

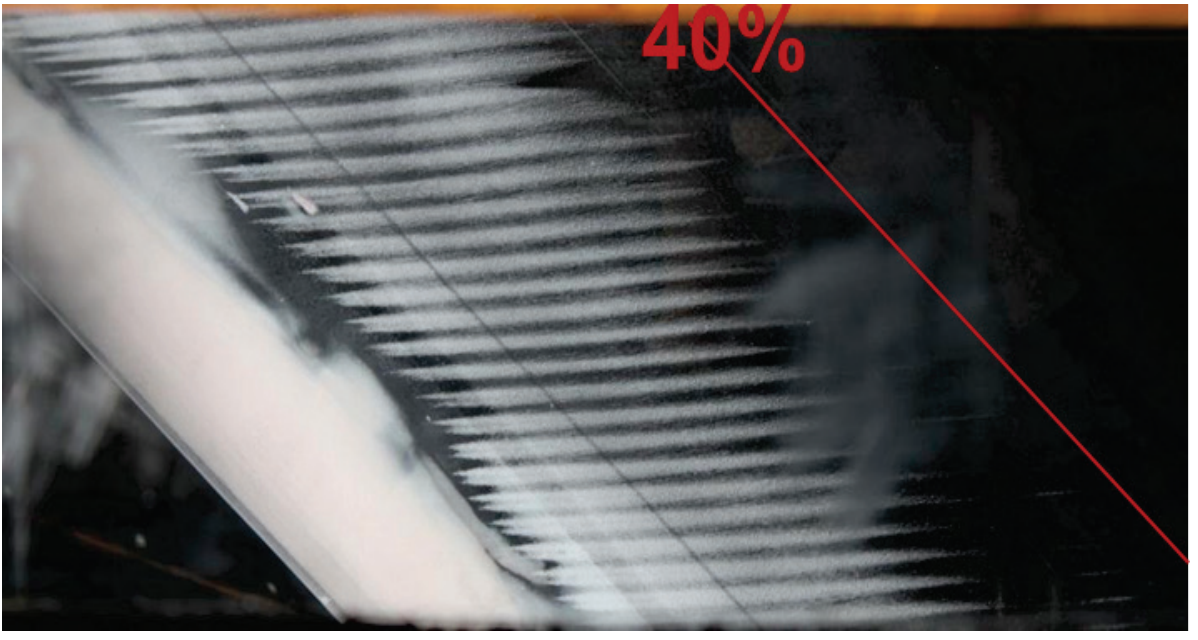

(b)

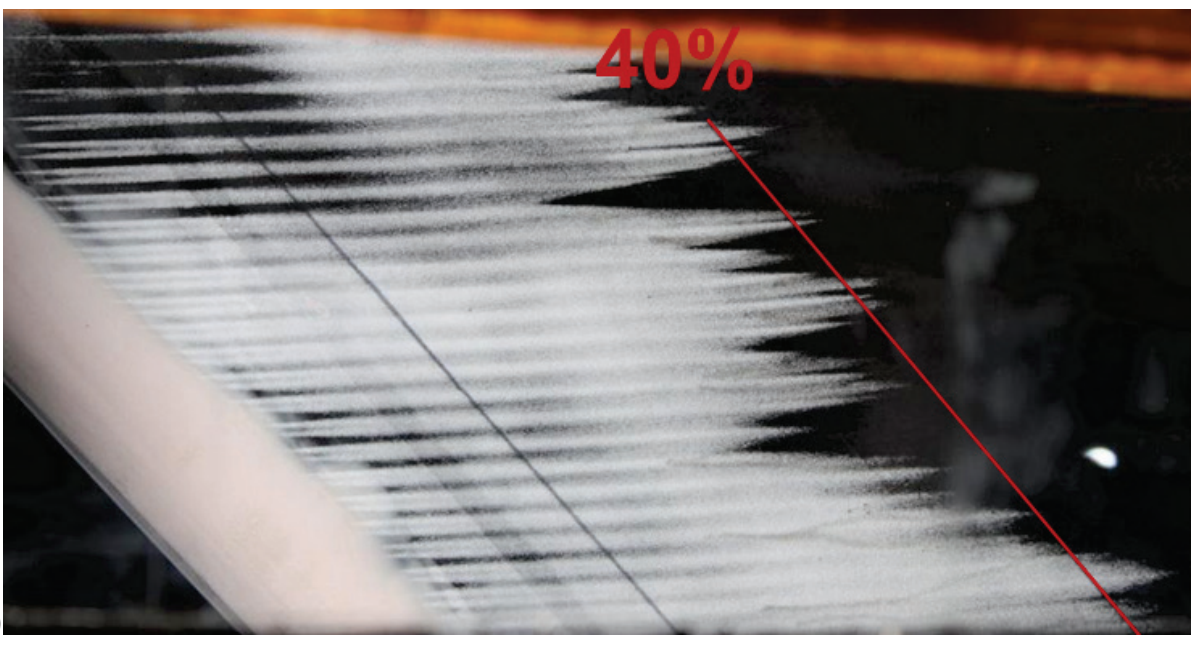

Figure 8-6. NFV for [36 $\mid 12]$ roughness: $x / c=0.029, R_{c}=3.2 \times 10^{6} ;$ (a) no tape, (b) with tape- higher turbulence. 
(a)
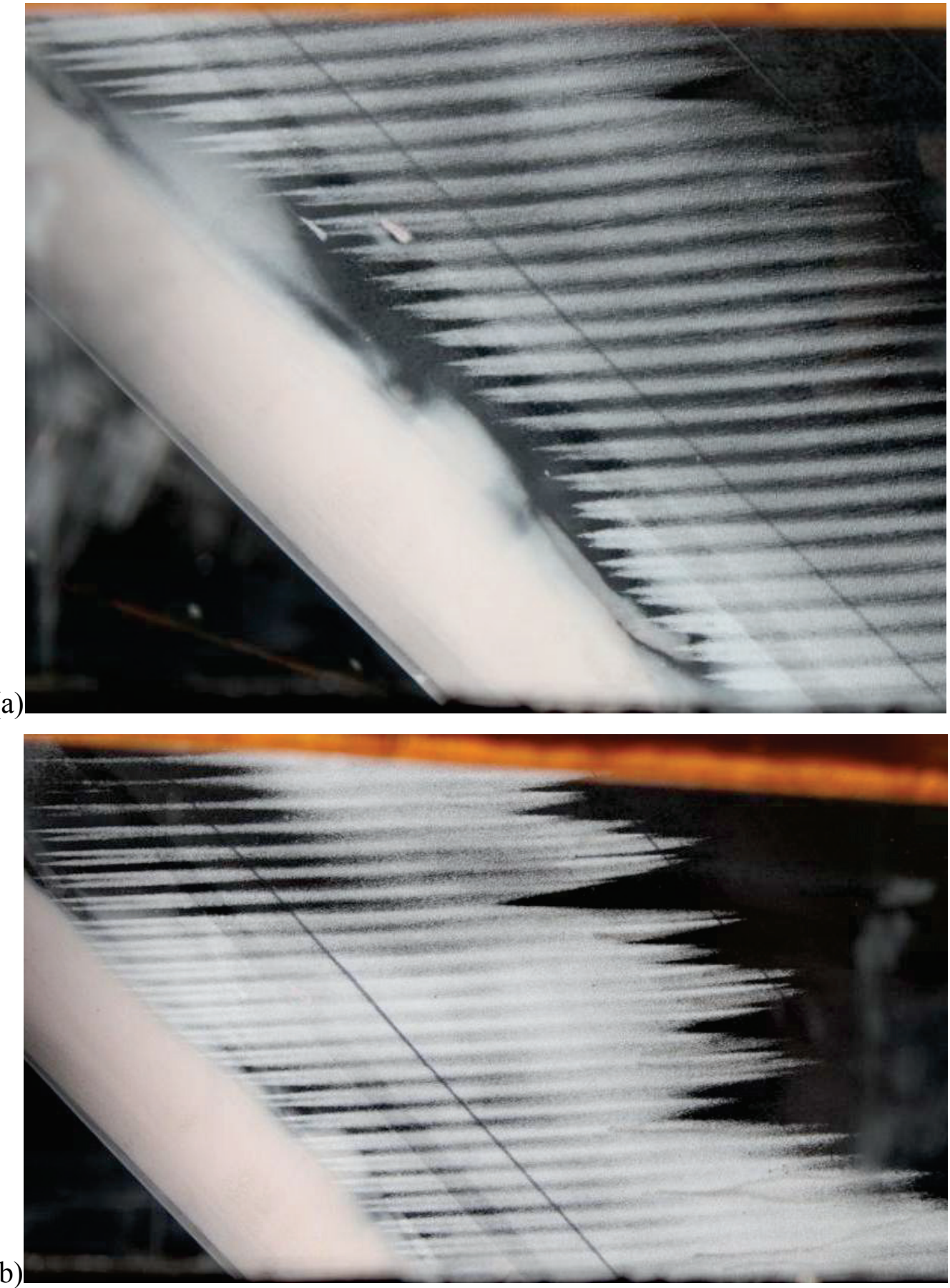

Figure 8-7. Close-up of naphthalene flow visualization for $\operatorname{Re}_{c}=3.2 \times 10^{6},[36 \mid 12]$ roughness. (a) No tape, (b) With tape- higher turbulence. 


\subsection{Hotwire Measurements, $15 \% x / c$}

Figures 8-8 - 8-10 and 8-13 - 8-15 display the boundary-layer velocity profiles, streamwise velocity contours and disturbance shapes for $R e_{c}=2.8 \times 10^{6}$ and $3.2 \times 10^{6}$ respectively. No significant difference between the original and increased turbulence cases is observed. A comparison of mode shapes with and without the increased turbulence is provided in Figures 8-11 and 8-16 for the two Reynolds numbers. For each test point, the maximum disturbance amplitude decreases slightly, as shown in Table 8-1. A decrease in maximum disturbance amplitude is consistent with previous NFV that show a slight transition delay when turbulence levels are increased. Again, this is contrary to expectations that higher turbulence levels would lead to larger initial disturbance amplitudes.

Table 8-1. Maximum stationary crossflow disturbance amplitudes at $15 \% x / c,[36 \mid 12]$ roughness.

\begin{tabular}{|c|c|c|}
\hline \multirow{2}{*}{$v_{r m s}{ }^{\prime} / U_{o}$} & \multicolumn{2}{|c|}{$\left[\left(u-u_{\text {avg }}\right) / U_{e}\right]_{\mathrm{rms}, \max }$} \\
\cline { 2 - 3 } & $R e_{c}=2.8 \times 10^{6}$ & $R_{c}=3.2 \times 10^{6}$ \\
\hline $0.020 \%$ & 0.0490 & 0.0756 \\
\hline $0.034 \%$ & 0.0473 & 0.0709 \\
\hline
\end{tabular}


Regardless of expectations, it is clear that even moderate changes in already low freestream turbulence levels have an impact on initial disturbance amplitude and the nature of the transition front for transition dominated by stationary crossflow waves. Figures 8-12 and 8-17 show the PSDs for the two Reynolds numbers. No significant change in the mode amplitude ratios occurs. Previous research indicated that once turbulence levels were below a threshold value low enough to encourage stationarywave-dominated transition, surface roughness was the primary factor in establishing initial disturbance amplitudes for stationary waves (Bippes 1999, Dagenhart \& Saric 1999). These results seem to indicate that freestream turbulence may still play an important role even in low-disturbance environments. Kurian et al. (2011) and Deyhle \& Bippes (1996) are two examples where freestream turbulence levels were increased to examine the impact on the crossflow instability. However, in these two examples, the freestream turbulence levels were no lower than $0.08 \%$ and as high as $0.7 \%$. In most of these tests, traveling waves dominated transition. To this author's knowledge, a thorough examination of the full impact of freestream turbulence on stationary crossflow waves for changes in freestream turbulence below $0.08 \%$ is still needed. 


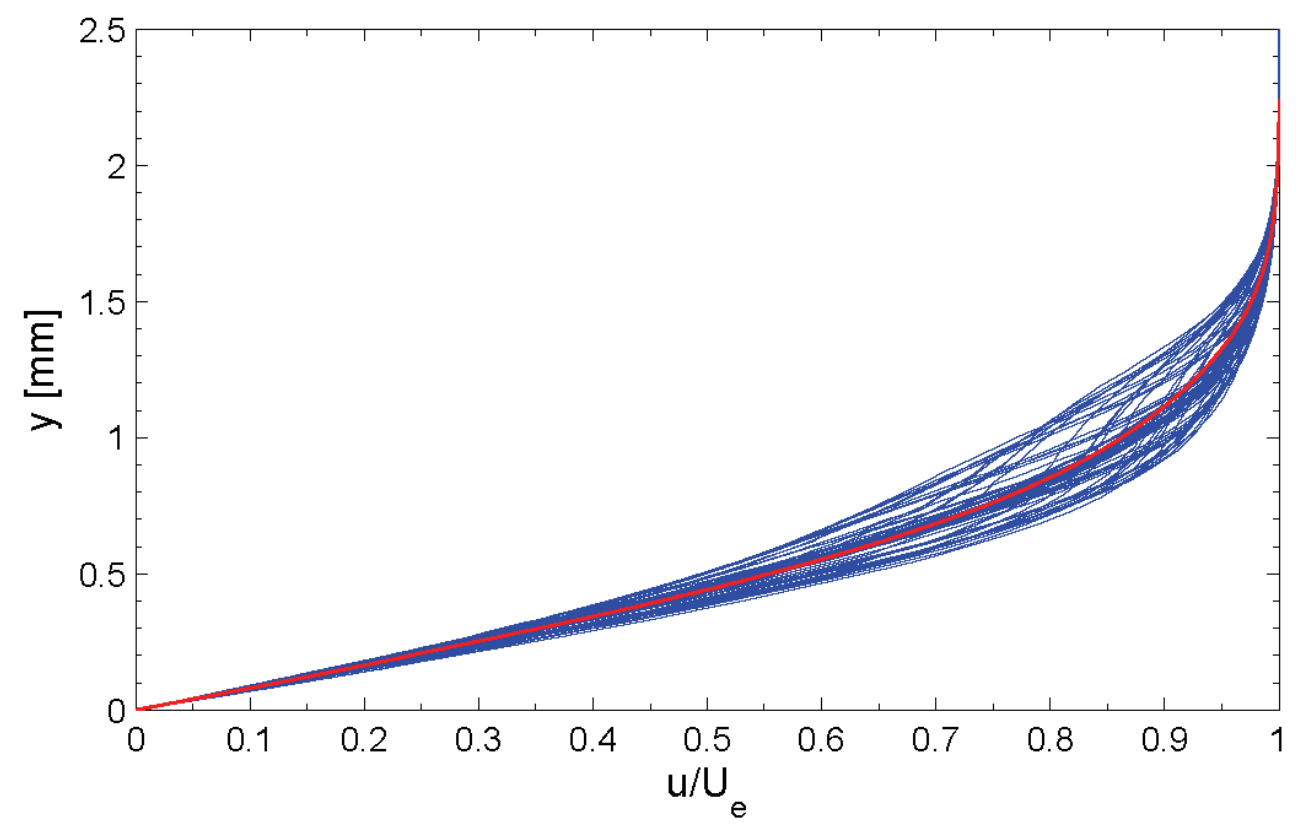

Figure 8-8. Spanwise array of 65 mean-flow boundary-layer profiles spanning $64 \mathrm{~mm}$ at $15 \% x / c$ (high turbulence). $R e_{c}=2.8 \times 10^{6},[36 \mid 12]$ roughness. Mean profile shown in red.

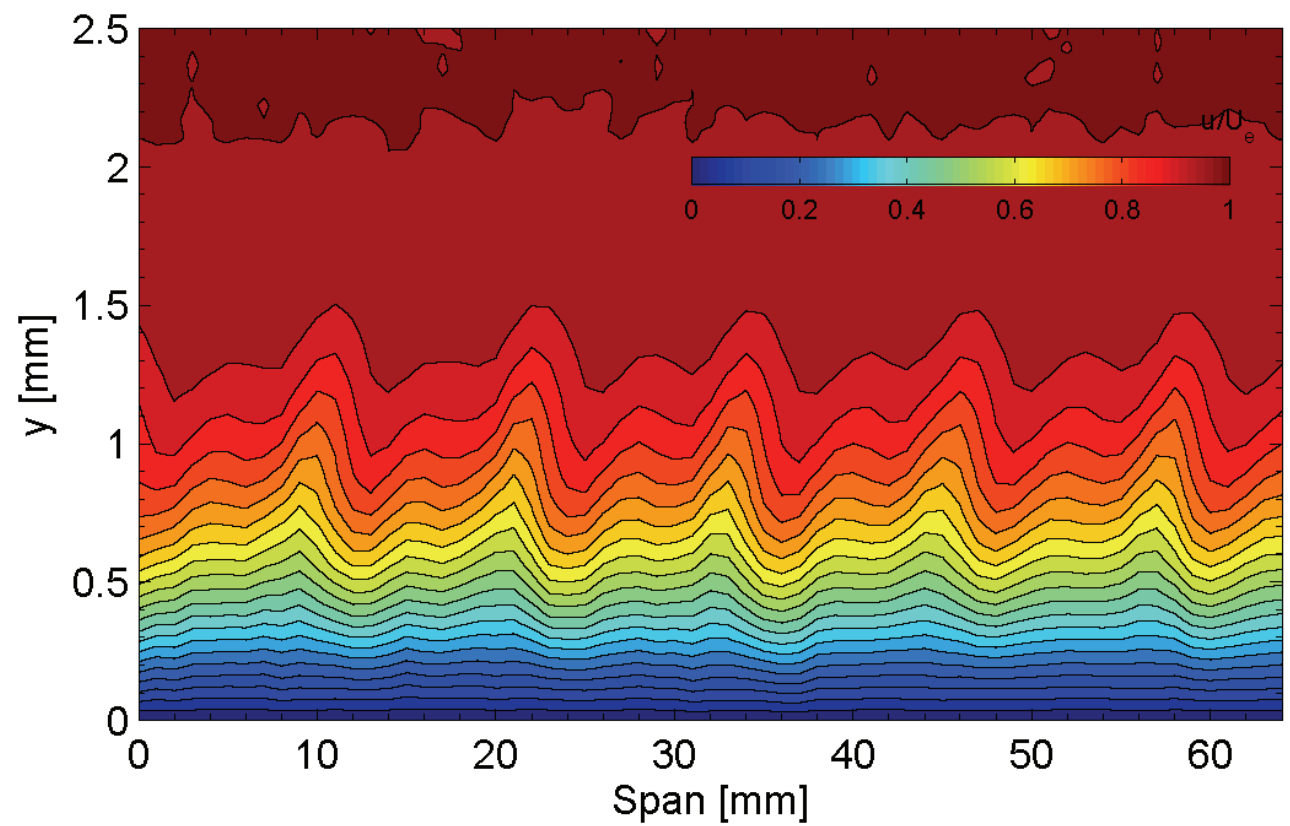

Figure 8-9. Streamwise velocity contours at $15 \% x / c$ (high-turbulence). $R e_{c}=2.8 \times 10^{6}$, [36|12] roughness. 


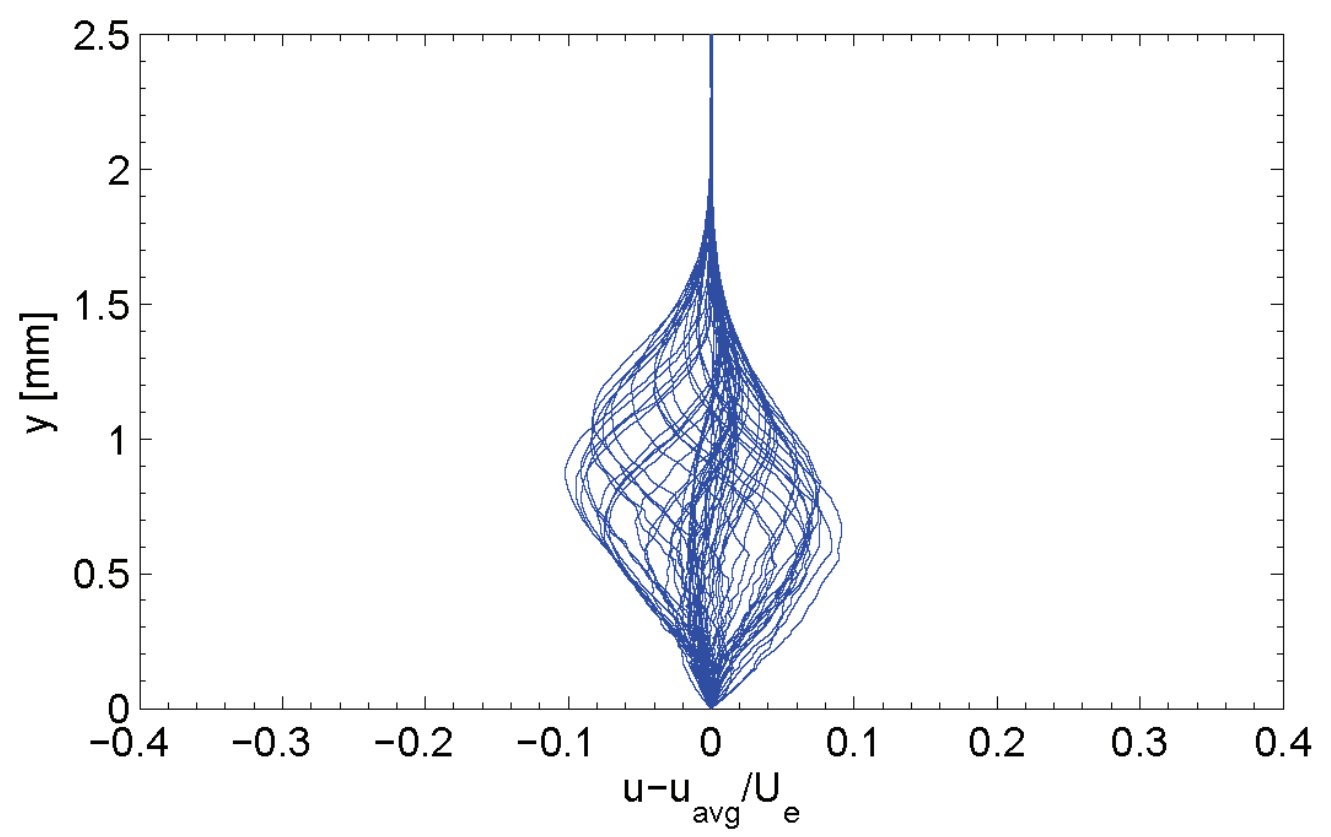

Figure 8-10. Spanwise array of 65 disturbance profiles spanning $64 \mathrm{~mm}$ at $15 \% x / \mathrm{c}$ (high turbulence). $R e_{c}=2.8 \times 10^{6},[36 \mid 12]$ roughness.

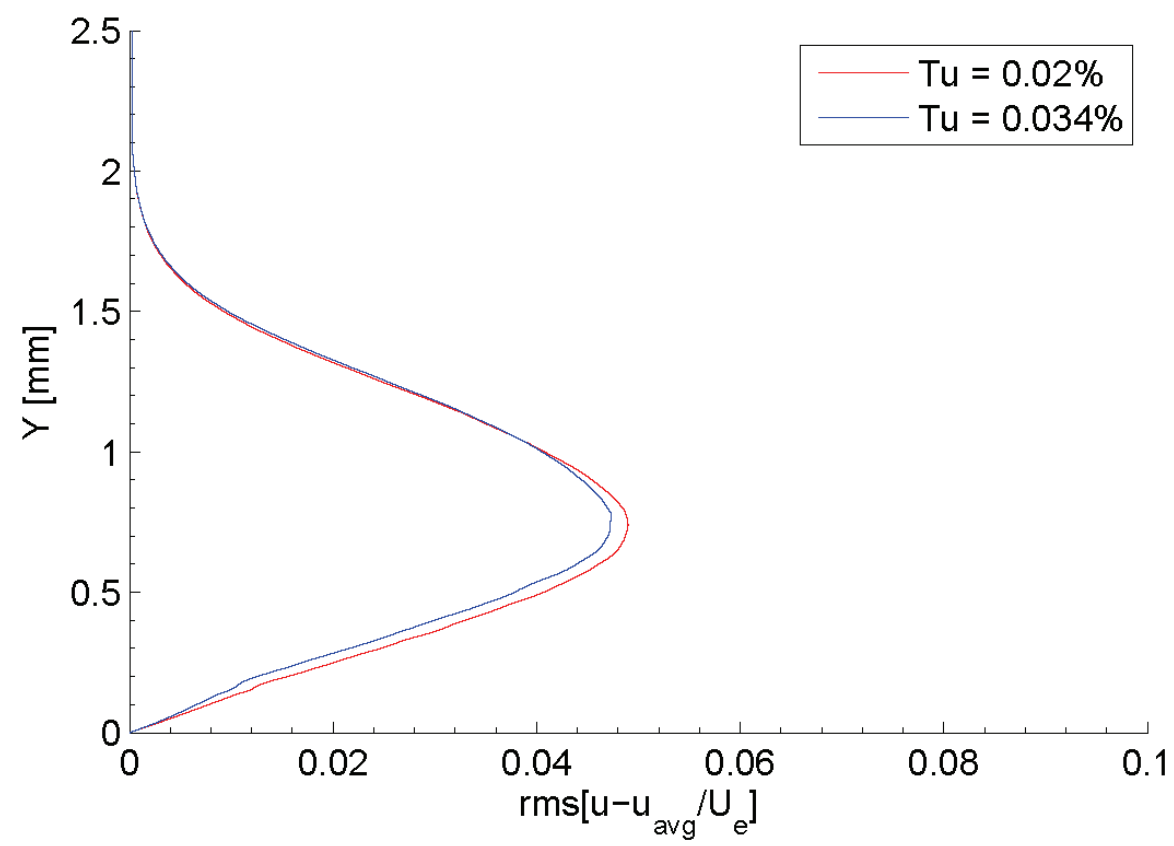

Figure 8-11. Stationary crossflow mode shape at $15 \% x / c . R e_{c}=2.8 \times 10^{6},[36 \mid 12]$ roughness. 


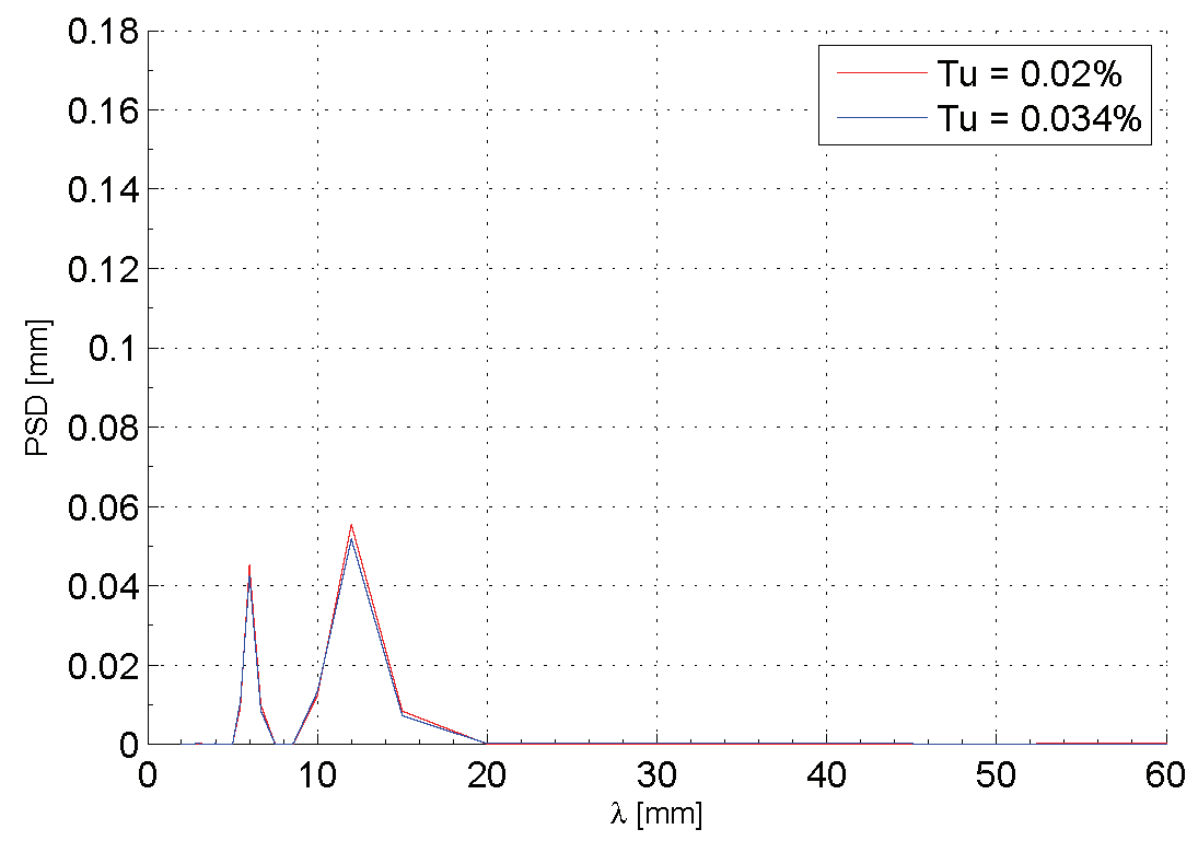

Figure 8-12. Power spectral density of spanwise hotwire scan at $15 \% x / c$. $R e_{c}=2.8 \times 10^{6},[36 \mid 12]$ roughness.

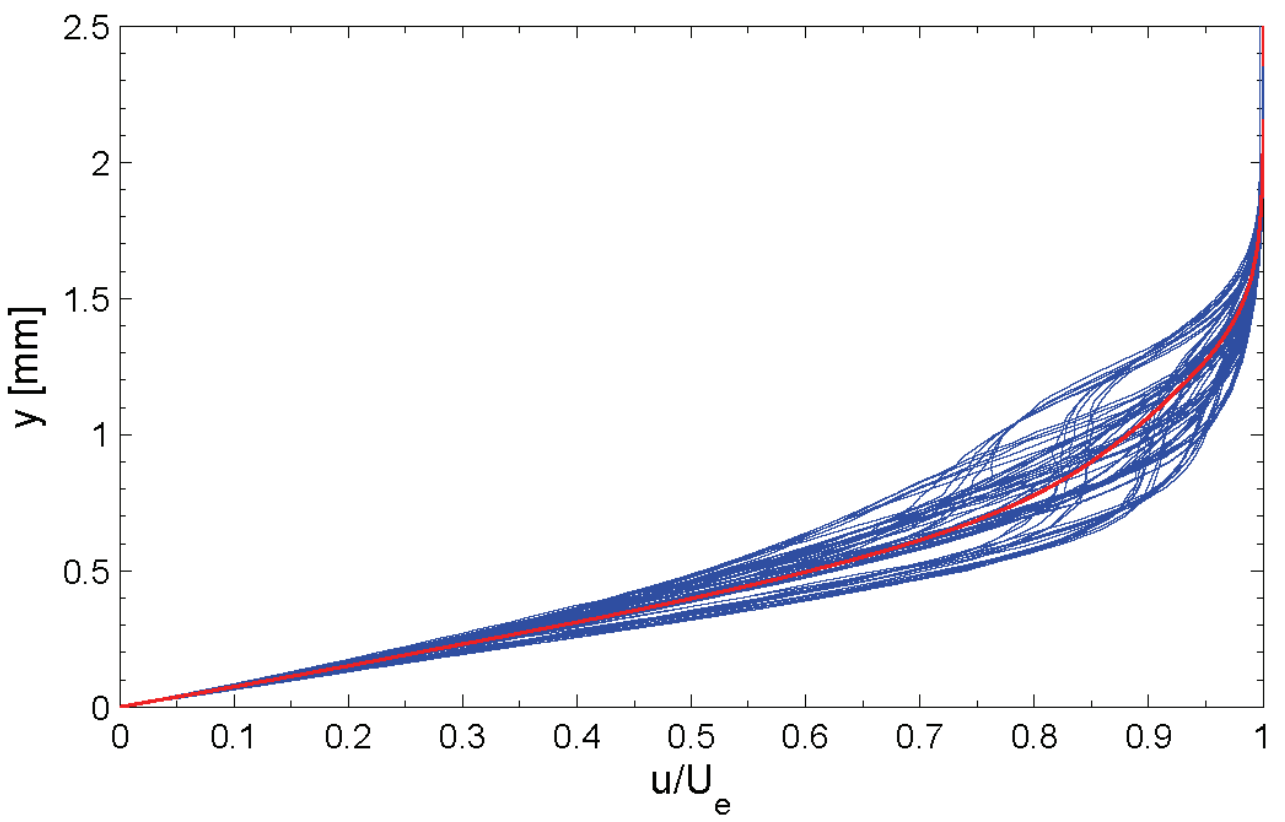

Figure 8-13. Spanwise array of 65 mean-flow boundary-layer profiles spanning $64 \mathrm{~mm}$ at $15 \% x / c$ (high turbulence). $R e_{c}=3.2 \times 10^{6},[36 \mid 12]$ roughness. Mean profile shown in red. 


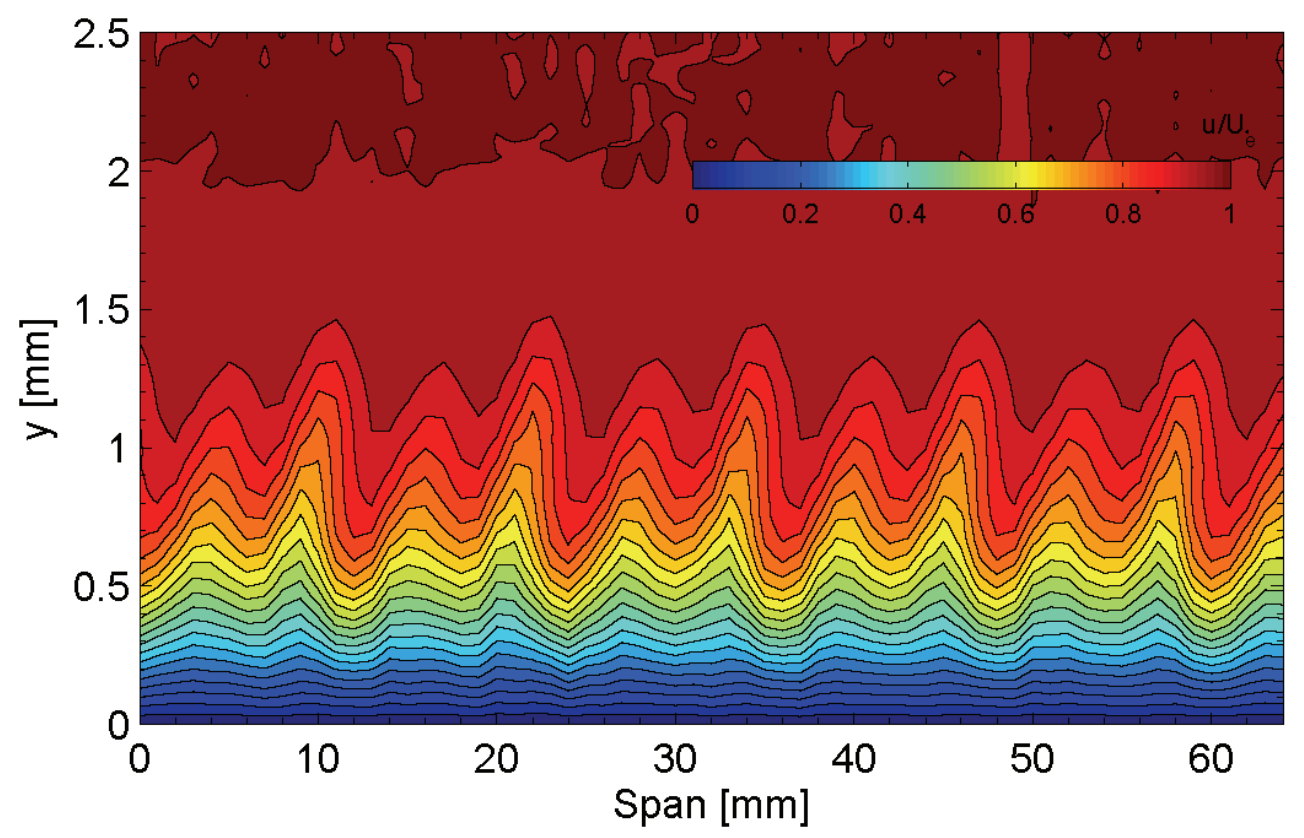

Figure 8-14. Streamwise velocity contours at $15 \% x / c$ (high turbulence). $R e_{c}=3.2 \times 10^{6}$, [36|12] roughness.

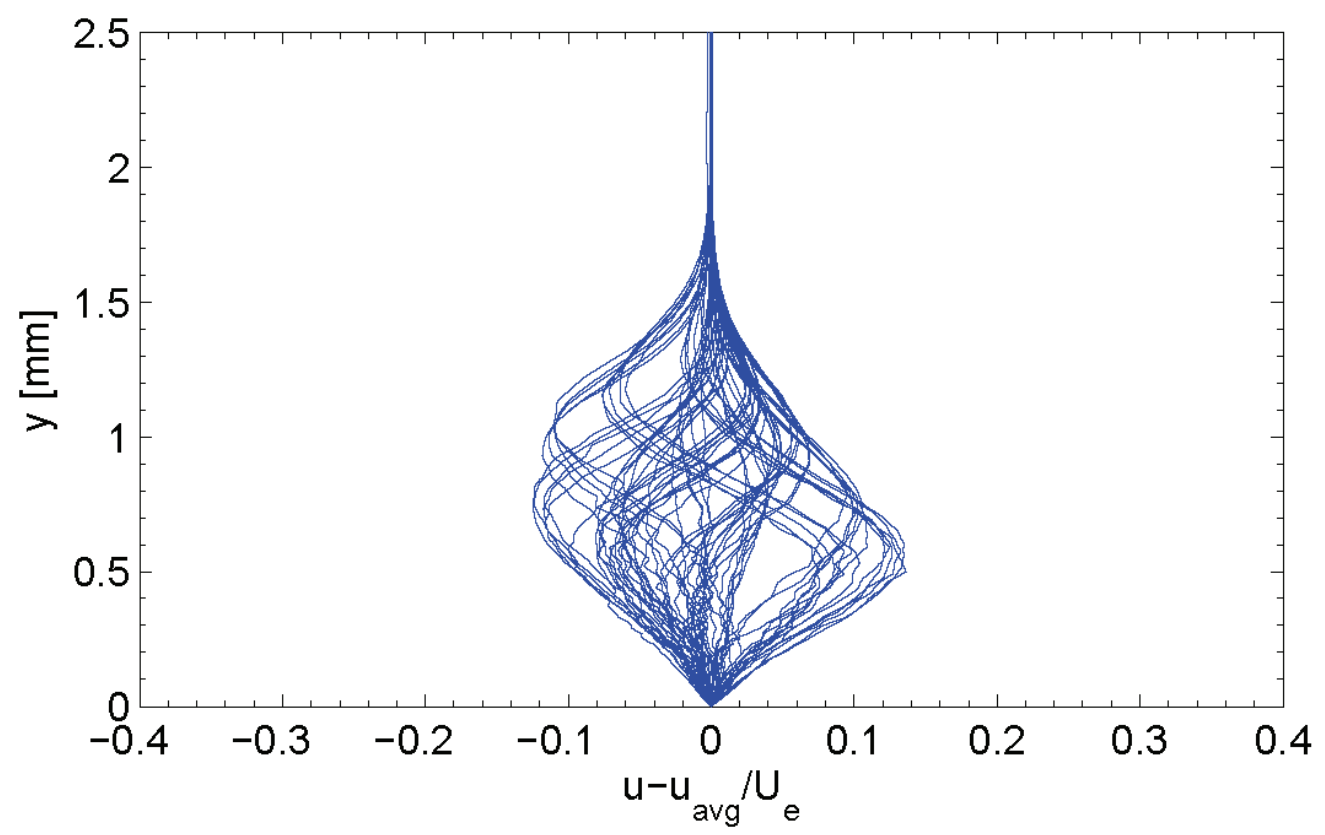

Figure 8-15. Spanwise array of 65 disturbance profiles spanning $64 \mathrm{~mm}$ at $15 \% x / c$ (high turbulence). $R e_{c}=3.2 \times 10^{6},[36 \mid 12]$ roughness. 


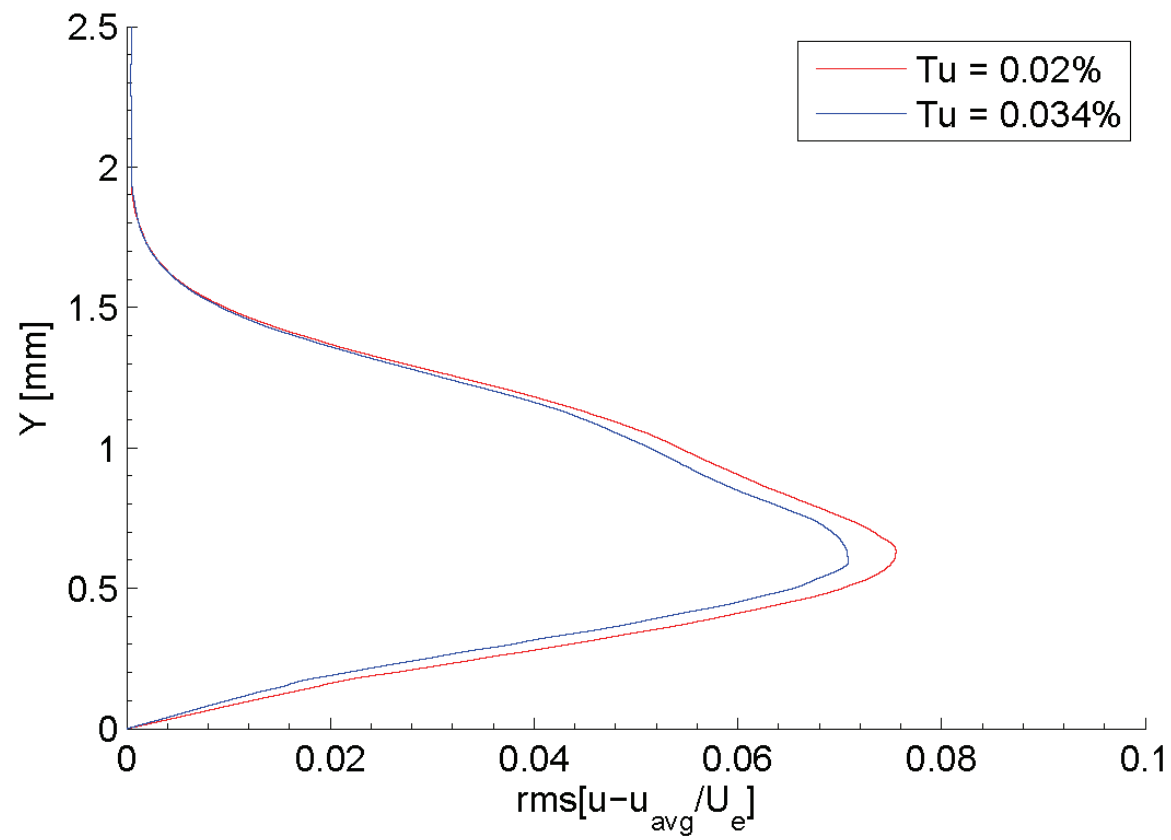

Figure 8-16. Stationary crossflow mode shape at $15 \% x / c . R e_{c}=3.2 \times 10^{6},[36 \mid 12]$ roughness.

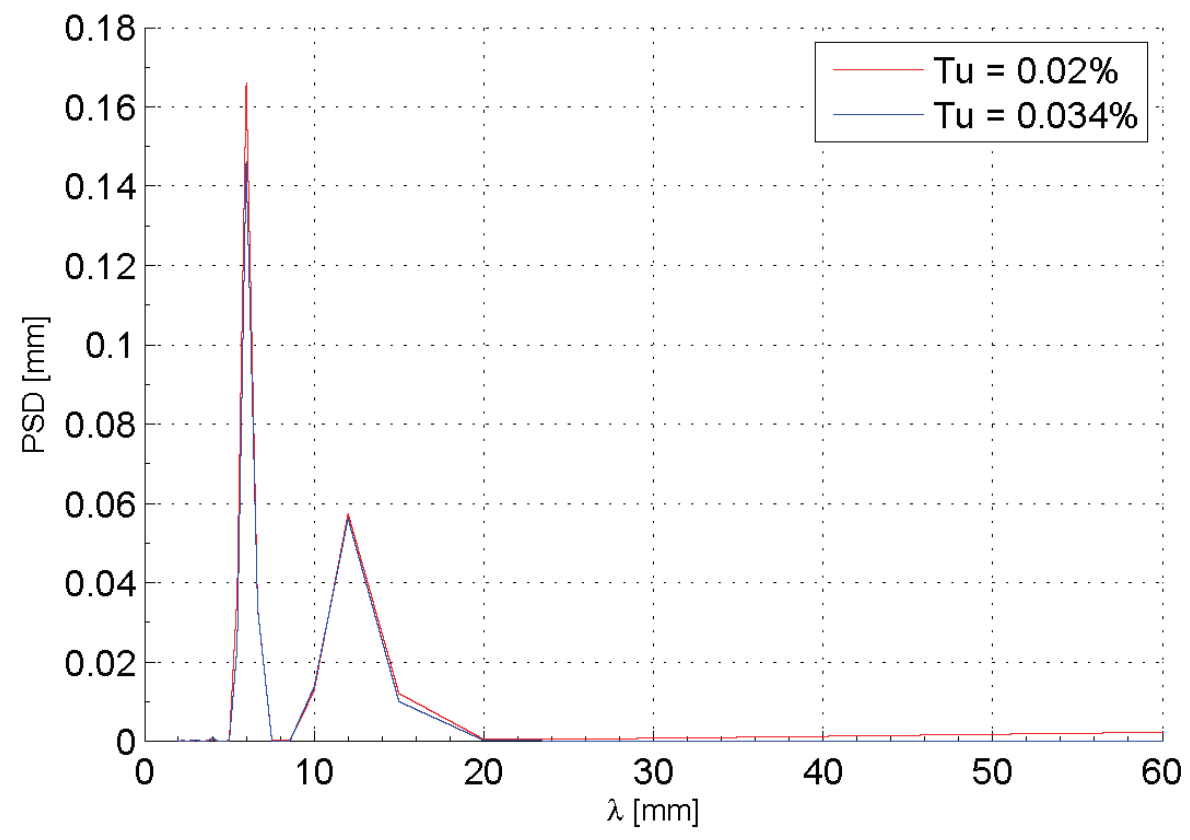

Figure 8-17. Power spectral density of spanwise hotwire scan at $15 \% x / c$. $R e_{c}=3.2 \times 10^{6},[36 \mid 12]$ roughness. 


\section{DISCUSSION OF RESULTS}

\subsection{Initial Disturbance Amplitudes}

The data shown in the four preceding sections provide insight into the receptivity process for a swept-wing dominated by the stationary crossflow instability and highlight aspects which differ from previous testing. Using a critical wavelength spacing, for both $R e_{c}=2.4 \times 10^{6}$ and $2.8 \times 10^{6}$, the normalized disturbance amplitudes show an almost linear relationship as roughness height increases. This relationship becomes slightly more nonlinear for the subcritical spacing. Tables 9-1 and 9-2 summarize the normalized disturbance amplitudes for the three Reynolds numbers tested at $\lambda=12 \mathrm{~mm}$ and $\lambda=6 \mathrm{~mm}$ respectively.

Table 9-1. Summary of normalized stationary crossflow disturbance amplitudes for $\lambda=12 \mathrm{~mm}$ at $15 \% x / c$.

\begin{tabular}{|c|c|c|c|}
\hline \multirow{2}{*}{$\begin{array}{c}k \\
{[\mu \mathrm{m}]}\end{array}$} & \multicolumn{3}{|c|}{$\left[\left(u-u_{\text {avg }}\right) / U_{e}\right]_{\mathrm{rms}, k / k o}$} \\
\cline { 2 - 4 } & $R e_{c}=2.4 \times 10^{6}$ & $R e_{c}=2.8 \times 10^{6}$ & $R e_{c}=3.2 \times 10^{6}$ \\
\hline 12 & 1.00 & 1.00 & 1.00 \\
\hline 24 & 1.01 & 1.00 & 0.94 \\
\hline 36 & 0.92 & 0.94 & $0.82^{*}$ \\
\hline 47 & 0.95 & 0.89 & $0.71^{*}$ \\
\hline
\end{tabular}

* Indicates nonlinear stability present

Table 9-2. Summary of normalized stationary crossflow disturbance amplitudes for $\lambda=6 \mathrm{~mm}$ at $15 \% x / c$.

\begin{tabular}{|c|c|c|c|}
\hline \multirow{2}{*}{$\begin{array}{c}k \\
{[\mu \mathrm{m}]}\end{array}$} & \multicolumn{3}{|c|}{$\left[\left(u-u_{\text {avg }}\right) / U_{e}\right]_{\mathrm{rms}, k / k o}$} \\
\cline { 2 - 4 } & $R e_{c}=2.4 \times 10^{6}$ & $R e_{c}=2.8 \times 10^{6}$ & $R e_{c}=3.2 \times 10^{6}$ \\
\hline 14 & 1.00 & 1.00 & 1.00 \\
\hline 27 & 0.93 & 0.98 & $0.98^{*}$ \\
\hline 42 & 0.84 & 0.85 & - \\
\hline 56 & 0.74 & $0.71^{*}$ & - \\
\hline
\end{tabular}

* Indicates nonlinear stability present 
This result is quite interesting since it indicates that the roughness Reynolds number defined by $R e_{k}=U(y=k) k / v$ may play only a weak role in the receptivity since its dependence is on $k^{2}$. A similar use of scaling using $R e_{k}$ is shown in White et al. (2005), although in that instance, initial disturbance amplitudes scale by $R e_{k}^{2}$. Differences in the amplitude drop-off between critical and subcritical spacing also indicate that any scaling should be approached with caution. Moreover, TAMU flight testing (Woodruff et al. 2011) and computations by Rizzetta et al. (2010) suggest that DRE shape also plays a role in generating initial disturbance amplitudes. Flight experiments showed that appliqué cylinders were more effective than pneumatic bumps. The work by Rizzetta et al. provided similar computational results and also showed that rectangles were more effective than cylinders. Scaling parameters and roughness shape are two different aspects of the receptivity problem, but they highlight the need to examine a larger parameter space before a cohesive receptivity theory is resolved.

Although earlier experiments focused on understanding later stages of the crossflow instability growth (i.e. primary instability growth and secondary mechanisms), they are all dependent on the initial disturbance amplitude and wavelength. Many of the earlier ASU test conditions, then, can also provide insight into the receptivity process. In particular, Reibert (1996) and Carrillo (1996), compiled an extensive database of hotwire scans similar to those described for this experiment over a wide range of chord locations and roughness heights. Table 9-3 provides a summary of measurements made by Reibert (R) and Carrillo (C) at 10\%, 15\% and 20\% x/c since these are the primary measurement locations for this study. Note that these authors acquired data at several other chord 
locations aft of $20 \%$. However, data at these locations are not relevant to the present study since effects from the receptivity process are often obscured by nonlinear stability activity. Reibert primarily focused on wavelengths equal to the most unstable mode, while Carrillo expanded the test matrix to include subcritical and supercritical spacing. With the exception of one roughness case at $R e_{c}=3.2 \times 10^{6}$, all scans were taken at $R e_{c}=2.4 \times 10^{6}$. It is important to emphasize that while the Reibert and Carrillo data were taken using the $\mathrm{NLF}(2)-0415$ under the same experimental configuration, the current data were acquired using the ASU(67)-0315 which has a different airfoil geometry and angle of attack. Although qualitative, comparisons with previous experiments may still highlight trends as a function of roughness height and Reynolds number. For all cases, the most unstable wavelength is $12 \mathrm{~mm}$.

Table 9-3. Summary of measurement points for Reibert (1996) and Carrillo (1996).

\begin{tabular}{|c|c|c|l|c|}
\hline \multicolumn{3}{|c|}{ Roughness } & \multicolumn{2}{|c|}{ Measurement Chord Location $(\% \times / c)$} \\
\hline $\begin{array}{c}k \\
{[\mu \mathrm{m}]}\end{array}$ & $\begin{array}{c}\lambda \\
{[\mathrm{mm}]}\end{array}$ & $\begin{array}{c}d \\
{[\mathrm{~mm}]}\end{array}$ & \multicolumn{1}{|c|}{$R e_{c}=2.4 \times 10^{6}$} & $R e_{c}=3.2 \times 10^{6}$ \\
\hline 0 & - & - & $\mathrm{C}(10 \%, 15 \%, 20 \%)$ & \\
\hline 6 & 8 & 3.7 & $\mathrm{C}(10 \%, 15 \%, 20 \%)$ & \\
\hline 48 & 8 & 3.7 & $\mathrm{C}(10 \%, 15 \%, 20 \% *)$ & \\
\hline 6 & 12 & 3.7 & $\mathrm{R}(10 \%, 15 \%, 20 \%)$ & $\mathrm{R}(10 \%, 15 \%, 20 \%)$ \\
\hline 18 & 12 & 3.7 & $\mathrm{R}(10 \%, 15 \%, 20 \%)$ & \\
\hline 48 & 12 & 3.7 & $\mathrm{R}(10 \%, 15 \%, 20 \%)$ & \\
\hline 6 & 18 & 3.7 & $\mathrm{C}(10 \%, 15 \%, 20 \%)$ & \\
\hline 12 & 18 & 3.7 & $\mathrm{C}(45 \%)$ & \\
\hline 18 & 18 & 3.7 & $\mathrm{C}(45 \%)$ & \\
\hline 6 & 36 & 3.7 & $\mathrm{R}(15 \%, 20 \%)$ & \\
\hline
\end{tabular}

*Chord-locations exhibiting signs of nonlinear stability activity 
Using the critical wavelength of $12 \mathrm{~mm}$, Reibert took measurements for roughness heights of $6 \mu \mathrm{m}, 18 \mu \mathrm{m}$ and $48 \mu \mathrm{m}$ at $R e_{c}=2.4 \times 10^{6}$. Table 9-4 shows the maximum disturbance amplitudes taken from plots in Reibert's thesis followed by the normalized amplitudes in Table 9-5. These values are best estimates from graphs in Reibert (1996); the original data are not available for use.

Table 9-4. Maximum stationary crossflow disturbance amplitudes for $\lambda=12 \mathrm{~mm}$ at $R e_{c}=2.4 \times 10^{6}$, estimated from Reibert (1996).

\begin{tabular}{|c|c|c|c|}
\hline \multirow{2}{*}{$k[\mu \mathrm{m}]$} & \multicolumn{3}{|c|}{$\left[\left(u-u_{\text {avg }}\right) / U_{e}\right]_{\mathrm{rms}, \max }$} \\
\cline { 2 - 4 } & $x / c=10 \%$ & $x / c=15 \%$ & $x / c=20 \%$ \\
\hline 6 & 0.0075 & 0.0150 & 0.0300 \\
\hline 18 & 0.0175 & 0.0375 & 0.0800 \\
\hline 48 & 0.0275 & 0.0625 & 0.1150 \\
\hline
\end{tabular}

Table 9-5. Normalized stationary crossflow disturbance amplitudes for $\lambda=12 \mathrm{~mm}$ at $R e_{c}=2.4 \times 10^{6}$, estimated from Reibert (1996).

\begin{tabular}{|c|c|c|c|}
\hline \multirow{2}{*}{$k[\mu \mathrm{m}]$} & \multicolumn{3}{|c|}{$\left[\left(u-u_{\text {avg }}\right) / U_{e}\right]_{\mathrm{rms}, k / k o}$} \\
\cline { 2 - 4 } & $x / c=10 \%$ & $x / c=15 \%$ & $x / c=20 \%$ \\
\hline 6 & 1.00 & 1.00 & 1.00 \\
\hline 18 & 0.78 & 0.83 & 0.89 \\
\hline 48 & 0.46 & 0.52 & 0.48 \\
\hline
\end{tabular}

Even taking into account error associated with estimating disturbance amplitudes from plots, there is a large decrease in initial disturbance amplitude as roughness height increases for all measured chord locations. For each of the roughness configurations at $10 \%, 15 \%$ and $20 \% x / c$, the mode shapes do not display any convex curvature or secondary lobes. Further inspection of the mean-boundary-layer profile for each case does not show signs of distortion. By these definitions then, the data in Table 9-5 are in 
the linear stability range and their values indicate that in this case, receptivity is a highly nonlinear process. By the time roughness height reaches $48 \mu \mathrm{m}$, the maximum disturbance amplitude is approximately half of what it would be for linear receptivity. Two roughness heights with the subcritical spacing are available from Carrillo. Table 9-6 shows the maximum estimated disturbance amplitudes followed by the normalized values in Table 9-7.

Table 9-6. Maximum stationary crossflow disturbance amplitudes for $\lambda=8 \mathrm{~mm}$ at $\operatorname{Re}_{c}=2.4 \times 10^{6}$, estimated from Carrillo (1996).

\begin{tabular}{|c|c|c|c|}
\hline \multirow{2}{*}{$k[\mu \mathrm{m}]$} & \multicolumn{3}{|c|}{$\left[\left(u-u_{\text {avg }}\right) / U_{e}\right]_{\mathrm{rms}, \max }$} \\
\cline { 2 - 4 } & $x / c=10 \%$ & $x / c=15 \%$ & $x / c=20 \%$ \\
\hline 6 & 0.0100 & 0.0250 & 0.0550 \\
\hline 48 & 0.0438 & 0.0925 & 0.1325 \\
\hline
\end{tabular}

Table 9-7. Normalized stationary crossflow disturbance amplitudes for $\lambda=8 \mathrm{~mm}$ at $\operatorname{Re}_{c}=2.4 \times 10^{6}$, estimated from Carrillo (1996).

\begin{tabular}{|c|c|c|c|}
\hline \multirow{2}{*}{$k[\mu \mathrm{m}]$} & \multicolumn{3}{|c|}{$\left[\left(u-u_{\text {avg }}\right) / U_{e}\right]_{\mathrm{rms}, k / k o}$} \\
\cline { 2 - 4 } & $x / c=10 \%$ & $x / c=15 \%$ & $x / c=20 \%$ \\
\hline 6 & 1.00 & 1.00 & 1.00 \\
\hline 48 & 0.55 & 0.46 & $0.30 *$ \\
\hline
\end{tabular}

* Indicates nonlinear stability present

Similar to the critical spacing case in Reibert (1996), the control wavelength displays a nonlinear relationship between roughness height and initial disturbance amplitude, as measured by Carrillo (1996). For $10 \%$ and $15 \% x / c$, no signs of nonlinear stability in the mode shape or boundary-layer profiles are present. Data that include disturbance amplitudes from multiple roughness heights for higher Reynolds numbers or 
supercritical spacing at early chord locations were not acquired at ASU. The reason behind the difference in normalized disturbance amplitudes between experiments at the UWT and KSWT is not understood at this time.

One other difference between the two sets of experiments is the presence of harmonics. For $\lambda=12 \mathrm{~mm}$, a $6 \mathrm{~mm}$ mode is present for all roughness heights and Reynolds numbers tested in the current experiment at $15 \% x / c$. Table $9-8$ shows a summary of the ratio of $12 \mathrm{~mm}$ to $6 \mathrm{~mm}$ disturbance amplitudes as a function of roughness height and Reynolds number at $15 \% x / c$. Variations up to $10 \%, 17 \%$ and $26 \%$ in amplitude ratio occur for the $2.4 \times 10^{6}, 2.8 \times 10^{6}, 3.2 \times 10^{6}$ cases respectively. Table 9-9 shows the amplitude ratio for measurements made by Reibert (1996) at $R e_{c}=2.4 \times 10^{6}$

Table 9-8. Summary of stationary crossflow disturbance amplitude ratios at $15 \% x / c$ for $\lambda=12 \mathrm{~mm}$.

\begin{tabular}{|c|c|c|c|}
\hline \multirow{2}{*}{$k[\mu \mathrm{m}]$} & \multicolumn{4}{|c|}{$\left[\left(u-u_{a v g}\right) / U_{e}\right]_{\mathrm{rms}, \lambda=12} /\left[\left(u-u_{\text {avg }}\right) / U_{e}\right]_{\mathrm{rms}, \lambda=6}$} \\
\cline { 2 - 4 } & $\mathrm{Re}_{\mathrm{c}}=2.4 \times 10^{6}$ & $\mathrm{Re}_{\mathrm{c}}=2.8 \times 10^{6}$ & $\mathrm{Re}_{\mathrm{c}}=3.2 \times 10^{6}$ \\
\hline 12 & 2.06 & 1.18 & 0.74 \\
\hline 24 & 1.94 & 1.18 & 0.55 \\
\hline 36 & 2.27 & 1.09 & $0.59^{*}$ \\
\hline 47 & 2.15 & 0.97 & $0.49^{*}$ \\
\hline
\end{tabular}

* Indicates nonlinear stability present

Table 9-9. Stationary crossflow disturbance amplitude ratios at $R_{c}=2.4 \times 10^{6}$ for $\lambda=12 \mathrm{~mm}$, estimated from Reibert (1996).

\begin{tabular}{|c|c|c|c|}
\hline \multirow{2}{*}{$k[\mu \mathrm{m}]$} & \multicolumn{3}{|c|}{$\left[\left(u-u_{\text {avg }}\right) / U_{e}\right]_{\mathrm{rms}, \lambda=12} /\left[\left(u-u_{\text {avg }}\right) / U_{e}\right]_{\mathrm{rms}, \lambda=6}$} \\
\cline { 2 - 4 } & $x / c=10 \%$ & $x / c=15 \%$ & $x / c=20 \%$ \\
\hline 6 & - & - & - \\
\hline 18 & 3.67 & 4.00 & 6.40 \\
\hline 48 & - & 8.33 & 5.11 \\
\hline
\end{tabular}


In the ASU configuration, the $6 \mathrm{~mm}$ wave is not detected until $30 \%$ chord when $k=6 \mu \mathrm{m}$. For the higher roughness cases, the ratio of disturbance amplitude for the $12 \mathrm{~mm}$ and $6 \mathrm{~mm}$ waves is higher than the current data. Interestingly, Yeates \& Saric (1984), also report a strong first harmonic, similar to the current results even though the NFV only showed streaking at the fundamental wavelength. For both the current data set and in Carrillo (1996), the first harmonic is not present at $15 \% x / c$ and below when subcritical roughness spacing is used. The differences in the results obtained from the previous ASU UWT tests and the current experiment suggest that there are perhaps other factors, not yet well established, that are influencing generation of the initial disturbance amplitudes.

\subsection{Environmental Influence}

Beyond the relationship between roughness height and initial disturbance amplitude, another objective of this experiment is to provide some insight into the differences between wind tunnel testing at ASU and flight tests at TAMU discussed in Section 1. The NFV data acquired in the KSWT reveal previously unobserved results. For these experimental conditions, transition Reynolds number does decrease as roughness height increases. These data use roughness heights $(12 \mu \mathrm{m}-47 \mu \mathrm{m})$ in a range similar to those tested at $\mathrm{ASU}(6 \mu \mathrm{m}-48 \mu \mathrm{m})$ and in both cases, the most unstable wavelength, $12 \mathrm{~mm}$ is forced. A forward marching transition front would be expected in cases where transition occurs before amplitude saturation has been reached. This could occur when initial disturbance amplitudes are too small to achieve saturation based on the given 
growth rates or conversely, where the saturation amplitude has increased for a given set of experimental conditions. These are two different viewpoints of the same issue. Starting first with the latter explanation, Bippes (1999) notes that increasing freestream turbulence decreased the saturation amplitude of the stationary modes. It is possible that the higher freestream turbulence levels in the UWT resulted in a lower saturation threshold compared to the KSWT configuration. Thus, even small amplitude roughness elements whose initial amplitudes are smaller were able to reach saturation before transition occurred. With lower turbulence levels in the KSWT, the saturation amplitude might not be achievable for the given initial disturbance amplitudes and growth rates. Freestream turbulence was not a parameter in the ASU crossflow saturation studies by Reibert (1996). Gladden (2001) examined the effect of elevated freestream turbulence in the UWT using the ASU(67)-0315 and found lower stationary amplitudes in a high turbulence $(T u=0.3 \%)$ environment, compared to the low-turbulence environment in the Reibert study. However, the comparison uses two different models at two different angles-of-attack. Additionally, for both Gladden and Bippes, the increased turbulence levels resulted in traveling-wave dominated transition for almost all cases. Studies comparing relative disturbance amplitudes based on changes in freestream turbulence in cases where stationary waves dominate have not been completed to date.

The preliminary study presented in Section 8 regarding modest changes in freestream turbulence $(0.02 \%$ to $0.034 \%)$ indicated that initial disturbance amplitudes might also be impacted. Maximum disturbance amplitude form hotwire scans decreased slightly when freestream turbulence was increased. The NFV showed a delay in 
transition under these conditions and a more ragged profile at higher Reynolds numbers, similar to what was previously observed at ASU. Thus, the answer to the question of whether freestream turbulence impacts saturation amplitude or initial disturbance amplitude may be both. Regardless, it is clear that a more thorough examination of the effect of changes in freestream turbulence is needed.

As a side note, until the differences in flight and wind tunnel tests are resolved, the TAMU research group also recognizes the importance of remeasuring turbulence levels for each new measurement campaign regardless of whether the facility is the same as previous studies. Both Dagenhart (1992) and Gaponenko et al. (2002) indicate that turbulence levels increased when their models and wall liners were installed in the tunnel. Bippes (1999) also recognized that one possibility for differences in reported turbulence values from current and previous DLR tests may be due to screen contamination. This is especially true if the flow visualization techniques that require smoke, oil or seeding are used. The data presented here suggest that even small changes in turbulence levels can impact results, reinforcing the importance of continuously monitoring freestream conditions. 


\section{SUMMARY AND CONCLUSIONS}

The purpose of this experimental investigation is to quantify the relationship between roughness height and the crossflow-wave disturbance amplitude for a spanwiseperiodic DRE array under conditions where a stationary crossflow instability dominates transition. This was accomplished under conditions were the crossflow wave was still dominated by linear stability. A secondary objective is to illuminate some of the differences in observed results for previous wind tunnel and flight tests as it relates to the operation and efficiency of these roughness arrays and the development of the crossflow instability.

To accomplish these goals, attention was first given to experimental design. The Klebanoff-Saric Wind Tunnel is the selected test facility. Previously known as the Arizona State University Unsteady Wind Tunnel, it was moved to Texas A\&M University and underwent significant flow quality improvements during the reconstruction effort. Flow quality measurements presented in this study indicate freestream turbulence levels are on the order of $0.02 \%$ for all three velocity components over the full speed range of the tunnel. Subsequent measurements after model and wall liner installation indicate that freestream turbulence levels are not substantially altered in the current experimental set-up. These turbulence levels may be as much as half the original values measured at ASU with models installed. The ASU(67)-0315 model, designed at ASU to have crossflow dominated transition, was sanded and polished to provide a $0.27 \mu \mathrm{m}$-rms surface finish. Installed at a swept angle of attack of $-2.9^{\circ}$, the 
configuration also includes wall liners on the test section floor and ceiling that match the inviscid streamlines for the given angle of attack to simulate an infinite swept-wing. Pressure distribution measurements from upper and lower span rows of pressure ports confirmed the validity of this assumption.

Once the freestream environment and experimental configuration were deemed suitable, detailed boundary-layer scans and naphthalene flow visualization commenced. Baseline NFV without any artificial roughness shows transition aft of $70 \% x / c$ for Reynolds numbers up to $2.8 \times 10^{6}$. This large extent of laminar flow for the case of natural roughness was never previously observed in any of the ASU configurations. Each naphthalene image showed a large usable measurement region along the span, indicating that surface preparation was sufficient for the experimental needs. Circular roughness elements $3 \mathrm{~mm}$ in diameter were set at $2.9 \% x / \mathrm{c}$ with a spacing equal to the most unstable wavelength, $\lambda=12 \mathrm{~mm}$. Roughness heights varied from $12 \mu \mathrm{m}-47 \mu \mathrm{m}$ depending on the test point. NFV showed transition moving forward as roughness height increased. This contrasts previously observed results at ASU, where transition location did not change for the same range of roughness heights. In addition, the transition front was more uniform compared to NFV tests at ASU. Boundary-layer scans using hotwire anemometry show a near linear dependence on roughness height in generating the initial disturbance amplitudes for all three Reynolds numbers tested. Strong presence of the first harmonic is also detected in all the hotwire results.

Similar tests were conducted with the roughness elements spaced at the control wavelength, $\lambda=6 \mathrm{~mm}$ and for roughness heights ranging from $14 \mu \mathrm{m}-56 \mu \mathrm{m}$. For these 
cases, NFV showed transition moving forward rather than aft. However, with the large extent of laminar flow present for the baseline case (no artificial roughness), it is suggested that the disturbance amplitudes introduced by the DREs were too large for control purposes. Experimental evidence from ASU also supports this conclusion. Hotwire measurements show at most, a weakly nonlinear dependence on roughness height for this wavelength spacing. For both the critical and control wavelengths, the normalized amplitude decrease is much less than originally measured at ASU.

Finally, preliminary tests with increased freestream turbulence levels indicate that even in low-disturbance environments, turbulence impacts generation and development of the stationary crossflow wave. This contrasts previously held beliefs that once the turbulence levels were low enough for the stationary wave to dominate transition, the only factor of concern was surface roughness. A more thorough examination of modest changes in freestream turbulence is needed before definitive conclusions can be drawn.

To summarize then, the original objective of providing quantitative receptivity data for DRE arrays was completed. Data for four roughness heights at three different Reynolds numbers and two wavelengths are provided. More generally, the results underscore several important details regarding the receptivity process for the stationary crossflow instability. First and foremost, it is clear that freestream turbulence levels do play a role in creating initial disturbance amplitudes. To this author's knowledge, this is the first experiment where trends based on different freestream turbulence levels are observed for stationary crossflow dominated transition. In previous studies on the effect of varying freestream turbulence, traveling waves dominate transition instead. The data 
here show that transition location does change with roughness height, in contrast to results observed at ASU and when modest changes in freestream turbulence occur, disturbance amplitudes and the transition front pattern also change. This is an initial step in understanding the difference in results between the ASU wind tunnel tests and TAMU flight tests. However, additional testing is required to determine the full nature or extent of influence, especially when different roughness shapes are involved. Until the role of freestream turbulence is fully understood, turbulence intensities and spectra should be reported for all experiments in order to understand the results within the context of the freestream environment. Second, the current experimental configuration and test matrix indicate that when the measurements are clearly done in the region of linear stability, the relationship between roughness height and initial disturbance amplitude is nearly linear and not quadratic as if it depended on $R e_{k}$. If true for all test conditions, it would simplify the modeling process greatly. However, the role of roughness shape and freestream turbulence requires further exploration and comparison of computational simulations of this experiment are needed before final conclusions regarding the receptivity process are drawn. 


\section{REFERENCES}

Arnal D, Archambaud JP. 2009. Laminar-turbulent transition control: NLF, LFC, HLFC. In Advances in Laminar-Turbulent Transition Modeling, VKI Lecture Series, pp. 15-1 - 15-22. Brussels, Belgium: Von Karman Institute for Fluid Dynamics

Arnal D, Houdeville R, Séraudi A, Vermeersch O. 2011. Overview of laminar-turbulent transition investigations at ONERA Toulouse. Presented at AIAA Fluid Dyn.Conf. Exhib., 41 ${ }^{\text {st }}$, Honolulu, AIAA Pap. No. 2011-3074

Barlow JB, Rae WH Jr, Pope A. 1999. Low-Speed Wind Tunnel Testing, $3^{\text {rd }}$ edn. New York: Wiley-Interscience

Bearman, PW. 1971. Corrections for the effect of ambient temperature drift on hot-wire measurements in incompressible flow. DISA Information 11:25-30

Bertolotti FP. 2000. Receptivity of three-dimensional boundary-layers to localized wall roughness and suction. Phys. Fluids 12(7):1799-1809

Bippes H. 1999. Basic experiments on transition in three-dimensional boundary layers dominated by crossflow instability. Prog. Aero. Sci. 35(4):363-412

Bruun HH. 1995. Hot-Wire Anemometry: Principles and Signal Analysis. New York: Oxford University Press

Bushnell DM. 1990. Notes on initial disturbance fields for the transition problem. In Instability and Transition to Turbulence, Vol. I, ed. MY Hussaini, RG Voigt, pp. 217-232. New York: Springer-Verlag

Carpenter AL. 2009. In-flight receptivity experiments on a 30-degree swept-wing using micron-sized discrete roughness elements. Ph.D. dissertation. Texas A\&M Univ. College Station, TX

Carpenter AL, Saric WS, Reed HL. 2009. In-flight receptivity experiments on a 30degree swept-wing using micron-sized discrete roughness elements. Presented at AIAA Aerosp. Sci. Meet., 47 , Orlando, AIAA Pap. No. 2009-0590

Carpenter AL, Saric WS, Reed HL. 2010. Roughness receptivity in swept-wing boundary layers - experiments. Int'l J. Eng. Sys. Modeling and Simulation, 2(1): 128-38

Carrillo RB Jr. 1996. Distributed-roughness effects on stability and transition in sweptwing boundary-layers. M.S. thesis. Arizona State Univ. Tempe AZ 
Chang CL, Choudhari MM. 2005. Boundary-layer receptivity and integrated transition prediction. Presented at AIAA Aerosp. Sci. Meet., $43^{\text {rd }}$, Reno, AIAA Pap. No. 2005-0526

Choudhari MM. 1993. Roughness-induced generation of crossflow vortices in threedimensional boundary layers. NASA Contractor Rpt. 4505, High Tech. Corp., Hampton, VA

Coleman WS. 1959. The characteristics of roughness from insects as observed for twodimensional, incompressible flow past airfoils. J. Aero. Sci. 26: 264-68

Collis SS, Lele SK. 1999. Receptivity to surface roughness near a swept leading edge. $J$. Fluid Mech. 380:141-68

Crouch JD. 1993. Receptivity of three-dimensional boundary layers. Presented at AIAA Aerosp. Sci Meet., 31 ${ }^{\text {st }}$, Reno, AIAA Pap. No. 93-0074

Crouch JD. 1994. Theoretical studies on the receptivity of boundary layers. Presented at AIAA Fluid Dyn. Conf. Exhib., 25 ${ }^{\text {th }}$, Colorado Springs, AIAA Pap. No. 94-2224

Crouch JD, Ng LL. 2000. Variable N-factor method for transition prediction in threedimensional boundary layers. AIAA J. 38(2):211-16

Dagenhart JR. 1992. Crossflow stability and transition experiments in a swept-wing flow. Ph.D. dissertation. Virginia Polytechnic Institute and State Univ. Blacksburg, VA

Dagenhart JR, Saric WS. 1999. Crossflow stability and transition experiments in a swept-wing flow. NASA TP 1999-209344, NASA LaRC, Hampton, VA

Deyhle H, Bippes H. 1996. Disturbance growth in an unstable three-dimensional boundary layer and its dependence on initial conditions. J. Fluid Mech. 316:73113

Ellingsen T, Palm E. 1975. Stability of linear flow. Phys. Fluids 18:487-88

Gaponenko VR, Ivanov AV, Kachanov YS, Crouch JD. 2002. Swept-wing boundarylayer receptivity to surface non-uniformities. J. Fluid Mech. 461:93-126

Gladden RD. 2001. Crossflow transition in elevated freestream turbulence. M.S. thesis, Arizona State Univ. Tempe, AZ

Gray WE. 1952. The effect of wing sweep on laminar flow. Tech. Rep. Aero 255, RAE, Hampshire, UK 
Green JE. 2008. Laminar flow control- back to the future?. Presented at AIAA Fluid Dyn. Conf. Exhib., $38^{\text {th }}$, Seattle, AIAA Pap. No. 2008-3738

Gregory N, Stuart JT, Walker WS. 1955. On the stability of three-dimensional boundary layers with applications to the flow due to a rotating disk. Phil. Trans. R. Soc. London Ser. A 248:155-99

Haynes TS, Reed HL. 2000. Simulation of swept-wing vortices using nonlinear parabolized stability equations. J. Fluid Mech. 405:325-49

Herbert T. 1997. Parabolized stability equations. Ann. Rev. Fluid Mech. 29:245-83

Hunt LE, Downs RS, Kuester MS, White EB, Saric WS. 2010. Flow-quality measurements in the Klebanoff-Saric Wind Tunnel. Presented at the AIAA Aero. Meas. Tech. Grnd. Test., $27^{\text {th }}$, Chicago, AIAA Pap. No. 2010-4538

Janke E. 2001. Receptivity and transition control of swept-wing boundary-layers; effects of surface curvature and nonlinearity. Presented at AIAA Fluid Dyn. Conf. Exhib., $31^{\text {st }}$, Anaheim, AIAA Pap. No. 2001-2980

Janke E, Balakumar P. 2000. On the secondary instability of three-dimensional boundary layers. Theoret. Comput. Fluid Dyn. 14:167-94

Joslin RD. 1998. Aircraft laminar flow control. Annu. Rev. Fluid Mech. 30:1-29

Kachanov YS. 1994. Physical mechanisms of laminar-boundary-layer transition. Ann. Rev. Fluid Mech. 26:411-82

Kachanov YS. 2000. Three-dimensional receptivity of boundary layers. Eur. J. Mech. BFluids. 19:723-44

Kawakami M, Kohama Y, Okutsu M. 1999. Stability characteristics of stationary crossflow vorticies in three-dimensional boundary layer. Presented at AIAA Aerosp. Sci. Meet., 37 ${ }^{\text {th }}$, Reno, AIAA Pap. No. 99-0811

Kohama Y, Saric WS, Hoos JA. 1991. A high-frequency secondary instability of crossflow vortices that leads to transition. In Proceedings of the Royal Aeronautical Society: Boundary Layer Transition and Control (ed. M. Gaster), pp. $4.1-4.13$. Royal Aeronautical Society, Cambridge, England, UK

Kurian T, Fransson JHM, Alfredsson PH. 2011. Boundary layer receptivity to freestream turbulence and surface roughness over a swept flat plate. Phys. Fluids 23:034107

Mack LM. 1984. Boundary-layer linear stability theory. AGARD Report No. 709, Brussels, Belgium 
Malik MR, Li F, Choudhari MM, Chang CL. 1999. Secondary instability of crossflow vortices and swept-wing boundary layer transition. J. Fluid Mech. 399:85-115

Martin ML, Carpenter AL, Saric WS. 2008. Swept-wing laminar flow control studies using Cessna O2-A test aircraft. Presented at U.S. A.F. T\&E Days, Los Angeles, AIAA Pap. No. 2008-1636

Morkovin, MV. 1969. Critical evaluation of transition from laminar to turbulent shear layers with emphasis on hypersonically traveling bodies. Air Force Flight Dyn. Lab. Rep. AFFDL-TR-68-149, Baltimore, MD

Morkovin MV, Reshotko E, Herbert T. 1994. Transition in open flow systems- a reassessment. Bull. Am. Phys. Soc. 39(9):1-31

Mousseux MC. 1988. Flow quality improvements in the Arizona State University Unsteady Wind Tunnel. M.S. thesis. Arizona State Univ. Tempe, AZ

Müller B, Bippes H. 1988. Experimental study of instability modes in a threedimensional boundary layer. In Proc. AGARD Symp. on Fluid Dynamics of Three-Dimensional Turbulent Shear Flows and Transition, AGARD CP-438, pp. 13-1-13-15, Cesme, Turkey

Naguib AM, Gravante SP, Wark CE. 1996. Extraction of turbulent wall-pressure timeseries using an optimal filtering scheme. Exp. Fluids 22(1):14-22

$\mathrm{Ng}$ LL, Crouch JD. 1999. Roughness-induced receptivity to crossflow vortices on a swept wing. Phys. Fluids 11(2):432-38

Perry, AE. 1982. Hot-Wire Anemometry. New York: Oxford University Press

Piot E, Content C, Casalis G. 2008. Receptivity of crossflow instabilities to a periodic roughness array on swept cylinder: investigation of the roughness size influence. Presented at AIAA Aerosp. Sci. Meet., 46 ${ }^{\text {th }}$, Reno, AIAA Pap. No. 2008-0502

Radeztsky RH Jr. 1994. Growth and development of roughness-induced stationary crossflow vortices. Ph.D. dissertation. Arizona State Univ. Tempe, AZ

Radeztsky RH, Reibert MS, Takagi S. 1993. A software solution to temperature-induced hot-wire voltage drift. In Proc. of the Third International Symposium on Thermal Anemometry. Washington DC: ASME Fluids Engineering Division

Radeztsky RH Jr, Reibert MS, Saric WS. 1999. Effect of isolated micron-sized roughness on transition in swept-wing flows. AIAA J. 37(11):1370-77

Reed HL, Saric WS. 1989. Stability of three-dimensional boundary layers. Ann. Rev. Fluid Mech. 21:235-84 
Reed HL, Saric WS. 2008. Transition mechanisms for transport aircraft. Presented at AIAA Fluid Dyn. Conf. Exhib., $38^{\text {th }}$, Seattle, AIAA Pap. No. 2008-3743

Reed HL, Saric WS, Arnal D. 1996. Linear stability theory applied to boundary layers. Ann. Rev. Fluid Mech. 28:389-428

Reibert MS. 1996. Nonlinear stability, saturation, and transition in crossflow-dominated boundary layers. Ph.D. dissertation. Arizona State Univ. Tempe, AZ

Reibert MS, Saric WS, Carrillo RB Jr., Chapman KL. 1996. Experiments in nonlinear saturation of stationary crossflow vortices in a swept-wing boundary layer. Presented at AIAA Aerosp. Sci Meet., 34 ${ }^{\text {th }}$, Reno, AIAA Pap. No. 96-0184

Reshotko E. 1975. Recent developments in boundary-layer transition research. AIAAJ. $13: 261-65$

Reshotko E. 1976. Boundary-layer stability and transition. Ann. Rev. Fluid Mech. 8:31149

Reshotko E, Saric WS, Nagib HM. 1997. Flow quality issues for large wind tunnels. Presented at AIAA Aerosp. Sci. Meet., 35 th , Reno, AIAA Pap. No. 97-0225

Riedel H, Sitzmann M. 1997. In-flight investigations of atmospheric turbulence. Aero. Sci. Tech. 5:301-19

Rizzetta DP, Visbal MR, Reed HL, Saric WS. 2010. Direct numerical simulation of discrete roughness on a swept wing leading edge. AIAA J. 48(11):2660-73

Saric WS. 1992. The ASU transition research facility. Presented at AIAA Aerosp. Grnd. Test., $17^{\text {th }}$, Nashville, AIAA Pap. No. 92-3910

Saric WS. 2007. Boundary-layer stability and transition. In Springer Handbook of Experimental Fluid Mechanics, ed. C. Tropea, A. Yarin, J.F. Foss, pp. 886-96. Berlin Heidelberg: Springer-Verlag

Saric WS, Carpenter AL, Reed HL. 2011. Passive control of transition in threedimensional boundary layers, with emphasis on discrete roughness elements. Phil. Trans. R. Soc. A 369:1352-64

Saric WS, Carrillo RB, Reibert MS. 1998. Leading-edge roughness as a transition control mechanism. Presented at AIAA Aerosp. Sci. Meet., 36 ${ }^{\text {th }}$, Reno, AIAA Pap. No. 98-0781

Saric WS, Reed HL. 2003. Crossflow instabilities- theory and technology. Presented at AIAA Aerosp. Sci. Meet., 41 ${ }^{\text {st }}$, Reno, AIAA Pap. No. 2003-0771 
Saric WS, Reed HL. 2004. Toward Practical Laminar Flow Control- remaining challenges. Presented at AIAA Fluid Dyn. Conf., 34 ${ }^{\text {th }}$, Portland, AIAA Pap. No. 2004-2311

Saric WS, Reed HL, Kerschen EJ. 2002. Boundary-layer receptivity to freestream disturbances. Ann. Rev. Fluid Mech. 34:291-319

Saric WS, Reed HL, White EB. 2003. Stability and transition of three-dimensional boundary layers. Ann. Rev. Fluid Mech. 35:413-40

Saric WS, Reshotko E. 1998. Review of flow quality issues in wind tunnel testing. Presented at AIAA Fluid Dyn. Conf., 29 ${ }^{\text {th }}$, Albuquerque, AIAA Pap. No. 98-2613

Saric WS, Takagi S, Mousseux MC. 1988. The ASU Unsteady Wind Tunnel and fundamental requirements for freestream turbulence measurements. Presented at AIAA Aerosp. Sci. Meet., 26 ${ }^{\text {th }}$, Reno, AIAA Pap. No. 88-0053

Saric WS, Yeates LG. 1984. Generation of crossflow vortices in a three-dimensional flat-plate flow. In Laminar-Turbulent Transition, Vol. II, ed. V. Kozlov, pp. 42937. Berlin: Springer-Verlag

Schrader LU, Brandt L, Henningson DS. 2009. Receptivity mechanisms in threedimensional boundary-layer flows. J. Fluid Mech. 618:209-41

Smith AMO, Gamberoni N. 1956. Transition, pressure gradient and stability theory. Rep. No. ES. 26388, Douglas Aircr. Co., Inc, Long Beach, CA

Tan-Atichat J, Nagib HM, Drubka RE. 1980. Effects of axisymmetric contractions on turbulence of various scales. NASA-CR-165136, Illinois Institute of Tech., Chicago, IL

van Ingen JL. 1956. A suggested semi-empirical method for the calculation of the boundary layer transition region. Rep. Nos. VTH 71 and 74, Dept. Aeronaut. Eng., Univ. Technol., Delft, Netherlands

von Doenhoff AE, Braslow AL. 1961. The effect of distributed surface roughness on laminar flow. In Boundary Layer Control, Vol 2, ed. V. Lachmann, pp. 657-81. Oxford: Pergamon Press

Wasserman P, Kloker M. 2002. Mechanisms and control of crossflow-vortex induced transition in a 3-D boundary layer. J. Fluid Mech. 456:49-84

Watmuff JH. 1998. Detrimental effects of almost immeasurably small freestream nonuniformities generated by wind-tunnel screens. AIAA J. 36(3):379-386 
Watmuff JH. 2006. Effects of weak free stream nonuniformity on boundary layer transition. J. Fluids Eng. 128:247-257

White EB. 2000. Breakdown of crossflow vortices. Ph.D. dissertation. Arizona State Univ. Tempe, AZ

White EB, Rice JM, Ergin FG. 2005. Receptivity of stationary transient disturbances to surface roughness. Phys. Fluids 17:064109

White EB, Saric WS. 2000. Application of variable leading-edge roughness for transition control on swept wings. Presented at AIAA Aerosp. Sci. Meet., $38^{\text {th }}$, Reno, AIAA Pap. No. 2000-0283

White EB, Saric WS. 2005. Secondary instability of crossflow vortices. J. Fluid Mech. 525:275-308

White EB, Saric WS, Gladden RD, Gabet PM. 2001. Stages of swept-wing transition. Presented at AIAA Aerosp. Sci. Meet., 39 ${ }^{\text {th }}$, Reno, AIAA Pap. No. 2001-0271

Woodruff MJ, Saric WS, Reed HL. 2011. Receptivity Measurements on a Swept-Wing Model. Presented at AIAA Fluid Dyn. Conf. Exhib., 41 ${ }^{\text {st }}$, Honolulu, AIAA Pap. No. 2011-3882 


\section{APPENDIX A}

ASU(67)-0315 Airfoil Coordinates 
Table A -1. ASU(67)-0315 Coordinates (normal to the leading edge).

\begin{tabular}{|c|c|c|c|c|c|c|c|}
\hline \multicolumn{4}{|c|}{ Suction Side } & \multicolumn{4}{|c|}{ Pressure Side } \\
\hline $\mathrm{x} / \mathrm{c}$ & $\mathrm{y} / \mathrm{c}$ & $\mathrm{x}$ [in] & y [in] & $\mathrm{x} / \mathrm{c}$ & $\mathrm{y} / \mathrm{c}$ & $\mathrm{x}$ [in] & y [in] \\
\hline 0 & 0 & 0 & 0 & 0 & 0 & 0 & 0 \\
\hline 0.00014 & 0.00325 & 0.0071 & 0.1655 & 0.00014 & -0.00163 & 0.0071 & -0.0830 \\
\hline 0.00056 & 0.00515 & 0.0285 & 0.2622 & 0.00056 & -0.00336 & 0.0285 & -0.1711 \\
\hline 0.00125 & 0.00708 & 0.0636 & 0.3605 & 0.00125 & -0.00531 & 0.0636 & -0.2703 \\
\hline 0.00223 & 0.00900 & 0.1135 & 0.4582 & 0.00223 & -0.00721 & 0.1135 & -0.3671 \\
\hline 0.00358 & 0.01098 & 0.1823 & 0.5590 & 0.00358 & -0.00913 & 0.1823 & -0.4648 \\
\hline 0.00532 & 0.01297 & 0.2708 & 0.6603 & 0.00532 & -0.01103 & 0.2708 & -0.5616 \\
\hline 0.00765 & 0.01518 & 0.3895 & 0.7728 & 0.00765 & -0.01299 & 0.3895 & -0.6613 \\
\hline 0.01073 & 0.01772 & 0.5463 & 0.9022 & 0.01073 & -0.01519 & 0.5463 & -0.7733 \\
\hline 0.0144 & 0.02026 & 0.7331 & 1.0315 & 0.01440 & -0.01740 & 0.7331 & -0.8859 \\
\hline 0.01879 & 0.02282 & 0.9566 & 1.1618 & 0.01879 & -0.01956 & 0.9566 & -0.9958 \\
\hline 0.02433 & 0.02561 & 1.2387 & 1.3038 & 0.02433 & -0.02179 & 1.2387 & -1.1094 \\
\hline 0.03147 & 0.02879 & 1.6022 & 1.4657 & 0.03147 & -0.02422 & 1.6022 & -1.2331 \\
\hline 0.04030 & 0.03228 & 2.0517 & 1.6434 & 0.04030 & -0.02678 & 2.0517 & -1.3634 \\
\hline 0.05134 & 0.03620 & 2.6138 & 1.8430 & 0.05134 & -0.02956 & 2.6138 & -1.5049 \\
\hline 0.06454 & 0.04047 & 3.2858 & & 0.06454 & -0.03248 & 3.2858 & -1.6536 \\
\hline 0.07987 & 0.04491 & 4.0663 & 2.2864 & 0.07987 & -0.03541 & 4.0663 & -1.8028 \\
\hline 0.09788 & 0.04958 & 4.9832 & 2.5242 & 0.09788 & -0.03834 & 4.9832 & -1.9520 \\
\hline 0.11880 & 0.05444 & 6.0483 & 2.7716 & 0.11880 & -0.04126 & 6.0483 & -2.1006 \\
\hline 0.14261 & 0.05942 & 7.2605 & 3.0251 & 0.14261 & -0.04410 & 7.2605 & -2.2452 \\
\hline 0.16932 & 0.06442 & 8.6204 & 3.2797 & 0.16932 & -0.04680 & 8.6204 & -2.3827 \\
\hline 0.19885 & 0.06932 & 10.1237 & 3.5292 & 0.19885 & -0.04930 & 10.1237 & -2.5099 \\
\hline 0.23092 & 0.07400 & 11.7565 & 3.7675 & 0.23092 & -0.05154 & 11.7565 & -2.6240 \\
\hline 0.26574 & 0.07841 & 13.5292 & 3.9920 & 0.26574 & -0.05348 & 13.5292 & -2.7228 \\
\hline 0.30285 & 0.08245 & 15.4186 & 4.1977 & 0.30285 & -0.05509 & 15.4186 & -2.8047 \\
\hline 0.34128 & 0.08594 & 17.3751 & 4.3753 & 0.34128 & -0.05630 & 17.3751 & -2.8663 \\
\hline 0.38092 & 0.08882 & 19.3932 & 4.5220 & 0.38092 & -0.05706 & 19.3932 & -2.9050 \\
\hline 0.42188 & 0.09107 & 21.4786 & 4.6365 & 0.42188 & -0.05738 & 21.4786 & -2.9213 \\
\hline 0.46248 & 0.09258 & 23.5456 & 4.7134 & 0.46248 & -0.05723 & 23.5456 & -2.9137 \\
\hline 0.50279 & 0.09332 & 25.5978 & 4.7511 & 0.50279 & -0.05660 & 25.5978 & -2.8816 \\
\hline 0.54243 & 0.09330 & 27.6160 & 4.7501 & 0.54243 & -0.05550 & 27.6160 & -2.8256 \\
\hline 0.58065 & 0.09252 & 29.5618 & 4.7103 & 0.58065 & -0.05387 & 29.5618 & -2.7426 \\
\hline 0.61700 & 0.09103 & 31.4125 & 4.6345 & 0.61700 & -0.05170 & 31.4125 & -2.6321 \\
\hline 0.65082 & 0.08888 & 33.1343 & 4.5250 & 0.65082 & -0.04908 & 33.1343 & -2.4987 \\
\hline
\end{tabular}


Table A-1. (continued)

\begin{tabular}{|c|c|c|c|c|c|c|c|}
\hline \multicolumn{4}{|c|}{ Suction Side } & \multicolumn{4}{|c|}{ Pressure Side } \\
\hline $\mathrm{x} / \mathrm{c}$ & $\mathrm{y} / \mathrm{c}$ & $x[$ in $]$ & $\mathrm{y}[\mathrm{in}]$ & $\mathrm{x} / \mathrm{c}$ & $\mathrm{y} / \mathrm{c}$ & $x$ [in] & $\mathrm{y}[\mathrm{in}]$ \\
\hline 0.68147 & 0.08612 & 34.6947 & 4.3845 & 0.68147 & -0.04617 & 34.6947 & -2.3506 \\
\hline 0.70901 & 0.08276 & 36.0968 & 4.2134 & 0.70901 & -0.04293 & 36.0968 & -2.1856 \\
\hline 0.73404 & 0.07874 & 37.3712 & 4.0088 & 0.73404 & -0.03928 & 37.3712 & -1.9998 \\
\hline 0.75720 & 0.07395 & 38.5503 & 3.7649 & 0.75720 & -0.03511 & 38.5503 & -1.7875 \\
\hline 0.77986 & 0.06815 & 39.7039 & 3.4696 & 0.77986 & -0.03029 & 39.7039 & -1.5421 \\
\hline 0.80317 & 0.06119 & 40.8907 & 3.1153 & 0.80317 & -0.02493 & 40.8907 & -1.2692 \\
\hline 0.82799 & 0.05302 & 42.1543 & 2.6993 & 0.82799 & -0.01917 & 42.1543 & -0.9760 \\
\hline 0.85302 & 0.04427 & 43.4286 & 2.2539 & 0.85302 & -0.01359 & 43.4286 & -0.6919 \\
\hline 0.87452 & 0.03658 & 44.5232 & 1.8623 & 0.87452 & -0.00920 & 44.5232 & -0.4684 \\
\hline 0.89340 & 0.02995 & 45.4844 & 1.5248 & 0.89340 & -0.00585 & 45.4844 & -0.2978 \\
\hline 0.91043 & 0.02418 & 46.3514 & 1.2310 & 0.91043 & -0.00333 & 46.3514 & -0.1695 \\
\hline 0.92637 & 0.01904 & 47.1630 & 0.9694 & 0.92637 & -0.00144 & 47.1630 & -0.0733 \\
\hline 0.94119 & 0.01453 & 47.9175 & 0.7397 & 0.94119 & -0.00013 & 47.9175 & -0.0066 \\
\hline 0.95500 & 0.01056 & 48.6206 & 0.5376 & 0.95500 & 0.00069 & 48.6206 & 0.0351 \\
\hline 0.97500 & 0.00531 & 49.6388 & 0.2703 & 0.97500 & 0.00112 & 49.6388 & 0.0570 \\
\hline 0.99000 & 0.00186 & 50.4025 & 0.0947 & 0.90009 & 0.00076 & 50.4025 & 0.0387 \\
\hline 1 & 0 & 50.9116 & 0 & 1 & 0 & 50.9116 & 0 \\
\hline
\end{tabular}




\section{APPENDIX B}

ASU(67)-0315 Pressure Distribution 


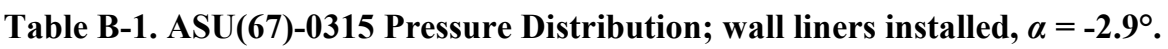

\begin{tabular}{|c|c|c|c|c|c|c|c|c|c|c|c|c|}
\hline \multicolumn{13}{|c|}{ Upper Port Row } \\
\hline \multirow{4}{*}{$x / c$} & \multicolumn{12}{|c|}{$R e_{c}$ - Experimental $C_{p, 3 D}$} \\
\hline & \multicolumn{3}{|c|}{$2.40 \mathrm{E}+06$} & & \multicolumn{3}{|c|}{$2.80 \mathrm{E}+06$} & & \multicolumn{3}{|c|}{$3.20 \mathrm{E}+06$} & \\
\hline & & $\operatorname{Abs}(\min )$ & $\operatorname{Abs}(\max )$ & & & $\operatorname{Abs}(\min )$ & $\operatorname{Abs}(\max )$ & & & $\operatorname{Abs}(\min )$ & $\operatorname{Abs}(\max )$ & \\
\hline & Avg & scatter & scatter & Uncert & Avg & scatter & scatter & Uncert & Avg & scatter & scatter & Uncert \\
\hline 0.000 & -0.036 & 0.005 & 0.005 & 0.021 & -0.038 & 0.005 & 0.005 & 0.015 & -0.040 & 0.005 & 0.005 & 0.012 \\
\hline 0.001 & 0.438 & 0.008 & 0.008 & 0.021 & 0.438 & 0.007 & 0.005 & 0.015 & 0.435 & 0.006 & 0.006 & 0.012 \\
\hline 0.002 & 0.490 & 0.007 & 0.008 & 0.021 & 0.490 & 0.008 & 0.006 & 0.015 & 0.488 & 0.007 & 0.007 & 0.012 \\
\hline 0.003 & 0.516 & 0.017 & 0.016 & 0.021 & 0.513 & 0.017 & 0.013 & 0.015 & 0.514 & 0.014 & 0.014 & 0.012 \\
\hline 0.005 & 0.512 & 0.008 & 0.008 & 0.021 & 0.513 & 0.009 & 0.007 & 0.015 & 0.513 & 0.006 & 0.006 & 0.012 \\
\hline 0.010 & 0.464 & 0.007 & 0.007 & 0.021 & 0.464 & 0.008 & 0.006 & 0.015 & 0.465 & 0.006 & 0.006 & 0.012 \\
\hline 0.015 & 0.408 & 0.007 & 0.007 & 0.021 & 0.408 & 0.007 & 0.006 & 0.015 & 0.408 & 0.006 & 0.006 & 0.012 \\
\hline 0.020 & 0.362 & 0.008 & 0.007 & 0.021 & 0.360 & 0.007 & 0.005 & 0.015 & 0.362 & 0.006 & 0.005 & 0.012 \\
\hline 0.025 & 0.330 & 0.007 & 0.006 & 0.021 & 0.329 & 0.006 & 0.006 & 0.015 & 0.330 & 0.005 & 0.005 & 0.012 \\
\hline 0.030 & 0.304 & 0.007 & 0.005 & 0.021 & 0.303 & 0.005 & 0.005 & 0.015 & 0.304 & 0.004 & 0.005 & 0.012 \\
\hline 0.050 & 0.273 & 0.026 & 0.027 & 0.021 & 0.272 & 0.031 & 0.037 & 0.015 & 0.281 & 0.022 & 0.027 & 0.012 \\
\hline 0.075 & 0.139 & 0.005 & 0.004 & 0.021 & 0.140 & 0.005 & 0.005 & 0.015 & 0.143 & 0.005 & 0.005 & 0.012 \\
\hline 0.100 & 0.136 & 0.005 & 0.004 & 0.021 & 0.136 & 0.004 & 0.004 & 0.015 & 0.136 & 0.005 & 0.004 & 0.012 \\
\hline 0.150 & 0.018 & 0.004 & 0.004 & 0.021 & 0.020 & 0.006 & 0.005 & 0.015 & 0.021 & 0.004 & 0.005 & 0.012 \\
\hline 0.200 & -0.042 & 0.005 & 0.004 & 0.021 & -0.041 & 0.004 & 0.005 & 0.015 & -0.040 & 0.004 & 0.004 & 0.012 \\
\hline 0.250 & -0.072 & 0.004 & 0.004 & 0.021 & -0.070 & 0.004 & 0.005 & 0.015 & -0.068 & 0.004 & 0.004 & 0.012 \\
\hline 0.300 & -0.102 & 0.004 & 0.004 & 0.021 & -0.100 & 0.004 & 0.004 & 0.015 & -0.098 & 0.003 & 0.004 & 0.012 \\
\hline 0.350 & -0.139 & 0.004 & 0.005 & 0.021 & -0.137 & 0.004 & 0.005 & 0.015 & -0.136 & 0.004 & 0.005 & 0.012 \\
\hline 0.400 & -0.161 & 0.004 & 0.004 & 0.021 & -0.158 & 0.003 & 0.004 & 0.015 & -0.156 & 0.004 & 0.004 & 0.012 \\
\hline 0.450 & -0.206 & 0.005 & 0.005 & 0.021 & -0.204 & 0.004 & 0.005 & 0.015 & -0.203 & 0.004 & 0.006 & 0.012 \\
\hline 0.500 & -0.225 & 0.005 & 0.005 & 0.021 & -0.223 & 0.005 & 0.005 & 0.015 & -0.220 & 0.004 & 0.006 & 0.012 \\
\hline 0.550 & -0.284 & 0.005 & 0.006 & 0.021 & -0.281 & 0.005 & 0.006 & 0.015 & -0.278 & 0.005 & 0.006 & 0.012 \\
\hline 0.600 & -0.278 & 0.005 & 0.007 & 0.021 & -0.275 & 0.006 & 0.006 & 0.015 & -0.271 & 0.005 & 0.007 & 0.012 \\
\hline 0.650 & -0.303 & 0.006 & 0.007 & 0.021 & -0.301 & 0.005 & 0.006 & 0.015 & -0.298 & 0.005 & 0.007 & 0.012 \\
\hline 0.700 & -0.314 & 0.014 & 0.012 & 0.021 & -0.311 & 0.013 & 0.014 & 0.015 & -0.307 & 0.015 & 0.013 & 0.012 \\
\hline 0.750 & -0.309 & 0.007 & 0.006 & 0.021 & -0.306 & 0.006 & 0.006 & 0.015 & -0.303 & 0.005 & 0.007 & 0.012 \\
\hline 0.800 & -0.202 & 0.005 & 0.005 & 0.021 & -0.200 & 0.005 & 0.006 & 0.015 & -0.199 & 0.005 & 0.006 & 0.012 \\
\hline 0.850 & -0.080 & 0.005 & 0.005 & 0.021 & -0.079 & 0.005 & 0.005 & 0.015 & -0.078 & 0.004 & 0.005 & 0.012 \\
\hline 0.900 & 0.009 & 0.004 & 0.004 & 0.021 & 0.010 & 0.005 & 0.005 & 0.015 & 0.011 & 0.004 & 0.004 & 0.012 \\
\hline
\end{tabular}




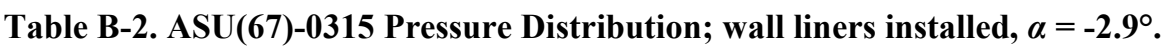

\begin{tabular}{|c|c|c|c|c|c|c|c|c|c|c|c|c|}
\hline \multirow{4}{*}{$\mathrm{x} / \mathrm{c}$} & \multicolumn{12}{|c|}{ Lower Port Row } \\
\hline & \multicolumn{12}{|c|}{$R e_{c}$ - Experimental $C_{p, 3 D}$} \\
\hline & \multicolumn{4}{|c|}{$2.40 \mathrm{E}+06$} & \multicolumn{4}{|c|}{$2.80 \mathrm{E}+06$} & \multicolumn{4}{|c|}{$3.20 \mathrm{E}+06$} \\
\hline & Avg & $\begin{array}{c}\text { Abs }(\min ) \\
\text { scatter }\end{array}$ & $\begin{array}{c}\text { Abs(max) } \\
\text { scatter }\end{array}$ & Uncert & Avg & $\begin{array}{c}\text { Abs(min) } \\
\text { scatter }\end{array}$ & $\begin{array}{c}\text { Abs }(\max ) \\
\text { scatter }\end{array}$ & Uncert & Avg & $\begin{array}{l}\text { Abs(min) } \\
\text { scatter }\end{array}$ & $\begin{array}{c}\text { Abs }(\max ) \\
\text { scatter }\end{array}$ & Uncert \\
\hline 0.00 & -0.048 & 0.005 & 0.003 & 0.021 & -0.051 & 0.003 & 0.003 & 0.015 & -0.057 & 0.003 & 0.003 & 0.011 \\
\hline 0.00 & 0.424 & 0.005 & 0.006 & 0.021 & 0.423 & 0.005 & 0.006 & 0.015 & 0.421 & 0.006 & 0.006 & 0.011 \\
\hline 0.00 & 0.481 & 0.006 & 0.007 & 0.021 & 0.481 & 0.005 & 0.007 & 0.015 & 0.479 & 0.007 & 0.006 & 0.011 \\
\hline 0.00 & 0.504 & 0.007 & 0.007 & 0.021 & 0.502 & 0.007 & 0.007 & 0.015 & 0.504 & 0.008 & 0.006 & 0.011 \\
\hline 0.01 & 0.505 & 0.007 & 0.007 & 0.021 & 0.506 & 0.006 & 0.007 & 0.015 & 0.507 & 0.007 & 0.007 & 0.011 \\
\hline 0.01 & 0.455 & 0.005 & 0.006 & 0.021 & 0.456 & 0.006 & 0.007 & 0.015 & 0.459 & 0.007 & 0.006 & 0.011 \\
\hline 0.02 & 0.393 & 0.006 & 0.006 & 0.021 & 0.394 & 0.006 & 0.005 & 0.015 & 0.398 & 0.007 & 0.006 & 0.011 \\
\hline 0.02 & 0.350 & 0.005 & 0.005 & 0.021 & 0.352 & 0.005 & 0.005 & 0.015 & 0.355 & 0.005 & 0.005 & 0.011 \\
\hline 0.03 & 0.314 & 0.006 & 0.005 & 0.021 & 0.315 & 0.005 & 0.004 & 0.015 & 0.318 & 0.005 & 0.005 & 0.011 \\
\hline 0.03 & 0.416 & 0.007 & 0.008 & 0.021 & 0.418 & 0.008 & 0.008 & 0.015 & 0.420 & 0.009 & 0.006 & 0.011 \\
\hline 0.05 & 0.195 & 0.004 & 0.005 & 0.021 & 0.198 & 0.004 & 0.004 & 0.015 & 0.201 & 0.004 & 0.004 & 0.011 \\
\hline 0.08 & 0.124 & 0.004 & 0.004 & 0.021 & 0.127 & 0.004 & 0.003 & 0.015 & 0.131 & 0.004 & 0.003 & 0.011 \\
\hline 0.10 & 0.074 & 0.013 & 0.012 & 0.021 & 0.076 & 0.015 & 0.017 & 0.015 & 0.081 & 0.011 & 0.011 & 0.011 \\
\hline 0.15 & -0.027 & 0.005 & 0.005 & 0.021 & -0.025 & 0.004 & 0.004 & 0.015 & -0.022 & 0.004 & 0.003 & 0.011 \\
\hline 0.20 & -0.053 & 0.004 & 0.005 & 0.021 & -0.051 & 0.005 & 0.004 & 0.015 & -0.047 & 0.004 & 0.005 & 0.011 \\
\hline 0.25 & -0.090 & 0.006 & 0.007 & 0.021 & -0.088 & 0.006 & 0.006 & 0.015 & -0.085 & 0.006 & 0.006 & 0.011 \\
\hline 0.30 & -0.126 & 0.006 & 0.008 & 0.021 & -0.124 & 0.007 & 0.006 & 0.015 & -0.121 & 0.006 & 0.006 & 0.011 \\
\hline 0.35 & -0.146 & 0.007 & 0.009 & 0.021 & -0.143 & 0.007 & 0.008 & 0.015 & -0.141 & 0.007 & 0.007 & 0.011 \\
\hline 0.40 & -0.182 & 0.007 & 0.010 & 0.021 & -0.179 & 0.008 & 0.009 & 0.015 & -0.187 & 0.007 & 0.008 & 0.011 \\
\hline 0.45 & -0.221 & 0.007 & 0.009 & 0.021 & -0.218 & 0.008 & 0.007 & 0.015 & -0.233 & 0.007 & 0.010 & 0.011 \\
\hline 0.50 & -0.236 & 0.007 & 0.010 & 0.021 & -0.234 & 0.008 & 0.008 & 0.015 & -0.233 & 0.007 & 0.008 & 0.011 \\
\hline 0.55 & -0.268 & 0.008 & 0.008 & 0.021 & -0.266 & 0.009 & 0.008 & 0.015 & -0.277 & 0.007 & 0.008 & 0.011 \\
\hline 0.60 & -0.279 & 0.007 & 0.009 & 0.021 & -0.273 & 0.008 & 0.008 & 0.015 & -0.269 & 0.007 & 0.007 & 0.011 \\
\hline 0.65 & -0.306 & 0.007 & 0.010 & 0.021 & -0.303 & 0.009 & 0.007 & 0.015 & -0.300 & 0.007 & 0.007 & 0.011 \\
\hline 0.70 & -0.339 & 0.007 & 0.009 & 0.021 & -0.338 & 0.009 & 0.008 & 0.015 & -0.334 & 0.006 & 0.008 & 0.011 \\
\hline 0.75 & -0.329 & 0.008 & 0.009 & 0.021 & -0.326 & 0.009 & 0.008 & 0.015 & -0.322 & 0.007 & 0.007 & 0.011 \\
\hline 0.80 & -0.206 & 0.006 & 0.009 & 0.021 & -0.203 & 0.008 & 0.006 & 0.015 & -0.200 & 0.006 & 0.006 & 0.011 \\
\hline 0.85 & -0.090 & 0.006 & 0.008 & 0.021 & -0.086 & 0.007 & 0.006 & 0.015 & -0.083 & 0.006 & 0.005 & 0.011 \\
\hline 0.90 & 0.002 & 0.007 & 0.008 & 0.021 & 0.003 & 0.007 & 0.006 & 0.015 & 0.004 & 0.005 & 0.005 & 0.011 \\
\hline
\end{tabular}


Table B-3. Freestream Parameters for Tables B-1 and B-2.

\begin{tabular}{|lccccc|}
\hline $\begin{array}{l}\text { Pressure } \\
\text { Port Row }\end{array}$ & $\begin{array}{c}\boldsymbol{R e}_{\boldsymbol{c}} \\
\text { Upper }\end{array}$ & $\begin{array}{c}\boldsymbol{p}_{\infty} \\
{[\text { torr] }}\end{array}$ & $\begin{array}{c}\boldsymbol{q} \\
{[\text { torr] }}\end{array}$ & $\begin{array}{c}\boldsymbol{T} \\
{\left[{ }^{\circ} \mathbf{C}\right]}\end{array}$ & $\begin{array}{c}\boldsymbol{U}_{\infty} \\
{[\mathbf{m} / \mathbf{s}]}\end{array}$ \\
Upper & $2.40 \mathrm{E}+06$ & 754.6 & 1.800 & 21.9 & 20.1 \\
Upper & $2.80 \mathrm{E}+06$ & 754.2 & 2.468 & 22.5 & 23.6 \\
Lower & $3.20 \mathrm{E}+06$ & 753.8 & 3.242 & 23.3 & 27.0 \\
Lower & $2.40 \mathrm{E}+06$ & 754.8 & 1.817 & 22.9 & 20.2 \\
Lower & $2.80 \mathrm{E}+06$ & 754.4 & 2.486 & 23.5 & 23.7 \\
\hline
\end{tabular}

Table B-4. ASU(67)-0315 Pressure Distribution for White \& Saric (2005); wall liners installed, $\alpha=-3.4^{\circ}, R e_{c}=2.4 \times 10^{6}$.

\begin{tabular}{|crr|}
\hline $\boldsymbol{x} / \boldsymbol{c}$ & Upper & Lower \\
0.00 & 0.125 & -0.138 \\
0.00 & 0.472 & 0.368 \\
0.00 & 0.511 & 0.445 \\
0.00 & 0.307 & 0.477 \\
0.01 & 0.481 & 0.492 \\
0.01 & 0.409 & 0.458 \\
0.02 & 0.342 & 0.405 \\
0.02 & 0.292 & 0.363 \\
0.03 & 0.266 & 0.330 \\
0.03 & 0.247 & 0.203 \\
0.05 & 0.177 & 0.174 \\
0.08 & 0.093 & 0.150 \\
0.10 & 0.056 & 0.111 \\
0.15 & -0.023 & 0.011 \\
0.20 & -0.070 & -0.015 \\
0.25 & -0.081 & -0.060 \\
0.30 & -0.117 & -0.105 \\
0.35 & -0.148 & -0.129 \\
0.40 & -0.176 & -0.176 \\
0.45 & -0.219 & -0.219 \\
0.50 & -0.232 & -0.237 \\
0.55 & -0.276 & -0.271 \\
0.60 & -0.287 & -0.286 \\
0.65 & -0.330 & -0.316 \\
0.70 & -0.315 & -0.351 \\
0.75 & -0.347 & -0.333 \\
0.80 & -0.234 & -0.205 \\
0.85 & -0.101 & -0.090 \\
0.90 & 0.000 & 0.001 \\
\hline & & \\
\hline
\end{tabular}




\section{APPENDIX C}

Power Spectral Densities for Empty Test Section Flow Quality Measurements 


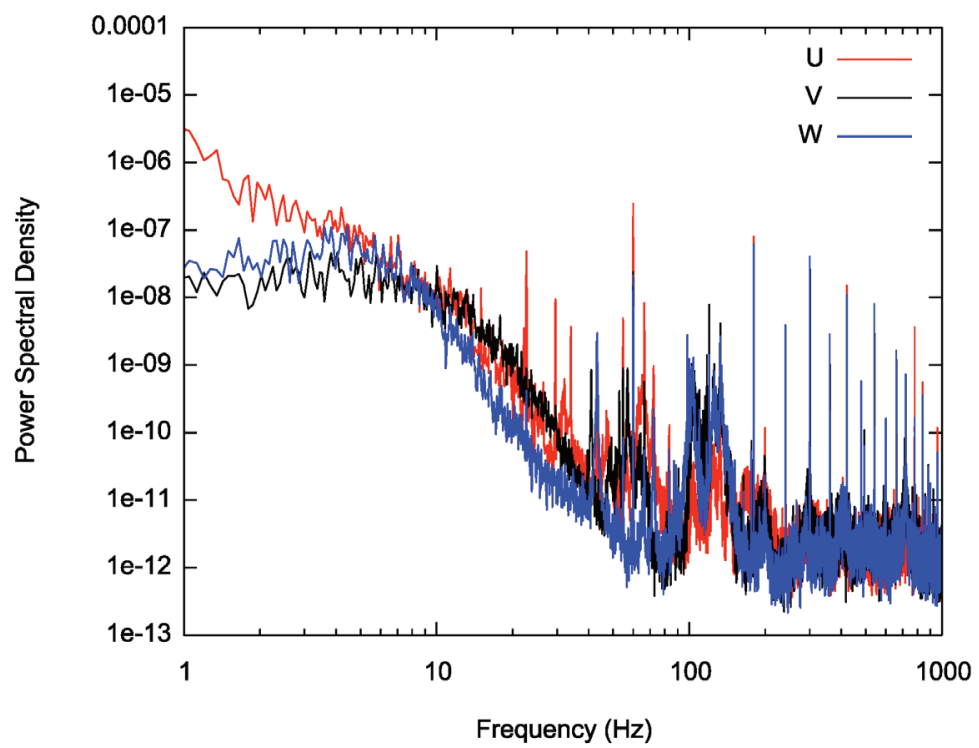

Figure C-1. Spectra from crosswire measurements at $(0.035,0.204,0.593)$ for $10 \mathrm{~m} / \mathrm{s}$.

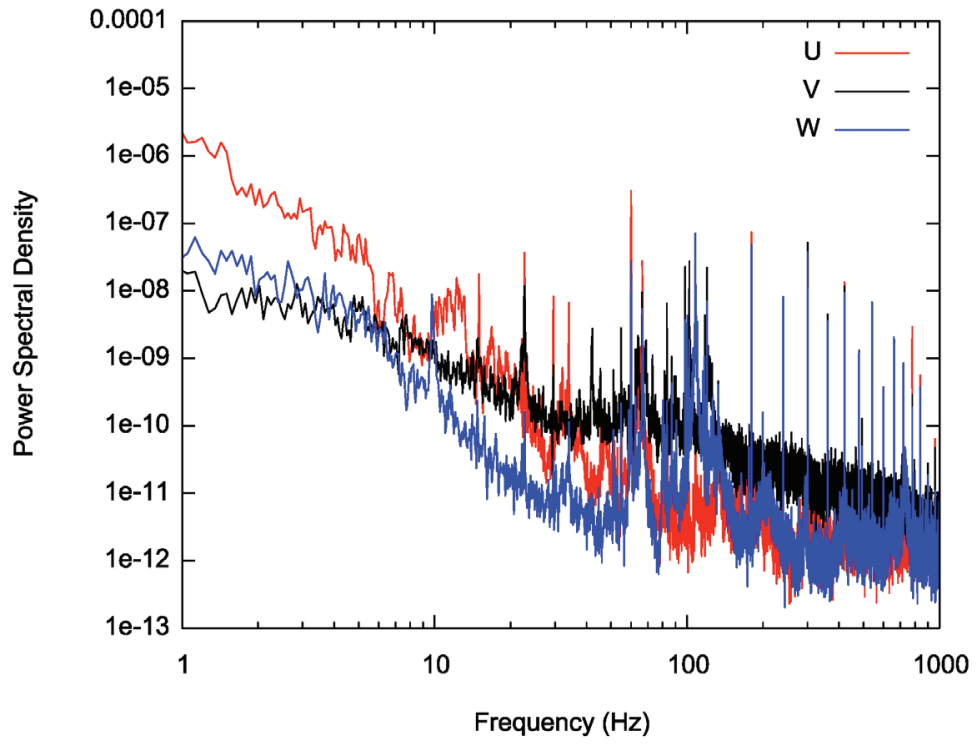

Figure C-2. Spectra from crosswire measurements at $(0.035,0.204,-0.036)$ for $10 \mathrm{~m} / \mathrm{s}$. 


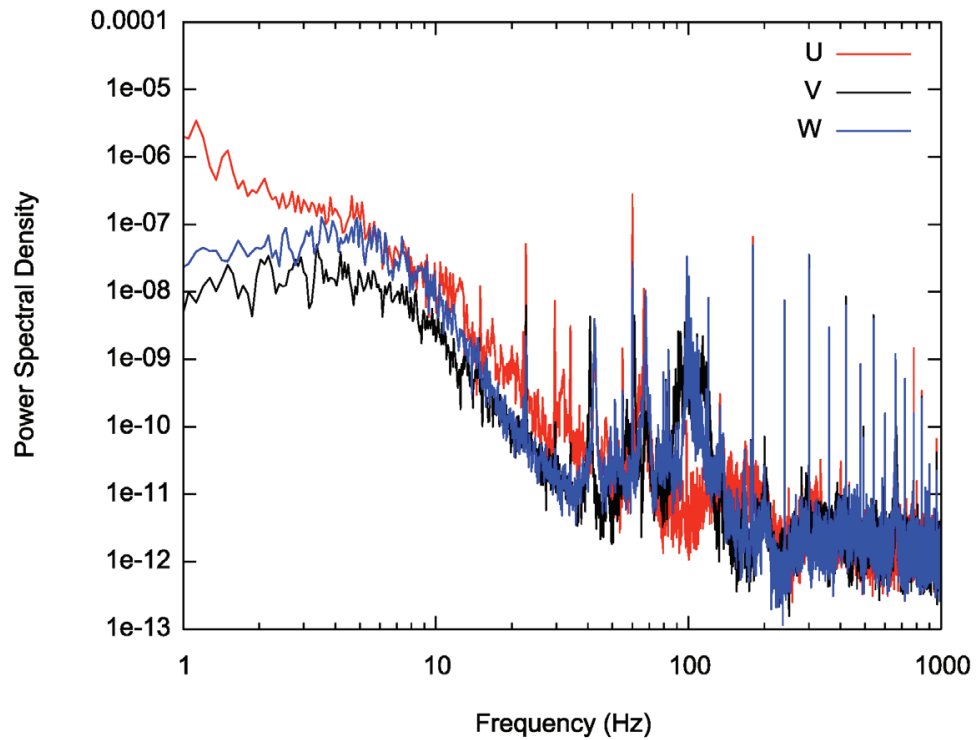

Figure C-3. Spectra from crosswire measurements at $(\mathbf{0 . 0 3 5}, \mathbf{0 . 2 0 4}, \mathbf{- 0 . 5 9 3 )}$ for $10 \mathrm{~m} / \mathrm{s}$.

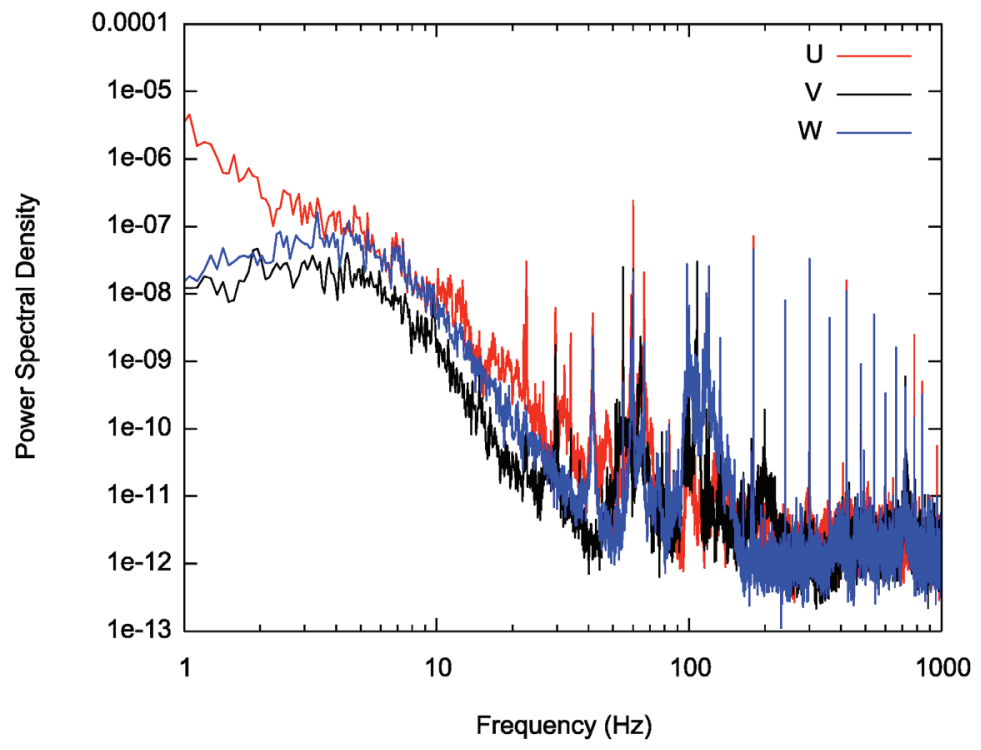

Figure C-4. Spectra from crosswire measurements at $(0.035,0.519,0.593)$ for $10 \mathrm{~m} / \mathrm{s}$. 


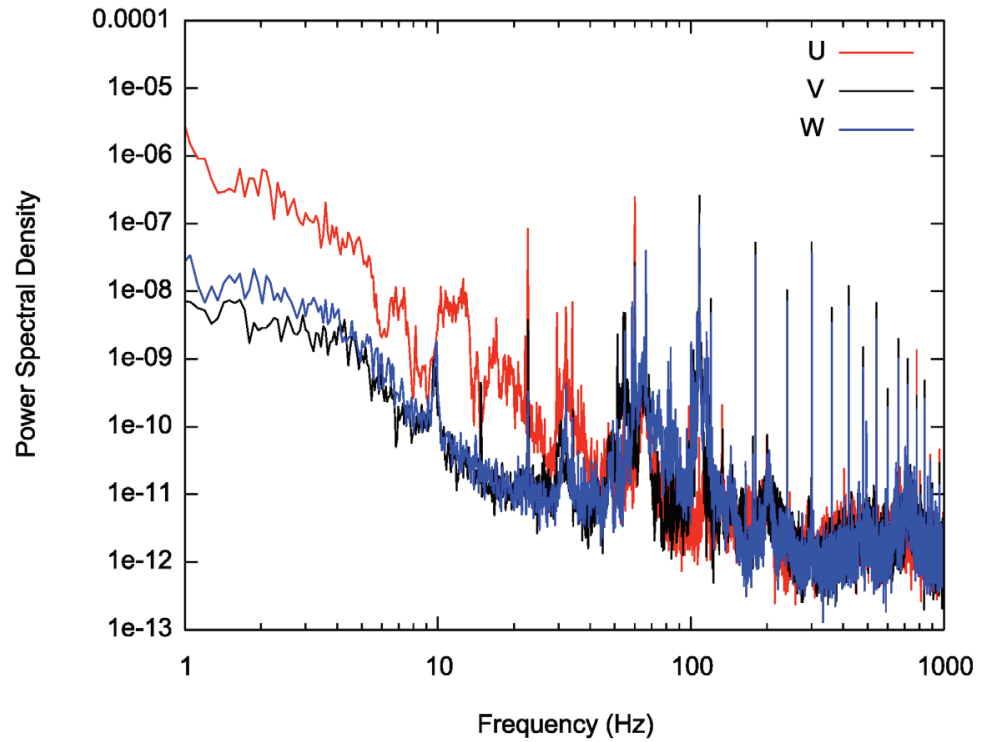

Figure C-5. Spectra from crosswire measurements at $(0.035,0.519,-\mathbf{0 . 0 3 6})$ for $10 \mathrm{~m} / \mathrm{s}$.

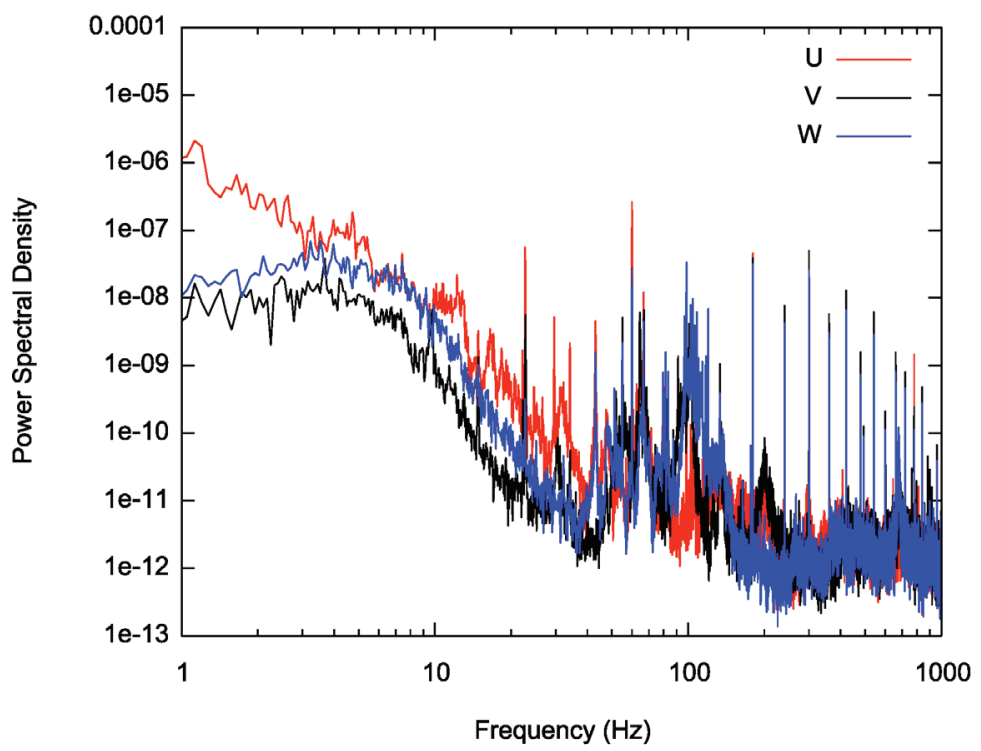

Figure C-6. Spectra from crosswire measurements at $(0.035,0.519,-0.593)$ for $10 \mathrm{~m} / \mathrm{s}$. 


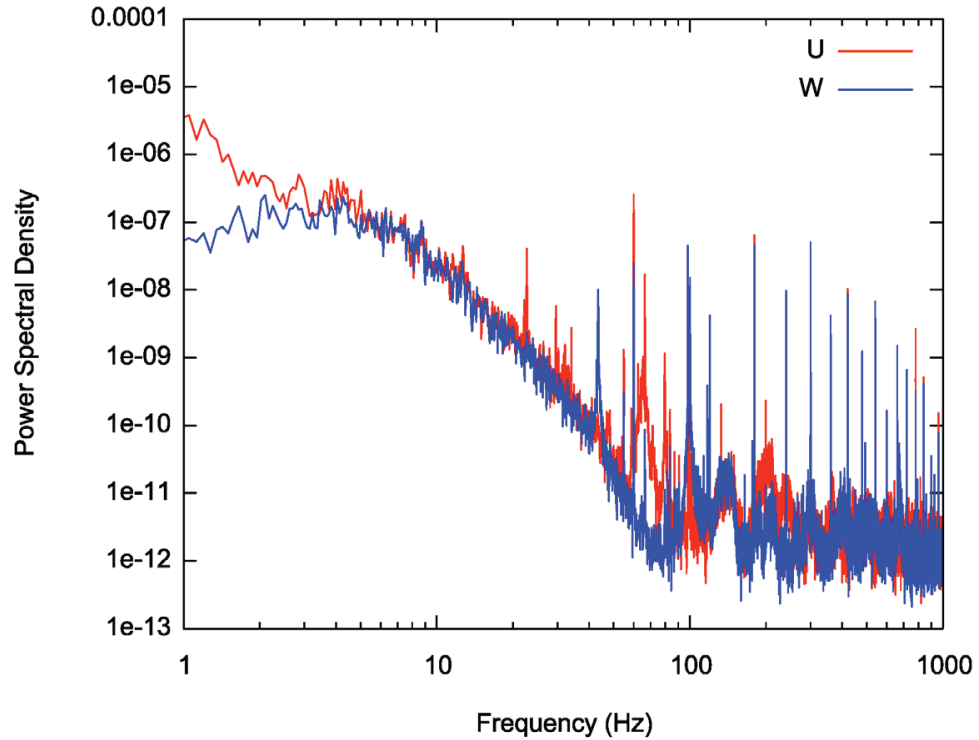

Figure C-7. Spectra from crosswire measurements at $(0.035,0.838,0.593)$ for $10 \mathrm{~m} / \mathrm{s}$.

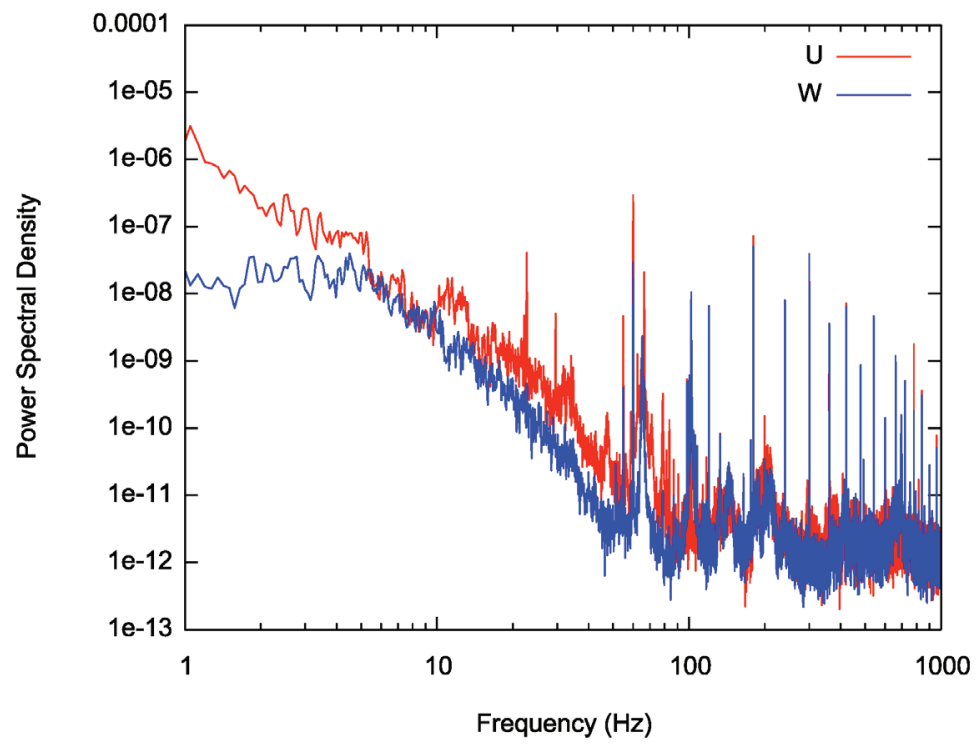

Figure C-8. Spectra from crosswire measurements at $(0.035,0.838,-0.036)$ for $10 \mathrm{~m} / \mathrm{s}$. 


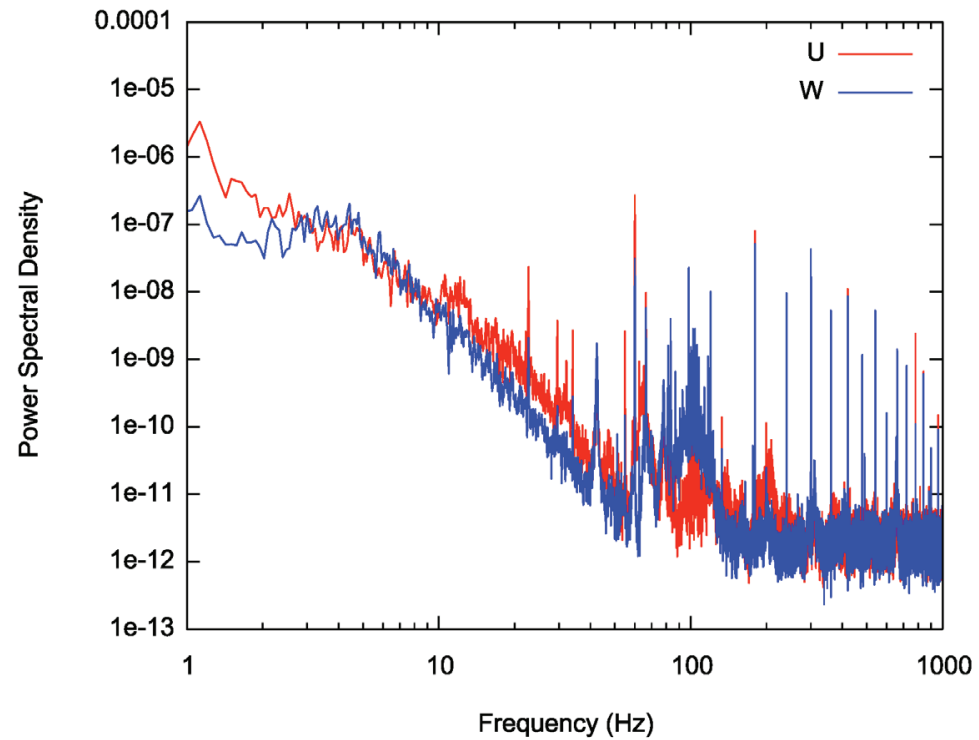

Figure C-9. Spectra from crosswire measurements at $(\mathbf{0 . 0 3 5}, \mathbf{0 . 8 3 8}, \mathbf{- 0 . 5 9 3 )}$ for $10 \mathrm{~m} / \mathrm{s}$.

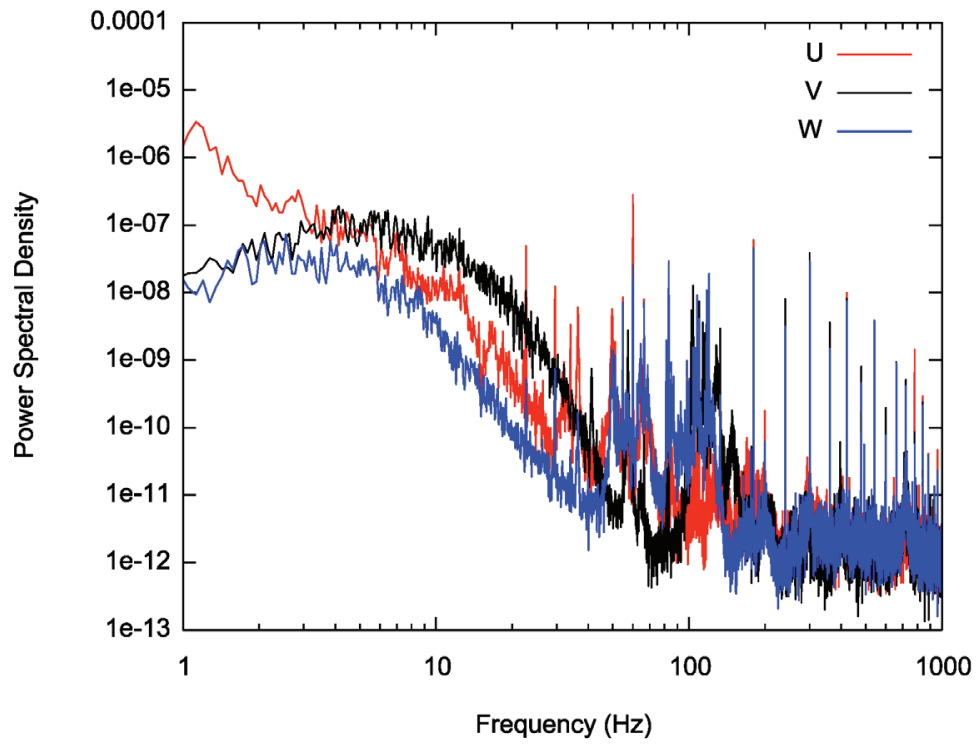

Figure C-10. Spectra from crosswire measurements at $(0.406,0.204,0.598)$ for $10 \mathrm{~m} / \mathrm{s}$. 


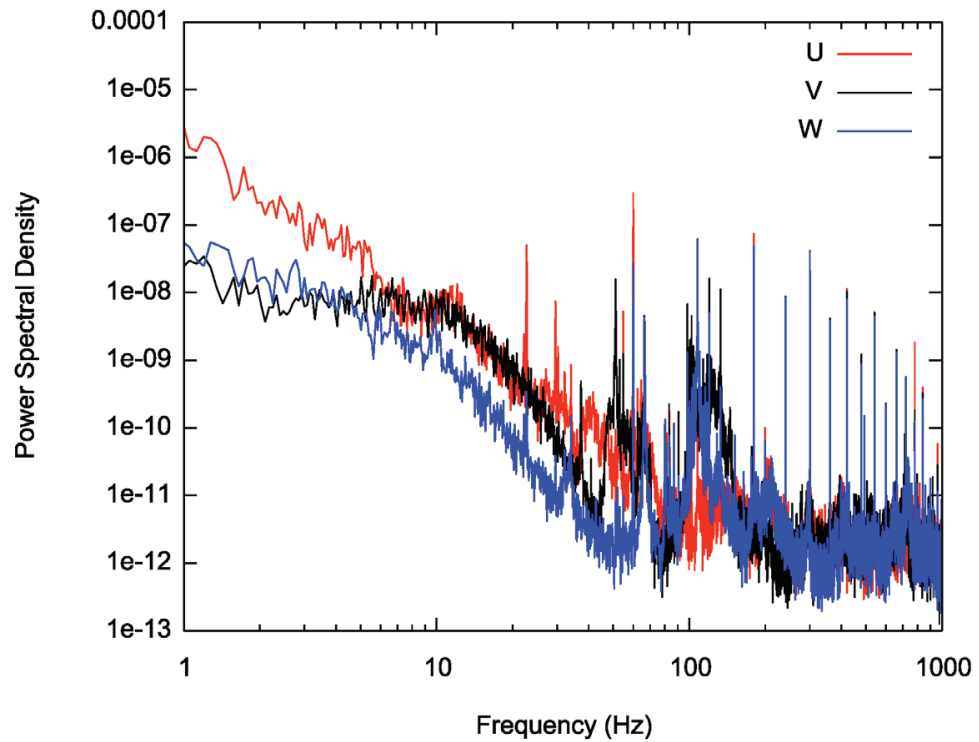

Figure C-11. Spectra from crosswire measurements at $(0.406,0.204,-0.024)$ for $10 \mathrm{~m} / \mathrm{s}$.

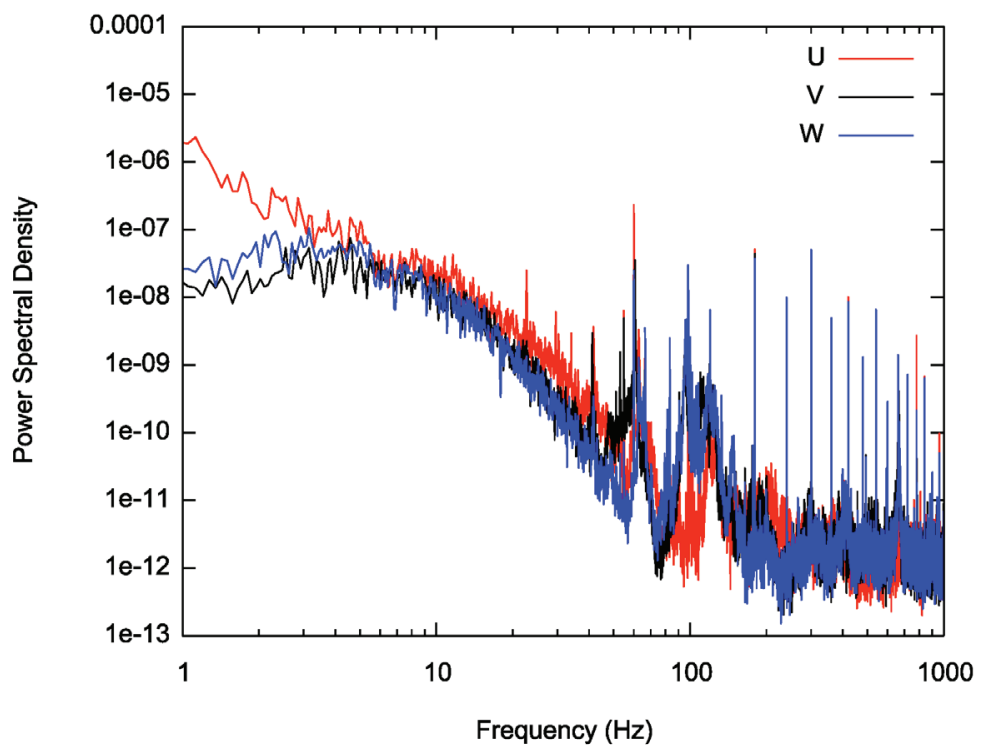

Figure C-12. Spectra from crosswire measurements at $(0.406,0.204,-0.598)$ for $10 \mathrm{~m} / \mathrm{s}$. 


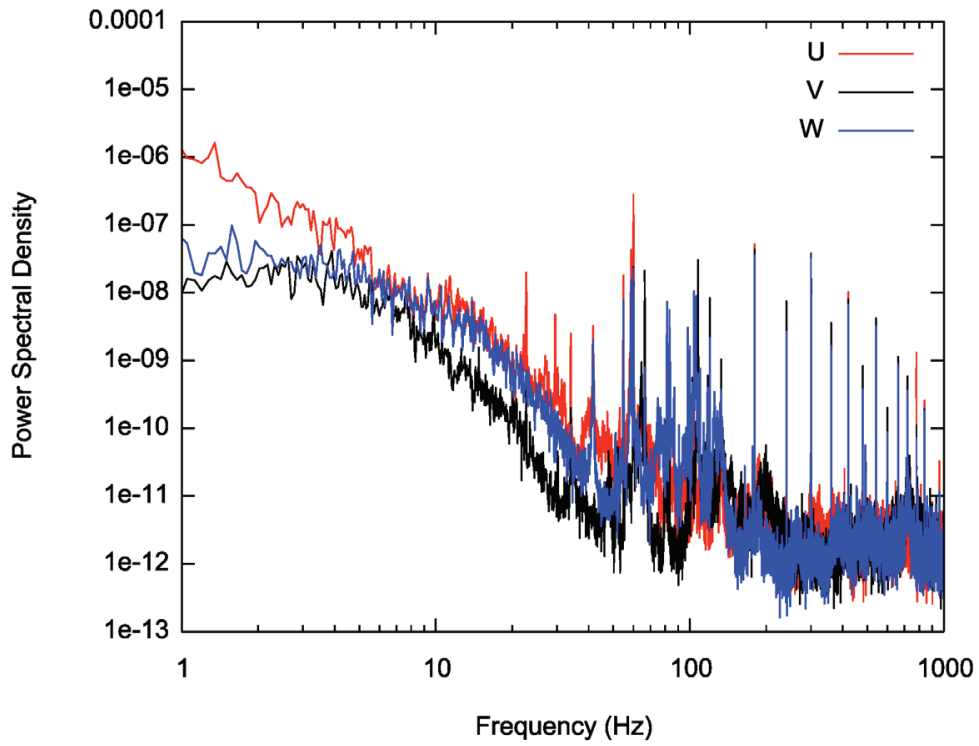

Figure C-13. Spectra from crosswire measurements at $(0.406,0.519,0.598)$ for $10 \mathrm{~m} / \mathrm{s}$.

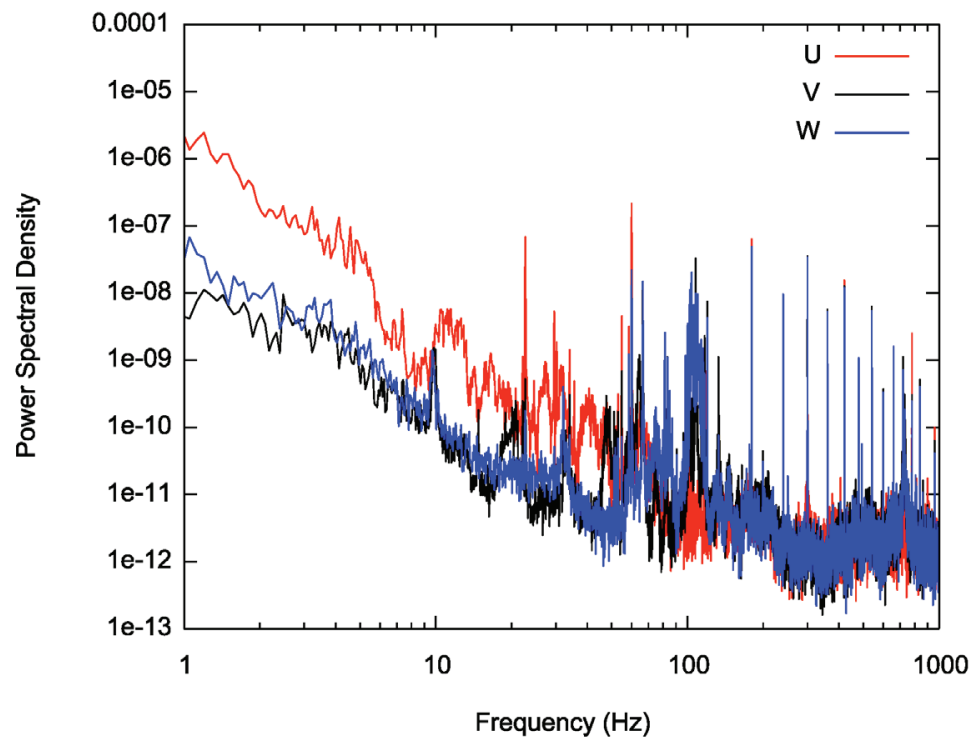

Figure C-14. Spectra from crosswire measurements at $(0.406,0.519,-0.024)$ for $10 \mathrm{~m} / \mathrm{s}$. 


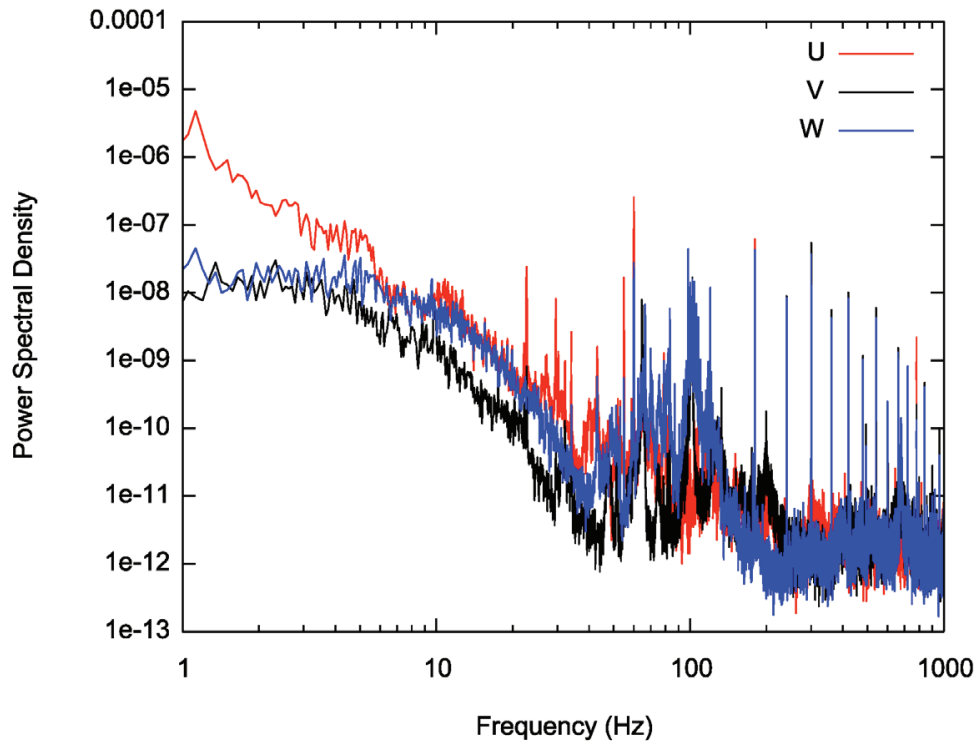

Figure C-15. Spectra from crosswire measurements at $(0.406,0.519,-0.598)$ for $10 \mathrm{~m} / \mathrm{s}$.

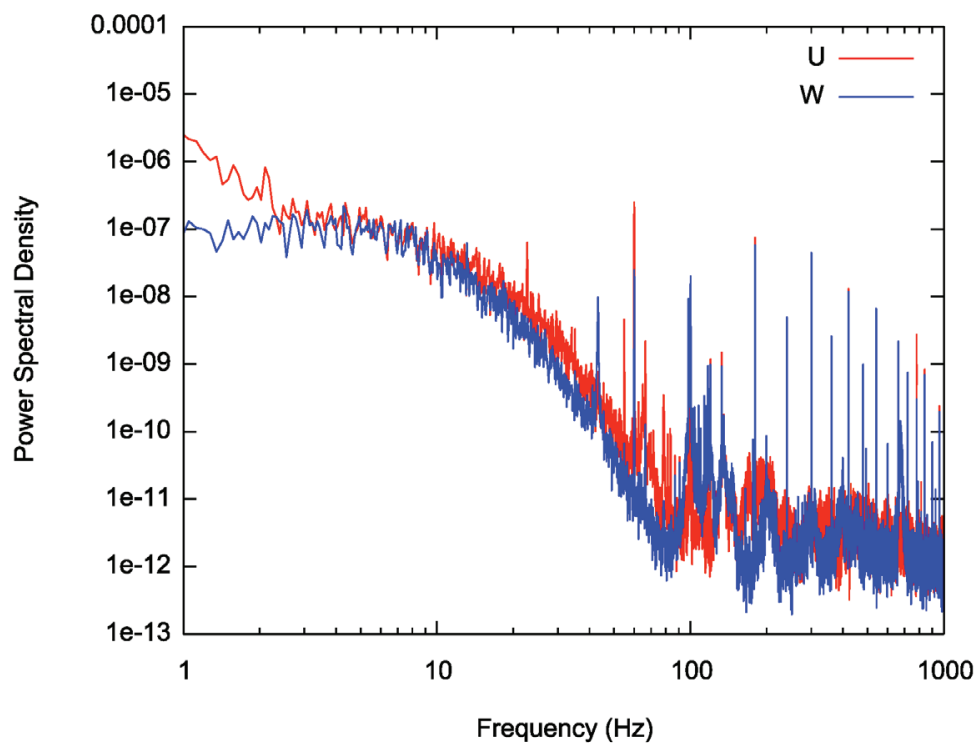

Figure C-16. Spectra from crosswire measurements at $(0.406,0.838,0.598)$ for $10 \mathrm{~m} / \mathrm{s}$. 


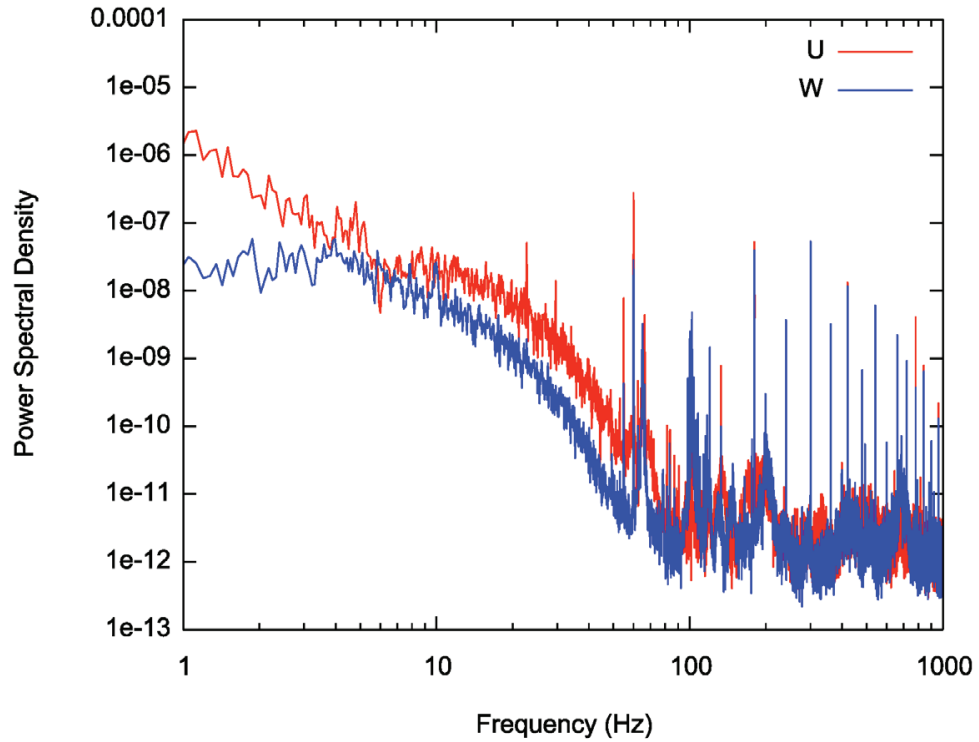

Figure C-17. Spectra from crosswire measurements at $(0.406,0.838,-0.024)$ for $10 \mathrm{~m} / \mathrm{s}$.

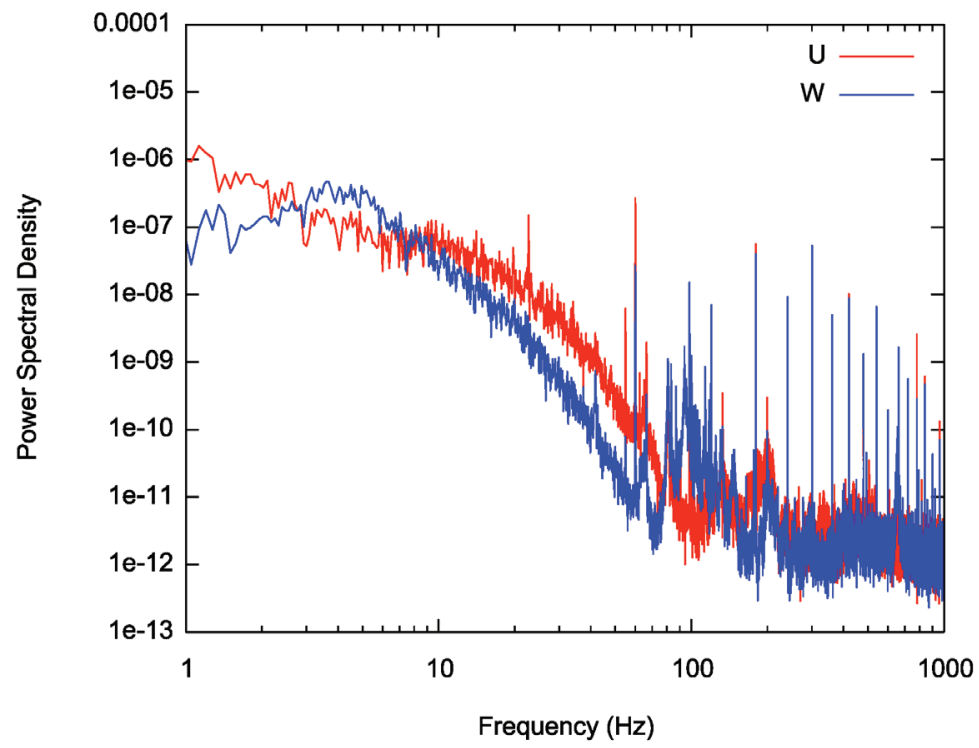

Figure C-18. Spectra from crosswire measurements at $(0.406,0.838,-0.598)$ for $10 \mathrm{~m} / \mathrm{s}$. 


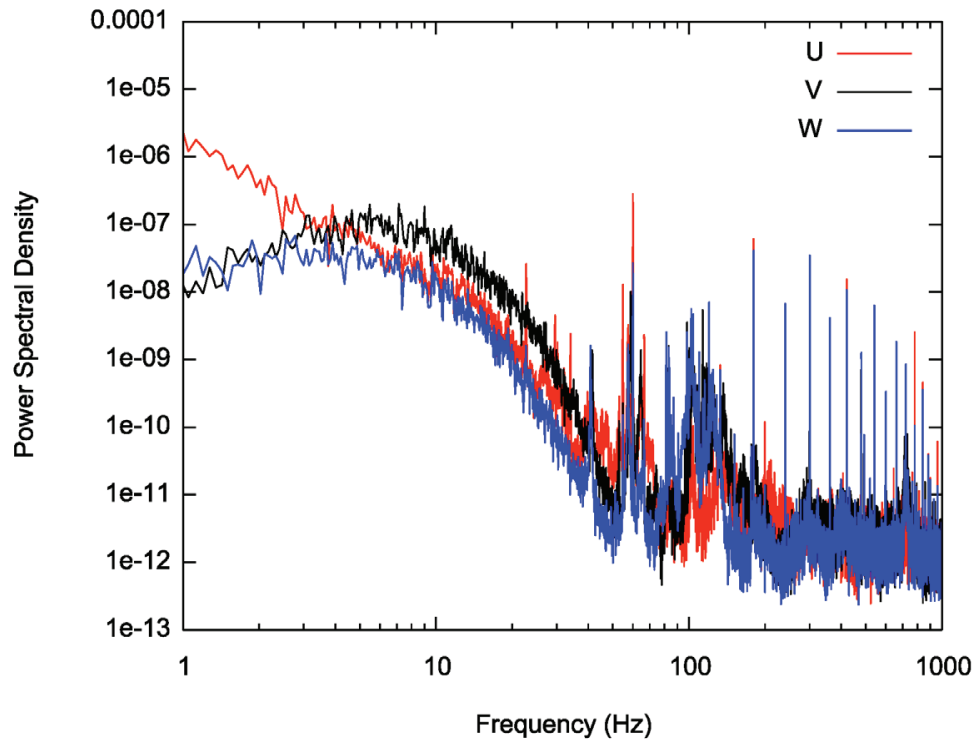

Figure C-19. Spectra from crosswire measurements at $(0.750,0.204,0.602)$ for $10 \mathrm{~m} / \mathrm{s}$.

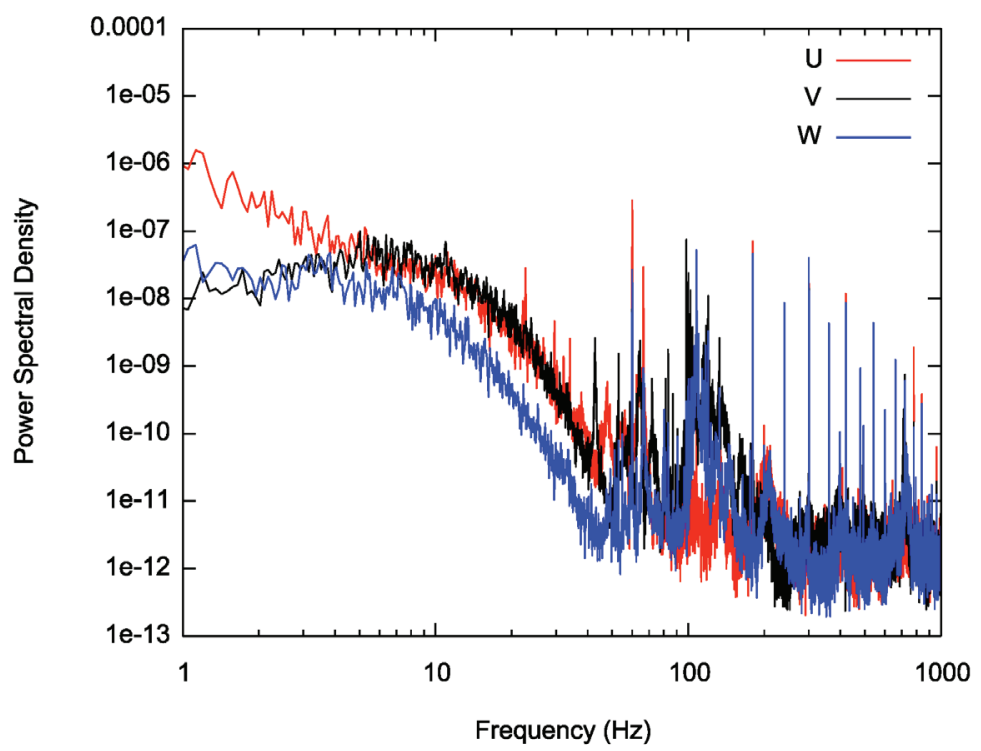

Figure C-20. Spectra from crosswire measurements at $(0.750,0.204,-0.013)$ for $10 \mathrm{~m} / \mathrm{s}$. 


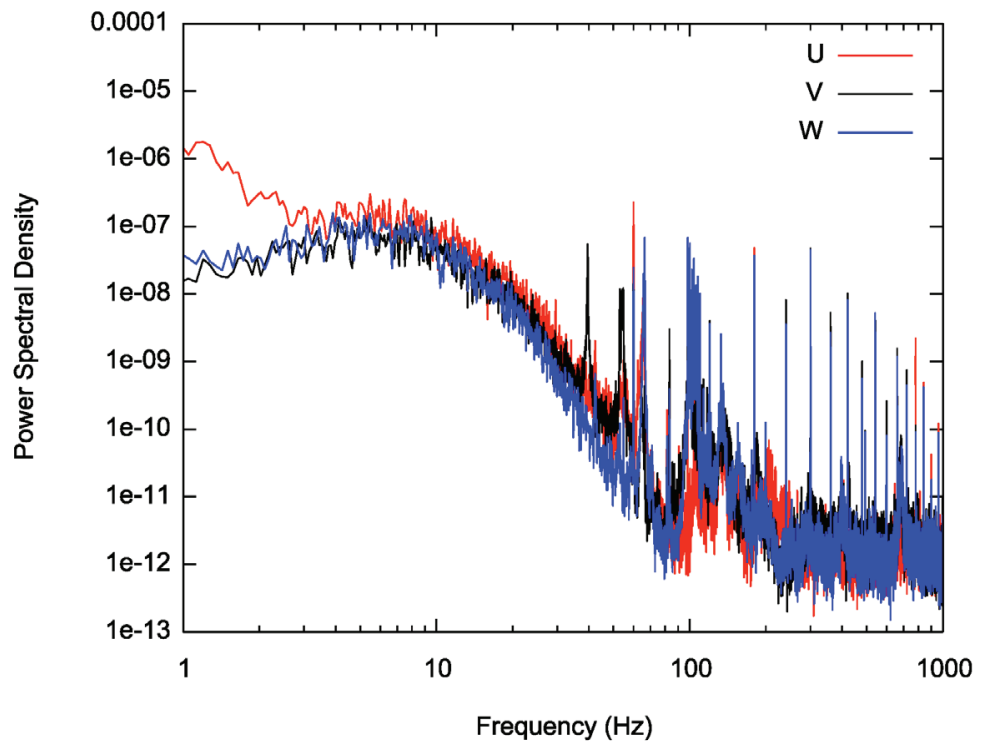

Figure C-21. Spectra from crosswire measurements at $(0.750,0.204,-0.602)$ for $10 \mathrm{~m} / \mathrm{s}$.

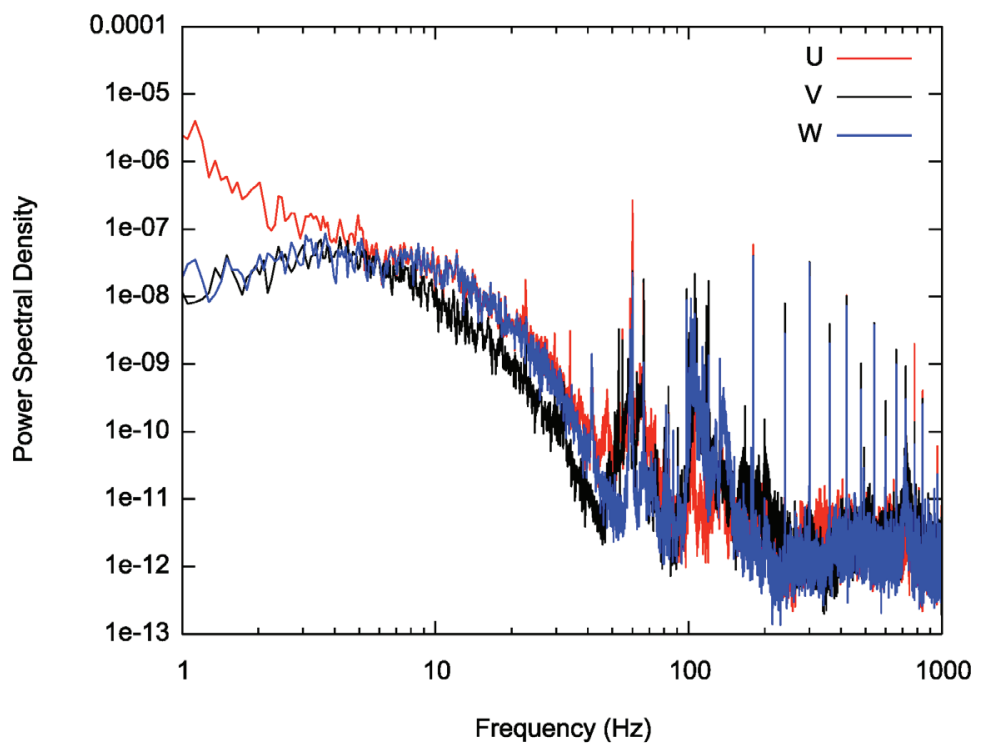

Figure C-22. Spectra from crosswire measurements at $(0.750,0.519,0.602)$ for $10 \mathrm{~m} / \mathrm{s}$. 


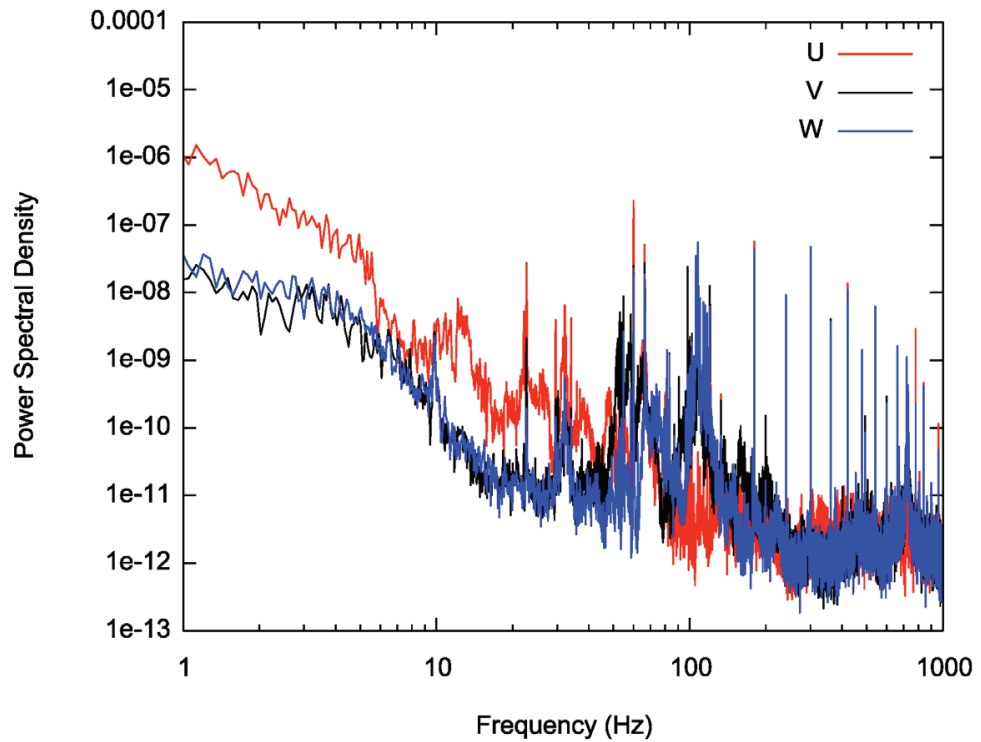

Figure C-23. Spectra from crosswire measurements at $(0.750,0.519,-0.013)$ for $10 \mathrm{~m} / \mathrm{s}$.

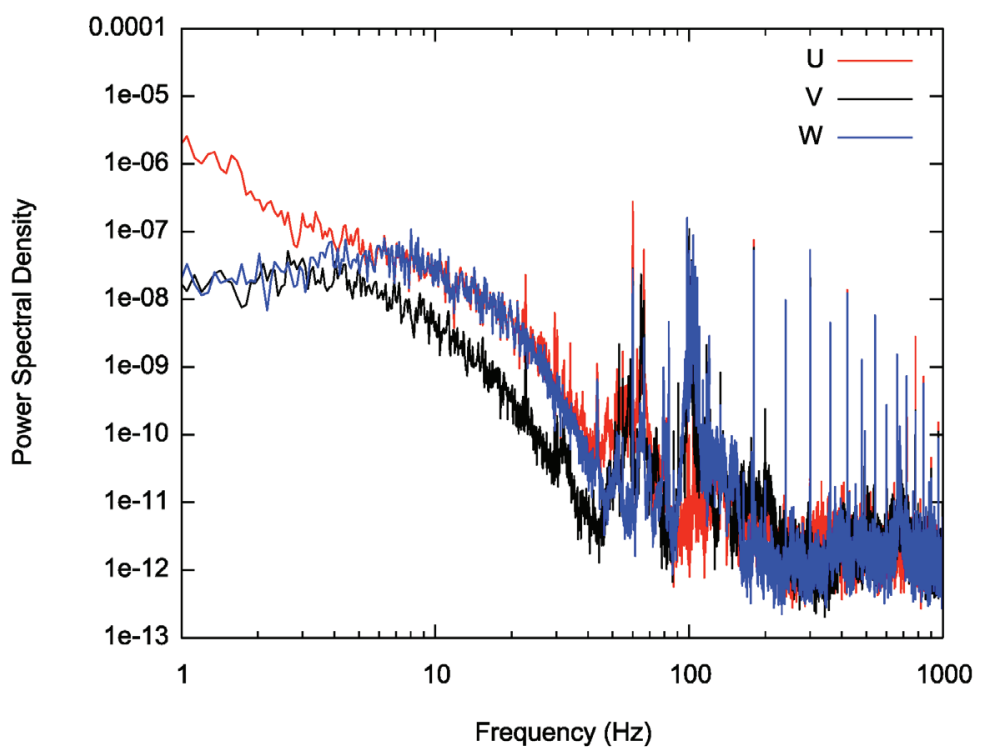

Figure C-24. Spectra from crosswire measurements at $(0.750,0.519,-0.602)$ for $10 \mathrm{~m} / \mathrm{s}$. 


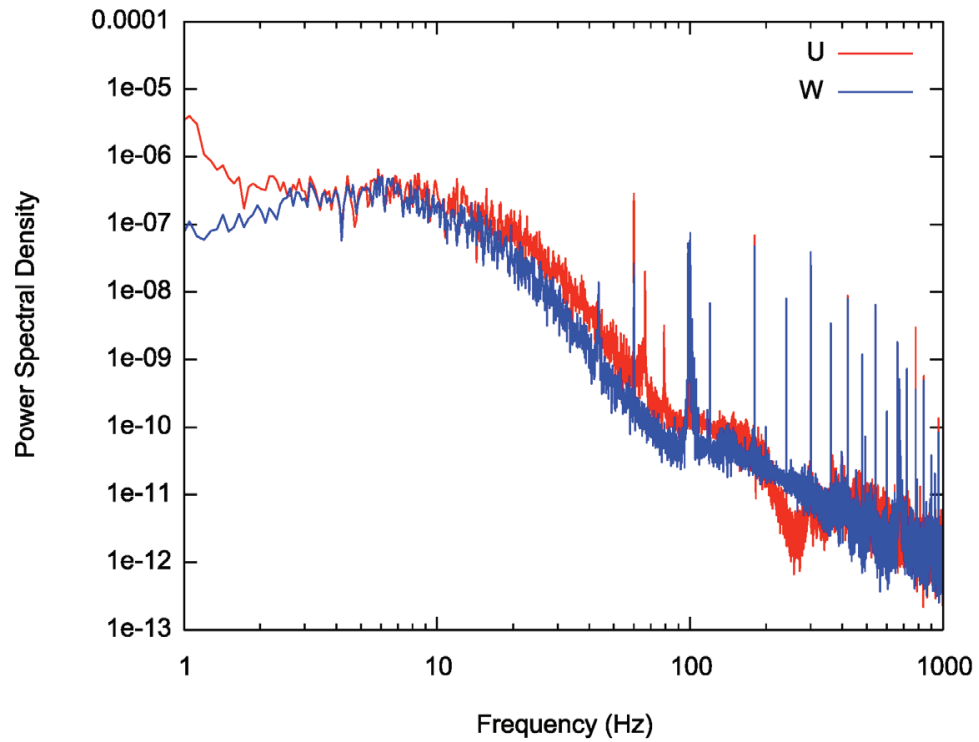

Figure C-25. Spectra from crosswire measurements at $(0.750,0.838,0.602)$ for $10 \mathrm{~m} / \mathrm{s}$.

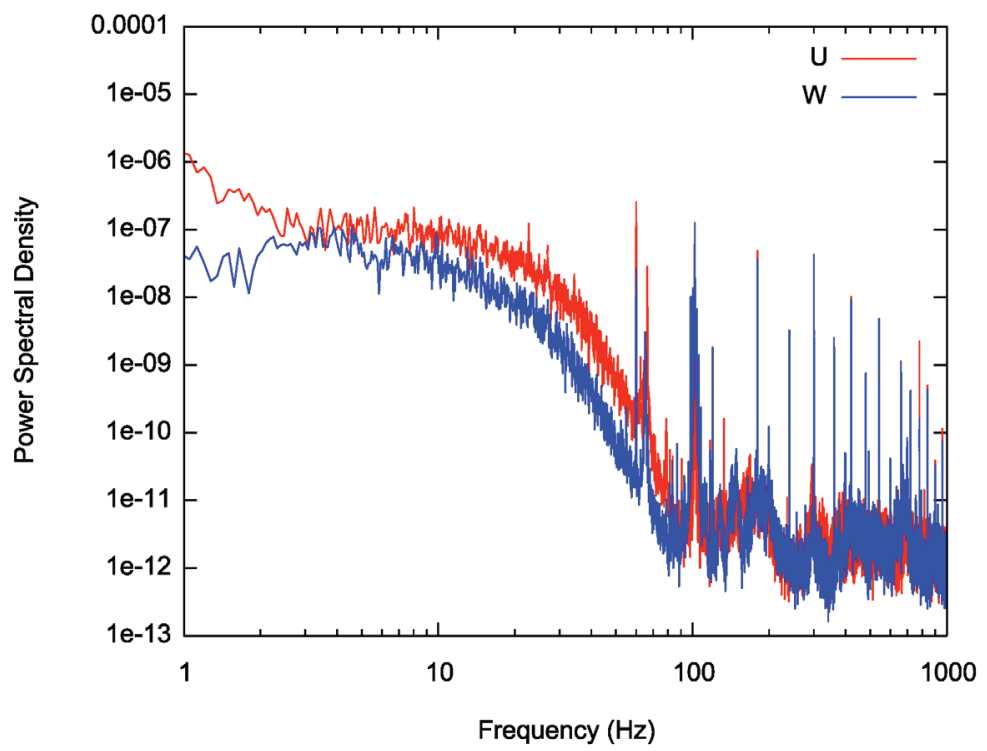

Figure C-26. Spectra from crosswire measurements at $(0.750,0.838,-0.013)$ for $10 \mathrm{~m} / \mathrm{s}$. 


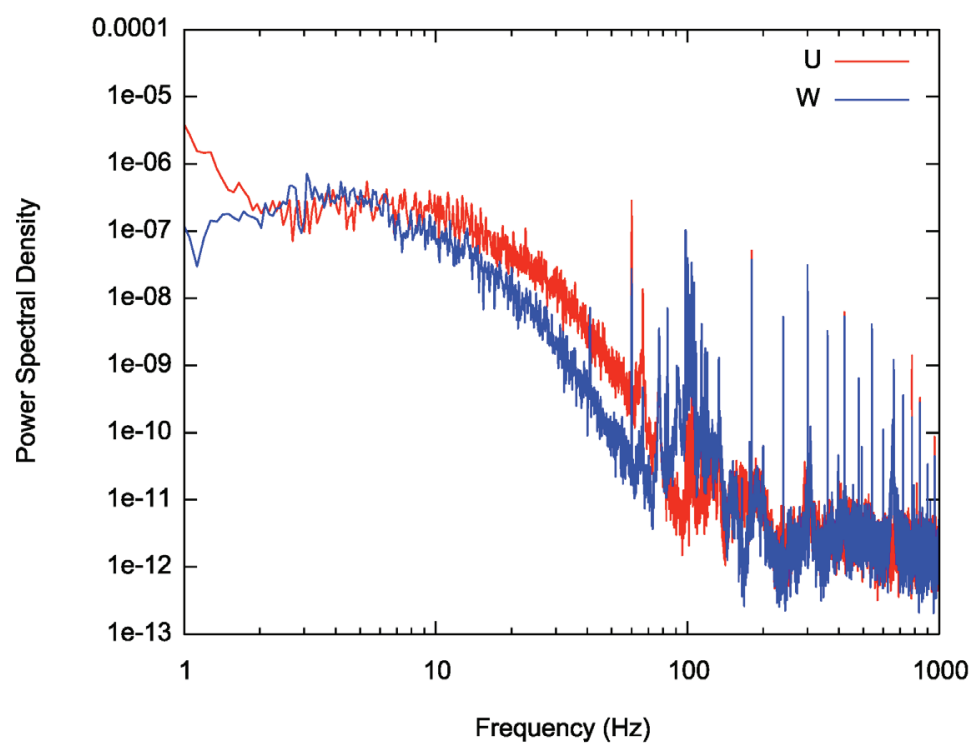

Figure C-27. Spectra from crosswire measurements at $(0.750,0.838,-0.602)$ for $10 \mathrm{~m} / \mathrm{s}$.

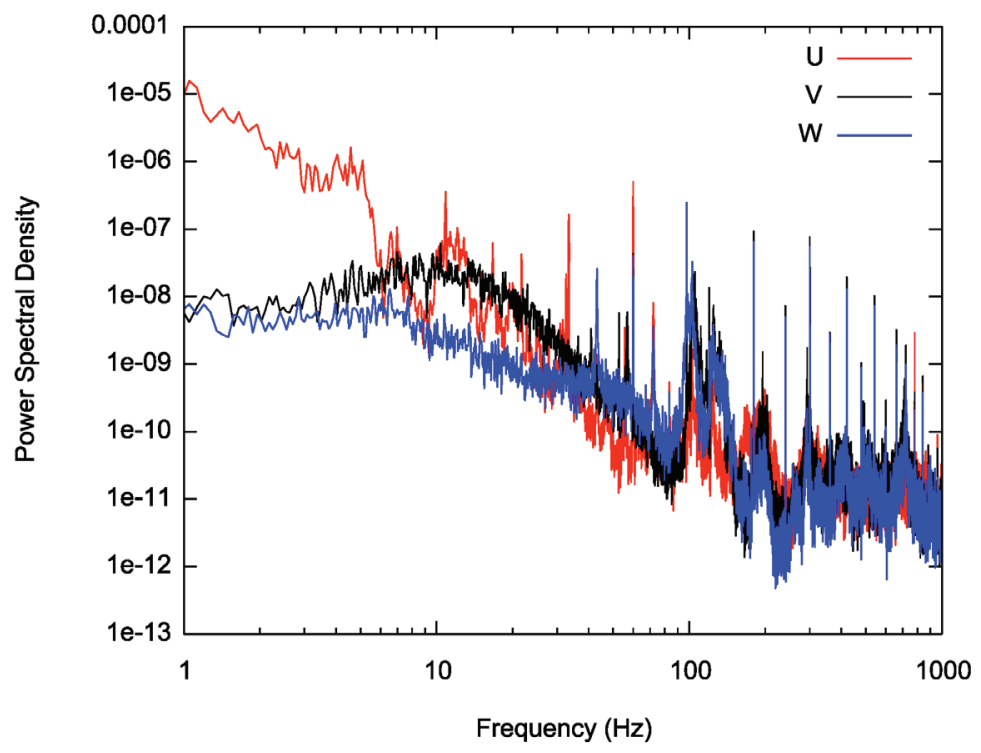

Figure C-28. Spectra from crosswire measurements at $(0.035,0.204,0.593)$ for $15 \mathrm{~m} / \mathrm{s}$. 


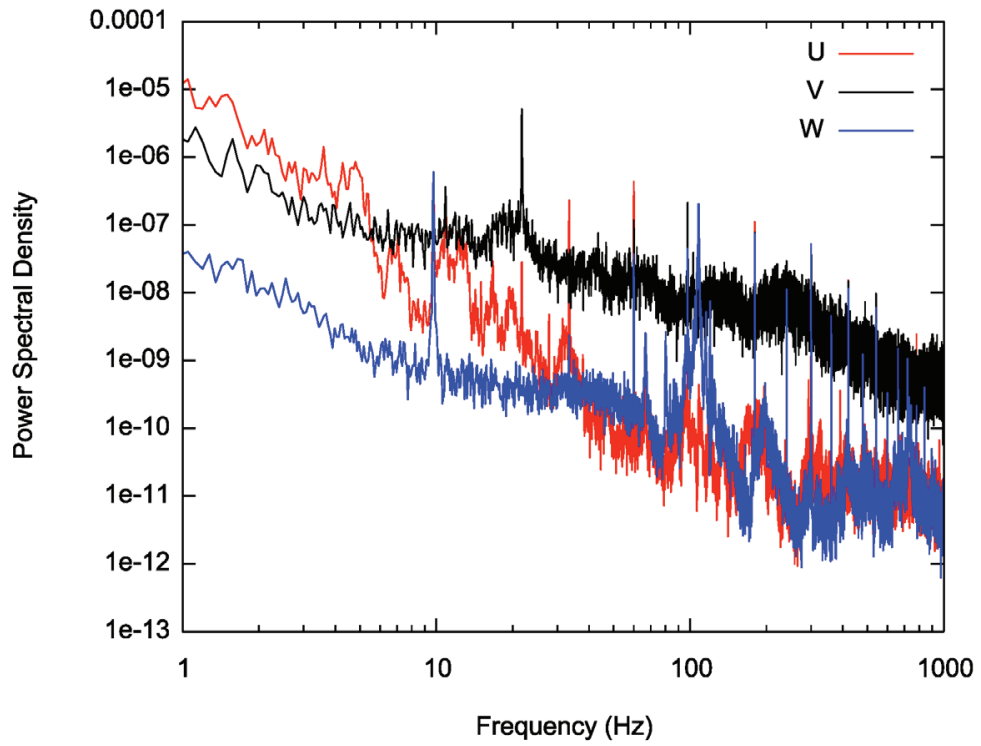

Figure C-29. Spectra from crosswire measurements at $(0.035,0.204,-0.036)$ for $15 \mathrm{~m} / \mathrm{s}$.

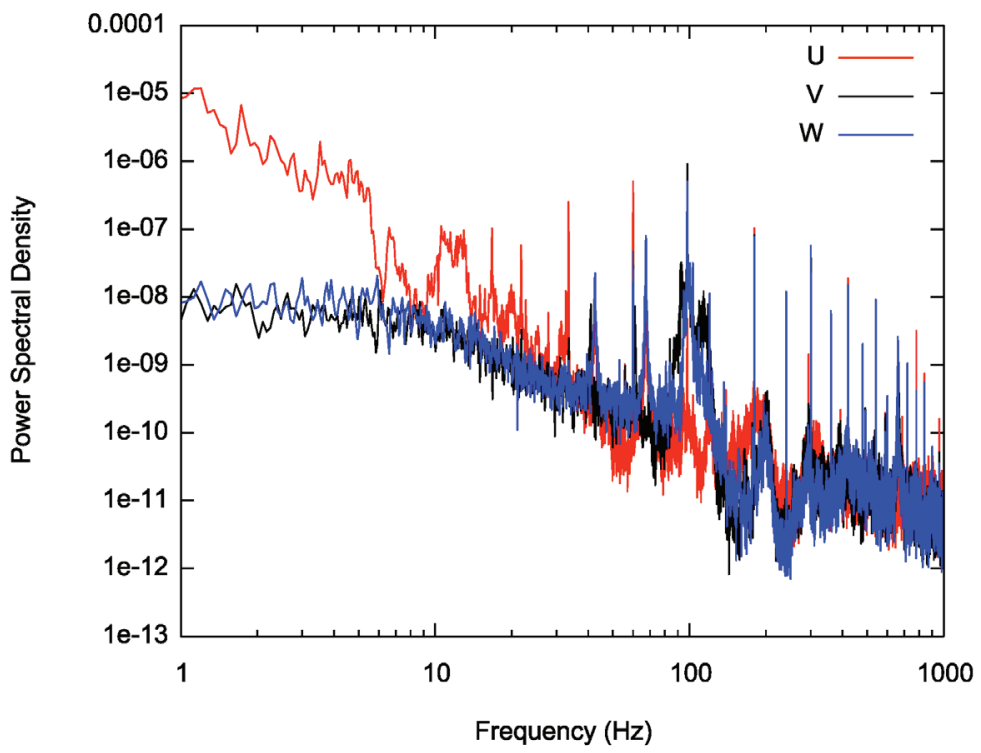

Figure C-30. Spectra from crosswire measurements at $(0.035,0.204,-0.593)$ for $15 \mathrm{~m} / \mathrm{s}$. 


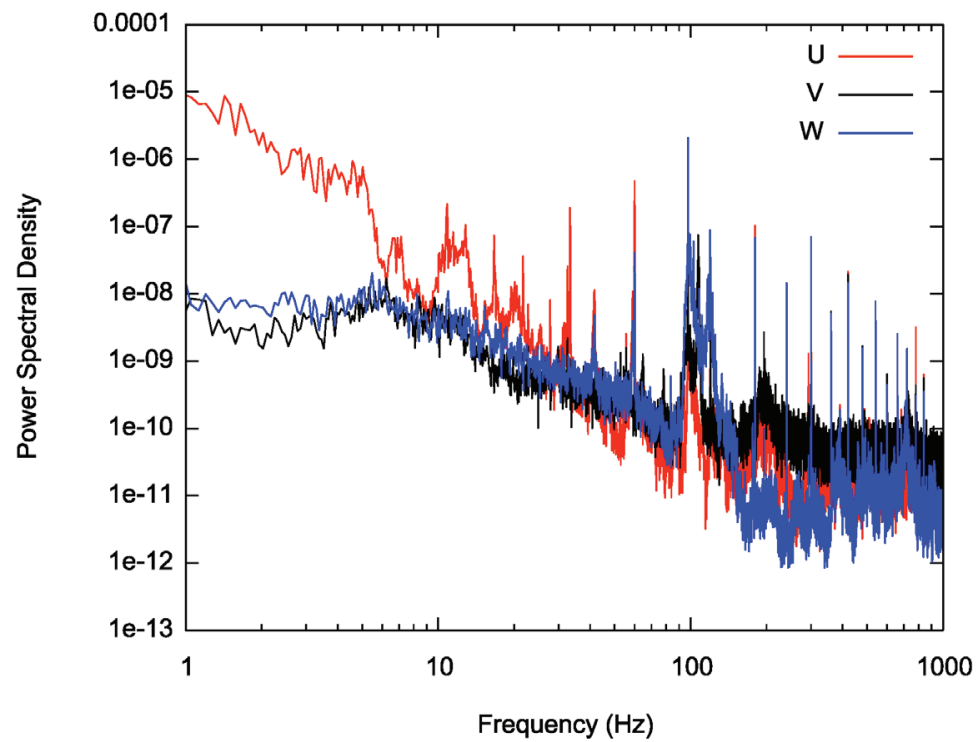

Figure C-31. Spectra from crosswire measurements at $(0.035,0.519,0.593)$ for $15 \mathrm{~m} / \mathrm{s}$.

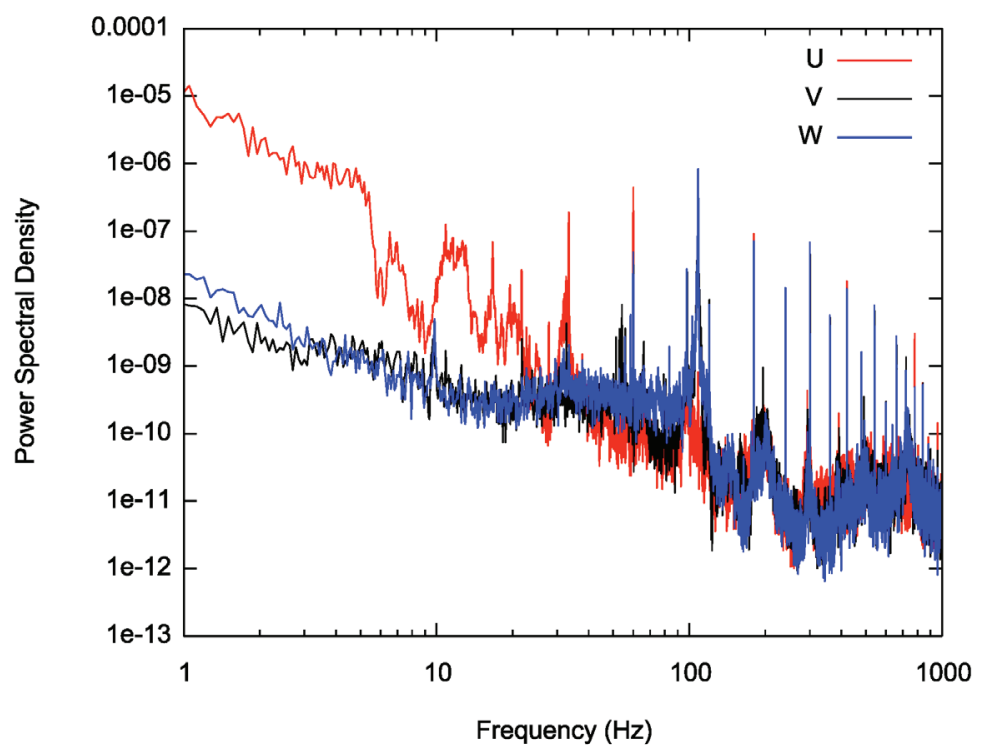

Figure C-32. Spectra from crosswire measurements at $(0.035,0.519,-0.036)$ for $15 \mathrm{~m} / \mathrm{s}$. 


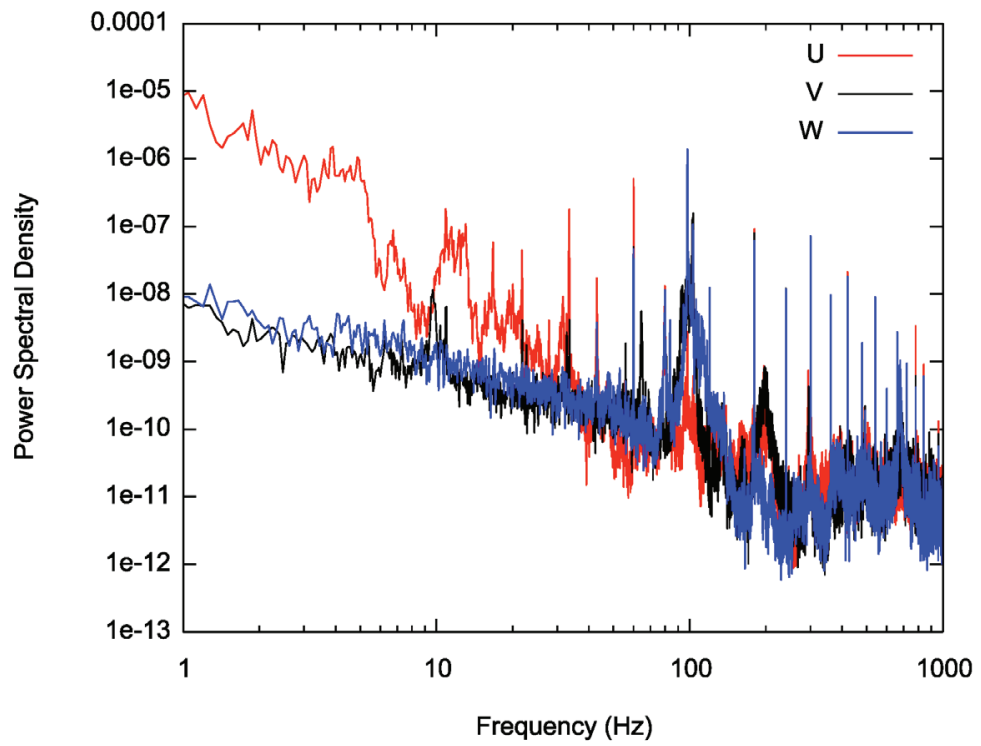

Figure C-33. Spectra from crosswire measurements at $(0.035,0.519,-0.593)$ for $15 \mathrm{~m} / \mathrm{s}$.

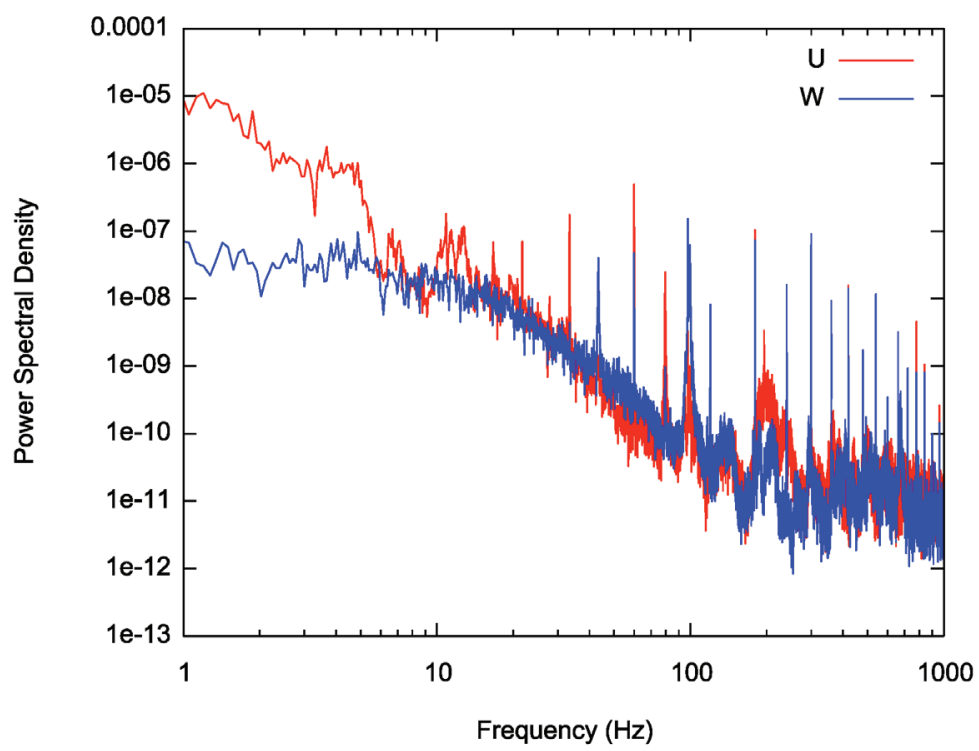

Figure C-34. Spectra from crosswire measurements at $(0.035,0.838,0.593)$ for $15 \mathrm{~m} / \mathrm{s}$. 


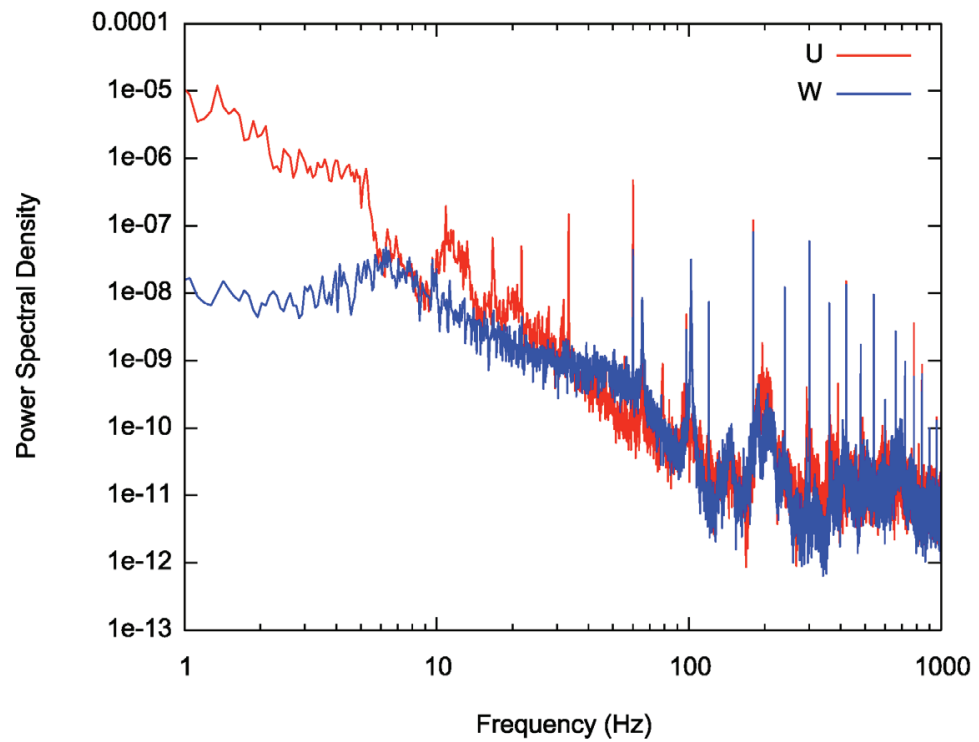

Figure C-35. Spectra from crosswire measurements at $(0.035,0.838,-0.036)$ for $15 \mathrm{~m} / \mathrm{s}$.

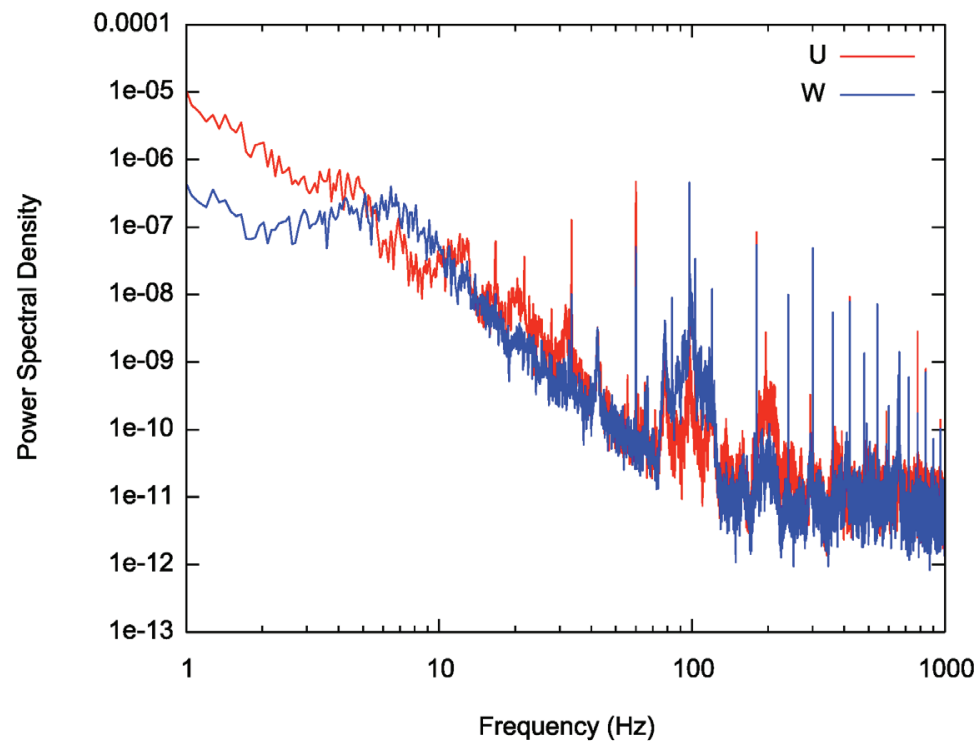

Figure C-36. Spectra from crosswire measurements at $(0.035,0.838,-0.593)$ for $15 \mathrm{~m} / \mathrm{s}$. 


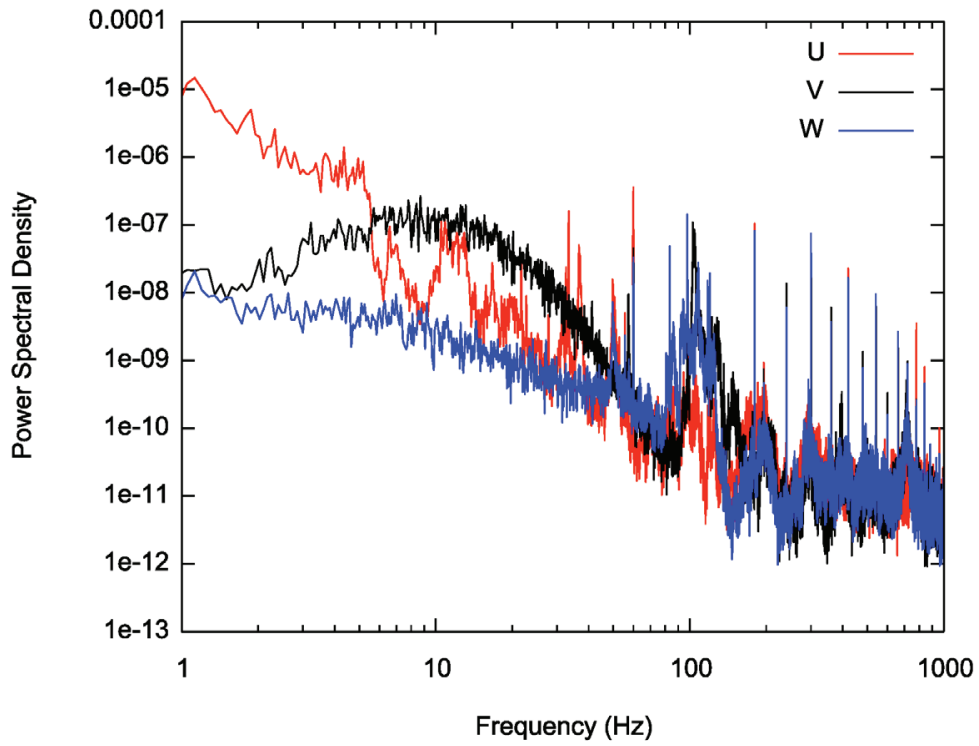

Figure C-37. Spectra from crosswire measurements at $(0.406,0.204,0.598)$ for $15 \mathrm{~m} / \mathrm{s}$.

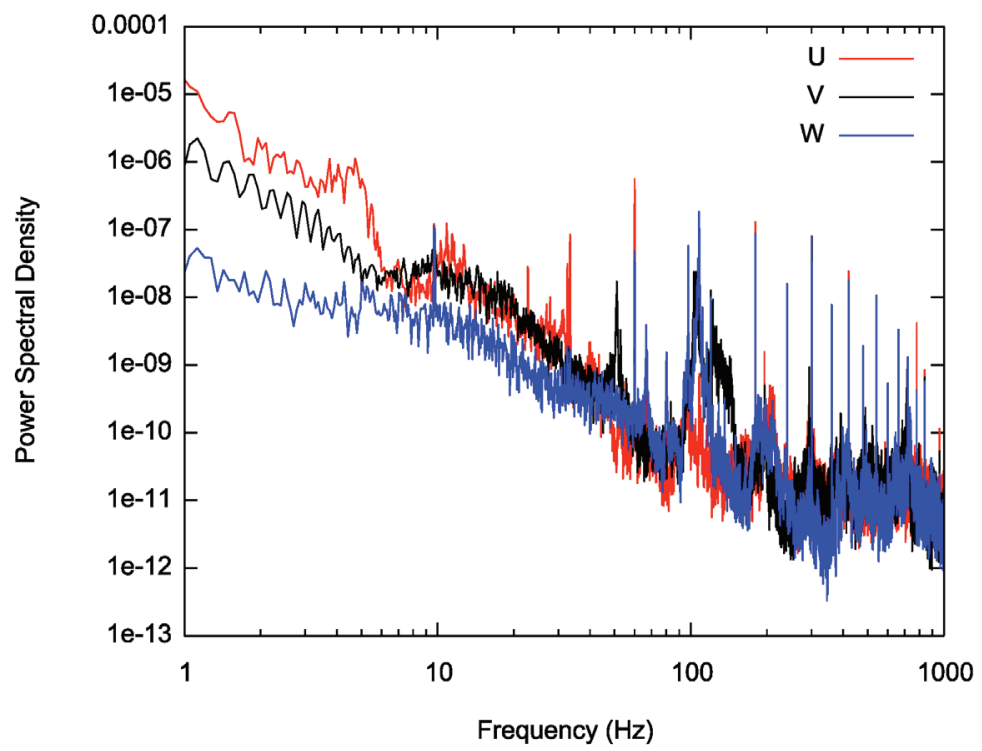

Figure C-38. Spectra from crosswire measurements at $(0.406,0.204,-0.024)$ for $15 \mathrm{~m} / \mathrm{s}$. 


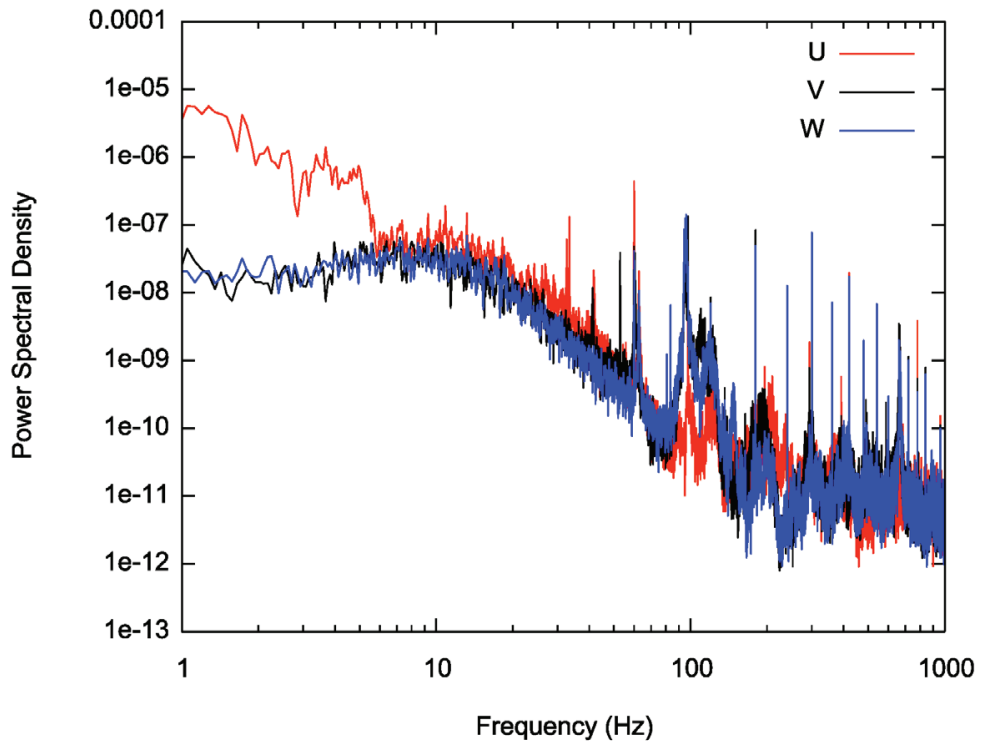

Figure C-39. Spectra from crosswire measurements at $(0.406,0.204,-0.598)$ for $15 \mathrm{~m} / \mathrm{s}$.

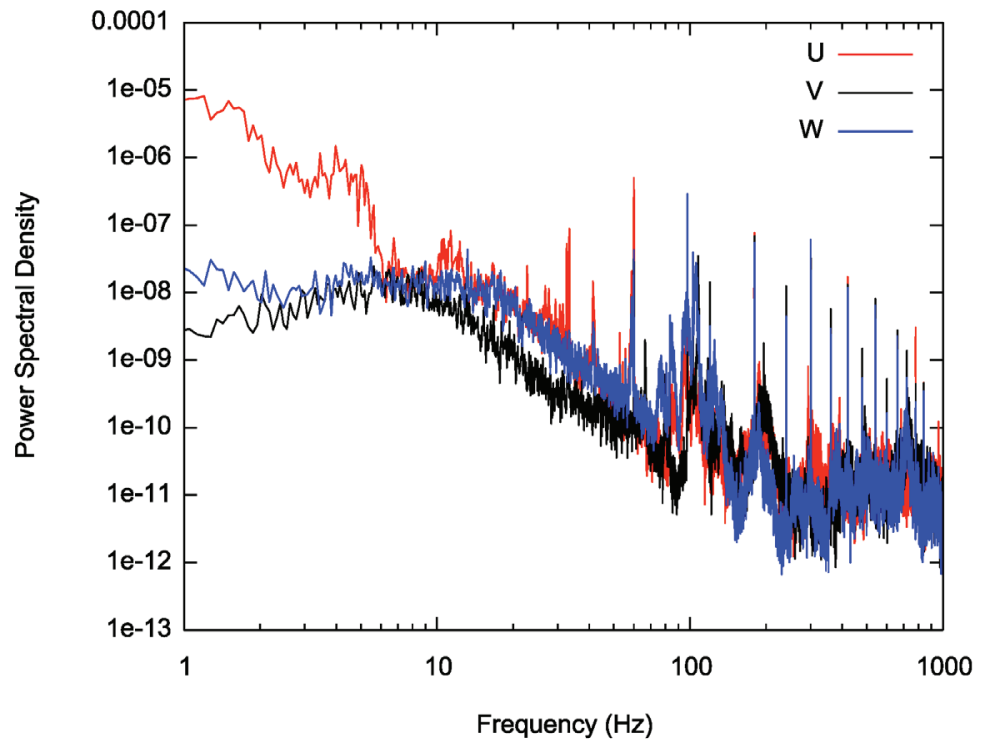

Figure C-40. Spectra from crosswire measurements at $(0.406,0.519,0.598)$ for $15 \mathrm{~m} / \mathrm{s}$. 


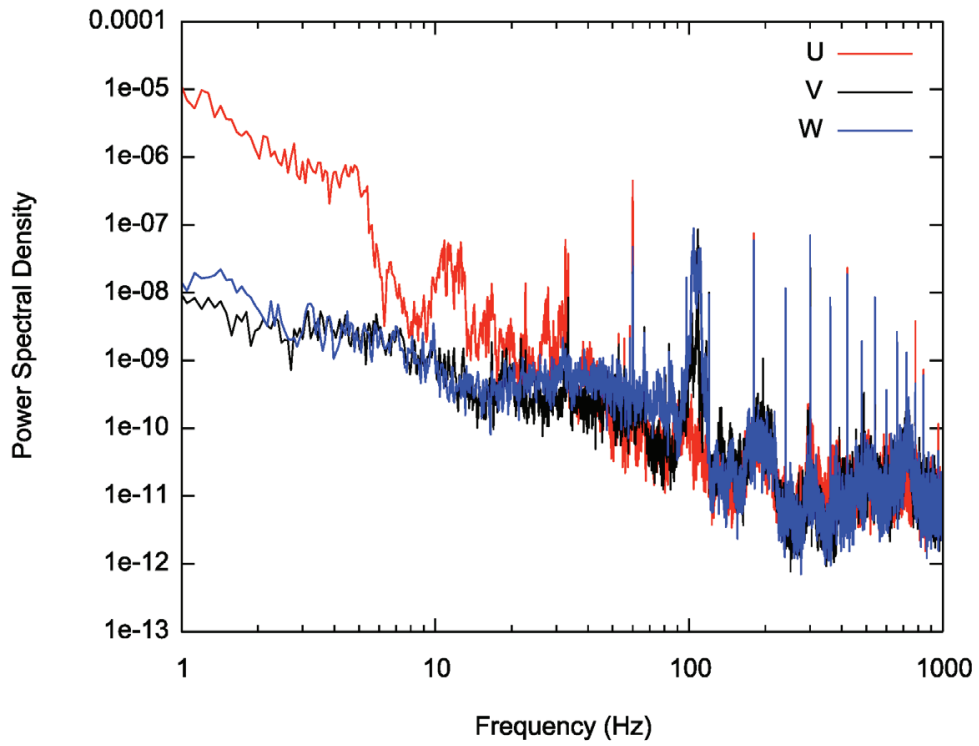

Figure C-41. Spectra from crosswire measurements at $(0.406,0.519,-0.024)$ for $15 \mathrm{~m} / \mathrm{s}$.

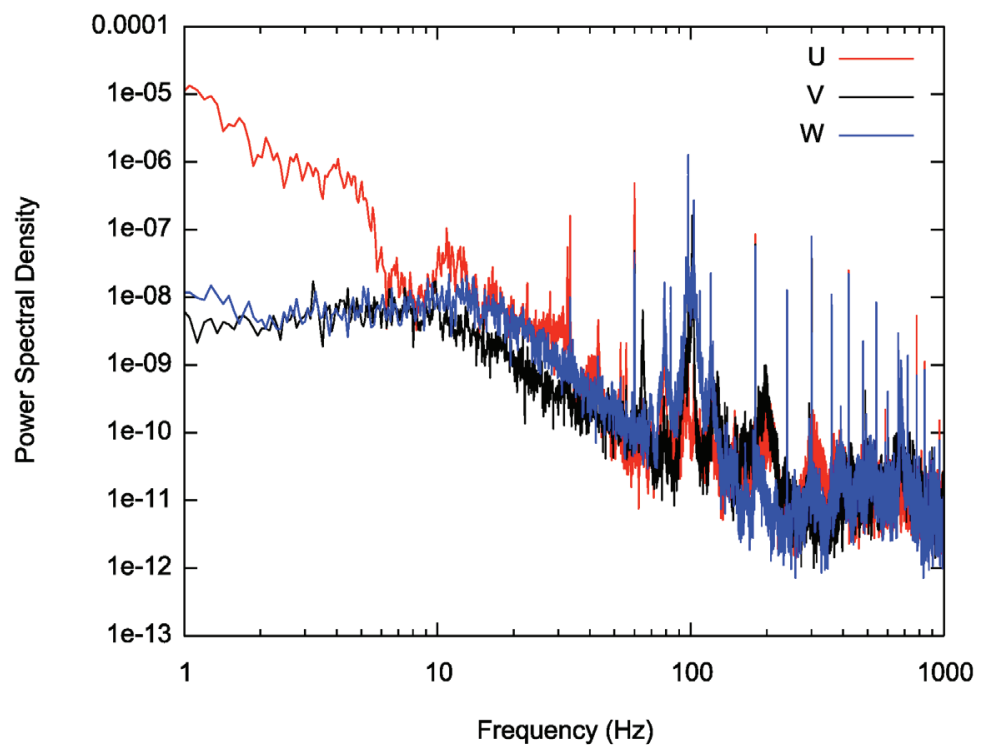

Figure C-42. Spectra from crosswire measurements at $(0.406,0.519,-0.598)$ for $15 \mathrm{~m} / \mathrm{s}$. 


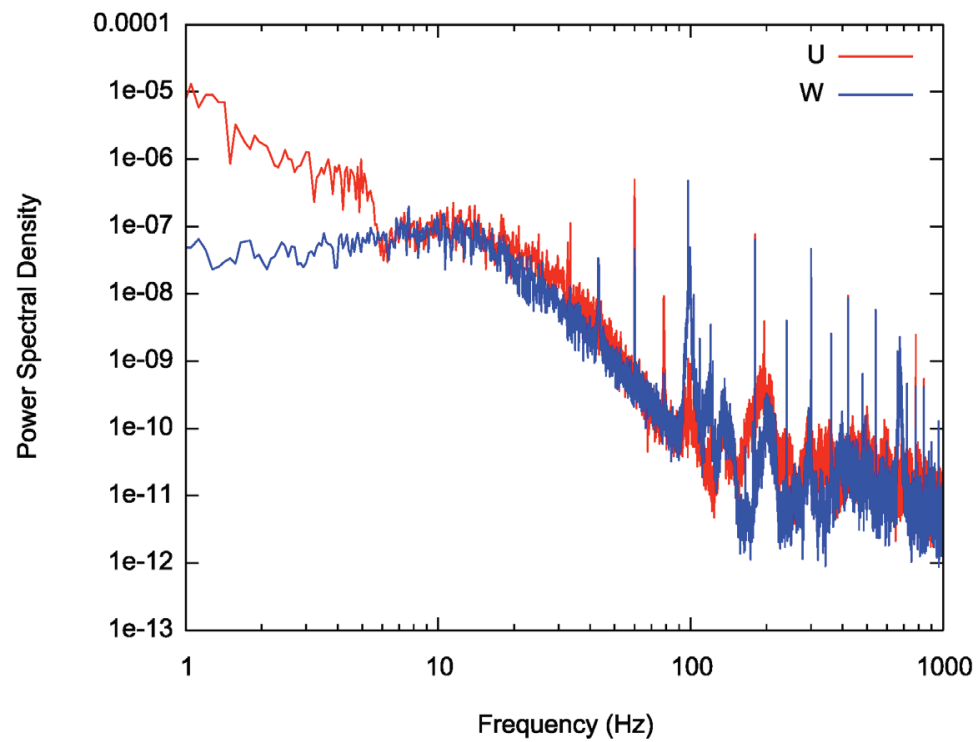

Figure C-43. Spectra from crosswire measurements at $(0.406,0.838,0.598)$ for $15 \mathrm{~m} / \mathrm{s}$.

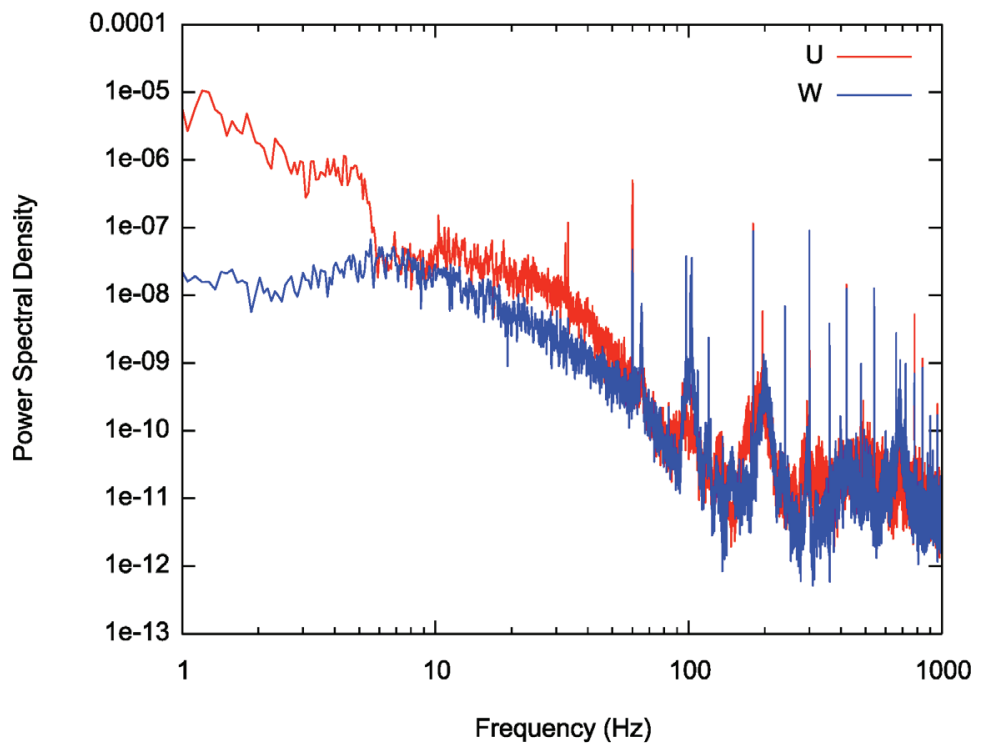

Figure C-44. Spectra from crosswire measurements at $(0.406,0.838,-0.024)$ for $15 \mathrm{~m} / \mathrm{s}$. 


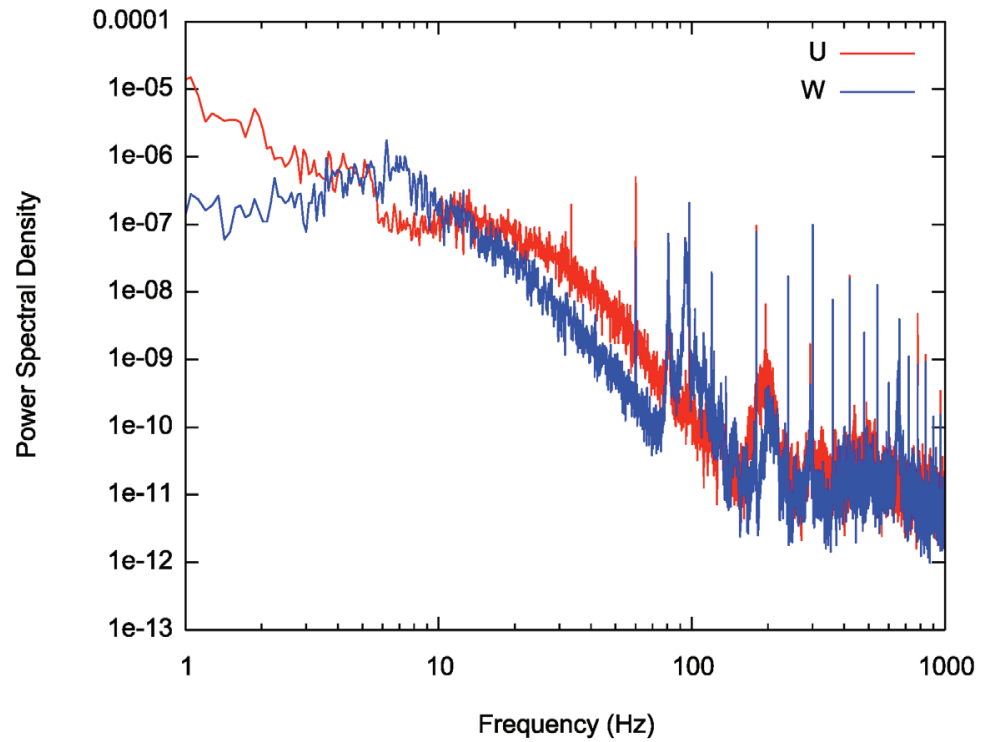

Figure C-45. Spectra from crosswire measurements at $(0.406,0.838,-0.598)$ for $15 \mathrm{~m} / \mathrm{s}$.

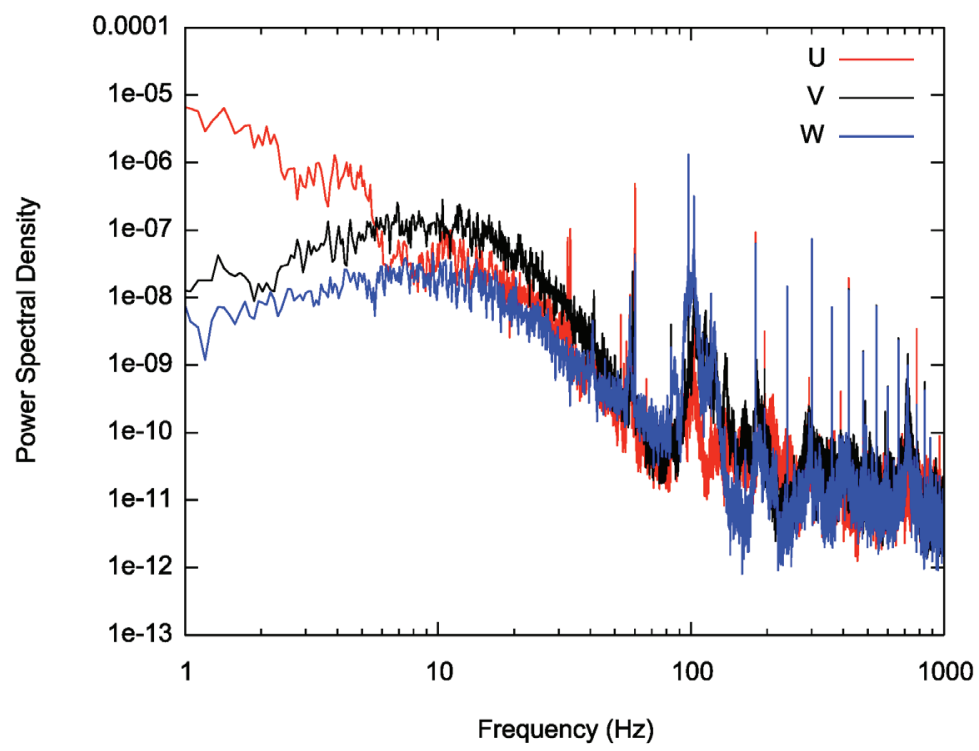

Figure C-46. Spectra from crosswire measurements at $(0.750,0.204,0.602)$ for $15 \mathrm{~m} / \mathrm{s}$. 


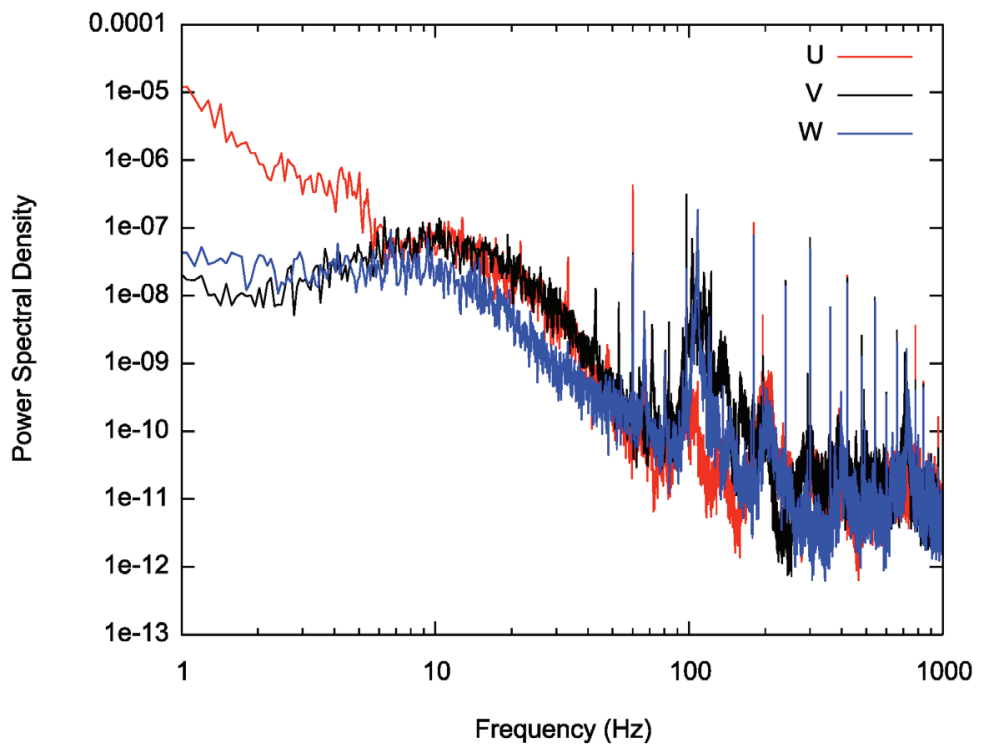

Figure C-47. Spectra from crosswire measurements at $(0.750,0.204,-0.013)$ for $15 \mathrm{~m} / \mathrm{s}$.

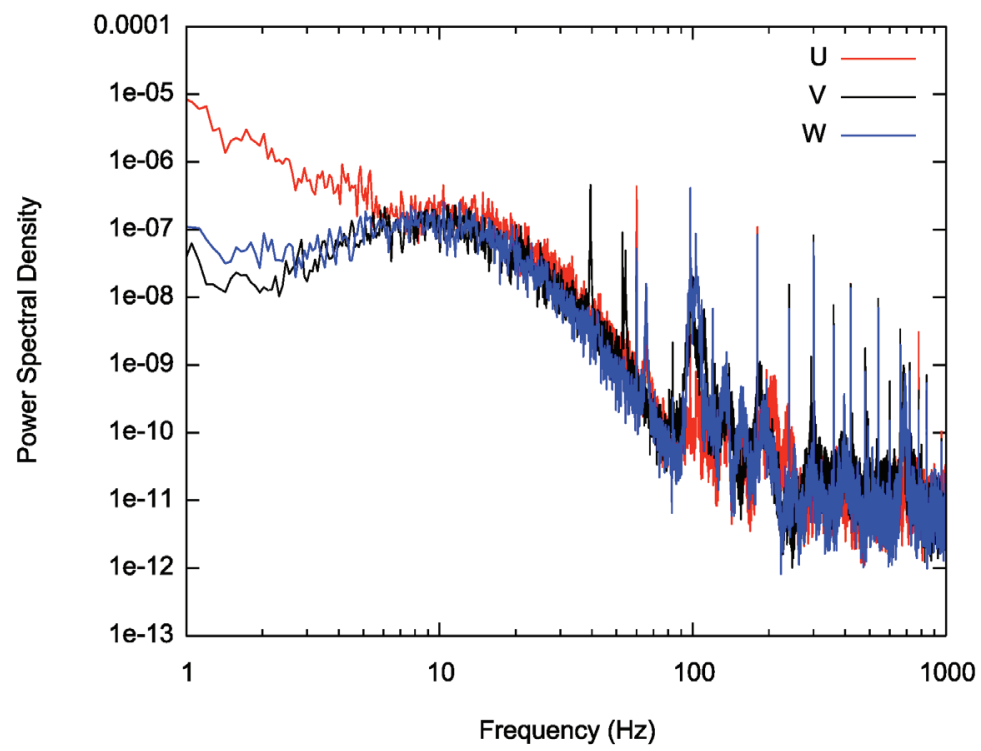

Figure C-48. Spectra from crosswire measurements at $(0.750,0.204,-0.602)$ for $15 \mathrm{~m} / \mathrm{s}$. 


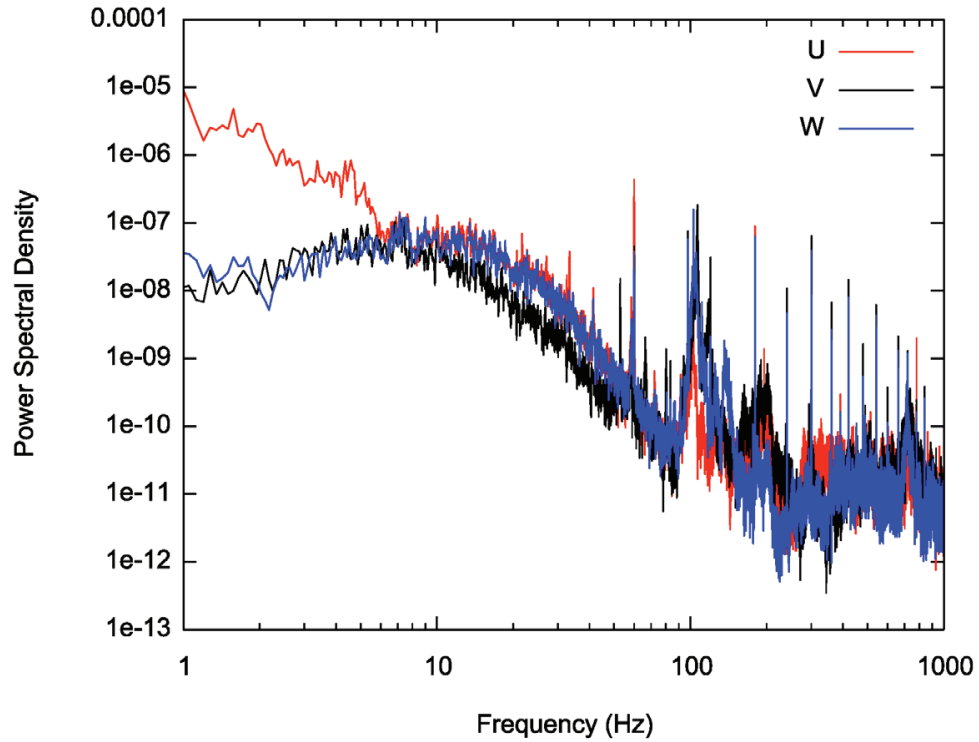

Figure C-49. Spectra from crosswire measurements at $(0.750,0.519,0.602)$ for $15 \mathrm{~m} / \mathrm{s}$.

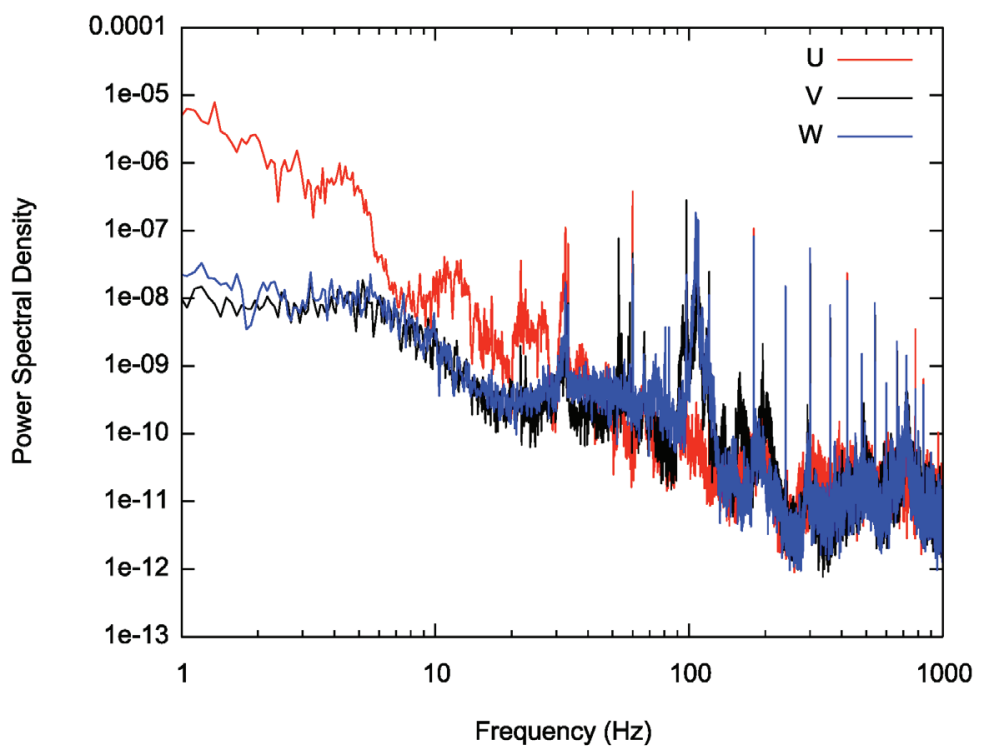

Figure C-50. Spectra from crosswire measurements at $(0.750,0.519,-0.013)$ for $15 \mathrm{~m} / \mathrm{s}$. 


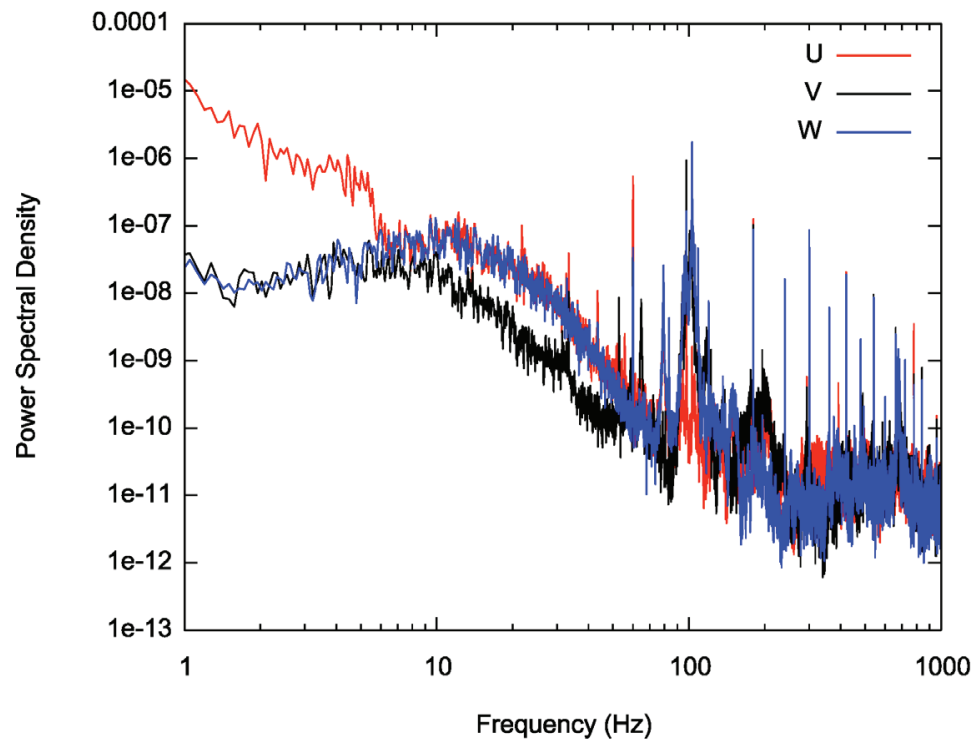

Figure C-51. Spectra from crosswire measurements at $(0.750,0.519,-0.602)$ for $15 \mathrm{~m} / \mathrm{s}$.

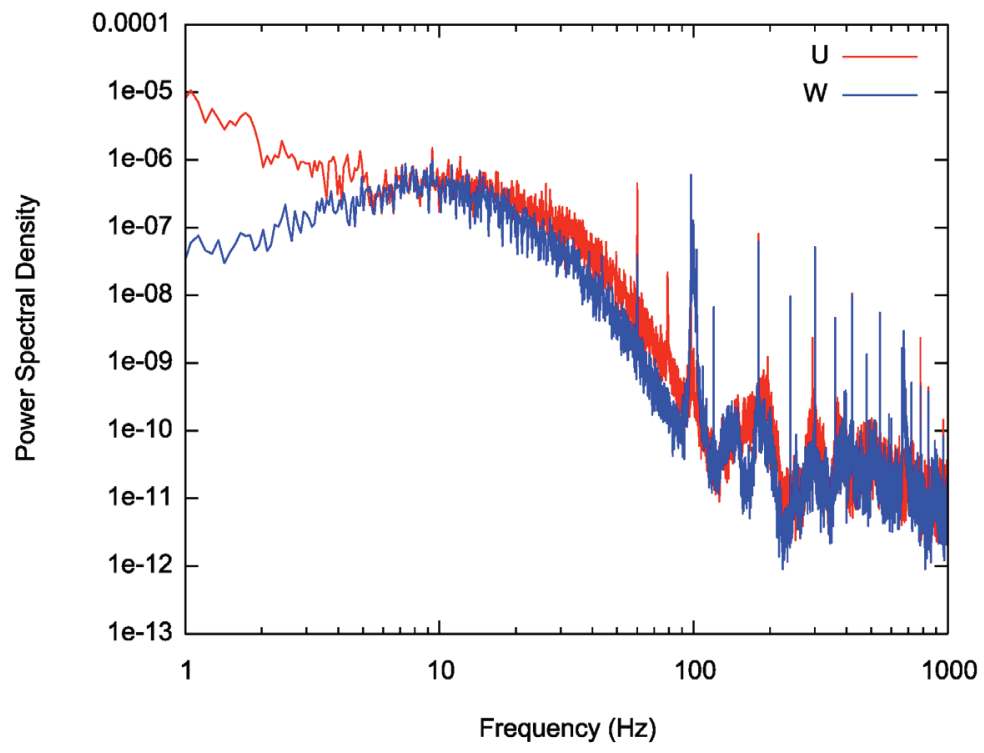

Figure C-52. Spectra from crosswire measurements at $(0.750,0.838,0.602)$ for $15 \mathrm{~m} / \mathrm{s}$. 


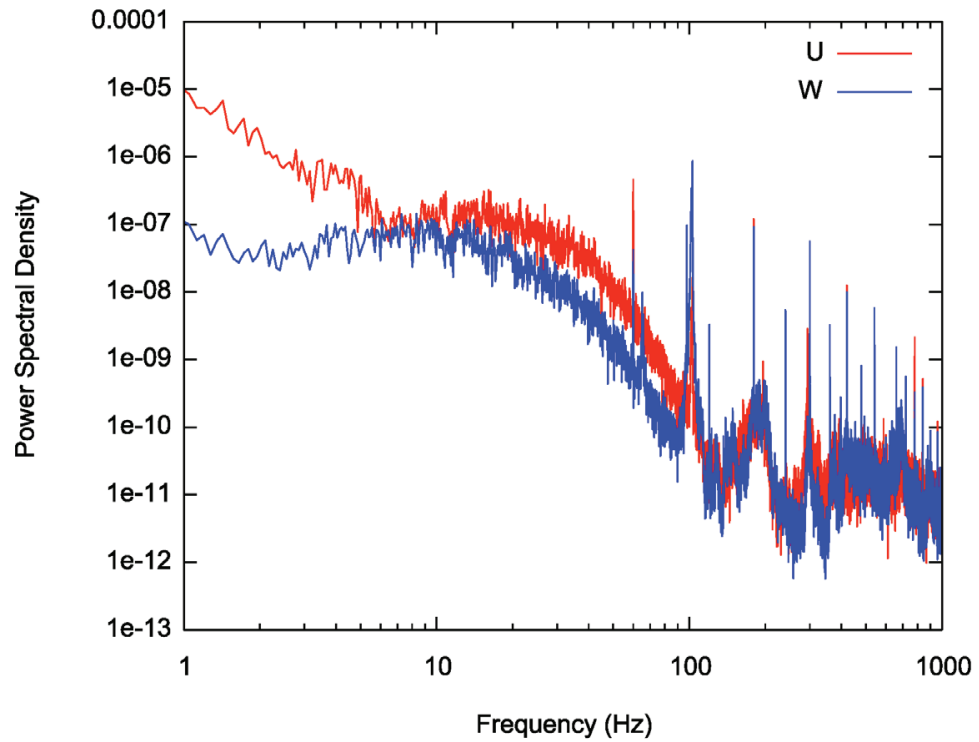

Figure C-53. Spectra from crosswire measurements at $(0.750,0.838,-0.013)$ for $15 \mathrm{~m} / \mathrm{s}$.

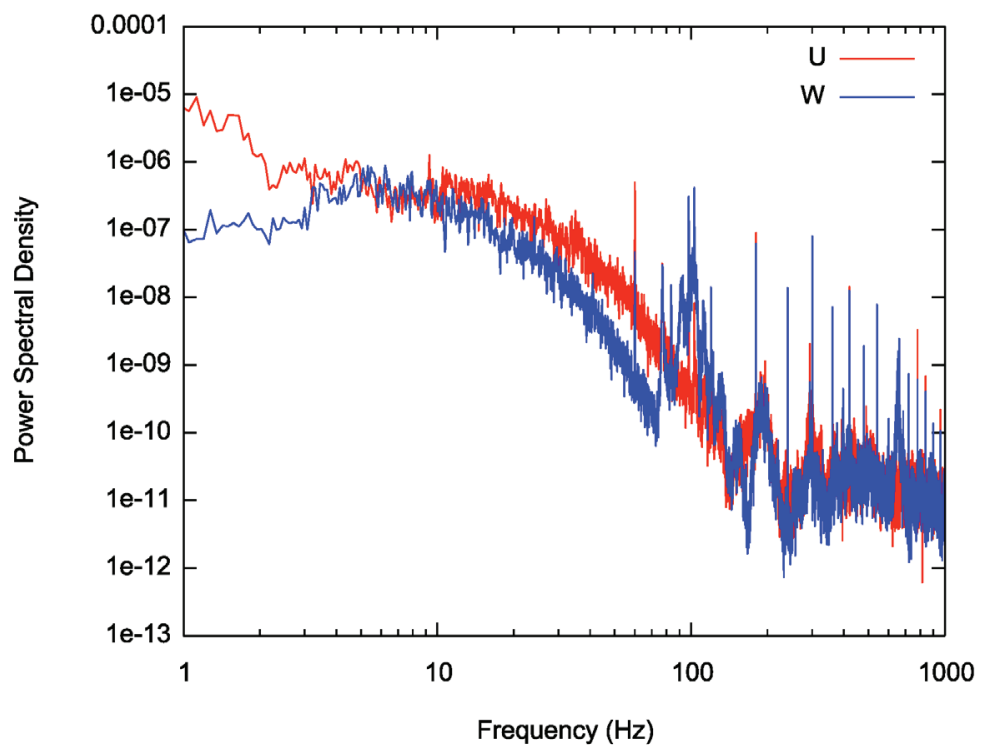

Figure C-54. Spectra from crosswire measurements at $(0.750,0.838,-0.602)$ for $15 \mathrm{~m} / \mathrm{s}$. 


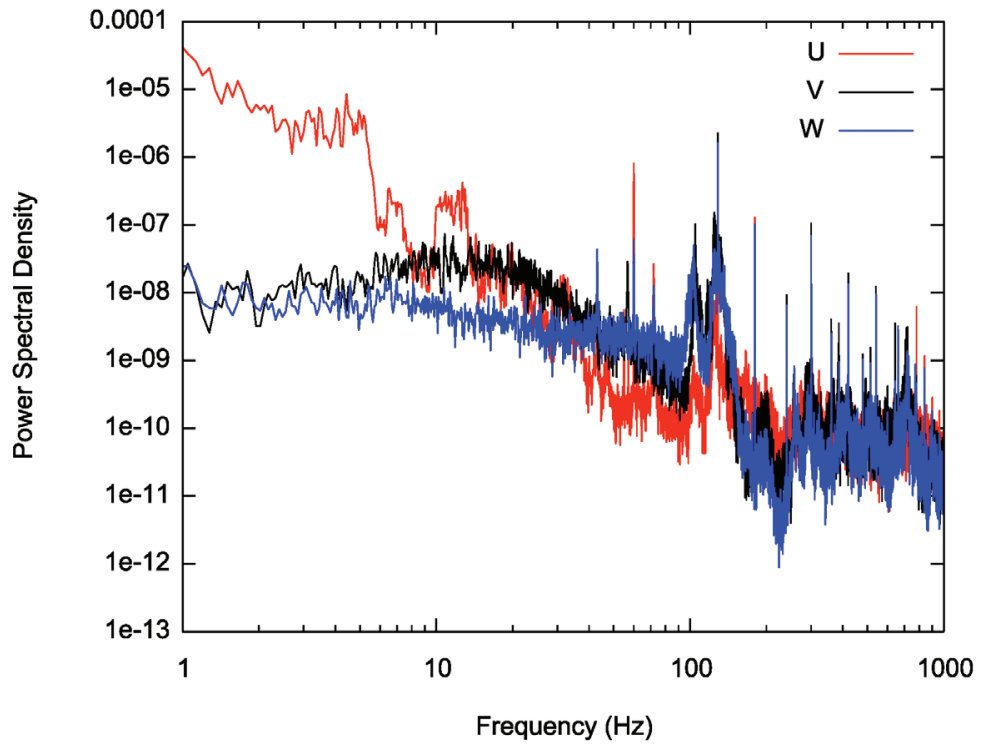

Figure C-55. Spectra from crosswire measurements at $(0.035,0.204,0.593)$ for $20 \mathrm{~m} / \mathrm{s}$.

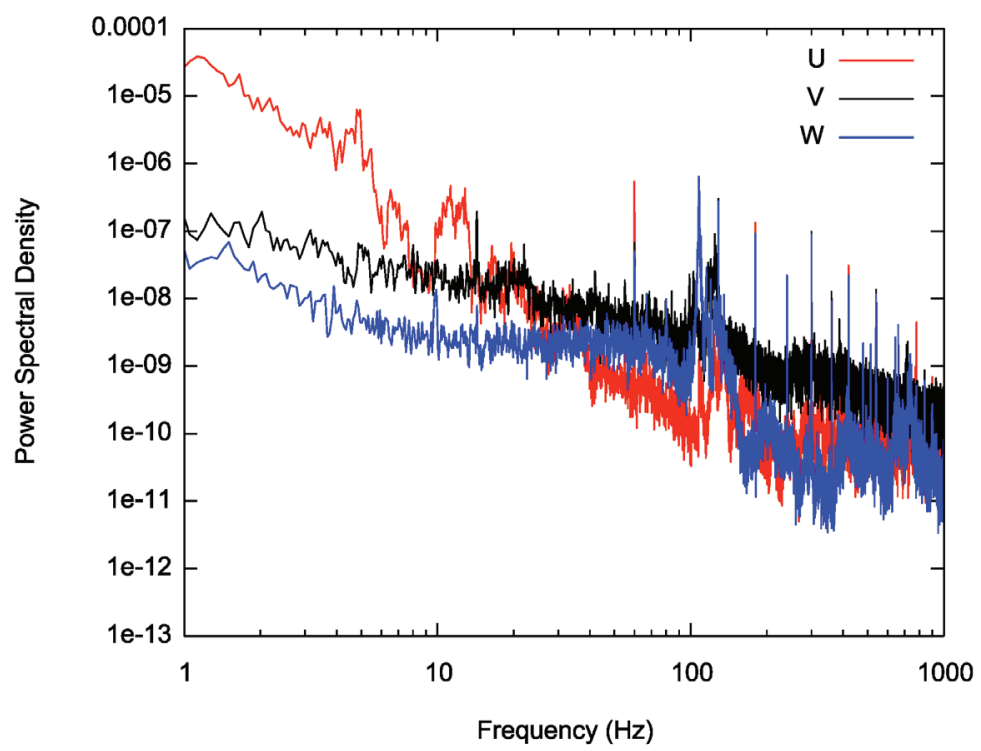

Figure C-56. Spectra from crosswire measurements at $(0.035,0.204,-0.036)$ for $20 \mathrm{~m} / \mathrm{s}$. 


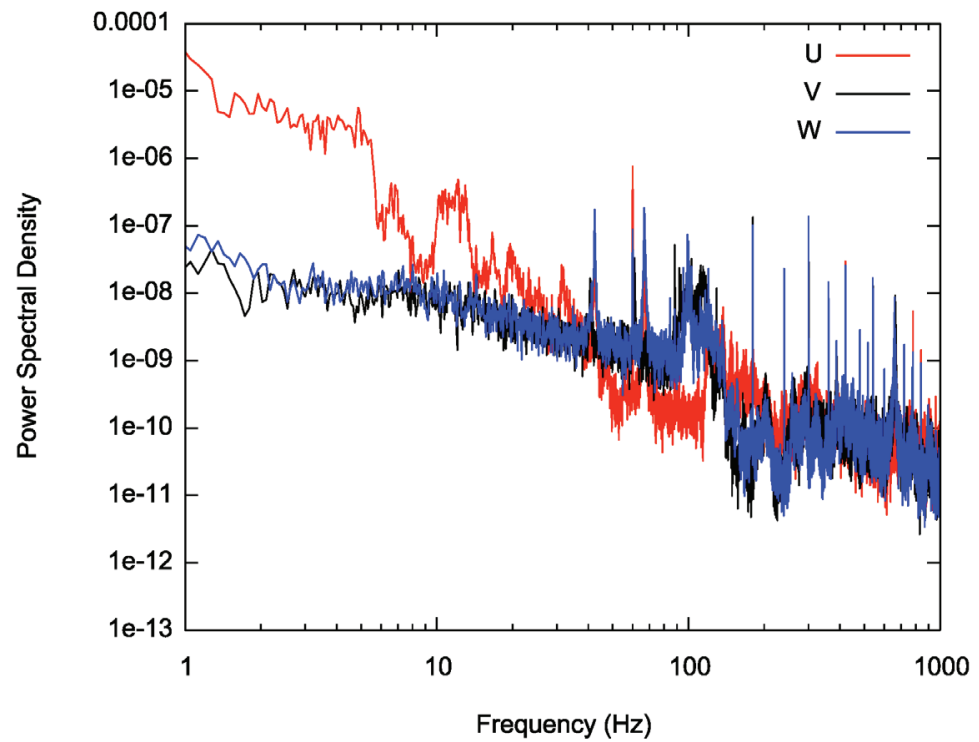

Figure C-57. Spectra from crosswire measurements at $(0.035,0.204,-0.593)$ for $20 \mathrm{~m} / \mathrm{s}$.

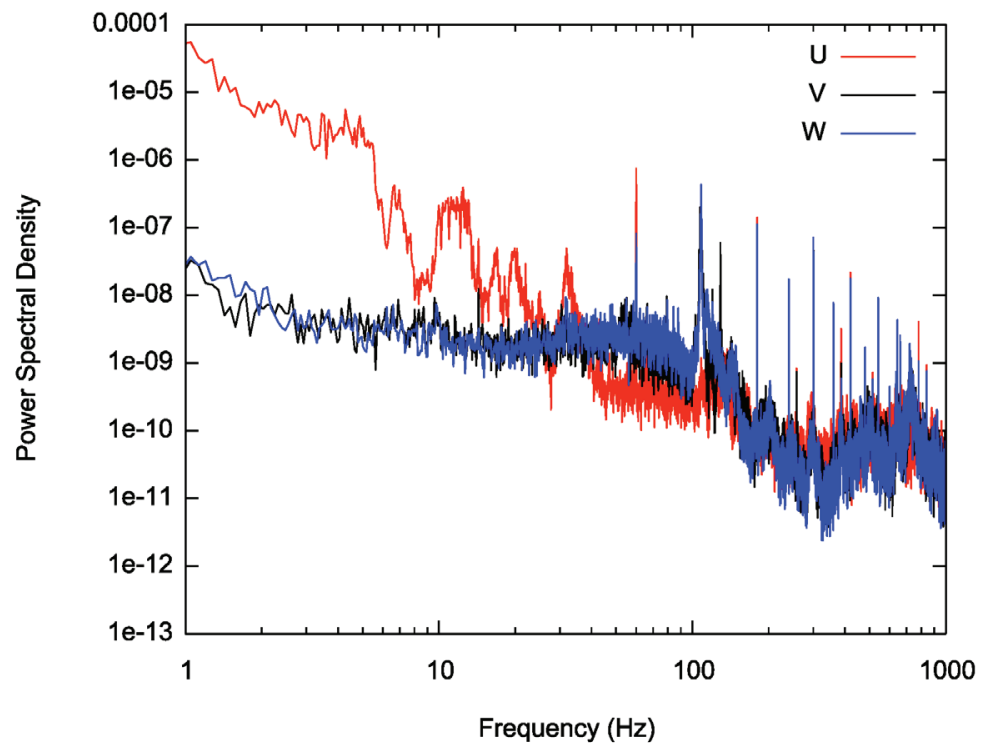

Figure C-58. Spectra from crosswire measurements at $(0.035,0.519,0.593)$ for $20 \mathrm{~m} / \mathrm{s}$. 


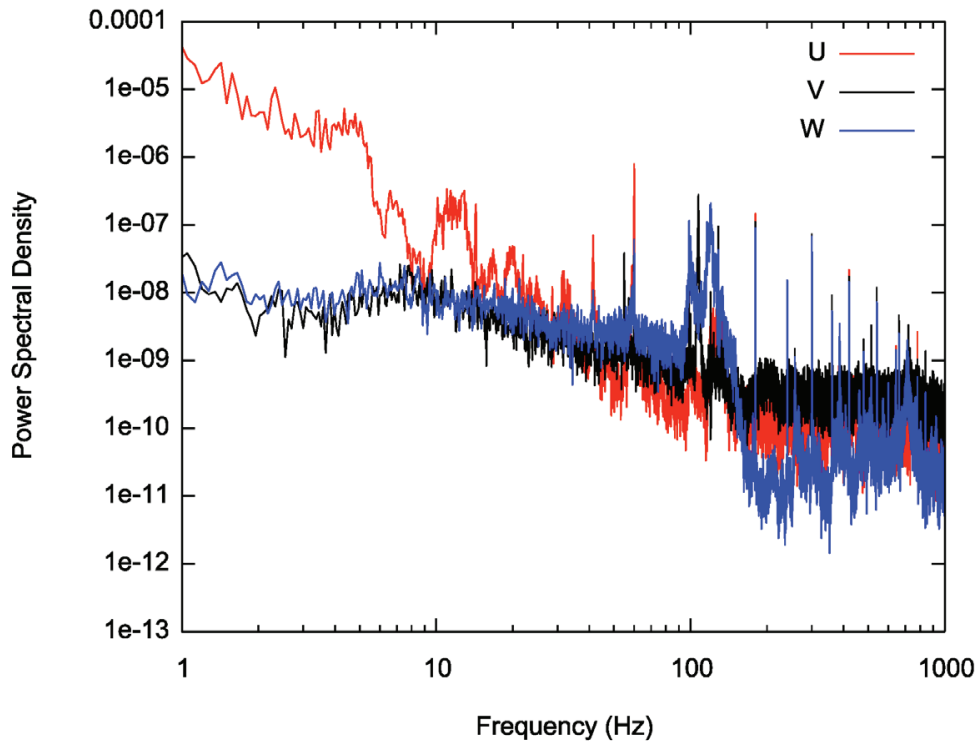

Figure C-59. Spectra from crosswire measurements at $(0.035,0.519,-0.036)$ for $20 \mathrm{~m} / \mathrm{s}$.

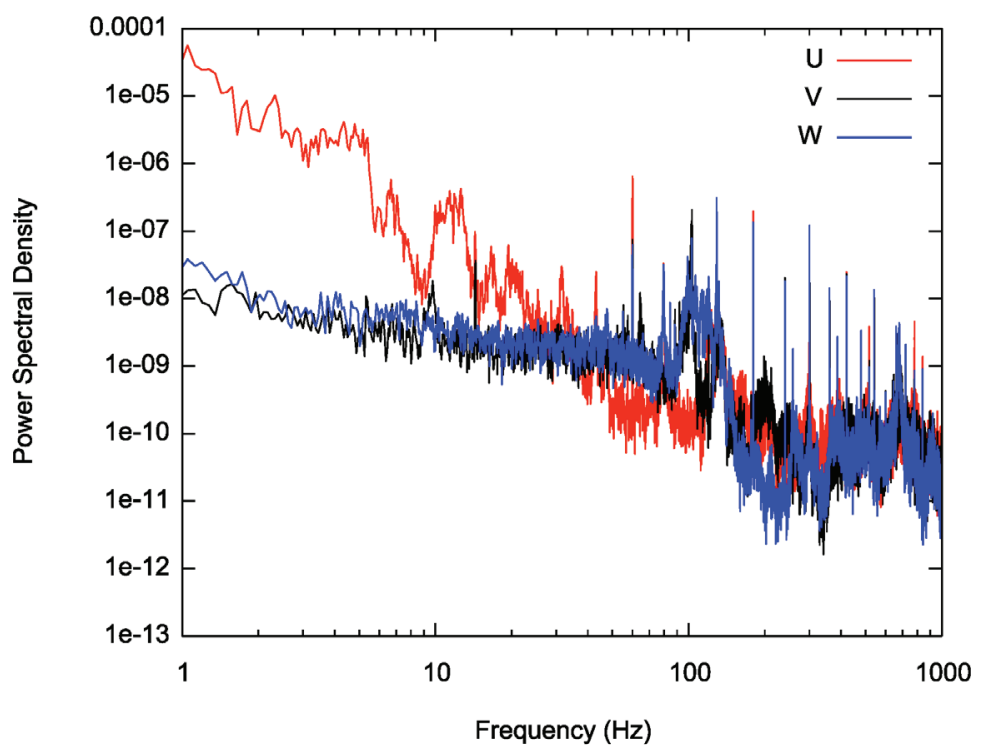

Figure C-60. Spectra from crosswire measurements at $(0.035,0.519,-0.593)$ for $20 \mathrm{~m} / \mathrm{s}$. 


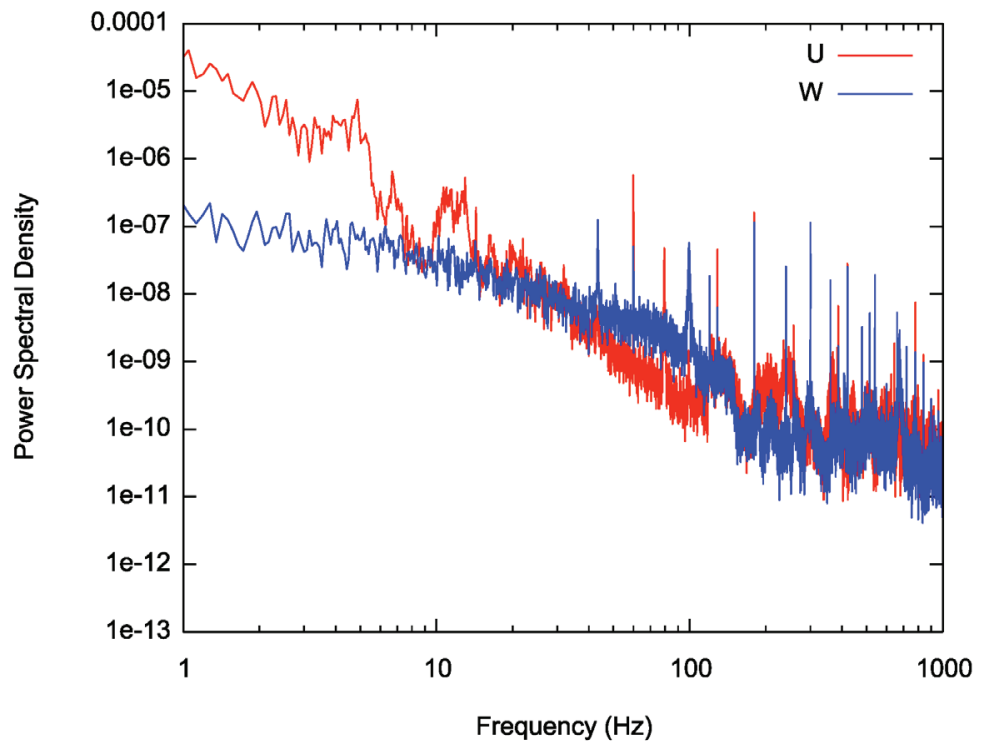

Figure C-61. Spectra from crosswire measurements at $(0.035,0.838,0.593)$ for $20 \mathrm{~m} / \mathrm{s}$.

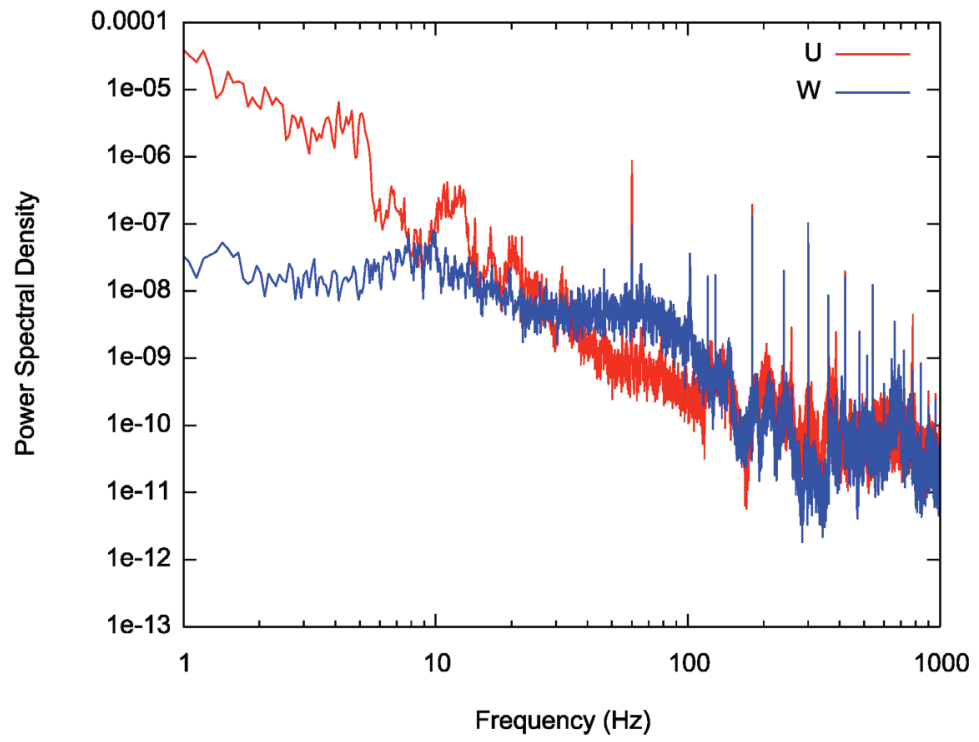

Figure C-62. Spectra from crosswire measurements at $(0.035,0.838,-0.036)$ for $20 \mathrm{~m} / \mathrm{s}$. 


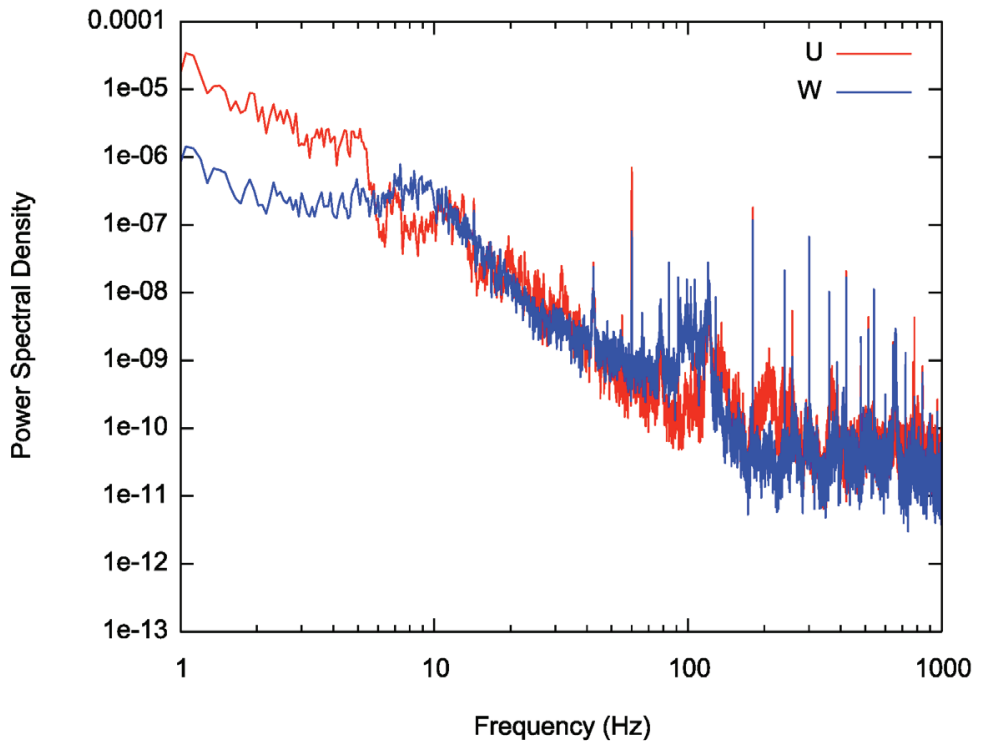

Figure C-63. Spectra from crosswire measurements at $(0.035,0.838,-0.593)$ for $20 \mathrm{~m} / \mathrm{s}$.

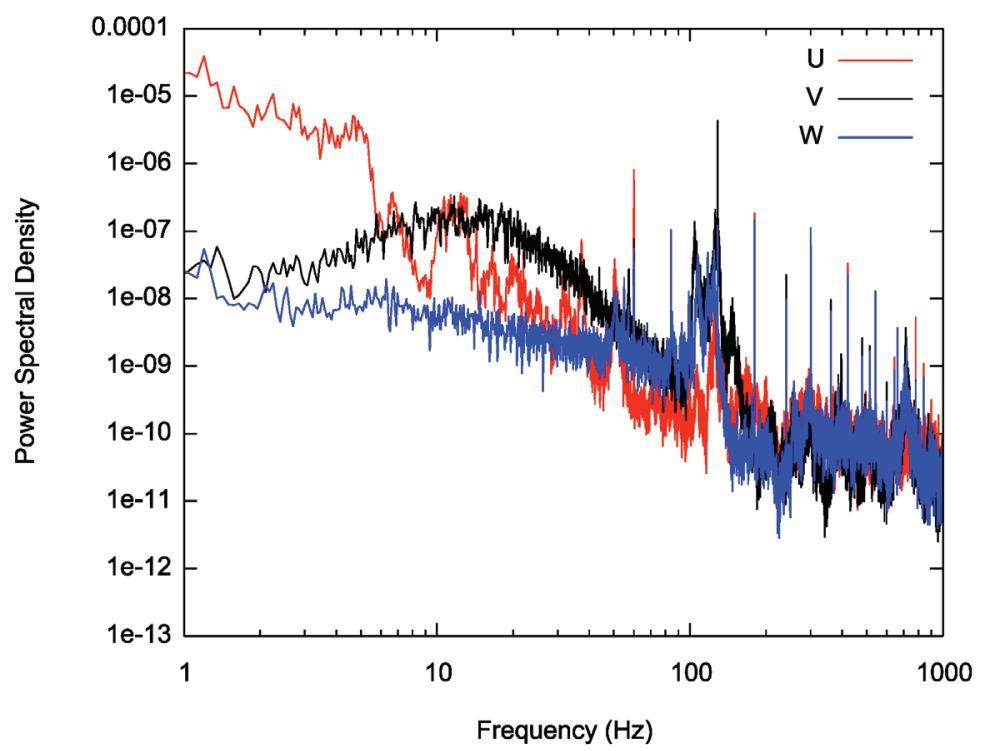

Figure C-64. Spectra from crosswire measurements at $(0.406,0.204,0.598)$ for $20 \mathrm{~m} / \mathrm{s}$. 


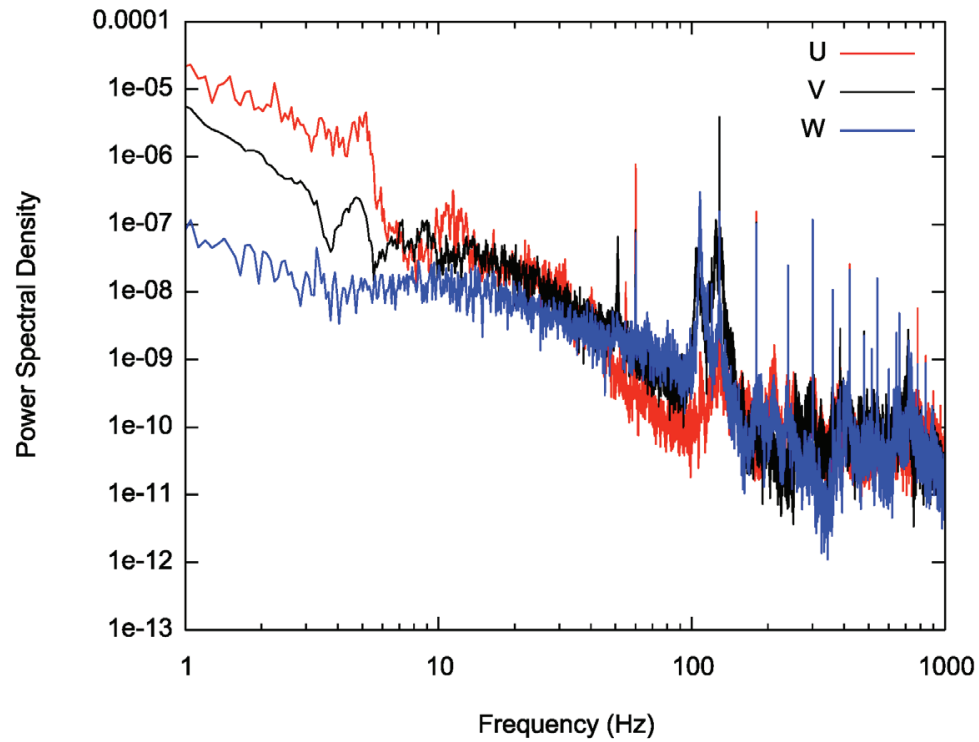

Figure C-65. Spectra from crosswire measurements at $(0.406,0.204,-0.024)$ for $20 \mathrm{~m} / \mathrm{s}$.

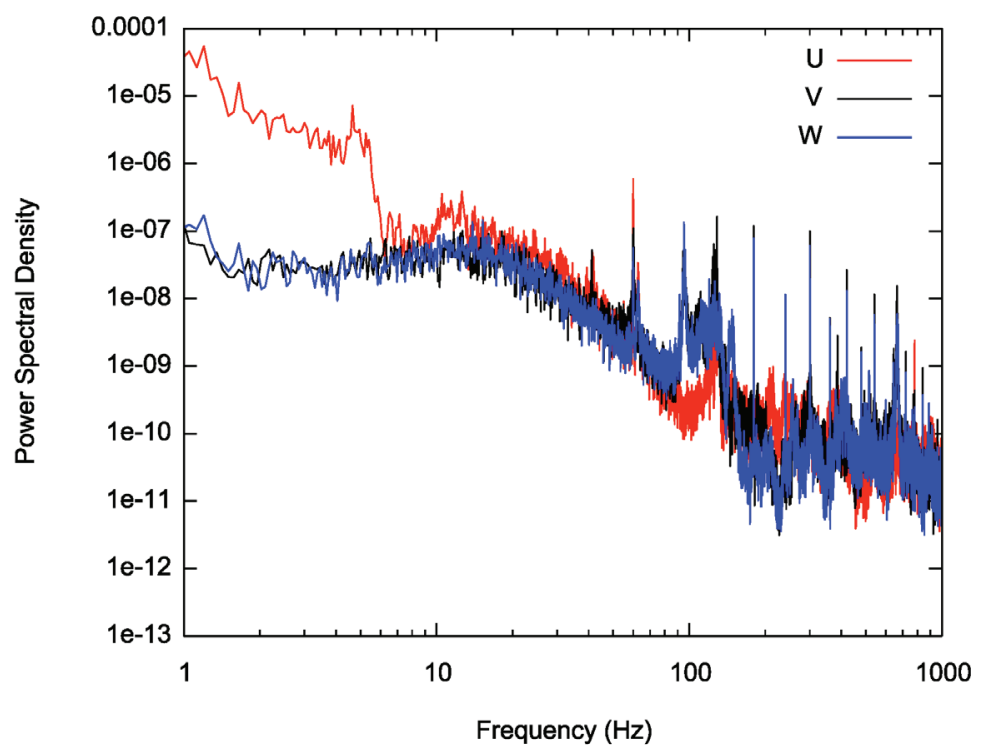

Figure C-66. Spectra from crosswire measurements at $(0.406,0.204,-0.598)$ for $20 \mathrm{~m} / \mathrm{s}$. 


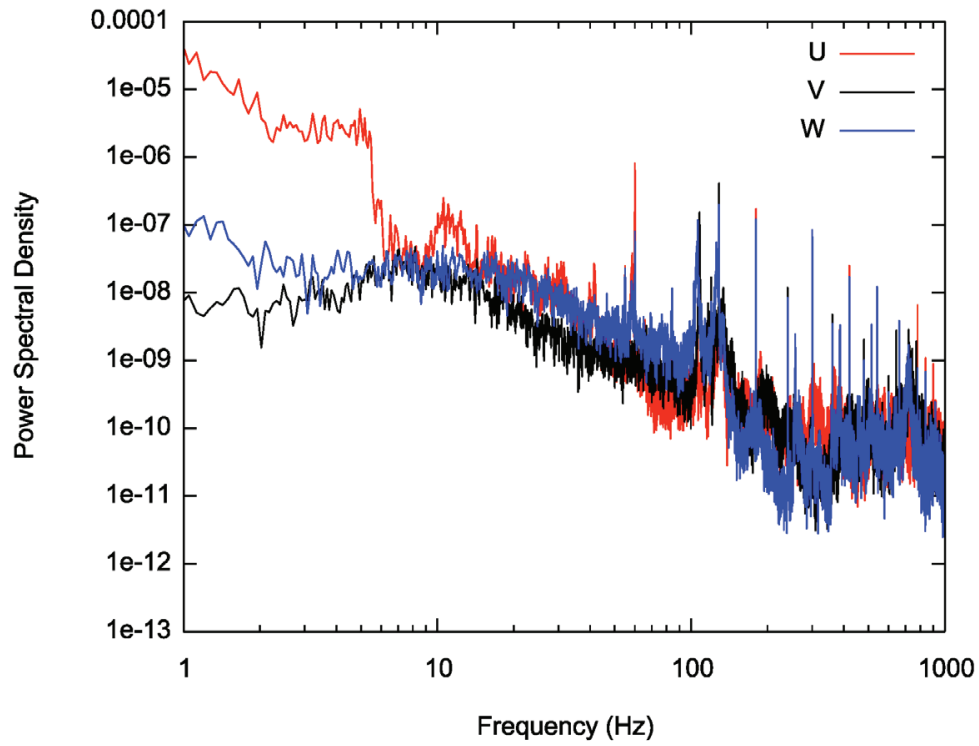

Figure C-67. Spectra from crosswire measurements at $(0.406,0.519,0.598)$ for $20 \mathrm{~m} / \mathrm{s}$.

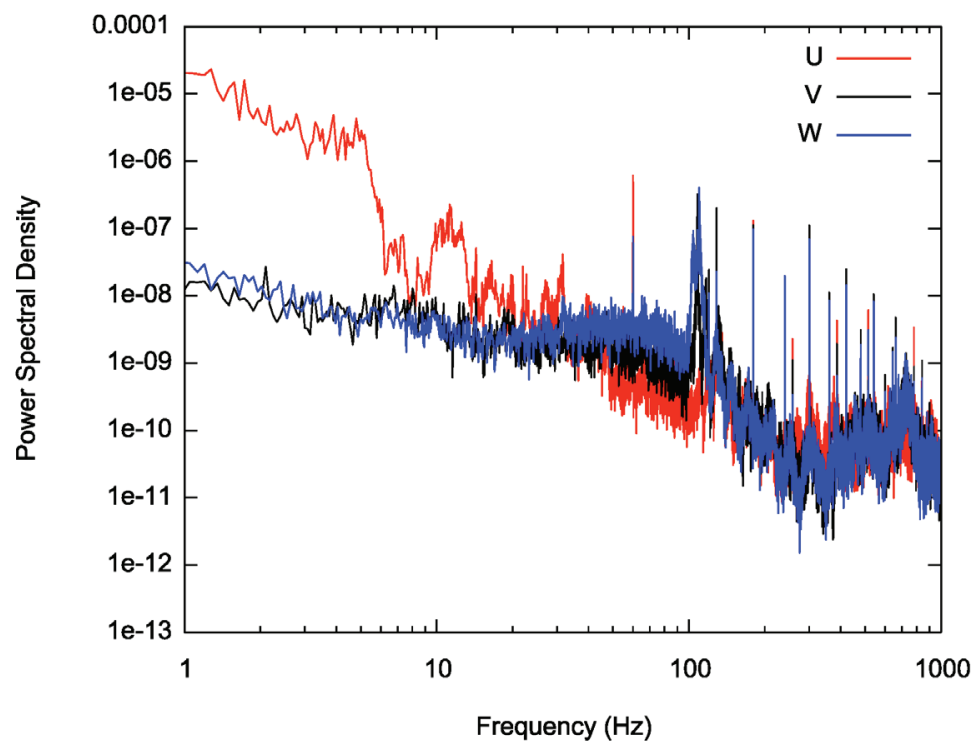

Figure C-68. Spectra from crosswire measurements at $(0.406,0.519,-0.024)$ for $20 \mathrm{~m} / \mathrm{s}$. 


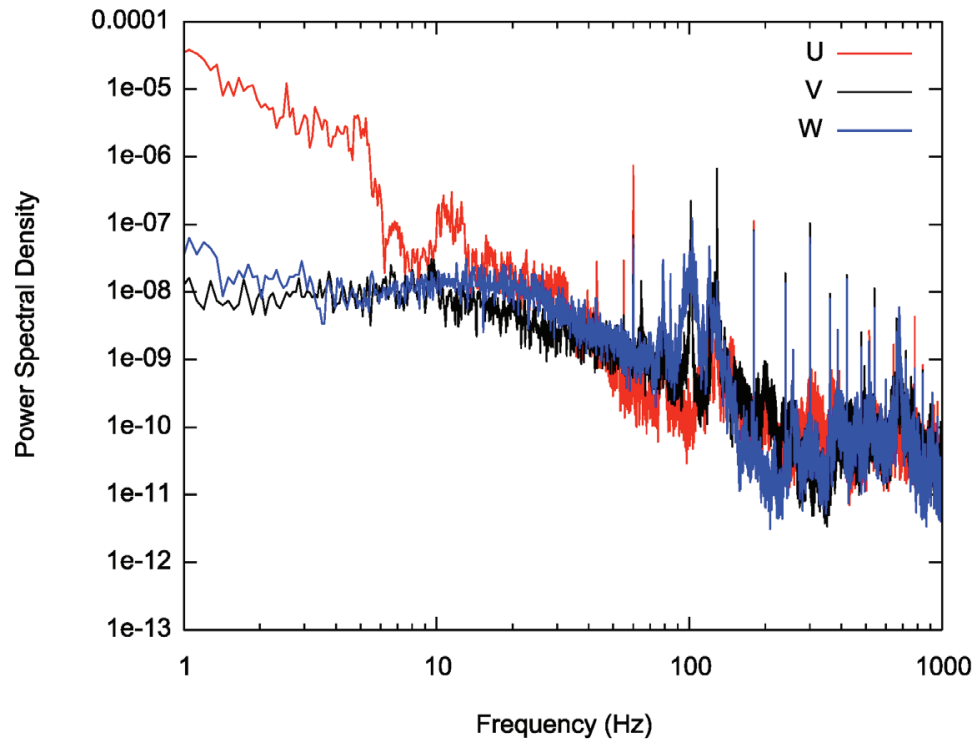

Figure C-69. Spectra from crosswire measurements at $(0.406,0.519,-0.598)$ for $20 \mathrm{~m} / \mathrm{s}$.

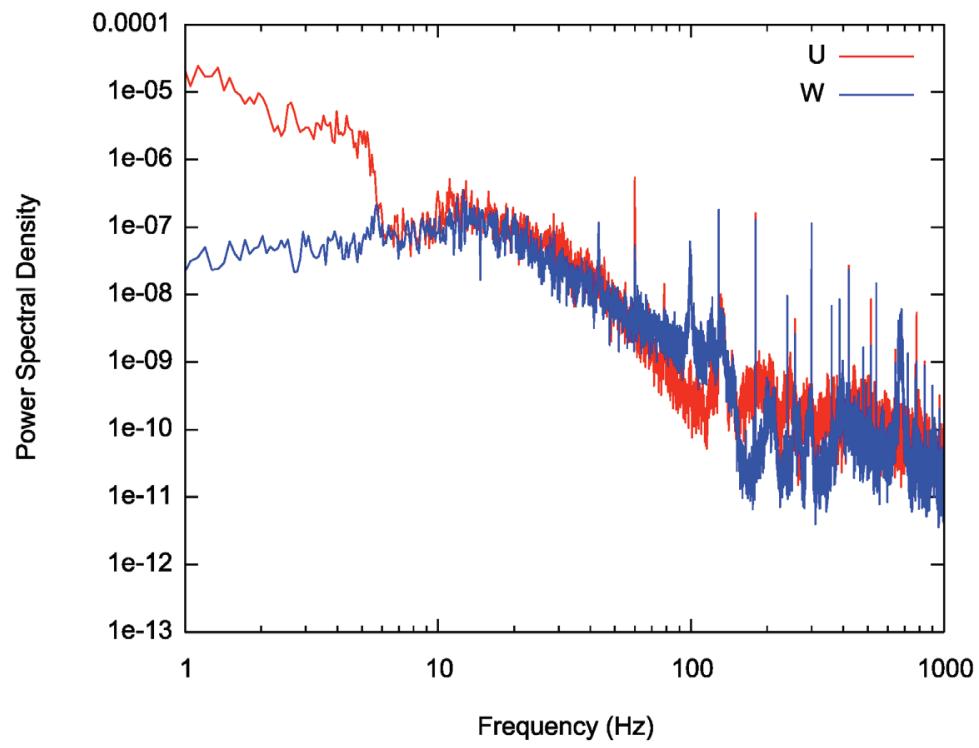

Figure C-70. Spectra from crosswire measurements at $(0.406,0.838,0.598)$ for $20 \mathrm{~m} / \mathrm{s}$. 


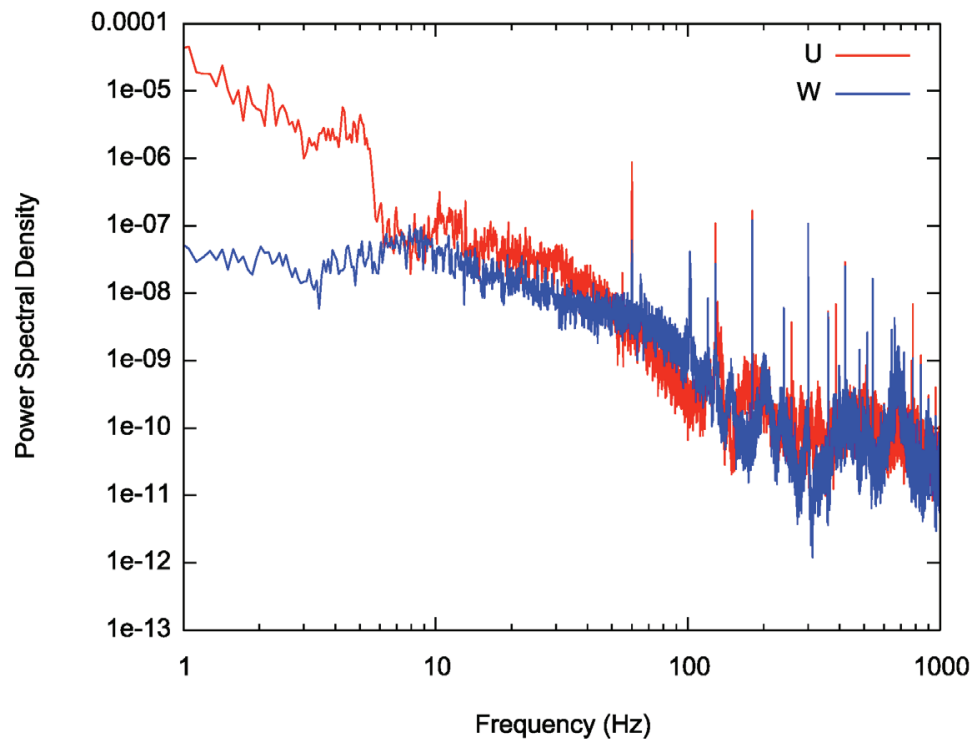

Figure C-71. Spectra from crosswire measurements at $(0.406,0.838,-0.024)$ for $20 \mathrm{~m} / \mathrm{s}$.

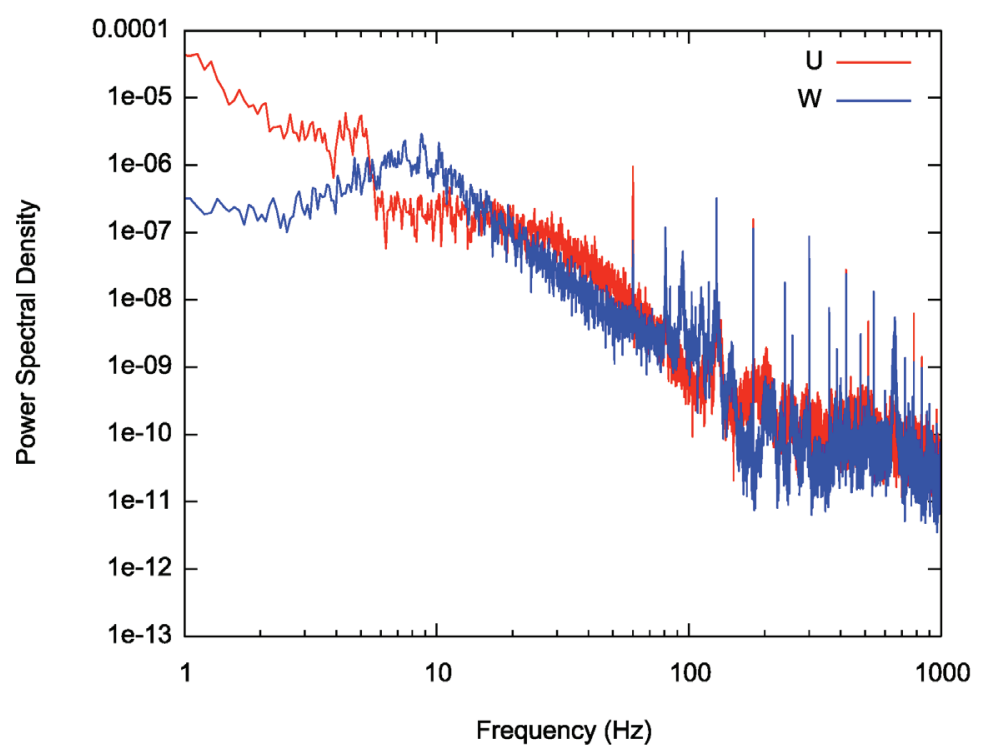

Figure C-72. Spectra from crosswire measurements at $(0.406,0.838,-0.598)$ for $20 \mathrm{~m} / \mathrm{s}$. 


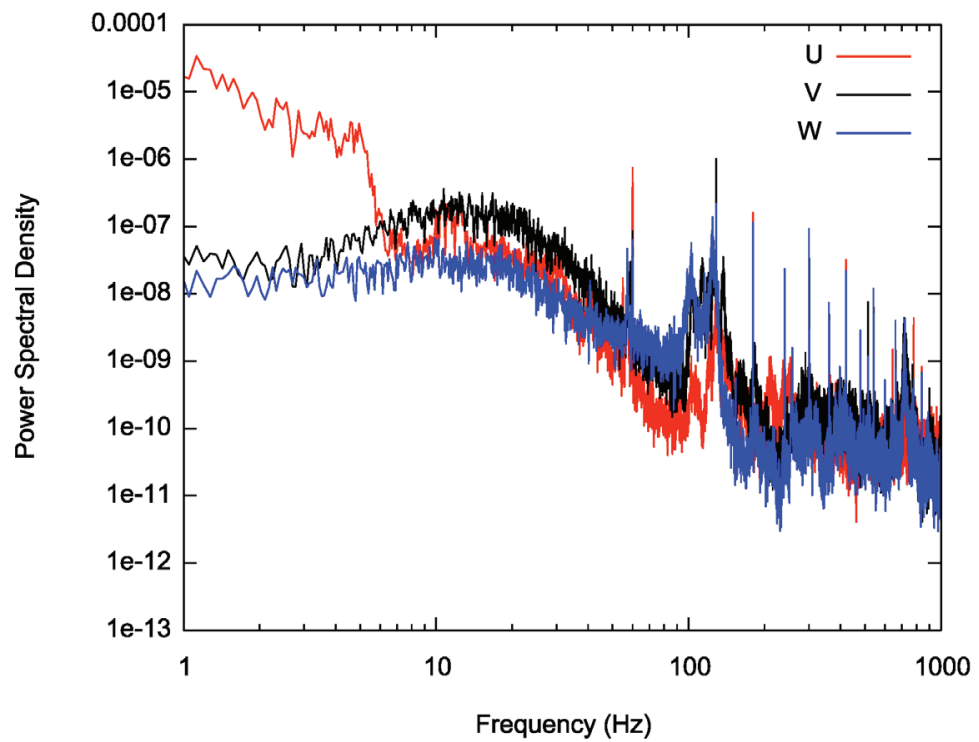

Figure C-73. Spectra from crosswire measurements at $(0.750,0.204,0.602)$ for $20 \mathrm{~m} / \mathrm{s}$.

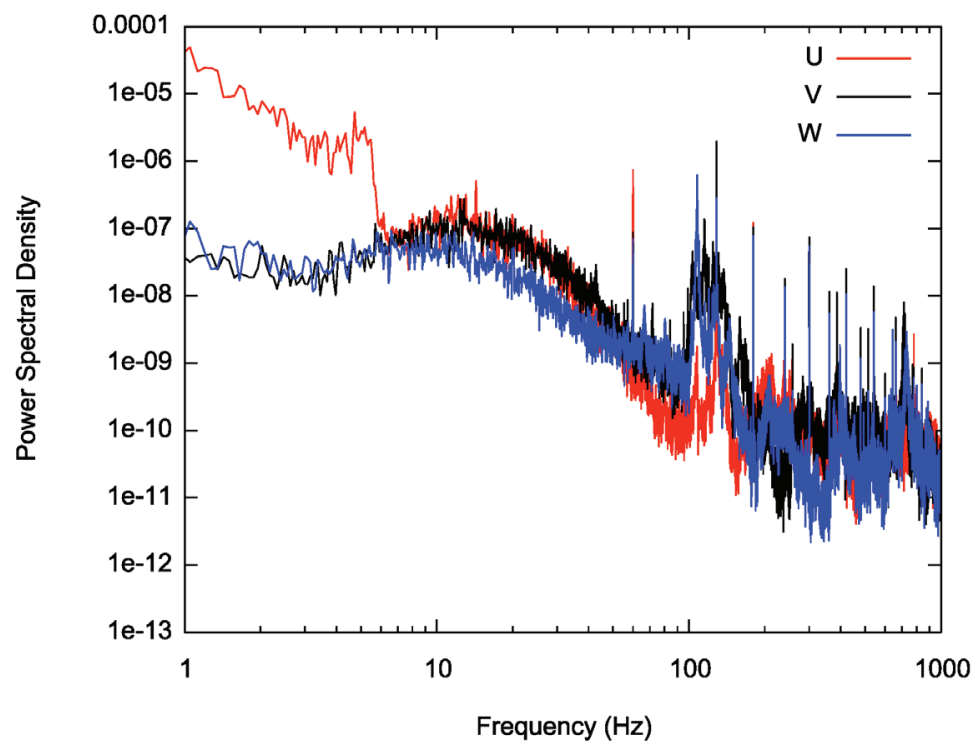

Figure C-74. Spectra from crosswire measurements at $(0.750,0.204,-0.013)$ for $20 \mathrm{~m} / \mathrm{s}$. 


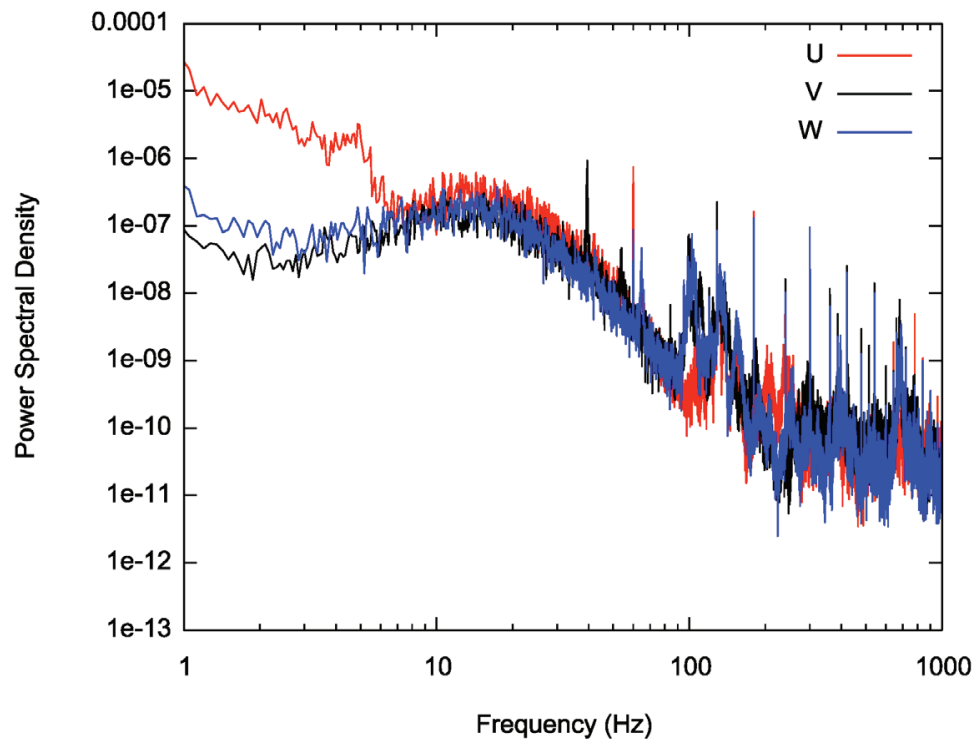

Figure C-75. Spectra from crosswire measurements at $(0.750,0.204,-0.602)$ for $20 \mathrm{~m} / \mathrm{s}$.

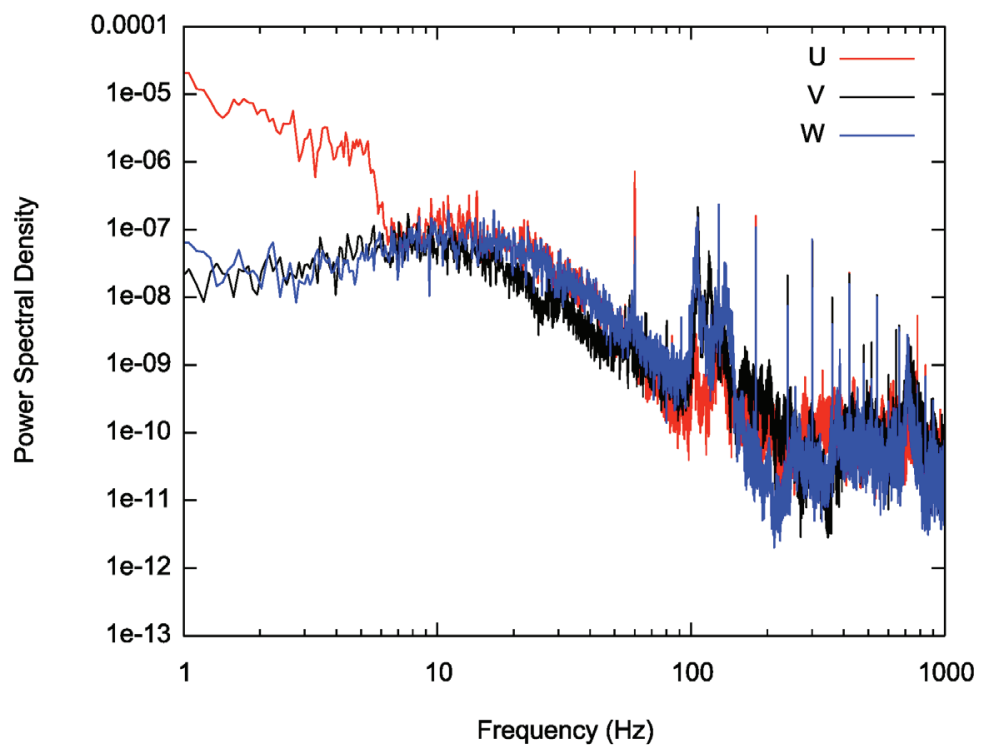

Figure C-76: Spectra from crosswire measurements at $(0.750,0.519,0.602)$ for $20 \mathrm{~m} / \mathrm{s}$. 


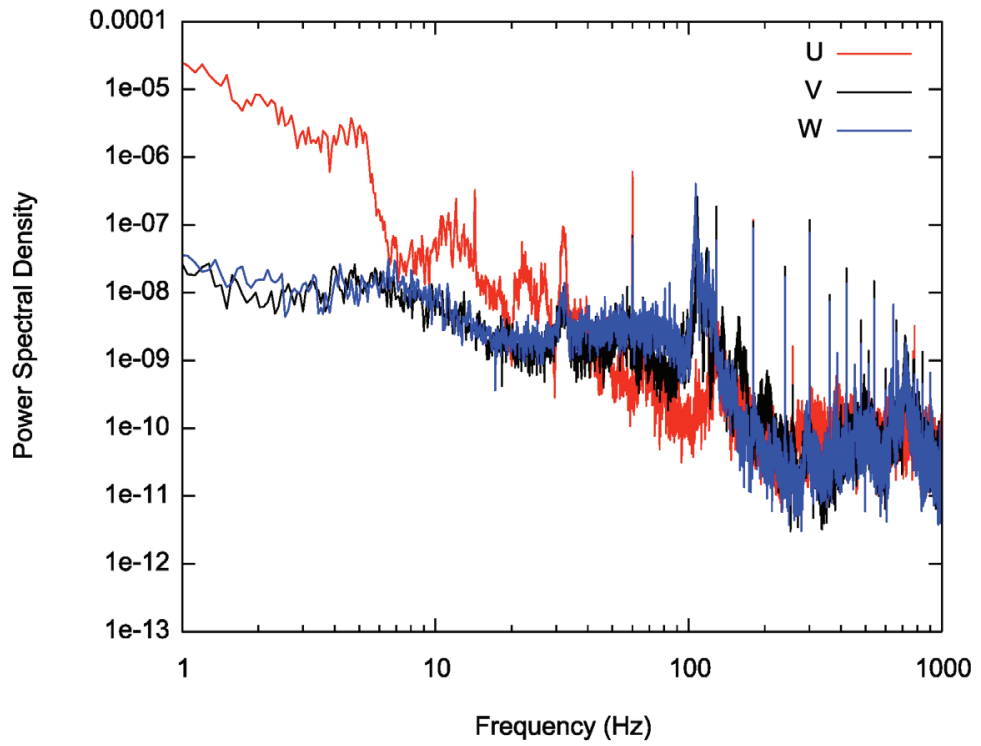

Figure C-77. Spectra from crosswire measurements at $(0.750,0.519,-0.013)$ for $20 \mathrm{~m} / \mathrm{s}$.

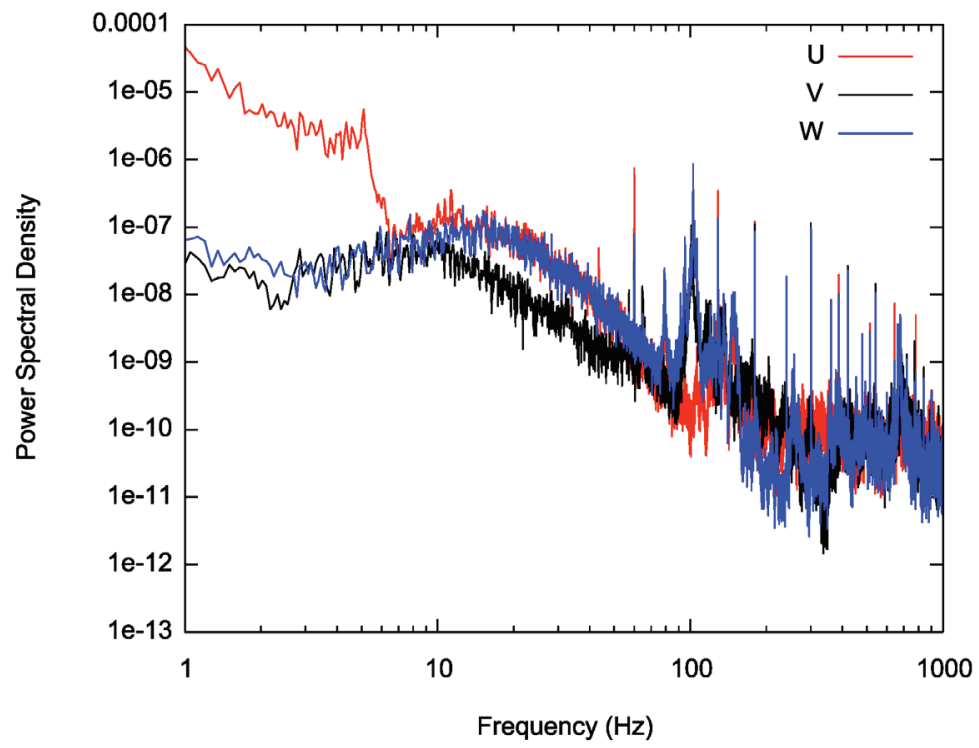

Figure C-78. Spectra from crosswire measurements at $(0.750,0.519,-0.602)$ for $20 \mathrm{~m} / \mathrm{s}$. 


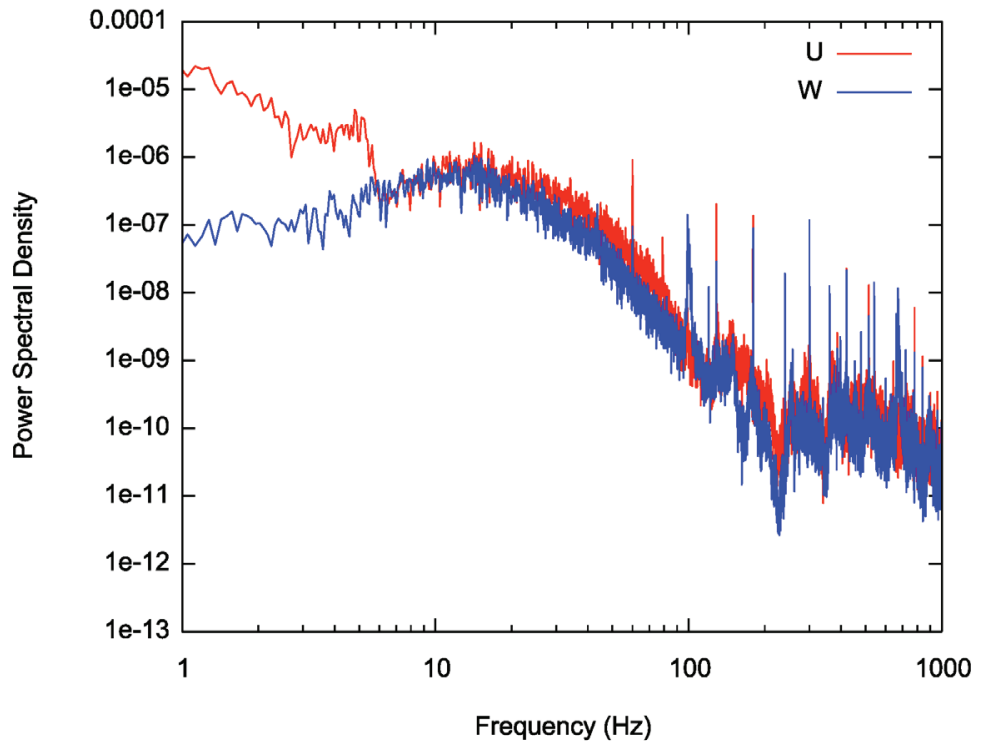

Figure C-79. Spectra from crosswire measurements at $(0.750,0.838,0.602)$ for $20 \mathrm{~m} / \mathrm{s}$.

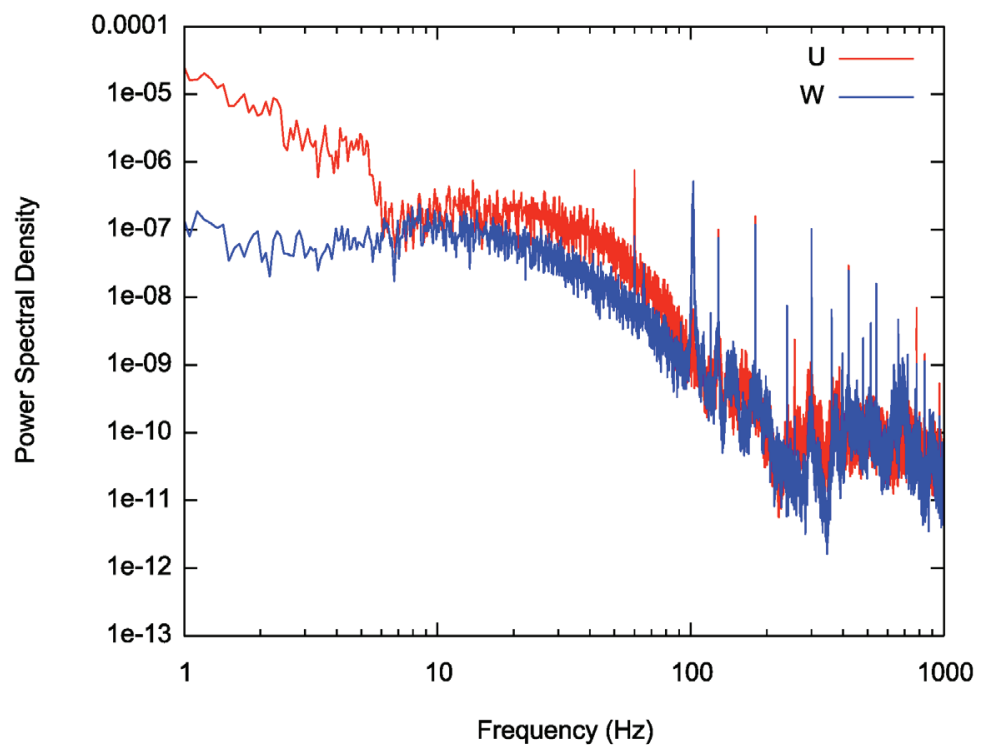

Figure C-80. Spectra from crosswire measurements at $(0.750,0.838,-0.013)$ for $20 \mathrm{~m} / \mathrm{s}$. 


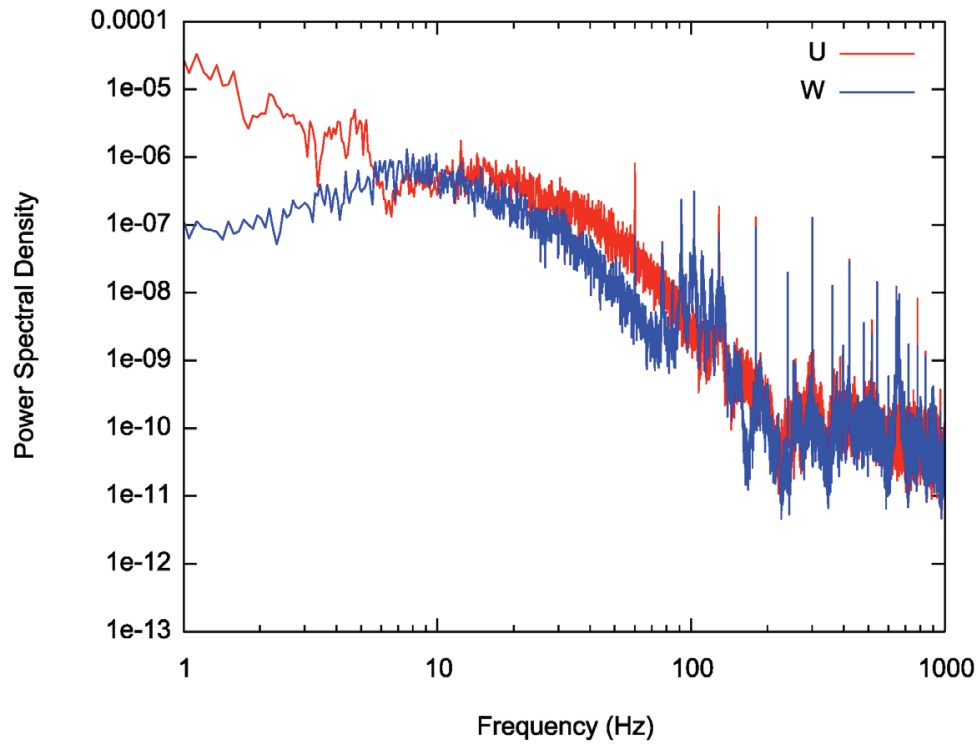

Figure C-81. Spectra from crosswire measurements at $(0.750,0.838,-0.602)$ for $20 \mathrm{~m} / \mathrm{s}$.

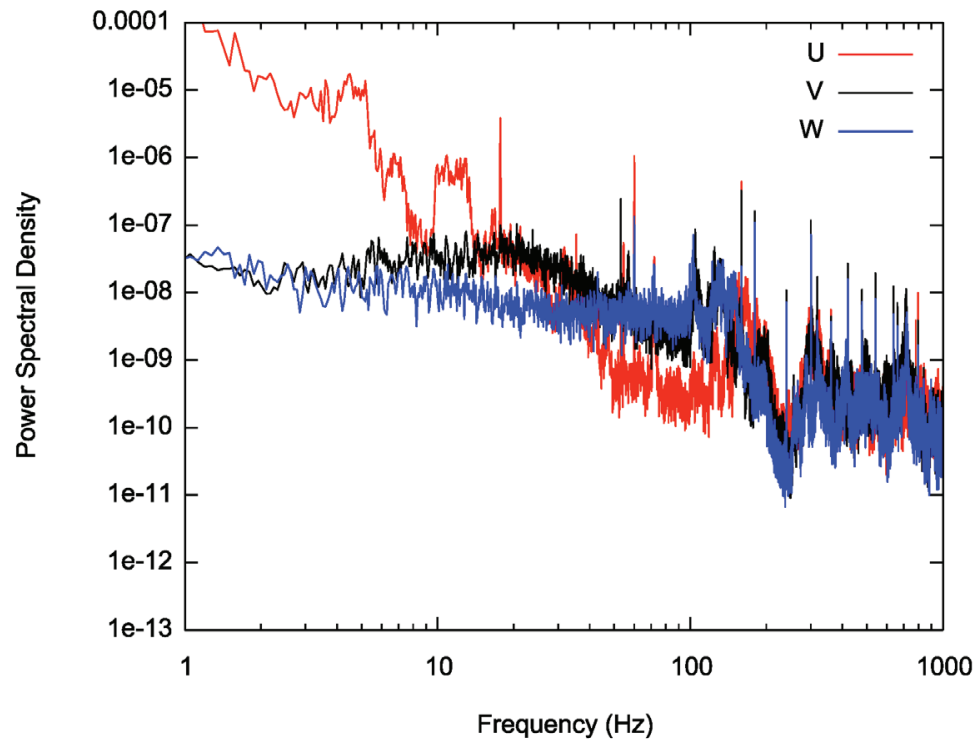

Figure C-82. Spectra from crosswire measurements at $(0.035,0.204,0.593)$ for $25 \mathrm{~m} / \mathrm{s}$. 


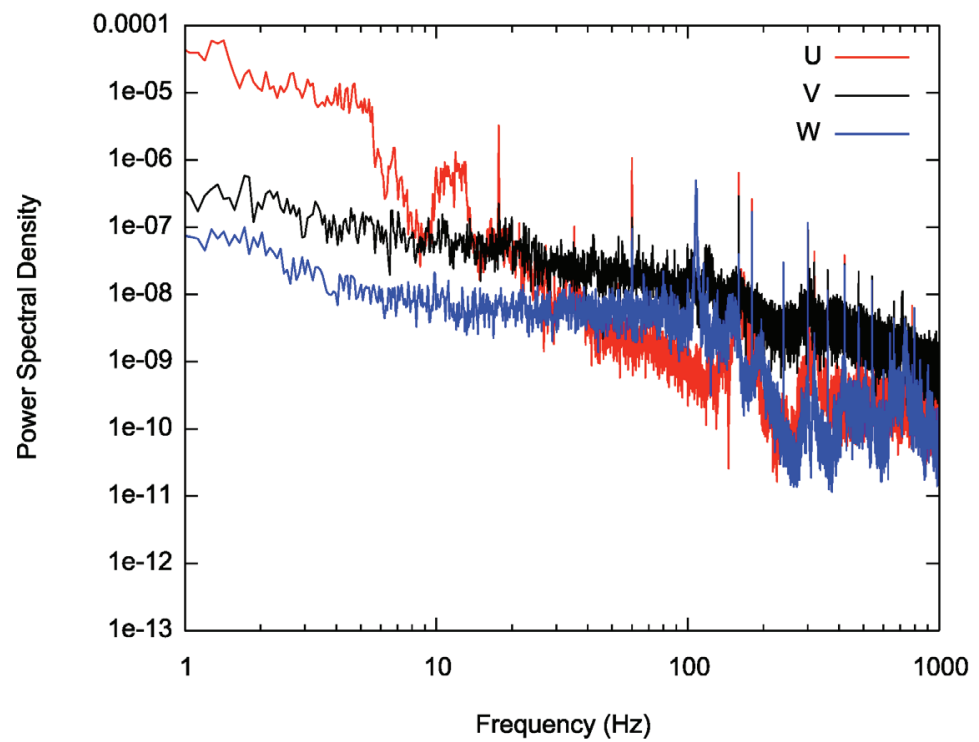

Figure C-83. Spectra from crosswire measurements at $(0.035,0.204,-0.036)$ for $25 \mathrm{~m} / \mathrm{s}$.

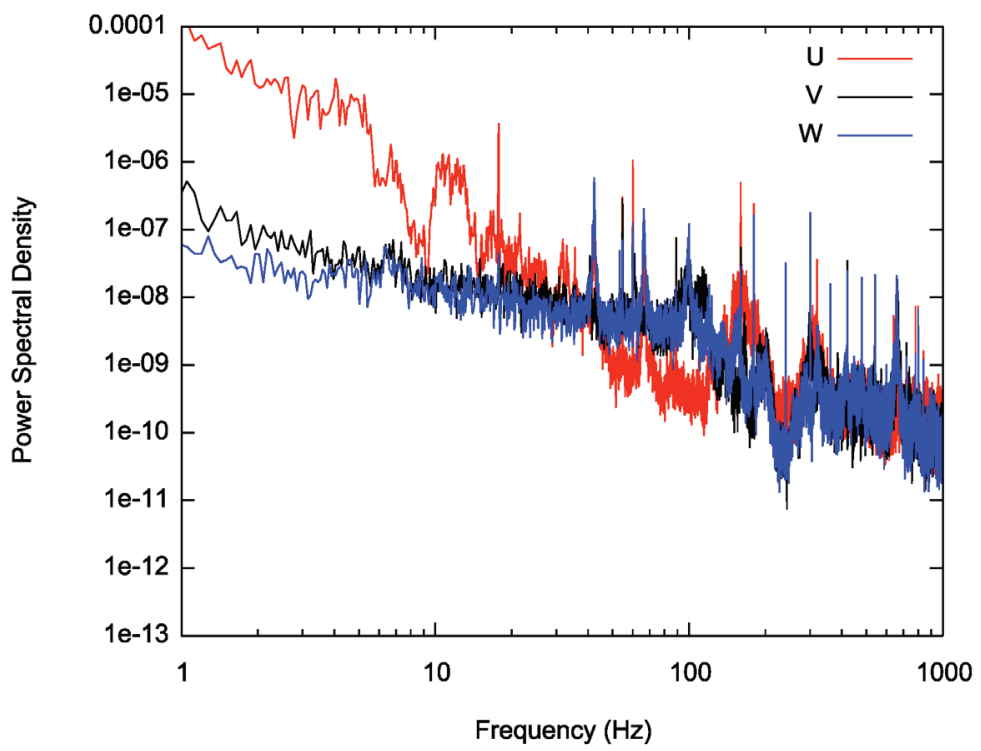

Figure C-84. Spectra from crosswire measurements at $(0.035,0.204,-0.593)$ for $25 \mathrm{~m} / \mathrm{s}$. 


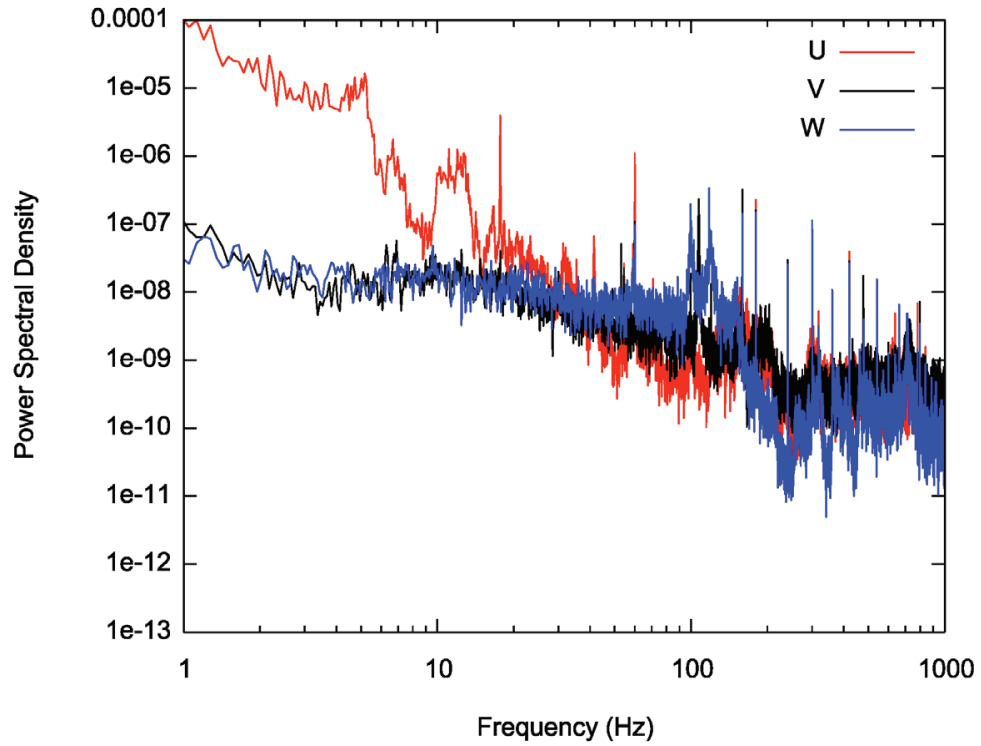

Figure C-85. Spectra from crosswire measurements at $(0.035,0.519,0.593)$ for $25 \mathrm{~m} / \mathrm{s}$.

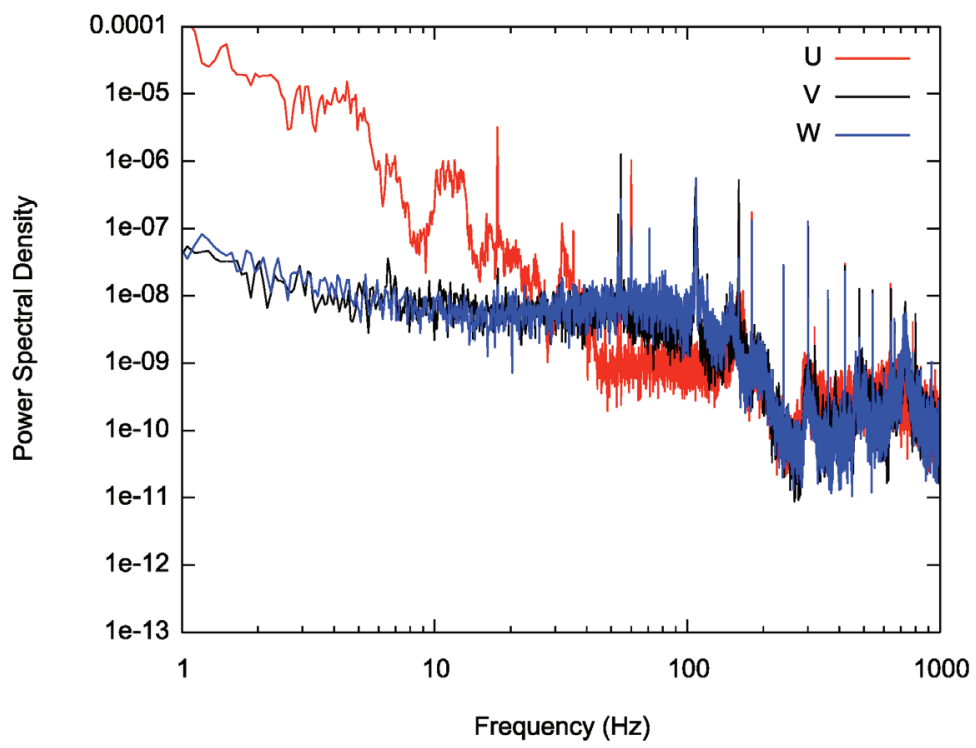

Figure C-86. Spectra from crosswire measurements at $(0.035,0.519,-0.036)$ for $25 \mathrm{~m} / \mathrm{s}$. 


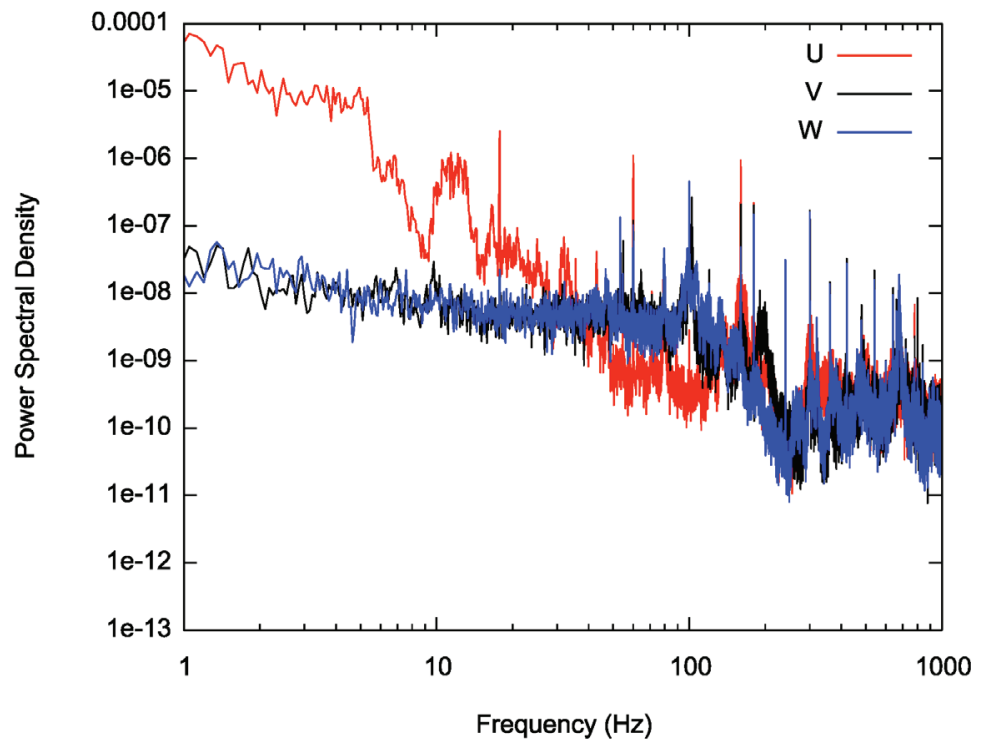

Figure C-87. Spectra from crosswire measurements at $(0.035,0.519,-0.593)$ for $25 \mathrm{~m} / \mathrm{s}$.

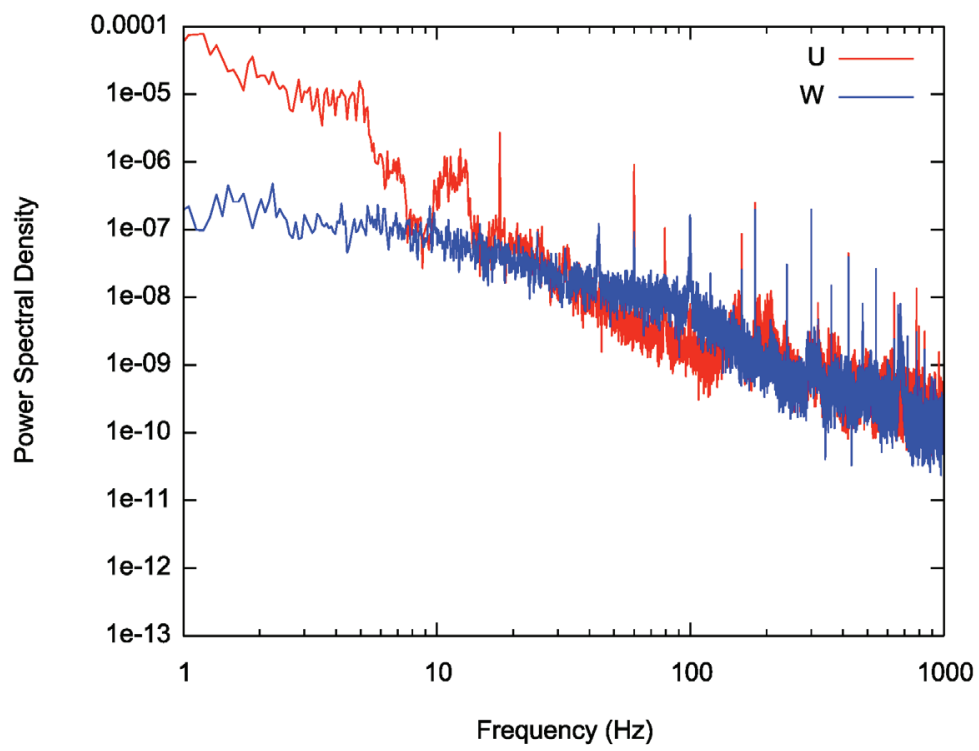

Figure C-88. Spectra from crosswire measurements at $(0.035,0.838,0.593)$ for $25 \mathrm{~m} / \mathrm{s}$. 


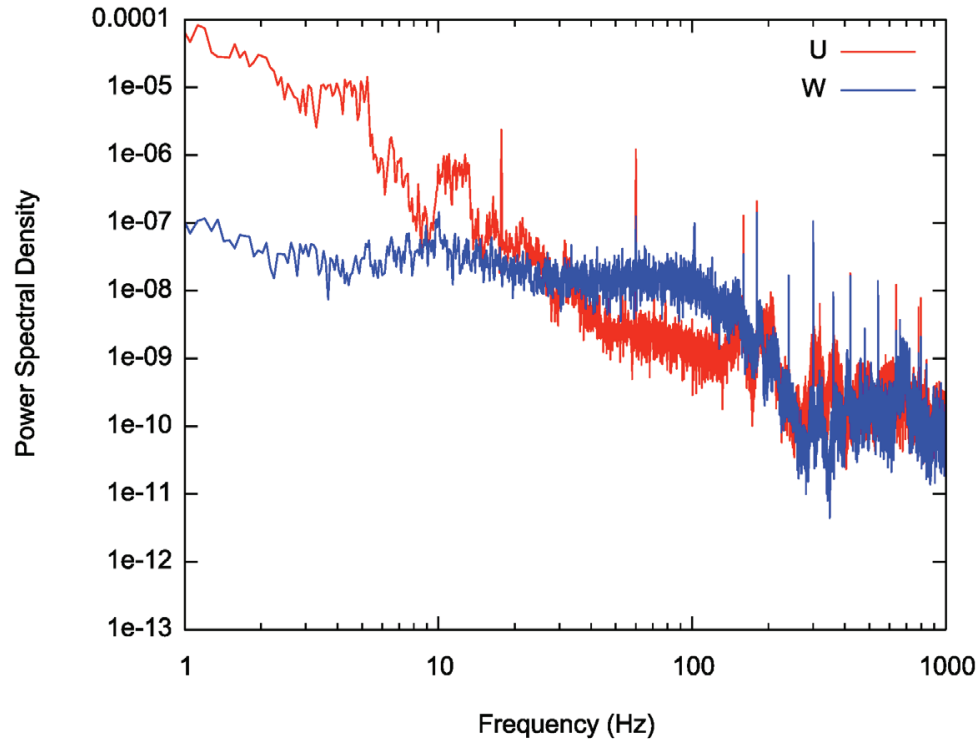

Figure C-89. Spectra from crosswire measurements at $(0.035,0.838,-0.036)$ for $25 \mathrm{~m} / \mathrm{s}$.

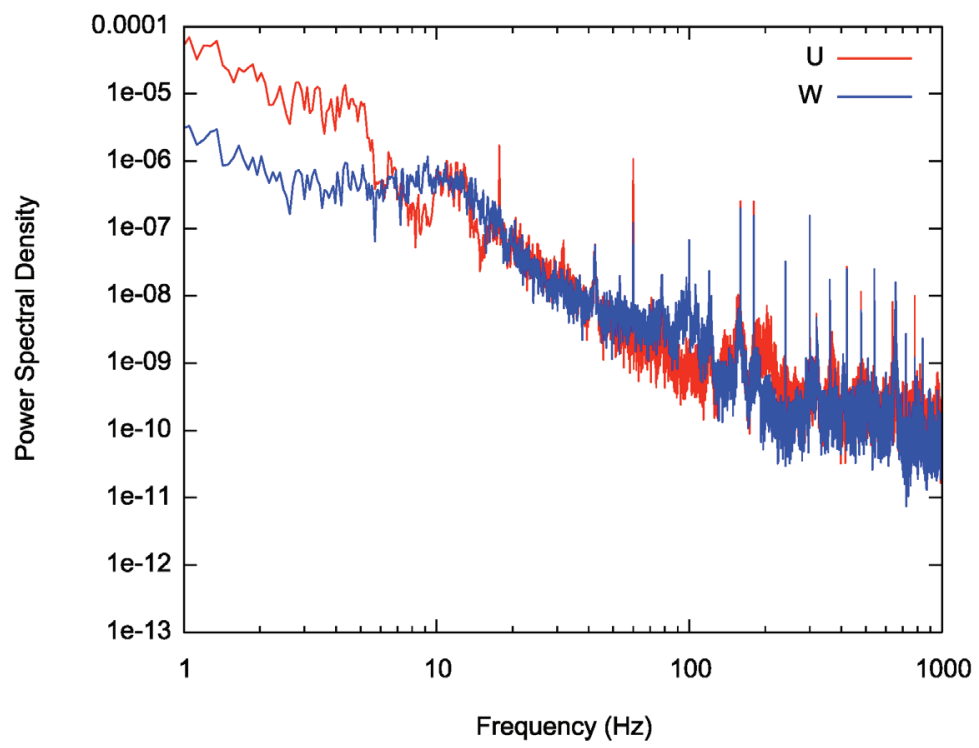

Figure C-90. Spectra from crosswire measurements at $(0.035,0.838,-0.593)$ for $25 \mathrm{~m} / \mathrm{s}$. 


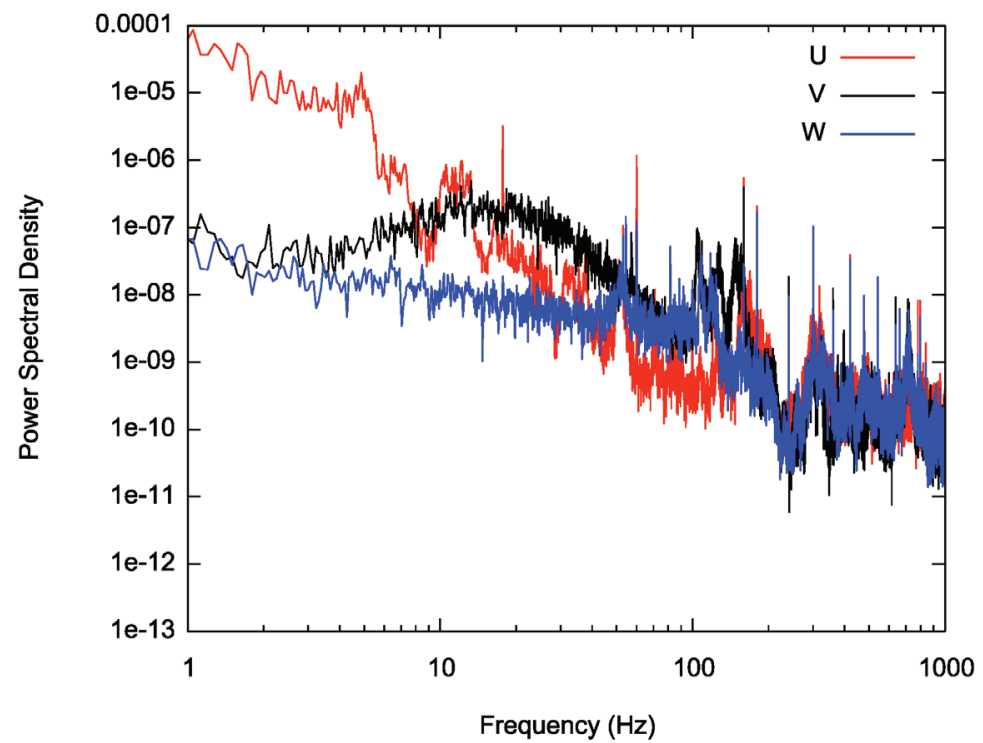

Figure C-91. Spectra from crosswire measurements at $(0.406,0.204,0.598)$ for $25 \mathrm{~m} / \mathrm{s}$.

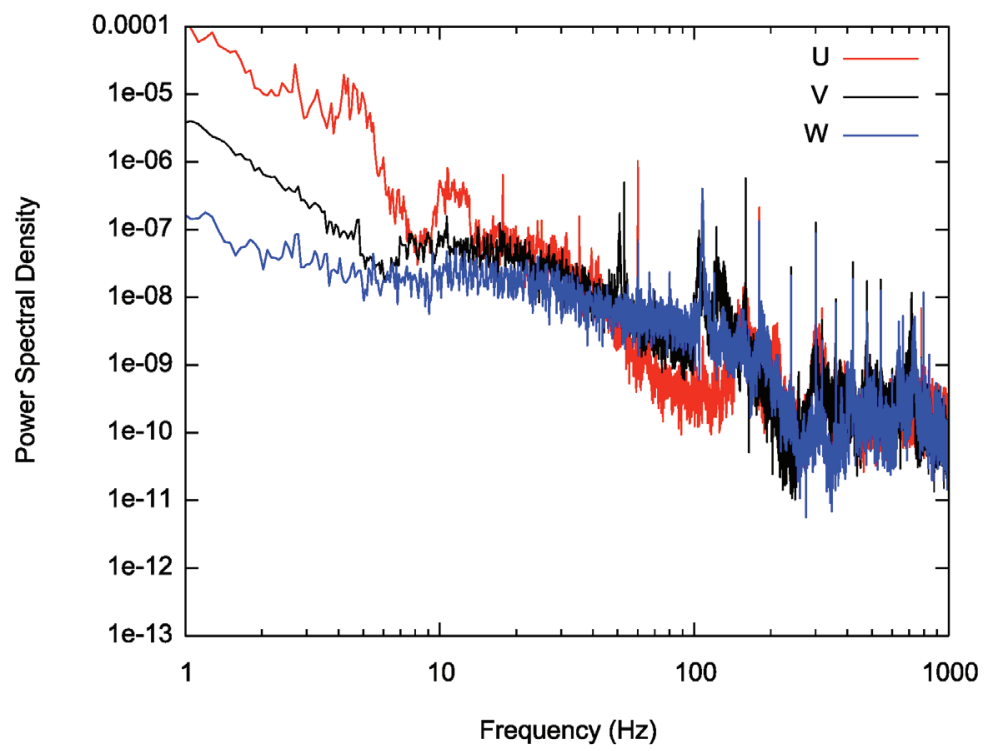

Figure C-92. Spectra from crosswire measurements at $(0.406,0.204,-0.024)$ for $25 \mathrm{~m} / \mathrm{s}$. 


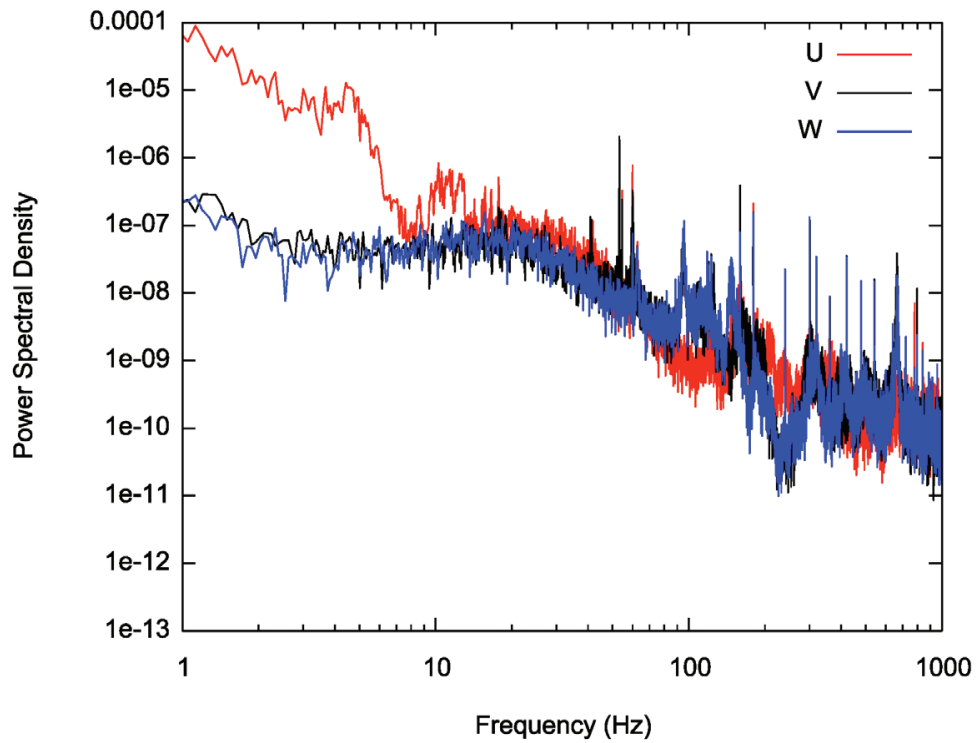

Figure C-93. Spectra from crosswire measurements at $(0.406,0.204,-0.598)$ for $25 \mathrm{~m} / \mathrm{s}$.

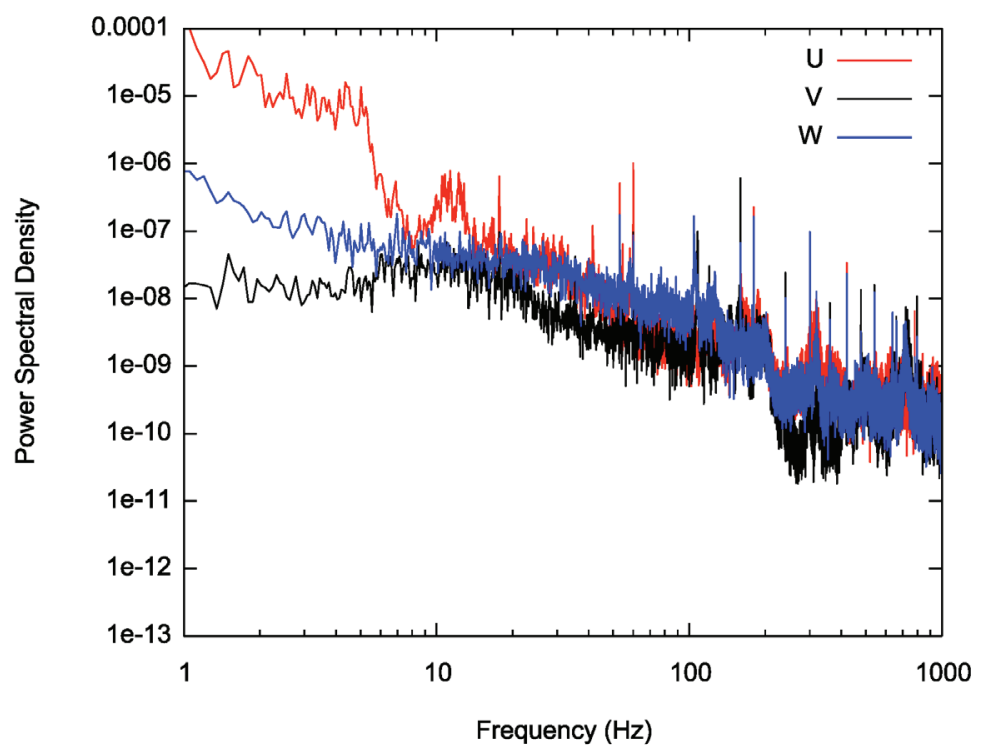

Figure C-94. Spectra from crosswire measurements at $(0.406,0.519,0.598)$ for $25 \mathrm{~m} / \mathrm{s}$. 


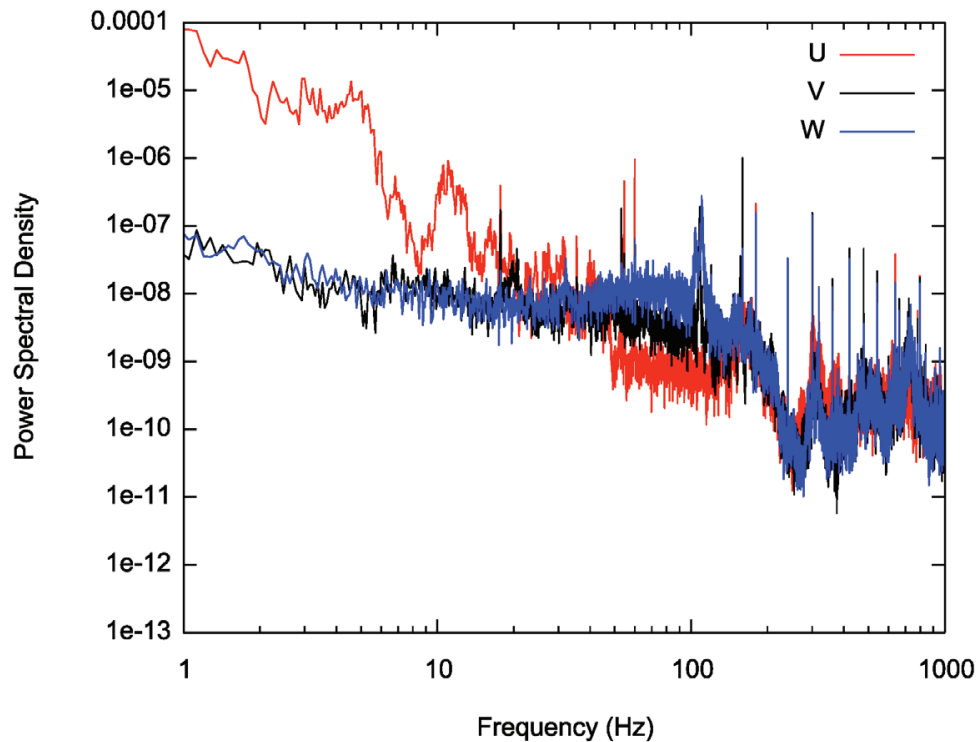

Figure C-95. Spectra from crosswire measurements at $(0.406,0.519,-0.024)$ for $25 \mathrm{~m} / \mathrm{s}$.

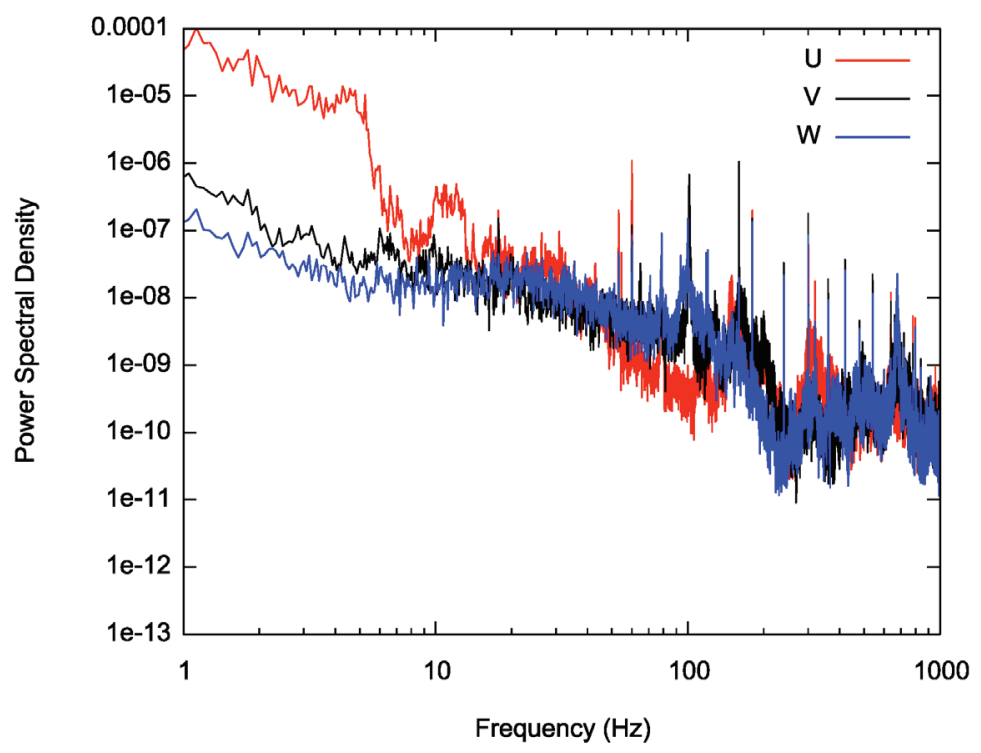

Figure C-96. Spectra from crosswire measurements at $(0.406,0.519,-0.598)$ for $25 \mathrm{~m} / \mathrm{s}$. 


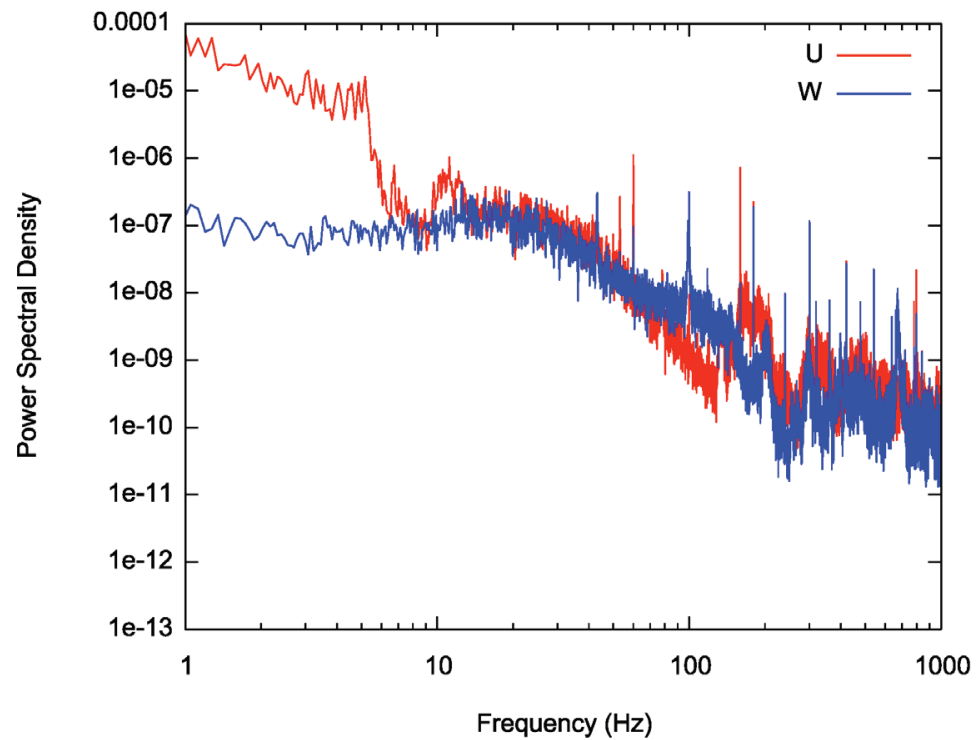

Figure C-97. Spectra from crosswire measurements at $(0.406,0.838,0.598)$ for $25 \mathrm{~m} / \mathrm{s}$.

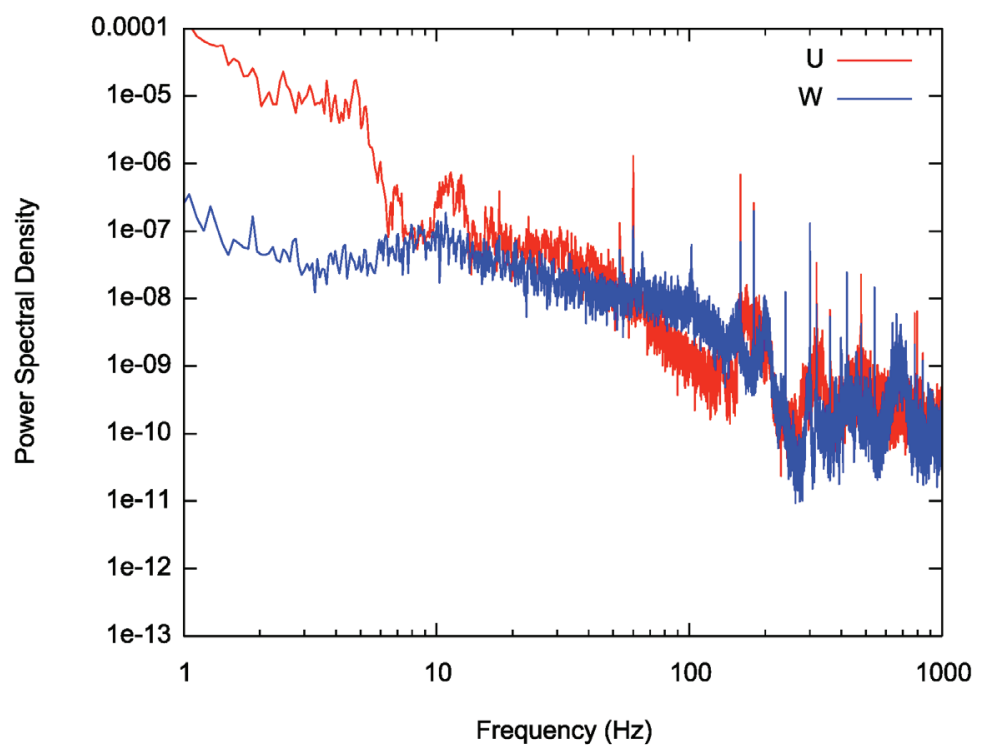

Figure C-98. Spectra from crosswire measurements at $(0.406,0.838,-0.024)$ for $25 \mathrm{~m} / \mathrm{s}$. 


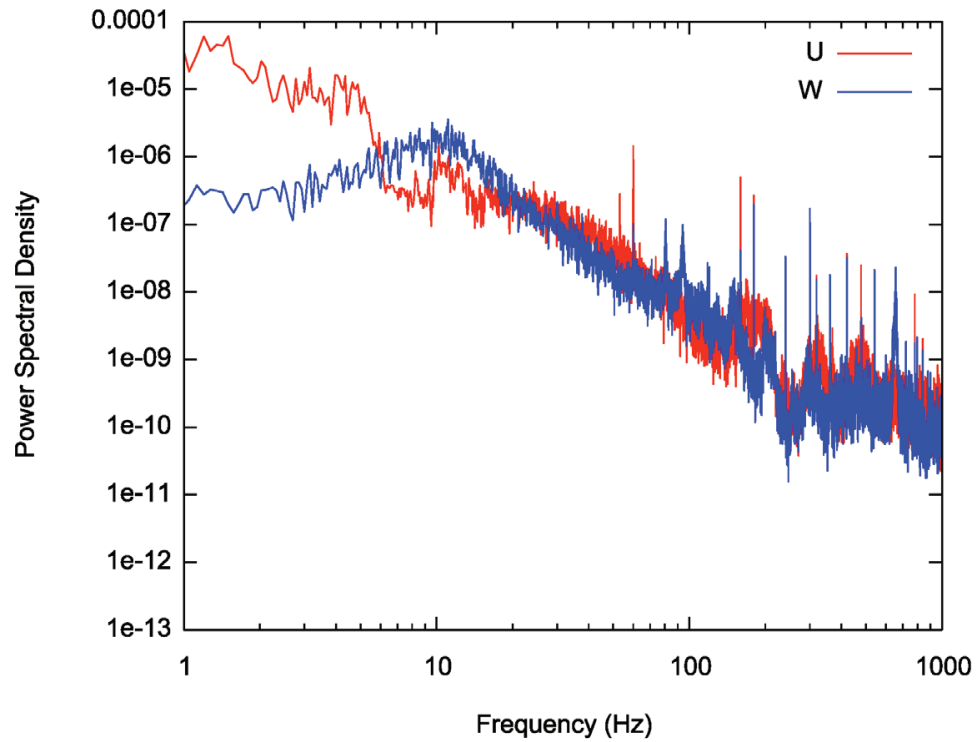

Figure C-99. Spectra from crosswire measurements at $(0.406,0.838,-0.598)$ for $25 \mathrm{~m} / \mathrm{s}$.

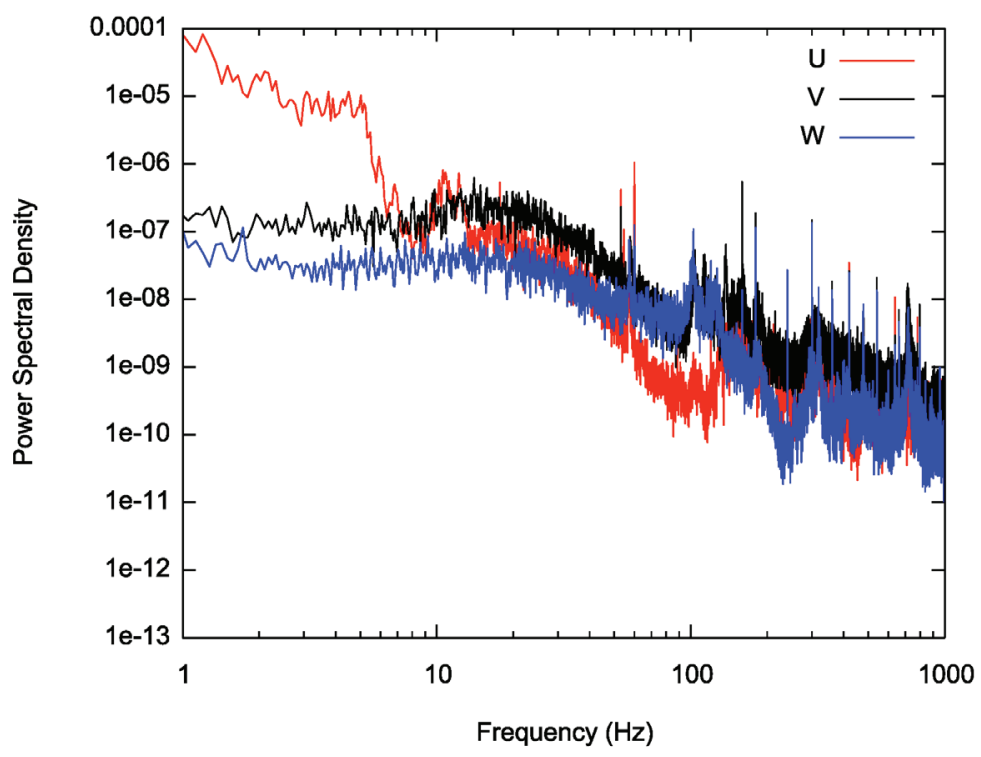

Figure C-100. Spectra from crosswire measurements at $(0.750,0.204,0.602)$ for $25 \mathrm{~m} / \mathrm{s}$. 


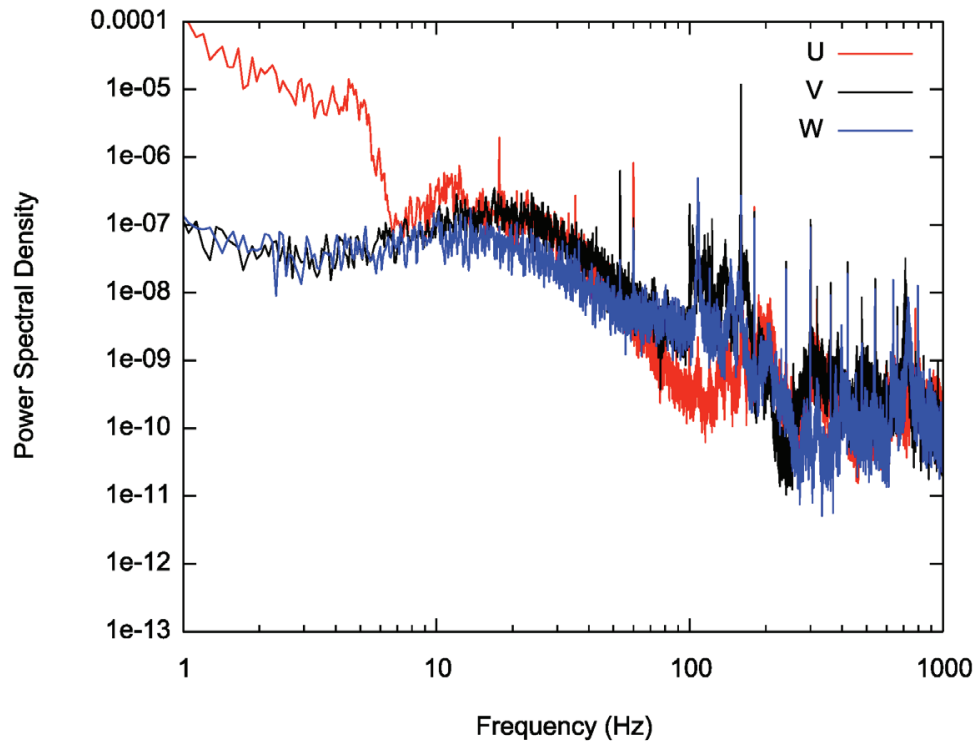

Figure C-101. Spectra from crosswire measurements at $(0.750,0.204,-0.013)$ for $25 \mathrm{~m} / \mathrm{s}$.

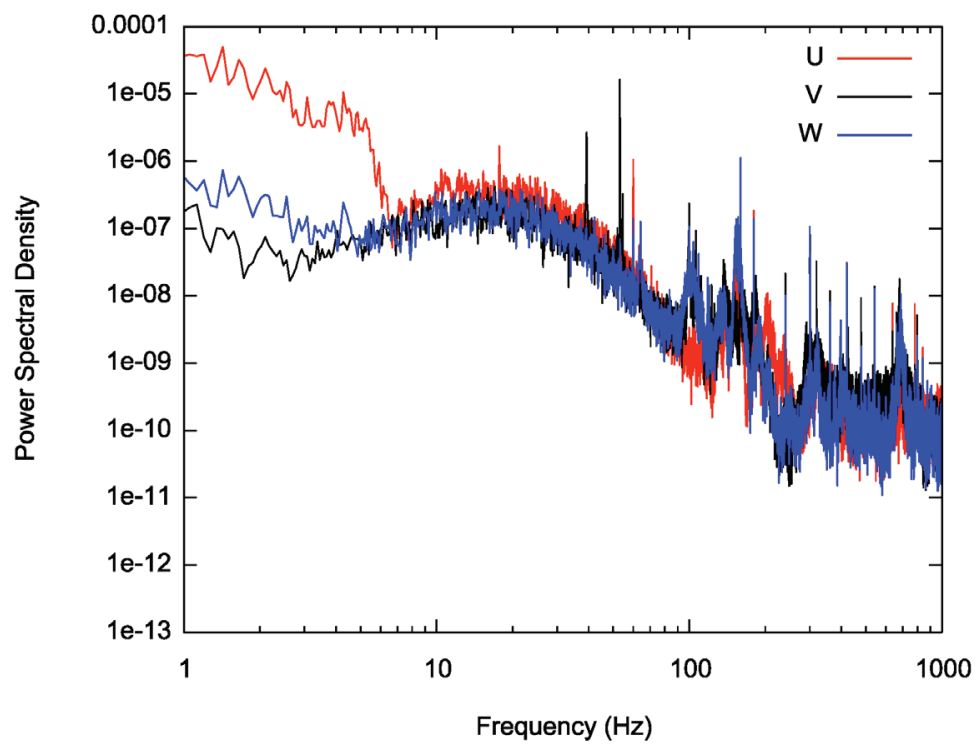

Figure C-102: Spectra from crosswire measurements at $(0.750,0.204,-0.602)$ for $25 \mathrm{~m} / \mathrm{s}$. 


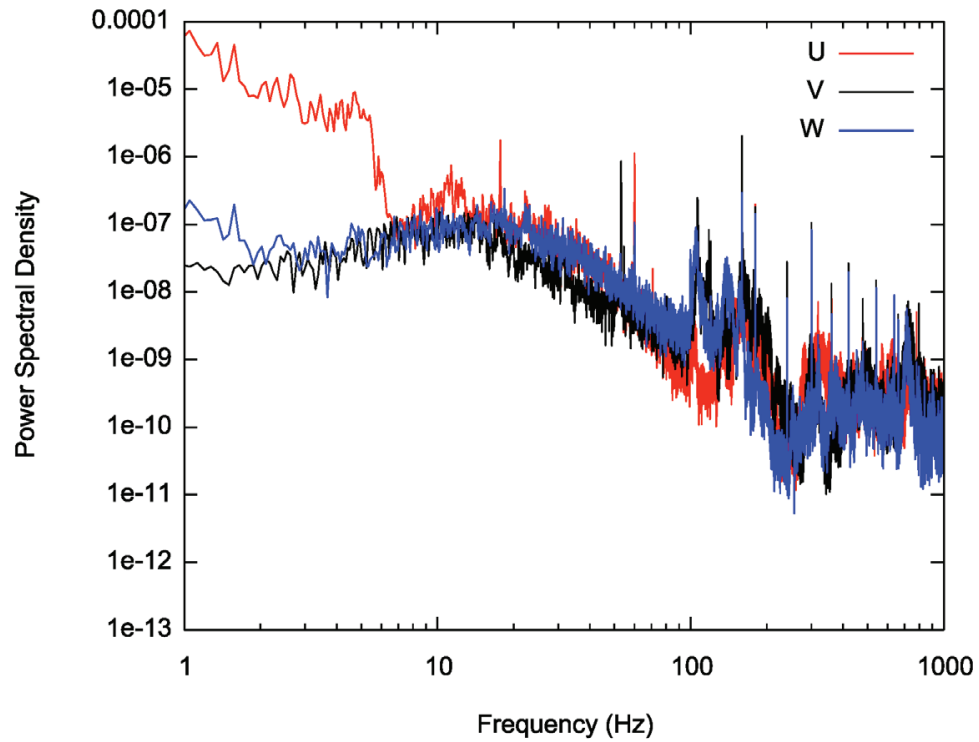

Figure C-103. Spectra from crosswire measurements at $(0.750,0.519,0.602)$ for $25 \mathrm{~m} / \mathrm{s}$.

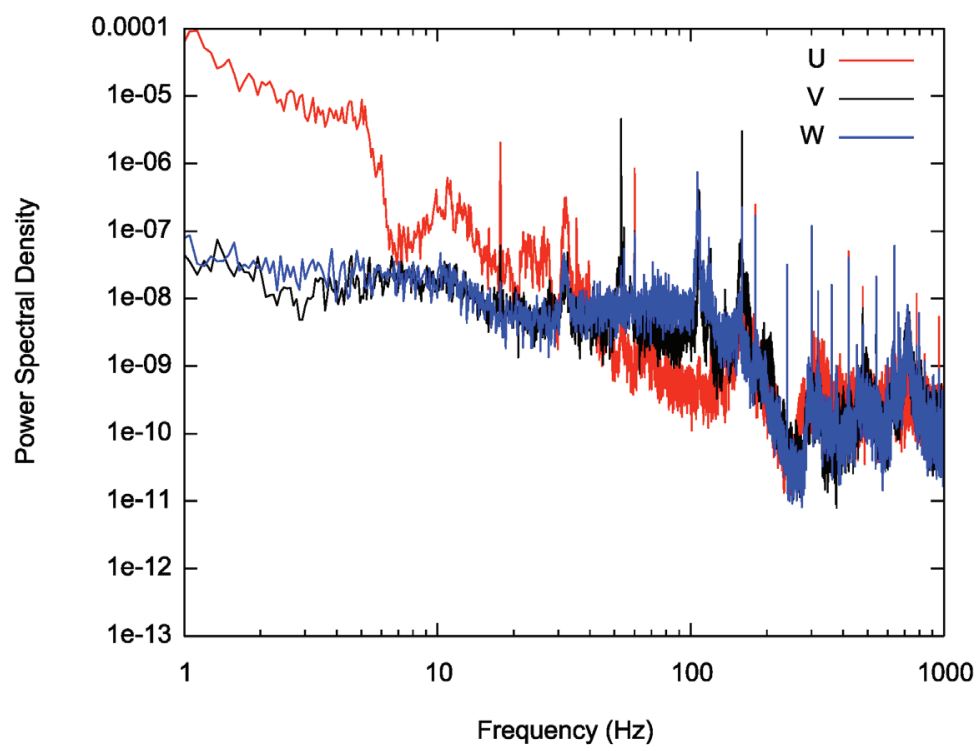

Figure C-104. Spectra from crosswire measurements at $(0.750,0.519,-0.013)$ for $25 \mathrm{~m} / \mathrm{s}$. 


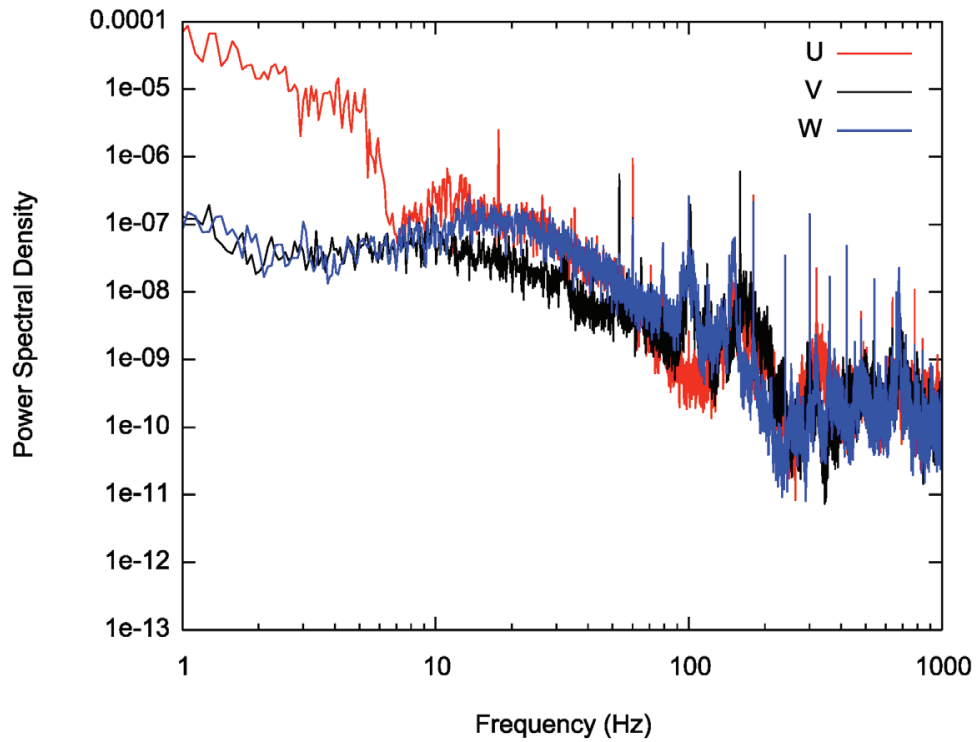

Figure C-105. Spectra from crosswire measurements at $(0.750,0.519,-0.602)$ for $25 \mathrm{~m} / \mathrm{s}$.

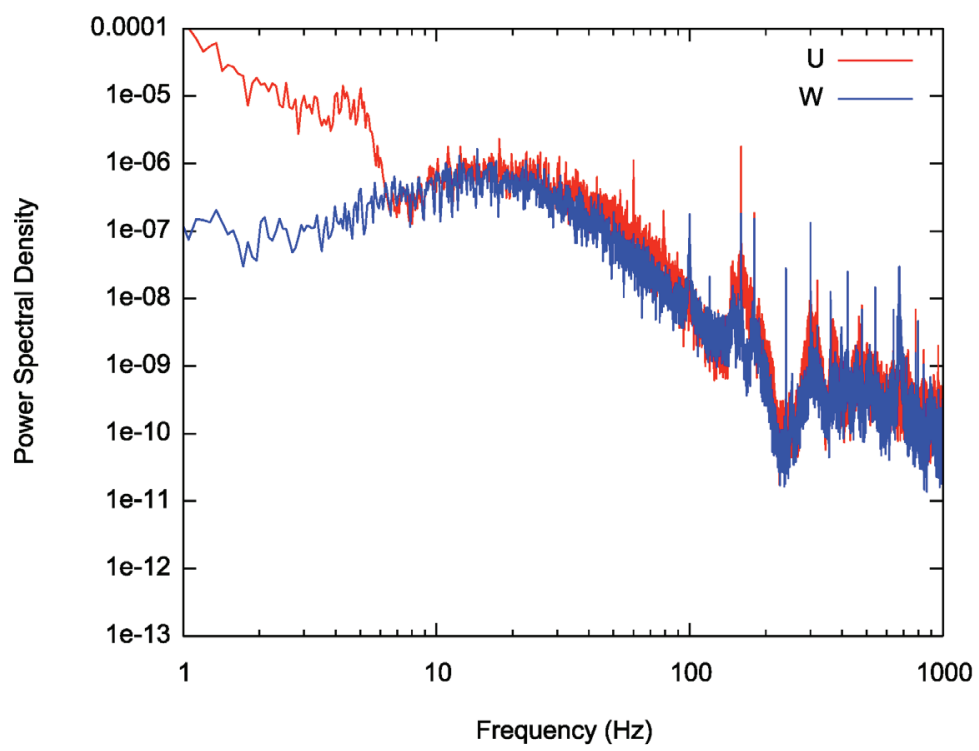

Figure C-106. Spectra from crosswire measurements at $(0.750,0.838,0.602)$ for $25 \mathrm{~m} / \mathrm{s}$. 


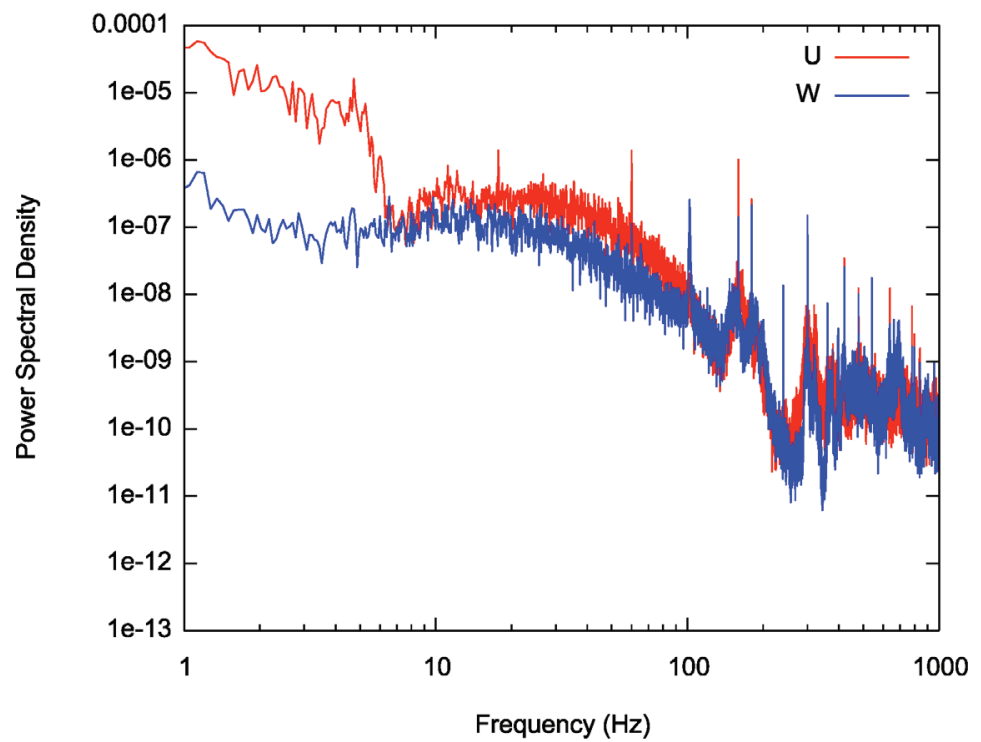

Figure C-107. Spectra from crosswire measurements at $(0.750,0.838,-0.013)$ for $25 \mathrm{~m} / \mathrm{s}$.

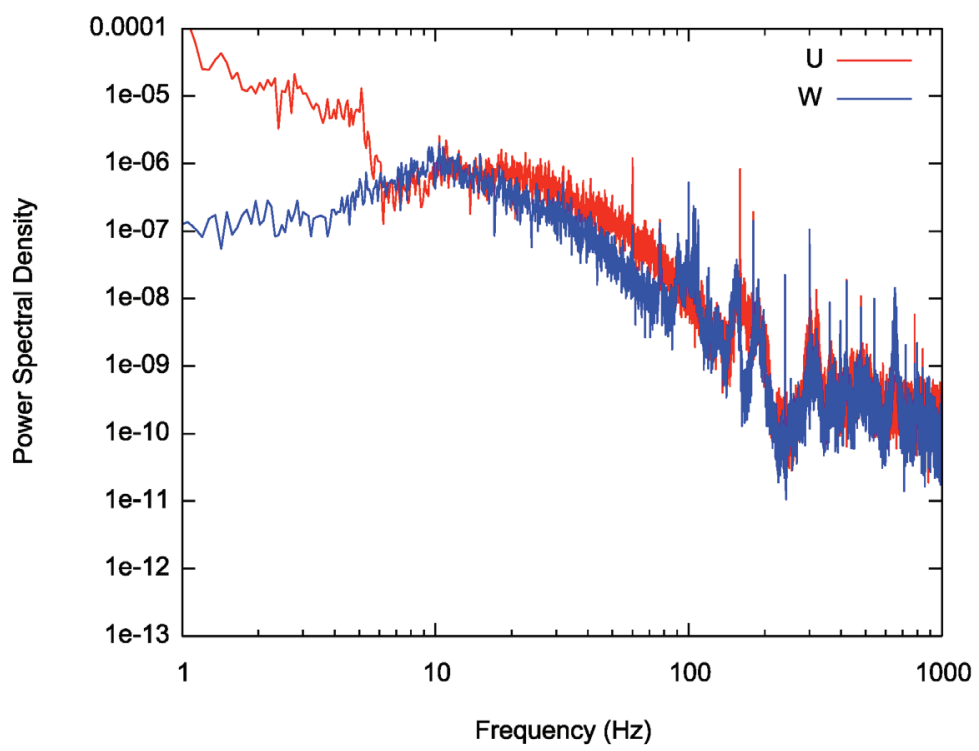

Figure C-108. Spectra from crosswire measurements at $(0.750,0.838,-0.602)$ for $25 \mathrm{~m} / \mathrm{s}$. 


\section{VITA}

Name: $\quad$ Lauren Elizabeth Hunt

Address: $\quad$ Texas A\&M University, Department of Aerospace Engineering H.R. Bright Building, Rm. 701, TAMU 3141

College Station, TX 77843-3141

Email Address: lauren_hunt314@yahoo.com

Education: $\quad$ B.S., Chemical Engineering, Arizona State University, 2004

Ph.D., Aerospace Engineering, Texas A\&M University, 2011 